\title{
15 Jahre Panelstudie zur (Medien-)Sozialisation
}

Wie leben die Kinder von damals heute als junge Erwachsene? 
Lebensweltbezogene Medienforschung:

Angebote-Rezeption - Sozialisation

Herausgegeben von

Ingrid Paus-Hasebrink

Sascha Trültzsch-Wijnen

Uwe Hasebrink

Band 10 
Ingrid Paus-Hasebrink | Philip Sinner

15 Jahre Panelstudie zur

(Medien-)Sozialisation

Wie leben die Kinder von damals

heute als junge Erwachsene?

Nomos 
() Titelbild: Guitafotostudio - stock.adobe.com

Die Deutsche Nationalbibliothek verzeichnet diese Publikation in der Deutschen Nationalbibliografie; detaillierte bibliografische Daten sind im Internet über http://dnb.d-nb.de abrufbar.

\section{Auflage 2021 \\ (c) Die Autoren \\ Publiziert von}

Nomos Verlagsgesellschaft $\mathrm{mbH} \&$ Co. KG

Waldseestraße 3-5 | 76530 Baden-Baden

www.nomos.de

Gesamtherstellung:

Nomos Verlagsgesellschaft $\mathrm{mbH} \& \mathrm{Co}$. KG

Waldseestraße 3-5 | 76530 Baden-Baden

ISBN (Print): 978-3-8487-8382-3

ISBN (ePDF): 978-3-7489-2772-3

DOI: https://doi.org/10.5771/9783748927723

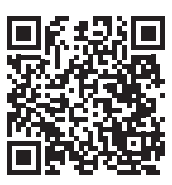

Onlineversion Nomos elibrary

\section{(C) $(\Theta \Theta \Theta$}

Dieses Werk ist lizenziert unter einer Creative Commons Namensnennung

- Nicht kommerziell - Keine Bearbeitungen 4.0 International Lizenz. 
Gewidmet unseren Familien 


\section{Vorwort der Herausgeberin und der Herausgeber zu Band 10 der Reihe „Lebensweltbezogene Medienforschung: Angebote, Rezeption, Sozialisation“}

Der vorliegende Band knüpft an die Bände 2 und 5 der Reihe Lebensweltbezogene Medienforschung mit den Titeln „Praxeologische Mediensozialisationsforschung. Fallbeispiel sozial benachteiligte Heranwachsende“ (2014) sowie „Langzeitstudie zur Rolle von Medien in der Sozialisation sozial benachteiligter Heranwachsender. Lebensphase Jugend“ (2017) an. Erneut steht damit der im Kontext der Reihe wichtige Begriff der Sozialisation im Mittelpunkt, beleuchtet vor dem Hintergrund medialer Wandlungsprozesse. Medialer Wandel ist tief in die Prozesse des gesellschaftlichen Wandels integriert und bleibt damit nicht ohne Konsequenzen für die Lebensführung von Menschen, mithin auch für Sozialisationsprozesse. Die Sozialisation eines Menschen ist ein lebenslanger Prozess, der sich in verschiedenen sozialen Zusammenhängen vollzieht, an denen das Individuum beteiligt ist und die sein Spielfeld zum Aufbau von Identität und Handlungskompetenz im Alltag bestimmen. Leben heute heißt Leben mit Medien; so vollzieht sich die lebenslange Sozialisation eines Individuums auch als mediatisierte Sozialisation, denn Medien sind Teil unserer Alltagspraxis, sie konstituieren den Alltag mit und bringen neue Praktiken hervor. (Medien)Sozialisationsforschung bedarf, wie auch der neue Band der Langzeitstudie mit dem Titel „15 Jahre Panelstudie zur (Medien-)Sozialisation. Wie leben die Kinder von damals heute als junge Erwachsene?“ deutlich zeigt, des Blicks auf die Lebensbedingungen von Individuen und der ihnen nahestehenden Personen. Denn Sozialisationsprozesse finden stets im Kontext ihrer Lebensführung und Alltagspraktiken an ihrem je spezifischen sozialen Ort statt. Vor diesem Hintergrund zielt der zehnte Band der Reihe darauf, die von sozialen und, darin eingelagert, medialen Wandlungsprozessen induzierten Bedingungen der Sozialisation der zu Beginn der Studie 2005 fünf Jahre alten jungen Menschen nunmehr als junge Erwachsene zu rekonstruieren: Was ist aus den Kindern der (Medien-)Sozialisationsstudie in sozial benachteiligten Familien geworden, und wie haben sich ihre sozio-ökomischen und sozio-emotionalen Bedingungen entwickelt? Welche Rolle weisen sie nun als junge Erwachsene Medien in ihrem Alltag zu? Diesen Fragen geht das Buch auf Basis einer siebten, im Jahr 2020 durchgeführten, Erhebungswelle nach; es beleuchtet 
die Veränderungen, die sich im Leben der jungen Menschen sowohl privat als auch beruflich vollzogen haben und macht - mit Blick auf ihr gesamtes bisheriges Leben - deutlich, wie sich ihr Mediengebrauch von der frühen Kindheit bis heute im jungen Erwachsenenalter verändert hat und welche Bedeutung sie nunmehr medialen Angeboten zuweisen. Theoretisch und methodisch baut der Band auf dem von Paus-Hasebrink konzipierten und im Laufe der Studie weiterentwickelten praxeologisch ausgerichteten Ansatz integrativer Mediensozialisationsforschung auf, in dessen Mittelpunkt die Frage nach dem subjektiven Sinn des (Medien-)Handelns von Individuen vor dem Hintergrund ihrer lebensweltlichen Kontexte steht.

Ingrid Paus-Hasebrink, Sascha Trültzsch-Wijnen, Uwe Hasebrink 


\section{Inhaltsverzeichnis}

Vorwort und Einführung zur Fortsetzung der

(Medien-)Sozialisationsstudie

1 Theoretische und methodische Basis

1.1 Praxeologische (Medien-)Sozialisationsforschung als Schlüssel zur Untersuchung des jungen Erwachsenalters

1.2 Sozialisation - ein lebenslanger Prozess

1.3 Lebensführung, sozialer Ort und feine Unterschiede in der Lebensbewältigung - drei analytische Konzepte zur Rekonstruktion (kommunikativer) Praktiken

1.4 Methodenrepertoire der Panel-Langzeitstudie

1.4.1 Das Methodendesign der ersten sechs Erhebungsphasen

1.4.2 Das Erhebungsrepertoire in der siebten Erhebungsphase - Leitfadeninterviews über Telefon, WhatsApp-Call oder Skype

1.4.3 Auswertungsmethoden der Panel-Langzeitstudie $\quad 37$

1.4.4 Zur Typenbildung $\quad 40$

2 Die Fallbeschreibungen - die jungen Menschen der PanelLangzeitstudie auf dem Weg zum Erwachsenwerden und wie sie heute leben

2.1 Kontextuelle Analyse und Familientypen

2.2 Die jungen Erwachsenen von Typ 1: Massive sozioökonomische Probleme als multiple Deprivation: Die rundherum überforderten Familien

2.2.1 Olivia Fein (im Jahr 201615 Jahre alt) und ihre Mutter: Eine vielfältig schwer belastete Familie

2.2.2 Timo Landinger (20 Jahre alt) und seine Familie: Schwierigste sozio-emotionale und sozio-ökonomische Bedingungen belasten den Jungen und hemmen seine Entwicklung 
2.2.3 Manfred Oblinger (21 Jahre alt) und seine Eltern:

Vielfältige sozio-emotionale wie sozio-ökonomische

Belastungen kennzeichnen die Familie, erst spät treten

Verbesserungen ein

2.2.4 Viktoria Öllinger (bald 20 Jahre alt) und ihre Mutter:

Die stark sozio-ökonomisch und sozio-emotional

belastete Familie stabilisiert sich

2.3 Die jungen Erwachsenen von Typ 2: Die sozio-

ökonomisch nicht mehr bzw. wenig belasteten, aber wegen

problematischer sozio-emotionaler Beziehungsstrukturen

überforderten Familien

2.3.1 Mario Hirtner (20 Jahre alt) und seine

Mutter: Große sozio-ökonomische Herausforderungen

nach einer zwischenzeitlichen Verbesserung und

phasenweise sozio-emotionale Schwierigkeiten prägen

das Familienklima

2.3.2 Benedikt Holzner (20 Jahre alt): Ein Junge aus einer sozio-emotional stark belasteten Familie erhält staatliche Unterstützung und geht seinen Weg in die Selbstständigkeit

2.3.3 Isabelle Rohringer (21 Jahre alt) und ihre Mutter: Aufgewachsen in einem vor allem sozio-emotional belasteten Familienklima und nunmehr mit guten Chancen zu mehr Selbständigkeit

2.3.4 Alfons Weiss (19 Jahre alt) und seine Mutter: Sozioökonomischer Aufstieg kennzeichnet die Familie. Die sozio-emotionale Beziehung verschlechtert sich dramatisch und eskaliert

2.4 Die jungen Erwachsenen von Typ 3: Die zwar sozioökonomisch, aber sozio-emotional weniger belasteten, relativ kompetenten Familien

2.4.1 Amelie Aufbauer (20 Jahre alt) und ihre Mutter:

Eine sozio-ökonomisch stark belastete Familie, die sich sozio-emotional mit den Jahren stabilisierte

2.4.2 Gregor Boll (21 Jahre alt) und seine Mutter: Sozioemotionale Verwerfungen und die Trennung der Eltern führen zu einem erfolgreichen Neustart in Deutschland 
2.4.3 Elisabeth Ebner (19 Jahre alt) und ihre Eltern: Eine trotz erheblich eingeschränkter Handlungsoptionen emotional stabile, den Alltag recht kompetent bewältigende Familie

2.4.4 Torsten Kaiser (20 Jahre alt) und seine Mutter: Sozio-emotionale Stabilität in der Familie kann zum Teil herausfordernde sozio-ökonomische Bedingungen kompensieren

2.4.5 Simone Stab (20 Jahre alt) und ihre Mutter: Eine sozioökonomisch stark belastete Familie, der es dennoch gelingt, ihren Alltag weitgehend zu meistern

2.5 Die jungen Erwachsenen von Typ 4: Keine belasteten sozioökonomischen Bedingungen mehr und unbelastete sozioemotionale Beziehungsstrukturen - Die relativ kompetenten >Aufsteiger

2.5.1 Gudrun Dornbacher (19 Jahre alt) und ihre Eltern: Eine Familie, die den Aufstieg schafft und in einem guten Familienklima lebt

2.5.2 Erich Grubert (21 Jahre alt) und seine Eltern: Eine Patchwork-Familie festigt ihre sozio-emotionalen Beziehungsstrukturen und schafft sozio-ökonomische Sicherheit

2.5.3 Helmut Pfortner (19 Jahre alt) und seine Eltern: Weitestgehend stabile sozio-emotionale Beziehungen kennzeichnen die Familie, die zunächst herausfordernden sozio-ökonomischen Bedingungen bessern sich

2.5.4 Susanne Scheib (21 Jahre alt) und ihre ehemals alleinerziehende Mutter: Eine junge Frau mit großem Zugewinn an Handlungsentwürfen und Handlungskompetenzen

2.5.5 Norbert Zarbl (20 Jahre alt) und seine Familie: In einer funktionalen Patchwork-Familie verbesserten sich die sozio-ökonomischen Bedingungen

3 Fallübergreifende Entwicklungen im Panel - Ergebnisse der fokussierten Analyse

3.1 Schule, Ausbildung, Studium und erste Arbeitsstelle - der Eintritt in die berufliche Zukunft 
3.2 Beziehungen und Pläne für (den Ausbau von)

Partnerschaften

3.3 Wohnbedingungen und Zukunftspläne

3.4 Mediengebrauch und Freizeitverhalten

3.5 Zwischenfazit: Die Lebensführung der jungen Erwachsenen

3.6 Veränderungen im Leben der Eltern

3.6.1 Typ 1: Massive sozio-ökonomische Probleme als multiple Deprivation: Die rundherum überforderten Familien (Landinger, Oblinger, Öllinger)

3.6.2 Typ2: Die sozio-ökonomisch nicht mehr bzw. wenig belasteten, aber wegen problematischer sozio-emotionaler Beziehungsstrukturen überforderten Familien (Hirtner, Holzner, Rohringer Weiss)

3.6.3 Typ 3: Die zwar sozio-ökonomisch, aber sozioemotional weniger belasteten, relativ kompetenten Familien (Aufbauer, Boll, Ebner, Kaiser, Stab)

3.6.4 Typ 4: Keine belasteten sozio-ökonomischen Bedingungen mehr und unbelastete sozio-emotionale Beziehungsstrukturen - Die relativ kompetenten >Aufsteiger (Dornbacher, Grubert, Pfortner, Scheib, Zarbl)

3.7 Veränderungen des Mediengebrauchs der Eltern

4 Perspektiven sozial benachteiligter junger Menschen und ihrer Eltern auf gesellschaftliche Krisen und die Rolle von Medien

4.1 Perspektiven sozial benachteiligter Familien auf Geflüchtete

4.1.1 Wahrnehmung und Perspektiven zum Thema

Migration und Flucht - Erfahrungen in den ersten fünf Erhebungswellen (2005 bis 2014)

4.1.2 Der Alltag mit Geflüchteten in der sechsten Erhebungswelle - Themen in den Panelfamilien

4.1.3 Gemeinsamkeiten und Unterschiede in den Wahrnehmungen und Perspektiven zwischen Eltern und Kindern

4.1.4 Umgang mit Medien und Kritik an ihrer Berichterstattung 
4.1.5 Wahrnehmung von Geflüchteten mit Blick auf die Familientypologie

4.1.6 Ausgewählte Fallbeispiele

4.1.6.1 Familien von Typ 1: Das Fallbeispiel Familie Öllinger

4.1.6.2 Familien von Typ 4: Das Fallbeispiel Familie Dornbacher

4.1.7 Zusammenfassung

4.1.8 Reflexion und Conclusio zu den Wahrnehmungen und Perspektiven auf Geflüchtete

4.2 Perspektiven auf die Covid-19-Pandemie im Kontext sozialer Benachteiligung

4.2.1 Rahmung der Erhebung

4.2.2 Lebensweltliche Herausforderungen in der Pandemie

4.2.3 Information und Kommunikation über die Covid-19Pandemie

4.2.3.1 Fernsehen und öffentlich-rechtlicher Rundfunk

4.2.3.2 (Online-)Zeitungen mit besonderem Blick auf regionale Angebote

4.2.3.3 YouTube als wichtige Informationsplattform

4.2.3.4 Soziale Medien und ihre Risiken

4.2.3.5 Das Radio - ein Informationsmedium

5 Qualitative Längsschnittforschung: Vielfältige Herausforderungen auf unterschiedlichen Ebenen

5.1 15 Jahre Panel-Langzeitstudie: Herausforderungen für die Forschung

5.2 15 Jahre Panel-Langzeitstudie: Was sagen die Panel-

Angehörigen selbst dazu?

5.2.1 „Nie jemand für eigene Wünsche interessiert“: Elternaussagen zur Langzeitstudie

5.2.2 „Es war vor allem auch mal was anderes“: Wie blicken die jungen Erwachsenen auf ihre Teilnahme an der Studie zurück? 
6 Zusammenfassung, Diskussion und Konsequenzen

6.1 Von der allmählichen Verfertigung eines Forschungsansatzes 281

6.1.1 Ausgangspunkt und Ziele

6.1.2 Aufriss der theoretischen Fundierung

6.1.3 Zur methodischen Fundierung

6.2 Von der (Medien-)Sozialisationsforschung als integrativer Familienforschung hin zu einer breiteren Perspektive auf Sozialisationskontexte

6.2.1 Familie im Zentrum der Sozialisationskontexte von Kindern und Jugendlichen

6.2.2 Die jungen Erwachsenen und ihr Beziehungsnetzwerk

6.2.3 Relevanz individueller Passungen beim Einstieg ins Erwerbsleben der jungen Erwachsenen

6.3 Die jungen Erwachsenen und ihr Mediengebrauch 296

6.4 Gesellschaftliche Konsequenzen aus der Panel-Langzeitstudie 300

7 Literaturverzeichnis 


\section{Vorwort und Einführung zur Fortsetzung der (Medien-)Sozialisationsstudie}

Neugier und Leidenschaft zu forschen gehören eng zusammen. Hinzu kommt oft auch der Wunsch, mit dem Erforschten etwas für die Belange der Menschen zu bewirken, mit denen und für die geforscht wurde. Im vorliegenden Fall sind dies junge Erwachsene, die bereits seit ihrem fünften Lebensjahr Teil einer Langzeitstudie sind, die 2005 mit der Frage begann, welche Rolle Medien in der Lebenswelt sozial benachteiligter Kinder spielen. Darin integriert war die Frage nach der Rolle der zentralen Sozialisationskontexte, allen voran der Familie, das heißt der Kernbeziehungsgruppe, in der ein Kind allein oder mit Geschwistern aufwächst und in dem es lernt, seinen Standort in der Familie und später in anderen Sozialisationskontexten, Kindergarten und Schule, in Peer-Groups und Freundschaften, zu finden und sich als (möglichst unverwechselbares) Individuum zu positionieren. Die Studie hatte von Beginn an das Anliegen - und dies ist auch das heutige Credo -, im Sinne von Norbert Elias engagierte Sozialforschung $\mathrm{zu}$ betreiben. Auf Basis empirischer Forschungsergebnisse sollten und sollen Zusammenhänge zwischen Lebensweltbedingungen und (Medien-)Sozialisation sozial benachteiligter Heranwachsender aufgezeigt und mögliche Wege zum Ausbau von Handlungsressourcen, Alltagsund Medienkompetenz der Betroffenen, umrissen werden. Nun, mehr als 15 Jahre nach Beginn der empirischen Arbeit an der Studie, handelt es sich bei den Kindern von damals um junge Erwachsene, die zwischen 20 und 21 Jahre alt sind, zum Teil noch zu Hause bei ihren Eltern oder allein bzw. in einer Partnerschaft leben und bemüht sind, ihren Weg privat und beruflich zu finden und zu gehen.

In bisher insgesamt sechs Erhebungswellen und einer Nachbefragung, die sich an wichtigen Entwicklungsphasen, wie Kindergartenzeit (2005), Einschulung (2007), mittlerer Kindheit (2010), Adoleszenz und Jugendalter (2012 sowie 2015 und 2016/2017), orientierten, wurden 20 (ab der zweiten Erhebungsphase noch 18) junge Menschen gemeinsam mit ihren engsten Beziehungspersonen in einer Panel-Langzeitstudie wissenschaftlich begleitet. Die Studie hatte sich die Aufgabe gestellt, so die Formulierung im ersten Förderantrag, „die mit dem sozialen Wandel einhergehenden sozialisatorischen Bedingungen speziell für 
Kinder in sozial schwächeren bzw. anregungsärmeren Milieus zu beschreiben und ihre Bedeutung für den Prozess der Sozialisation“ aufzuzeigen. Es ging vor allem darum, das Verhältnis von Medien zu anderen Sozialisationskontexten zu untersuchen und zu eruieren, welche Relevanz diesen bei der Identitätskonstruktion, dem Aufbau von Wissen und in der Wertevermittlung zukommt. Ausgehend von der Frage nach den von sozialen und, darin eingelagert, medialen Wandlungsprozessen induzierten Bedingungen von Sozialisation bei sozial benachteiligten Heranwachsenden ging es zudem darum, die Dynamik der Kindheitsund Jugendentwicklung mit zu berücksichtigen und im Zusammenhang damit die sich verändernde Rolle der Sozialisationskontexte selbst im Laufe der Studie in den Blick zu nehmen. Die ersten vier Phasen schlugen sich in zwei Buchpublikationen nieder, die gemeinsam mit Michelle Bichler (Paus-Hasebrink/ Bichler 2008) und Jasmin Kulterer (Paus-Hasebrink/ Kulterer 2014) verfasst wurden.

2017 wurde die Langzeitstudie mit einer dritten deutschsprachigen (Paus-Hasebrink 2017) und einer englischsprachigen Buchpublikation (Paus-Hasebrink/ Kulterer/ Sinner 2019) vorerst abgeschlossen. Fragen von interessierten Kollegen und Kolleginnen, aber auch nicht-wissenschaftlich orientierten Freunden und Freundinnen und vielen anderen, wie es denn wohl den Jungen und Mädchen als Erwachsenen und ihren Eltern gehen möge, ob und wie es ihnen gelingt bzw. gelingen kann, sich in der neuen Lebensphase zurecht zu finden, dienten der eigenen Neugier und Forscherinnenleidenschaft als weitere Nahrung. So entstanden die Idee und der Wunsch, in noch einer weiteren Erhebungswelle, der siebten Welle, zu erfahren, wie sich das Leben der mittlerweile jungen Erwachsenen weiter entwickelt hat und wie sie sich heute positionieren: Wie sehen ihre Handlungsoptionen, Handlungsentwürfe und Handlungskompetenzen aus? Welche Rolle weisen die jungen Erwachsenen in ihrem Alltag nunmehr Medien zu? Und welche Relevanz kommt dabei ihren engsten Bezugspersonen aus der Familie, vor allem ihren Eltern, ihren Geschwistern und ihren Partnern und Partnerinnen sowie deren Mediengebrauch in diesem Kontext zu? Ein weiteres mit der Länge der Panelstudie über mehr als 15 Jahre verbundenes Anliegen der Studie wird in der Frage aufgegriffen, wie die jungen Menschen und ihre Eltern die lange Zeit der Forschung selbst wahrgenommen haben. Vor diesem Hintergrund wurden schließlich die Panelangehörigen erneut kontaktiert, um sie zu motivieren, erneut an der Fortsetzung der Studie teilzunehmen. 17 von 18 Familien sind dieser Einladung gefolgt. 
Aufgrund des eigentlich schon 2017 erfolgten Abschlusses des Projekts und der damit endenden Förderung durch den Jubiläumsfonds der Oesterreichischen Nationalbank, der dankenswerter Weise die Studie von 2005 bis 2017 nach insgesamt dreimaliger Antragstellung (2004, 2009, 2013) und jeweils erfolgreich abgeschlossenem Peer-Review-Verfahren finanziell erst möglich gemacht hat, lag der neuen Erhebungswelle und dem auf dieser Basis entstandenen Buch zwar nur ein geringes Budget - aber dafür eine umso größere Portion an persönlichem Engagement zu Grunde, unterstützt insbesondere durch Philip Sinner, der von 2012 bis zum Projektende mitgearbeitet hat und als Co-Autor dieser Publikation fungiert, und von Nadja Haupt, die sich in den ersten Projektphasen, der Erhebung und der Datenauswertung 2020, intensiv in die Projektarbeit eingebracht hat.

Ingrid Paus-Hasebrink

Worum geht es nun konkret in der Fortsetzung der (Medien-)Sozialisationsstudie? Im Mittelpunkt der Publikation steht die Frage nach der Rolle von Medien in der Übergangsphase der jungen Menschen von der Jugend ins Erwachsenenleben und als junge Erwachsene, geprägt von Schulende, Lehre und Ausbildung, von neuen partnerschaftlichen und romantischen Beziehungen, von Beziehungen zu Freunden und Freundinnen, zu Kollegen und Kolleginnen, privat, und auch im Arbeits- und Berufsumfeld. Wie hat sich das Leben der jungen Männer und Frauen angesichts neuer gesellschaftlicher Herausforderungen im Privatleben und vor allem auch im Beruf entwickelt und wie sehen nun ihre Lebensentwürfe aus? Und wie gehen die nunmehr jungen Erwachsenen und ihre Eltern mit den vielfältigen und zunehmend sozio-ökonomisch und sozio-emotional schwieriger werdenden gesellschaftlichen, aber auch ganz privaten Herausforderungen in Pandemie-Zeiten um? Zudem wird ein Blick auf die Wahrnehmungen und Umgangsweisen der Panelangehörigen mit Geflüchteten in den Jahren 2015 und 2016 geworfen und die Rolle von Medien in den Kontexten dieser gesellschaftlichen Krisensituationen nachgezeichnet.

Zunächst wird in Kapitel 1 die theoretische und methodische Basis fortgeschrieben. In Kapitel 2 folgen mit Blick auf die Veränderungen der jungen Erwachsenen und ihrer Eltern Fallbeschreibungen zu den Panelangehörigen, um ihre jeweils individuelle Situation im Kontext 
nachvollziehbar zu machen. In Kapitel 3 werden die Veränderungen mit Blick auf alle Panelangehörigen hinweg reflektiert und diskutiert.

Kapitel 4 zeichnet die Wahrnehmungen und Umgangsweisen der Familien mit gesellschaftlichen Krisensituationen nach. Kapitel 5 ist dem Rückblick auf 15 Jahre Panelstudie gewidmet, zum einen in Bezug auf die Forschenden und zum anderen auf die an der Panelstudie beteiligten Kinder und ihre Eltern. Das 6. und letzte Kapitel beinhaltet Diskussion und Fazit zur Langzeitstudie und formuliert notwendige gesellschaftliche Konsequenzen.

Ingrid Paus-Hasebrink und Philip Sinner 


\section{Theoretische und methodische Basis}

\subsection{Praxeologische (Medien-)Sozialisationsforschung als Schlüssel zur Untersuchung des jungen Erwachsenalters}

Die Diskussion um Sozialisation und was sie ausmacht, hat schon eine lange Tradition (Veith 2015). Ebenso lang und auch kontrovers geführt verläuft die Debatte um eine möglichst leistungsstarke Sozialisationstheorie$^{1}$, die den Blick auf Gesamtzusammenhänge richtet. Allzu oft verliert sich sozialwissenschaftliche Forschung darin, sich auf Randphänomene zu kaprizieren und Einzelphänomene, stets, auch methodisch, feiner austariert, in den Mittelpunkt ihres Forschungsinteresses zu rücken. Damit läuft sie Gefahr, den bunten Kühen, wie man sie nennen könnte, mehr Aufmerksamkeit zu widmen als drängenden, gesellschaftlich brisanten Themen. Um Sozialisationsprozesse in ihrer hohen Komplexität auch im Rahmen der (Medien-)Sozialisationsforschung analytisch zu erfassen und empirisch operationalisierbar zu machen, bedarf es jedoch vor allem eines begrifflichen Rahmens, der es erlaubt, soziale Zusammenhänge sowohl im Sinne von individuellen biografischen als auch gesellschaftlichen Veränderungen möglichst ganzheitlich zu untersuchen. Dazu bieten sich praxeologische Ansätze an. Sie sind geeignet, die Komplexität und die Verflochtenheit der angesprochenen Prozesse gleichzeitig in den Blick zu nehmen (Bettinger/ Hugger 2020): „Theorien sozialer Praktiken verweisen auf eine epistemologische Position, die Sozialität als etwas begreift, das aus Bündeln von ,Doings' und ,Sayings' (Schatzki 2002: 73) besteht und demnach im handelnden Vollzug hervorgebracht wird. Praxistheorien wenden sich von strukturalistischen sowie subjektivistischen Ansätzen ab und schlagen einen Weg ein, der individuelles, Tätigsein' und gesellschaftliche Ordnungen als unmittelbar aufeinander bezogene Komponenten des Sozialen in den Mittelpunkt rücken” (Bettinger/ Hugger 2020: 1f.). Sie ermöglichen es, Sozialisation weder als „kausale(n) Prozess der gesellschaftlichen Prägung noch als Impuls für individuelle Persönlichkeitsentwicklung“ aufzufassen. Stattdessen, so Grundmann und Höppner (2020: 9), wird davon ausgegangen, „dass Sozialisation als ein Oszillieren von sich verwebenden

1 Siehe dazu Abschnitt 2 „Modelle und Theorien“ im Handbuch Sozialisationsforschung (Hurrelmann et al. 2015: 144-299). 
und verbindenden (kooperativen und koordinierenden) Aktivitäten und Tätigkeiten zu verstehen ist und dass sich in diesen praktischen Vollzügen Entitäten konstituieren“. Sozialisation wird so „als ein ergebnisoffener Prozess des Miteinander-Lebens, des stetigen Vollziehens gemeinsamer Lebensführung und der stetigen Gestaltung sozialer Bezugspraxis konzipiert und analysiert, ohne in die bekannten Dualismen, Reduktionismen und Kausalitätsannahmen zu verfallen“ (ebd.). Dies bedeutet, „Sozialisation in einem weiten Sinne als die Gesamtheit jener Praktiken und Prozesse" zu begreifen, ,in denen Dispositionen und Dispositionsnetze angelegt und fortlaufend transformiert werden, die in konkreten Praktiken der Subjektivierung angesprochen, mobilisiert und ausgeformt werden (können)“ (Alkemeyer 2020: 100). Denn um das Verhältnis von Gesellschaft und Individuum angemessener $\mathrm{zu}$ begreifen und $\mathrm{zu}$ beschreiben, bedarf es nach Elias (2006a) dynamischer und prozesshafter Begriffe (siehe dazu Alikhani 2020: 106f.). ${ }^{2}$ Mit Hilfe von dynamischen und prozesshaften Begriffen kann es besser gelingen, so Alikhani (2020: 10) mit Bezug auf Elias (2006: 170), ,gleichzeitig die beiden verschiedenen, jedoch voneinander untrennbaren individuellen und sozialen Ebenen der menschlichen Existenz (zu) erfassen“. Tasheva (2020: 153) hebt hervor, dass „die Existenz des einzelnen Menschen (...) nicht einfach in der Allgemeinheit einer Gesellschaft (geschieht), sondern in vielfältigen sozialen Beziehungen zu konkreten anderen einzelnen Menschen. Weder ist die Gesellschaft der Daseinsgrund des Individuums noch ist das Individuum ein autonomes ,Fürsichsein““.

2 Norbert Elias (2006: 101) betont, wie Alikhani (2020: 106f.) herausarbeitet, dass „viele soziologische Theorieansätze das Problem des Verhältnisses von ,Individuum' und ,Gesellschaft' weitgehend ungelöst (lassen), obwohl die entsprechende Fragestellung zu den Kernfragen der soziologischen Theoriebildung gehört“. Der auf Elias zurückgehende Figurationsbegriff steht im Mittelpunkt des Konzepts der Kommunikativen Figurationen von Hepp und Hasebrink (siehe dazu Hasebrink 2014; Hepp/ Hasebrink 2014). Dieses dient für Kammerl und Lampert in ihrer Langzeitstudie „Sozialisation in einer sich wandelnden Medienumgebung (Socialisation in a Changing Media Environment)“ (siehe Kammerl et al. 2020) auch als Ausgangspunkt zur Untersuchung von Interaktionsbeziehungen in der Sozialisation. Der Begriff der Figuration lenkt den Blick darauf, dass Familien, so Hasebrink (2014: 228), als „kommunikativ konstruiertes Sozialgebilde“ aufgefasst werden können; im Mittelpunkt stehen nicht Verwandtschaftsverhältnisse, die bestimmen, wer zur Familie gehört, dies ergibt sich vielmehr ,aus der konkreten kommunikativen Praxis“ (ebd.: 228). Hasebrink und Hepp nutzen diesen Begriff, wie dies Hasebrink formuliert, „als eine konzeptionelle Brücke zwischen dem kommunikativen Handeln der beteiligten Individuen und dem sozialen Zusammenhang (...), der sich aus diesem Handeln ergibt, dieses aber zugleich auch prägt" (Hasebrink 2014: 227). 
Sozialisation als „Beziehungspraxis“ (Grundmann 2015: 162) zu verstehen, hebt die große Bedeutung der sich dynamisch vollziehenden Prozesshaftigkeit von Sozialisation hervor.

So geht es in der Panel-Langzeitstudie nicht um fest gefügte Entitäten, etwa des Individuums oder der Gesellschaft bzw. um das Woher und das Wohin; vielmehr steht in der praxeologisch ausgerichteten (Medien-)Sozialisationsforschung das Wie sich prozessual vollziehender Sozialisation im Laufe des „prozessualen Hervorbringens einer gemeinsamen Lebensführung" (Grundmann 2015: 167) von Subjekten im Mittelpunkt. Da diese Sichtweise bereits ausführlich dargelegt wurde (siehe insbesondere PausHasebrink 2017a, 2019, 2021), werden im Folgenden nur einige wenige Aspekte erneut benannt, um den Argumentationsgang der Langzeitstudie ins Gedächtnis zu rufen. Praxeologische (Medien-)Sozialisationsforschung geht von der Frage aus, welche Relevanz Medien im Laufe des Sozialisationsprozesses von Individuen - im vorliegenden Fall vom Kindergartenalter bis hin zum Beginn des Erwachsenalters - in Bezug auf die Lebensführung und Alltagsbewältigung von Menschen zukommt. Im Kontext der Sozialisation bestimmen spezifische soziale Zusammenhänge das Spielfeld der Möglichkeiten für ein Individuum, Identität auszubilden und Handlungskompetenz im Alltag zu erwerben und diese möglichst auszubauen. Der Studie liegt die Überzeugung zu Grunde, dass sich mit einer praxeologischen Perspektive rekonstruieren lässt, wie Individuen und Gruppen leben und in ihren je verschiedenen sozialen Milieus den veränderten medialen Bedingungen und Möglichkeiten im Rahmen ihres Alltags praktischen Sinn verleihen.

Mit dem Blick auf die Methoden der Sinngebung, das Tableau alltagspraktischer Orientierungsmuster, d.h. wie Menschen - und dies mit Hilfe von Medien - ihren Handlungen im Alltag Sinn geben, wird (Medien-)Sozialisation weder aus einer subjektiven noch objektiven Perspektive erforscht. Die Untersuchung erfolgt vielmehr aus der Perspektive des praktischen Sinns der auf Bourdieus Theorie der Praxis (1977) fußenden Praxeologie. Ins Visier genommen wird dabei das je individuelle, aber dennoch über den/ die Einzelne/n hinausweisende Lebensumfeld, in dem er bzw. sie agiert bzw. agieren kann. Mitbetrachtet werden also auch die sozialen Räume bzw. das soziale Milieu, in dem jeweils bestimmte Handlungsziele von Individuen und ihre jeweiligen Ressourcen wirksam werden und in dem bestimmte Handlungsmuster „am Platz“ (Weiß 2000: 47) sind. Der Blick gilt Menschen in ihrer jeweiligen Lebensführung in ihrem je spezifischen Alltag und den ihnen tatsächlich oder symbolisch zur Verfügung 
stehenden sozialen Räumen. Dazu zählen auch mediale Räume, alte wie neue Medienangebote (Paus-Hasebrink 2017a: 25ff.).

Die Studie zielt auf die Analyse des Mediengebrauchs ${ }^{3}$ von Individuen im Kontext von Mediatisierungsprozessen (siehe dazu auch Kapitel 6). ${ }^{4}$ Mediengebrauch wird zum einen mitbestimmt von den Grundkoordinaten der täglichen Lebensbewältigung von Individuen, zum anderen aber auch vom Medienangebot und seinen spezifischen Darbietungsformen selbst. Dies bedeutet, im Kontext von (Medien-)Sozialisationsforschung auch die sich im Laufe des Sozialisationsprozesses des Individuums verändernden und von Medienunternehmen unterschiedlicher Couleur zur Verfügung gestellten Mediendienste und -anwendungen mit zu beachten. Schließlich kann nur genutzt werden, was auf dem Markt zur Verfügung steht.

Medien zählen zu den zentralen Ausdrucks- und Verständigungsmitteln von Menschen, die mit ihrer Hilfe Bedeutungen konstruieren und sich ihrer aktiv als Ausdrucks- und als Verständigungsmittel bedienen. Um die Veränderungen durch eine kaum mehr überschaubare Fülle digitaler Medien mit Blick auf ihre Angebots- und Gebrauchsweisen erfassen zu können, bietet sich als gedanklicher Ausgangspunkt der Mediatisierungsansatz (Krotz/ Hepp 2013; Livingstone/ Lunt 2014) an. Der Begriff der Mediatisierung beschreibt, wie soziale Veränderungen auftreten „when

3 Die im Text verwendeten Begriffe Medienhandeln sowie Mediengebrauch (media usage) sind als Synonyme zu verstehen; sie beschreiben, wie Individuen oder Gruppen auf spezifische Weise mit Medien umgehen und wie sie Medien als Teil sozialen Handelns einsetzen, um ihren Alltag zu leben. Das Verständnis des Medienhandelns als Gebrauch gründet in Wittgensteins „Gebrauchstheorie“ der Sprache, wonach sich die Bedeutung des Gesagten erst im konkreten Gebrauch vollzieht, in der Praxis des Sprechens, seiner Kontextgebundenheit und damit in konkreten gesellschaftlichen Verwendungszusammenhängen (siehe dazu Paus-Hasebrink 2017a). Der Begriff der kommunikativen oder medialen Praktiken (communicative or media practices) weist auf die jeweiligen Ausprägungen des medialen Handelns hin und erfasst die spezifischen Umgangsweisen, die zusammengenommen den Mediengebrauch bzw. das Medienhandeln kennzeichnen. Der im Text ebenfalls vorkommende Begriff Mediennutzung (media use) dient als Oberbegriff; darunter wird jeglicher Kontakt mit Medien subsumiert.

4 Kommunikation wird dabei als ein Grundkonstituens menschlicher Existenz gesehen; ein menschliches Wesen stellt sich in der Kommunikation dar (Mead 1988 [1934]). Kommunikation ist laut Reichertz (1998: 9) „wechselseitig organisierte und symbolisch vermittelte Interaktion" und stets als eine Form sozialen Handelns und als symbolvermitteltes Handeln von Menschen für Menschen, in bestimmten Situationen und bestimmten Soziallagen und oft auch mit bestimmten Medien zu verstehen. 
communication patterns are transformed due to the communication tools and technologies“ (Lundby 2014: 3). Mediatisierung macht „den Medienwandel zum Ausgangspunkt“ und nimmt damit „den Wandel des sozialen Subjekts in seinem Alltag, seinen Kommunikationsformen und Identitätskonstruktionen in den Blick" (Krotz 2017: 23).

Im Kontext medialer Dynamisierungsprozesse ${ }^{5}$ ist es mehr denn je wichtig, nicht allein auf den Umgang mit einem Medienangebot zu schauen, sondern zu verstehen, wie sich jeweils individuell bestimmte Mediengebrauchsweisen zusammensetzen und wie sie sich im Laufe der lebenslangen Sozialisation verändern, d.h. auch wie Kinder und Erwachsene in ihren privaten und beruflichen Alltagskontexten agieren und in ihrem Mediengebrauch Medienangeboten vor dem Hintergrund jeweils spezifischer, sich dynamisch verändernder medialer Bedingungen praktischen Sinn verleihen. Mit Hilfe des Konzepts der Medienrepertoires (Hasebrink/ Domeyer 2012) richtet sich der Blick auf das gesamte von Medienhandelnden zusammengestellte persönliche Repertoire und wie diese unterschiedliche neue Medienangebote entsprechend ihren Bedürfnissen in ihre bereits bestehenden Repertoires integrieren.

\subsection{Sozialisation - ein lebenslanger Prozess}

Im Kontext der praxeologischen Perspektive auf (Medien-)Sozialisationsprozesse ist es wichtig, Sozialisation stets als einen dynamisch-interaktiven Prozess $^{6} \mathrm{zu}^{\mathrm{z}}$ verstehen, der aktiv vom Individuum mitbestimmt und mitkonstruiert wird und in dem dieses seine Identität im Zusammenhang seiner lebensweltlichen Verankerung bearbeitet und in dem, wie dies oben bereits angesprochen wurde, das Verhältnis von Gesellschaft und Individuum stets als dynamische und prozesshafte verstanden wird. Mitbedacht werden muss ebenso, dass Sozialisation nicht mit der Kindheit und der Jugend endet, auch wenn in der Sozialisationsforschung noch immer

5 Siehe dazu auch die Langzeitstudie von Röser et al. (2019) zur Verhäuslichung des Internets. Zentrales Ziel der Studie war es, „das Zusammenspiel von medialem und nicht-medialem Handeln, die Einbettung der Mediennutzung in zeitliche, räumliche und soziale Strukturen, die Interaktion von Medienhandeln und Beziehungsleben in der Paarkonstellation“ (ebd.: 2) zu erfassen. Röser et al. zeigen deutlich auf, wie Medienkommunikation den häuslichen Alltag durchdringt.

6 Siehe dazu auch Hurrelmann und Bauer (2015: 146) sowie als Überblick zur Mediensozialisation Aufenanger (2008) sowie Vollbrecht und Wegener (2010) und speziell zur Mediensozialisation Heranwachsender Süss (2004). 
die Fokussierung auf diese Altersstufen dominiert (Böhnisch/ Lenz 2015: 871). Sozialisation ist vielmehr ein lebenslanger Prozess (Fromme 2007). Sozialisationsprozesse sind damit „auch feste Bestandteile des Erwachsenenalters" (Böhnisch/ Lenz 2015: 871). Nach Böhnisch und Lenz (ebd.) ist das Erwachsenenalter heute einer „offenen Entwicklungsdynamik“ unterworfen und von andauernden biografischen Bewältigungsproblemen gekennzeichnet. Diese Dynamik vollzieht sich in verschiedenen sozialen Zusammenhängen, an denen das Individuum beteiligt ist und die sein Spielfeld zum Aufbau von Identität und Handlungskompetenz im Alltag mitbestimmen. Mit Bezug auf Heinz (2003) betonen Böhnisch und Lenz (2015: 872), dass „Sozialisationsprozesse im Erwachsenenalter (...) strukturiert (sind) durch das immer wiederkehrende Streben nach biografischer Handlungsfähigkeit in der institutionell und lebensweltlich vermittelten Spannung zu den Bewältigungsaufgaben, wie sie in den gesellschaftlichen Entgrenzungsprozessen freigesetzt werden". Auch in diesem Zusammenhang kann als nähere Erklärung, wie sich die Bewältigung vollzieht bzw. vollziehen kann, das von Robert Havighurst $(1948,1972)$ entwickelte Konzept der Entwicklungsaufgaben ${ }^{7}$ weiterhelfen. Es dient zur Erklärung individuell-biografischer, sich im Zusammenhang mit sozialen Kontexten der jeweiligen Lebenswelt und Lebensführung von Individuen vollziehender dynamischer Entwicklung. Es bietet einen wichtigen Ausgangspunkt für die Möglichkeit, wie dies Hans-Dieter Kübler fordert, „breit ansetzende, mehrperspektivische und -methodisch arbeitende Sozialisationsforschungen“ (...) um „die nötige individualistisch und biografisch konzentrierte Dimension zu ergänzen“ (Kübler 2010: 28).

Schon 1956 hatte der deutsche Entwicklungspsychologe Hans Thomae darauf hingewiesen, dass menschliches Leben „nicht einfach ein Zunehmen an physischen und psychischen Einheiten und Eigenheiten bedeutet, sondern ein Sichwandeln, ein Anderswerden, das nicht zufällig eintritt, sondern auf gesetzte Weise, von inneren Impulsen gelenkt“ (Thomae 1956: 107). Vor der Überbetonung von Gesetzmäßigkeiten - so wie dies noch in den von Erikson gesetzten stufenförmigen Krisen bzw. in den Stufenmodellen von Piaget, Kohlberg und auch Selman zu finden ist - wird dabei

7 Stephan Niemand spricht in seiner Untersuchung zu „Alltagsumbrüchen und Medienhandeln“, einer qualitativen Panelstudie zum Wandel der Mediennutzung in Übergangsphasen, von Daseinsthematiken. Sie weisen auch eine Nähe zu den handlungsleitenden Themen nach Charlton und Neumann (1986) auf und stellen Basisthemen dar, „die in einer bestimmten Lebenssituation relevant werden und die als (geäußerte oder beobachtbare) 'Gedanken, Wünsche, Befürchtungen, Hoffnungen und Konflikte' (Thomae 1988: 53) sichtbar werden“" (Niemand 2020: 239f.). 
gewarnt und die „Einbettung des leibseelischen Geschehens in eine spezifische soziale Lage" (ebd.: 108) gefordert. In Havighursts theoretischem Konzept der Entwicklungsaufgaben (1972; siehe auch Mayseless/ Keren 2014: 63), das im Rahmen der Theorie des life span developments die Perspektive auf die situationsgebundene Auseinandersetzung des Individuums mit den Anforderungen in seinem Leben richtet, wird deutlich hervorgehoben - dies sei erneut betont -, dass der Mensch im Prozess der Identitätsentwicklung lebenslang einer Vielzahl von unterschiedlichen situativen Gegebenheiten ausgesetzt ist, in denen er seine Handlungskompetenz immer wieder neu unter Beweis stellen muss. „Die jeweils ausgeprägte Strukturierung der Handlungskompetenzen wirkt dabei als Steuerungsinstanz für das Handeln und Verhalten in den verschiedenen Situationen" (Hurrelmann 1990: 163). So versteht denn Hurrelmann auch Persönlichkeit als , die individuelle, in Interaktion und Kommunikation mit Dingen wie mit Menschen erworbene Organisation von Merkmalen, Eigenschaften, Einstellungen, Handlungskonzepten und Selbstkonzepten eines Menschen auf der Basis der natürlichen Anlagen und als Ergebnis der Bewältigung von Entwicklungs- und Lebensaufgaben zu jedem Zeitpunkt der Lebensgeschichte" (Hurrelmann 1990: 71). ${ }^{8}$ Entwicklungsaufgaben beschreiben, „welche Form die für verschiedene Altersabschnitte typischen körperlichen und psychischen aber auch kulturellen und sozialen Anforderungen und Erwartungen haben" (Hurrelmann/ Quenzel 2016: 24); sie werden gekennzeichnet durch die individuelle und eine soziale Dimension (ebd.: 25).

In der lebenslangen Sozialisation stellen die Ausbildung und stete Bearbeitung des Selbstbildes eine tägliche Herausforderung für das Individuum dar. Sozialisation findet statt im Prozess der Identitätsentwicklung eines Menschen und ist stets an eine Vielzahl von unterschiedlichen situativen Gegebenheiten gebunden, denen ein Mensch im Laufe seines Lebens ausgesetzt ist. Ein Mensch muss im Laufe seines Lebens seine Handlungskompetenz immer wieder neu unter Beweis stellen, indem er spezifische biografisch geprägte Entwicklungs- oder Lebensaufgaben bewältigt.

Das Konzept der Entwicklungs- bzw. Lebensaufgaben (siehe dazu PausHasebrink 2010) verbindet Individuum und Umwelt, setzt kulturelle Anforderungen mit individueller Leistungsfähigkeit in Beziehung und betont die Handlungsfähigkeit von Individuen. So betonen denn auch Hurrel-

8 Auch Heckhausen und Schulz (1999) weisen eindrücklich darauf hin, dass sich das Konzept der Entwicklungsaufgaben nicht auf die Lebensphasen Kindheit und Jugend beschränkt. 
mann und Bauer (2021: 12): „Auch wenn die Einflüsse der sozialen Umwelt, also des jeweiligen sozialen, wirtschaftlichen und kulturellen Milieus, groß sind, auch wenn sie langanhaltend wirken und nachhaltige Spuren hinterlassen - dies allein reicht nicht aus, um eine Persönlichkeitsentwicklung vollständig vorherzubestimmen".

Welche Entwicklungs- und Lebensaufgaben stellen sich nun dem Individuum im jungen Erwachsenenalter?

Dazu ist es nötig, sich vor Augen zu führen, dass der Begriff des jungen Erwachsenenalters noch recht jung ist. Arnett wies 2000 darauf hin, dass in modernen Gesellschaften Heranwachsende eine längere Phase der Orientierung brauchen (Arnett 2000; siehe auch Fortuin/ van Geel/ Vedder 2016: 1). Das Konzept des jungen Erwachsenenalters entwickelte sich, so Guglhör-Rudan (2012), erst in den letzten Jahrzehnten. Die nunmehr als eine neue Lebensphase umrissene und als eigenständig klassifizierte beruhe auf grundlegenden in Bezug auf das Jugendalter entwickelten und auf das junge Erwachsenenalter übertragene entwicklungstheoretischen und soziologischen Betrachtungen und Theorien. „Die individuelle Entwicklung vom Jugend- ins Erwachsenenalter ist durch keine klaren Trennlinien voneinander abgrenzbar, so dass aus entwicklungspsychologischer Sicht eine Betrachtung des jungen Erwachsenenalters ihren Anfang in der Darstellung der Entwicklungsschritte des Jugendalters nimmt. Auch aus soziologischer Sicht kann das junge Erwachsenenalter nur eingebettet in den Lebensverlauf der Menschen sinnvoll betrachtet werden" (Guglhör-Rudan 2012: 19).

Zur Veranschaulichung, um welche Aspekte es dabei handelt, sei auf die von Hurrelmann und Quenzel (2016: 25) in vier zentralen Dimensionen gefassten Aspekte einer lebenslang stattfindenden Sozialisation hingewiesen: Qualifizieren, Binden, Konsumieren und Partizipieren. Auf der individuellen Ebene bedeutet die Entwicklungsaufgabe Qualifizieren die Entwicklung der intellektuellen und sozialen Kompetenzen. Binden umreißt die Entwicklung der Körper- und Geschlechtsidentität und der Bindungsfähigkeit. Unter Konsumieren wird die Fähigkeit, „emotional erfüllende und stabile Freundschafts- und Sozialkontakte zu knüpfen, einen angemessenen Lebensstil zu entwickeln und zu einem souveränen Umgang mit Freizeitund Konsumangeboten zu kommen“ (ebd.) verstanden. Dazu zählen nach Hurrelmann und Quenzel (ebd.) auch die „selbstbestimmte Nutzung von Medien zur individuellen Bereicherung“. Die Entwicklungsaufgabe Partizipieren bezieht sich auf „die Entfaltung eines persönlichen Systems von Werten und ethischen Prinzipien der Lebensführung, die mit der körperlichen und psychischen Konstitution und den eigenen Verhaltens- und 
Handlungsmöglichkeiten in Übereinstimmung stehen und eine sinnvolle und persönlich erfüllende Lebensführung ermöglichen“ (ebd.: 26f.).

In Bezug auf ihre gesellschaftliche Dimension, die bei jungen Erwachsenen in einer ihnen bisher nicht bekannten Weise relevant wird, da es nun heißt, sich möglichst selbständig als Mitglied einer Gesellschaft zu behaupten, lassen sich diese Entwicklungsaufgaben folgendermaßen skizzieren: Im Zentrum stehen die mehr denn je wichtigen, gar notwendigen Aspekte der eigenen Handlungsfähigkeit und Handlungskompetenz (Hurrelmann/ Quenzel 2016: 27). Denn nach der Beendigung der Schule stellt sich für junge Menschen die Herausforderung, sich für die gesellschaftliche Mitgliedsrolle eines Berufstätigen zu qualifizieren und entsprechende kognitive und soziale Fähigkeiten sowie berufsrelevante Fachkenntnisse zu erwerben, um im Zuge einer Berufstätigkeit den nötigen Lebensunterhalt selbstständig finanzieren zu können. Dabei spielt das Ausbildungsumfeld „als einer der wichtigsten funktionalen Ausschnitte im Leben junger Menschen“ (Ortner 2014: 78) eine wichtige Rolle. „Ab dem Eintritt ins Berufsleben bietet zudem das Arbeitsumfeld Gelegenheit für einschlägige Erfahrungen. Das Augenmerk bei diesen beiden Bereichen gilt ebenfalls den Personen, d.h. den Lehrenden, Arbeitgebern und Arbeitgeberinnen, Kollegen und Kolleginnen, mit denen junge Menschen in den unterschiedlichen Stufen ihres schulischen und beruflichen Werdegangs interagieren" (ebd.; Hervorheb. im Original).

Mit Blick auf eine weitere zentrale Entwicklungs- und Lebensaufgabe, die Kompetenz zur Bindung, ist nunmehr die Herausforderung verbunden, sich emotional und sozial von den Eltern zu lösen und eigene, tragfähige Kontakte zu Freunden und Gleichaltrigen aufzubauen sowie, wenn gewünscht, eine „liebevolle, intime Partnerschaft“ (Hurrelmann/ Quenzel 2016: 27) einzugehen.

Daneben sind junge Menschen als Erwachsene erstmals herausgefordert, im Wirtschaftsleben zurecht zu kommen und Handlungskompetenz im „Konsumieren“ zu erlangen, um, wie Hurrelmann und Quenzel (2016: 28) dies beschreiben, „einen selbstständigen und an den eigenen Bedürfnissen und Interessen ausgerichteten Umgang mit allen Angeboten des Wirtschafts-, Freizeit- und Mediensektors und seinen vielfältigen Entspannungs-, Selbsterfahrungs- und Unterhaltungsprogrammen einschließlich seiner finanziellen Kosten einzuüben“. Die Rolle als mündiger Bürger bzw. mündige Bürgerin zu erlangen, erfordert als weitere Entwicklungsund Lebensaufgabe, sich als politischer Bürger oder politische Bürgerin zu begreifen und die Kompetenz des Partizipierens zu erwerben und aus- 
zubauen. „Hier geht es darum, die Fähigkeit zur aktiven Beteiligung an Angelegenheiten der sozialen Gemeinschaft zu erlangen“ (ebd.).

Ähnlich wie Hurrelmann und Quenzel umreißen auch Krampen und Reichle (2008) die zentralen Entwicklungsaufgaben im „frühen Erwachsenenalter" - wie sie diese Lebensphase nennen - bei jungen Menschen zwischen 18 und 29 Jahren und führen die folgenden wesentlichen Aufgaben an: Ablösung von der Herkunftsfamilie, Berufsausbildung und Berufseintritt, Intensivierung und Differenzierung sozialer Beziehungen sowie Verantwortlichkeiten (wie etwa Partnerwahl und Elternschaft). Sie definieren die Lebensphase des frühen Erwachsenalters insgesamt als „Zeit der Beziehungs- und Verantwortungsentwicklung" (Krampen/ Reichle, 2008: 338), und dies mit Bezug auf die persönlichen und sozialen Kontexte, vor allem im Privatleben wie Partnerschaft und Freundschaft, aber auch in gesellschaftlichen Gruppen und im Beruf (siehe auch Heismann 2012: 41).

Guglhör-Rudan weist darauf hin, dass vor allem die Kategorisierung der Autonomie von Hofer und Pikowsky (2002) zum Verständnis des jungen Erwachsenenalters Erklärungskraft zukommen. Hofer und Pikowsky benennen dazu vier Teilbereiche: die emotionale Autonomie, Verhaltensautonomie, kognitive Autonomie und die ökonomische Autonomie. „Die verhaltensmäßige und ökonomische Autonomie ist nun ein wichtiger Entwicklungsbereich, verglichen mit der emotional-kognitiven Autonomieentwicklung des Jugendalters. Erwachsene unterscheiden sich von Jugendlichen bzw. jungen Erwachsenen dadurch, dass sie in allen eben genannten Bereichen Autonomie entwickelt haben (Reinders 2003)" (Guglhör-Rudan 2012: 51). Allerdings setzen, wie Guglhör-Rudan (ebd.) mit Bezug auf Baumrind (1991), Hofer und Pikowsky (2002) sowie Schneewind (1999) hervorhebt, „Eltern der Autonomieentwicklung Grenzen, die im Idealfall von der Kindheit (Erziehung) über das Jugendalter bis ins junge Erwachsenenalter (z. B. Ratschläge, Bedingungen) langsam schwächer werden, um irgendwann die Verantwortung den (erwachsenen) Personen selbst zu überlassen": Die Beziehung zwischen Eltern und Jugendlichen entwickele sich allmählich in Richtung einer gleichberechtigteren Partnerschaft; dabei bauen die jungen Menschen auf Erfahrungen mit Gleichaltrigen auf.

Arnett (2004; Hervorheb. im Original) umreißt die Phase zwischen Jugendlichen und Erwachsenen in seiner Studie als Emerging Adulthood und beschreibt sie als heterogen; junge Erwachsene haben oft noch nicht ihren Beruf gefunden, sondern sind noch in einer Ausbildung und daher auch noch nicht finanziell völlig unabhängig. Dennoch leben einige bereits nicht mehr zu Hause bei ihren Eltern, einige bereits in Partnerschaften 
(Arnett 2004) und sind stärker als noch im Jugendalter herausgefordert, ihren Alltag selbst zu organisieren und eigene Pläne, Wünsche und Ideen zu entwickeln. Zur Einordnung dieses Konzepts gibt Arnett (2016: 234; Hervorheb. im Original) zu bedenken, dass ,all human life stage concepts are socially, culturally, and historically grounded, rather than being biologically based and universal." Ohne diesen Faktor als den entscheidenden zu klassifizieren, macht Arnett deutlich, dass Unterschiede im sozio-ökonomischen Status und in den Lebensbedingungen relevant sind: „Variations in socioeconomic status and life circumstances also determine the extent to which a given young person may experience emerging adulthood, even within a country that is affluent overall“ (Arnett 2004: 22). Arnetts Untersuchungen zeigen die Relevanz des sozialen Hintergrunds für die im Mittelpunkt der Sozialisationsstudie stehenden sozial benachteiligten jungen Erwachsenen: "Young people in the lower social classes experience their late teens and $20 \mathrm{~s}$ as a time of struggling to enter an unpromising and unwelcoming labor market (...). They look at work not as a form of selfexpression and identity fulfillment but as a way to make a living and seek only to get a stable job that pays a decent wage. When they look to the future, they see not a wide open expanse of possibilities but only a succession of closed doors." (Arnett 2016: 227)

Für die Sozialisationsstudie stellt sich vor diesem Hintergrund die Frage, ob sich dieses auf Studien in den Vereinigten Staaten von Amerika zurückgehende Forschungsergebnis auch bei den jungen Erwachsenen des Panels nachweisen lässt, oder ob das in Österreich und Deutschland weitaus stärker ausgeprägte Netz des Sozialstaates auch ihnen größeren Freiraum und persönliche Entfaltungsmöglichkeiten mit Blick auf die Wahl eines gewünschten Berufs ermöglicht. Wie die Ergebnisse der Bildung von Familientypen gezeigt haben (Paus-Hasebrink/ Kulterer/ Oberlinner 2017; Paus-Hasebrink/ Kulterer/ Sinner 2019), ließen sich innerhalb des Panels klar erkennbare Unterschiede in Bezug auf die sozio-ökonomischen und sozio-emotionalen Bedingungen und die jeweils damit verbundene Alltagsbewältigung der Familien feststellen. Bleiben diese Unterschiede nunmehr auch für die jungen Erwachsenen gültig? Oder lassen sich Veränderungen hin zu einem anderen Typ identifizieren, der von der vorherigen Typenzuordnung der Familien abweicht? Spielen Unterschiede zwischen den Geschlechtern dabei eine Rolle? Um diesen Fragen differenziert nachzugehen, bedarf es eines theoretischen Ansatzes, der in der Lage ist, feine Unterschiede im Sinne Bourdieus dingfest zu machen. Dazu werden im 
Folgenden erneut die im Laufe der Studie entwickelten analytischen Konzepte benannt ${ }^{9}$.

\subsection{Lebensführung, sozialer Ort und feine Unterschiede in der Lebensbewältigung - drei analytische Konzepte zur Rekonstruktion (kommunikativer) Praktiken}

Sozialisationsprozesse sind an die Lebenswelt eines Individuums gebunden. Wo und wie ein Mensch seit frühester Kindheit aufwächst, welche sozio-ökonomischen, aber auch welche sozio-emotionalen Ressourcen ihm in seiner Familie bzw. Kernbeziehungsgruppe zur Verfügung stehen, ist - dies hat sich im Laufe der Langzeitstudie mehr und mehr herauskristallisiert - von höchster Relevanz.

Lebenswelt realisiert sich in der alltäglichen Lebensführung (Kudera 2001). Dieser Ansatz stellt das Subjekt in den Mittelpunkt, dies allerdings ohne strukturelle Dispositionen der alltäglichen Lebensführung zu missachten. Sie müssen stets mitgedacht werden; in ihnen schlagen sich die feinen Unterschiede nieder, wie dies Bourdieu $(1989,1996)$ nennt. Diese entwickeln sich in spezifischen sozialen Milieus und prägen sich als spezifischer Habitus in grundlegenden Anschauungsweisen aus, die die Angehörigen desselben Milieus weitgehend teilen und in denen sie sich von den Angehörigen anderer sozialer Milieus unterscheiden. Das soziale Milieu markiert den sozialen Ort, an dem Kinder und ihre Eltern ihre Erfahrungen machen - mediale wie nicht-mediale -, Kinder ihre Identität aufbauen, Handlungskompetenz erwerben und unter Beweis stellen, sich selbst und ihre Umwelt beurteilen, bewerten und einordnen. Das soziale Milieu wird nach Stefan Hradil (1999: 28; siehe auch Hradil 2008) geprägt durch die soziale Lage der Familie und die sie kennzeichnenden Faktoren Einkommen, Beruf bzw. ausgeübte Arbeit, formale Bildung und Wohnsituation der Erziehungsberechtigten, bei denen das Kind aufwächst.

Das Individuum, auch bereits im Kindes- oder Jugendalter, mehr noch im jungen Erwachsenalter legt sein praktisches Handeln als einen Versuch an, kraft seiner bzw. ihrer Kapitalien die ins Auge gefassten Chancen des jeweiligen sozialen Ortes zu verwirklichen (Habermas 1988: 473; Weiß

9 Herleitung und Ausdifferenzierung der theoretischen Fundierung der Studie wurden bereits an unterschiedlichen Stellen ausführlich dargelegt; deshalb soll darauf an dieser Stelle nicht erneut tiefergehend eingegangen werden (siehe zum theoretischen Ansatz insbesondere Paus-Hasebrink 2017a und 2019). 
2000: 48). Dazu setzt es als soziale und symbolische Kapitalien auch (digitale) Medien ein, um seinen persönlichen Nutzen im Kontext seiner Entwicklungsaufgaben, etwa in der Auseinandersetzung mit dem eigenen Selbstbild oder zur Pflege von Kontakten mit Peers, zu maximieren.

Auf diese Weise wird „die objektive Struktur sozial ungleicher Handlungsbedingungen transformiert in die subjektive Struktur divergierender Lebensentwürfe" (Weiß 2000: 49). Dabei handelt es sich um einen komplexen, von der formalen Bildung, dem Geschlecht und damit verbundener Körperlichkeit des je Einzelnen mitbestimmten psycho-sozialen Prozess der Identitätsbildung und Identitätsbehauptung.

Um Sozialisationsprozesse im Zusammenhang untersuchen zu können, wurden im Rahmen der Studie drei analytische Konzepte entwickelt: Handlungsoptionen, Handlungsentwürfe und Handlungskompetenzen (siehe Paus-Hasebrink 2017a; 2019).

- Handlungsoptionen bezeichnen das für das Individuum, ein Kind, seine Geschwister und seine Eltern in der Familie, faktisch existierende Arrangements der objektiven Merkmale der sozialen Lebenslage - sie bedeuten eine Anordnung von Ermöglichungen und Beschränkungen.

- Handlungsentwürfe kennzeichnen die Ziele und Pläne, die sich bei einem Kind und seinen Bezugspersonen in seiner Familie beobachten lassen. Handlungsentwürfe sind das, was einzelne Menschen aus dem Zusammenspiel der gegebenen Bedingungen und ihres jeweiligen ,Eigensinns' als ihren Lebensplan entwickeln.

- Handlungskompetenzen bezeichnen, wie sich die dem Individuum zugänglichen materiellen, kulturellen und sozialen Ressourcen seines Milieus zur Umsetzung seiner Lebensentwürfe in den kognitiven und motivationalen Voraussetzungen seines Handelns und damit in seinen Handlungspraktiken niedergeschlagen haben.

In diesem Zusammenhang ist es wichtig darauf hinzuweisen, dass die zentralen Analysekonzepte keine voneinander losgelöst zu verstehenden Konzepte sind; sie beschreiben vielmehr mit jeweils spezifischem Blick auf Handlungsoptionen, Handlungsentwürfe und Handlungskompetenzen die miteinander verbundenen Interaktionen zwischen den im Mittelpunkt stehenden jungen Erwachsenen und ihren jeweiligen Sozialisationskontexten in ihrem Alltag. 


\subsection{Methodenrepertoire der Panel-Langzeitstudie}

In der Panel-Langzeitstudie wurde ein regelmäßig sensibel weiterentwickeltes Mehr-Methoden-Design eingesetzt, das sich des Konzepts der Triangulation bedient (siehe Paus-Hasebrink/ Bichler 2008: 78ff.) und das es erlaubt, das Zusammenspiel von entwicklungspsychologischen Prozessen des Aufwachsens, d.h. die Relevanz der Entwicklungsaufgaben eines Kindes sowie die Lebensaufgaben seiner Eltern bzw. Erziehungsberechtigten und die sozio-ökonomischen (z.B. Ende der Arbeitslosigkeit, Verschlechterung der finanziellen Bedingungen) sowie die damit oft zusammenwirkenden sozio-emotionalen Faktoren seiner Familie (z.B. Trennung der Eltern, Schicksalsschläge in der Familie), aber auch die ganz spezifischen, das Individuum prägenden Wünsche und Interessen (seinen Eigen-Sinn) im Kontext zu analysieren und zu beschreiben.

Um die täglich ablaufenden Prozesse zentraler Sozialisationskontexte, allen voran der Familie und der Kernbeziehungsgruppe sowie später Partnerschaftsbeziehungen, näher umreißen und wissenschaftlich untersuchen zu können, wurden die Untersuchungen in Bezug auf makro-, meso- und mikroprozessuale Ebenen operationalisiert (Paus-Hasebrink et al. 2017: 45) und für die Fortsetzung der Panel-Langzeitstudie in Bezug auf die Lebenswelt junger Erwachsener ausdifferenziert:

Auf der Makro-Ebene finden sich die sozial-strukturell relevanten Faktoren wie eigenes Einkommen und eigene Bildung und berufliche Lage, aber auch die der Eltern und gegebenenfalls des Partners bzw. der Partnerin; sie bestimmen das soziale Milieu von Individuen, d.h. seine von der sozialen Lage geprägten Lebensbedingungen, in entscheidender Weise mit. Aber auch wirtschaftliche, soziale, kulturelle und mediale Kontexte eines Landes sind relevant.

Auf der Meso-Ebene, der Ebene der Familie bzw. Kernbeziehungsgruppe mit ihren spezifischen Beziehungsstrukturen zwischen Eltern und Kind(ern), Partnern und Partnerinnen sowie Geschwistern, aber auch Angehörigen der entfernteren Familie und Freunde und Freudinnen sowie Peers als Teile des sozialen Netzwerks, aber auch von Kollegen und Kolleginnen, die allesamt das Klima der Familie bzw. Kernbeziehungsgruppe mitprägen, stellt sich die Frage nach den sozialen Ressourcen jeder einzelnen Einheit in Abhängigkeit ihrer makro-strukturellen Verankerung. In diese Zusammenhänge bringt bzw. bringen sich das Individuum, im Fall der vorliegenden siebten Erhebungswelle der Langzeitstudie die jungen Erwachsenen, auf der Mikro-Ebene aus einer bestimmten Position heraus ein. 
Auf der Mikro-Ebene, also der Ebene des/der jeweiligen im Zentrum der Untersuchung stehenden jungen Erwachsenen, sind die Aspekte Alter und Geschlecht und die damit verbundenen spezifischen Lebensaufgaben relevant, ebenso wie der davon mitbestimmte Medienumgang des Individuums. Dieses Konglomerat stellt einen zentralen Teilaspekt des Gesamtforschungsfeldes dar.

\subsubsection{Das Methodendesign der ersten sechs Erhebungsphasen}

In allen vorangegangenen Panelphasen (siehe dazu Paus-Hasebrink/ Bichler 2008 sowie Paus-Hasebrink/ Kulterer 2014; Paus-Hasebrink et al. 2018) wurden als wichtigstes Erhebungsinstrument Leitfadeninterviews sowohl mit den Kindern, später Heranwachsenden, und mit ihren Eltern bzw. mit einem Elternteil eingesetzt. Dabei wurde stets darauf geachtet, dass ein Junge von einem männlichen und ein Mädchen von einem weiblichen Mitglied des Erhebungsteams befragt wurde (siehe dazu Habermehl 1992). Diese Vorgehensweise diente dazu, die Scheu vor einem Gespräch über gerade bei zunehmendem Alter der Kinder für sie eventuell heikle Erfahrungen und Erlebnisse, allen voran sexueller Art, zu reduzieren. Ziel der Interviews war es, zu untersuchen, welche Bedeutung die jungen Menschen ihrem eigenen sowie dem Medienumgang ihrer Eltern zuschrieben und wie - vice versa - die Eltern den Medienumgang ihrer Kinder wahrnahmen und wie sie ihre (Medien-)Erziehung verstanden. Gefragt wurde auch danach, welche Medienangebote die Eltern selbst in ihrem Alltag nutzen und welche Bedeutung sie ihnen zuschreiben.

Etwaige Differenzen zwischen den Eltern- und Kinderaussagen ließen auf der einen Seite Rückschlüsse auf den Umgang zwischen Eltern und Kindern in der Familie zu, mithin auf ihren Umgang miteinander in der Familie, das heißt ihr doing family ${ }^{10}$ und in diesem Zusammenhang auf das Familienklima. In diesem Zusammenhang war es auch relevant, wie die Familie (digitale) Medien gebraucht (Lange 2020) und wie Eltern mit den Medienpraktiken ihrer Kinder umgehen. Mascheroni, Ponte und Jorge (2018) sprechen dabei - so auch der Titel ihres Herausgeberwerks - von „digital parenting“. Mit Hilfe der Leitfadeninterviews ließ sich erheben, welche Relevanz die Heranwachsenden den sich während der Panelphasen im Kontext der Mediatisierung verändernden Medienangeboten und Mediendiensten zuwiesen und welchen Stellenwert diese tatsächlich in

10 Siehe dazu ausführlicher Paus-Hasebrink 2017a sowie Paus-Hasebrink 2019. 
ihrem Alltag einnahmen, d.h. wie sich der Mediengebrauch der Heranwachsenden im Kontext ihres zunehmenden Alters veränderte. Auch die medienerzieherischen Bemühungen der Eltern und welche Rolle der eigene Medienumgang der Eltern für die (Medien-)Sozialisation ihrer Kinder spielte, war Ziel der ersten sechs Erhebungsphasen. Die Interviews gaben Aufschluss darüber, wie Kinder und Eltern die über die Jahre der Erhebung mit zunehmendem Alter der Kinder, insbesondere in der Jugendphase und Pubertät aufs Engste verbundenen Abgrenzungsprozesse zu ihren Eltern bewältigten. Mit in den Blick genommen wurden in allen Erhebungsphasen auch andere Sozialisationskontexte, etwa die Freundschaften von Kindern und Eltern, ihre jeweiligen Peers, die Rolle von Kindergarten und Schule sowie der Beginn des Arbeitsprozesses, einer Lehre oder weiterführenden Schule.

Zusätzlich zu den Leitfadeninterviews wurde ein standardisierter, an die Eltern bzw. Erziehungsberechtigten gerichteter Fragebogen eingesetzt, um zentrale Aspekte lebensweltlicher Konstellationen der Familien des Panels kontinuierlich zu erheben und einen Vergleich zu den zuvor durchgeführten Erhebungswellen vornehmen zu können. Nach der Durchführung der Interviews im Rahmen eines Erhebungsbesuchs erhielten die Eltern einen schriftlichen Fragebogen ausgehändigt, in dem sie um Auskunft zu ihrer Lebens- und Wohnsituation der Familie gebeten wurden (Familienkonstellation; berufliche und finanzielle Situation; Wohnsituation der Familie etc.). Der Fragebogen diente neben den Interviews und Beobachtungsprotokollen dazu, die Handlungsoptionen der Familien zu beschreiben.

Ergänzt wurden die Angaben auf den Fragebögen durch Beobachtungsprotokolle, auf denen das Erhebungsteam strukturiert Eindrücke zur Lebensumgebung und Wohnsituation sowie zur Medienausstattung der Familien festhielt. Darüber hinaus bot das jeweilige Beobachtungsprotokoll auch die Möglichkeit, Notizen zum Umgang der Familienmitglieder miteinander zu notieren, aber auch Eindrücke zu den jeweiligen Untersuchungspersonen, Eltern wie Kindern, aber auch anderen anwesenden Familienmitgliedern, zu ihrem Auftreten, ihren Eigenheiten etc. zu vermerken. Die Beobachtungskriterien blieben über die gesamte Erhebungszeit stabil (Paus-Hasebrink/ Kulterer 2014: 381). In der Kombination mit den Beobachtungsprotokollen war es möglich, die Handlungsentwürfe der Panelangehörigen zu untersuchen und die Struktur der Lebensführung von Eltern und Kindern zu rekonstruieren sowie ihrem jeweils spezifischen Verhalten in der Familie nachzugehen, d.h. wie sie etwa Konflikten begegneten oder auch Nähe zueinander praktizierten und wie sie ihren Alltag in Peer Groups, Freundschaften, Schulklassen und in der Nachbarschaft 
gestalteten, welche Medien sie in welchen Situationen nutzten, welche Wünsche, Ängste, aber auch welche Zukunftspläne sie hatten. In der Folge von Bourdieu (1996: 110) und Weiß (2001: 12) wurden die Handlungskompetenzen mit Blick auf die materiellen, kulturellen und sozialen Ressourcen der Panelangehörigen, die ihnen als kognitive und motivationale Ausstattung zur Verfügung standen, erfasst. In diesem Kontext wurden Fragen nach den Einstellungen der Kinder und ihrer Eltern zu Kindergarten, Schule, Ausbildung und Beruf gestellt, die Eltern nach ihren Vorstellungen und Plänen wie auch ihren Kapazitäten in Bezug auf die Erziehung ihrer Kinder bzw. der Eltern und Kinder im Umgang mit Medien befragt. Beide Erhebungsinstrumentarien dienten während der ersten sechs Panelwellen der Langzeitstudie dazu, eine Globalcharakteristik der befragten Familie zu erstellen (siehe auch Schütze 1977; Charlton/ Neumann 1986; siehe Paus-Hasebrink/ Kulterer 2014: 57f. sowie Paus-Hasebrink et al. 2017: 50ff.).

Um den Entwicklungsprozessen und -aufgaben von jungen Menschen in der Lebensphase Jugend sowie den sich im Kontext der Mediatisierung veränderten Medienangeboten und -diensten und der nunmehr deutlich gestiegenen Bedeutung von Angeboten Sozialer Medien gerecht werden zu können, wurden zu den bereits erprobten Erhebungsinstrumenten ab der fünften Erhebungswelle drei neue hinzugefügt: die Erstellung von Netzwerkkarten (zur Erhebung der Relevanz von Medien und Bezugspersonen), Fotografien von zentralen Orten im eigenen Zimmer („bedroom culture" (Bovill/ Livingstone 2001: 3)) und die Methode des Lauten Denkens beim gemeinsamen Ansehen eines von den Probanden und Probandinnen ausgewählten Angebots aus dem Spektrum Sozialer Medien. In der Anordnung und spezifischen Kombination von Schreibtisch, Computer, Fernseher, Stereoanlage und Möbeln, dem Vorhandensein von Postern oder Deko-Artikeln drücken sich der Eigen-Sinn der Jugendlichen sowie die für sie relevanten sozialen Praktiken und die ihnen wichtigen Elemente ihrer Jugendkultur aus (siehe Lincoln 2013: 318f.).

Die Kombination des im Wesentlichen qualitativ ausgerichteten Methodeninstrumentariums diente dazu, die Handlungsoptionen, Handlungsentwürfe und Handlungskompetenzen von Eltern bzw. Erziehungsberechtigten und ihren Kindern möglichst transparent zu machen und die Lebenssituation und Lebensführung bestmöglich zu erfassen. 


\subsubsection{Das Erhebungsrepertoire in der siebten Erhebungsphase - Leitfadeninterviews über Telefon, WhatsApp-Call oder Skype ${ }^{11}$}

Schon als Abschluss der sechsten Erhebungsphase waren 2017 Telefoninterviews eingesetzt worden. Sie dienten dazu, die Daten dieser schon im Januar und Februar 2016 durchgeführten Erhebungen zu ergänzen. Ziel war es, einen möglichst aktuellen Stand zur Lebenssituation der Jungen und Mädchen sowie zumindest einer erziehungsberechtigten Person zu erhalten. In diesen Gesprächen, die erneut, wenn es um einen Jungen ging von einem Mitarbeiter des Teams, und bei Mädchen von einer Mitarbeiterin geführt wurden, konnten die von den Befragten als zentrale Veränderungen wahrgenommen Ereignisse sowie der aktuelle Stand ihrer beruflichen oder schulischen Situation erfasst werden. Einige Jungen und Mädchen sahen sich in der sechsten Erhebungswelle mit einer von Unsicherheit geprägten Lebenssituation konfrontiert; sie suchten nach einer Lehrstelle oder wussten noch nicht, ob sie die Schule beenden und in den Arbeitsmarkt gehen sollten. Bei einigen stand die Frage im Raum, ob sie die Arbeitslosigkeit nach der Beendigung der Schule würden überwinden können etc. Wichtig war auch die Frage nach der aktuellen Situation im Familien- und Freundeskreis, ob es seit der letzten Erhebung Veränderungen gegeben hat und ob aktuell eine Partnerschaft besteht. Außerdem wurde in diesen Telefoninterviews erneut nach den nun aktuellen Lieblingsmedien gefragt und wie die jungen Leute sie in ihren Alltag einbinden.

In der Fortsetzung 2020, der siebten Erhebungsphase, wurden Telefon-, WhatsApp-Call oder Skype-Interviews eingesetzt. ${ }^{12}$ Der fernmündliche Weg war auch deswegen sehr praktikabel, da vor der Erhebung nicht bekannt war, welche jungen Erwachsenen noch im Elternhaus anzutreffen wären und welche inzwischen eigene Hausstände haben. Im Mittelpunkt der niederschwelligen und flexiblen Erhebungsform stand die Frage, wie sich das Leben der mittlerweile jungen Erwachsenen der Panelstudie und ihrer Eltern entwickelt hat. Dazu wurde der Leitfadenkatalog reduziert und die Konzentration auf die Veränderungen in den vergangenen Jahren sowie die aktuelle Lebenssituation betreffende Fragen gelegt. Ergänzend wurde aber auch erhoben, wie die jungen Erwachsenen und ihre Eltern, rückblickend auf immerhin 15 Jahre Gesamtdauer der Studie, die Besuche

11 Wie auch in den vorherigen Erhebungswellen erhielten die Teilnehmenden eine Aufwandsentschädigung.

12 Der Leitfaden zur Erhebung ist unter folgendem Link einsehbar: https:/www.no mos-shop.de/isbn/978-3-8487-8382-3. 
des Teams der Sozialisationsstudie empfunden haben. Infolge der Covid-19Pandemie erwies sich die Entscheidung für diesen Erhebungsweg nicht nur als eine gute, sondern auch die einzig mögliche Strategie, da Besuche bei den jungen Erwachsenen und ihren Eltern aufgrund der mit der Pandemie verbundenen massiven Einschränkungen (etwa weitreichende Kontaktverbote) nicht mehr möglich waren und daher auf ein Face-toFace-Interview ebenso verzichtet werden musste wie auf eine teilnehmende Beobachtung. Um die Fragen zur aktuellen Lebenssituation möglichst umfassend zu gestalten, wurden in den Telefoninterviews auch Fragen zur Wahrnehmung und zum Umgang mit der Pandemie und die Rolle der Medien integriert. Da es sich mittlerweile um junge Erwachsene und nicht mehr um Kinder oder Jugendliche handelte, erschien zudem ein Telefoninterview aus methodologischer Perspektive nicht als problematisch.

Zwar gelten persönlich als Face-to-Face-Interviews geführte Befragungen in der qualitativen Forschung als das Nonplusultra - so erlauben sie, „die Stimme in Verbindung mit der Körpersprache berücksichtigen zu können" (Schulz/ Ruddat 2012: o. S), dennoch bieten auch telefonisch geführte Interviews Vorteile. Wie Schulz und Ruddat berichten, ermöglicht „die Durchführung telefonischer Leitfadeninterviews unter Umständen detailliertere und ehrlichere Einblicke in die Wünsche, Sorgen und Ängste der Befragten“ (Schulz/ Ruddat 2012: o. S). Als mögliche Gründe für die, wie sie berichten, „teilweise höhere Qualität der Telefoninterviews"13 führen sie die Anonymität der Gesprächssituation, den Ort des Interviews, das Telefon als selbstbestimmtes Medium sowie den verringerten Einfluss (etwa der Wegfall einer eventuellen mimischen und gestischen Einflussnahme) von Merkmalen der interviewenden Person.

\subsubsection{Auswertungsmethoden der Panel-Langzeitstudie}

Die Auswertung auch der siebten Erhebungsphase erfolgte nach der bereits seit Beginn der Studie eingesetzten Strategie (siehe dazu ausführlicher Paus-Hasebrink et al. 2018). Der Auswertungsprozess gliederte sich in

13 Schulz und Ruddat (2012) haben einen Vergleich zwischen Face-to-Face-Interviews und Telefoninterviews vorgenommen. Sie haben eine explorative qualitative Studie über die private und berufliche Lebenssituation von Wissenschaftlern und Wissenschaftlerinnen in kinderlosen Doppel-Karriere-Beziehungen vorgenommen; dabei fanden zwölf Interviews über Telefon und neun als Face-to-FaceInterviews statt. 
mehrere Phasen und Teilschritte, die in der folgenden Abbildung schematisch dargestellt sind. Der einzige Unterschied zu den vorherigen Analysen bestand darin, dass in der siebten Phase lediglich die Daten der Interviews zur Auswertung herangezogen wurden.

Alle Auswertungsphasen - insbesondere die Codierung und Auswertung - sind eng aufeinander bezogen und durch wechselseitige Rückbezüge gekennzeichnet, um ein zentrales Charakteristikum qualitativer Datenanalyse zu erfüllen. Konkret bedeutet dies, dass z.B. während der einzelnen Auswertungsschritte Recodierungen am Material vorgenommen werden können und auch das Ausgangsmaterial (Audio-Aufzeichnung) erneut herangezogen werden kann, um dann Klarheit zu schaffen, wenn für die Interpretation wichtige Nuancen durch die Verschriftlichung verloren gegangen sind oder Unklarheiten bestehen.

\begin{tabular}{|c|c|c|c|c|}
\hline & Phase: & \multicolumn{2}{|l|}{ Transkription der Einzelinterviews } & \multirow{2}{*}{ 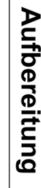 } \\
\hline 2. & Phase: & $\begin{array}{l}\text { Computerunterstützte Aufbereitung } \\
\text { des Interview-Materials }\end{array}$ & $\begin{array}{l}\text { Erstellung eines Codewortbaums } \\
\text { Themen- und sinnspezifische } \\
\text { Codierung des Materials } \\
\text { Pflege der Familienmatrizen }\end{array}$ & \\
\hline 3. & Phase: & Fokussierte Analyse & $\begin{array}{l}\text { Breite Auswertung aller Interviews } \\
\text { nach bestimmten Kategorien und } \\
\text { Dimensionen }\end{array}$ & \\
\hline & Phase: & Kontextuelle Einzelfallanalyse & $\begin{array}{l}\text { Detaillierte Auswertung jedes } \\
\text { Einzelinterviews } \\
\text { Erstellung einer } \\
\text { Globalcharakteristik jeder Familie }\end{array}$ & 胥 \\
\hline 5 & Phase: & Einzelfallbeschreibung & $\begin{array}{l}\text { Tiefgreifende Analyse und detaillierte } \\
\text { Darstellung von allen } 18 \text { Familien }\end{array}$ & อี \\
\hline 6 & Phase: & Typenbildung & $\begin{array}{l}\text { Überprüfung der Typenzugehörigkeit mit } \\
\text { Blick auf die Veränderungen bei den } \\
\text { Eltern und den jungen Erwachsenen }\end{array}$ & \\
\hline
\end{tabular}

Abbildung 1: Die aktualisierten Phasen der Aufbereitung und Auswertung der Daten aus der Panelstudie. Quelle: Eigene Darstellung.

Bei der Transkription der Einzelinterviews wurde erneut streng auf die Anonymisierung aller Eigennamen und Orte geachtet. Sie erfolgte in dialekt-bezogener Sprache und in literarischer Umschrift, da für die Analyse die inhaltliche Bedeutung des Gesagten von Bedeutung ist (siehe dazu und zur Transkription allgemein Kowal/ O'Connell 2005 sowie Dresing/ Pehl 2015) bedeutsam waren. Auf diese Weise konnte am besten die Authenti- 
zität der Darstellung der Gespräche gewährleistet werden. Nach der Transkription wurde das Material induktiv und deduktiv thematisch kodiert (Flick 2013), dies erfolgte computergestützt unter Verwendung des Programms MAXQDA (siehe dazu genauer Paus-Hasebrink et al. 2017). ${ }^{14}$ Die Materialauswertung folgte erneut der von Paus-Hasebrink und Keuneke entworfenen (siehe dazu Paus-Haase et al. 1999) und von Paus-Hasebrink in einer Reihe von Studien weiterentwickelten (u.a. Paus-Hasebrink et al. 2004; Paus-Hasebrink/ Schmidt/ Hasebrink $2011^{2}$ ) methodischen Vorgehensweise einer fokussierten und kontextuellen Analyse. Im Mittelpunkt der siebten Phase der Sozialisationsstudie stand nunmehr die vertiefende Einzelfallanalyse zu allen 17 beteiligten jungen Erwachsenen und einem Elternteil ( $\mathrm{n}=34$ ). Eine Familie (Familie Fein) nahm aus schwerwiegenden persönlichen Gründen nicht an der erneuten Befragung teil; doch auch sie wurde als Fallbeschreibung wie alle anderen auch mit Bezug auf die vorherigen Erhebungsphasen zumindest kurz vorgestellt. Der Schwerpunkt des in den bisherigen Panelphasen ebenfalls durchgeführten fokussierenden Analyseschritts lag nunmehr auf den beiden zentralen Parametern 1) Veränderungen seit der vorherigen Erhebungsphase in Bezug auf die private und berufliche Lebenssituation der jungen Erwachsenen und ihrer Eltern, mit Blick auf den Umgang mit Medien und dahinterstehende Strategien, sowie 2) der Wahrnehmung der aktuellen Lebenssituation mit Blick auf die Verfolgung von Plänen und Zielen für die Zukunft. Einer ähnlich sorgfältigen Analyse unterlagen auch die Aussagen der Befragten zum Umgang mit der Covid-19-Pandemie und die so genannten Meta-Fragen der Erhebung 2020, in denen die jungen Erwachsenen und ihre Eltern darum gebeten wurden, rückblickend zu ihren Erfahrungen mit der Teilnahme an einer so langandauernden Studie und den Besuchen des Forschungsteams Stellung zu nehmen.

In den kontextuellen Einzelfallanalysen standen detaillierte Auswertungen der jeweiligen Einzelinterviews der jungen Erwachsenen und ihrer Eltern im Vordergrund. Um die Veränderungen seit der Erhebungsphase 2016 bzw. 2017 beurteilen zu können, wurde bei jeder Fallbeschreibung auch ein Rückblick auf alle vorherigen Erhebungsphasen gegeben. Dazu dienten die Matrix aller vorherigen Erhebungsphasen sowie die Globalcharakteristiken (Schütze 1977) bzw. Familienprofile der vergangenen Jahre als Ausgangspunkt. Diese Matrix enthält über alle Erhebungsphasen hin-

14 Siehe dazu den Codewortbaum unter: https://www.nomos-shop.de/isbn/978-3-84 87-8382-3. 
weg zu allen Analysekategorien die Kernaussagen aus den Interviews, übersichtlich zu jeder Familie aufgeschlüsselt und ihr zugeordnet.

\subsubsection{Zur Typenbildung}

In den ersten Erhebungswellen hatte sich gezeigt, dass das Zusammenspiel der Handlungsoptionen, Handlungsentwürfe und Handlungskompetenzen der Familienmitglieder unterschiedlich ausfiel und damit zu unterschiedlichen Sozialisationskontexten führte. Diese zeigten sich darin, wie die Familienmitglieder vor dem Hintergrund ihrer jeweiligen Situation, sozio-ökonomisch und sozio-emotional, ihr Leben als Familie gestalteten und bewältigten. Diese Erkenntnis führte dazu, nach dem Ende der vierten Erhebungswelle Familientypen zu bilden (siehe dazu Paus-Hasebrink/ Kulterer 2014: 239 sowie Kapitel 2), die sich in ihren zentralen Schritten an dem von Kluge (2000) vorgeschlagenen Vorgehen orientierte. Die Typenbildung erfolgte vor dem Hintergrund des Zusammenspiels der Handlungsoptionen, Handlungsentwürfe und Handlungskompetenzen sowohl der Heranwachsenden als auch ihrer Eltern. Der Begriff des Zusammenspiels betont, dass es sich nicht um voneinander unabhängige Einzelkonzepte handelt. Vielmehr ergibt sich jedes der Einzelkonzepte bereits zum Teil aus dem Zusammenspiel mit den jeweils anderen. So bestimmen sich die Handlungsoptionen etwa einerseits aus den subjektiv feststellbaren Faktoren der sozio-ökonomischen Bedingungen, der sozialen Lage, andererseits daraus, wie diese subjektiv wahrgenommen werden, was wiederum von den Handlungsentwürfen und den Handlungskompetenzen des Einzelnen abhängt, diese jedoch auch ihrerseits wiederum mitprägen. Wie die Familienmitglieder ihr Leben führten, schlug sich nieder in ihren sozio-emotionalen Familienstrukturen, die ihr Zusammenleben als Familie prägten. In der Art, wie es den Familien gelang, ihr Leben als Familie zu meistern, ließen sich zwischen den Familien des Panels Unterschiede bzw. Gemeinsamkeiten identifizieren. Mit Blick auf die jeweiligen Besonderheiten ergab sich in einer kontrastierenden Gegenüberstellung die Zuordnung der Familien zu dem Typ, dessen Merkmalskombination sie am stärksten in ihrer Lebensführung charakterisierte. Abweichende Tendenzen hin zu anderen Typen während einer oder mehrerer Untersuchungswellen wurden in den Erläuterungen der Zugehörigkeit jeder einzelnen Familie zu einem Typus diskutiert. Die in diesem Kontext gebildeten vier Familientypen dienen in der vorliegenden Publikation als Strukturierungsmerkmal zur Einordnung der Fallbeschreibungen (siehe 
dazu Kapitel 2). Zudem werden sie als Folie herangezogen, auf der alle jungen Erwachsenen und auch ihre Mütter und Väter erneut mit Blick auf die drei Analysekonzepte, operationalisiert durch die sozio-ökonomischen und sozio-emotionalen Bedingungen der Panelbeteiligten sowie ihre jeweiligen Praktiken der Alltagsbewältigung, hin beleuchtet werden (siehe dazu Kapitel 3). 


\section{Die Fallbeschreibungen ${ }^{15}$ - die jungen Menschen der Panel- Langzeitstudie auf dem Weg zum Erwachsenwerden und wie sie heute leben}

\subsection{Kontextuelle Analyse und Familientypen}

In den folgenden 18 Fallbeschreibungen ${ }^{16}$ stehen die subjektbezogenen Kontexte der jungen Erwachsenen und ihrer Eltern im Mittelpunkt, um tiefere Bedeutungszusammenhänge zwischen den sozialen, familialen und medialen Einflüssen auf die Sozialisation der jungen Männer und Frauen nachzuzeichnen. In den kontextuellen Einzelfallanalysen geht es um detaillierte Auswertungen der jeweiligen Einzelinterviews der jungen Erwachsenen und ihrer Eltern - zumeist der Mütter. Die kontextuelle Materialauswertung aller Daten erfolgte für jeden jungen Mann und jede junge Frau bzw. für ihre Familie mit Hilfe einer vorgefertigten, thematisch strukturierten Matrix in Anlehnung an die Auswertungsmethode des the-

15 Im Folgenden ist von ,Fallbeispiel' die Rede, wenn es sich um im Rahmen der (Medien-)Sozialisationsstudie ausgewählte typische Beispiele handelt. Der Begriff ,Fallbeschreibung' wird dann gewählt, wenn es um eine Panel-Familie im Kontext aller geht, so wie bei den im vorliegenden Kapitel beschriebenen Darstellungen der Familien im Laufe der Gesamtstudie mit besonderer Berücksichtigung der siebten Erhebungswelle.

16 An der siebten Erhebungswelle 2020 beteiligten sich von den seit der zweiten Erhebungswelle insgesamt 18 beteiligten Familien (ursprünglich zählten $20 \mathrm{Fa}$ milien zum Panel der Sozialisationsstudie) noch 17 junge Männer und Frauen und ein Elternteil an der Erhebung. Lediglich in einem Fall war der Vater während des Interviews anwesend und kommentierte zuweilen Aussagen seiner Frau. Familie Fein konnte zu Beginn der siebten Erhebungswelle zwar kontaktiert werden, Mutter und Tochter nahmen jedoch - so die Aussage der Mutter aus schwerwiegenden Gründen nicht an den Interviews teil. Frau Fein betonte im Telefonat zur Aufnahme des erneuten Kontakts, dass die Familie seit der Erhebung 2016 „Schlimmes“ erlebt habe und sich daher nicht in der Lage fühle, erneut für ein Interview zur Verfügung zu stehen. Dennoch wurde mit Blick auf die vergangenen Erhebungswellen eine Fallbeschreibung zu Olivia Fein und ihrer Mutter - im Kontext aller anderen Fallbeschreibungen - erstellt, um auf diese Weise nachvollziehbar zu machen, welche Relevanz den sozio-ökonomischen und sozio-emotionalen Lebensbedingungen dieser Familie zukommt und wie ihr Mangel an adäquaten Bewältigungsstrategien die (Medien-)Sozialisation von Olivia Fein geprägt hat. 
matischen Kodierens nach Flick (2013, siehe dazu Kapitel 1 sowie insbesondere Paus-Hasebrink et al. 2018). Die Kernpunkte bzw. Kernaussagen aus den Interviews wurden anschließend übersichtlich zu jeder Familie aufgeschlüsselt und ihr zugeordnet und über alle Erhebungswellen hinweg thematisch nebeneinandergestellt, so dass sich die Veränderungen in den einzelnen Rubriken über die Jahre hinweg im Überblick erschließen.

Auf Grundlage der kontextuellen Analyse wurden nach Ende der ersten Projektphase (2007) neun besonders auffällige und aussagekräftige Einzelfälle ${ }^{17}$ als ,Fallbeispiele ${ }^{6}$ ausgewählt und mittels Einzelfallbeschreibungen dargestellt (siehe Paus-Hasebrink/ Bichler 2008, S. 192ff.). Nach späteren Erhebungswellen wurde eine weitere Familie (Familie Öllinger) hinzugenommen, da sich in dieser Familie einer alleinerziehenden Mutter und ihrer Tochter im Sinne der Forschungsfragen wichtige Veränderungen vollzogen hatten, die ausführlich in einem Fallbeispiel nachgezeichnet wurden. Diese zehn Fallbeispiele (siehe dazu Paus-Hasebrink/ Kulterer 2014, S. 78ff. sowie Kulterer/ Paus-Hasebrink 2017, S.69ff. sowie PausHasebrink/ Kulterer/ Oberlinner 2017, S. 129ff.) ließen in besonderer Weise den Zusammenhang zwischen dem sozialen und insbesondere familialen Hintergrund und dem Medienumgang der jungen Menschen im Laufe ihrer Sozialisation erkennen. Diese Fallbeispiele wurden nach allen Erhebungswellen in detaillierter Form hinweg fortgeschrieben.

$\mathrm{Zu}$ allen anderen Familien wurden seinerzeit Steckbriefe ${ }^{18}$ angefertigt und fortlaufend gepflegt, bei denen es sich um Zusammenfassungen der wichtigsten Familienmerkmale, Entwicklungsschritte der Familie und des Kindes sowie des Mediengebrauchs in der Familie handelte. Nach der siebten und letzten Erhebungswelle 2020 wurden zu allen erfassten Familien einheitliche und ausführliche Fallbeschreibungen erstellt. Ziel ist es, im Sinne der Forschungsfragen der erneuten Erhebung nachzuzeichnen, wie sich die Lebenswege der Jugendlichen hin zu jungen Erwachsenen vollzogen haben, ob und wie es den jungen Menschen gelingt, mittlerweile einen stärker selbstbestimmten Weg zu gehen und sich von ihren Familien, allen voran ihren Eltern, zu lösen und nunmehr durch andere

17 Die ausgewählten Familien waren: Aufbauer, Boll, Fein, Holzner, Grubert, Landinger, Oblinger, Stab und Weiss. Als Kriterien dienten dazu „Ein-Eltern-Familie“ (Weiss, Holzner, Aufbauer); „kinderreiche Familie“ (Oblinger, Boll, Landinger); „Familien mit Migrationshintergrund“ (Stab) sowie „Familien, in denen sich im Verlauf der Panelstudie die lebensweltlichen Hintergründe deutlich verändert hatten " (Fein, Grubert) (siehe Paus-Hasebrink/ Bichler 2008, S. 192).

18 Alle Fallbeschreibungen und Steckbriefe können kostenfrei unter folgender URL abgerufen werden: https://www.nomos-shop.de/isbn/978-3-8487-8382-3. 
Handlungsoptionen (eigene finanzielle Mittel in Folge von weiterer Ausbildung, Lehre bzw. Berufseinstieg etc.) eigene Handlungsentwürfe zu entwickeln und mit welchen Handlungskompetenzen sie ihren Alltag und darin eingelagert auch ihren Mediengebrauch gestalten. Es geht darum, deutlich zu machen, wie sich die sozio-ökonomischen und sozio-emotionalen Bedingungen der jungen Menschen als junge Erwachsene zwischen der sechsten und siebten Erhebungswelle entwickelt haben und wie diese zur Zeit der siebten Erhebungswelle (März bis Juni 2020) aussehen. Dabei wird möglichst genau nachgezeichnet, ob und wie es den jungen Erwachsenen gelingt, ihren Alltag zu meistern und welche Kontexte und Aspekte dabei eine entscheidende Rolle spielen. Dieses Ziel steht im Mittelpunkt der folgenden 18 Fallbeschreibungen. In ihnen lässt sich im Kontext nachverfolgen, wie sich die jungen Menschen über nunmehr 15 Jahre verändert haben und welche lebensweltlichen Faktoren sich bei ihrer Entwicklung als besonders bedeutsam erwiesen, welche Sozialisationskontexte neben ihrem Alter und ihrem Geschlecht an Einfluss gewonnen und wie sich mediale Vorlieben und Gebrauchsweisen entwickelt und verändert haben. Die Fallbeschreibungen leuchten tief in die Lebenswelt der jungen Erwachsenen (und ihrer Familien) hinein, um ihre individuellen Entwicklungen - und darin auch ihren Mediengebrauch - mithin ihre (Medien-)Sozialisation, vor dem Hintergrund ihrer lebensweltlichen, sich dynamisch verändernden sozio-ökonomischen und sozio-emotionalen Bedingungen im Kontext zu erfassen. Dies war die Motivation, nach etwa 15 Jahren Begleitung der jungen Menschen und ihrer Familien im Rahmen der Panel-Langzeitstudie erneut den Blick auf die Fülle unterschiedlicher Aspekte im Kontext der Lebensführung der jungen Männer und Frauen zu richten und ihnen im Rahmen einer Einzelfallanalyse, aufbereitet als Fallbeschreibung, näher nachzugehen. Um dies zu ermöglichen, beginnt jede Fallbeschreibung mit einer kurzen Zusammenfassung der Lebenssituation und der zentralen Entwicklungen und Veränderungen im Lebensalltag der an der Panelstudie beteiligten Familien seit Beginn der Langzeitstudie 2005 bis zur sechsten Erhebungswelle 2016. Im Anschluss daran wird die aktuelle Lebenssituation des bzw. der jeweils im Mittelpunkt stehenden jungen Erwachsenen beleuchtet sowie danach die ihrer Eltern (zumeist ihrer Mütter). Im letzten Schritt richtet sich das Augenmerk auf den Mediengebrauch und eventuelle neue Schwerpunktsetzungen sowie Veränderungen im Medienrepertoire der jungen Erwachsenen und ihrer Eltern. Ein Fazit schließt die Fallbeschreibungen ab. 
Im Verlauf der Studie ${ }^{19}$ war mehr und mehr deutlich geworden, dass die sozio-ökonomischen Bedingungen, die soziale Benachteiligung ausmachen (Paus-Hasebrink/ Bichler 2007: 132), die Lebensführung der Familien in zentraler Weise prägten, dies allerdings nicht auf eine eindimensionale Weise. Vielmehr wurde deutlich, dass die verschiedenen Faktoren, die eine soziale Benachteiligung begründeten, erst im individuell von den Betroffenen mitbestimmten Zusammenspiel der jeweiligen Handlungsoptionen, Handlungsentwürfe und Handlungskompetenzen der einzelnen Panelangehörigen insbesondere mit den ihr Familienleben und ihre Familienführung mitbestimmenden sozio-emotionalen Konstellationen auf eine jeweils spezifische Weise virulent wurden. Gleichwohl wiesen die verschiedenen Bewältigungsformen über Familien hinweg gewisse Gemeinsamkeiten auf, die die Existenz verschiedener Typen von Familien nahelegten, die wiederum jeweils für die (Medien-)Sozialisation unterschiedliche Voraussetzungen schafften. Über alle Untersuchungswellen hinweg zeigten sich jedoch bei den Kindern und später Jugendlichen der Panelstudie keine durchgängigen Muster in ihrer Mediensozialisation. Mediensozialisation erwies sich - das machen die Ergebnisse der kontextuellen Analyse eindrucksvoll deutlich - als ein von einer Fülle von Einflüssen mitbestimmtes, multifaktorielles Phänomen, das sich bei jedem Kind bzw. Jugendlichen in jeder Familie, je nach Zusammenspiel meso-strukturel-

19 So zeigte sich nach den ersten sechs Erhebungswellen, dass die Familien während der Panelstudie zahlreiche Veränderungen in ihrem Leben erfahren hatten; es fanden sich sowohl auf der sozio-ökonomischen als auch auf der sozio-emotionalen Beziehungsebene Verbesserungen bzw. Verschlechterungen in der Lebensführung der Familien. Diesen Veränderungen lagen unterschiedliche Auslöser zugrunde, etwa ein Umzug in eine andere Gegend oder auch in ein anderes Land, eine persönliche Tragödie oder ein Schicksalsschlag in der Familie, oder auch die Auflösung und/ oder Neugründung einer Lebensgemeinschaft bzw. einer Ehe, aus denen sich entsprechend neue sozio-ökomische und sozio-emotionale Strukturen ergeben. Fanden getrenntlebende Elternteile neue Partner bzw. neue Partnerinnen, hatte dies für die betroffenen Kinder keinesfalls dieselben Konsequenzen wie für die Eltern. So schienen sich zwar bei einigen Familien die sozio-ökonomischen Bedingungen und auf den ersten Blick auch die sozioemotionalen Beziehungsstrukturen in der Kernbeziehungsgruppe verbessert zu haben, doch einige Kinder litten gerade unter diesen neuen Beziehungsstrukturen. Im Rahmen der kontextuellen Analyse und der damit einhergehenden intensiven Auseinandersetzung mit den Familien wurde eindrucksvoll deutlich, mit welchen Veränderungen (positiven wie negativen), Krisen (persönlichen, gesundheitlichen, schulischen, beruflichen, finanziellen) und vielfältigen Herausforderungen im Alltag die jungen Erwachsenen und ihre Familien konfrontiert wurden und wie sie damit umgehen. 
ler, d.h. auf der Ebene der Familie angesiedelter Einflussgrößen wie der sozial-familialen Ressourcen, der Beziehungsstrukturen der Familie, des (Medien-)Erziehungsverhaltens der Eltern, und mikro-struktureller, d.h. das Kind in seinen persönlichen Voraussetzungen prägender Faktoren wie etwa Geschlecht, Entwicklungsaufgaben, ,Eigensinn“ und auch Medienvorlieben, unterschiedlich ausgestaltete. Mit zunehmendem Alter der Kinder weitete sich zudem ihr Lebensumfeld. Kindergarten, Schule, außerschulische Institutionen, Peers, Freunde und Freundinnen, Nachbarn und insgesamt die Infrastruktur auch der Wohnumgebung der Familie, spielten eine wichtige Rolle in der Sozialisation und bestimmten damit auch den Mediengebrauch der Kinder mit. Auf der Makro-Ebene galt jedoch zu Beginn der Studie 2005 für alle Familien die sozio-ökonomisch schlechtere Ausstattung, ihre soziale Lage, als das sie verbindende Strukturmerkmal; diese war ein zentraler Ausgangspunkt der Familienauswahl vor der ersten Erhebungswelle zu Beginn der Studie (siehe dazu Paus-Hasebrink/ Bichler 2008, S. 132ff.). Wie die Familienmitglieder ihr Leben führten - dies war über die ersten sechs Erhebungswellen hinweg sehr deutlich geworden -, schlug sich nieder in ihren sozio-emotionalen Familienstrukturen, die ihr Zusammenleben als Familie prägten. In der Art, wie es den Familien gelang, ihr Leben als Familie zu meistern, ließen sich zwischen den Familien des Panels Unterschiede bzw. Gemeinsamkeiten identifizieren. Mit Blick auf die jeweiligen Besonderheiten ergab sich in einer kontrastierenden Gegenüberstellung die Zuordnung der Familien zu dem Typ, dessen Merkmalskombination sie am stärksten in ihrer Lebensführung charakterisierte. Abweichende Tendenzen hin zu anderen Typen während einer oder mehrerer Untersuchungswellen wurden in den Erläuterungen der Zugehörigkeit jeder einzelnen Familie zu einem der vier Typen diskutiert (siehe dazu Paus-Hasebrink/ Kulterer/ Oberlinner 2017, S. 129ff.). Ausgehend von dieser Erkenntnis wurde 2014 und zuletzt 2017 eine Typologie aller Familien erstellt und die 18 Familien der Panel-Langzeitstudie einem der insgesamt vier Typen zugeordnet. Denn die einem Typ zugeordneten Familien ließen vor dem Hintergrund ihrer sozio-emotionalen Beziehungen im Umgang mit der sozialen Benachteiligung Muster erkennen, in die als Facetten auch ihr Mediengebrauch sowie in die im Mittelpunkt der Studie stehende (Medien-)Sozialisation der für die Untersuchung ausgewählten Kinder eingewoben waren; als typbildende Merkmale konnten sie jedoch nicht herangezogen werden. Es bleibt - siehe dazu Kapitel 3 zu überprüfen, ob und, wenn ja, in wieweit dies nach wie vor auch bei den jungen Erwachsenen der Fall ist. 
Die nach der vierten vorgenommene und nach der sechsten Erhebungswelle weiter geführte und aktualisierte Bildung von Familientypen (siehe Paus-Hasebrink/ Kulterer 2014, S. 239 sowie Paus-Hasebrink/ Kulterer/ Oberlinner 2017, S. 129ff., Paus-Hasebrink et al. 2018) dient im Folgenden als strukturgebendes Moment der Darstellung aller 18 Fallbeschreibungen. Zur besseren Einordnung des Vorgehens zunächst ein kurzer Rückblick auf die Vorgeschichte und die Vorgehensweise der Typenbildung.

Ziel der Typenbildung 2014 und 2017 war es, den komplexen und dynamischen Prozessen in der Lebensführung der Familien vor dem Hintergrund ihrer sozio-ökonomischen Situation und sozio-emotionalen Beziehungskonstellationen insbesondere in der Kernbeziehungsgruppe so gut wie möglich gerecht zu werden und sie nachvollziehbar abzubilden. Die Typenbildung erfolgte, dies sei erneut hervorgehoben, vor dem Hintergrund des Zusammenspiels der Handlungsoptionen, Handlungsentwürfe und Handlungskompetenzen sowohl der Heranwachsenden als auch ihrer Eltern. ${ }^{20}$

Die vier Typen und ihre Familien im Überblick:

Typ 1: Massive sozio-ökonomische Probleme als multiple Deprivation: Die rundherum überforderten Familien (Fein, Landinger, Oblinger, Öllinger)

Typ 2: Die sozio-ökonomisch nicht mehr bzw. wenig belasteten, aber wegen problematischer sozio-emotionaler Beziehungsstrukturen überforderten Familien (Hirtner, Holzner, Rohringer, Weiss)

Typ 3: Die zwar sozio-ökonomisch, aber sozio-emotional weniger belasteten, relativ kompetenten Familien (Aufbauer, Boll, Ebner, Kaiser, Stab)

Typ 4: Keine belasteten sozio-ökonomischen Bedingungen mehr und unbelastete sozio-emotionale Beziehungsstrukturen - Die relativ kompetenten >Aufsteiger`(Dornbacher, Grubert, Pfortner, Scheib, Zarbl)

20 Siehe dazu und im Folgenden Paus-Hasebrink/ Kulterer/ Oberlinner 2017: 129ff. 


\begin{tabular}{|c|c|c|c|c|}
\hline Merkmale & Typ 1 & Typ 2 & Typ 3 & Typ 4 \\
\hline $\begin{array}{c}\text { Sozio-ökonomische } \\
\text { Situation }\end{array}$ & Stark belastet & $\begin{array}{c}\text { Nicht mehr } \\
\text { bzw. gering } \\
\text { belastet }\end{array}$ & Belastet & $\begin{array}{c}\text { Nicht mehr } \\
\text { belastet }\end{array}$ \\
\hline $\begin{array}{c}\text { Sozio-emotionale } \\
\text { Bedingungen und } \\
\text { Beziehungsstrukturen }\end{array}$ & Stark belastet & Belastet & $\begin{array}{c}\text { Weniger } \\
\text { belastet }\end{array}$ & \begin{tabular}{c} 
Nicht belastet \\
\hline Bewältigungsstrategien
\end{tabular} \\
Überfordert & Überfordert & $\begin{array}{c}\text { Relativ } \\
\text { kompetent }\end{array}$ & $\begin{array}{c}\text { Relativ } \\
\text { kompetent }\end{array}$ \\
\hline
\end{tabular}

\section{Abbildung 2: Die Familientypen im Überblick. Quelle: Eigene Darstellung.}

Im Folgenden werden die 18 Fallbeschreibungen mit Blick auf die wichtigsten Facetten der Veränderung in der Lebensführung und damit den jeweiligen Handlungsoptionen, Handlungsentwürfen und Handlungskompetenzen der jungen Erwachsenen vorgestellt und danach gefragt, welche Parameter sich verändert haben und in welcher Weise sich der Prozess des Erwachsenwerdens der jungen Menschen bis etwa zu ihrem 19. bzw. 21. Lebensjahr vollzogen hat. Die Fallbeschreibungen werden dazu dem jeweiligen Familientyp, zu dem sie 2017 gehörten, zugeordnet und in alphabetischer Reihenfolge vorgestellt. Vor diesem Hintergrund folgt in Kapitel 3 aus einer stärker vergleichend-fokussierenden, fallübergreifenden Perspektive ein Überblick über die Veränderungen im Alltag aller jungen Erwachsenen der Panelstudie und welche Rolle dabei ihren wichtigen Bezugspersonen (Eltern, Partner und Partnerinnen, Freunde und Freundinnen, Arbeitgeber und Arbeitgeberinnen, Kollegen und Kolleginnen etc.) zukommt. Dabei steht im Mittelpunkt, ob und, wenn ja, in welcher Weise sich jeweils typbezogene Unterschiede zwischen den jungen Erwachsenen und ihren Eltern feststellen lassen. 


\subsection{Die jungen Erwachsenen von Typ 1: Massive sozio-ökonomische Probleme als multiple Deprivation: Die rundherum überforderten Familien}

Charakteristisch für die Familien von Typ 1 war, dass es sich bei ihnen entweder um Großfamilien oder Ein-Eltern-Familien handelte, die sich über die Erhebungszeit in der Bewältigung ihres Alltags massiv überfordert zeigten. Die sie kennzeichnende Deprivation ließ sich bei den Familien von Typ 1 auf mehreren Ebenen feststellen: $\mathrm{Zu}$ massiven sozio-ökonomischen Einschränkungen kamen im Laufe der Erhebung bei Eltern wie Kindern noch psychische und physische Erkrankungen hinzu, die bei den Eltern zur Arbeitsunfähigkeit führten und bei den Kindern zu Beeinträchtigungen ihrer Entwicklung. Alle Familien litten auch zum Ende der sechsten Erhebungswelle noch stark unter belastenden und ausweglos wirkenden sozio-ökonomischen Bedingungen. Die prekäre sozio-ökonomische Lage, die über die Erhebungszeit das Familienleben entscheidend prägte, zeigte sich auch in den jeweiligen Wohnunterkünften der Familien - entweder recht kleinen Wohnungen oder aber renovierungsbedürftigen Häusern, zum Teil in strukturell benachteiligten oder weit abgelegenen Wohngegenden. Die abgelegene Wohnlage war für einige Heranwachsende aus Typ 1 auch mit weiten Wegen und damit einhergehend großen zeitlichen Belastungen verbunden, um zur Schule, zu Praktikums- oder Lehrstellen zu kommen. Zum Ende der sechsten Erhebungswelle schien sich abzuzeichnen, dass es zumindest den Eltern nicht mehr gelingen würde, der mit ihren stark eingeschränkten Handlungsoptionen verbundenen massiven Überforderung zu entkommen. Die meisten Familien zeigten über die Erhebungszeit hinweg gegenüber ihren Kindern ein gewisses Maß an Pflichtbewusstsein - man müsse sich ja um seine Kinder kümmern -; dennoch war zumindest bei zwei der Familien des Typs (Landinger und Fein) zu keinem Zeitpunkt zu übersehen, dass sie ihre Kinder als Belastung erlebten. Die Spannung zwischen elterlichem Pflichtbewusstsein und eigenen Handlungsentwürfen (dem Wollen) und eigenen Handlungskompetenzen (dem Können) der Eltern, etwa in der Bewältigung ihrer Lebensaufgaben, persönlicher wie familienbezogener, allen voran der (Medien-)Erziehung der Kinder, blieb über den Erhebungszeitraum deutlich erkennbar. Dies zeigte sich in der gesamten Lebensführung der Familienmitglieder und in ihrem Zusammenleben als Familie, sowohl in den nicht-medialen als auch, wenn auch zuweilen weniger direkt, in den für die Sozialisation der Kinder bedeutsamen medialen Handlungspraktiken von Eltern und Kindern. 
2.2.1 Olivia Fein (im Jahr 201615 Jahre alt) und ihre Mutter: Eine vielfältig schwer belastete Familie

\section{5 bis 2016}

Olivia Fein lebte zu Beginn der Studie zusammen mit ihrer alleinerziehenden Mutter und ihrem jüngeren Bruder in einer $66 \mathrm{~m}^{2}$ großen, spärlich eingerichteten Reihenhaus-Mietwohnung in einem Randbezirk mit einem hohen Anteil an Migranten und Migrantinnen einer Großstadt. Olivias Mutter ist gelernte Friseurin, sie war jedoch die meiste Zeit während der Teilnahme an der Panelstudie arbeitslos. Die sozio-ökonomischen Bedingungen der Familie waren stets angespannt. Von Olivias Vater hatte sich Frau Fein schon vor Beginn der Sozialisationsstudie getrennt. Aufgrund ihres sehr geringen Haushaltseinkommens war Familie Fein auf staatliche Fördergelder angewiesen, insbesondere auf Zuschüsse zu den Mietkosten. Von der zweiten bis zur vierten Erhebungswelle hatte Frau Fein einen neuen Partner, einen gelernten Tischler, mit dem sie eine Tochter bekam und in einem Neu- bzw. Anbau seines Elternhauses in einem anderen Bundesland in einem Dorf lebte. Da sich der Lebensgefährte jedoch gerade in Umschulung befand und Frau Fein als Aushilfe vormittags in einem Tischlereibetrieb nur wenig verdiente, blieben die Einkommensverhältnisse der Familie auch in dieser Zeit prekär.

Olivia litt stark unter der Trennung ihrer Eltern, dem anschließenden Umzug, der damit verbundenen Trennung von ihren alten Freunden und Freudinnen und unter dem spannungsgeladenen Verhältnis zum neuen Lebensgefährten ihrer Mutter. Schon während des ersten Interviews mit Olivia 2005 war sie nicht in der Lage, sich auf das Gespräch zu konzentrieren, sprach ständig von Verlust(ängsten) oder Tod, davon in ihrem Zimmer eingesperrt zu sein und von niemandem geliebt zu werden. In der zweiten Erhebungswelle zeigte sich die nunmehr siebenjährige Olivia noch stärker verstört und unsicherer; Olivia war nicht nur psychisch sehr belastet, sondern zudem auch an Epilepsie erkrankt. Frau Fein war ihrer Tochter kaum eine Unterstützung; mit der Erziehung ihrer Kinder war sie massiv überfordert und sah sich nicht in der Lage, Olivia bei der Bewältigung ihrer Probleme zu helfen. Sie habe einfach, so Frau Fein, nicht genügend Zeit, um sich um ihre Kinder zu kümmern. Olivia kam mit den veränderten Lebensumständen der Familie nicht zurecht und wurde psychisch auffällig; sie hatte gar mit Selbstmord gedroht. Nach dem Eingreifen ihrer Großmutter mütterlicherseits wurde Olivia in eine betreute Wohngemeinschaft gebracht, wo sie sich gut einlebte. Das strukturierte Heimleben schien ihr gut zu bekommen. Nach einiger Zeit im Heim 
gab es aber auch dort gravierende Probleme und Verhaltensauffälligkeiten von Olivia, die, inzwischen zehnjährig, vor anderen Kindern masturbierte. Nachdem ihre Mutter kurz vor Beginn der vierten Erhebungswelle erneut das Sorgerecht für ihre Tochter beantragt hatte und ihr dies auch wieder zugesprochen worden war, verließ Olivia das Heim und kehrte nach Hause zu ihrer Mutter zurück. Das angespannte Verhältnis zwischen Olivia und ihrer Mutter schien sich jedoch durch die Trennung über die drei Jahre, die Olivia in der betreuten Wohngemeinschaft verbracht hatte, noch verschlechtert zu haben. So zeigte sich Frau Fein ihrer Tochter gegenüber sehr misstrauisch, und Olivia nutzte den Hinweis auf das Jugendamt oft als Druckmittel ihrer Mutter gegenüber und versuchte, sie damit zu erpressen. Gemeinsam besuchten Mutter und Tochter zwar eine Psychotherapeutin, die sie bei Erziehungsfragen unterstützen sollte, aber Frau Fein lehnte die Therapeutin ab: „(M)it der Frau komm i überhaupt net ei.“ Ihre Ratschläge berücksichtigte Frau Fein nicht. Olivia nahm die Betreuerin vom Jugendamt, wie ihre Mutter in einem Interview betonte, als eine „Freundin“ wahr. Die Mutter versuchte denn auch, ihrer Tochter dies auszureden: „Da sag i Olivia, ja aber net, dass des gleich wieder als en neiche Freindin siagst, des is deine neue Betreuerin, hab i gsagt, goi." Olivia suchte zwar des Öfteren auch Orientierung bei ihrer Mutter, etwa als sie ihre Regel bekam und Bauchschmerzen hatte, als diese ihr aber keinen Rat geben konnte, war Olivia sehr enttäuscht: „Maah, was bist denn du für Eine? Kannst mir als Mama net amal weiterhelfen!“ Die Zeit, die Olivia in der betreuten Wohngemeinschaft verbracht hat, wertete ihre Mutter als „verlorene Zeit“, die sie beide auseinandergebracht und ihre Tochter verändert hätte. So schwärmte sie von ihrem „alten Kind“, der Olivia, bevor diese ins Heim kam. Das Heim habe, so Frau Fein in der vierten Erhebungswelle, Olivia zu einem „Monster“ gemacht.

Zwischen der vierten und sechsten Erhebungswelle hatte sich Frau Fein von ihrem früheren Lebensgefährten getrennt. Aufgrund ihrer schlechten gesundheitlichen Situation - Frau Fein war zwischenzeitlich nicht nur wie ihre Tochter an Epilepsie erkrankt, sondern auch wegen ihrer Depression in der sechsten Erhebungswelle in psychotherapeutischer Behandlung wurde ihr mit 33 Jahren Frührente zugesprochen. Die finanzielle Situation der Familie erwies sich danach als besonders prekär. Nach der Trennung vom zeitweiligen Lebensgefährten war Frau Fein mit ihren nunmehr drei Kindern wieder zurück in die Stadt gezogen und wohnte dort in einer beengten Drei-Zimmerwohnung. An der fünften Erhebungswelle der Studie nahm Familie Fein nicht teil, da Frau Fein wegen starker psychischer Probleme dazu nicht in der Lage war. In der sechsten Erhebungswelle 
hatte sie einen neuen, doppelt so alten Lebensgefährten, der aber nicht in der gemeinsamen Familienwohnung lebte. Olivia besuchte in der sechsten Erhebungswelle eine Vor-Berufsschule und bezog dafür ein monatliches Taschengeld in Höhe von 80 Euro.

Familie Feins Familienklima war von Beginn der Panelstudie an bis zum Ende der Teilnahme der Familie stark belastet. Oberflächlich stellte Frau Fein ihre belastete Situation als schlicht gegeben hin und machte den Eindruck, ihr Leben recht pragmatisch - mit Blick auf sich selbst - auszurichten. Es wurde jedoch deutlich, dass dies Versuche des Überspielens waren und keine Bearbeitung oder tatsächliche Akzeptanz ihrer problematischen Lage. Die sozio-emotionalen Beziehungsstrukturen von Familie Fein wurden über die gesamte Erhebung von diesem Verhalten stark beeinträchtig. Frau Fein war nicht in der Lage, als Mutter für ihre Kinder da zu sein, sie ertrug weder körperliche noch emotionale Nähe zu ihnen und schien sich nicht für sie zu interessieren. Vielmehr war sie über die Zeit zunehmend mit den Herausforderungen ihres eigenen Lebens ausgelastet. Olivia beschrieb ihre Mutter als „kaltherzig“ und wünschte sich eine Bezugsperson, der sie vertrauen konnte. Das Mädchen litt unter dem Desinteresse ihrer Mutter und der Überforderung der Familie durch ihre sozio-ökonomische, aber besonders auch durch ihre sozio-emotionale Lebenssituation. Mit der Bewältigung ihrer persönlichen wie familienbezogenen Lebensaufgaben schien Frau Fein bis zum Ende ihrer Teilnahme an der Studie stark überfordert. Sie versuchte zwar immer wieder, persönliches Lebensglück durch neue Partnerschaften zu erlangen, in denen sie ihre Sexualität ausleben konnte - dies wurde sehr deutlich und auch von Olivia betont, da sie dieses Verhalten der Mutter oft als belastend erlebte - und die gleichzeitig die finanzielle Lage der Familie verbesserten, doch immer wieder scheiterte Frau Fein daran, ihre Handlungsentwürfe erfolgreich umzusetzen. Sexualität und der Wunsch nach finanzieller Stabilität avancierten in Familie Fein zu beherrschenden Familienthemen. So stellte Frau Fein ihre Tochter Olivia unter dauernden „Sex-Verdacht“, möglicherweise, weil sie sich selbst in diesem Zusammenhang als getrieben wahrnahm. Olivias Verhalten ließ erkennen, dass sie unter den wechselnden Partnerschaften ihrer Mutter litt; bis zum Ende ihrer Teilnahme an der Studie war erkennbar, dass es Olivia sehr schwerfiel, ein ausbalanciertes Verhältnis zu sich selbst und vor allem ihrem Körper und ihrer Sexualität zu finden. Olivias Lebenssituation blieb bis zum Schluss der Erhebung in allen Belangen von der Überforderung ihrer Mutter belastet, ihren Alltag gemeinsam mit ihren Kindern zu meistern. 
Olivia reagierte auf die Belastungen in ihrer Familie seit Beginn der Erhebungen psychisch stark auffällig und mit einem übermächtigen Wunsch nach Aufmerksamkeit; auch zum Ende der Erhebungen schwankte sie noch zwischen frühreifem Verhalten auf der einen und noch sehr kindlichen Wünschen auf der anderen Seite.

\section{0: Familie Fein nahm am Interview wegen „schlimmer Erlebnisse“ nicht teil}

In der siebten Erhebungswelle fand zwar ein kurzes Telefongespräch mit Frau Fein statt; sie lehnte es jedoch ab, an der Befragung teilzunehmen, da die Familie in den vergangenen Jahren, wie sie der Interviewerin sagte, „sehr Schlimmes" mitgemacht habe. Frau Fein war nicht bereit, näher darauf einzugehen, um was es sich dabei gehandelt hatte. Die Interviewerin vereinbarte mit Olivias Mutter, nach einiger Zeit nochmals anzurufen, um dann auch zu erfahren, ob vielleicht Olivia bereit sei für ein Telefoninterview (Olivia selbst war nicht erreichbar); bei einer Nachfrage lehnte Frau Fein eine Teilnahme jedoch für sich selbst und auch für Olivia erneut entschieden ab. Ob und inwiefern Frau Feins Absage in einem Zusammenhang mit einem während der sechsten Erhebungswelle schwelenden Gerichtsverfahren gegen ihren zeitweiligen Lebensgefährten stand, konnte nicht eruiert werden. In den Interviews der sechsten Erhebungswelle drängte sich der Verdacht auf, dass es zwischen ihm und Olivia zu sexuellen Handlungen gekommen war; sowohl Frau Fein als auch Olivia selbst deuteten dies an. Aufgrund sexueller Übergriffe auf die leibliche Tochter, Olivias jüngere Halbschwester, bestand für Frau Feins Lebensgefährten bereits ein gerichtliches Besuchsverbot.

\section{Medien im Leben von Olivia Fein und ihrer Mutter}

Olivia dienten Geschichten und Figuren aus den Medien, vor allem dem Fernsehen und später auch YouTube, als Orientierungsgeber und Alltagsbegleiter. In frühen Erhebungsphasen hatte Olivia eine Zeitlang für die Hauptfigur der Serie Hannab Montana geschwärmt und in diesem Zusammenhang behauptet, diese in einem Video mit sexuellen Inhalten gesehen zu haben. Zudem gefiel ihr der Medienstar Selena Gomez. Wenn sie so viel Geld verdienen könnte wie diese, würde Olivia, wie sie in der vierten Erhebungswelle erzählte, „shoppen, shoppen, shoppen, bis dass i koa Geld mehr hoab." Seit früher Kindheit dienten Olivia mediale Figuren, anfangs vor allem des Fernsehens, als Begleiter und Orientierungsgeber. Noch in der vierten Erhebungswelle erzählte sie, „funf bis zehn a halb Stund', pro Tag" fernzusehen. Olivia bevorzugte Krimiserien wie K11 oder Gerichtssendungen. Zusätzlich spielte Olivia auch zahlreiche Online-Spiele, von 
denen ihre Mutter nichts wusste, und surfte oft auf YouTube und sah sich dort Musikvideos von den Sängerinnen Miley Cyrus, Rihanna, Pink oder Lady Gaga an. Dabei, so Olivia, sähe sie auch oft nackte Menschen, das, so betonte sie, finde sie aber "ganz normal und schon langweilig“, viel lieber würde sie mehr von ihrer früher schon favorisierten Heldin Hannab Montana alias Miley Cyrus sehen. Vor allem in den letzten Erhebungswellen der Studienteilnahme flüchtete Olivia in mediale Familiengeschichten als Vorlagen für gelungenes Familienleben, sie interessierte sich auch sehr für Geschichten vom Leben der Stars, für Klatsch und Tratsch über Prominente, Reiche und Königshäuser. Zudem hing sie einer Fülle von Konsumfantasien nach, aber auch der Idee, eigene Handlungskompetenz durch einen baldigen Auszug aus ihrer Familie und die Gründung einer eigenen Familie zu erlangen. Olivias Handlungsentwürfe kreisten um eine möglichst baldige Unabhängigkeit; sie wünschte sich schon als 15-Jährige, bald einen Partner zu finden und mit ihm Kinder zu bekommen. Olivia schien sich nach einer geordneten Familie und Geborgenheit zu sehnen. Auch Soziale Medien-Angebote dienten Olivia in vielfältiger Weise als Chance, ihren Spannungsgefühlen Ausdruck zu verleihen. Dabei zielte sie darauf ab, sich möglichst als erotisch anziehende und attraktive Frau zu präsentieren, mit Hilfe dieser Angebote versuchte sie, sich Aufmerksamkeit zu verschaffen.

Zwar hatte Olivia in der ersten und zweiten Erhebungswelle, wie Olivias Mutter seinerzeit im Interview erzählte, gerne zusammen mit ihr Telenovelas, wie Sturm der Liebe und Rote Rosen sowie "echte“ Serien mit starken Frauen wie z.B. Charmed, angeschaut, Rat und Unterstützung im Umgang mit Medien fand Olivia bei ihrer Mutter aber zu keinem Zeitpunkt der Erhebung. In der vierten Erhebungswelle klagte sie im Interview:

Interviewerin: Und über die Sachen, die du aloa anschaust, redest du da drüber mit deiner Familie, dass du deiner Mama vielleicht erzählst, was'd angeschaut hast oda dass dei Mama fragt?

Olivia: Na, des interessiert sie gar ned.

Interviewerin: Fragt sie also gar ned, was'd angschaut hast?

Olivia: $\mathrm{Hm} \mathrm{hm}$ (verneinend).

Frau Fein bestätigte dies durch eine Aussage im Interview der zweiten Erhebungswelle; so hob sie, um ihr Desinteresse an Olivias Medienumgang $\mathrm{zu}$ kaschieren, fast entschuldigend hervor:

Es is ned nur so, dass das Fernsehen einen schlechten Einfluss hat, der hat auch an guad'n Einfluss. Und do, bei solche Sendungen, kann man vü lernen, find i. Do werden die Kinder einerseits auch selbstständiger, jo, und durch den Fernseher werden's schon auch reifer. Sie lernen des 
kennen, ohne dass man sich wirklich dafür Zeit nehmen muss, wenn man keine hat, sag i amoi.

Für Frau Fein selbst spielte das Fernsehen mehr und mehr eine wichtige Rolle; Zeitungen lese sie nur, wie sie meinte, „wenn gar nichts anderes mehr ginge“.

Fazit

Olivia, Tochter einer alleinerziehenden, zumeist arbeitslosen und schon mit 33 Jahren aufgrund schwerer gesundheitlicher Probleme auf Frührente angewiesenen Frau, litt von Beginn bis zum Ende der Teilnahme der Familie an der Panelstudie 2016 sehr unter den sozio-ökonomischen und insbesondere den sehr angespannten sozio-emotionalen Lebensbedingungen ihrer Mutter. Die Handlungsoptionen der Familie erwiesen sich während der gesamten Erhebungszeit als stark eingeschränkt. Der Verlust ihres leiblichen Vaters nach der Scheidung der Eltern - Olivia hatte mit ihm danach kaum mehr Kontakt (laut Frau Fein interessierte er sich danach nicht mehr für seine Kinder) - und die wechselnden Partnerschaften ihrer Mutter machten Olivia zu schaffen; aufgrund von Depressionen und einer Selbstmordandrohung lebte sie zwischenzeitlich drei Jahre in einer betreuten Wohneinrichtung. Das Verhältnis zu ihrer Mutter blieb trotz Begleitung durch das Jugendamt und eine Psychotherapie für Mutter und Tochter angespannt. Zwischenzeitlich hatte sich Olivias Mutter vom Vater ihrer jüngsten Tochter getrennt. Ihm gegenüber bestand ein gerichtliches Betretungsverbot der neuen Wohnung von Familie Fein aufgrund sexueller Handlungen gegenüber seiner leiblichen Tochter, Olivias jüngerer Halbschwester. Im Interview mit Olivia und ihrer Mutter in der sechsten Erhebungswelle war auch von sexuellen Vorkommnissen zwischen Olivia und dem ehemaligen Lebensgefährten die Rede. An der siebten Erhebungswelle nahm Familie Fein nicht mehr teil; Frau Fein begründete dies, wie sie es im Telefonat zur Interviewvereinbarung nannte, mit „schlimmen Erlebnissen“ in der Zwischenzeit.

Die angespannte sozio-ökonomische und sozio-emotionale Situation ihrer Mutter, deren stark auf sich selbst ausgerichteten Handlungsentwürfe, aber auch Olivias eigene belastete psychosexuelle Entwicklung, spiegelten sich von Beginn der Erhebung 2005 bis zum Ende der Teilnahme von Familie Fein 2016 an der Panelstudie in Olivias Mediengebrauch wider. In ihrem jeweiligen Medienrepertoire, den bevorzugten Geschichten und Figuren, suchte sich Olivia Stoff zur Bearbeitung ihrer Handlungsentwürfe und Orientierung im Ringen um eigene Handlungskompetenz. Bei ihrer überforderten Mutter fand Olivia keine Hilfe und Orientierung. 
2.2.2 Timo Landinger (20 Jahre alt) und seine Familie: Schwierigste sozio-emotionale und sozio-ökonomische Bedingungen belasten den Jungen und hemmen seine Entwicklung

2005 bis 2017

Timo ist das jüngste Kind in einer schwer deprivierten Großfamilie, bestehend aus Herrn und Frau Landinger sowie sechs Kindern. Herr Landinger ist der leibliche Vater der vier jüngsten Geschwister, während die beiden Ältesten einer früheren Beziehung von Frau Landinger entstammen. Die Familiensituation war über alle Jahre der Untersuchung hinweg von Instabilität und Konflikten sowie von physischer und psychischer Gewalt gekennzeichnet. Timo selbst steht unter der Überwachung von staatlichen Fürsorgestellen und verbrachte viele Jahre, auch entgegen dem Wunsch seiner Eltern, in stationärer Unterbringung. Während seine älteren Geschwister nach und nach das Elternhaus verlassen konnten, um sich ein eigenes Leben aufzubauen, hatte Timo diese Möglichkeit nicht. Die Perspektivlosigkeit lässt sein Leben sehr beengt und trostlos erscheinen, er selbst beklagte die Gesamtsituation jedoch nie.

Zwischen der zweiten und dritten Erhebungswelle floh Frau Landinger aus der Familienwohnung und lebte mit Timo und einem weiteren Kind im Frauenhaus. Einige Zeit später versöhnten sich die Eltern und wagten einen Neubeginn, aus dem auch eine gewisse Verbesserung der sozio-ökonomischen Situation und der Wohnbedingungen resultierte. Die Familie zog in ein anderes Bundesland und bewohnte von nun an ein Einfamilienhaus in einer sehr abgelegenen Gegend. Woher die finanziellen Ressourcen dafür stammten, konnte nicht geklärt werden, als am wahrscheinlichsten gilt eine Erbschaft. Insgesamt muss der Umzug als deutliche Verbesserung gewertet werden: Die zuvor bewohnte, nur $62 \mathrm{~m}^{2}$ große, Wohnung war heruntergekommen, hatte bauliche Mängel und es stank teilweise nach Urin. Mit $110 \mathrm{~m}^{2}$ bot das neue Haus weitaus mehr Platz, jedoch war es zu Beginn ebenfalls stark renovierungsbedürttig. Allerdings gelang es der Familie über die Jahre hinweg, den Zustand nachhaltig zu verbessern, dennoch blieb es im Gebäude immer sehr unordentlich und alles andere als sauber. Zudem roch es stark nach Zigarettenrauch. Timos persönliche Situation änderte sich durch den Umzug nur rudimentär. Er erhielt ein eigenes Zimmer, welches er nutzte, um sich im Rahmen seiner beschränkten Möglichkeiten zu entfalten. Er hielt sein Zimmer weitestgehend sauber und aufgeräumt und es schien so, als wolle er dadurch Ordnung und Struktur in sein Leben bringen. Dennoch blieb er psychisch beeinträchtigt, und seine Bedürfnisse wurden von den Eltern 
meist vernachlässigt, da sie einerseits mit finanziellen Herausforderungen, andererseits aber auch mit eigenen gesundheitlichen (psychischen wie physischen) Problemen beschäftigt waren. Herr Landinger ist ausgebildeter Steinmetz, jedoch war er über die gesamte Studiendauer hinweg arbeitslos und erschien nicht erpicht darauf, tatsächlich einen Job zu finden. Vielmehr war er bestrebt, so viele staatliche Unterstützungsmaßnahmen $\mathrm{zu}$ beziehen wie möglich. Um dies zu erreichen betrieb er umfangreiche Online-Recherchen und diagnostizierte bei sich selbst 62 verschiedene Erkrankungen. In Folge versuchte er diese offiziell anerkennen zu lassen, um so höhere Hilfszahlungen zu erhalten. Frau Landinger war zunächst 15 Stunden in der Woche als Telefonistin tätig, von der dritten bis zur fünften Erhebungswelle war aber auch sie arbeitslos oder befand sich aus gesundheitlichen Gründen in Karenz. Erst ab der sechsten Erhebungswelle war Frau Landinger wieder in ihrem erlernten Beruf als Bürokauffrau tätig.

Die sozio-ökonomischen und sozio-emotionalen Probleme der Eltern und die ständig ausgetragenen Konflikte entfalteten eine negative Wirkung und es gab kaum Zuneigung zwischen den Familienmitgliedern. Gerade Timo litt unter dem Mangel an Wärme und Zuneigung und führte in einem Interview an, dass er das Gefühl hatte, dass niemand ihn mochte, nicht einmal die Katzen der Familie. Dennoch nannte er seine Mutter die wichtigste Schutzinstanz gegenüber dem Vater. Denn das Familienklima war nachhaltig durch das bevormundende Verhalten von Herrn Landinger bestimmt, der alles besser zu wissen glaubte und seine Meinung durchzusetzen wusste. Um seine dominante Stellung aufrechtzuerhalten zögerte er nicht, körperliche Bestrafungen und psychische Demütigungen einzusetzen. Er selbst bestritt dies zwar, die Aussagen von Timo und seiner Mutter legten jedoch das Gegenteil nahe, und das Verhalten von Frau Landinger bestätigte diesen Schluss. Nach der Flucht ins Frauenhaus hatte sich das Paar zwar versöhnt, dennoch erschien Frau Landinger weiterhin von der Beziehung zu ihrem Mann belastet. In seiner Gegenwart wirkte sie ängstlich und zurückgezogen, während sie sich in seiner Abwesenheit im Rahmen ihrer Möglichkeiten herzlich und zugewandt zeigte. Timos kognitive und emotional-affektive Entwicklung litt nachhaltig unter den vielfältigen Problemen der Familie und der daraus resultierenden mangelnden Unterstützung und Zuwendung. Bei ihm wurden ADHS und andere psychische Erkrankungen diagnostiziert; Timo benötigte dauerhaft besondere Betreuung, die von seinen Eltern nicht geleistet werden konnte. Zum Teil verhinderten diese sogar aktiv eine weiterführende Behandlung, da sie es für die bessere Lösung hielten, wenn Timo im Familienverband 
aufwüchse. So mangelte es ihm zeitweise an adäquater Behandlung und Hilfe. 2005 ging Timo in einen allgemeinen Kindergarten, 2007 besuchte er eine Sonderschule und eine Nachbarschaftsbetreuung. Ergänzend dazu nahm die Familie ambulante therapeutische Familienbetreuung in Anspruch. Während der Zeit im Frauenhaus wurden all diese Maßnahmen unterbrochen. Nach dem Umzug besuchte Timo erneut eine Sonderschule, wurde aber bereits nach nur einer Woche suspendiert und dann für mehrere Monate zu Hause unterrichtet. 2010 wurde er für sieben Monate in die Kinderpsychiatrie eingewiesen.

Zwischen 2012 und 2014 war Timo schließlich in einer spezialisierten, sozialpädagogischen Wohneinrichtung untergebracht. Dort lebte er unter der Woche und wurde nur am Wochenende nach Hause geholt. So konnte er seine Pflichtschuljahre absolvieren und zeigte während der Zeit in der Einrichtung deutliche Fortschritte in seiner Entwicklung. Die medikamentöse Behandlung konnte erheblich reduziert werden und er profitierte deutlich von den stabilen Routinen, den regelmäßigen therapeutischen Übungen und strengen Richtlinien zur Mediennutzung. Auch der intensive Kontakt mit Kindern seines Alters wirkte sich positiv aus und er entwickelte erstmals Peer-Group-Beziehungen, die auf gemeinsamen Erlebnissen und Vertrauen beruhten. Dies spiegelte sich auch in der Interviewführung wider: Timo blieb zwar eher in sich gekehrt, er wirkte aber deutlich aufgeweckter als in den Jahren zuvor und trat sehr freundlich auf. Jedoch war er zu diesem Zeitpunkt bereits 15 Jahre alt, das Ende seiner Pflichtschulzeit rückte näher und somit die Frage nach seiner Zukunft. Herr Landinger wollte „Einmischungen von außen" soweit wie möglich vermeiden und wendete sich gegen eine Tätigkeit in einer geschützten Werkstätte, da damit der Status als „Behinderter“ einherginge. Stattdessen verfolgte er das Ziel, mit Hilfe des AMS, des österreichischen Services für Arbeitssuchende, eine reguläre Ausbildungsstätte für Timo zu finden. Timos eigene Handlungsentwürfe standen zu diesem Zeitpunkt in deutlichem Gegensatz zu seinen Handlungsoptionen und seinen Handlungskompetenzen. Obwohl er sich in der Wohneinrichtung wohlfühlte und ganz offensichtlich von der Betreuung profitierte, wollte er nach der Pflichtschulzeit wieder fest zu seinen Eltern ziehen und dort eine Berufsausbildung aufnehmen. Sein primärer Wunsch war es, Entwickler für Computerspiele zu werden. Jedoch hatte ihm eine Betreuerin in der Schule unmissverständlich klargemacht, dass er der Ausbildung nicht gewachsen wäre. Als Alternativen gab Timo auch eine Ausbildung zum Koch, eine Karriere beim Bundesheer oder den Betrieb eines eigenen Kebab-Standes an. 
In der sechsten Erhebungswelle lebte Timo wieder zu Hause und bewegte sich in seinem engen und anregungsarmen Mikrokosmos. Seine Freizeit verbrachte er ausschließlich mit Computerspielen, private Kontakte zu Freunden oder Gleichaltrigen hatte er keine. Während der Woche arbeitete er in einer betreuten Werkstatt, in der er verschiedene handwerkliche Tätigkeiten erlernte. Seine Eltern waren weitestgehend zufrieden mit der Einrichtung, da dort weniger pädagogische Konzepte verfolgt würden als in Timos bisheriger Schule. Noch immer hegten sie die Hoffnung, dass Timo zu einer „richtigen“ Lehrstelle gelangen könnte. Timo selbst, so schien es, konnte seine Chancen realistisch einzuschätzen. Zwar wünschte er sich eine Ausbildung als Koch, Tischler oder Verkäufer, jedoch wollte er dazu nicht die Berufsschule besuchen. Als Grund dafür führte er an, nicht „viel schreiben“ zu können und insgesamt ein „eher fauler Typ“ zu sein. Eigentlich wäre es sein Wunsch, ein angenehmes Leben mit einer Frau zu führen, die ihn liebe und nicht gleich wieder verlassen würde. Jedoch glaubte er nicht an eine Erfüllung, da er so süchtig nach Computerspielen sei und dies abschreckend wirken würde: „Joa, aber des hob i koa Chance, dass i jemals a Frau hob.“

2020: Timo Landinger - Eine perspektivlose Zukunft in Abhängigkeit von staatlicher Fürsorge

Die siebte und abschließende Erhebungswelle bringt einige Veränderungen im Leben der Familie mit sich. Herr Landinger ist noch immer arbeitslos, gibt sich aber sehr beschäftigt mit der Abwicklung des Erbes seiner kürzlich verstorbenen Mutter. Dies ist auch die Begründung, weshalb nicht er, sondern seine Ehefrau das Interview führt. Tatsächlich ist es seit dem im Frauenhaus geführten Interview in der dritten Erhebungswelle das erste Mal, dass Frau Landinger ausführlich Stellung beziehen kann. Herr Landinger unterstreicht ausdrücklich, dass seine Frau über alle nötigen Informationen verfüge und er inhaltlich nichts hinzuzufügen habe. Im Vergleich zu den vorherigen Erhebungswellen wirkt das Klima in der Familie deutlich verbessert und auch die Terminvereinbarung für das Interview erfolgte erstmals problemlos. Zum Zeitpunkt der Befragung war Frau Landinger noch in ihrem Beruf als Bürokauffrau tätig, freute sich aber bereits sehr auf den nun kurz bevorstehenden Eintritt in die Pension und die damit einhergehende Möglichkeit, mehr Zeit bei Spaziergängen in der Natur und im Wald zu verbringen und sich um den neu erworbenen Familienhund zu kümmern. Zu ihren Freuden zählt es Frau Landinger, den Enkelkindern beim Spielen zuzusehen und „im Großen und Ganzen" sei sie zufrieden mit der Gesamtsituation. Die Familie be- 
wohnt weiterhin ihr Haus und lebt sehr zurückgezogen. Soziale Kontakte außerhalb der Familie bestehen kaum. Dadurch bringen die Beschränkungen zur Eindämmung der Covid-19-Pandemie auch kaum Veränderungen für die Familie mit sich. Weder Herr noch Frau Landinger äußern sich kritisch über die staatlich verordneten Maßnahmen, ganz im Gegensatz zu Timo, der eine sehr ablehnende Position bezieht und beklagt, dass die Regierung die Wirtschaft zerstören würde.

Sowohl Timo als auch die gesamte Familie sind in der siebten Erhebungswelle weiterhin von staatlichen Sozialleistungen abhängig. Frau Landinger beklagt zwar, dass es hier aktuell zu Verzögerungen käme, jedoch beschränkt sie sich auf diese Aussage, und es werden keine Konflikte mit den zuständigen Stellen thematisiert. Es scheint so, als habe die Familie sich mit ihren Handlungsoptionen abgefunden und Frieden mit der Lebenssituation gemacht. Auch die formulierten Handlungsentwürfe stimmen damit überein und es werden keine Pläne und Ziele mehr thematisiert, die als unrealistisch betrachtet werden müssen. Dies spiegelt sich auch im Umgang mit Timos beruflicher Situation wider: Die noch 2016 und 2017 geäußerte Hoffnung auf eine „richtige“ Berufsausbildung hat sich nicht erfüllt. Vielmehr musste Timo auch seine Tätigkeit in zwei geschützten Werkstätten aufgeben und er ist derzeit ohne Beschäftigung. Ursache hierfür waren zuletzt Mobbingvorkommnisse in dem Betrieb. Timo gibt an, dass er sich "gemobbt vorkimma" sei und bezeichnet die Situation in der Werkstätte als „ned so ansprechend für mi.“ Ergänzend meint er, dass es zwar nicht so schlimm gewesen sei, er sich aber auch nicht so wirklich daran erinnern könne, was denn gewesen sei: „I was eigentlich jetzt so gar nimmer, was ma do ned so gtaugt hat." Auf die konkrete Nachfrage, ob es sich um Konflikte mit den Arbeitskollegen handelte, verneint er dies: „Ähm ne, also ich glaub mit den Kollegen wars ned... Egal. Irgendwas war's halt scho." Diese Aussage legt zumindest die Vermutung nahe, dass es Probleme mit den Betreuungspersonen gab, jedoch kann oder will auch Frau Landinger in dieser Sache keine Klarheit schaffen. In naher Zukunft soll Timo erneut in einem „Lernhaus“, also einer geschützten Werkstatt, untergebracht werden. Hierzu laufen bereits die Vorbereitungen mit der zuständigen Verwaltung und es gibt eine feste Zusage für einen Platz.

Hinsichtlich seiner Handlungsoptionen hat sich bei Timo inzwischen ein hohes Maß an Fatalismus breitgemacht, das auch weitestgehend seinen realistischen Perspektiven entspricht. Während er in früheren Erhebungswellen über hochtrabende wie unrealistische Berufswünsche gesprochen hatte, sind seine persönlichen Handlungsentwürfe in der siebten Erhe- 
bungswelle auf ein Mindestmaß zusammengeschrumptt und Timo nennt keine Pläne und Ziele mehr. Tatsächlich schätzt er seine Handlungskompetenzen inzwischen realistisch ein und ihm ist bewusst, dass frühere Berufswünsche nicht umsetzbar sind. Seine Aussagen lassen tief in Timos Perspektivlosigkeit blicken. So nennt er seine aktuelle berufliche Situation „ja eigentlich relativ eh in Ordnung“, ihm ist allerdings nicht bewusst, was ihn in seiner nächsten betreuten Arbeitsstelle erwartet. Dort werde er „joa arbeiten! (lacht). Einfach so halt." Auch auf Nachfrage, was er denn tun werde, gibt Timo an, dies "grad gar nicht auswendig“ zu wissen. Aus seinen Formulierungen geht hervor, dass ihm inzwischen bewusst ist, wie abhängig er von den Entscheidungen anderer ist, in seinem Fall auch von Stellen der staatlichen Fürsorge. Jedoch würde es das österreichische System von geschützten Einrichtungen auch für Timo erlauben, zumindest Wünsche hinsichtlich seiner beruflichen Tätigkeit zu hinterlegen. Dennoch nennt Timo keine Handlungsentwürfe für seine Zukunft oder zumindest Wünsche. Vielmehr „muss“ er einfach abwarten und „dann voi hackl'n", also einfach irgendetwas arbeiten. Daran zeigt sich, dass Timo offenbar kaum Ansprüche an seine eigene Zukunft stellt und es hingenommen hat, dass weitestgehend andere darüber entscheiden.

Timos Wohnsituation hat sich seit der telefonischen Nachbefragung 2017 deutlich verbessert. Er ist in den ersten Stock des Hauses gezogen, der zuvor von seiner Schwester und deren Familie bewohnt worden war. Dieser Familienteil scheint in ein eigenes Haus gezogen zu sein, jedoch besteht weiterhin viel Kontakt, da Frau Landinger davon berichtet, dass die Enkelkinder regelmäßig bei ihr im Garten spielen. Auf diese Weise verfügt Timo zwar nicht über eine eigene Wohnung, jedoch über ein deutlich größeres Zimmer als zuvor und mehr Privatsphäre. Zuvor grenzte sein Zimmer direkt an das Schlafzimmer seiner Eltern und er musste dieses jedes Mal passieren, um seine Räumlichkeiten zu betreten oder zu verlassen.

\section{Medien im Leben von Timo und seinen Eltern}

Medien spielten in Timos Leben von frühester Kindheit an eine zentrale Rolle, sie waren seine ständigen Begleiter. Als er älter wurde und die Wochentage im Internat verbrachte, erweiterte sich auch sein Horizont und er zeigte Interesse an Aktivitäten wie Besuchen auf einem Bauernhof oder praktische Erfahrungen. Seine Eltern unterstützten diesen Wissensdrang aber nur sehr eingeschränkt und verwiesen ihn darauf, dass bereits zu viel Zeit mit dem Pendeln zwischen Schule und Wohnort verbraucht würde, so dass kaum noch Kapazitäten für Unternehmungen blieben. So konnte 
Timo sich nur mit medialen Unterhaltungsangeboten beschäftigen. Waren es zunächst Fernsehangebote, so wendete er sich später fast ausschließlich PC- und Konsolenspielen zu. Sie boten ihm die Möglichkeit, eine Form der Selbstwirksamkeit zu erleben. Zu kämpfen, sich selbst zu beweisen und Erfolgserlebnisse zu genießen, waren seine primären Nutzungsmotive. Über den gesamten Studienzeitraum hinweg faszinierten ihn ganz besonders Themen wie Kampf, Tod, Gewalt und Zerstörung. Und seine Eltern gewährten ihm dazu Zugang, indem sie Timo regelmäßig mit gewalthaltigen Videospielen versorgten. Mit der Zeit besaß er eine umfassende Sammlung an Kampfspielen, die er oft von morgens bis abends spielte. Bereits 2012 bezeichnete Herr Landinger Videospiele als „die Welt“ seines Sohnes. Trotz der geradezu überwältigenden Spielpraxis von Timo muss dabei betont werden, dass er ein sehr schlechter Spieler blieb. Mehrere Male gewährte er den Interviewern einen Einblick in seine eingeschränkten Fähigkeiten zur Bewältigung der Spielanforderungen. 2016, Timo verbrachte erneut seine gesamte Freizeit zu Hause, bezeichnete er sich selbst als „süchtig“ nach Videospielen und gab an: „Ich träume nur von Spielen, Spielen, Spielen." An den Wochenenden spielte er demnach oft zwölf Stunden am Stück und auch unter der Woche war es das Erste, was er tat, wenn er aus seiner Bildungsstätte nach Hause kam. Bis 2016 durfte Timo das Internet zu Hause nicht nutzen, obwohl der Zugang zu einem PC mit Internet und später zu einem Smartphone bereits ab $2010 \mathrm{zu}$ Timos größten Wünschen zählten. Seine Eltern begründeten das Verbot damit, dass dies zu gefährlich sei. Ansätze zur gemeinsamen, begleiteten Internetnutzung wurden jedoch nicht thematisiert. Es besteht die Vermutung, dass die tatsächliche Motivation hinter diesem Verhalten darin lag, weiterhin Kontrolle über Timo und seinen Zugang zu Informationen zu behalten. Erst 2014 bekam Timo sein erstes Telefon. Es handelte sich um ein veraltetes Gerät, mit dem er lediglich seine Eltern anrufen und ihnen SMS schicken konnte.

2020 hat sich an Timos Konsum von Videospielen kaum etwas geändert. Shooter und Action-Rollenspiele sind weiterhin von zentraler Bedeutung für ihn und begleiten seinen gesamten Tagesablauf. Es sei „arg“, aber er „muss“, so sagt er, „immer" spielen. Aber es gibt eine grundlegende Veränderung in Bezug auf Timos Mediennutzung: Es scheinen keinerlei elterliche Reglementierungen mehr zu existieren, und Frau Landinger betont, keinen Einblick in die Inhalte der Mediennutzung ihres Sohnes zu haben. Neben seinen alten Konsolen besitzt er nun auch eine PlayStation 4 und spielt darauf leidenschaftlich Spiele der Monster Hunter Reihe online gegen andere. Konsolenspiele sieht Timo auch 2020 als seinen „einzigen 
Lebensinhalt". Zudem darf er nun den Laptop seiner Mutter verwenden und verfügt über sein eigenes Smartphone mit Vertrag, auf dem er uneingeschränkt alle Inhalte und Apps nutzen kann. Timo macht von diesen neuen Freiheiten Gebrauch, jedoch ohne elterliche Anleitung und begleitende Kontrolle. Eigenen Angaben zu Folge hat Timo Facebook, WhatsApp und YouTube, nutzt diese Angebote aber „eher passiv“. Ob er auch Instagram hat, muss Timo zunächst nachschauen, da er es nicht auswendig weiß. Dies veranschaulicht deutlich seinen Mangel an Medienkompetenz, mit dem ihn seine Eltern allein lassen. Neu ist auch die Nutzung des Internets zur Informationsbeschaffung, etwa über die Covid-19-Pandemie. Dazu, so Timo, verwendet er verschiedene Nachrichtenanbieter, in erster Linie jedoch „die App“, wohl eine auf dem Telefon vorinstallierte NewsZentrale.

Auch Frau Landinger hat ihre Mediennutzung angepasst. Sie verwendet inzwischen WhatsApp zur Kommunikation mit der Familie und nutzt das Internet zur Beschaffung von Nachrichten und Bastelanleitungen (YouTube). In erster Linie informiert sie sich aber über das Fernsehen und sucht dort auch Unterhaltung (ORF 1, Puls 4, Sat.1 Gold, n-tv). Schon früher diente das Fernsehen als wichtigstes Unterhaltungs- und Informationsmedium für die Eltern. Herr Landinger betonte regelmäßig, besonderen Wert auf die Rezeption von Dokumentationen und Wissenssendungen auf Sendern wie ZDF.info, 3sat und n-tv zu legen, aber er gab auch an, wie er sie bezeichnete, „Idiotensendungen“ wie Scrubs, How I Met Your Mother und Simpsons sowie Sportsendungen anzuschauen. Zudem las Herr Landinger Medizin- und Pflanzenkundebücher, während sich seine Frau Häkel- und Handarbeitszeitschriften zuwendete. Im Gegensatz zu Timo hatten seine Eltern bereits seit mehreren Jahren einen Internetanschluss und Smartphones, nutzten diese Möglichkeiten aber nur sehr eingeschränkt. Die wichtigsten Aspekte von Herrn Landingers Internetnutzung umfassten E-Mail-Korrespondenz, digitale Behördengänge sowie die Recherche, etwa nach Krankheiten.

Fazit

Sowohl das Familienklima als auch die finanzielle Situation von Familie Landinger haben sich zuletzt etwas stabilisiert und verbessert. Dennoch bleiben die sozio-ökonomischen und die sozio-emotionalen Bedingungen schwer belastet. Herr und Frau Landinger scheinen sich mit ihren Lebensbedingungen abgefunden und arrangiert zu haben. Insbesondere Frau Landinger blickt optimistisch nach vorn und freut sich auf einen geruhsamen Lebensabend mit ihren Enkelkindern und in der Natur. Der Leid- 
tragende bleibt Timo, der auch 2020 keine konkreten Handlungsentwürfe entwickelt und keinerlei eigene Perspektiven für eine bessere Zukunft hat. Dies liegt nicht zuletzt daran, dass seine Eltern in der Vergangenheit vielversprechende Therapie- und Bildungsmaßnahmen verhindert haben. So konnte er kaum eigene Handlungskompetenzen, die ansonsten vielleicht möglich gewesen wären, entwickeln. Auch seine Handlungsoptionen sind maximal eingeschränkt; Timo ist in seiner weiteren Entwicklung vollständig abhängig von anderen, insbesondere von Stellen der staatlichen Fürsorge. Zuletzt bestand der Plan, Timo erneut in einer geschützten Einrichtung unterzubringen; er selbst hat jedoch keine Kenntnis darüber, was ihn dort erwarten wird. Seine Lebensbedingungen hat Timo inzwischen weitestgehend realistisch erfasst und dahingehend seine Handlungsentwürfe angepasst, auf die schlimmste mögliche Weise: Denn er hat nicht nur seine Hoffnungen auf eine Beziehung abgeschrieben, sondern er formuliert auch keine Pläne und Ziele, in seinem Fall eher Wünsche, mehr für die Zukunft. Stattdessen geht er fatalistisch davon aus, irgendetwas zu arbeiten, das ihm von außen vorgeschrieben wird. Als einzige Ablenkung bleiben ihm, wie schon in seiner Kindheit und Jugend, Medienangebote, um seine Zeit auszufüllen. Während Timo früher auf Konsolen- und PCSpiele beschränkt war, hat er inzwischen auch die Möglichkeit, online gegen andere zu spielen, und so ein Mindestmaß an Kontakten zu pflegen. Zudem gestatten ihm seine Eltern inzwischen auch die Nutzung eines eigenen Smartphones und des Laptops seiner Mutter. Dabei ist er jedoch auf sich alleine gestellt und erhält keine Anleitung oder Begleitung.

2.2.3 Manfred Oblinger (21 Jahre alt) und seine Eltern: Vielfältige sozioemotionale wie sozio-ökonomische Belastungen kennzeichnen die Familie, erst spät treten Verbesserungen ein

2005 bis 2017

Manfred Oblinger war zu Beginn der Studie sechs Jahre alt. Er ist der Drittjüngste aus einer Großfamilie mit insgesamt sechs Kindern, die drei Ältesten stammen aus einer früheren Ehe seiner Mutter. Manfred kam für die jüngeren Geschwister somit die Rolle des großen Bruders zu, die er auch aktiv annahm. In diesem Kontext zeigte er auch immer wieder sehr deutlich seine Zuneigung zu seinen jüngeren Geschwistern, trat für sie ein und betonte, dass er „für sie sterben würde“ (2014). Dennoch war das Verhältnis über den gesamten Erhebungszeitraum auch immer wieder von Spannungen belastet, die sowohl Manfred als auch seine Mutter of- 
fen einräumten. Dies lag einerseits daran, dass Manfred die Geschwister „elterlich erziehen“ (2007) und immer „das Sagen haben“ (2010) wollte, andererseits aber auch daran, dass er kaum seine Ruhe hatte und sich nicht zurückziehen konnte, da die Jüngeren sehr oft seine Nähe suchten und dabei auch immer ungefragt und ohne anzuklopfen in sein Zimmer kamen. Erschwerend kam hinzu, dass beide, ebenso wie Manfred selbst, an ADHS litten. Als Ausgleich besuchte Manfred häufig seine älteren Geschwister, die im selben Haus, beziehungsweise in der näheren Umgebung wohnten, um sich mit ihnen auszutauschen und dort auch Ruhe zu finden.

Seit Beginn der Studie lebte die Familie immer in einer geförderten $100 \mathrm{~m}^{2}$ großen Mietwohnung, in der Manfred auch ein eigenes Zimmer hatte. Dieses musste er sich aber oft mit seinem Vater teilen, wenn dieser spät aus der Arbeit kam oder es Spannungen zwischen den Eltern gab. Die Familie war kurz vor Beginn der Studie aufs Land gezogen, weil sie dort die dringend benötigte, leistbare größere Wohnung in einer sehr abgelegenen und infrastrukturell benachteiligten ländlichen Gegend bekommen konnte. Die Wohnung befand sich während der Laufzeit der Studie in einem recht guten Zustand und war zumeist sauber. Alle Familienmitglieder fühlten sich dort, so die Mutter und auch Manfred selbst, wohl. Die neue Wohngegend hingegen betrachtete vor allem die Mutter in den ersten drei Erhebungswellen sehr kritisch, sie störte sich an den „ausländischen Nachbarskindern“. Später lebte sich die Familie jedoch ein, und auch Frau Oblinger pflegte intensive freundschaftliche Kontakte, auch mit Nachbarinnen mit Migrationshintergrund. Zeitweise engagierte sie sich ehrenamtlich bei der örtlichen Tafel. Manfred selbst wollte 2016 gerne ,immer dort leben“.

Die Oblingers zählten im Panel stets zu den sozio-ökonomisch am stärksten belasteten Familien und waren über alle Erhebungswellen hinweg immer als armutsgefährdet einzustufen. Neben der geförderten Wohnung bezog die Familie 2005 Sozialhilfe und 2014 Sozialhilfe, Notstandshilfe und Wohnbeihilfe, 2016 waren beide Elternteile auf Arbeitslosengeld angewiesen. Die belastenden Faktoren waren dabei vielfältig: Frau Oblinger verfügt nur über einen Hauptschulabschluss und war immer Hausfrau, da sie mit 16 Jahren ihr erstes Kind bekommen hatte. Herr Oblinger ist ausgebildeter Koch, arbeitete zunächst aber als technischer Zeichner. In der ländlichen Gegend fand er jedoch in diesem Beruf keine Beschäftigung und begann wieder, zum Teil in Vollzeit, gelegentlich aber auch nur in Teilzeit, in verschiedenen Betrieben als Koch zu arbeiten. Seine Arbeitszeiten waren unregelmäßig, und er musste oft mehrere Tage oder Wochen fernab der Familie wohnen, was sich auch negativ auf das Familienklima 
auswirkte. Erschwerend belasteten auch gesundheitliche Probleme beide Elternteile. Herr Oblinger war immer wieder schwer erkrankt und musste operiert werden. 2014 war er in Folge einer schweren Depression nicht arbeitsfähig. Frau Oblinger erlitt zwischen 2010 und 2012 zwei Schlaganfälle und hat seither körperliche und kognitive Einschränkungen.

Die finanzielle Lage beeinflusste das Freizeitverhalten der Familie, da kaum Geld für Unternehmungen übrigblieb. Gemeinsame Aktivitäten beschränkten sich auf Kartenspiele am Abend oder ein gemeinsames Mittagessen von Manfred mit seinen Eltern. Oft aßen die Familienmitglieder jedoch auch getrennt voneinander. Vor allem zu Beginn der Erhebung hatte es deutlich Anzeichen dafür gegeben, dass die Beziehung der Eltern belastet war. Auch das Verhältnis zwischen den Geschwistern bezeichnete Frau Oblinger als nicht gut, es wurde viel gestritten. So wollte Manfred gerne über die jüngeren Geschwister bestimmen, was die sich aber nicht gefallen ließen, sodass es häufig zu Streit kam. Manfred habe zudem, wie seine Mutter erzählte, seine Ruhe gebraucht und habe es überhaupt nicht ertragen können, wenn die Geschwister ihn im Zimmer störten. Zu seinem Vater hatte Manfred allerdings ein gutes Verhältnis und bezeichnete sich als „Papakind“. Das Verhältnis zu seiner Mutter wurde 2016 stark erschüttert, da Manfred mit einem Freund bei einem Diebstahl erwischt wurde und 60 Sozialstunden ableisten musste.

Wie seine Eltern war Manfred gesundheitlich immer stark eingeschränkt. Neben ADHS wurde bei ihm Asthma diagnostiziert, dennoch rauchte er als Jugendlicher. Zudem war er stark übergewichtig und nahm im Verlauf der Studie immer weiter zu. Seine körperlichen Probleme führten dazu, dass er kaum sportliche Freizeitaktivitäten mit Gleichaltrigen wahrnahm. Da Manfred später auch als Jugendlicher kein Interesse daran zeigte auszugehen, blieben ihm in seiner Freizeit fast ausschließlich seine Computerspiele. Diese nutzte er jedoch intensiv zum Austausch mit anderen Spielern weltweit. Darüber hinaus traf er sich selten mit Freunden. Erstmals in der dritten Erhebungswelle hatte Manfred einen besten, türkischstämmigen Freund. Später berichtete er von Aktivitäten mit Freunden, aber nie von einer romantischen Beziehung.

$\mathrm{Zu}$ Manfreds Lage trug auch bei, dass er nicht nur aufgrund seiner Körperfülle immer wieder zum Opfer von Mobbing wurde, sondern auch wegen seines Verhaltens, denn Manfred gilt nach ärztlichen Diagnosen als hochbegabt. Seine thematischen Interessen, etwa für Politik, Geschichte und Zeitgeschehen, waren eher ungewöhnlich für sein Alter. Lange Zeit hatte Manfred für den rechtsgerichteten Politiker Jörg Haider geschwärmt, da dieser, wie Manfred betonte, nicht so viele Ausländer ins Land lassen 
würde. In seiner strukturschwachen Wohngegend erhielt er jedoch nie eine entsprechende Förderung und zu einem, immer wieder diskutierten, Umzug konnte sich die Familie nicht entschließen. Nach der Volksschule besuchte Manfred zunächst erfolgreich eine Neue Mittelschule und dann eine Handelsakademie, die er jedoch bereits nach zwei Monaten wieder abbrach, da er kein „Schultyp“ sei. Vielmehr wollte er möglichst schnell ins Berufsleben einsteigen und einen Lohn für seine Arbeit bekommen. Dieser Wunsch steht in enger Verbindung zur wirtschaftlich sehr angespannten Situation der Familie, die Manfred auch als jüngeres Kind sehr wohl wahrgenommen hatte: „So liaba hob i im Kühlschrank wos, g'scheider der Kühlschrank is voi, Mama, mir hom wos zum Essen, ois wia i hon jetz a Puma-Leiberl.“ Der Berufseinstieg gestaltete sich jedoch äußerst schwierig, und Manfred erhielt auch in einem erweiterten Umkreis um seinen Wohnort mehrere Absagen zu seinen Bewerbungen auf Lehrstellen. In der sechsten Erhebungswelle 2016 verbrachte er somit sehr viel Zeit zu Hause vor dem Computer und hatte noch keine berufliche Zukunftsperspektive.

2020: Manfred Oblinger - einem hochbegabten Jungen gelingt der Start ins Berufsleben

In der siebten Erhebungswelle 2020 kommt es zu grundlegenden Veränderungen, die zu weitreichenden Verbesserungen führen: Im Gegensatz zu 2016 ist die Familie nicht mehr auf Sozialhilfe angewiesen. Frau Oblinger ist nach vielen Jahren im Krankenstand und in Arbeitslosigkeit als Küchengehilfin wieder ins Berufsleben eingestiegen. Derzeit überlegt sie, eine Umschulung zur Pflegehelferin zu absolvieren. Herr Oblinger befindet sich zwar wegen einer Wirbelsäulenversteifung aktuell im Krankenstand, ist jedoch als Küchenchef in der Nähe des Wohnortes fest angestellt. Manfreds Leben hat sich völlig verändert. Nach langer und erfolgloser Suche hat er, mit Hilfe des AMS, eine Lehrstelle zum EDV-Kaufmann gefunden und ist dazu in die Nähe einer Großstadt gezogen. Beim Umzug und der Eingewöhnung in der neuen Umgebung hat ihn sein Vater unterstützt. Manfred bezeichnet sich noch immer als „absolutes Papakind“ und pflegt ein extrem enges Verhältnis zu seinen Eltern. Neben gegenseitigen Besuchen ruft er beide Elternteile im Laufe des Tages auch viele Male an. Seine Arbeitsstelle bezeichnet er als „Traumberuf“, in dem er sich weiter entwickeln möchte. Nach der bevorstehenden Lehrabschlussprüfung soll Manfred fest übernommen werden und umfangreiche interne Fortbildungsmaßnahmen erhalten. Somit kann Manfred nun erstmals auch längerfristige Pläne machen, Ziele festsetzen und konkrete Handlungsentwür- 
fe verfolgen - eine Folge der neuen Chance zur Ausbildung und der damit verbundenen Möglichkeit, neue Handlungskompetenzen zu entwickeln. Mit seinen Kollegen und seinem Chef hat Manfred, wie er erzählt, ein sehr gutes Verhältnis. Dies wirkt sich auch positiv auf seine Handlungsoptionen aus, denn er werde nicht nur beruflich gefördert, sondern könne auch zu sehr günstigen Konditionen in einer Betriebswohnung in einer Wohngemeinschaft mit zwei Kollegen wohnen, die er als enge Freunde und wichtige Bezugspersonen bezeichnet. Insgesamt ist Manfred, in seinen Worten, „richtig aufgegangen“ im Leben nahe der Stadt. Über seinen Umzug, das Zusammenleben mit seinen Kollegen und seine Arbeit äußert er sich sehr zufrieden. Früher habe er sehr zurückgezogen gelebt, wollte sich richtiggehend „daham verkriachen“. Noch immer sei er niemand, der ständig auf Partys gehe, aber er sei viel und gern draußen unterwegs und besuche auch gemeinsam mit Freunden Kneipen; Alkohol trinke er allerdings nicht. Auch Frau Oblinger berichtet, dass Manfred nun ein ganz anderer Mensch sei. Im Gegensatz zu früher sei er draußen unterwegs, träfe sich mit Freunden, führe mit dem Rad und ginge sogar ins Schwimmbad oder an den See. Ausschlaggebend dafür war eine Magenverkleinerung, die Manfred gemeinsam mit seinem Vater hatte vornehmen lassen. Infolge dessen konnte er sein Gewicht stark reduzieren und ein ganz neues Lebensgefühl und Lebensfreude entwickeln. Zu seinen weiteren Wünschen für die nahe Zukunft gehört es, noch mehr als bereits jetzt unter Menschen zu gehen. Dazu will er sich weiter vom „PC distanzieren“, bis er „ein Verhältnis von 75:25“ erreicht habe.

\section{Medien im Leben von Manfred und seinen Eltern}

Trotz ihrer eingeschränkten finanziellen Mittel war Familie Oblinger immer sehr gut mit Mediengeräten ausgestattet. Dazu zählten neben mehreren Fernsehern mit Kabelanschluss auch ein Sky-Abonnement, mehrere Computer, Smartphones und zahlreiche Spielekonsolen (PlayStation 2, 3 und 4, XBox, Xbox360). Computer nahmen dabei immer eine zentrale Rolle im Leben von Manfred ein. Bereits in der vierten Erhebungswelle bezeichnete er seinen Laptop als das mit Abstand wichtigste Gerät, und er verbrachte Tag für Tag viele Stunden vor dem Bildschirm: „Ja Laptop - Platz 1, Platz 2: Laptop. Platz 3: Laptop. Platz 4: Laptop.“ Später übernahm diese Rolle ein leistungsstärkerer Stand-PC, den er technisch immer weiter aufrüstete, unter anderem mit neuen Bildschirmen und Grafikkarten, und den er in erster Linie für zumeist gewalthaltige Spiele nutzte. Seine Mutter unterstützte ihn und versorgte Manfred bereits im Alter von sechs Jahren mit Spielen für 16- bis 18-Jährige, nicht zuletzt um ihm 
von Aktivitäten mit „Ausländerkindern“ in der Nachbarschaft abzuhalten. Neben dem Spielen an sich entwickelte sich später auch der intensive Austausch mit anderen Spielern weltweit zu einem zentralen Nutzungsmotiv für Manfred. Dazu verwendete er sowohl die Chatfunktion von Spielen als auch Teamspeak und Skype. Auf diese Weise gelang es Manfred, seine Interessen mit Gleichgesinnten zu teilen und, zumindest in Ansätzen, seinen Mangel an Freundschaften und Freizeitaktivitäten zu kompensieren. Darüber hinaus nutzte er seinen PC auch zur Informationsbeschaffung (Wikipedia, Google, gutefrage.at, eBooks), für Video- und Musikstreaming (YouTube, Mediatheken, Radio) und zum Konsum von pornografischen Inhalten (auch gemeinsam mit Freunden). Bereits als Kind faszinierten ihn Animés wie Dragon Ball $Z$, in deren Welt er eintauchte und mit deren Figuren er sich identifizierte. Später erweiterte er sein Repertoire um Infotainment-Angebote und wiederum meist gewalthaltige Computerspiele, Filme, Krimis und Serien: „I bin eher so da Action Typ mit Bluat.“ (2016) Sein Smartphone nutzte Manfred, im Gegensatz zu seinen Geschwistern und vielen Gleichaltrigen, dagegen nur in einem überschaubaren Umfang. Auch auf Facebook war Manfred in der vierten Erhebungsweise zeitweise vertreten, nahm dann aber wieder Abstand von Sozialen Medien und schaut sich heute nur selten etwas auf Twitter an. 2020 nutzt er sein Smartphone in erster Linie, um per Telefon und WhatsApp mit seinen Eltern Kontakt zu halten und um Rezepte auf YouTube zu suchen, denn gesund $\mathrm{zu}$ kochen ist eine seiner neuen Leidenschaften. Noch immer bezeichnet Manfred Computerspiele als einen „großen Teil“ von sich. Jedoch möchte er deren Bedeutung reduzieren und nennt folgende Aufteilung für seine Freizeit: Je „a Drittel so Serien schau'n (Amazon Prime, Netflix, YouTube), Freind treffen und zock'n." Zu Informationszwecken, etwa über das Weltgeschehen, nutzt Manfred das Internet dagegen ausdrücklich nicht (mehr), dies würde ihn „schlichtweg nicht interessieren“.

Auch für Manfreds Eltern haben Medien im Alltag eine hohe Relevant. Bereits in der zweiten Erhebungswelle beurteile Frau Oblinger den Stellenwert von Medien auf einer Skala von 1 bis 10 mit einer 10. In der Nutzung der Eltern dominierten lange Zeit jedoch eher traditionelle Medienangebote. Die bevorzugten Inhalte der Eltern (Dokumentationen, Krimis, Heimatfilme, Sport- und Nachrichtensendungen) veränderten sich dabei über die Erhebungswellen hinweg kaum. Für Frau Oblinger sind auch nach wie vor das Radio sowie die Tageszeitung sehr wichtig geblieben. Obwohl sie inzwischen gerne und oft das Internet nutzt, sieht sie keine Veranlassung dazu, zu Gunsten von Online-Zeitungen auf die gedruckte Ausgabe zu verzichten. Aufgrund der gesundheitlichen Belastungen beider Eltern dienten 
ihnen Medien im Laufe der Jahre nicht nur zur Information, sondern mehr und mehr auch als zentrale Freizeitfüller. Vor allem Herr Oblinger nutzte Medien früher meist nur abends nach der Arbeit, dies hatte sich infolge seiner Erkrankungen und der daraus resultierenden Berufsunfähigkeit jedoch stark geändert. Für Frau Oblinger kommt Medien gar eine therapeutische Bedeutung zu, denn sie spielt seit ihren Schlaganfällen gerne Gedächtnisspiele auf Facebook oder Candy Crush auf dem Smartphone, „um ihren Geist wach zu halten“. Zudem nutzt Frau Oblinger gerne auch Soziale Medien wie Facebook und WhatsApp, um mit Freunden in Kontakt zu bleiben und sich auszutauschen. In der siebten Erhebungswelle hat sich Facebook für sie jedoch auch zu einer wichtigen Informationsquelle abseits der medialen Berichterstattung entwickelt. Den dort kommunizierten Informationen, etwa zur Covid-19-Pandemie, scheint sie vielfach mehr Glauben zu schenken als jenen in den Fernsehnachrichten und in der Zeitung.

\section{Fazit}

Familie Oblingers Handlungsoptionen blieben über viele Jahre hinweg stark eingeschränkt; die Familie litt jedoch nicht nur unter schweren sozio-ökonomischen, sondern auch unter sozio-emotionalen Belastungen. Hinzu kamen schwerwiegende gesundheitliche Einschränkungen, denen Herr und Frau Oblinger auch mit einer verstärkten Mediennutzung begegneten. Zeitweiligen sozio-emotionalen Konflikten zum Trotz versuchten die Familienmitglieder zumeist, im Rahmen ihrer Möglichkeiten, untereinander für ein möglichst gutes Klima zu sorgen und füreinander einzutreten. Daraus resultiert bis heute ein sehr enges Verhältnis zwischen Manfred und seinen Eltern, das, wie sich in Manfreds Alltag zeigt, auch eine räumliche Trennung aushält. Aus eigener Kraft gelang es der Familie jedoch nicht, den hochbegabten Sohn entsprechend zu fördern oder Förderung aktiv zu suchen, um ihm so eine entsprechende Schulbildung als Start ins Berufsleben zukommen zu lassen. Dazu war schließlich die Unterstützung durch eine staatliche Stelle nötig. So ist es ganz konkret den Maßnahmen des AMS zu verdanken, dass Manfred schließlich eine Lehrstelle vermittelt wurde, die seinen Kompetenzen und Interessen entspricht. Der dafür nötige Umzug in eine größere Stadt ermöglichte Manfred neue Handlungsentwürfe und eine Perspektivenerweiterung, die er für einen erfolgreichen Neustart zu nutzen wusste. Auch im Sinne der Förderung seiner Gesundheit und der sozialen Aktivität war ein externer Eingriff als Auslöser nötig. Erst nach einer invasiven Magenverkleinerung gelang es Manfred, deutlich an Gewicht zu verlieren und in der Folge neue Freizeit- 
aktivitäten aufzunehmen und Freundschaften zu entwickeln. In diesem Zusammenhang versucht Manfred nun auch seine überbordende Nutzung von Computerspielen zu reduzieren und seine Handlungskompetenzen auf andere Tätigkeiten zu richten. Es darf jedoch nicht außer Acht gelassen werden, dass ihm seine umfassenden Kenntnisse in den Bereichen Computer, Internet und Online-Kommunikation auch in seiner Ausbildung zu Gute kommen.

\subsubsection{Viktoria Öllinger (bald 20 Jahre alt) und ihre Mutter: Die stark sozio-ökonomisch und sozio-emotional belastete Familie stabilisiert sich}

\section{5 bis 2017}

Die Familienkonstellation von Familie Öllinger veränderte sich während der Studie mehrfach. Zu Beginn der Erhebung im Jahr 2005 war Frau Öllinger von Viktorias leiblichem Vater geschieden, hatte aber einen neuen Partner, den Viktoria als Vater bezeichnete. Zwei Jahre später lebte sie wieder allein, nach drei weiteren Jahren wieder in einer Ehe. Seit ihrer erneuten Scheidung 2012 und einigen weiteren gescheiterten Beziehungen lebt Frau Öllinger allein mit ihrer Tochter Viktoria, die unter der Trennung ihrer Eltern emotional stark litt. Zu Beginn der Erhebung war Frau Öllinger bereits nicht mehr in ihrem Beruf tätig, sie war gelernte Köchin und hatte später auch eine Zeitlang als Bürokauffrau gearbeitet, sondern arbeitete als Putzhilfe, später in einem Callcenter, dann als Schulbusfahrerin. Seit der vierten Erhebungswelle (2012) konnte Frau Öllinger wegen schwerer Krankheiten keiner beruflichen Tätigkeit mehr nachgehen. Aus finanziellen Gründen war die Familie einige Male umgezogen. Zu Beginn der Erhebung hatte sie eine $69 \mathrm{~m}^{2}$ große Mietwohnung bewohnt, in der dritten Untersuchungswelle lebten Mutter und Tochter mit dem neuen Mann in einer größeren Mietwohnung in einer anderen Wohngegend als zuvor. In der vierten Erhebungswelle zogen Frau Öllinger und ihre Tochter erneut um; da sie die neue Wohnung bald als zu klein empfanden, erfolgte im selben Sommer ein weiterer Umzug. 2016 bewohnten Frau Öllinger und ihre Tochter eine neue geförderte Gemeindewohnung in Stadtnähe.

Die sozio-ökonomische und sozio-emotionale Situation der Familie war aufgrund einer Privatinsolvenz infolge des unredlichen Verhaltens des damaligen Partners von Frau Öllinger während ihres langen Krankenhausaufenthaltes lange Zeit sehr stark angespannt. Der Mann hatte sie nicht 
nur mit einer anderen Frau betrogen, sondern auch mit Internetbestellungen auf ihren Namen hohe Schulden angehäuft. In der sechsten Erhebungswelle (2016) erhielt Frau Öllinger die ersehnte Invalidenrente, um „über die Runden zu kommen“. Viktoria, die sich mehr und mehr für ihre Mutter verantwortlich zeigte, versuchte mit zunehmendem Alter die finanziellen Schwierigkeiten der Familie durch eigenen Verzicht zu reduzieren. In der sechsten Erhebungswelle zeigte sie Anzeichen von Magersucht.

Viktoria war mit der Zeit zur entscheidenden Ansprechpartnerin ihrer Mutter in nahezu allen Belangen geworden. Noch zum Ende der Erhebung 2016 und der telefonischen Nachbefragung Anfang 2017 lebte Viktoria in einem stark ausgeprägten sozio-emotionalen Spannungsfeld: Einerseits sorgte sie sich um ihre Mutter; ihr lag daran, dieser weiterhin in allen Belangen beizustehen und sie zu unterstützen. Deshalb hatte sie lange Zeit versucht, ihr das Gefühl zu vermitteln, noch nicht erwachsen zu werden. Andererseits zog es sie von zu Hause fort; sie wünschte sich, nach der Schule die Welt kennenzulernen und damit endlich auch eigene Handlungsentwürfe entwickeln und ein eigenständiges Leben führen $\mathrm{zu}$ können.

Familie Öllinger war nicht nur in ihren Handlungsoptionen und Handlungsentwürfen stark belastet, sondern Mutter wie Tochter auch in ihren Handlungskompetenzen sehr eingeschränkt und kaum in der Lage, ihren Alltag zu bewältigen.

2020: Viktoria Öllinger und ihre alleinerziehende, kranke Mutter: Ausgeprägte Parentifizierung, doch mehr Eigenständigkeit

2020 hat sich Viktorias Lage sehr verändert, ihre Mutter ist jedoch nach wie vor eine zentrale Bezugsperson in ihrem Leben; Viktoria kümmert sich rührend um die mittlerweile im Rollstuhl sitzende und auf umfassende Pflege angewiesene Frau. Viktoria hat den Besuch einer Fachschule für Mode abgebrochen, da sie sich dort unter Lernstress gefühlt und zu viel Druck empfunden habe. Danach hat sie eine Handelsschule besucht - eine Ausbildung, die es ihr ermöglicht, nun im Personalmanagement in einem großen Klinikum in der Stadt zu arbeiten, in der sie mit ihrer Mutter lebt. Viktoria erzählt, dass sie ihren dringenden Wunsch, ein Jahr im Ausland zu verbringen, aufgegeben habe, weil sie, wie sie sagt, ihre Mutter „nicht allein lassen wollte“. So schafft sie es aufgrund einer Reduzierung auf 30 Stunden trotz aller alltäglichen Herausforderungen in dem Krankenhaus, weiterhin noch ihre Mutter zu pflegen und auch emotional für sie da zu sein. Unterstützt wird sie durch einen täglich in die Wohnung kommenden Sozialdienstleister, der den Wechsel des Dauerkatheters vor- 
nimmt und Frau Öllinger beim Duschen hilft. Sie selbst unterstütze ihre Mutter, so auch beim Duschen und bei der Reinigung des künstlichen Darmausgangs, wenn der Sozialdienstleister nicht kommen könne.

Das bereits seit Viktorias zehntem Lebensjahr zu beobachtende umgekehrte Mutter-Tochter-Verhältnis - Viktoria nahm mehr und mehr die Rolle der sich kümmernden Mutter ein - hat sich in der siebten Erhebungswelle 2020 noch weiter in Richtung einer Parentifizierung entwickelt. Aber Viktorias Handlungsentwürfe werden nicht länger allein vom Spannungsverhältnis zwischen starker verantwortlicher Gebundenheit an ihre Mutter und ihrer Sehnsucht nach Ferne und dem damit verbundenen Wunsch, nach der Schule die Welt kennenzulernen und dann endlich auch eigene Handlungsentwürfe zu entwickeln und ein eigenständiges Leben führen zu können, beeinflusst. Ihre zwischenzeitlichen Bestrebungen, der Mutter gegenüber nicht als junges Mädchen in der $\mathrm{Pu}-$ bertät zu gelten, weil diese große Angst vor dem Erwachsenwerden ihrer Tochter äußerte, da damit möglicherweise eine, auch räumliche, Ablösung von ihr verbunden sein könnte, scheint Viktoria ganz überwunden zu haben. Seit zwei Jahren hat Viktoria einen festen Partner, mit dem sie eine sehr vertrauensvolle und auch sie selbst stärkende Beziehung unterhält. Nach dem klaren Entschluss, die Fachschule für Mode zu beenden und eine Handelsschule zu besuchen und dem Einstieg in die Arbeitswelt, gelang es Viktoria, eigenständiger zu werden und auch mehr emotionale Freiheit von der Mutter zu erlangen. So bemühte sie sich sehr darum, ihre Mutter zu mehr Selbständigkeit zu ermuntern, dies auch zuweilen gegen den offen geäußerten Widerstand der Mutter („Boah, bist du gemein.“). Zwischenzeitlich musste Viktoria auch versuchen, die mit Tränen einhergehenden Zusammenbrüche ihrer Mutter und deren „Drohungen“, „alles hinschmeißen" und nicht mehr leben zu wollen, zu kompensieren. Wie Viktoria sagt, sei ihre Mutter wieder Kind geworden, „sie is“, so Viktoria wörtlich, „mei Kind word'n, sozusag'n.“. Ihre Mutter habe sie bei allem gebraucht und sei „trotzig wie ein Kleinkind“ gewesen und habe ihr „das Leben schwer gemacht“. Nach einer Zeit der gegenseitigen Anfeindung hätten aber beide gelernt, die aktuelle Lage zu akzeptieren und nun auch zu bewältigen, wie Viktoria im Interview erzählt.

Von ihrem leiblichen Vater erhielt und erhält Viktoria nach wie vor keine emotionale Unterstützung. Seit ihrem 18. Geburtstag, zu dem er sich immerhin gemeldet hatte, hat sie ihn nicht mehr privat gesehen. Danach sei sie jedoch noch einmal zu ihm gefahren, um mit ihm zu sprechen und ihn nicht wegen nicht gezahlter Alimente verklagen zu müssen. Ihre Mutter und sie hätten das ihnen zustehende Geld dringend gebraucht, da 
die Querschnittslähmung der Mutter hohe finanzielle Ausgaben nach sich gezogen habe. Ihr Vater sei aber auch unter den nunmehr für Viktoria emotional verschärften Bedingungen nicht bereit gewesen, sie zu trösten und zu unterstützen; sie habe von ihm nur Ablehnung erfahren und Sätze gehört, wie: „Is doch mir scheiß egal, wann du auf der Stroß'n land'st mit deiner Mama. Und dann hob i hoit wieder gwusst, ok, er hot si hoit net gändert. Und des tuat hoit scho weh, wannst des von erm hearst."

Sozio-ökonomisch haben sich Viktorias Lebensbedingungen mit dem Einstieg in ihren Beruf verbessert, sie verdient nach dem Abschluss der Schule eigenes Geld. Aber, wie Viktoria bedauert, seien dadurch wichtige finanzielle Befreiungen für sie und ihre Mutter weggefallen, wodurch ihr Leben deutlich teurer geworden sei. Denn nach wie vor wohnt sie bei ihrer Mutter in der mittlerweile behindertengerecht umgebauten kleinen Mietwohnung. Die damit für sie verbundenen Abzüge seien auch ein Grund, warum sie überlege, innerhalb der nächsten zwei Jahre auszuziehen.

Frau Öllinger: Schwere gesundheitliche Herausforderungen, Rollstubltanzen als Lebenselixier

Frau Öllingers sozio-ökonomische Bedingungen sind weiterhin sehr schlecht, da sie aufgrund ihrer schweren, wie sie erzählt durch einen ärztlichen Kunstfehler nach einer 2018 bei einer Lumbalpunktion entstandenen Querschnittlähmung noch weniger als vorher einer beruflichen Tätigkeit nachgehen könne. Frau Öllinger ist vollends auf staatliche Unterstützung angewiesen; sie erhält eine Berufsunfähigkeitspension (909 Euro) und Pflegegeld für die Stufe 4. Ihre eigenen Handlungsentwürfe hat sie dennoch um eine ihr selbst wichtige Aktivität erweitert; mit Freude hat sie das Angebot angenommen, in einer Rollstuhltanzgruppe mit zu tanzen. Den Freund ihrer Tochter, von dem sie im Interview sagt, „des is a goanz Liaba“, nimmt sie nicht als „Konkurrenz“ wahr, nicht zuletzt, weil sich Viktoria aufopfernd um ihre Mutter kümmert und auch noch weiterhin mit in der Wohnung lebt. Sehr froh ist Frau Öllinger denn auch, dass ihre Tochter zur Zeit des Interviews aufgrund der mit der Covid-19-Pandemie verbundenen Einschränkungen zu Hause, in ihrer unmittelbaren Nähe, arbeiten könne.

\section{Medien im Leben von Viktoria und ibrer Mutter}

Medien, allen voran das Fernsehen und dabei in erster Linie Angebote auf RTL, spielten in Viktorias Leben stets eine große Rolle; schon zur Zeit der zweiten Erhebungswelle 2007 gehörten Daily Soaps, wie allen voran 
Gute Zeiten - Schlechte Zeiten, zu Viktorias Lieblingsrepertoire. Sie boten ihr eine symbolische Vorlage, sich auf spielerisch-unterhaltende Weise mit den wechselnden Beziehungen und unterschiedlichen Alltagsbegebenheiten und Alltagsproblemen auseinanderzusetzen, wie sie auch das Leben ihrer Mutter mitbestimmten. Auch in ihrer Mediennutzung passte sich Viktoria lange Zeit den Wünschen ihrer Mutter an. So rezipierte sie nicht nur mit ihr gemeinsam die Serie Sex and the City, sie folgte auch deren Wunsch, sie an allen Einträgen auf ihrem Facebook-Account partizipieren zu lassen. Ein wenig Eigenständigkeit versuchte Viktoria zum Ende der sechsten Erhebungswelle mit Hilfe von Soziale Medien-Anwendungen zu gewinnen, so kommunizierte sie etwa mit ihrer besten Freundin über WhatsApp. Heute nutzt Viktoria vor allem WhatsApp zur Kommunikation mit ihrer engen Freundin und mit Peers. Zudem schaut sie sich auf YouTube, wie sie sie nennt, „Schicksals-Stories“ an - eine Möglichkeit sich mit eigenen Herausforderungen und Problemen auf informativ-unterhaltsame Weise auseinanderzusetzen und zu sehen, dass auch andere Menschen Probleme zu bewältigen haben. Facebook schaut sie lediglich „mal durch“. Vom Fernsehen sei sie auf Netflix „umgestiegen“, weil sie da schauen könne, was und wann sie wolle und dort keine Werbung gezeigt werde. Allen voran rezipiert sie - und dies auf ihrem Smartphone - gern die Serien Haus des Geldes, Elite, Breaking Bad oder auch Riverdale. Die früher bevorzugten Daily Soaps im Fernsehen, die Viktoria insbesondere in den Erhebungswellen 2014 und insbesondere 2016 vor allem auf Wunsch ihrer Mutter mit dieser gemeinsam angesehen hatte, spielen für Viktoria nun keine Rolle mehr.

Bei Frau Öllinger haben Medien mittlerweile eine noch größere Bedeutung gewonnen als schon in den vorherigen Erhebungswellen. So schaut sie viel Fernsehen, von den Nachrichten über die Daily Soaps Unter Uns, Alles was zählt, GZSZ und diverse Talkshows. Zu ihren bevorzugten Sendern gehören RTL und ORF 2. Daneben nimmt ihr Smartphone einen hohen Stellenwert in ihrem Alltag ein; wichtig ist ihr vor allem ein Online Nachrichtendienst aus der Stadt, in der sie lebt - dies nicht zuletzt der aktuellen Lage zur Zeit der siebten Erhebungswelle im März zu Beginn der Covid-19-Pandemie geschuldet. Wichtig sind Frau Öllinger aber auch unterschiedliche Online-Spiele sowie WhatsApp und Facebook. Instagram habe dagegen für sie an Bedeutung verloren. Der Fernseher, so Frau Öllinger, sei aber ihr alltäglicher Begleiter; der „äuft daheim immer“, und auch ihr Handy habe sie „immer bei sich“, ob zur Unterhaltung oder Information, insbesondere aber zur Kommunikation. Mit ihrer Tochter 
spricht Frau Öllinger aktuell recht viel über Themen aus den Medien, vor allem rund um die Pandemie.

Fazit

Nach schweren Jahren, gekennzeichnet von stark begrenzten Handlungsoptionen aufgrund massiver sozio-ökonomischer Probleme und einer extrem engen sozio-emotionalen Beziehung, die durch die zunehmende Erkrankung und damit einhergehend der physischen und psychischen Abhängigkeit der Mutter von ihrer Tochter zu einer ausgeprägten Parentifizierung führte, erweist sich Viktoria in der siebten Erhebungswelle als engagierte junge Frau. Sie ist mittlerweile in der Lage, das über die Jahre der Gesamterhebung überaus enge emotionale Verhältnis zu ihrer Mutter ein wenig zu lockern, um sich selbst mehr Raum für eigene Handlungsentwürfe zu schaffen. Dabei helfen ihr sowohl ihre berufliche Situation - die Stelle in der Verwaltung eines Klinikums entspricht ihren Handlungskompetenzen und gibt ihr Stabilität und Selbstbewusstsein als auch ihr Partner und nach wie vor ihre beste Freundin, die ihr beide sehr wichtig sind. Viktoria scheint sich nach den vielen wechselnden Lebenslagen, mitbestimmt von den verschiedenen Ehen, Beziehungen und Umzügen ihrer Mutter, aus den schwierigen sowohl sozio-ökonomischen als auch sozio-emotionalen Einschränkungen und der damit verbundenen Enge lösen zu können. Es gelingt ihr mittlerweile, ihre Lebensführung insoweit handlungskompetent zu gestalten, als sie nunmehr bewusst die Rolle der Stärkeren in der Familie einnimmt und ihre Mutter als das eher hilfsbedürftige Kind betrachtet, das es zu ermutigen, aber auch zuweilen zurechtzuweisen gilt. Seit früher Kindheit nehmen dabei Medien, entsprechend den sich Viktoria stellenden Entwicklungsaufgaben, einen wichtigen Stellenwert ein. Sie dienten und dienen ihr zum Austausch und zur Teilhabe, aber auch zur Stabilisierung und Selbstvergewisserung. Für ihre Mutter sind Medien zu einem zentralen Teil ihrer Alltagsgestaltung geworden.

\subsection{Die jungen Erwachsenen von Typ 2: Die sozio-ökonomisch nicht mehr} bzw. wenig belasteten, aber wegen problematischer sozio-emotionaler Beziehungsstrukturen überforderten Familien

Kennzeichen der Familien von Typ 2 waren neue Familienkonstellationen bzw. Trennungserfahrungen und im Zuge dessen auch ein stark angespanntes Familienklima. Im Gegensatz zu den Familien von Typ 1 erwie- 
sen sich zwar die sozio-ökonomischen Bedingungen der Familien von Typ 2 in der fünften und sechsten Erhebungsphase als deutlich verbessert, ihre sozio-emotionalen Beziehungsstrukturen blieben dagegen weitgehend problematisch oder verschlechterten sich noch. Die Familienmitglieder dieser Familien zeigten sich in ihrer Lebensführung überfordert. Zwar hatte beispielsweise Frau Holzner mit ihrem neuen, finanziell besser gestellten Partner zwischenzeitlich eine neue Familie gegründet und war danach sozio-ökonomisch bessergestellt, das Zusammenleben der ehemaligen Kernfamilie, zu der ihr Sohn Benedikt zählte, war aber weiterhin sehr belastet. Auch Frau Weiss konnte durch eine Erbschaft und über Mieteinnahmen aus ebenfalls geerbten Immobilien ihre finanzielle Situation verbessern, ihr Verhältnis zu ihrem Sohn Alfons besserte sich dadurch jedoch nicht. Weder die neu gebundene Frau Holzner noch die von ihrem Ex-Mann getrenntlebende Frau Weiss brachten während der Erhebungszeit genügend Interesse für ihre Söhne auf, die Beziehungen zu ihnen erwiesen sich als deutlich belastet. Bei Familie Rohringer und Familie Hirtner veränderten sich zwar die sozio-ökonomischen Bedingungen weiter zum Positiven, wenn auch nicht in derselben Weise wie bei Familie Holzner und Weiss. Ähnlich wie bei ihnen war aber das Zusammenleben der Familie belastetet. Bei Familie Hirtner, die zuvor in deutlich besseren sozio-emotionalen Beziehungen miteinander gelebt hatte, verschlechterte sich aber im Vergleich zu vorher, vor allem in der sechsten Erhebungsphase, das Familienklima, nachdem sich Frau Hirtner von ihrem Partner getrennt hatte und - insbesondere im Zuge seiner Suche nach einer Ausbildungsstelle starken Druck auf ihren Sohn ausübte. Alle vier Familien von Typ 2 ließen aufgrund ihrer schlechten sozio-emotionalen Beziehungsstrukturen klare Anzeichen von Überforderung in der Bewältigung ihres Alltags als Kernfamilie erkennen, die sich auch in der (Medien-)Erziehung ihrer Kinder niederschlug, etwa in Desinteresse an den Belangen ihrer Kinder. Auffällig ist, dass es sich in drei der vier Familien (Weiss, Hirtner und Rohringer) um alleinziehende Mütter handelt. Auch Frau Holzner war lange Zeit alleinerziehend; bei Benedikt und seinen älteren Geschwistern, die alle seit der dritten Erhebungswelle in einer betreuten Wohngemeinschaft außerhalb der Familie lebten, handelt es sich um einen Sohn aus der Beziehung mit dem vorherigen Partner. 
2.3.1 Mario Hirtner (20 Jahre alt) und seine Mutter: Große sozioökonomische Herausforderungen nach einer zwischenzeitlichen Verbesserung und phasenweise sozio-emotionale Schwierigkeiten prägen das Familienklima

\section{5 bis 2017}

Während der Rekrutierungsphase lebte die Familie, bestehend aus Mario (2005 fünf Jahre alt), seiner Mutter und deren festem Lebensgefährten sowie seinem Bruder Lukas (18), in einer nur $45 \mathrm{~m}^{2}$ großen Wohnung. $\mathrm{Zu}$ Beginn der Studie war die Familie dann bereits in eine $106 \mathrm{~m}^{2}$ große Mietwohnung ganz in der Nähe des Bahnhofs einer Großstadt gezogen, die vom Staat gefördert wurde. Anfangs mussten sich die Brüder ein Zimmer teilen, später verzichtete die Familie auf das Wohnzimmer, so dass es zwei Kinderzimmer gab. Die Familie schätzte die Wohnung und deren infrastrukturelle Anbindung immer sehr, lediglich der Mangel eines Balkons und der zeitweilige Lärm einer nahen Großbaustelle wurden beklagt. Während der Krise um Fluchtmigration 2015 und 2016 sorgten die Nähe des Bahnhofs und die damit verbundenen Mengen an Geflüchteten bei Frau Hirtner für Unbehagen. Sie begründete dies auch mit dem Umstand, selbst vor der Haustüre angegriffen worden zu sein. Bei diesem Überfall wurde sie niedergeschlagen, und der Täter versuchte ihr die Handtasche wegzureißen. Als sie bereits auf dem Boden lag, trat der Angreifer weiter auf sie ein, bevor er flüchtete. $\mathrm{Ob}$ es sich dabei tatsächlich um einen Geflüchteten handelte, konnte auch von der hinzugezogenen Polizei nicht geklärt werden.

Bis zur vierten Erhebungswelle (2012) war die Wohnung immer sauber und ordentlich, wobei Frau Hirtner merklich rauchte. 2010 betonte der damals elfährige Mario sogar, dass die Wohnung deshalb so sauber wäre, da alle Familienmitglieder immer gemeinsam putzen würden. Ganz anders präsentierte sich die Wohnsituation in der sechsten Erhebungswelle (2016): Die Einrichtung war abgewohnt, alle Räume waren unordentlich und schmuddelig, es roch stark nach Rauch, und die beiden Hauskatzen wirkten sehr ungepflegt. Zu diesem Zeitpunkt wurde unübersehbar, dass Frau Hirtner ein Alkoholproblem hatte und stark trank.

Die finanzielle Situation war gerade zu Beginn der Erhebung sehr prekär, da sowohl Frau Hirtner als auch ihr Lebensgefährte arbeitslos waren und die Familie auf Sozialhilfe angewiesen war. Im Verlauf verbesserte sich die sozio-ökonomische Lage etwas, da beide Festanstellungen in Vollzeit fanden. Frau Hirtner, die bis heute über keinerlei Berufsausbildung verfügt, verlor jedoch mehrfach ihre Arbeitsstelle und arbeitete in 
wechselnden gastronomischen Betrieben. Über den gesamten Erhebungszeitraum hinweg blieb die Familie armutsgefährdet. Kostenintensive Freizeitaktivitäten waren somit seltene Ausnahmen, dennoch versuchte die Familie viel Zeit gemeinsam zu verbringen und etwa nach draußen oder zum Schwimmen zu gehen. Kurz nach der vierten Erhebungswelle (2012) trennten sich Frau Hirtner und ihr langjähriger Partner. Dies mag auch einer der Auslöser für ihren dann verstärkten Alkoholkonsum gewesen sein. Die Trennung war auch für Mario ein harter Schlag, denn er hatte niemals Kontakt zu seinem leiblichen Vater und war immer davon ausgegangen, dass der Partner seiner Mutter sein Vater wäre. Die Wahrheit erfuhr er erst mit dem Bruch zwischen „seinen Eltern“. Die Enttäuschung wurde noch verstärkt, da es nach der Trennung, entgegen vorher gemachter Versprechungen, bald zu einem völligen Kontaktabbruch kam. Diesen Erlebnissen zum Trotz akzeptierten sowohl Mario als auch sein Bruder den bald folgenden neuen Lebensgefährten ihrer Mutter rasch, und bauten erneut ein Vertrauensverhältnis zu ihm auf. Diese neue Partnerschaft begann bereits kurze Zeit nach der Trennung.

Das Verhältnis zwischen Mutter und Sohn war über viele Jahre sehr eng und von Vertrauen gekennzeichnet. Beide betonten stets, auch über schwierige Themen oder Unstimmigkeiten offen sprechen zu können, um eine Lösung zu finden. Frau Hirtner war ihren Söhnen zwar immer zugewandt, jedoch machte sie ihnen auch kaum Vorgaben und interessierte sich nur sehr eingeschränkt für deren mediale und non-mediale Freizeitaktivitäten. Ihr Erziehungsstil entsprach insgesamt stark einer Laissez-faire-Haltung. Nach der fünften Erhebungswelle verschlechterte sich das Verhältnis zu Mario und wurde distanzierter. Frau Hirtner, so schien es, fühlte sich von Marios Bedürfnis nach Nähe überfordert und betonte, dass sie in seinem Alter schon von zu Hause ausgezogen wäre. Auch Mario bestätigte die zunehmenden Konflikte mit seiner Mutter und berichtete gar von einer Situation, in der diese sehr betrunken war und ihm gegenüber aggressiv wurde. Trotzdem meinte Mario auch in der sechsten Erhebungswelle, dass „eigentlich alles passt“ und die Familienmitglieder sich gut verstünden. Damals war er 16 Jahre alt und befand sich in der Pubertät. Dennoch betonte er, sich auch mit Fragen zum Thema Sexualität an seine Mutter wenden zu können. Mario war damals mehreren Stressfaktoren ausgesetzt. Einerseits sorgte er sich um seine Mutter, die kurz zuvor überfallen worden war, und litt unter der zunehmenden Distanzierung. Andererseits stand er unter Druck und musste sich seiner Zukunftsplanung zuwenden und entsprechende Handlungsentwürfe entwickeln. Nach dem Hauptschulabschluss hatte Mario zunächst eine Ausbildung zum Elektro- 
installateur begonnen. Diese musste er jedoch kurze Zeit später wieder abbrechen, da er unter Höhenangst litt. In der Folge besuchte er Kurse des AMS und war auf der Suche nach einer neuen, passenden Lehrstelle. Seine Mutter übte in dieser Zeit großen Druck auf ihn aus, zusätzlich auch arbeiten zu gehen, um Geld zu verdienen. Es war zu vermuten, dass sie dies mit guten Absichten tat, denn es war ihr ausdrücklicher Wunsch, dass Mario aktiv bliebe und nicht „faul zu Hause“ säße. Als Reaktion darauf lenkte Mario seine Pläne um und versuchte so zu „funktionieren“, wie seine Mutter es von ihm verlangte. Emotional zog er sich jedoch zurück, reduzierte die Kontakte zu seiner Mutter und zu Freunden und widmete sich verstärkt Videospielen und dem Erlernen von Programmiersprachen am Computer. Auf diese Weise gelang es ihm, sich wichtige Fähigkeiten für den Arbeitsmarkt anzueignen und seine Handlungskompetenzen auszuweiten. Schließlich konnte Mario eine Ausbildungsstelle im IT-Bereich finden und wurde eingestellt. Dadurch ergab sich eine Verbesserung seiner Handlungsoptionen und es bedeutete für ihn eine Chance, unabhängiger zu werden und weiterführende Handlungsentwürfe verfolgen zu können. Obwohl Mario in der sechsten Erhebungswelle optimistischer in seine Zukunft blicken konnte, machte er dennoch deutlich, dass ihn die angespannte Situation zu Hause weiterhin belastete.

2020: Mario Hirtner - ein IT-begeisterter junger Mann startet im Beruf durch und begründet mit seiner Freundin einen eigenen Hausstand

In der siebten Erhebungswelle (2020) präsentiert sich die Lebenssituation der einzelnen Mitglieder der Familie Hirtner höchst divergent. Mario ist inzwischen fast 21 Jahre alt und konnte sich sowohl beruflich als auch privat etablieren. Im Juli 2019 hat er seine Ausbildung zum IT-Techniker mit ,ausgezeichnetem Erfolg“ abgeschlossen und wurde von seinem Ausbildungsbetrieb unbefristet übernommen. Dort fühlt er sich sehr wohl, und er hatte bereits frühzeitig den Wunsch auf Übernahme hinterlegt. Mario hat Spaß an der Arbeit und will den Betrieb auch in Zukunft nicht verlassen, „weil, des war ja eigentlich eh schon immer des, was i machen wollt". Darüber hinaus stellt ihm sein Arbeitgeber seit Anfang 2020 eine stark vergünstigte Mietwohnung zur Verfügung, die Mario seitdem gemeinsam mit seiner Freundin bewohnt, mit der er bereits seit etwa drei Jahren zusammen ist. Die Wohnung befindet sich direkt auf dem Betriebsgelände und bietet kurze Wege, zudem ist sie auch nur fünf Minuten von der Wohnung seiner Mutter entfernt. Mario zeigt sich äußerst glücklich, sowohl über die eigene Wohnung als auch über das harmonische Zusammenleben mit seiner Freundin. Eine eigene Wohnung mache 
zwar auch viel mehr Arbeit, so Mario, böte aber auch viel Gutes, wie etwa mehr Freiheit. Insgesamt hat Mario die Chancen genutzt, welche ihm seine zweite Ausbildungsstelle geboten hat. Er hat seine Ziele und Pläne zielstrebig verfolgt, und durch seinen ausgezeichneten Lehrabschluss neue Handlungskompetenzen erworben. Auf diese Weise hat er seine Handlungsoptionen nachhaltig verbessert, und kann, davon ausgehend, nun erweiterte Handlungsentwürfe für seine berufliche aber auch für seine private Zukunft entwerfen. Ähnlich wie Mario, ist es auch seinem jüngeren Bruder, nach dem Abbruch einer ersten Ausbildung, inzwischen gelungen, eine für ihn passende Lehrstelle zu finden. Lukas sei, so seine Mutter, „voi a Braver", und der Wechsel des Ausbildungsplatzes habe ihm gutgetan. Aktuell befindet er sich im dritten Lehrjahr zum Einzelhandelskaufmann und arbeitet in einem Baumarkt in einer nahegelegenen Kleinstadt. Er wohnt noch zu Hause und pendelt jeden Tag in die Arbeit.

Ganz anders als bei ihren beiden Söhnen zeigt sich die aktuelle Lebenssituation von Frau Hirtner. Ihre seit 2012 andauernde Partnerschaft hat sie beendet und lebt derzeit allein. Darüber zeigt sie jedoch kein Bedauern, und sie geht davon aus, dass auch wieder neue Beziehungen kommen werden. Beruflich befindet sie sich dagegen in einer schweren Krise, und auch ihre finanzielle Situation sehe „scheiße aus“. Frau Hirtner ist erneut arbeitslos, weil ihr nach längerer Krankheit, kurz vor der siebten Erhebungswelle, gekündigt wurde. Sie war zuvor nach 27 Jahren in der Gastronomie in den Lebensmitteleinzelhandel gewechselt; die neue Arbeit machte ihr sehr viel Freude. Zudem habe sie in dem halben Jahr bei ihrem bisherigen Arbeitgeber, einem Bio-Supermarkt, sehr viel gelernt und es sei dort „sehr cool“ gewesen. Der Verlust des Arbeitsplatzes und die Sorgen um die Zukunft, insbesondere in Zeiten der Covid-19-Pandemie einen neuen Job im Lebensmittelbereich zu finden, belasten Frau Hirtner sehr. Erschwerend kommt hinzu, dass sie unter Asthma leidet und dadurch zusätzlich eingeschränkt ist. Aktuell fühlt sie sich sehr überfordert und perspektivlos und kann sich kaum Vorstellungen für eine bessere Zukunft machen. Dennoch äußert sie unter Tränen die Hoffnung, dass es „auf jeden Fall“" wieder einen Arbeitsplatz für sie geben werde.

Noch in der sechsten Erhebungswelle war die Beziehung zwischen Mario und seiner Mutter vielfältig belastet. Inzwischen scheinen sich die sozio-emotionalen Bedingungen entspannt zu haben, dennoch unterscheiden sich die Perspektiven von Mario und seiner Mutter. Mario selbst bezeichnet das Verhältnis als "gut" und schätzt es, dass die Wohnungen nicht weit voneinander entfernt liegen. So sei es trotz der Arbeit möglich, sich zumindest „ab und zu“ zu sehen. Mit seinem Bruder verbringt er dagegen 
mehr Zeit und die beiden unternehmen viel gemeinsam, etwa Shoppingtouren. Frau Hirtner beschreibt das Verhältnis zu Mario dagegen als „sehr gut" und den Auszug ihres „Sohnesmannes“ empfindet sie als tiefgreifende Veränderung. Sie bedauert die räumliche Trennung und räumt lachend ein, eine „sehr traurige Mutter“ zu sein. Bezeichnender Weise erwähnt Mario weder die gesundheitlichen Probleme seiner Mutter, noch deren erneuten Jobverlust und die damit verbundene Perspektivlosigkeit. Auch Frau Hirtner erwähnt nicht, dass sie in dieser Situation Hilfe und Unterstützung von ihrem Sohn bekommen würde. Mario hatte jedoch noch in der sechsten Erhebungswelle seiner Mutter versprochen, dass sie bei ihm wohnen könnte, wenn es ihr mal irgendwann ganz schlecht gehen sollte, und dass er sie dann pflegen würde. Frau Hirtner macht aber deutlich, sich ein enges Verhältnis zu ihrem nun erwachsenen Sohn zu wünschen. In den Interviews mit Mutter und Sohn in der siebten Erhebungswelle wurde deutlich, dass die Differenzen zwischen beiden noch immer nicht gänzlich überwunden sind.

\section{Medien im Leben von Mario und seiner Familie}

Sowohl die Familie als Gemeinschaft als auch die Kinder selbst waren seit der dritten und vierten Erhebungswelle sehr umfangreich mit Medien ausgestattet. Die beiden Jungen hatten neben ihren Mobiltelefonen und MP3Playern bereits zwei Exemplare der PlayStation 2, einen Nintendo DS und jeweils eigene Fernseher mit Kabelanschluss. Mario verfügte in seinem Zimmer außerdem bereits über einen Laptop mit Internetzugang, einen DVD-Player und eine Wii. In der sechsten Erhebungswelle besaß er zudem einen umfangreich ausgestatteten "Gaming-PC“ und ein neues Smartphone. Medien hatten seit Beginn der Erhebung einen sehr hohen Stellenwert für Mario, und seine Mutter ließ ihm diesbezüglich fast immer alle Freiheiten. Bereits in der ersten Erhebungswelle dominierte das Fernsehen seine Freizeitgestaltung (Spongebob und Confetti TV, später Simpsons, Family Guy, South Park, Naruto sowie Dokumentationen und der Sender Comedy Central), ab der dritten Erhebungswelle gewannen auch Videospiele auf dem Nintendo DS an Bedeutung. Ab der vierten, vor allem aber ab der sechsten, Erhebungswelle spielten das Internet und Computerspiele (Minecraft, Pokémon, League of Legends) die größte Rolle für Mario, da er auch beruflich mit Computern arbeiten wollte und in der Beschäftigung damit zudem eine Weiterbildungsmaßnahme sah. In diesem Kontext ist hervorzuheben, dass er bereits seit der vierten Erhebungswelle mittels einer Paysafe-Card fünf Euro im Monat für einen Minecraft-Server bezahlte und diesen anderen Spielern gegen Bezahlung zur Verfügung stellte. Ergänzend 
lud er bei YouTube Werbe- und Informationsvideos über Minecraft hoch. Auch in der Schule hatte Mario frühzeitig viel Kontakt mit Computern und Internet, und im Informatikunterricht wurde der Umgang mit Office-Programmen gelehrt. In der Schule war IT sein Lieblingsfach. Bereits an der Hauptschule konnte er Erfahrungen im Programmieren sammeln und an der Polytechnischen Schule dann ein ECTL-Training absolvieren. Mit zunehmendem Alter konzentrierte sich Marios Mediennutzung auf die Internetdienste Google, YouTube, Wikipedia und auf das, zum Teil illegale, Streaming von Inhalten. Zudem hatte das Internet einen hohen Stellenwert in seinem Medienrepertoire, um über Chatprogramme wie Team-Speak, Skype und auch Facebook mit Freunden und Schulkollegen in Kontakt zu bleiben. Ähnlich ist dies auch in der siebten Erhebungswelle: Kommunikationsmittel wie Telefon, SMS und WhatsApp sind Mario sehr wichtig, um in „ständigem Kontakt“ mit seinen Freunden zu sein, sich auszutauschen und zu erfahren, wie es ihnen geht. Soziale Medien bezeichnet er dagegen als „eigentlich gor nicht wichtig“, da er auf Facebook und Twitter, wenn überhaupt, nur „lese“. Instagram, Snapchat und TikTok lehnt Mario dagegen ab. Eine Ausnahme bildet weiterhin YouTube. Diese Plattform nutzt Mario nicht nur zur Unterhaltung, sondern auch umfassend zur Information, insbesondere über Covid-19. Insgesamt hat sich sein Informationsverhalten deutlich verändert. Spielten Information und Nachrichten in der sechsten Erhebungswelle noch so gut wie gar keine Rolle, so nutzt Mario inzwischen nicht nur Online-Zeitungen wie DerStandard.at oder Krone.at, sondern auch verschiedene News-Apps auf seinem iPhone. Unverändert hoch im Kurs stehen bei ihm Spiele auf dem Computer, nicht jedoch Konsolenspiele. Dabei schätzt er inzwischen in erster Linie Aufbausimulationen wie Civilization VI, Minecraft und Tropical Odyssey, aber auch Pokémon und League of Legends sind weiterhin beliebt. Wie bei vielen anderen jungen Erwachsenen im Panel auch, ist die Bedeutung des Fernsehens für Mario deutlich zurückgegangen, während Netflix an Relevanz gewonnen hat. Mario betont jedoch, dass er eigentlich niemals alleine schaue, sondern immer mit anderen zusammen. In diesem Kontext hat auch seine Beziehung zu einer Veränderung seiner Mediennutzung geführt, denn gemeinsam mit seiner Freundin entdeckt er gerne Neues. Feste Genre-Vorlieben haben sie dabei nicht, aber beide mögen Serien sehr. Seine Freundin liebt zudem Horrorfilme, die sie sich dann gemeinsam ansehen.

Nicht nur für Mario, sondern auch für seine Mutter, hatten Medien immer einen hohen Stellenwert. Neben Informationsangeboten wie der Kronenzeitung oder Punkt 12 auf RTL, schätzte Frau Hirtner auch immer 
Unterhaltungssendungen im Fernsehen. Ihr ist bewusst, und sie verbalisiert dies auch, dass es sich bei den von ihr rezipierten Medieninhalten meist um eher seichtere Unterhaltungsangebote handelt. Sie stand aber immer offen zu ihren Vorlieben für Frauentausch, DSDS, Supertalent sowie für Sitcoms, Serien und auch Filme. 2020 schaut sie noch mehr fern als früher. Einerseits stehen ihr aufgrund der Covid-19-Pandemie kaum andere Freizeitangebote zur Verfügung, andererseits hat sie wegen der erneuten Arbeitslosigkeit sehr viel Zeit: „Ich weiß scho gar nimmer, was i schau'n soll." Ab der sechsten Erhebungswelle genießen auch Internet und Smartphone eine sehr große Bedeutung für Frau Hirtner. Neben Computerspielen (Bingo, Bowling) betrieb sie zeitweilig auch Online-Glückspiele und setzte dabei echtes Geld ein. Darüber hinaus nutzt sie Online-Banking sowie Telefonie, SMS und WhatsApp, um mit ihren Söhnen und Freunden in Kontakt zu bleiben. Auch Facebook nutzt Frau Hirtner weiterhin aktiv, sowohl zum Austausch und zur Selbstdarstellung als auch zur Information. Neu von ihr genutzte Informationsangebote sind die Nachrichtensendungen des ORF und von n24.

\section{Fazit}

Familie Hirtners Leben war immer von sozio-ökonomischen Einschränkungen bestimmt. Wiederkehrende Phasen der Arbeitslosigkeit sorgten für zusätzliche Einschnitte, aber auch in Zeiten der Vollbeschäftigung blieb die Familie zumeist armutsgefährdet. Eine Ursache für Frau Hirtners schwierigen Stand auf dem Arbeitsmarkt war und ist das Fehlen einer Berufsausbildung. Sie scheint eine fleißige und hart arbeitende Frau zu sein, dennoch blieben ihre Handlungsoptionen eingeschränkt. Zwar arbeitete sie sich immer wieder in neue Arbeitsstellen ein, sie entwickelte jedoch nie Handlungsentwürfe, wie etwa Ziele oder Pläne, um ihre Handlungskompetenzen durch eine Fortbildung oder gar eine Berufsausbildung zu erweitern. Umso wichtiger erschien es ihr, sowohl Mario als auch seinen Bruder Lukas zu fleißig arbeitenden Menschen zu erziehen und sie in Ausbildungen unterzubringen. Um dies zu erreichen, übte sie auch erheblichen Druck auf Mario aus, was schließlich zu einer zeitweiligen Entfremdung zwischen ihnen führte. Auch wenn Frau Hirtner zuletzt ihren Sohn und die Bedeutung ihrer Beziehung hervorhebt, gibt es dennoch Indizien dafür, dass nicht alle Konflikte gänzlich aufgelöst und verarbeitet werden konnten. Die Übernahme durch seinen Ausbildungsbetrieb, der Einstieg ins Berufsleben und die Gründung eines eigenen Hausstandes in einer angemieteten Betriebswohnung waren für Mario entscheidende Schritte auf dem Weg in seine Selbstständigkeit. Neben 
den verbesserten Handlungsoptionen ist seine langjährige Freundin für Marios Entwicklung von großer Bedeutung. Mit ihr ist er Anfang 2020 dann auch den nächsten Schritt in eine gemeinsame Wohnung gegangen. Mit dem Abschluss seiner Ausbildung gelang es Mario nicht nur, seine Handlungskompetenzen zu erweitern, sondern auch seine Handlungsoptionen grundsätzlich zu verbessern. Auf diese Weise löste er sich von seiner Mutter und den eingeschränkten Verhältnissen seiner Familie und kann nun weiterführende Handlungsentwürfe für sein zukünftiges Leben entwickeln. Dabei half ihm sein schon früh geäußertes großes Interesse an Computern, Programmieren und am Internet. Dieses baute er, zunächst in der Schule und im Selbststudium, später auch in Fortbildungskursen des AMS, aus und erarbeitete sich so die erforderlichen Qualifikationen für eine Ausbildungsstelle als IT-Techniker, die er auch mit „ausgezeichnetem Erfolg" abschloss. Auch sein privater Mediengebrauch ist durch einen selektiven Umgang mit medialen Unterhaltungs-, Informations- und insbesondere mit Kommunikationsangeboten, allem voran mit seiner Freundin und mit Freunden, gekennzeichnet.

2.3.2 Benedikt Holzner (20 Jahre alt): Ein Junge aus einer sozio-emotional stark belasteten Familie erhält staatliche Unterstützung und geht seinen Weg in die Selbstständigkeit

\section{5 bis 2017}

Die frühe Kindheit von Benedikt (2005 fünf Jahre alt), seiner leiblichen Schwester (22) und seines leiblichen Bruders (24) war von sozio-ökonomischen Herausforderungen und gravierenden sozio-emotionalen Belastungen gekennzeichnet. Gemeinsam mit ihrer Mutter lebten sie in beengten Verhältnissen zur Untermiete in einer von ihnen ungeliebten Wohngegend, die laut Aussagen von Frau Holzner durch „einen hohen Ausländeranteil“" geprägt war. Zudem gab es immer wieder Probleme mit den Nachbarn. Zu Beginn der Studie war Frau Holzner arbeitslos und befand sich in Umschulung, in der zweiten Erhebungswelle (2007) war sie in Teilzeit als Verkäuferin tätig und bezog weiterhin Sozialhilfe, um das Auskommen abzusichern. Die ganze Familie litt unter der Gewalt des Ex-Partners von Frau Holzner, ganz besonders Benedikt, der durch frühere körperliche Misshandlungen durch den Ex-Partner der Mutter gezeichnet war und sich nur schwer konzentrieren konnte. Zu dieser Zeit wurde bei ihm ADHS diagnostiziert, und er litt unter unkontrollierbaren Wutanfällen. Seine Therapeutin bescheinigte ihm den Entwicklungsstand eines Dreijährigen; 
sein Defizit war auch in den ersten Interviews mit dem Jungen deutlich erkennbar. Benedikts Familie war in dieser Notsituation nicht in der Lage, ihm die notwendige Hilfe, Sicherheit und Stabilität zu geben, um seine Traumatisierung zu bewältigen.

Kurz nach der zweiten Erhebungswelle kam es zu einem tiefen Einschnitt im Leben von Familie Holzner, der sich rückblickend als eine wegweisenden Richtungsentscheidung für die weitere Entwicklung von Benedikt erweisen sollte. Sowohl Benedikt als auch seine beiden älteren Geschwister wurden nach dem Einschreiten des Kinderschutzbundes aus der Familie genommen und in speziellen betreuten Wohneinrichtungen untergebracht. Obwohl zunächst als zeitlich befristete Maßnahme geplant, sollte Benedikt nicht wieder dauerhaft nach Hause zurückkehren. Im Alter von 16 Jahren entschied er sich freiwillig, weiter in seiner liebgewonnenen Wohngemeinschaft zu bleiben, später begründete er am selben Ort einen eigenen Hausstand. Der staatlichen Maßnahme zum Trotz blieben die Verhältnisse in Familie Holzner auch in den folgenden Jahren schwierig. An der dritten Erhebungswelle (2010) nahm nur Frau Holzner teil, ein Interview mit Benedikt war auf Grund der Unterbringung nicht möglich, außerdem besuchte er zu diesem Zeitpunkt nur selten seine Familie in der Stadt. In der vierten Erhebungswelle (2012) konnte weder mit Frau Holzner noch mit Benedikt ein Interview geführt werden. Die Absage wurde mit einem laufenden Gerichtsverfahren begründet, das Frau Holzner und die Kinder betraf. Weitere Erklärungen zu den Entwicklungen in der Familie, auch telefonisch, wurden kategorisch abgelehnt. Jedoch kam es in dieser Zeit erneut zu einer grundlegenden Veränderung für die Familie: Bereits 2010 hatte Frau Holzner ihren neuen Lebensgefährten geheiratet und war anschließend gemeinsam mit ihm in eine Eigentumswohnung gezogen, die er noch mit seiner Ex-Partnerin erworben hatte. Ab der fünften Erhebungswelle (2014) haben die beiden einen gemeinsamen Sohn, und in der sechsten Erhebungswelle (2016) war ein weiterer Junge gerade auf die Welt gekommen. Die älteren Kinder von Frau Holzer wohnten weiterhin in verschiedenen betreuten Wohneinrichtungen, besuchten die Mutter nun jedoch öfters „zu Hause“ und brachten auch Besuch mit. 2014 war Benedikt häufig an Wochenenden zu Besuch, und auch 2016 nahm er an Wochenenden oft die mehrere Stunden lange Fahrt in die Stadt und zurück zu seiner Schule auf sich, obwohl er sich dort ein Zimmer mit seinen beiden Halbbrüdern teilen musste. Zur Begründung verwies er darauf, dass ihn dies nicht stören würde und er „ja wegen der Kleinen herkomme“. 
Durch die Heirat und den Umzug in eine Eigentumswohnung hat sich die sozio-ökonomische Lage der Familie grundlegend verbessert. Frau Holzners Ehemann arbeitet in Vollzeit als Facharbeiter im Frachtzentrum an einem Flughafen, und auch Frau Holzner begann, unterbrochen von Zeiten in Mutterschutz und Elternkarenz, zunächst ebenfalls in Teilzeit als Arbeiterin im Frachtzentrum und später in einem Reinigungsbetrieb am Flughafen zu arbeiten. Auch der Wechsel des Stadtteils und somit der Wohngegend wurde von der Familie grundsätzlich als positive Veränderung wahrgenommen. Dennoch wurde auch weiterhin der hohe „Ausländeranteil" kritisiert, und besonders während der starken Fluchtbewegung 2015 und 2016 beklagte sich Frau Holzner über ,viele Ausländer und die Flüchtlinge aus einer nahegelegenen Unterkunft“. Benedikt hat sich in seiner Wohngemeinschaft eingelebt und ist später auch freiwillig dortgeblieben, obwohl er hätte ausziehen können. 2014 teilte er sich dort noch ein Zimmer mit einem Mitbewohner, 2016 bewohnte er schließlich ein Einzelzimmer. Er äußerte sich durchweg positiv über das Verhältnis unter den Mitbewohnern. Es seien „echte Freundschaften“ entstanden, und ein älteres Mädchen bezeichnete er auch als „Schwester“. Sehr positiven Einfluss übten auch die Betreuerinnen und Betreuer in der Wohngemeinschaft aus, die zwar zum Teil streng waren und das Einhalten von Regeln einforderten, jedoch immer verlässliche Ansprechpersonen und vertrauenswürdige Instanzen auch bei Problemen waren. Benedikt äußerte sich sehr deutlich darüber, dass ihm sowohl allgemeine Verhaltensregeln als auch Vorschriften zur Medien- und Smartphone-Nutzung sehr dabei geholfen hätten, sich besser auf die Schule und Hausaufgaben konzentrieren und sich weiterentwickeln zu können. An der ländlichen Lage der Wohngemeinschaft schätzte er die vielen Freizeit- und Sportmöglichkeiten in der Region und er entwickelte sich zu einem begeisterten Skifahrer. Wintersport und Skifahren trugen nicht nur erheblich zu seiner Persönlichkeitsentwicklung bei, er entschied sich im Jahr 2016 auch dazu, sich eine Ausbildungsstelle im Bereich Seilbahntechnik zu suchen und absolvierte dazu mehrere Betriebspraktika, die er sich selbstständig und ohne Unterstützung durch seine Mutter organisierte. Benedikts Plan war es, die Pflichtschulzeit zu absolvieren und so bald wie möglich eine Berufsausbildung zu beginnen, um unabhängig zu werden und sein eigenes Geld zu verdienen. Seine Handlungsentwürfe waren in dieser Hinsicht sehr klar und geradlinig, und er wirkte beim Interview in der sechsten Erhebungswelle sehr erwachsen, konzentriert und reflektiert. Diese Entwicklung von Benedikt veranschaulicht den positiven Einfluss seiner Entfernung aus der Kernfamilie auf seine Handlungsoptionen und den damit einhergehenden 
sich entwickelnden Handlungsentwürfen für sein zukünftiges Leben. Unterstützt durch die Förderung seiner Betreuerinnen und Betreuer sowie der Lehrkräfte in seiner Schule konnte Benedikt auch die nötigen Handlungskompetenzen entwickeln, um seine Pläne allen Widrigkeiten und Herausforderungen zum Trotz erfolgreich in die Tat umzusetzen. Neben dem schulischen Umfeld trug auch eine romantische Beziehung zur persönlichen Entwicklung von Benedikt bei, über die er 2016 berichtete. Er führte zu diesem Zeitpunkt eine Beziehung zu einem Mädchen, das ganz in der Nähe seiner Wohngemeinschaft lebte. Mit ihr teilte er verschiedene gemeinsame Interessen hinsichtlich der Freizeitgestaltung und der Mediennutzung. Als weiterer positiver Faktor kam hinzu, dass die Familie seiner Freundin ihn mit offenen Armen aufnahm und er auch in diesem Umfeld Sicherheit und Stabilität erlebte, die ihm in seiner eigenen Familie fehlte.

Benedikts positive Entwicklung war so zunächst nicht zu erwarten, da seine Mutter sich nach seiner Unterbringung nicht nur widersprüchlich, sondern auch negativ über seine Wohngemeinschaft und seine schulischen Leistungen äußerte. Tatsächlich hatte Frau Holzner ihren Sohn nie in seiner Wohngemeinschaft besucht oder seine Schule gesehen und an einem Elternabend teilgenommen. Vielmehr war sie sich nicht einmal über den genauen Standort sicher und konnte nur die Region grob bestimmen. Sie zeigte kaum Interesse an irgendeinem Aspekt seines Lebens und ging sogar so weit, ihn entgegen offensichtlicher Tatsachen zu verunglimpfen. Während Frau Holzner Benedikt wiederholt als faul, unmotiviert und ungepflegt beschrieb, präsentierte dieser sich in den Interviews stets freundlich, engagiert und zugänglich und zunächst als gepflegter Teenager und später als sehr ordentlicher junger Mann. Gleichzeitig machte sich seine Mutter aber nie die Mühe, sich mit der Schule oder den Betreuern in der Wohneinrichtung in Verbindung zu setzen, um mehr über die Entwicklung ihres Sohnes zu erfahren und sich mit seinen Bezugspersonen auseinanderzusetzen. Vielmehr gab sie in den Interviews an, dass sie keine hohe Meinung von den Betreuern habe; so äußerte sie sich einmal besonders abfällig über die Betreuerinnen. Ganz besonders in den Interviews der sechsten Erhebungswelle und der telefonischen Nachbefragung 2017 wurde sehr deutlich, dass Frau Holzner sich nicht für Benedikt und seine Entwicklung zu einem jungen Mann interessierte. So war sie kaum in der Lage, Auskunft über ihn, seine Interessen, seine Mediennutzung, seine Zukunftspläne oder gar seine Beziehung zu seinem leiblichen Vater, ihrem Ex-Partner, zu geben. Frau Holzner schien sich ausschließlich auf ihre neue Familie und die beiden kleinen Söhne zu konzentrieren und 
ihre ganze Energie da zu investieren. Benedikt wurde dagegen eher als Besucher in der Familie wahrgenommen, der an den Wochenenden ab und zu seine Halbgeschwister besuchte und sich dann intensiv mit ihnen befasste.

Das Desinteresse und die despektierlichen Äußerungen seiner Mutter stehen in einem deutlichen Kontrast zum Verhalten Benedikts, der sich seit 2014 niemals negativ über seine Mutter geäußert oder über mangelnde Zuwendung geklagt hatte. Bei Benedikt scheint es, dass die Behandlung und Therapie, die er im Laufe der Jahre erhielt, kombiniert mit einem positiven Lernumfeld in seiner Wohneinrichtung, zu einer allgemeinen Verbesserung seiner Entwicklung geführt haben. Von seinen früheren Entwicklungsdefiziten war in den späteren Erhebungswellen nichts mehr wahrzunehmen. Vielmehr präsentierte er sich als reifer, freundlicher und gut erzogener junger Mensch mit Plänen für die eigene Zukunft. Sobald er selbst davon berichten konnte (ab 2014) war klar, dass Benedikt sich in seiner neuen Wohnsituation wohl fühlte und dass er von der Intervention in seine zuvor problematischen Lebensumstände immens profitierte. Die Einrichtung bot ihm die Stabilität und die verfügbaren Bezugspersonen, die ihm zu Hause fehlten, und er scheint sein ganzes Leben um diese Einrichtung und die Schule herum gebaut zu haben und sie tatsächlich als sein "Zuhause“ zu betrachten. Da Benedikt zu Beginn der Studie Anlass zur Sorge gab, kann seine durchweg positive und selbstgestaltete Entwicklung als besonders erfreulich angesehen werden. Benedikt war das einzige Kind aus einer der sehr belasteten Familien im Panel, das bereits im jungen Erwachsenenalter auffallend selbstbewusst, ausgeglichen und selbstsicher wirkte.

2020: Benedikt Holzner - ein selbstbewusster junger Mann mit klaren Handlungsentwürfen und den nötigen Handlungskompetenzen setzt sich durch

In der siebten Erhebungswelle (2020) wird sehr deutlich, was sich bereits zuvor angekündigt hatte. Benedikts Leben muss weitestgehend losgelöst von seiner Familie betrachtet werden. Bereits 2017 hatte Frau Holzner eingeräumt, dass seit 2016 kaum noch Kontakt besteht. Auch die Besuche bei seinen jüngeren Halbgeschwistern hat Benedikt inzwischen drastisch eingeschränkt. Er erklärt dies jedoch nicht mit Ablehnung oder einem schlechten Verhältnis zu den Kindern, sondern führt zur Begründung seine eingeschränkten zeitlichen Ressourcen während Berufsschule und Lehre an. Wie zuvor äußert er sich zwar nicht negativ über seine Mutter, jedoch bezeichnet er das Verhältnis zu seiner Familie skeptisch und beschreibt es als „nicht wirklich übertrieben vui“. 
Seine in der sechsten Erhebungswelle verbalisierten Ziele hat Benedikt alle in die Tat umgesetzt. Er hat seine Wunschausbildung als Maschinenbautechniker im Seilbahn-Bereich erfolgreich absolviert und leistet seinen Zivildienst beim Rettungsdienst ab. Sowohl auf den Abschluss der Berufsschule und die Ausbildung im Betrieb noch auf den Start seines Zivildienstes hatte die Covid-19-Pandemie bislang Einfluss. Den Unterricht konnte Benedikt noch vor Einstellung der Präsenzlehre beenden, und in seinem Betrieb lief das Tagesgeschäft, unter Beachtung der neuen Hygiene- und Abstandsregeln, weiter; gerade der Rettungsdienst ist in Zeiten der Pandemie auf den Zivildienst angewiesen, so dass sein Dienstantritt nicht ausgesetzt wurde. Ganz in der Nähe seiner ehemaligen Schule hat er vor einiger Zeit eine eigene Wohnung bezogen, die er auch während des Zivildienstes auf Grund der Mietkostenübernahme durch den Staat behalten kann. In diesem Punkt zeigt sich deutlich das Desinteresse bzw. die Unkenntnis von Frau Holzner über das Leben ihres Sohnes. Von dessen eigener Wohnung scheint sie nichts zu wissen, vielmehr berichtet sie überzeugt und ohne zu zögern, dass Benedikt noch immer in der betreuten Wohngemeinschaft leben würde.

Beruflich stehen für Benedikt nach dem Zivildienst erneut grundlegende Änderungen an. Er plant zwar auch in Zukunft im Bereich der Seilbahntechnik zu arbeiten, seinen Ausbildungsbetrieb möchte er jedoch verlassen. Dies begründet er mit dem Betriebsklima und den Qualitäten der Unternehmensführung, die sich zwar nach außen als erfolgreiches und sympathisches Unternehmen präsentiere, diesen Versprechen nach innen aber nicht gerecht werde. Für seine weitere berufliche Entwicklung hat er zwei Optionen, die er sich gleichermaßen gut vorstellen kann, eine klare Richtungsentscheidung hatte er 2020 aber noch nicht getroffen. Einerseits kann er sich vorstellen, in Österreich zu bleiben und bei einem Mitbewerber einzusteigen. Alternativ dazu sieht er jedoch auch eine Perspektive darin, zumindest zeitweise nach Kanada auszuwandern und sich dort beruflich zu orientieren und zu etablieren. Benedikt hat Verwandte in Kanada, die diesen Schritt ermöglichen und unterstützen würden.

\section{Medien im Leben von Benedikt}

Bereits in seiner frühen Kindheit hatte Benedikt Medien intensiv genutzt. Sie waren zeitweise seine Hauptinformationsquelle, seine Begleiter und oft seine einzige Freizeitbeschäftigung. Zu Beginn der Studie verbrachte er die meiste Zeit des Tages im Kindergarten, aber er und seine Geschwister sahen oft schon am frühen Morgen fern und nahmen diese Tätigkeit wieder auf, sobald sie am Nachmittag aus dem Kindergarten nach Hause 
kamen. Sie schauten oft bis $21 \mathrm{Uhr}$ fern, am Wochenende sogar noch länger. Benedikt selbst behauptete 2007, dass er „immer“ fernsehe. Zudem besaß er eine breite Palette an Merchandise-Artikeln und bezeichnete seine DVDs, CDs, den Fernseher und Videospiele als seine „liebsten Dinge im Leben“. Seine Mutter zeigte aufgrund der alltäglichen Anforderungen an sie Laissez-faire-Praktiken; sie erwartete dementsprechend, dass Kindergarten und Schule bzw. Medien Mediation betreiben würden. All dies änderte sich drastisch, als Benedikt nach einer Intervention des Kinderschutzbundes aus der Familie genommen wurde und in eine betreute Wohngemeinschaft zog. Aufgrund der fehlenden Informationen aus den Jahren 2010 und 2012 und weil Frau Holzner größtenteils keine Angaben zur Mediennutzung ihres Sohnes machen konnte, abgesehen davon, dass sie ihn für „mediensüchtig“ (2012) hielt, konnte seine tatsächliche Nutzung erst durch spätere Interviews rekonstruiert werden, aber es wurde deutlich, dass sie in der Einrichtung akribisch kontrolliert und geregelt wurde. Gleichzeitig lernte er dort, Medien selektiv zu nutzen, was sich später in Benedikts reflektierter und zielgerichteter Mediennutzung niederschlug. Zwar spielten Medien weiterhin eine wichtige Rolle in seinem Leben, aber auf eine ganz andere Art und Weise und auf einem anderen Niveau als zu Beginn der Studie. Wie die meisten Teenager im Panel interessierte sich Benedikt sehr für verschiedene Soziale Medien-Dienste, wie YouTube, WhatsApp, Instagram und Snapchat - allerdings nutzte er Facebook lange Zeit nicht. Neben Online- und Computerspielen, wie Age of War, Prince of Persia, FIFA und GTA, interessierte er sich auch für TVSerien, für Sportübertragungen und für Dokumentationen. Sein Zugang zu Spielkonsolen war durch die Regeln der Wohngemeinschaft jedoch ebenso eingeschränkt wie der freie Internetzugang. Zudem mussten sich die Mitbewohner und Mitbewohnerinnen auch darüber einig werden, was sie im Fernsehen anschauen wollten. In der sechsten Erhebungswelle gab Benedikt an, dass sein Smartphone sein wichtigstes Mediengerät sei, weil er damit mit seinen Freunden in Kontakt treten könne und der Aspekt der Kommunikation für ihn sehr wichtig sei. Da er oft zwischen seiner Wohngemeinschaft und der Wohnung seiner Familie pendelte, war das Smartphone auch sein wertvollstes Mediengerät, mit dem er seine Lieblingsdienste und -inhalte überall nutzen konnte. Gleichzeitig übergab er sein Telefon oft freiwillig an die Betreuer, um sich besser auf seine Hausaufgaben konzentrieren zu können. Deutlich intensiver aber auch zielgerichteter als andere Jungen im Panel nutze Benedikt Soziale Medien zum Zwecke seines Identitätsmanagements. Dabei unterschied er deutlich zwischen den verschiedenen Angeboten. So hatte er bei Instagram etwa 
viele Follower und schränkte diese nicht ein, während er Snapchat ganz bewusst nur für einen eingeschränkten Adressatenkreis von „maximal 100 Personen" nutzte. Ganz bewusst inszenierte er sich dort beim Sport und besonders beim Skifahren, um als junger, sportlicher und aktiver Mann wahrgenommen zu werden. In Folge wurden ihm auf Snapchat auch mehrfach Nacktbilder (Sexting) zugeschickt, was er aber eigenen Angaben zu Folge ignorierte; wohl auch, da er zu diesem Zeitpunkt eine Beziehung pflegte. Neben Sozialen Medien nutzte Benedikt insbesondere 2016 Medien auch intensiv, um sich breitgefächert zu informieren: Er las in der Wohngemeinschaft die Lokalzeitung, hörte die Nachrichtenangebote verschiedener Radiosender und nutze sowohl Nachrichten-Apps als auch Wikipedia und Google zur zielgerichteten Recherche.

In diesen beiden Punkten, Nutzung Sozialer Medien und Information, zeigt sich ein deutlicher Wandel der Mediennutzung von Benedikt. 2020 spielen beide Aspekte kaum noch eine Rolle. Benedikt schaut kaum noch fern, hört kein Radio und liest auch nicht mehr die Zeitung. Auch Soziale Medien haben für ihn stark an Reiz verloren, insbesondere die früher stark praktizierte Selbstpräsentation spielt kaum noch eine Rolle. WhatsApp dient ihm zur Kommunikation mit Freunden, und er nutzt inzwischen auch Facebook, vornehmlich zur Information. Darüber hinaus ist sein einziges genutztes Informationsangebot die App eines lokalen Online-Portals auf seinem Smartphone. Auch Konsolenspiele haben keine Bedeutung mehr für Benedikt, stattdessen spielt er inzwischen sehr intensiv am PC, unter anderem die Spiele Counter Strike: Global Offensive, Call of Duty und Escape from Tarkov. Dabei spielt er sehr intensiv online gegen Freunde und nimmt auch an einer Counter Strike Online-Liga teil.

\section{Frau Holzner - eine berufstätige Mutter mit neuer Familie}

Frau Holzner ist 2020 zufrieden mit ihrem Leben und zeigt sich deutlich offener und reflektierter als in den vorherigen Erhebungswellen. Als größte Veränderungen betrachtet sie den gänzlichen Wegzug der älteren Kinder und das familiäre Zusammenleben mit den beiden Nachzüglern und ihrem Ehemann. Sie arbeitet inzwischen Vollzeit in einem Reinigungsbetrieb am Flughafen und ist während der Covid-19-Pandemie nicht von Kurzarbeit oder Arbeitslosigkeit betroffen. Im Gegensatz dazu ist ihr Mann in Kurzarbeit, da der Flugverkehr drastisch zurückgegangen ist und nur noch wenige Flugzeige abgefertigt werden müssen. Die finanziellen EinbuBen der Familie beziffert Frau Holzner auf etwa 200 Euro im Monat. Darin sieht sie jedoch kein Problem, da gleichzeitig Kosten für Kindergarten und Schule in etwa gleicher Höhe entfallen. Obwohl der Lockdown und die 
Schließung der Bildungseinrichtungen die Söhne hart treffen, kann sie der Situation dennoch auch etwas Positives abgewinnen: Sie empfindet es als gute Erfahrung, dass sie und ihr Mann aufgrund der Covid-19-Pandemie zeitweise die Rollen getauscht haben und er verstärkt bei den Kindern zu Hause bleibt. Dies habe ihm „nicht geschadet“ und er habe „auf dem Tableau präsentiert bekommen, wie die bei mir so sand.“

Auch Frau Holzners Lebenssituation hat sich nachhaltig gewandelt. Sie verfügt inzwischen über grundlegend veränderte Handlungsoptionen, lebt in gesicherten Verhältnissen in einer Eigentumswohnung und führt mit ihrem Ehemann eine harmonische Beziehung. Ihre Handlungsentwürfe beziehen sich stark auf ihre neue Familie und zielen darauf ab, den beiden Jungen eine gute und sichere Kindheit mit ausreichend Freizeitangeboten zu bieten. Dieses Ansinnen spiegelt sich auch in der familialen Mediennutzung wider. Frau Holzner hat sich seit Beginn der Studie weiterentwickelt und wendet sich ihren beiden „Nachzüglern“ in ganz anderer Weise zu als dies bei den drei älteren Kindern der Fall war. Im Zusammenspiel mit der nachhaltig verbesserten sozio-ökonomischen Lage der Familie stehen somit auch Handlungskompetenzen zur Verfügung, um, ohne ein erneutes Eingreifen des Staates, die Handlungsentwürfe in die Tat umzusetzen.

\section{Medien im Leben von Frau Holzner}

Sowohl Frau Holzner als auch ihr Mann wenden sich intensiv verschiedenen Medienangeboten zu. Im Mittelpunkt stehen dabei die Motive der Information, der Kontaktpflege (besonders bei Frau Holzner) und die Bereitstellung von kindgerechten Informationen für die beiden Kinder. Während der Mann von Frau Holzner intensiv das Online-Angebot der Kronenzeitung nutzt, bedient sich Frau Holzner eines recht breiten Informationsrepertoires: Neben Krone.at nutzt sie auch das Online-Angebote der örtlichen Bundeslandzeitung sowie eines Stadtportals und hat darüber hinaus auch ein Abonnement für das Online-Angebot der Zeitung aus einem anderen Bundesland. Sowohl auf dem Smartphone als auch inzwischen verstärkt im Radio verfolgt sie die Informationsangebote des ORF. Darüber hinaus rezipiert sie Dokumentationen und Diskussionsrunden im privaten Servus-TV. Im Vergleich zu früheren Erhebungswellen hat sich die Mediennutzung von Frau Holzner 2020 stark von Unterhaltung hin zur Information verschoben, und sie scheint, im Gegensatz zu früher, auch den Medienumgang ihrer Kinder stärker zu reflektieren und positiv beeinflussen zu wollen. Insbesondere ihretwegen setzt die Familie nun verstärkt auf den Streaming-Anbieter Netflix, um sie bewusst von den Nachrichten in den Medien über die Covid-19-Pandemie abzuschirmen. So suchen 
Frau Holzner und ihr Mann Angebote auf Netflix, die eine „kindliche Version von dem Virus“ zeigen. Im Gegensatz zu früher scheinen die beiden Kinder auch keinen freien Zugang mehr zu Fernsehen, Netflix oder Computerspielen zu haben.

\section{Fazit}

Benedikt hat sich zu einem selbstbewussten, selbstständigen jungen Mann entwickelt. Dazu hat in erheblichem Maße auch die Entfernung aus seiner Familie und die Unterbringung in einer betreuten Wohngemeinschaft beigetragen. Während sich seine Mutter kaum für ihn interessierte und ihn nicht unterstützte, lernte er dort einerseits strenge Regeln und Strukturen, andererseits aber auch Aufmerksamkeit, Unterstützung, Zuwendung und Sicherheit kennen. Vor dem Hintergrund seiner Schulausbildung entwickelte Benedikt für sich und seine Bedürfnisse passende Handlungsentwürfe, die er im Rahmen seiner Handlungskompetenzen auch umsetzen konnte. 2020 wohnt Benedikt in seiner eigenen Wohnung und kann für den weiteren Lebensweg auf seine erfolgreiche Berufsausbildung als Maschinenbautechniker im Seilbahn-Bereich bauen; sie bietet ihm eine Chance für deutlich verbesserte Handlungsoptionen. Seine Zukunft sieht er in Österreich oder als Auswanderer in Kanada, wobei es sich bei dieser Option nicht um eine Träumerei von der großen weiten Welt handelt, sondern um einen durchaus plausiblen Plan, den er basierend auf seiner Ausbildung und seinen verwandtschaftlichen Beziehungen in Kanada umsetzen könnte. Im Verlauf der Studie und unter Begleitung und Anleitung in seiner Wohngemeinschaft, hat sich Benedikt einen reflektierten Mediengebrauch angeeignet.

Frau Holzner, deren Handlungsoptionen sich durch die neue Ehe mit einem besser gestellten Partner deutlich verbessert haben, hat sich im Laufe der Zeit immer weiter von Benedikt distanziert. Bereits 2016 räumte sie ein, kaum noch Kontakt zu ihrem Sohn zu haben. 2020 bestätigt sich diese Selbsteinschätzung, denn Frau Holzner weiß über ganz zentrale Entwicklungen im Leben ihres Sohnes, wie etwa den Bezug der ersten eigenen Wohnung, nicht Bescheid. Jedoch scheint Frau Holzner sehr zufrieden mit ihrem Leben zu sein. Sie äußert kein Bedauern über die Entfremdung. Im Gegensatz zu früher geht sie engagiert in ihrer Rolle als Mutter von Benedikts vier- und siebenjährigen Halbbrüdern auf, um die sie sich verantwortungsvoll gemeinsam mit ihrem Ehemann kümmert. In diesem Kontext richtet sie ihre Handlungsentwürfe auf ein gutes Aufwachsen der beiden Kinder aus und orientiert daran auch den eigenen Mediengebrauch sowie jenen der neuen Kernfamilie. Aus anfänglich sozio-ökonomisch stark be- 
lasteten Verhältnissen konnte sich Frau Holzner befreien und auch im Berufsleben erneut etablieren. Dazu trug maßgeblich auch die Eheschließung und der Umzug in eine Eigentumswohnung bei.

\subsubsection{Isabelle Rohringer (21 Jahre alt) und ihre Mutter: Aufgewachsen in einem vor allem sozio-emotional belasteten Familienklima und nunmehr mit guten Chancen zu mehr Selbständigkeit}

\section{5 bis 2017}

Das Familienklima bei Familie Rohringer, zu der Tochter Isabelle und ihr drei Jahre jüngerer Bruder sowie ein älterer Halbbruder gehören der älteste Sohn aus Frau Rohringers erster Ehe lebt seit der vierten Erhebungswelle (2012) nicht mehr im selben Haushalt - war stark angespannt. Es war gekennzeichnet durch eine neue Familienkonstellation und damit verbundene Trennungserfahrungen, die zu schwierigen sozio-emotionalen Belastungen führten. Die Lebensführung der Familie erwies sich dadurch als deutlich beeinträchtigt. Aufgrund der starken Alkoholerkrankung von Isabelles Vater, der beruflich als Metzger arbeitete, kam es in der zweiten Erhebungswelle (2007) zur Trennung der Eltern. Isabelle litt unter der Trennung von ihrem Vater, da ihr die Kommunikation mit ihm sehr wichtig war. Da sie ihrem Vater wegen seiner Alkoholprobleme helfen und ihn beschützen wollte, war sie emotional überfordert; später kümmerte sich der Vater kaum mehr um sie. In der dritten Erhebungswelle (2010) lernte Frau Rohringer einen neuen Mann kennen, in dessen Firma sie mittlerweile Vollzeit als Bürokraft arbeitete und bei dem sie zusammen mit ihren Kindern die Wochenenden verbrachte; in der Woche lebte die Familie in ihrer eigenen Wohnung. Isabelle beschrieb das Verhältnis zum Lebensgefährten ihrer Mutter als „mal sehr gut und mal sehr schlecht“. Kritik von ihm konnte sie kaum ertragen, zuweilen zeigte sie dessen älterer Tochter gegenüber Eifersuchtsgefühle, da der Lebensgefährte, wie sie meinte, seine leibliche Tochter bevorzugte. Wenn der „Stiefvater" sie kritisierte, bereitete dies Isabelle große psychische Probleme. Zwar erklärte sie in der vierten Erhebungswelle (2010), dass sie sehr wohl wisse, „was sie an ihm hat" und wie es der Familie ohne ihn ergangen wäre. Bei Streit zwischen ihrer Mutter und dem neuen Lebensgefährten stellte sie sich jedoch auf die Seite der Mutter und meinte, diese solle „ihn rauswerfen“. Später in der sechsten Erhebungswelle wurde der Lebensgefährte der Mutter auch für Isabelle als Gesprächspartner wichtig; sie verstand sich grundsätzlich gut mit ihm, aber ab und zu flögen doch „die Fetzen“, vor allem dann, wenn sie, wie 
sie im Interview angab, infolge der Wochenendbesuche zu wenig Zeit zum Lernen hatte oder der Lebensgefährte, wie auch ihre Mutter, Kritik an ihrer Handynutzung äußerte. Papa nannte sie den Lebensgefährten der Mutter allerdings nicht, sondern sprach ihn mit seinem Vornamen an.

Frau Rohringer, die zu Beginn der Studie 2005 als Hausfrau und zusätzlich als Tagesmutter geringfügig beschäftigt war und später auch als Kellnerin die Familie miternährte, wurde in den besonders prekären sozio-ökonomischen Phasen finanziell von den Großeltern unterstützt. Ab der vierten Erhebungswelle hatte sich das Einkommen der Familie zwar etwas verbessert, es lag jedoch weiterhin knapp unter dem Äquivalenzeinkommen, bis zu dem Armutsgefährdung angenommen wird. Die alleinerziehende Mutter ließ Anzeichen von Überforderung in der Bewältigung ihres Alltags erkennen; dies schlug sich auch in der (Medien-)Erziehung ihrer Kinder nieder. Frau Rohringer beurteilte zwar die Mediennutzung ihrer Kinder zuweilen kritisch (so hielt sie wenig vom „Hartz-IV“-Fernsehkonsum ihrer Tochter), feste Nutzungsregeln setzte sie jedoch nicht. Fehlverhalten sanktionierte sie zuweilen mit Hausarrest oder mit „HandyEntzug“, dies sei „die einzige Strafe, „die noch was bringt“. Gleichzeitig schätzte sie das Fernsehen für Isabelle als einen guten „Lehrmeister“ ein, damit sie „die deutsche Sprache besser lernt“, Isabelle habe aufgrund ihrer Legasthenie und Lernschwierigkeiten mit erheblichen Konzentrationsproblemen zu kämpfen. Zwar kritisierte ihre Mutter einige Medienangebote, gegen die Sendung Die Super Nanny hatte sie jedoch nichts Negatives einzuwenden. So betonte sie im Interview in der zweiten Erhebungswelle (2007), diese nutze ihre Tochter gern, „weil sie dann sieht, wie brav sie selbst im Vergleich zu den anderen Kindern" ist. Noch als Isabelle schon Jugendliche war, schaute Frau Rohringer kritisch, wenn auch nicht konsequent, auf die Mediennutzung ihrer Tochter. In der sechsten Erhebungswelle überzog sie ihre Tochter zuweilen mit willkürlich gesetzten Kontrollaktionen, insbesondere bei ihrer SMS- und WhatsApp-Kommunikation. Frau Rohringer nutzte auch Isabelles Facebook-Account über ihr eigenes Mobiltelefon und über ihren Laptop. Sie las regelmäßig Isabelles Einträge, woraufhin es zwischen Mutter und Tochter, so Isabelle in der sechsten Erhebungswelle, oft Streit gab. Sie verlangte denn auch von ihrer Mutter, diese sollte sich selbst bei Facebook anmelden, sobald sie selbst das 18. Lebensjahr erreicht habe, danach habe sie kein Recht mehr, sie zu kontrollieren. 
2020: Isabelle und ibrer Mutter gelingt es nun besser, ibre Handlungsentwürfe unabhängiger voneinander zu entwickeln und umzusetzen

Isabelles Leben hat sich mittlerweile sehr verändert; so wohnt sie nunmehr mit ihrem Freund zusammen in einer eigenen Wohnung, nutzt jedoch zuweilen die Möglichkeit, bei ihrer Mutter zu schlafen, wenn z.B. die Wetterverhältnisse schlecht sind und die Anfahrt zur Arbeit morgens nicht so gut zu bewältigen wäre. Denn Isabelle arbeitet in dem Ort, in dem ihre Mutter lebt. Frau Rohringer wohnt mit ihrem jüngeren Sohn nunmehr in einem Haus der Schwester ihres Lebensgefährten, weil es dieser zu groß war (als Ausgleich hatte Familie Rohringer für sie eine $50 \mathrm{~m}^{2}$-Eigentumswohnung gesucht). Isabelle hat ihre Ausbildung zur Kinderpflegerin abgeschlossen und arbeitet in einem Hotel in der Kinderbetreuung, und zur Zeit der Befragung wegen der Covid-19-Pandemie nur online. Das Familienklima hat sich bei Familie Rohringer in der siebten Erhebungswelle entspannt; auch Frau Rohringer wirkt ausgeglichener. Sie war zwischen der sechsten und siebten Erhebungswelle an einem Hirntumor erkrankt und hatte danach ihr Leben, wie sie sagt, sehr bewusst umgestaltet, zudem seien die Kinder während ihres Krankenhausaufenthaltes „schlagartig erwachsen geworden“.

\section{Medien im Leben von Isabelle und ibrer Mutter}

Isabelle nutzt heute Medien eher selektiv und zumeist mit ihrem Freund gemeinsam, etwa Serien und Filme wie z.B. Türkisch für Anfänger auf Amazon Prime; das Radio läuft bei ihr „daheim immer nebenbei“, und auf Snapchat spielt sie mit einer Freundin gern Spiele. WhatsApp dient Isabelle zur Kommunikation mit der Mutter und mit Peers. Zudem ist ihr Google wichtig, wo, wie es ihre Mutter beschreibt, ihre Kinder schlicht alles nachschauten („die googlen alles“).

Das Thema Medienerziehung spielt zwischen Isabelle und ihrer Mutter nun keine Rolle mehr, dennoch weist diese ihre Tochter noch darauf hin, das Handy zur Seite zu legen, wenn sie bei ihr ist. „Handysüchteln“ kritisiert Frau Rohringer auch heute noch. Noch immer hat sie einen Zugang zum Facebook-Account ihrer Tochter, den sie bis zur Volljährigkeit von Isabelle auch stets im Blick hatte. Doch heute schaue sie die Einträge nur noch „aus Langeweile“ durch oder um zu sehen, was in der „Verkaufsbörse" angeboten werde und nicht mehr, um ihre Tochter zu kontrollieren.

Heute nutzt Frau Rohringer Medien mehr noch als schon in den vorherigen Erhebungswellen, sehr intensiv, sowohl beruflich als auch privat. Besonders wichtig ist ihr das Handy, auf dem sie WhatsApp, E-Mails, Pinterest und eine Skifahr-App nutzt, aber auch der PC, den sie für die 
Arbeit, zum Lesen von Tageszeitungen, fürs Onlinebanking und Onlineshopping einsetzt. Eine wichtige Rolle spielt für Frau Rohringer auch das Fernsehen, in dem sie vor allem Nachrichten anschaut, gern aber auch Angebote wie Bergdoktor, „Schnulzen“, Skifahren sowie die Sendungen Heimatleuchten und Pfusch am Bau. Daneben ist Frau Rohringer die Smarthome-Anwendung Amazon Alexa sehr wichtig, etwa fürs Radiohören, um bestimmte Lieder zu suchen oder Informationen übers Wetter oder andere Informationen abzufragen. Nach der Erkrankung sieht sie viele Vorteile in der Nutzung von Medien, da sie damit nun auch „Organisatorisches digital“ erledigen könne. Allgemein aber empfindet sie nach wie vor jegliche Mediennutzung als zu viel und in der Lage, „abhängig“ zu machen.

Fazit

Familie Rohringers Handlungsoptionen verbesserten sich im Laufe der Erhebung zwar, da die Mutter nunmehr im Unternehmen ihres neuen Lebensgefährten arbeitete und sich im Zuge dessen die sozio-ökonomische Lage der Familie etwas entspannte. Das Familienklima blieb in der Kernfamilie jedoch weiterhin angespannt. Frau Rohringers Handlungsentwürfe richteten sich vor allem auf ihre Beziehung zum neuen Lebensgefährten. Sie blieb dennoch für Isabelle bis zum Ende der Erhebung 2020 eine sehr wichtige Bezugsperson, wenn auch deren Beziehung zu ihrer dominanten Mutter bis zum Ende der sechsten Erhebungswelle keinesfalls konfliktfrei war. Als besonders belastend erlebte das in ihrer Handlungskompetenz aufgrund einer Legasthenie und Lernschwäche eingeschränkte Mädchen die nahezu vollständig anmutende und willkürliche Kontrolle ihrer SMSund WhatsApp-Nachrichten und der Einträge auf Facebook durch die Mutter, die noch am Ende der sechsten Erhebungswelle andauerte. Isabelle entwickelte während dieser Erhebungsphase den Wunsch, aus der gemeinsamen Wohnung auszuziehen. In der siebten Erhebungswelle ist es Isabelle auch gelungen, ihre Handlungsentwürfe stärker von ihrer Mutter und auch ihrem leiblichen, alkoholkranken Vater zu lösen. Dieser hatte sich nach der Trennung von der Mutter nach der zweiten Erhebungswelle kaum mehr um Isabelle gekümmert, ihr aber betrunken durch Anrufe und Treffen mit ihr in seiner Stammkneipe emotional sehr zugesetzt. Isabelle musste sich aus diesem Grund sogar einer Psychotherapie unterziehen. In der siebten Erhebungswelle ist Isabelle mit ihrem Freund in eine eigene Wohnung gezogen und hatte auch ihre Ausbildung als Kinderpflegerin abgeschlossen. Zum Zeitpunkt der Erhebung arbeitet sie in der Kinderbetreuung eines Hotels. Frau Rohringer war zwischen der sechsten und siebten Erhebungswelle an Krebs erkrankt und längere Zeit in einem 
Krankenhaus gewesen - ein einschneidendes Erlebnis für die Kernfamilie. Zum Ende der Erhebung erweist sich das Familienklima der Familie deutlich entspannter als in den vorherigen Erhebungsphasen, es scheint Mutter und Tochter nun besser zu gelingen, ihre jeweiligen Handlungsentwürfe unabhängiger voneinander zu entwickeln und auch so umzusetzen, dass diese nicht - wie zuvor - eher zu Lasten von Isabelle gehen. Medien spielen in der letzten Erhebungsrolle wie auch schon in den Wellen davor für Isabelle insbesondere eine Rolle, um soziale Kontakte aufzubauen und zu pflegen. So liegt ihr nun besonders daran, mit ihrem Freund gemeinsam Amazon Prime-Videos anzuschauen.

\subsubsection{Alfons Weiss (19 Jahre alt) und seine Mutter: Sozio-ökonomischer Aufstieg kennzeichnet die Familie. Die sozio-emotionale Beziehung verschlechtert sich dramatisch und eskaliert}

\section{5 bis 2017}

Seit Beginn der Studie lebte Alfons (2005 vier Jahre alt) gemeinsam mit seiner alleinerziehenden Mutter und seinem jüngeren Halbbruder Christian (16) am Stadtrand einer Kleinstadt mit guter infrastruktureller Anbindung im Zentralraum. Von 2005 bis 2012 lebte die Familie in einer $72 \mathrm{~m}^{2}$ großen und immer sehr ordentlichen und sauberen Eigentumswohnung. Dort teilten sich die Brüder, zu Alfons Leidwesen, ein Zimmer. Kurz nach der vierten Erhebungswelle (2012) zog Familie Weiss in eine $110 \mathrm{~m}^{2}$ Doppelhaushälfte, nur einige Straßenzüge entfernt, und blieb dort bis zum Ende der Studie wohnen. Nun verfügten alle Familienmitglieder über eigene Zimmer und gerade die Kinderzimmer waren bei allen Besuchen sehr ordentlich. In der fünften Erhebungswelle (2014) lebte zudem ein Pflegekind, gegen Finanzierung durch das Jugendamt, bei der Familie. Das Mädchen entschloss sich jedoch freiwillig, das Haus wieder zu verlassen.

Seit dem Beginn der Studie bis zur vierten Erhebungswelle war die Familie immer armutsgefährdet und verfügte nur über geringe Geldmittel. In der ersten Erhebungswelle befand sich Frau Weiss in Karenz, in den folgenden Jahren arbeitete sie geringfügig oder in Teilzeit (30h) als Angestellte in einem Baumarkt. Dennoch beteuerte Frau Weiss in allen Interviews, beide Immobilien selbst finanziert zu haben und dies durch fleißige Arbeit und eiserne Sparsamkeit erreicht zu haben. Es ist jedoch zu vermuten, dass ihre Eltern sie maßgeblich finanziell unterstützt hatten. $\mathrm{Ab}$ 2014, einhergehend mit dem Tod ihrer Eltern, verbesserte sich die finanzielle Situation von Frau Weiss nachhaltig. Einerseits verfügte sie nun 
über ein nicht näher definiertes „Vermögen“, bestehend aus „Geldanlagen und Wertpapieren“, andererseits erbte sie auch Immobilien und bezog Einkünfte aus deren Vermietung, die sich 2016 auf monatlich etwa 1.000 Euro beliefen. Zugleich wechselte Frau Weiss zu dieser Zeit den Arbeitgeber und war nun bei einer gemeinnützigen Wohnungsbaugesellschaft tätig, dort verdiente sie eigenen Angaben zufolge nun „das Doppelte“. In der sechsten Erhebungswelle (2016) war Frau Weiss „krankheitsbedingt“ arbeitslos und bezog monatlich 900 Euro vom AMS. In Kombination mit ihren Mieteinnahmen, Alimenten und der Familienbeihilfe bezifferte sie ihr Einkommen auf 2.801 bis 3.100 Euro monatlich und war somit auch in der Arbeitslosigkeit nicht armutsgefährdet.

Die zum Teil eingeschränkte sozio-ökonomische Situation konnte die Familie, auch mit Unterstützung der Großeltern mütterlicherseits, gut kompensieren. Im Haushalt schien es an nichts zu mangeln, und die Familie unternahm auch vergleichsweise regelmäßig Ausflüge, etwa zum Schwimmen oder, weitaus kostenintensiver, zum Skifahren. Alfons war auch über viele Jahre zunächst im Turnverein und bei den Pfadfindern aktiv und unternahm dort viele Reisen, später bei der Freiwilligen Feuerwehr. Zudem spielte er Gitarre und erhielt privaten Unterricht. Stark belastet war die Familie dagegen immer auf sozio-emotionaler Ebene, auch wenn Frau Weiss stets bemüht war, diese Konflikte herunterzuspielen und nach Außen eine weitestgehend heile Familie zu zeigen. Frau Weiss war während der gesamten Studiendauer immer alleinerziehend, obwohl sie von der ersten Erhebungswelle an eine feste Partnerschaft mit einem Bahnbeamten führte. Das Paar lebte aber niemals zusammen. Mit dem Lebensgefährten seiner Mutter kam Alfons immer gut aus, und dieser war auch eine Bezugsperson für den Jungen. Vereinzelt unternahmen die beiden auch in der Freizeit etwas gemeinsam. Alfons wichtigstes Vorbild war jedoch immer sein leiblicher Vater. Dieser lebte, gemeinsam mit seinen eigenen Eltern, auf einem Bauernhof in einer vergleichsweise weit entfernten und sehr ländlich geprägten Gebirgsgegend. Dort verbrachte Alfons bevorzugt seine Ferien und auch oft die Wochenenden. Sein Vater unternahm viel mit ihm (Skifahren, Ausflüge, Basteln und Handwerken), zudem genoss Alfons dort aber auch viel größere Freiheiten als bei seiner Mutter und konnte viel Zeit mit Freunden verbringen. Tatsächlich äußerte Alfons von Beginn der Studie an immer wieder nachdrücklich den Wunsch, dass er lieber fest bei seinem Vater leben wollte. Als Kind verbot Frau Weiss aber diesen Umzug, und auch als Jugendlichem gelang es Alfons nicht, seinen Wunsch durchzusetzen. Es ist zu vermuten, dass Frau Weiss einerseits in einer Machtposition gegenüber dem Vater ihres Erstgeborenen 
stehen wollte, andererseits aber auch finanzielle Beweggründe (Alimente, Kindergeld) hinter ihrer Verweigerungshaltung standen. Denn ihr Ansinnen, Alfons an sich zu binden, stand in einem starken Widerspruch zu dem Verhältnis zwischen Mutter und Sohn, das in allen Erhebungswellen belastet und distanziert und von gegenseitigem Desinteresse geprägt war. Gerade in der fünften und sechsten Erhebungswelle fiel auf, dass Frau Weiss das Gespräch immer wieder auf den jüngeren Halbbruder zu lenken versuchte und diesen, ohne entsprechende Leistungen, in den höchsten Tönen lobte, während sie an Alfons wenig Interesse zeigte und dies auch im Interview recht offen so kommunizierte. Alfons spürte das Desinteresse seiner Mutter und die Bevorzugung des jüngeren Halbbruders und zog sich immer weiter von familiären Unternehmungen und von seiner Mutter zurück.

Frau Weiss führte Probleme mit Alfons immer auch auf einen „Impfschaden" zurück, den dieser angeblich als Baby erlitten haben soll. In der vierten Erhebungswelle berichtete die Mutter zudem, dass Alfons in einer Einrichtung der „Lebenshilfe“ auf seine geistige Entwicklung hin untersucht und dabei eine 40-prozentige geistige Beeinträchtigung festgestellt wurde. Tatsächlich war Alfons zu Beginn der Erhebung ein sehr zurückhaltendes und schüchternes Kind mit einigen Verhaltensauffälligkeiten, diese waren jedoch in späteren Erhebungswellen nicht mehr zu erkennen. Alfons blieb zwar schüchtern, aber er trat immer sehr freundlich und offen auf und berichtete reflektiert über sein Leben und seine Interessen. Zudem besuchte er, mit mittelmäßigen Leistungen, immer eine Regelschule. Ganz im Gegensatz zu seinem Halbbruder Christian, der aufgrund von Einschränkungen und Lerndefiziten in der Schule über mehrere Jahre hinweg als „Integrationskind“ geführt wurde. In der sechsten Erhebungswelle, mit inzwischen 15 Jahren, besuchte Alfons eine Hauptschule und stand kurz vor dem Schulabschluss. Zu diesem Zeitpunkt plante er zunächst den weiteren Schulbesuch auf einer Polytechnischen Schule und überlegte, im Anschluss daran eine Lehre als Schlosser zu absolvieren. Erstmals berichtete er nicht mehr davon, zu seinem Vater ziehen zu wollen, da es dort keine berufliche Perspektive gäbe, und er lieber gemeinsam mit einem Freund an seinem derzeitigen Wohnort eine Ausbildung absolvieren wollte.

2020: Alfons Weiss - ein junger Mann aus zerrütteten Verhältnissen muss einen Neubeginn wagen und seinen eigenen Weg erst noch finden

Aus beruflicher Perspektive und hinsichtlich ihrer sozio-ökonomischen Bedingungen hat sich Frau Weiss in der siebten Erhebungswelle (2020) erneut verbessert. Sie hat eine einjährige Ausbildung zur Justizwachebe- 
amtin absolviert und arbeitet als Gerichtsdienerin in ihrer Heimatstadt. Zusätzlich arbeitet sie, mit Genehmigung ihres Dienstherrn, im Sommer am Wochenende und an freien Tagen in der Eisdiele eines Bekannten. In ihrer Freizeit verbringt sie viel Zeit am See oder mit Freunden und verreist mit ihrem jüngeren Sohn. Beide wohnen noch immer gemeinsam in der Doppelhaushälfte, und Frau Weiss ist voll des Lobes über ihn, über seine Freundlichkeit und Zuverlässigkeit und seine Entwicklung. Christian macht inzwischen in Deutschland eine Ausbildung im Hotelgewerbe und besucht begleitend eine „normale“ Berufsschule im Regelbetrieb, wie seine Mutter betont.

Bereits in früheren Erhebungswellen hatte sich Frau Weiss kritisch bis herablassend über, wie sie stets formulierte, „Ausländer“ geäußert. So thematisierte sie etwa zu Beginn der Studie die vielen „Sozialfälle“ in ihrer damaligen Wohnsiedlung, und während der starken Fluchtmigration 2015 und 2016 positionierte sie sich stark ablehnend gegen Unterstützungsmaßnahmen und die Aufnahme von Geflüchteten. 2020 scheinen sich diese Ansichten verstärkt und radikalisiert zu haben. In einem persönlichen Vorgespräch zum Telefoninterview äußert sich Frau Weiss mehrfach sehr negativ über „Ausländer“ im Allgemeinen und über Muslime im Besonderen. Sie beklagt sowohl die Überfremdung der Gesellschaft als auch den Umstand, dass man in der nahegelegenen Großstadt, die als eher beschaulich und sicher bezeichnet werden kann, durch den Zuzug von „Ausländern“ jetzt quasi an „jeder Hausecke Drogen kaufen“ könne. Als ein sehr positives Gegenbeispiel für eine Gesellschaft führt sie einen Urlaub in Slowenien an, wo „keine Ausländer“ gewesen seien, sondern „nur Slowenen, Deutsche und Österreicher". Gemeint sind wohl erneut Muslime, denn sie betont auch, dass es ja dann „keine Probleme mit Ausländern“ gäbe, wenn diese aus katholischen Ländern stammten.

Nicht zuletzt ihre Ansichten zu Migration und Drogen führten kurz nach der sechsten Erhebungswelle zu einem schwerwiegenden Streit mit Alfons und einer völligen Eskalation der Beziehung zu ihrem Sohn. 2017 warf Frau Weiss den noch minderjährigen Jungen aus dem Haus. Auslöser dafür war der Verdacht, dass Alfons Drogen konsumiert und mit diesen gehandelt haben könnte. Er spricht dagegen von einem Konflikt über Alkohol und den Konsum von „ein paar Bier“, den seine Mutter „in ihrer Welt" nicht tolerierte. In der Folge wartete Frau Weiss, so ihre Beteuerung, darauf, dass ihr Sohn nach Hause käme, um sich zu entschuldigen. Aber Alfons kam nicht nach Hause, sondern lebte, eigenen Angaben zufolge, „Zwei Monate“ lang auf der Straße in der Nähe des Bahnhofs. In dieser Zeit bezog er keinerlei staatliche Unterstützungsmaßnahmen, sondern 
sammelte Pfandflaschen, wenn er Geld zum Überleben benötigte. Später ersuchte Alfons den Bürgermeister seiner Heimatstadt um Hilfe, der für ihn eine geförderte Wohnung organisierte. Die nötige Kaution übernahm Frau Weiss, da Alfons sonst weiterhin auf der Straße hätte leben müssen. In dieser Wohnung lebt Alfons auch aktuell und bezieht monatlich 180 Euro an Wohnbeihilfe. Zusätzlich erhält er 300 Euro aus der Vermietung einer Wohnung, die er gemeinsam mit seinem Bruder von seinem Großvater geerbt hat. Zudem, so Frau Weiss, erhält er Arbeitslosenunterstützung. Dies verneint Alfons jedoch und verweist darauf, dass er als ungelernter Arbeiter in einem Lager in der Kommissionierung arbeite und dies auch noch ein oder zwei Jahre machen möchte, um sich dann neu zu orientieren. Über sein Einkommen kann Alfons jedoch nicht frei verfügen, da er unter der Aufsicht eines gerichtlich bestellten Sachwalters steht. Diese Maßnahme beruht auf seiner ehemaligen Obdachlosigkeit, dem Verdacht auf Drogenmissbrauch und der Verschwendung von Geld. Alfons selbst bestätigt diese Maßnahme und begründet sie damit, dass er „im letzten Jahr zu viel Geld ausgegeben" habe. Laut Frau Weiss hatte Alfons zahlreiche Verträge im Internet abgeschlossen, unter anderem einen Handyvertrag und Mitgliedschaften bei Partnerbörsen für 300 Euro im Monat. In einem Jahr habe er zwar 18.000 Euro verdient (Herkunft unbekannt, Frau Weiss deutet Drogen an), bereits nach zwei Monaten sei aber alles ausgegeben gewesen. In Folge hat er keinen Zugriff auf sein Konto und kann nicht mit Bankomatkarte bezahlen. Auf Vertrauensbasis erhält er monatlich ein Taschengeld ausgezahlt, mit dem er selbstständig Einkäufe des täglichen Bedarfs tätigen kann. Mit seinem neuen Leben zeigt sich Alfons recht zufrieden, und er will daran auch nichts ändern: „Will meine Ruhe haben! Meine eigene Wohnung." Das schon immer schwierige Verhältnis zu seiner Mutter ist durch die Vorkommnisse und ihr fehlendes Verständnis massiv belastet. Dennoch berichtet Alfons davon, dass es zuletzt zu einer gewissen Verbesserung gekommen ist: „Ja, jetzt ist's besser. Sie wäscht mir jetzt meine Wäsche und so.“

\section{Medien im Leben von Alfons und seiner Familie}

Alfons nutzte von Kindesbeinen an sehr intensiv Medien, in erster Linie das Fernsehen. Seine Mutter schränkte ihn dabei auch kaum ein. Seinem Alter entsprechend nutzte Alfons zunächst Kinderprogramme, dies allerdings mit hoher zeitlicher Intensität, im Lauf der Zeit wendete er sich aber davon ab. Alfons entwickelte eine große Leidenschaft für Sportsendungen, die sich auch in seinem Interesse für verschiedene Fußballvereine und Skirennläufer widerspiegelte. Zudem schaute er gerne verschiedene Serien, 
die auch bei anderen Jungen in seinem Alter beliebt waren. Zuletzt gab er an, im Fernsehen gerne Skirennen, Skispringen, Fußball, DSDS, The Voice of Germany, Spiderman und James Bond zu sehen. Genutzte Sender waren DMAX, ORF 1, ORF 2, RTL, RTL2, Sat1 und ProSieben. Wenn er Informationssendungen und Nachrichten verfolgte, dann über ORF und RTL. Speziell Fernsehnachrichten stufte Alfons immer als glaubwürdig ein und nutzte sie unter anderem, um sich über die „Flüchtlingsthematik“ zu informieren, da er sehr daran interessiert war, wieso die Menschen nach Österreich kommen wollten. Online-Angebote, und insbesondere Soziale Medien, spielten in Alfons Leben lange gar keine oder eine nur sehr untergeordnete Rolle. Darin unterschied er sich deutlich von den anderen Heranwachsenden im Panel. Erst in der sechsten Erhebungswelle nutzte er Facebook, nicht jedoch Snapchat oder Instagram. Auf seinem Smartphone setzte er stark auf Telefonie, YouTube und WhatsApp. Aus den WhatsApp-Gruppen der Schule hatte er sich auch bald zurückgezogen, da er dort von Mitschülern „verarscht" worden sei. Auch hinsichtlich des Konsums von Videospielen unterschied sich Alfons lange von seinen Altersgenossen. Zu seinen bevorzugten Angeboten zählten Spiele wie Subway Surfer, Landwirtschafts-Simulator, Soccer Stars oder Geometry Dash. Bei seinem Vater und bei Freunden spielte Alfons auch ohne das Wissen seiner Mutter Konsolenspiele, die erst ab 18 Jahren freigegeben sind, wie GTA 5 und Call of Duty. Daheim hatte Alfons dagegen keine eigene Konsole. 2020 erweist sich seine Mediennutzung als unklar. Frau Weiss vermutet, dass ihr Sohn den ganzen Tag an einer Konsole Fußball spiele, fernsähe und sich mit Telefon und WhatsApp beschäftige. Alfons selbst betont dagegen, dass er nicht mehr an der Konsole spiele und lediglich über ein Billardspiel auf dem Handy verfüge. Darüber hinaus schaue er viel fern und widme sich intensiv Netflix und Amazon Prime. Um Fußballspiele auf Sky ansehen zu können, besucht Alfons, in Ermangelung eines Abonnements, Wettbüros. Zur Information greift er auf die Kronenzeitung, die Bildzeitung, Google News und verschiedene Online-Zeitungen zurück. Noch immer nutzt Alfons WhatsApp und schaut gelegentlich auf Facebook, jedoch gibt er auch an, inzwischen oft persönliche Unterhaltungen der Kommunikation via Smartphone vorzuziehen.

Die Mediennutzung von Frau Weiss war über die Jahre hinweg vergleichsweise gering und sehr konstant. Insbesondere das Fernsehen diente ihr zur Rezeption von Informationssendungen, Dokumentationen und Nachrichten. Die Sendung Musikantenstadl blieb dabei ein ständiger Begleiter. Ihr Smartphone nutzte sie dagegen lange Zeit nur für Telefonie und SMS, nicht aber zur Online-Nutzung und für Apps. Zeitweise spielte 
das Internet am Computer aber eine wichtige Rolle, um sich über Impfthemen $\mathrm{zu}$ informieren und um Mitglied in entsprechenden Mailinglisten zu sein - dieses Interesse ebbte aber im Zeitverlauf immer weiter ab. Auch 2020 zeigt sich Frau Weiss wenig versiert im Umgang mit digitalen Medien. Im Kontext der Vorbereitungen für die Distanz-Lehre ihres Sohnes Christian betonte sie, wie wenig sie selbst, aber auch der 16-Jährige, sich mit Computern, Internet und Druckern auskennen würden; deshalb habe sich diese Umstellung als große Herausforderung erwiesen. Auch den Fernseher hat sie „schon seit einer Ewigkeit nicht mehr eingeschaltet“, da sie „des eigentlich gar ned gebraucht (hat), die Unterhaltung mit Medien“. Arbeit, Nebenjob und Freizeit mit Freunden würden sie, so Frau Weiss, ausreichend beschäftigen. Im Gegensatz zu früher nutzt Frau Weiss inzwischen aber (soziale) Medien auf dem Smartphone, um zu kommunizieren und um sich zu informieren. Als Beispiele nennt sie WhatsApp sowie Facebook, YouTube und Nachrichten-Apps wie Stadt A.com zur Information sowie eine Wetter-App.

\section{Fazit}

Die Handlungsoptionen der Familie haben sich im Zuge der Stabilisierung ihrer sozio-ökonomischen Situation mit der Zeit nachhaltig verbessert. Das schon immer angespannte Verhältnis zwischen Frau Weiss und ihrem Sohn Alfons hat sich dagegen im Laufe der Jahre immer weiter verschlechtert, und zuletzt waren die sozio-emotionalen Bedingungen so stark belastet wie in keiner anderen Familie im Panel. Gelang es in früheren Jahren noch, die Konflikte zu verdecken und sich gegenseitig weitestgehend zu ignorieren, so hat der Streit über mutmaßlichen Drogenmissbrauch zu einer nachhaltigen Eskalation geführt. Als absoluter Tiefpunkt erwies sich der Rauswurf des minderjährigen Jugendlichen aus dem Elternhaus und eine daran anschließende, mehrmonatige Obdachlosigkeit. Inzwischen hat sich die Situation, durch die nun sozio-ökonomisch zumindest abgesicherten Lebensverhältnisse von Alfons, etwas beruhigt und normalisiert. Dennoch ist der inzwischen 19-Jährige nur eingeschränkt geschäftsfähig und seine Handlungsoptionen sind stark eingeschränkt. Alfons verfügt über keinerlei Berufsausbildung, was sich maßgeblich auf seine Handlungskompetenzen auswirkt, und er hat derzeit auch keine Pläne, um dies in absehbarer Zeit zu ändern. Zwar äußert er den Wunsch „ein Arbeitsplatz“ zu finden, „was mir gefällt so“, aber er entwickelt keine glaubhaften Handlungsentwürfe, die eine entsprechende Perspektive realistisch erscheinen lassen. Die direkte Frage nach einem Wunscharbeitsplatz oder Traumjob beantwortet er mit „Ja, so 'nen Traktor fahren!“ Dazu gibt es aber keinen 
realen Bezug in seiner Lebenswelt, denn die früher betriebene Nebenerwerbslandwirtschaft seiner Großeltern existiert nicht mehr. Alle Tiere und Gerätschaften sind verkauft, und der Grund ist verpachtet. In seiner Freizeit wendete sich Alfons immer bevorzugt Bewegtbildangeboten zu. Waren es früher nur das Fernsehen und insbesondere Sportangebote, so spielt inzwischen auch Netflix eine zentrale Rolle. Computerspiele und Soziale Medien hatten für ihn dagegen immer eine geringere Bedeutung als für andere Heranwachsende im Panel. Auffällig ist, dass sich Alfons, auch unter schwierigen Lebensbedingungen, stark Informationsangeboten zuwendet und dazu verschiedene Zeitungen nutzt. Für Frau Weiss hat die Bedeutung insbesondere des Fernsehens im Verlauf der Studie dagegen abgenommen, Online-Angebote und dabei vor allem Soziale Medien zur Information und Kommunikation haben dagegen deutlich an Relevanz gewonnen.

\subsection{Die jungen Erwachsenen von Typ 3: Die zwar sozio-ökonomisch, aber sozio- emotional weniger belasteten, relativ kompetenten Familien}

Die Familien von Typ 3 wiesen zwar weiterhin belastete sozio-ökonomische Strukturen auf, es gelang ihnen aber über die Erhebungswellen hinweg dennoch, ihre sozio-emotionalen Bedingungen zu verbessern. Sie zeigten sich daher trotz eingeschränkter sozio-ökonomischer Bedingungen zum Ende der sechsten Erhebung in der Bewältigung ihres Alltags weniger belastet als die Familien von Typ 1 und 2. Den Familien von Typ 3 gelang es zunehmend besser, mit ihren schweren sozio-ökonomischen Bedingungen umzugehen. Sie nahmen zwar ihre Handlungsoptionen als deutliche Einschränkung wahr, ihnen gelang es aber aufgrund ihrer ausgeglichenen und deutlich stabileren Beziehungen in der Kerngruppe, die damit verbundenen Herausforderungen für das Familienleben zu meistern und ihren Alltag zu bewältigen. Die Familien von Typ 3 erschienen größtenteils recht zufrieden mit ihrer Lebenssituation. Darin unterschieden sie sich klar von den Familien aus Typ 1, aber auch aus Typ 2. Den Familien Stab, Ebner und Kaiser aus Typ 3 gelang es schon seit Beginn der Studie, recht gut mit ihren erschwerten sozio-ökonomischen Bedingungen umzugehen, anders wiederum als den Familien Boll und Aufbauer, die sich erst während der fünften und sechsten Erhebungswelle sozio-emotional stabilisieren und infolge dessen relativ kompetent mit ihrer sozialen Lage umgehen und den Alltag bewältigen konnten. Die Familien aus Typ 3, allen voran die Mütter, bemühten sich um Harmonie in der Familie - ein Grund, wes- 
halb sie ihren Kindern auch im Umgang mit Medien relativ viel Freiheit ließen und ihnen kaum Regeln setzten. Ihnen war eine offene, gute Kommunikation miteinander und gegenseitige Unterstützung wichtig; zum Ende der sechsten Erhebungswelle fühlten sich Amelie Aufbauer, Gregor Boll, Elisabeth Ebner, Torsten Kaiser und Simone Stab in ihren Familien weitgehend wohl und aufgehoben, wenngleich einige ihrer Geschwister zwischenzeitlich mit erheblichen Belastungen zu kämpfen hatten, wie die ältere Schwester von Amelie bzw. zum Schluss der Studie der jüngere Bruder von Simone. Doch auch bei ihnen schafte es die Familie als Ganzes, Lösungen zu finden und sich bei der Bewältigung der Probleme der Geschwister als weitgehend kompetent zu erweisen, wie dies in Familie Stab der Fall war.

\subsubsection{Amelie Aufbauer (20 Jahre alt) und ihre Mutter: Eine sozio-} ökonomisch stark belastete Familie, die sich sozio-emotional mit den Jahren stabilisierte

\section{5 bis 2017}

Familie Aufbauer, zu der neben Amelie noch ihre ältere Halbschwester und zwei jüngere Halbgeschwister gehören, zählte seit Beginn der Studie zu den sozio-ökonomisch stark belasteten Familien, wenn sich auch in der sechsten Erhebungswelle die finanzielle Lage der alleinerziehenden Mutter durch das Kinderbetreuungsgeld für ihren jüngsten Sohn etwas verbessert hatte. Über alle Erhebungswellen hinweg war die Familie zwar stets auf unterschiedliche staatliche Hilfen angewiesen, sie verstand es jedoch, mit dem wenigen Geld recht gut zurecht zu kommen. Frau Aufbauer wirkte dennoch vor allem in den ersten Erhebungswellen in der Alltagsbewältigung stark überfordert. So musste Amelie zusammen mit ihren Geschwistern schon früh Verantwortung für ihren Tagesablauf übernehmen; die Kinder kümmerten sich oft selbst darum, rechtzeitig für die Schule aufzustehen, sich ihr Frühstück zu machen, ihre Hausaufgaben zu erledigen und auch andere Alltagsbelange zu bewältigen. Dies war auch dann der Fall, wenn ihre Mutter wegen Arbeitslosigkeit, wie in der ersten und zweiten Erhebungswelle, zu Hause war. In der sechsten Erhebungswelle befand sich Frau Aufbauer in Karenz. Wie schon in den Jahren zuvor legte die alleinerziehende Mutter nur wenig oder gar keinen Wert auf den Kontakt zu den Vätern ihrer Kinder. Während der gesamten Erhebungszeit war die Familie des Öfteren umgezogen, zumeist dann, wenn die Wohnbeihilfe für eine Wohnung ausgelaufen war. So hatte Amelie nicht nur unter häu- 
figen Umzügen und den damit verbundenen Schulwechseln (etwa elf bis zwölf, wie sich Amelie erinnerte) zu leiden, sondern auch unter einem angespannten Familienklima, welches zumeist mit den Konflikten zwischen Amelies Mutter und ihrer älteren Schwester zusammenhing.

Im Laufe der vierten Erhebungswelle glätteten sich die früher häufig offen ausgetragenen Konflikte in der Familie, die auch zwischen den Geschwistern zu viel Streit geführt hatten. Nach dem Auszug der älteren Schwester vor der sechsten Erhebungswelle, hatte sich das Familienklima für alle Familienmitglieder deutlich verbessert, da die Wohnung vorher für die Familie zu klein war und auch die große Nähe zu Konflikten geführt hatte. Amelie zeigte sich aber recht enttäuscht darüber, dass ihre Schwester sich nach ihrem Auszug aus der Familie stärker zurückzog und kaum mehr nach Hause zu Besuch kam. Amelies eigene Beziehung zu ihrer Mutter hatte sich aber durch den Auszug der älteren Schwester deutlich verbessert. Sie war ihr, wie Amelie sagte, „eine beste Freundin“ geworden. Mit ihrem leiblichen Vater hatte Amelie bereits seit der zweiten Erhebungswelle keinen Kontakt mehr, er war psychisch schwer krank und hatte seine Tochter wegen der Einnahme starker Medikamente bei einigen ihrer wenigen zufälligen Begegnungen kaum erkannt. Wie sehr diese Tatsache Amelie wirklich belastete, ließ sich nur schwer beurteilen. Auffällig war, dass Amelie schon früh ältere Partner hatte, eine Zeitlang eine Beziehung zu einem fünf Jahre älteren Jungen, zu dem sie eine starke Abhängigkeitsbeziehung entwickelt hatte; mit Hilfe ihrer Mutter gelang es ihr aber, sich daraus wieder zu befreien. Auch in der sechsten Erhebungswelle hatte Amelie erneut einen älteren Partner. Auch ihm gegenüber verhielt sich das recht intelligent und in den Interviews recht selbstbewusst wirkende Mädchen sehr unsicher. Es gelang Amelie zuweilen nur schwer, eigene Handlungsentwürfe zu entwickeln.

Die Lebensführung von Amelies Mutter, einer gelernten Goldschmiedin, war über weite Strecken der Studie gekennzeichnet von Ruhelosigkeit; Frau Aufbauer schien stets auf der Suche nach etwas Neuem zu sein. So pflegte sie zu den Vätern ihrer Kinder nie längere Beziehungen, auch zu dem Vater ihres jüngsten Sohnes bestand bereits kurze Zeit nach dessen Geburt kein Kontakt mehr. Der Mann stammte aus Afrika und sei, so Frau Aufbauer, bestimmt schon wieder zurück in seiner Heimat, sie wisse dies aber nicht. Während der Erhebungszeit trennte sich Frau Aufbauer stets recht bald von ihren neuen Lebensgefährten, da es mit ihnen, wie sie erzählte, immer wieder zu neuen Konflikten kam und dann der Partner die gemeinsame Wohnung verließ. In der fünften Erhebungswelle betonte Frau Aufbauer, nunmehr allein, ohne einen neuen Partner leben zu 
wollen. Auch ihre berufliche Situation veränderte sich sehr häufig und war mitbestimmt von Arbeitslosigkeit, Karenzzeiten (auch in der sechsten Erhebungswelle befand sie sich wegen ihres 18 Monate alten jüngsten Kindes in Karenz), aber auch unterschiedlichen Teilzeitbeschäftigungen. Mit der Erziehung ihrer Kinder schien Frau Aufbauer stark überfordert. So war auch Medienerziehung für sie zu keiner Zeit ein relevantes Thema, auch Kontrolle, geschweige denn kommunikative Begleitung, gab es in Familie Aufbauer anfangs so gut wie gar nicht. Später änderte sich dies; so kam es in den letzten Erhebungswellen zwar zwischen Frau Aufbauer und Amelie des Öfteren wegen ihrer intensiven Smartphone-Nutzung zum Streit, und Amelie zog sich dann in ihr Zimmer zurück, aber Mutter und Tochter führten $\mathrm{ab}$ und zu Gespräche, etwa über Gefahren im Internet.

2020: Amelie: Auf der Suche nach einem eigenen Weg bei verbesserten sozioemotionalen Beziehungen zur Mutter

Amelie hat mittlerweile eine eigene Wohnung ganz in der Nähe ihrer Familie bezogen; die Entscheidung, auszuziehen, betrachtet sie „als beste Entscheidung"; sie genießt es, allein zu wohnen. Bevor Amelie wieder in die Großstadt, in der nun auch seit einiger Zeit wieder ihre Mutter mit ihren beiden jüngeren Halbgeschwistern wohnt, zurückgezogen war, hatte sie eine Zeitlang in einer anderen Großstadt gelebt. Wie ihre Mutter im Interview erzählt, war sie „wegen der Liebe (...) abgehauen“ und habe die Handelsakademie, die sie vorher besucht hatte, abgebrochen und in der Großstadt „an der Kasse gearbeitet“. Anfang 2019 ist Amelie wieder zurückgekommen und besucht derzeit noch eine Abendschule, um ihre Matura nachzuholen. Amelie selbst spricht nicht über den Grund ihres Aufenthalts in der anderen Großstadt. Amelie weiß bislang noch nicht, was sie nach der Matura studieren möchte. Sie macht den Eindruck, noch auf der Suche nach einem eigenen Weg zu sein. Ihre Mutter betont im Gespräch jedoch, dass ihre Tochter gerne einen Doktortitel hätte und erzählt, dass in Gesprächen mit ihrer Tochter zuletzt von Medizin die Rede gewesen sei.

Das Verhältnis zwischen Mutter und Tochter hat sich nach Amelies Auszug in eine eigene Wohnung weiter verbessert; sie geht auch regelmäBig zum Essen zur Familie. Zur Zeit der Erhebung hat Amelie seit etwa einem Jahr einen neuen Partner; sie scheut sich im Interview jedoch, dies als „Beziehung“ zu bezeichnen; mit ihm unternimmt sie viel, aber jeder habe, wie sie betont, „seine Freiheiten“. Abwechselnd wohnt Amelie bei ihrem Freund oder dieser bei ihr. Ihre finanziellen Ausgaben deckt die 
Schülerin durch staatliche Beihilfe; sie brauche, wie sie sagt, „nicht viel Geld, lediglich für ihre Fixkosten und Essen“.

Amelies nach wie vor alleinerziehende Mutter wohnt nach dem Auszug ihrer Tochter (Amelies ältere Halbschwester war schon vor längerer Zeit ausgezogen) zusammen mit ihren beiden jüngsten Kindern erneut in einer geförderten Mietwohnung. Der Mietvertrag dafür ist gerade für zwei Jahre verlängert worden, doch danach, darin ist sich Frau Aufbauer sicher, müsse sie wieder „etwas Neues suchen, da der Vertrag voraussichtlich nicht nochmal verlängert wird“. Amelies Mutter hat zwischenzeitlich in einem Hotel gearbeitet und war danach ein Jahr lang selbstständig als „Aufräum-Coach“ tätig; zur Zeit der siebten Erhebung arbeitet sie Teilzeit im Verkauf in einem Secondhand-Laden und schreibt viermal im Jahr eine Kolumne für ein Magazin zum Thema „Aufräumen“. Klar äußert Frau Aufbauer im Interview ihre schon in vorherigen Erhebungswellen geäußerte Überzeugung, dass sie „keinen Mann mehr ihm Haus“ haben möchte. Sie wünscht sich vor allem weiterhin eine geförderte Mietwohnung, da sie sich andere Wohnungen kaum leisten könne. Frau Aufbauers Handlungsentwürfe scheinen nach Jahren ökonomischer Instabilität und wechselnden Partnerschaften, zahlreichen Umzügen und Arbeitswechseln um mehr Stabilität zu kreisen, im Mittelpunkt steht dabei die Förderung einer Mietwohnung; mit ihrer derzeitigen Wohnung und auch mit der Umgebung und den Nachbarn zeigt sie sich zur Zeit sehr zufrieden.

\section{Medien im Leben von Amelie und ihrer Mutter}

Noch bis zur sechsten Erhebungswelle blieben Medien und in den Medien präsente Stars, wie etwa die mutige Hexe Phoebe aus der Sendung Charmed, als Orientierungsgeber für Amelie hoch relevant; sie dienten ihr als eine Möglichkeit, die instabilen Rahmenbedingungen ihrer Familie, ihre stets sozio-ökonomisch, aber über viele Jahre hinweg auch sozio-emotional angespannte Lage, unter denen sie oft stark litt, zu bearbeiten und auch mit Hilfe medialer Angebote Halt zu gewinnen. Amelie bevorzugte medial solche Themen, die sie aufgrund ihrer Lebenssituation beschäftigten. So interessierte sie sich für Themen, die auf Kämpfen, Starksein und Selbstständigkeit ausgerichtet waren, aber auch Brutalität enthielten (Chucky die Mörderpuppe) und die sich ums, Tough'-Sein drehten. Amelie gefielen Sendungen, in denen es darum ging, den eigenen Weg zu finden, aber auch Casting-Sendungen, die sozialen Aufstieg dokumentierten und symbolisierten. Auch das Real Life Format mieten, kaufen, wohnen gehörte zu ihren Favoriten; die Sendung hatte sie sogar dazu bewogen, über eine Maklerkarriere nachzudenken. Amelie nutzte auch entsprechende Ratge- 
berliteratur, um virtuell Wege zu mehr finanzieller Sicherheit auszuloten. In der vierten Erhebungswelle skizzierte Frau Aufbauer Amelies Fernsehkenntnisse folgendermaßen:

Oiso, die Amelie woaß des gonze Fernsehprogramm auswendig. (...) Oiso, i hob koa Tele oda so wia die Heftln hoaßn, sondern i brauch nur die Amelie frogn, Amelie, wos is heit $20.15 \mathrm{Uhr}$, konns ma eigentlich so von fuchzehn Sendern sogn 20.15, Sat 1 des, auf ORF 1 des, auf ORF 2 des, auf RTL.

Auch heute spielen Medien in Amelies Alltag noch eine wichtige Rolle, wenn sie sie auch, wie sie sagt, insgesamt kontrollierter nutze. Amelie bezeichnet ihr Smartphone als das am häufigsten genutzte Medium; sie zieht es vor allem zur Kommunikation und Information, etwa zur OnlineRecherche, für Online-Nachrichten (z.B. DerStandard.at, ZiB20-Seite auf Instagram) heran, nutze es aber auch aus Langeweile zur Unterhaltung. Im Vordergrund steht dabei WhatsApp zum Schreiben und Telefonieren; Facebook sei ihr als „Eventkalender“ wichtig, Instagram zum Durchschauen. Insgesamt habe ihre Handy-Nutzung, wie sie sagt, abgenommen. Sie schränke sie bewusst ein und bezeichnet sie „als Zeitverschwendung und Stressfaktor“. Für Snapchat interessiert sie sich „so gut wie gar nicht mehr“. Spiele auf der Xbox gehören dagegen fest zu Amelies Alltag. Dort gefällt ihr vor allem das Spiel ARK Survival Evolved, da sich der Spieler bzw. die Spielerin ,als einziger Mensch auf der Erde im Zeitalter der Dinosaurier ein Leben aufbauen" muss. Amelies Mutter ist sogar davon überzeugt, dass ihre Tochter gemeinsam mit ihrem Freund teils die Nächte durchspielt. Netflix gehört auch zu Amelies Medienrepertoire, etwa Serien wie Stranger Things und Expanse. Oft nutze sie Netflix täglich, manchmal gebe es dann aber auch wieder monatelange Pause, und oft schaue sie „stundenlang durch“, höre dann aber auch nach kurzer Zeit wieder auf, weil ihr die Angebote dann doch nicht gefallen.

Amelies Mutter nutzt heute mehr Medien als früher und diese auch öfter. Seit drei Jahren hat Frau Aufbauer ein Smartphone, danach sei ihr Mediengebrauch, wie sie im Interview erzählt, stark gestiegen, er habe sich „wahrscheinlich verdoppelt“. Das Handy sei ihr für die Kommunikation wichtig, für WhatsApp und fürs Telefonieren, aber auch zur Information, so vor allem für YouTube und Instagram, zur Wissensaneignung, Online-Recherche, Online-Zeitungen. Information sucht sie mittlerweile nur noch übers Handy. So sind ihr Instagram und YouTube z.B. für Tutorials über Pflanzen oder Brotbacken wichtig, aber sie nutzt auch verschiedene Apps zur „Schulorganisation“ ihrer jüngeren Kinder, auch das Fernsehen, 
etwa zum gemeinsamen Anschauen von Supertalent mit ihrem jüngsten Sohn, iTunes und auch DVD zur Unterhaltung. Ihren Laptop benötigt sie insbesondere für ihre Aufräum-Kolumnen. Bücher seien dagegen mittlerweile „fast ganz aussortiert worden“, erzählt Frau Aufbauer, sie habe kaum noch welche zu Hause, auch keine Print-Zeitungen. Computerspiele gehören ebenfalls zum Medienrepertoire von Frau Aufbauer, an die Namen der Spiele erinnert sie sich jedoch nicht, früher habe sie Die Sims gespielt.

\section{Fazit}

Nach sehr bewegten, oft auch unruhigen, von wechselnden Partnerschaften der Mutter und häufigen Wohnungs- und Wohnortwechseln gekennzeichneten Zeiten, hat sich die Situation von Familie Aufbauer mittlerweile stabilisiert. Die Amelie stark belastenden emotionalen Spannungen in der Familie haben sich gelöst, und die alleinerziehende Mutter von vier Kindern scheint nicht mehr mit der Alltagsbewältigung in ihrer Familie überfordert zu sein. Dazu beigetragen haben der Auszug der älteren Halbschwester von Amelie und ganz besonders ihr eigener vor der letzten Erhebung; Amelie wohnt nun in einer eigenen Wohnung in der Nähe der Mutter. Bis zum Ende der Erhebung blieben aber die Handlungsoptionen der Familie sehr angespannt. Nach ebenfalls wechselnden Partnerschaften des jungen Mädchens, vor allem mit älteren Freunden, von der eine nahe$\mathrm{zu}$ an Abhängigkeit grenzte, und einem recht abrupten Wechsel in eine andere Großstadt, wie Amelies Mutter sagte, „aus Liebe“, hat sich Amelie mittlerweile aber psychisch stabilisiert, sodass sie in der Lage war, neue, weiterführende Handlungsentwürfe zu entwickeln. Sie holt ihre Matura in einer Abendschule nach und plant danach ein Studium. Amelie, die schon als Kind sehr aufgeweckt wirkte und schon früh um Autonomie zu ringen schien - so suchte sie im Fernsehen nach starken Vorbildern und rezipierte später bevorzugt Sendungen, in denen es um persönlichen, aber auch finanziellen Aufschwung ging - macht auch heute noch den Eindruck, auf der Suche nach einem eigenen Weg zu sein, dies mittlerweile jedoch mit mehr Handlungskompetenz, auch im Umgang mit Medien. 
2.4.2 Gregor Boll (21 Jahre alt) und seine Mutter: Sozio-emotionale Verwerfungen und die Trennung der Eltern führen zu einem erfolgreichen Neustart in Deutschland

\section{5 bis 2017}

Familie Boll ist eine Großfamilie mit turbulenter Vergangenheit, die zu Beginn der Studie aus den Eltern und zehn Kindern bestand - vier Mädchen und sechs Jungen, wobei der älteste Sohn bereits ausgezogen war. Gregor ist der Drittjüngste im Bunde und war 2005 fünf Jahre alt. Anfangs wohnte die Familie in einem heruntergekommenen Bauernhaus im ländlichen Norden Österreichs. Ihr Eigenheim in einer nahegelegenen Stadt wurde kurz zuvor zwangsversteigert. Mangels direkter Nachbarn erlebten die Kinder der Familie eine gewisse Isolation und pflegten kaum soziale Kontakte außerhalb der Familie. Gregor zeigte sich in Gesprächen zunächst sehr introvertiert und scheu gegenüber Fremden. Er besuchte nicht den Kindergarten und konnte erst mit dem Eintritt in die Grundschule Peer-Group-Beziehungen aufbauen, wobei er seine Freunde fast nur in der Schule, nicht aber in seiner Freizeit sah. Gregors Eltern hatten kaum Zeit für die Betreuung ihrer Kinder und „parkten“ diese oft vor Medien, besonders dem Fernseher, oder überließen sie sich selbst. Als Hauptgrund dafür wurde die finanziell angespannte Lage der zwölfköpfigen Familie angeführt. Frau Boll war zu Beginn der Untersuchung Hausfrau und kümmerte sich um die neun Kinder. Zunächst gelang es Herrn Boll einigermaßen, die Familie über die Runden bringen. Später gab er seine bezahlte Tätigkeit als Masseur auf, um sich voll auf die Hunde- und Pferdezucht und die Bewirtschaftung des Bauernhofs zu konzentrieren. Somit entfiel eine wichtige Einnahmequelle, und Frau Boll sah sich gezwungen, neben der Erziehung der Kinder eine Erwerbstätigkeit aufzunehmen. In der zweiten Erhebungswelle arbeitete sie daher in Teilzeit als Reinigungskraft im Lokal ihres ältesten Sohnes.

Zur dritten Erhebungswelle 2010 erlebte die Familie eine Krise, in deren Folge sich die Familiensituation drastisch änderte. Frau Boll erkrankte schwer an Krebs, bekam eine Chemotherapie und musste operiert werden. Während sie gegen die Krankheit kämpfte, betrog ihr Mann sie mit der Volksschullehrerin von Gregor und drei seiner Geschwister. Frau Boll zog die Konsequenzen und lies sich nach 22 Jahren Ehe scheiden. Sie verließ das Heim der Familie und zog zunächst mit Gregor und drei seiner Geschwister in eine Mietwohnung in einer nahegelegenen Stadt, während Gregors damals 15-jährige Zwillingsbrüder beim Vater blieben. Neben einer privaten Berufsunfähigkeitsrente bezog Frau Boll Unterhalt 
für die bei ihr lebenden Kinder, und man arrangierte sich zunächst mit der neuen Situation. In dieser Phase war Gregor in eine Hauptschule gewechselt, fühlte sich dort wohl und hatte ein gutes Verhältnis mit seinen Mitschülern und Mitschülerinnen und auch seinen Lehrern und Lehrerinnen. Die Trennung seiner Eltern und die Sorgen um die Gesundheit seiner Mutter gingen jedoch nicht spurlos an ihm vorüber. Die Noten des bisherigen Einser-Schülers verschlechterten sich, und Gregor begann, wie seine Mutter erzählte, als Folge des psychischen Stresses mit dem Bettnässen. Auch Freundschaften litten unter seiner emotional angespannten Situation; erneut stellten Geschwister und Mutter seine zentralen Bezugspersonen. Auch Herr Boll bemühte sich um Kontakt, und das Verhältnis zwischen Vater und Sohn wurde von allen Beteiligten als gut bezeichnet. Dennoch gab es Konflikte über Besuchszeiten. Insgesamt fehlte es an Beständigkeit in Gregors Leben. Frau Boll kämpfte weiter gegen die Folgen ihrer Krebserkrankung an, und Herr Boll arbeitete viel auf seinem Bauernhof. Das Familienklima war durch die gesundheitlichen Probleme und die ungeklärten Verhältnisse der Eltern stark belastet.

Um sich aus dieser Situation zu befreien und um die als einengend und belastend empfundene Wohngegend zu verlassen, zog Frau Boll zwischen der dritten und der vierten Erhebungswelle einen Schlussstrich und übersiedelte mit sechs Kindern in ihre alte Heimat nach Deutschland. Als einzige Sicherheit und finanzielle Ressource blieb der Familie zunächst nur die Berufsunfähigkeitsrente von Frau Boll, da sie bereit war, auf jegliche Unterhaltszahlungen durch ihren Ex-Mann zu verzichten. Im Gegenzug machte er keine Ansprüche auf das Sorgerecht für die Kinder geltend. Als wichtige emotionale Ressource erwies sich der Zusammenhalt in der neuen Kernfamilie: Auch die inzwischen 16-jährigen Zwillinge folgten Frau Boll nach Deutschland und trugen einen wichtigen Teil zur Festigung der sozio-emotionalen Bedingungen bei. Gerade für Gregor war diese Anwesenheit seiner Brüder ein Stabilitätsanker, da er in ihnen nicht nur Vorbilder, sondern auch einen Vaterersatz sah. Nach dem Umzug und als Folge des nunmehr sehr sporadischen Kontakts verschlechterte sich Gregors Verhältnis zu seinem Vater. Der Junge beteuerte bald nachhaltig, dass er nicht mehr nach Österreich zurückkehren wolle, sondern jetzt eine neue Heimat gefunden habe. Der Schulwechsel verlief problemlos, und seine Noten stabilisierten sich. Auch das Bettnässen verschwand, und Gregor entwickelte sich zu einem fröhlichen Teenager. Im ersten Interview nach dem Umzug zeigte er sich begeistert von seiner neuen Schule und guten Lehrern und Lehrerinnen. Erstmals sprach er auch von vielen und engen Freunden, mit denen er sich außerhalb der Schule traf und bei denen er 
Vertrauen und Stabilität erlebte. Ab diesem Zeitpunkt sollten enge und intensive Peer-Group-Kontakte zu einem zentralen Charakteristikum von Gregors Leben als Jugendlicher und später junger Erwachsener werden.

Allen finanziellen Schwierigkeiten zum Trotz schien Frau Boll sehr glücklich über den Neuanfang zu sein und sich über den Kontakt zu ihren alten Jugendfreunden zu freuen. Die Familie bezog ein $110 \mathrm{~m}^{2}$ großes Haus, das sich die Mutter mit Unterstützung von Freunden für 25.000 Euro kaufen und innerhalb der folgenden Jahre auch komplett abbezahlen konnte. Um zusätzliche Einkünfte zu generieren, baute Frau Boll ihre Hundezucht wieder auf und erweiterte diese Tätigkeit nachhaltig. Es gelang der Familie, ihre sozio-ökonomische Situation zu verbessern, und ab 2014 war Frau Boll, wie sie betonte, „schuldenfrei“. Die Gewinne aus ihrer Hundezucht ermöglichten auch größere Investitionen, wie den Kauf von Heizöl oder von Reparaturen sowie regelmäßige Ausflüge und jährliche Urlaube auf den Nordseeinseln. Dennoch fühlte sich Frau Boll stets finanziell stark gefordert, da ihr daran lag, den Kindern auch Wünsche zu erfüllen. Für den Kauf eines Smartphones für Gregor, dem sehr daran lag, in seiner Peer-Gruppe nicht zurückzustehen, war es dennoch nötig, dass die ganze Familie sich daran beteiligte.

Der Kontakt zu Herrn Boll verschlechterte sich mit der Zeit weiter und brach nach einem Streit um Unterhalt und einige Gerichtsprozesse gänzlich ab. Frau Boll berichtete, dass Gregor „fassungslos" darüber war, dass der Vater nicht nur seine Frau, sondern auch seine Kinder völlig im Stich gelassen habe. Dennoch bezeichnete Gregor das Verhältnis zu seinem Vater 2014 noch als gut, ab 2016 schien es jedoch, als habe der Vater bedingt durch sein „schäbiges“ Verhalten jede Chance auf Kontakt zu seinem Sohn verwirkt.

Aufgrund guter schulischer Leistungen legten Gregors Lehrkräfte ihm den Besuch einer weiterführenden Schule nahe; ein Plan, den auch Frau Boll gerne unterstützt hätte. Im Raum standen auch die fachgebundene Hochschulreife und ein FH-Studium. Gregor konnte sich dies gut vorstellen, jedoch haderte er mit seinen Zukunftsplänen und ließ sich stark von seinen Erfahrungen materieller Nöte leiten. Für ihn dominierte immer der Wunsch, bald eigenes Geld zu verdienen, finanziell aufzusteigen und sich Statussymbole finanzieren zu können. 2017 begann er, nach wie er sagte „durchschnittlichem“ Realschulabschluss, eine Ausbildung zum Einzelhandelskaufmann in einem Baumarkt. Mit der Entscheidung zeigte er sich sehr zufrieden, und auch das Verhältnis zu seinen neuen Arbeitskollegen erwies sich als gut. Frau Boll sprach mit viel Stolz über ihren Sohn, der sich trotz vieler problematischer Ereignisse in der Vergangenheit sehr 
positiv entwickelt habe. Heute komme er mit der Trennung der Eltern klar und wisse, wo er stehe und was er wolle, meint sie.

2020: Gregor Boll - unterstützt durch seine Mutter entwickelt er sich zu einem erfolgreichen jungen Mann mit Plänen für die private und berufliche Zukunft

In der siebten Erhebungswelle 2020 hat sich im Leben von Gregor Vieles verändert, er steht sowohl im Privaten als auch im Berufsleben fest auf eigenen Beinen. Seine Ausbildung hat er erfolgreich abgeschlossen, konnte jedoch vom Betrieb nicht übernommen werden. Da der Eigentümer keinen Nachfolger finden konnte, hat er sein Unternehmen abgewickelt und seine Angestellten mit einer Abfindung ausbezahlt. Aufgrund seiner guten Verankerung in der örtlichen Gemeinschaft gelang es Gregors jedoch bald, eine Festanstellung bei einem internationalen Chemiekonzern in der nahen Umgebung zu erhalten, die ihm ein Bekannter vermittelt hatte; dort fühlt sich Gregor sehr wohl. Aktuell arbeitet er im Unternehmen als Ungelernter in der Logistik und verdient, wie er sagt, „gutes Geld“ (1.600 Euro netto). Dennoch hat Gregor angesichts der sich ihm bietenden guten Situation in der Firma neue Handlungsentwürfe entwickelt: Er möchte sich bei seinem Arbeitgeber etablieren und dort eine zweite Ausbildung absolvieren, um sowohl in der Betriebshierarchie als auch finanziell $(2.300$ Euro netto) aufzusteigen. Zum Zeitpunkt des Interviews bestand bereits die Vereinbarung mit seinem Arbeitgeber zu einem Bewerbungsgespräch, um die weitere Planung zu besprechen. Finanzielle Sicherheit und Statussymbole, auch im Bereich Medien, sind Gregor heute sehr wichtig. Obwohl er inzwischen über ein eigenes Einkommen verfügt, unterstützt Frau Boll ihren Sohn auch weiterhin bei größeren Anschaffungen.

2017 war Gregor zunächst mit einem seiner Brüder in eine WG gezogen. Inzwischen lebt er mit seiner festen Freundin in einer eigenen Wohnung ganz in der Nähe seiner Mutter. Gregor ist nicht nur mit seiner Wohnung, sondern auch mit der Region sehr zufrieden und möchte dort „niemals wegziehen“. So sieht es laut Gregor auch seine Freundin, die derzeit noch Lehramt studiert. Ihre Zukunft planen beide gemeinsam: Wir „wollen in dem Dorf bleiben und auch bauen. Ist zwar verdammt teuer, aber mein Gott." Im Vater seiner Freundin sehe Gregor, so Frau Boll, seinen „Ersatzvater“. Zu seinem leiblichen Vater hat Gregor keinen Kontakt mehr, und er erwähnt ihn auch nicht, obwohl inzwischen auch Herr Boll schwer an Krebs erkrankt ist und möglicherweise bald sterben wird. Während die Töchter, wie Frau Boll erzählt, Mitleid mit ihrem Vater zeigen, könne und wolle Gregor ihm nicht verzeihen, und er werde daher auch seiner Beerdigung in Österreich fernbleiben. Auch Frau Boll 
zeigt kein Mitleid mit ihrem Ex-Mann und sieht dessen Krebserkrankung unverhohlen als „ausgleichende Gerechtigkeit“.

Auch sie selbst hatte es in der jüngsten Vergangenheit nicht leicht und war mit mehreren Schicksalsschlägen konfrontiert. Nach dem Tod ihrer Mutter Anfang 2020 sei auch ihre beste Freundin plötzlich - wohl aufgrund eines Schlaganfalls - „aus dem Leben gerissen“ worden. Sie war Frau Bolls engste Bezugsperson und einer der Gründe für die Rückkehr in die alte Heimat. Sie hatte die Familie tatkrättig unterstützt. Wenige Zeit nach dem Tod der Freundin erlitt Frau Boll eine schwere Embolie und verbrachte zehn Tage auf der Intensivstation. Dieses Erlebnis bezeichnet sie als „Schuss vor den Bug“, es habe zu einem „Umdenken“ geführt. Sie lasse nun Vieles ruhiger angehen als früher und wisse das Hier und Jetzt viel mehr zu schätzen. Von ihren Kindern verlange sie nun viel mehr Selbstständigkeit und Unterstützung im Haushalt. Inzwischen ist Frau Boll, wie sie erzählt, mit Medikamenten gut eingestellt und hat keine Einschränkungen mehr, jedoch ermahnen ihre behandelnden Ärzte sie zu mehr Bewegung und Sport. Zur Zeit der Covid-19-Pandemie, in der sie, wie sie im Interview berichtet, weitestgehend zurückgezogen zu Hause lebe, konzentriere sie sich auf ihre sehr erfolgreiche Hundezucht. Gregor selbst bezeichnet seine Mutter aufgrund ihrer Krebserkrankung zwar als Teil der Risikogruppe, ihre erneute schwere Erkrankung, ebenso wie die seines Vaters, spricht er aber nicht an.

\section{Medien im Leben von Gregor und seiner Mutter}

In den ersten beiden Erhebungswellen verbrachten Gregor und seine Geschwister viel Zeit im Freien. Dennoch wurden sie von ihren Eltern auch oft vor dem Fernseher „geparkt“, damit die Eltern ihren beruflichen Verpflichtungen nachkommen konnten. Nach der Trennung seiner Eltern nahm die Intensität von Gregors Mediennutzung, auch altersbedingt, leicht zu. Durch den Umzug nach Deutschland gewannen gerade Soziale Medien an Bedeutung, um den Kontakt mit Freunden und den älteren Brüdern aufrecht zu halten. Diverse Kommunikationstools spielen aber auch eine wichtige Rolle in seinem aktuellen lokalen Freundeskreis. Gerade dem Smartphone kommt dabei große Bedeutung zu, auch als Symbol für Status und Teilhabe. Gregor nutzt es intensiv zur Kommunikation, zur Information und für Unterhaltungsangebote. Auch pornografische Inhalte rezipierte er, wie er freimütig im Interview erzählt, über sein Smartphone. Gelegentlichen Pornokonsum bezeichnete er als „normal“ für einen Jungen in seinem Alter. In den späteren Erhebungswellen zeichnete sich eine zunehmende Affinität zu Computerspielen ab (Call of Duty, Black Ops 
3, Far Cry 4, FIFA). Dies hängt vermutlich mit dem Einfluss der älteren Zwillingsbrüder zusammen, die sich sehr für Computerspiele interessieren und mit denen Gregor viel Zeit verbringt.

Mittlerweile hat Gregors Medienbesitz deutlich zugenommen, und seine Wohnung ist sehr gut mit entsprechender Technik ausgestattet. Neben einem Fernseher mit Zugang zu Amazon Prime, Netflix und Disney+, einem neuen Computer für Gaming, Tablet und iPhone verfügt Gregor auch in allen Räumen über Amazon Alexa. Mediengeräte sind inzwischen sehr wichtig für ihn: „Es ist jetzt nicht so, dass ich immer kucke: Was kommt jetzt für ein neuer heißer Scheiß auf den Markt? Ich hab“ ja früher immer gesagt, es ist mir scheiß egal, was ich mir hole, Hauptsache es funktioniert halbwegs, mittlerweile ist es aber schon geil, wenn man diesen Luxus an den Mediengeräten hat, ne." Gregor trifft sich normalerweise regelmäßig mit seinen Freunden, dennoch hält er mit ihnen auch über Telefon, WhatsApp und Facebook engen Kontakt. Zur Information und Unterhaltung dienen ihm auch Angebote auf YouTube, Online-Nachrichtenseiten und Livestreams auf Twitch.tv. Zur Zeit des Interviews in der ersten Phase der Covid-19-Pandemie nutzt er auch regelmäßig die App Houseparty, um trotz der Kontaktbeschränkungen mit seinen Freunden "eine gute Zeit" verbringen zu können. Gemeinsam mit seiner Freundin sind ihm auch Streamingdienste (Breaking Bad, Black List, Supernatural, Sounds of Anarchy, Krimiserien) wichtig, mit denen er sich gern und regelmäßig beschäftigt. Ihm gefielen nahezu alle Inhalte, wie er sagt, insbesondere aber Actionfilme. Gemeinsam mit seiner Freundin schaue er auch gerne Liebesfilme, was er als „schwul“ bezeichnet: „Ich kuck Action, ich kuck genauso mit meiner Freundin gerne Liebesfilme, so schwul das klingt, aber, alles (lacht), alles kuck ich." Wie in vorherigen Erhebungswellen ist weiterhin das Smartphone, inzwischen ein aktuelles iPhone, sein wichtigstes Gerät: „Ohne Handy“, so Gregor, sei man „für Kommunikation und Entertainment aufgeschmissen“.

Die Mediennutzung von Frau Boll ist seit geraumer Zeit stark vom Motiv der Informationsbeschaffung und Teilhabe, vereinzelt auch von den Motiven Zerstreuung und Information gekennzeichnet. Konkret genutzte Angebote sind dabei die lokale Tageszeitung, die sie vorwiegend für Hintergrundinformationen zum örtlichen Geschehen liest, Bücher und im Fernsehen diverse Quiz-Shows (Wer wird Millionär) und Doku-Soaps (z.B. Frauentausch), über letztere könne sie sich „totlachen“, sowie Krimis (Tatort). Darüber hinaus schaut sie gerne politische Talkshows wie Titel, Thesen, Temperamente, Anne Will, Markus Lanz, Menschen bei Maischberger, hart aber fair mit Frank Plasberg und drei nach neun im WDR. Ihren 
Laptop nutzt Frau Boll vorwiegend für diverse Online-Aktivitäten wie die Informationssuche mit Google und Wikipedia zu medizinischen Themen, für E-Mails und das Versenden von Hundebildern an Interessenten.

2020 verweist Frau Boll stärker als früher auf den hohen Stellenwert von Medien. Gerade WhatsApp ist ihr wichtig geworden, um in der Covid-19Pandemie Kontakt mit den Familienmitgliedern zu halten. Dabei denkt sie besonders an ihre Söhne in Österreich, ihre Tochter in der Schweiz und ihre beiden Enkelkinder, die 2019 auf die Welt gekommen sind, und deren Aufwachsen sie auf diese Weise miterleben könne. Gesteigerten Wert legt Frau Boll inzwischen auch auf die Nutzung von verschiedenen Online-Zeitungen, um über den Verlauf der Pandemie in Deutschland und Österreich auf dem Laufenden zu bleiben. Obwohl sie sich lange dagegen verwehrt hatte, verfügt sie inzwischen über zwei Handys: Ein privates Smartphone und ein zusätzliches geschäftliches Gerät, um dem deutlich angestiegenen Interesse an Hunden aus ihrer Zucht begegnen zu können.

\section{Fazit}

Nach der Trennung seiner Eltern, die ihn stark erschüttert hatte, und dem damit verbundenen Umzug nach Deutschland, war Gregor mit einer völlig neuen Lebenssituation konfrontiert. Er sah sich, wie auch seine Mutter, herausgefordert, neue Pläne und Ziele zu entwickeln und seine Handlungsentwürfe ganz neu auszurichten. Durch den Zusammenhalt in der Kernfamilie und durch die Unterstützung von Freunden gelang es Familie Boll, nicht nur neue Pläne zu entwickeln, sondern diese auch privat und beruflich umzusetzen und im Falle von Gregor nach dem erfolgreichen Abschluss der Schule und einer Ausbildung neue Handlungskompetenzen zu erwerben. Gregor hat sich gemeinsam mit seiner Freundin etabliert und plant mit ihr bereits den Bau eines eigenen Hauses. Auch beruflich verfolgt er weitreichende Ziele; er möchte eine weitere Ausbildung absolvieren und sich so die nötigen Kompetenzen für einen Aufstieg in der Unternehmenshierarchie seines Arbeitgebers zu sichern. Gregor und seiner Mutter ist es gelungen, sich einen bescheidenen Wohlstand zu erarbeiten; beide blicken infolge der nunmehr etwas verbesserten Handlungsoptionen zum Ende der Studie optimistisch in die Zukunft. Medien spielten für Gregor immer eine wichtige Rolle, sowohl zur Unterhaltung und Information als auch zur Kommunikation und als Mittel zur Teilhabe in der PeerGroup - daran hat sich auch im jungen Erwachsenenalter nichts geändert. Inzwischen praktiziert er allerdings einen reflektierten Mediengebrauch, und die gemeinsame Mediennutzung ist auch Teil seiner Beziehung und 
im Rahmen von Freizeitaktivitäten mit seinen Brüdern und Freunden. Mehr denn je betrachtet Gregor inzwischen hochpreisige Mediengeräte auch als Statussymbole; er verfügt inzwischen über ein eigenes Einkommen und ihm liegt daran, auch größere Summen in seine Medienausstattung zu investieren.

2.4.3 Elisabeth Ebner (19 Jahre alt) und ihre Eltern: Eine trotz erheblich eingeschränkter Handlungsoptionen emotional stabile, den Alltag recht kompetent bewältigende Familie

2005 bis 2017

Elisabeth Ebner lebte zusammen mit ihrem zwei Jahre älteren Bruder seit Beginn der Studie 2005 in einer Kernfamilie; 2013 heirateten ihre Eltern. Herr und Frau Ebner hatten aber schon vor der Geburt ihrer Kinder zusammengelebt. Die Familie litt unter stark eingeschränkten sozio-ökonomischen Bedingungen, die sie über nahezu alle Erhebungswellen hinweg belasteten. Lediglich in der vierten Erhebungswelle lag das Einkommen der Familie knapp über der Armutsgefährdung. Herr Ebner, gelernter Landwirt, bewirtschaftete seit Beginn der Studie einen kleinen Hof. Elisabeths Mutter hatte zwar eine Lehre als Köchin absolviert, war jedoch neben ihrer Mitarbeit auf dem Hof von der ersten bis zur vierten Erhebungswelle geringfügig als Reinigungskraft angestellt. Ab der fünften Erhebungswelle widmete sie sich ausschließlich der Mitarbeit in der Landwirtschaft. Elisabeth war die schwierige sozio-ökonomische Lage ihrer Familie schon früh bewusst. So erzählte sie einmal, dass sie kein Taschengeld bekomme: „I griag's hoid vielleicht erst später, wenn i nochand von da Schui draußen bin. Owa wahrscheinlich brauch i eh kan's, wenn i nochand eh de Lehr moch, griag i eh mei Göd söwa."

Familie Ebner wohnte in der ersten Erhebungswelle in einem stark renovierungsbedürttigen Bauernhaus ohne Heizung auf dem Land, das einen ungepflegten, wenn nicht schmutzigen Eindruck machte und von Schimmel befallen war. Der Schimmelbefall hatte bei Elisabeths Bruder Asthma ausgelöst, sodass die Wohnsituation für die Familie nicht länger tragbar war. Ab der zweiten Erhebungswelle bewohnte die Familie ein neues Wohnhaus direkt neben dem alten - eine positive Veränderung im Leben der Familie. Obwohl der Bauernhof abgelegen auf dem Land lag, fühlte sich Familie Ebner dort sehr wohl. Im Gegensatz zur belasteten sozio-ökonomischen Situation erwiesen sich, von Beginn der Studie an bis zum Ende, die Familienstrukturen als sehr gefestigt und das Familien- 
klima als sehr gut. Die Eltern führten eine stabile Beziehung und später Ehe. Elisabeth hatte über alle Erhebungswellen hinweg ein sehr gutes Verhältnis zu ihrem Vater, und auch das Verhältnis zu ihrer Mutter blieb stets sehr herzlich und harmonisch. Elisabeth bezeichnete sie als ihre wichtigste Bezugsperson und auch als ihr Vorbild. Auch zwischen Elisabeth und ihrem Bruder gab es kaum Konflikte. Obwohl die Eltern sehr viel arbeiteten und die Familie deshalb nur selten etwas gemeinsam unternehmen konnte, lag den Mitgliedern dennoch daran, so viel Zeit wie eben möglich miteinander zu verbringen. Da dennoch ihre Eltern wenig Zeit für die Kinder hatten, wurde für Elisabeth auch ihre Tante zu einer wichtigen Begleiterin im Alltag. Mit ihr verbrachte Elisabeth viel Zeit.

Familie Ebner gelang es trotz ihrer erheblich eingeschränkten Handlungsoptionen über die gesamte Erhebungszeit hinweg, ihre Handlungsentwürfe jeweils mit Blick auf die gesamte Familie hin auszurichten; ihr Familienklima erwies sich als stabil und intakt - eine wichtige Basis für Elisabeth und ihren Bruder, ihre Entwicklungsaufgaben gut zu bewältigen und Handlungskompetenz zu erwerben. Die Erziehung in Familie Ebner lag über die Jahre hauptsächlich bei Elisabeths Mutter; sie ließ ihren Kindern viele Freiheiten, setzte aber stets auch klare Regeln. Herrn und vor allem Frau Ebner lag daran, ihren Kindern im eigenen Tun möglichst Vorbild zu sein und mit ihren Kindern offen zu kommunizieren.

Elisabeth hatte zudem feste Bindungen in ihrem Freundeskreis aufgebaut und in der fünften Erhebungswelle einen Ausbildungsplatz in ihrem Wunschberuf als Floristin bekommen, der ihr viel Freude bereitete.

2020: Elisabeth - eine junge Floristin mit klaren Handlungsentwürfen und ausgeglichenen sozio-emotionalen Beziehungen zu ibren Eltern und ibrem Freund

Elisabeth Ebner, die zum Zeitpunkt der siebten Erhebungswelle 19 Jahre alt ist, wohnt zwar noch bei ihren Eltern, sie verbringt aber die meiste Zeit des Tages nach der Arbeit und auch die Nächte bei ihrem Freund, einem 25 Jahre alten Forstfacharbeiter, mit dem sie bereits dreieinhalb Jahre zusammen ist und der eine eigene Garconniere in der Nähe seiner Eltern bewohnt. Elisabeth betont im Interview 2020, „total glücklich und verliebt“" zu sein und eine gemeinsame Zukunft mit ihm zu planen. Auch Elisabeths Eltern schätzen ihren Freund, weil er so denke wie sie. Geplant ist, dass Elisabeth eine eigene Garconniere in der Garage in der Nähe des Elternhauses bekommt, in die sie dann mit ihrem Freund einzieht. Zur Zeit der siebten Erhebung wohnen noch ihr Bruder und dessen Familie dort. 
Elisabeth kommt, wie sie erzählt, auch mit ihren Arbeitskolleginnen und -kollegen gut zurecht. Mit einer Kollegin treffe sie sich auch in der Freizeit gemeinsam mit ihrem Freund zu „Pärchen-Aktivitäten“. Ihre beste Freundin kennt sie aus der Berufsschule, viele neue und ältere Freunde habe sie über ihren Freund kennengelernt. Mit ihren Eltern spricht Elisabeth auch in der siebten Erhebungsphase nach wie vor viel, so fahre sie vor der Arbeit bei ihnen vorbei, weil sie dort noch alle ihre Sachen habe. Den Feierabend verbringt sie bei ihrem Freund meist sehr ruhig, mit Fernsehen, mit dem Hund spazieren gehen oder mit Gesprächen mit seiner Familie. Nach wie vor pflegt Elisabeth eine gute Beziehung zu ihren Eltern. Die Lebensführung ihrer Eltern hat in den vergangenen Jahren kaum geändert; die Familie steht bei ihnen trotz eingeschränkter sozio-ökonomischer Bedingungen nach wie vor im Mittelpunkt.

\section{Medien im Leben von Elisabeth und ihren Eltern}

Elisabeths Mediennutzung diente mit zunehmendem Alter des Mädchens vor allem der Kontaktpflege. Ihre frühere Vorliebe für Fernsehen und Computerspiele nahm später $\mathrm{ab}$ - in der zweiten Erhebungswelle hatte sie Spiele auf Online-Spieleportalen wie beispielsweise Spieleaffe präferiert, später dagegen machten ihr auch komplexere Spiele wie Minecraft und Spiele auf ihrem Smartphone, wie Candy Crush oder Clash of Clans Spaß. An „Schießer-Spielen“, die der Bruder bevorzugte, habe sie aber nie Interesse gehabt, erzählte Elisabeth; auf der Wii spielte sie lediglich Spiele ohne gewalthaltige Inhalte wie Super Mario Galaxy. Zum Ende der sechsten Erhebungswelle interessierte sie sich für Sendungen wie Shopping Queen oder How I Met Your Mother, außerdem für Animes, Liebesfilme und „traurige Filme“. Ihre TV-Lieblingssender waren Nickelodeon, Super RTL, Lieblingssendungen Hanna Montana, Big Time Rush, iCarly, Hotel Zack und Cody. Gern schaute sie auch Zeichentrickfilme und Mangas.

Später zählten Mangas nicht mehr zu ihrem favorisierten Angebot, da ihr Freund kein Interesse an ihnen zeigte. Alleine würde sie sie noch anschauen, da sie aber nur mit ihrem Freund fernsehe, spielten diese Sendungen keine Rolle mehr für sie. Elisabeths Mediennutzung dreht sich in der siebten Erhebungswelle um ihr Handy, aber auch ums Fernsehen; besonders gern nutzt sie die Streaming-Sender TLC, der insbesondere amerikanische Serien und Filme bereithält, und Comedy Central. Gern verfolgt sie auch die Reality-Soap-Opera Berlin Tag und Nacht auf RTL II sowie American Dad und Family Guy auf YouTube, aber auch Crime-Serien, Tier- und Naturdokumentationen. Elisabeth schätzt ihre Mediennutzung „mittel bis hoch“ ein; Medien erleichtern die Kommunikation, ist sie 
überzeugt, aber sie könnte sich ein Leben auch ohne Medien vorstellen. Elisabeth befürchtet, „dass alle immer abhängiger davon werden und sie eben auch, was sie nicht möchte. " $\mathrm{Zu}$ ihren großen favorisierten medialen Aktivitäten zählt auch - dies betont ihre Mutter im Interview des Öfteren kritisch - häufiges und intensives Online-Shopping.

Für ihr Hobby Fotografieren hat sich Elisabeth eine Spiegelreflexkamera und das Huawei P30 Pro Smartphone gekauft, um noch bessere Fotos machen zu können. Zuweilen lade sie ihre Bilder auch bei Instagram hoch, erzählt sie - sowohl auf ihren privaten Account für Fotografie als auch auf den Firmen-Account des Blumenladens. Aber ihre Bilder mache sie aber vor allem für sich selbst.

Elisabeths Eltern nutzen vor allem das Smartphone und das Fernsehen. Insbesondere Elisabeths Mutter ist ihr Smartphone sehr wichtig, ein Leben ohne kann sie sich eigentlich nicht mehr vorstellen, weder privat noch beruflich. Sie ist auch begeistert von den Nutzungsmöglichkeiten des Internets, vor allem schätzt sie die Suchmöglichkeiten auf Google und Google Maps, aber sie nutzt auch YouTube und willhaben.at, das österreichische Äquivalent zu eBay Kleinanzeigen. Gefahren sieht Frau Ebner vor allem im Online-Shopping, sie befürchtet, dass dies zu Geschäftesterben im Dorf führt. Ebenso problematisch schätzt sie es ein, wenn man Krankheitssymptome google, „weil man sich dadurch nur noch mehr Sorgen macht“. Radio und Fernsehen nutzt Elisabeths Mutter auch, das Radio allerdings über ihr Smartphone, Fernsehen werde eher selten am Abend genutzt, sie selbst, erzählt Frau Ebner, schlafe dabei oft schnell ein. WhatsApp spielt zum Austausch mit der Familie und der Kirchenchor-Gruppe eine Rolle. Über Medien und mit ihnen einhergehende Risiken kommunizieren die Eltern mit ihren Kindern nun nur noch sehr selten, etwa wenn sie selbst Hilfe von den Kindern brauchen. So hatte Frau Ebner einmal eine SpamSMS bekommen und sich Rat bei ihrem Sohn geholt.

\section{Fazit}

Familie Ebners finanzielle Situation blieb über nahezu alle sieben Erhebungswellen konstant schwierig; auch zum Ende der Studie ist die Familie noch als armutsgefährdet anzusehen. Trotz der belasteten Handlungsoptionen in ihrer Familie war Elisabeth schon als Kind in der Lage, eigene Handlungsentwürfe zu entwickeln; auch als junge Frau verfügt sie über gute Handlungskompetenzen, ihr Leben eigenständig zu gestalten. Auch mit Medien geht Elisabeth insgesamt recht kompetent um, ihr gelang es zumeist recht gut, sie in ihr Leben zu integrieren, ohne sich von ihnen bestimmen zu lassen. 
Mittlerweile hat Elisabeth ihre Ausbildung in ihrem Wunschberuf als Floristin abgeschlossen und auch schon erfolgreich an Wettbewerben teilgenommen. Ihre Handlungsentwürfe richten sich nun auf den Meisterabschluss bei einem renommierten Floristen, den sie gern im Januar 2021 abschließen möchte. Zwar koste diese Ausbildung ca. 15.000 Euro, nach langen Überlegungen habe sie sich aber, wie sie erzählte, dafür entschieden, denn, so Elisabeth, „an der Bildung sollte man nicht sparen“. Mit ihrem Freund hat sie klare Zukunftspläne; in ihrer Familie fühlt sie sich nach wie vor sehr wohl. Elisabeths Leben scheint in für sie sehr zufriedenstellenden Bahnen zu verlaufen.

\subsubsection{Torsten Kaiser (20 Jahre alt) und seine Mutter: Sozio-emotionale Stabilität in der Familie kann zum Teil herausfordernde sozio- ökonomische Bedingungen kompensieren}

\section{5 bis 2017}

Familie Kaiser bestand zunächst aus Herrn und Frau Kaiser sowie den drei Söhnen Torsten, Marius und Jakob, die zu Beginn der Studie fünf, drei und ein Jahr alt waren. Herr Kaiser war Beamter und arbeitete zusätzlich als Tanzlehrer, während der zweiten Erhebungswelle absolvierte er die Abendmatura. Frau Kaiser verfügt über einen Realschulabschluss und eine abgeschlossene Lehre zur Amtshelferin, befand sich in der ersten Erhebungswelle aber nach der Geburt ihrer Söhne noch in Karenz. Von der zweiten bis zur dritten Erhebungswelle arbeitete sie in Teilzeit (15h) als Verkäuferin. Nach einer Fortbildung konnte sie in der vierten Erhebungswelle ihre Stundenzahl auf $20 \mathrm{~h}$ erhöhen und war seitdem als medizinische Fachangestellte in einem Krankenhaus tätig. Das Einkommen der Familie lag jedoch während der gesamten Laufzeit der Studie unter dem Äquivalenzeinkommen von $60 \%$ des durchschnittlichen Einkommens der österreichischen Bevölkerung; sie galt daher als armutsgefährdet. Zwischen der zweiten und dritten Erhebungswelle ließen sich Torstens Eltern scheiden, und der Vater verließ die gemeinsame Wohnung. Nach der Trennung von ihrem Ex-Mann ging Frau Kaiser, deren psychische Probleme sich nach der Trennung verstärkten, keine neue Beziehung mehr ein und sie beteuerte, dass „ihre Jungs“ als Familie ausreichten. Die Familie wohnte seit jeher in einer $90 \mathrm{~m}^{2}$ großen Eigentumswohnung in einer guten und urban erscheinenden Wohngegend, deren Erwerb während der ersten und zweiten Erhebungswelle durch Wohnbauförderung ermöglicht wurde. Die Wohnung befand sich stets in einem sehr sauberen und ordentlichen 
Zustand und war liebevoll eingerichtet. Zwar war die Familie nach der Scheidung aufgrund des geringen Einkommens von Frau Kaiser weiterhin armutsgefährdet, durch die abbezahlte Eigentumswohnung bestand jedoch kein belastender Mangel an finanziellen Ressourcen. So konnte sich die Familie auch zusätzliche Ausgaben leisten, wie etwa ein Patentier auf einem Gnadenhof. Auch für gemeinsame Aktivitäten und, ab der vierten Erhebungswelle 2012, „Kuschelfernsehabende“ fand die Familie Zeit, wenn auch nur am Wochenende und nach der Scheidung der Eltern seltener als zuvor. Oft nahm Torsten an diesen Unternehmungen nicht teil. Er erzählte, dass es nur mit dem Vater „richtige Unternehmungen“ gebe, der auch immer etwas geplant habe (Spielhalle, Museum, Baden gehen). Die Unternehmungen mit seiner Mutter seien dagegen weniger spannend, man fahre dann manchmal zu den Großeltern oder verbringe auch einen schönen Tag zu Hause.

Seit der dritten Erhebungswelle verfügte Torsten über ein eigenes Zimmer, das er nach seinen Wünschen selbst einrichten durfte. Es diente ihm als Rückzugsort, wenn er seine Ruhe haben wollte. Denn das Verhältnis zwischen den drei Brüdern war laut Frau Kaiser zwar recht gut, die Kinder verbrachten gemeinsam viel Zeit und standen füreinander ein, dennoch gab es auch Rivalitäten und Raufereien. Obwohl Torsten der Älteste der drei Brüder ist, musste er sich dennoch immer wieder gegenüber seinen Brüdern behaupten, wobei gerade Marius die Rolle des „Anführers“ stets einforderte und zum Teil auch übernahm. Auch in der sechsten Erhebungswelle, mit inzwischen 16, 14 und zwölf Jahren, trugen die drei Konflikte zum Teil handgreiflich aus. Ab der vierten Erhebungswelle gibt es auch eine kleine Halbschwester aus der zweiten Ehe von Herrn Kaiser, die, so Frau Kaiser, ein recht enges Verhältnis zu ihren drei Halbbrüdern entwickelte.

Sowohl Torsten als auch seine Mutter zeigten sich immer sehr zufrieden mit der Wohnung sowie mit der Wohngegend und der vorhandenen Infrastruktur. Torsten wollte, wie er betonte, aus der Gegend daher nie wegziehen und hatte deshalb eine interessante Lehrstelle auf dem Land abgelehnt. Dennoch war die Wohngegend problembehaftet, und die Situation eskalierte in der fünften Erhebungswelle. Ein Nachbar stalkte Frau Kaiser und belastete ihre Psyche damit schwer. Über ein Gewaltschutzzentrum bekam sie Hilfe, der Nachbar darf sie seither nicht mehr kontaktieren. Neben diesem Stalking-Vorfall belasteten auch andere Faktoren die sozio-emotionalen Bedingungen der Familie schwer. Bereits früher gab es Konflikte mit Nachbarn, und Frau Kaiser befand sich bereits vor der Scheidung wegen psychischer Probleme in Behandlung, die sich durch die 
Trennung noch verstärkten. 2016 war Frau Kaiser über drei Monate im Krankenstand und absolvierte einen stationären Krankenhausaufenthalt. Einen weiteren Klinikaufenthalt wollte sie in Zukunft aber unbedingt vermeiden, da ihr Ex-Mann, so Frau Kaiser, ihre Abwesenheit zu seinen Gunsten ausnützen würde. Insgesamt war das Verhältnis zwischen den geschiedenen Elternteilen dauerhaft belastet und verschlechterte sich mit der Zeit zunehmend. Frau Kaiser berichtete, dass es immer mehr Spannungen gäbe und der Vater versuchte, sie aus dem Leben der Kinder zu drängen. Sie kritisierte zudem auch, dass Herr Kaiser den Kindern zu große Freiheiten einräumte, etwa durch den Zugang zu gewalthaltigen und nicht altersgemäßen Videospielen. Auch unterschiedliche Ansichten über Torstens Schulausbildung sorgten immer wieder für Konflikte. Die Scheidung der Eltern belastete Torsten sehr, aber sein Vater blieb präsent und übernahm auch eine wichtige Rolle als Vorbild. Torsten war es dabei gerade im Jugendalter sehr wichtig, dass sein Vater stolz auf ihn und seine Brüder war. Persönlichen Differenzen zum Trotz gelang es Herrn und Frau Kaiser zumeist, gemeinsam für die Kinder als Eltern zur Verfügung zu stehen. Dazu zählte es auch, dass die Kinder regelmäßig alle 14 Tage Zeit mit ihrem Vater verbrachten und in diesem Rahmen auch besondere Unternehmungen stattfanden. Noch in der sechsten Erhebungswelle betonte Torsten, dass er mit seinen Eltern zwar nicht über alles reden könne, seine Mutter und sein Vater aber seine wichtigsten Bezugspersonen seien.

Eine wichtige Bezugsperson war für Torsten ab der dritten Erhebungswelle sein persönlicher Mentor, Pfarrer Peter. Diesen lernte er im Rahmen eines Programms der Kinder- und Jugendanwaltschaft kennen und bezeichnete ihn auch in der sechsten Erhebungswelle noch als seinen besten Freund. Ab der fünften Erhebungswelle betreute der Pfarrer auch Marius und Jakob. Ältere Freunde spielten für Torsten immer eine wichtige Rolle, wobei er selbst in der fünften Erhebungswelle angab, dass seine Freunde meist über 45 Jahre alt seien und er sich mit ihnen besser als mit Gleichaltrigen verstehe. Dies bestätigte auch Frau Kaiser, die ihren Sohn als „Einzelgänger" bezeichnete. Vor diesem Hintergrund ist es überraschend, dass Torsten bereits im Alter von zehn Jahren von einer Freundin sprach und mit zwölf Jahren von einer "Geliebten“, die ihn jedoch „nicht wollte“. In der fünften Erhebungswelle berichtete er davon, für zwei Monate eine Freundin gehabt zu haben, die in einer betreuten Wohneinrichtung wohnte und deshalb nur selten Zeit für ihn hatte. Zudem wollte er sich damals nicht zu viel mit Pornografie beschäftigen, da es auch „reale Mädchen“ gäbe. In der sechsten Erhebungswelle bestätigte er aber, dass es niemanden mehr in seinem Leben gäbe. Er bezeichnete sich selbst als „nicht so ein 
Typen für One-Night-Stands“, sondern eher für langfristige Beziehungen. Diese Aussagen veranschaulichen, wie die Themenkreise Liebe und Sexualität in der Pubertät zunehmend an Bedeutung für Torsten gewannen. Sein Interesse an Sexualität zeigte sich dabei auch in seiner Mediennutzung, denn tatsächlich berichtete Torsten immer wieder auch von der Rezeption pornografischer Inhalte im Internet.

Torstens schulische Bildungsbiografie war wenig geradlinig. Er zählte immer zu den jüngsten Kindern in der Klasse und besuchte nach der Volksschule zunächst eine Mittelschule. 2014 brach er jedoch die Schule ab, besuchte in der sechsten Erhebungswelle einen Lehrvorbereitungskurs und schrieb Bewerbungen. Als Grund für den Abbruch nannte Frau Kaiser Torstens schlechten Leistungen, die vielen Fehltage durch Schnupperpraktika, Probearbeiten und Lehrlingstests und seine fehlende Motivation zu lernen. Der vorläufige Abbruch der Schule sorgte auch für erhebliche Unstimmigkeiten in der Familie, da Frau Kaiser sich wünschte, dass ihr Sohn die Schulbildung fortführen möge, während der Vater gegen die Matura war und Torsten zu einer Ausbildung riet. Torsten selbst hatte ein ambivalentes Verhältnis zur Schule. Einerseits fand er sie langweilig, andererseits störte er sich aber auch an den steigenden Anforderungen. Nach dem Ende der Pflichtschulzeit war er zunächst froh über die viele Freizeit. Torstens Orientierungslosigkeit war auch ein Beleg dafür, dass ihm entsprechende Handlungsentwürfe für seine eigene Zukunft fehlten. Die Uneinigkeit der Eltern trug ihren Teil zu seiner Unsicherheit bei. Dies spiegelte sich auch in den sehr unterschiedlichen Berufswünschen wieder, die Torsten nannte: Politiker, Kindergärtner und Jurist. Zudem hat er auch im Rahmen eines FSJ Erfahrungen im Bereich Altenpflege gesammelt. Welche Schulabschlüsse und Ausbildungen für seine Wünsche jeweils nötig wären, ordnete Torsten jedoch nie entsprechend ein.

2020: Torsten Kaiser - ein HAK-Absolvent bleibt zunächst beruflich orientierungslos

Frau Kaiser hat sich letztlich mit ihrem Wunsch durchgesetzt: Torsten hat 2020, nach „schwerem Zittern und Dahintun“, die Handelsakademie ohne Matura abgeschlossen und verfügt nun über eine Berufsausbildung als Bürokaufmann. Wie auch schon in den Jahren zuvor ist es Torstens Wunsch, nun ins Berufsleben zu starten und einen Job zu finden, der ihm Spaß machen und "gutes Geld“ bringen möge. In welche Richtung es dabei gehen soll, weiß er aber noch nicht. Obwohl er ausgebildeter Bürokaufmann ist, kann er sich eher eine Tätigkeit in einem Altersheim vorstellen, wie im FSJ (Freiwilliges Soziales Jahr). Alternativ schwebt ihm vor, eine „einjähri- 
ge Zusatzausbildung als Pflegeassistenz" zu machen, um später beruflich Kaufmännisches und Pflege kombinieren zu können. Während andere Jungen im Panel die Weichen für ihre berufliche Zukunft schon gestellt haben oder sogar bereits ins Berufsleben gestartet sind, setzt sich Torstens Orientierungslosigkeit zunächst fort. Dennoch zeigt er sich aktuell erleichtert über seinen Schulabschluss und blickt optimistisch in die Zukunft. Eine Beziehung führt Torsten aktuell nicht und strebt dies auch nicht an, da er einer Freundin nicht die nötige „Aufmerksamkeit schenken“ könne. Dies führt er einerseits auf das viele Lernen für die Schule zurück und andererseits würden der „Führerschein und die Arbeitssuche“ jetzt so viel Zeit beanspruchen, dass dies „weder für mich noch für meine Freundin von Vorteil“" wäre.

Frau Kaiser hat ihre psychischen Probleme überwunden, und ihr ist es jetzt das Wichtigste, dass sich die Söhne positiv entwickeln. Neben Torstens Schulabschluss berichtet sie hocherfreut auch von den Entwicklungen bei ihren beiden jüngeren Söhnen: Jakob (16) besucht weiterhin das Gymnasium, ist aber von einer naturwissenschaftlichen Schule auf eine musisch-künstlerisch ausgerichtete gewechselt. Marius (18) hat eine Ausbildung bei einem befreundeten Fotografen in einer nahegelegenen Kleinstadt aufgenommen und wohnt dort auch unter der Woche. Aus Interesse an der Materie hat auch Frau Kaiser eine Nebentätigkeit im Büro von dessen Atelier aufgenommen und arbeitet dort nun zusätzlich an zwei Nachmittagen in der Woche. Da sie weiterhin $20 \mathrm{~h}$ in der Woche im Krankenhaus arbeitet, wirkt sich dies dennoch erleichternd auf die angespannte sozio-ökonomische Situation der Familie aus. Darüber hinaus will Frau Kaiser keine großen Pläne machen, denn „was beruflich kommt, das könne man eh nicht wissen“. Stattdessen lebe sie „im Hier und Jetzt“, wolle aber, wenn die Kinder aus dem Gröbsten heraus sind, wieder etwas mehr Zeit für sich selbst haben.

Im Gegensatz zu früher thematisieren weder Frau Kaiser noch Torsten Streitigkeiten und Konflikte zwischen den Geschwistern. Auch die zuletzt immer angespanntere Situation zwischen den geschiedenen Eltern findet keine Erwähnung mehr. Das Verhältnis zwischen Frau Kaiser und ihren inzwischen jugendlichen und erwachsenen Söhnen scheint dagegen weiterhin sehr eng und vertraut zu sein. Torsten plant, auch im Falle des Berufseinstiegs, zunächst weiterhin zu Hause zu wohnen. Zudem hat die Familie sich einen Hund gekauft, der von allen vier Familienmitgliedern gleichermaßen und zuverlässig versorgt wird. Auch das über viele Jahre gepflegte Projekt Gnadenhof setzt die Familie fort. Nachdem das alte Pa- 
tentier vor kurzer Zeit gestorben war, wurde erneut gemeinschaftlich eine Patenschaft übernommen.

\section{Medien im Leben von Torsten und seiner Mutter}

Die Familie war seit Beginn der Erhebung gut mit Medien ausgestattet. Bereits ab der ersten Erhebungswelle gab es einen Computer mit Internetzugang, dieser war jedoch durch ein Passwort geschützt, und das Internet stand bis zur vierten Erhebungswelle nur sehr eingeschränkt und kontrolliert zur Verfügung. Mit der Zeit wurde der Bestand an Geräten immer weiter aufgestockt, neben Fernsehern, Smartphones, Computern und Laptops auch um eine PlayStation 4 und eine Wii. Auch Torsten selbst besaß stets viele Mediengeräte. Neben einem eigenen Fernseher mit Amazon Fire TV-Stick, Gaming-Laptop und Smartphone hatte er auch einen Nintendo 3DS und eine PSP sowie zahlreiche Spiele, unter anderem DayZ, Star Wars: The Clone Wars, World of Warcraft und viele Pokémon-Spiele.

Bereits in der dritten und vierten Erhebungswelle erzählte Torstens Mutter, dass Medien für ihren Sohn eine enorm hohe Bedeutung hätten und er noch viel mehr beziehungsweise den ganzen Tag vor dem Fernseher oder mit Videospielen verbringen würde, wenn er denn nur dürfte. Dieser Stellenwert ist in der fünften und sechsten Erhebungswelle nochmals gewachsen, doch im Jugendalter hinderte ihn Frau Kaiser offenbar nicht mehr daran, „die ganze Freizeit am Computer“ zu verbringen. Zu Familienaktivitäten kam Torsten in dieser Zeit demnach nur noch, wenn er seinen Laptop mitbringen durfte. Auf Unternehmungen außer Haus verzichtete er zum Teil ganz, um zu Hause spielen zu können. Diesen zum Teil drastischen Schilderungen steht jedoch entgegen, dass Torsten, wie er selbst in allen Jahren berichtete, auch immer Freizeitaktivitäten draußen praktiziert hat und auch an Unternehmungen teilnahm. Sein Medienrepertoire war inhaltlich breit und reichte von Gewalt und Krieg, über Wissenschaft bis Sexualität und Fantasy. Ihn begeisterten Computerspiele mit einer Altersfreigabe ab 16 oder 18 Jahren, wie Call of Duty, Assassin's Creed oder World of Warcraft, zu denen sein Vater ihm auch Zugang verschaffte. Bereits ab der fünften Erhebungswelle wandte sich Torsten ganz bewusst ausgewählten YouTubern wie Gronkb oder Key-Sar zu. Insgesamt gewann YouTube für ihn stark an Bedeutung, und auch unterwegs schaute er auf seinem Smartphone viele Videos an und kommentierte diese teilweise. Andere Soziale Medien-Plattformen spielten dagegen nur eine untergeordnete Rolle: Bis zur sechsten Erhebungswelle war Torsten nur auf Facebook angemeldet, legte dort sehr großen Wert auf strikte Einstellungen zur Privatsphäre und hatte zudem nur sehr wenige und ausgewählte Freunde: „Ich bin jetzt 
nicht so einer, der jetzt quasi wie wild Freunde sammelt." Schon in der fünften Erhebungswelle beschäftigte sich Torsten ausgiebig mit Themen wie Datenschutz, Privatsphäre und der NSA. Auch 2020 setzen sich diese Entwicklungen fort: Neben Facebook hat Torsten inzwischen auch einen Account bei Instagram, beide Angebote nutze er jedoch, wie er sagt, de facto nicht. Für seine Freunde, die Soziale Medien intensiv verwenden, sei dies in Ordnung, da sie ihn auf WhatsApp immer erreichen könnten. Torsten selbst bevorzugt zur Kommunikation sein Smartphone und die Angebote WhatsApp und Facetime sowie Telefonate. Auch für Computerspiele nutzt Torsten inzwischen fast ausschließlich sein Smartphone. Dies begründet er damit, dass er so auch mal schnell zwischendrin spielen könne, etwa „im Bus kleine Kartenspiele“ oder wenn er nur „kurz Zeit“" habe. Für andere Spiele, auch Rollenspiele und MMORPGs, nimmt er sich dagegen auch zu Hause länger Zeit, um diese am Smartphone zu spielen. Auch seiner Vorliebe für YouTube ist Torsten treu geblieben und nutzt die Video-Plattform nun intensiv auch zur Information. Insbesondere folgt er Kanälen, die sich auch mit politischen Themen befassen, wie Mister Wissen 2 Go und jungenaiv, darin unterscheidet sich Torsten deutlich von den meisten anderen jungen Erwachsenen im Panel. Neben YouTube nutzt Torsten zum Streaming inzwischen auch intensiv Netflix und verbringt damit viel Zeit. Lineares Fernsehen konsumiere er, wie er sagt, nur mehr in Ausnahmefällen, etwa wenn ein „guter Film“ wie Fluch der Karibik komme. Aufgrund seiner Kompetenzen im Umgang mit Computer und Internet gelang es Torsten in den letzten Monaten seiner Schulzeit zudem gut, den Umstieg auf die Distanz-Lehre zu meistern. Im Gegenzug beklagte er sich über die mangelnden Kompetenzen unter den Lehrkräften.

Frau Kaiser selbst hat grundsätzlich eine positive Einstellung gegenüber Medien und findet insbesondere das Internet sehr nützlich. Daher hat sie ihren Söhnen auch immer den Zugang ermöglicht, wenn auch in jüngeren Jahren nur stark reglementiert. Insbesondere der exzessiven Mediennutzung, wie Torsten sie einige Jahre betrieben habe, stehe sie, wie sie betont, kritisch gegenüber, verhindert habe sie sie jedoch nicht. Gerade soziale Netzwerkplattformen betrachtet sie skeptisch, da sie selbst in der füntten Erhebungswelle bereits negative Erfahrungen mit Stalking bei Facebook gemacht hatte, dennoch löschte sie ihren Account nicht dauerhaft und blieb der Seite treu. Dies hatte jedoch auch andere Gründe: Seit Torsten bei Facebook angemeldet war, war sie dort mit ihm befreundet, um ihn etwas kontrollieren zu können. Obwohl sie sich gegen zu umfangreichen Medienkonsum ausspricht, sieht auch Frau Kaiser selbst jeden Tag mehrere Stunden fern, spielt Spiele auf ihrem Smartphone und 
nutzt das Internet intensiv für Facebook, WhatsApp, E-Mails, Online-Banking, Online-Bestellungen, eBay, Inforecherche, YouTube und Amazon. Im Fernsehen bevorzugte sie lange Zeit Dokumentationen, Gerichtsshows, Real Life-Formate wie Familien im Brennpunkt, Unterhaltungsangebote wie Achtung Kontrolle, K11, Niedrig und Kubnt sowie Filme wie Star Wars und Dirty Dancing oder Produktionen von und mit Til Schweiger. In der siebten Erhebungswelle tritt der Unterhaltungsaspekt beim Fernsehen etwas zurück, nun rezipiere sie dort viele Nachrichtensendungen und verfüge mittlerweile auch über ein Wochenendabonnement der Kronenzeitung. Zur Unterhaltung streame sie nun verschiedene Serien und greife auf die Mediathek von Sat.1 Gold zurück. Ein wichtiger Aspekt ihrer Smartphone-Nutzung bezieht sich auf den Austausch und die Kommunikation mit ihren Söhnen, besonders mit Marius, der unter der Woche nicht mehr zu Hause wohnt. Dafür nutzt sie Facetime und WhatsApp. Insgesamt bezeichnet sich Frau Kaiser selbst als „starke“ Mediennutzerin.

\section{Fazit}

Familie Kaiser ist es trotz ihrer stark eingeschränkten Handlungsoptionen aufgrund einer über die Jahre durchgängigen Armutsgefährdung und auch einiger Krisensituationen nach der Trennung der Eltern gelungen, sozio-emotional als Familie mit einem zumeist recht guten Familienklima zusammenzubleiben. Der Wunsch der Eltern, sich trotz der eigenen Differenzen und der daraus resultierenden Scheidung gemeinsam um die drei Söhne zu kümmern und für diese da zu sein, war dafür die Basis. Zur relativen sozio-ökonomischen Stabilität hat dabei auch der Umstand beigetragen, dass die Familie in einer geförderten und abbezahlten Eigentumswohnung lebt und sich somit keine Gedanken um steigende Mietkosten und drohende Umzüge machen musste.

Obwohl seine Eltern beide für ihn da waren und auch die regelmäßigen Besuchszeiten bei seinem Vater eingehalten wurden, litt Torsten unter der Scheidung. Hilfe suchte und fand er auch in einem Mentorenprogramm der Kinder- und Jugendanwaltschaft, dessen Erfolg aber nicht abschließend geklärt werden konnte. Über viele Jahre hinweg blieb Torsten orientierungslos, was seine Schulausbildung und seinen möglichen Berufsweg angeht. Auch zum Ende der Studie hat er noch keine klaren Handlungsentwürfe entwickelt, die seinen Handlungskompetenzen entsprechen. Da$\mathrm{zu}$ hat auch beigetragen, dass sich Herr und Frau Kaiser uneinig darüber waren, welche Schulbildung ihr Sohn erhalten sollte. 2020 verfügt Torsten nun zwar über einen HAK-Abschluss (Handelsakademie) und eine Berufsausbildung als Bürokaufmann, wohin sein Weg ihn aber führen soll, ist 
noch immer unklar. Medien spielten und spielen in Torstens Familie und auch bei ihm selbst eine große Rolle. Trotz einer zuweilen sehr intensiven Nutzung geht Torsten recht kritisch und kompetent mit Medien um. Dabei wendet er sich auch ausgewählten Informationsangeboten zu und setzt sich intensiv mit komplexen Themenbereichen auseinander.

\subsubsection{Simone Stab (20 Jahre alt) und ihre Mutter: Eine sozio-ökonomisch stark belastete Familie, der es dennoch gelingt, ihren Alltag weitgehend zu meistern}

2005 bis 2017

Simone Stab ist die Tochter einer Osteuropäerin, die zu Beginn der Studie bereits 14 Jahre in Österreich lebte. Simones Vater, ein gebürtiger Österreicher, hatte sich schon zwei Jahre nach Simones Geburt wieder von seiner Frau getrennt. Simone hatte über die Erhebungen hinweg einmal mehr, einmal weniger Kontakt zu ihrem leiblichen Vater. In der fünften Erhebungswelle kam es zu einem regelmäßigen Kontakt, der vom Vater ausging, worüber Simone sich sehr erfreut zeigte. In der sechsten Erhebungswelle wurde aber deutlich, dass sie ihn mittlerweile zwar noch immer regelmäßig traf, die Beziehung jedoch durch die neue Frau des Vaters - die sich aber zum Zeitpunkt der Erhebung, wie es hieß, wohl wieder scheiden lassen wollte - nicht sehr innig war. Die neue Frau des Vaters zeigte sich offenbar eifersüchtig; sie wollte es nicht zulassen, dass Simone Zeit alleine mit ihrem Vater verbringen konnte. $\mathrm{Zu}$ ihrer Mutter hatte Simone dagegen stets eine sehr enge Beziehung. In der Pubertät legte sie zwar Wert auf etwas mehr Eigenständigkeit, sie bezeichnete ihre Mutter dennoch als „,beste Mutter der Welt“, und dies, obwohl Frau Stab aufgrund der sehr angespannten sozio-ökonomischen Lage der Familie und als alleinerziehender Mutter, die den Lebensunterhalt verdienen musste, kaum Zeit für ein intensives familiäres Miteinander blieb. Darüber klagte jedoch Frau Stab selbst des Öfteren. In der fünften Erhebungswelle (2014) hatte Simone neben einer besten Freundin ihren ersten festen Freund. Mit ihm verbrachte sie zunächst sehr viel Zeit, jedoch trennten sie sich nach zwei Jahren Beziehung bereits wieder. Zu ihrem Halbbruder hatte Simone stets ein zwiespältiges Verhältnis: Einerseits gab sie an, ihn zu lieben und zu ihm zu stehen, andererseits stellte sein zuweilen schwieriges Verhalten für sie ein Problem dar, vor dem sie in der fünften Erhebungswelle oft aus der Wohnung floh. Nach einer Zeit in der Neuen Mittelschule hatte Simone vor der sechsten Erhebungswelle einen Schulwechsel vollzogen 
und besuchte nun eine Höhere Technische Lehranstalt (HTL) mit dem Schwerpunkt Bildhauerei. Für ihre Peers hatte sie dann nur noch wenig Zeit, da die Arbeitsbelastung durch die Schule sehr hoch war.

Die sozio-ökonomische Situation der Familie blieb während der gesamten Erhebungszeit stark belastet. Frau Stab hatte zwar in ihrem Heimatland eine Schule mit der Allgemeinen Hochschulreife abgeschlossen, dieser Abschluss war ihr aber in Österreich nicht als Matura anerkannt worden. Diese Kränkung begleitete Frau Stab durch alle Erhebungswellen, dennoch unternahm sie keinen Versuch, die Matura in Österreich noch zu erwerben. So schwankte sie immer wieder zwischen Arbeitslosigkeit und geringfügigen Beschäftigungen bzw. Teilzeitbeschäftigung in weniger gut bezahlten Tätigkeitsbereichen, wie etwa als Kellnerin. Besondere Anschaffungen, wie ein neues Auto, das in der vierten Erhebungswelle notwendig wurde, stellten die Familie daher immer wieder vor große finanzielle Herausforderungen. Frau Stab entwickelte zwar den Wunsch sich selbständig zu machen und hatte auch eine Umschulung zur Masseurin absolviert, den Schritt in die dauerhafte Selbständigkeit allerdings nicht geschafft. Zum Ende der sechsten Erhebungswelle (2016) war sie weiterhin als Kellnerin und Masseurin in Teilzeit beschäftigt. Simone erhielt zwar kein festgelegtes Taschengeld, aber ihre Mutter sorgte stets dafür, die Grundbedürfnisse der Familie zu decken, für Extrawünsche blieb jedoch kein Geld übrig. Größere Anschaffungen, wie etwa einen Laptop, finanzierte Simones Vater. Ihre ganz persönlichen Wünsche, vor allem im Hinblick auf andere Mediengeräte, konnte sich Simone aber nicht erfüllen.

Während der Studie war die Familie einige Male umgezogen. So etwa zwischen der ersten und zweiten Erhebungswelle, um in eine staatlich geförderte Wohnung in einer kleinen Gemeinde zu ziehen, da die Mutter die Mietkosten allein nicht mehr aufbringen konnte. Auch die kleine Wohnung, die die Familie von der dritten bis zur sechsten Erhebungswelle bewohnte, lag auf dem Land, wo sich vor allem Frau Stab selbst wohl fühlte. Zwischen der sechsten und siebten Erhebungswelle war Familie Stab erneut umgezogen, nachdem sich Frau Stab wegen der lauten Gartenarbeiten der Gärtner in der Wohnanlage mit der Hausverwaltung angelegt hatte. Ein Mann habe durch häufiges Rasenmähen „den Pflanzen keine Chance gegeben, zu wachsen; da stand der Typ da mit dem Rasenmäher, braun war die Erde. Und der stand da und hat gebrummt a halbe Stunde. Über Flecken, wo noch nicht a'mal Gras gewachsen ist." Und die Betriebskosten in der Miete, die wegen der intensiven Gartenarbeit ,immer wieder erhöht" worden seien, habe sie auch nicht mehr bezahlt und sich eigenmächtig ein Beet angelegt. Nach telefonischen Beschwerden habe sie 
daraufhin einen der Gärtner direkt konfrontiert und sei „rabiat“ geworden. Daraufhin sei sie für zwei Wochen in „eine Nervenklink zwangseingewiesen“ worden. Da sich anschließend die Stimmung in ihrem Wohnumfeld insgesamt verschlechtert hatte, zog die Familie für ein halbes Jahr in einen anderen Wohnort.

Zur Zeit der sechsten Erhebungswelle lebte Simones Bruder in der Woche nicht mehr zu Hause, sondern in einer betreuten sozialpädagogischen Wohneinrichtung. Frau Stab hatte sich selbst darum gekümmert, weil sie mit ihrem Sohn nur schwer zurechtkam und sich dieser mehr und mehr in Computer-Spielwelten zurückgezogen hatte.

2020: Simone Stab und ibre Mutter - eine um Handlungskompetenz bemübte junge Frau einer alleinerziehenden Migrantin, deren Leben sich um ibre Kinder dreht

Noch in der telefonischen Nachbefragung Anfang 2017 plante Simone einen Work-and-Travel-Aufenthalt über zwei Wochen in Island; später wollte sie dann vielleicht nach Südamerika oder Indien reisen. Diese Reisepläne konnte Simone jedoch nicht verwirklichen. Zur Zeit der siebten Erhebungswelle freut sich Simone aber auf eine Ausbildung zur Steinbildhauerin in Italien und hoff, dass ihr die Covid-19-Pandemie und die damit verbundenen Einschränkungen keinen Strich durch ihre Wünsche machen. Finanziell wird diese Ausbildung durch das Erasmus+ Programm der Europäischen Union unterstützt, da eine eigenständige Finanzierung des Auslandsaufenthaltes die Möglichkeiten der Familie überstiegen hätte. Simones großer Wunsch ist es, nach dem Abschluss zurückzukehren und in einer österreichischen Großstadt am Marionettentheater zu arbeiten. Zum Zeitpunkt der Erhebung wohnt Simone jedoch noch zusammen mit ihrer Mutter und auch wieder mit ihrem Halbbruder zu Hause; der Beginn der Ausbildung ist für den Herbst geplant. Der Kontakt zu ihrem Vater blieb auch in den vergangenen Jahren unregelmäßig und eher schlecht. Lange hat Simone, wie sie erzählt, unter dem Verhalten des Vaters, sich nicht richtig um sie zu kümmern, gelitten. Seit einer, wie sie es im Interview nannte, „Selbstfindungsphase“ hat sie diese Kränkungen mittlerweile im Großen und Ganzen verarbeitet und versteht sich nun grundsätzlich gut mit ihm. Kontakt gibt es allerdings eher selten, aber mehr wolle sie auch nicht, wie Simone betonte. Denn mit seiner Freundin hat ihr Vater vor einem Jahr ein Kind bekommen, und das sei, wie Simone erzählt, für sie zunächst ein Schock gewesen, denn für sie sei er auch nie dagewesen. Das Verhältnis zu ihrem Bruder habe sich jedoch inzwischen gebessert, seitdem sie nicht mehr die Rolle der vermittelnden „Mutter“ zwischen 
ihm und ihrer gemeinsamen Mutter einnehme; Streitereien gebe es aber nach wie vor noch.

Seit drei Jahren hat Simone wieder einen festen Freund, auch wenn es, wie ihre Mutter erzählt, zur Zeit der Erhebung Konflikte zwischen beiden gebe. Auch Simone berichtete von Problemen mit ihrem Freund; sie seien sehr unterschiedlich und es gebe "viele Turbulenzen, aber jetzt fühlt es sich gut an“. Auch zu Freundinnen hat Simone weiterhin Kontakt, besonders gern sei sie an den Wochenenden und ihnen und ihrem Freund am See in der Nähe ihrer Wohnung.

Frau Stab arbeitet zur Zeit der siebten Erhebung in einem durch die Europäische Union geförderten Job als Gärtnerin; dabei verdiene sie zwar nicht viel, aber diese Arbeit mache ihr „viel Spaß“. Weiterhin ist sie auf staatliche Beihilfen und Transferleistungen angewiesen. Ihre finanzielle Situation, so erzähle sie, „stresst sie jeden Monat, da sie bisher noch Alleinverdienerin im Haushalt ist". Ihr Alltag wird bestimmt durch den Kampf um die Finanzierung des Lebensunterhalts ihrer Familie. Vom Sozialamt bezieht sie auch 2020 Wohnbeihilfe. Nach einem halben Jahr in einer anderen Gemeinde ist sie an den alten Wohnort auf dem Land zurückgekehrt und aktuell mit ihrer neuen Wohnsituation zufrieden. Es besteht nun sogar die Möglichkeit, im gemeinschaftlichen Garten selbst etwas anzupflanzen. Mit ihrer Tochter und deren Entwicklung zeigt sich Frau Stab im Interview sehr zufrieden, weniger dagegen mit ihrem Sohn. Er hat zwar die Schule abgeschlossen, aber noch keine Lehrstelle gefunden. Frau Stab moniert, dass er sich nicht intensiv genug darum kümmere und zu wenig Planungen für seine Zukunft unternähme. In diesem Zusammenhang ärgert sie sich darüber, dass ihr Sohn jetzt schon 18 Jahre alt ist, sie aber keinerlei Geld mehr für ihn bekomme, da er sich aktuell nicht mehr in Ausbildung befindet. Gleichzeitig leiste er aber auch noch nicht den dringend benötigten eigenen Beitrag zum Lebensunterhalt der Familie.

Ihre eigenen Handlungsentwürfe drehen sich in der siebten Erhebungswelle mittlerweile eher um Freundschaften mit einigen Frauen:

Die hab' ich nach wie vor, ähm fast die gleichen. Is` alles gut. Männer... hab i jetzt eine dreijährige Beziehung g'habt, aber die hab' i jetzt auch beendet. Hat da nicht gepasst, genau. Bin i wieder solo quasi. (lacht) Ähm beziehungstechnisch, keine Ahnung. Schaff i net so." Dies mache sie zwar unglücklich; dass sie "net in eine Beziehung ankommen kann, weil, mittlerweile bin i 51, und i habe keine lange Beziehung g'habt bis jetzt.

Über die gesamte Erhebungszeit wurde deutlich, dass ihre Kinder in ihren Lebensentwürfen stets an erster Stelle standen. Frau Stab lag in besonderem Maße daran, ihnen grundlegende Werte wie (Nächsten-)Liebe 
und den Glauben an Gott zu vermitteln. Ihre ganz persönlichen Handlungsentwürfe konnte Frau Stab jedoch zu keinem Zeitpunkt der Erhebung umsetzen; ihr gelang es nicht, einen passenden neuen Partner zu finden, obwohl sie sich nach einer glücklichen und erfüllenden Beziehung sehnte. Als Mutter von zwei Kindern sei dies aber, wie Frau Stab betonte, nicht einfach zu realisieren. Auch alle Bemühungen im Bereich der Partnersuche auf diversen Online-Dating-Plattformen blieben ohne nachhaltigen Erfolg. Frau Stab legt auch in der siebten Erhebungswelle noch großen Wert auf Harmonie und Liebe. Insbesondere zu ihrer Tochter Simone hat sie nach wie vor ein sehr enges Verhältnis und ist stolz auf sie. Ihre eigenen Wünsche kreisen im Interview um einen Garten, eigene Tiere und um eine verlässliche Partnerschaft mit einem Mann. Während der gesamten Erhebungszeit folgte Frau Stab klaren Erziehungsidealen, sie war auch stets darum bemüht, diese in ihrer Erziehung entsprechend $\mathrm{zu}$ vermitteln. Sie zeigt sich denn auch mit Simones Erziehung zufrieden, nicht jedoch mit der ihres Sohnes. In seinem Fall, so Frau Stab, sei sie mit der Erziehung überfordert gewesen, und dem Jungen habe ein Vater gefehlt.

Frau Stab betrachtete das Leben zur Zeit der siebten Erhebung, gerade auch mit Blick auf die Covid-19-Pandemie, eher etwas resignativ:

Ja, so im Großen und Ganzen bin ich zufrieden und dankbar für die Kraft, die i g'habt habe, weil es war doch nicht leicht in de letzten Jahre. Und die Erkenntnis aber daraus, dass wohl ähm nix zu ändern is, und das gilt für die ganz Menschheit, glaub ich, allein schon diese Corona-Gschichte. I merk immer wieder, wenn die Menschen oder auch ich, auf der auf der ähm so dagegen sand, ja. Irgendwas auflösen wollen oder dagegen sand, ja. Dass das keine gute Kraft ist, keine gute Energie. Wir dürfen nix bekämpfen. Wir können nur hin schau'n. (...) Das war meine Lektion (damit spricht sie ihre Erfahrung mit der Zwangseinweisung in eine Nervenklink an; Anm. d. Verf.). (...) Egal was uns nicht gefällt, wir dürfen nie etwas bekämpfen (...).

\section{Medien im Leben von Simone und ibrer Mutter}

In den ersten Erhebungswellen hatte Simone in Fernsehfiguren noch nach Vorbildern Ausschau gehalten. Besonders der Verlust des Vaters durch die Trennung der Eltern und die manchmal fehlende Stütze der Mutter, die sie stets als angespannt und belastet erlebt hatte, da sie damit beschäftigt war, die finanziellen Probleme der Familie zu lösen, hatten bei ihr in der frühen Kindheit zu großer Trauer und zu Verlustängsten geführt Gefühle, die sie mit Hilfe von Medienangeboten zu bearbeiten versuchte. 
Ihr gefielen mediale Protagonisten die Waisenkinder waren, allen voran Mogli aus dem Dschungelbuch oder auch Protagonisten mit viel Geld, etwa London aus Hotel Zack und Cody („Die ist eigentlich strohdumm... aber voll reich.“) oder später die Serienfigur Hannah Montana, die durch ihren Gesang berühmt geworden war und viel Geld verdiente. In der sechsten Erhebungswelle spielten diverse Castingshows, die sich mit dem Thema sozialer Aufstieg befassten, für Simone eine bedeutsame Rolle.

2020 geht Simone, wie auch ihre Mutter meint, sehr bewusst mit Medien um; sie seien aber, wie sie selbst sagt, „sehr in ihrem Leben integriert“. Einen hohen Stellenwert schreibt sie den Medien aber nicht mehr zu. Sie sei der Meinung, dass man hinterfragen solle, wie und wozu man Medien nutzt. So hat Simone über ein Jahr ein altes Tastenhandy benutzt, um zu prüfen, ob ihr das ausreicht. Sie fand diese selbstauferlegte Einschränkung sogar „richtig gut“, habe sich aber wegen schulischer Angelegenheiten schließlich doch wieder ein Smartphone zugelegt. Denn sie nutzt oft WhatsApp, insbesondere für Schulangelegenheiten und zum Vereinbaren von Treffen, und, wie sie es zusammenfasst, „nicht mehr zur langen Kommunikation, sondern eher für Organisatorisches“. Zudem telefoniere sie damit hin und wieder und nutze es auch, zumeist am Wochenende, für Instagram und YouTube, zum Musik hören, um YouTuber zu verfolgen und um sich Dokumentationen anzusehen. Auch über ihren Laptop nutze sie YouTube und Netflix, allen voran für Liebes-, Action- und Fantasyfilme, aber auch für anspruchsvolle Anwendungen wie Photoshop, Illustrator und InDesign. Zwar meint Frau Stab, dass die Mediennutzung beider Kinder gestiegen sei, Simone selbst aber schätzt ihre Mediennutzung hingegen „als mittel“ ein. Wie ihre Mutter hält auch sie Medien für „manipulativ; man solle lieber im Jetzt sein und sich nicht von Medien berieseln und ablenken lassen“. Medien seien, so Simone, eine „Ablenkung vom richtigen Leben“.

Auch für ihre Mutter nahmen Medien stets einen wichtigen Stellenwert ein, so etwa das Internet für die intensive Partnersuche, aber auch zur Erledigung von Behördenangelegenheiten, etwa für die Unterlagen an das Sozialamt oder um sich auf YouTube Videos anzusehen oder Online-Banking zu tätigen. Frau Stab, die in ihrer Heimat ohne Strom und damit auch ohne elektronische Medien aufgewachsen war, hatte Medien lange Zeit sehr skeptisch gegenübergestanden, lernte deren Vorteile jedoch mit der Zeit schätzen. Zum Ende der sechsten Erhebungswelle zeigte sie sich allerdings wieder sehr kritisch, so erwähnte sie gesundheitliche Auswirkungen durch die Strahlenbelastung von Bildschirmen. Sie wollte sich am liebsten den 
Medien entziehen, auch wegen der oft negativen Nachrichten, die es zu hören oder zu sehen gebe.

Frau Stabs Mediennutzung sei, wie sie im Interview der siebten Erhebungswelle meint, "gleichgeblieben“ und insgesamt eher als gering einzuschätzen. Medien seien zwar wichtig zur „Vernetzung der Welt und zum Austausch von Informationen“, doch ihr persönlich „sind es zu viele Informationen“. Ihr Fernsehgerät hatte Frau Stab schon vor langer Zeit abgeschafft und überlegte sich auch, nun ganz auf ein Handy zu verzichten, das sie „eher nervt“.

Fazit

Simones Handlungsoptionen waren stets von der schwierigen sozio-ökonomischen Lage ihrer Familie gekennzeichnet; ihre Mutter, die aus einem osteuropäischen Land stammt, musste als alleinstehende Frau die Familie auch allein ernähren. Simones gestaltete mit der Zeit ihre Lebensführung selbständiger und hat sich eine langjährige, wenn auch komplizierte Partnerschaft mit ihrem festen Freund aufgebaut. Noch immer aber ist ihre Mutter, die ihre Handlungsentwürfe stets auf die Familie ausgerichtet hat, die zentrale Bezugsperson in ihrem Leben. Mit der Enttäuschung über ihren Vater und seine tendenzielle Vernachlässigung hat sie sich dagegen als junge Erwachsenen abgefunden. Mit ihrem Bruder versteht sie sich besser und auch im Großen und Ganzen mit ihrem Freund, mit dem sie seit drei Jahren eine Beziehung führt. Simone steht kurz vor dem Schulabschluss an einer HTL und hat danach ein einjähriges Stipendium an einer Steinbildhauerschule zur Weiterbildung in Südtirol in Aussicht. Das Schulgeld erhält sie im Rahmen des Erasmus+ Programms der Europäischen Union und alle notwendigen Vereinbarungen hat sie bereits unterzeichnet. Aktuell wohnt sie aber noch zu Hause bei ihrer Mutter auf dem Land, die weiterhin mit sozio-ökonomischen Herausforderungen sehr zu kämpfen hat, da auch der mittlerweile volljährige Sohn noch nicht zum Lebensunterhalt beiträgt. Simones Handlungsentwürfe kreisen um den Abschluss der Bildhauerschule und ihren großen Wunsch, anschließende eine Anstellung am Marionettentheater einer Stadt in Österreich zu bekommen. Simone gelingt es, ihre Handlungskompetenzen im Zusammenspiel zwischen Schule, Freund und Freundinnen sowie ihrer Mutter und ihrem Bruder gut einzusetzen. Dies gelingt auch ihrer Mutter, die halbtags als Gärtnerin arbeitet, aber aufgrund ihrer stark eingeschränkten Handlungsoptionen - nach wie vor sorgt sie sich täglich um den Lebensunterhalt der Familie - sehr belastet ist. Frau Stabs Wunsch für die Zukunft ist, 
dass i ein Platzl finde, ein Haus mit Garten. Dass i mi wohl fühle, dass i auch ein paar Tiere hab'n kann, so wie Hühner oder ein Schwein oder so. Des wäre so mein Wunsch. Und eine funktionierende, ein lieber Mann, eine funktionierende Partnerschaft für mein letztes halbes Leben, sozusagen. Des sind so meine Wünsche.

Medien spielen bei Mutter und Tochter eher eine funktionale Rolle. Beide, vor allem aber Frau Stab, betrachten gar einen hohen Medienkonsum als problematisch. Simone nutzt zwar unterschiedliche Medien, fürchtet aber, sie könnten manipulieren und vom Leben ablenken.

\subsection{Die jungen Erwachsenen von Typ 4: Keine belasteten sozio-ökonomischen Bedingungen mehr und unbelastete sozio-emotionale Beziehungsstrukturen - Die relativ kompetenten , Aufsteiger}

Die Familien von Typ 4 lebten schon seit einiger Zeit in derselben Familienkonstellation zusammen. Aufgrund maßgeblich veränderter sozio-ökonomischer Bedingungen, etwa durch Heirat oder durch eine feste Stelle bzw. eine neue besser bezahlte Arbeit des Vaters bzw. der Mutter oder auch den Zuverdienst durch die Mutter, erfuhren sie eine sozio-ökonomische Verbesserung. Sie erwiesen sich als `Aufsteiger . In diesem Kontext veränderten sich zuweilen auch ihre sozio-emotionalen Rahmenbedingungen so maßgeblich, dass die Familien insgesamt am Ende der sechsten Erhebungswelle einen recht stabilen Eindruck machten. Die Familien von Typ 4 zeichnen sich in ihrem Alltag durch kompetente Bewältigungsstrategien aus. Die Eltern charakterisiert, dass sie sich ihren Kindern gegenüber zugewandt verhielten und sich interessiert an ihren (medialen) Wünschen und Belangen zeigten sowie dass ihnen daran lag, die einmal erreichte sozio-ökonomische und auch sozio-emotionale Stabilität in ihren Familien nicht zu gefährden. Vielmehr richteten die Eltern ihre Handlungsentwürfe darauf aus, das gute Familienklima zu stabilisieren und ihre bessere soziale Position auch für ihre Kinder zu sichern und für diese da zu sein. Sie waren nicht länger darauf zurückgeworfen, mit den Herausforderungen zu kämpfen, die sich etwa aus der Verquickung der sozio-ökonomischen Bedingungen und schweren Krankheiten, gescheiterten Beziehungen oder nicht erfüllbaren eigenen Handlungsentwürfen ergaben. Die Eltern von Typ 4 hatten durch ihre verbesserten finanziellen und zeitlichen Ressourcen mehr Spielraum, ihren Kindern gegenüber Zuwendung und ein ausbalanciertes $\mathrm{Ma}$ an Nähe zu zeigen. So konnte es ihnen auch gelingen, durch eine wenig restriktive (Medien-)Erziehung den Kindern deren ganz 
spezifischem Eigen-Sinn gemäß Raum für sich selbst zu lassen. Diese Entwicklung ließ sich auch in den Handlungsentwürfen der Heranwachsenden und im Aufbau ihrer Handlungskompetenzen erkennen; sie ließen ein stabileres Selbstvertrauen erkennen und äußerten Pläne, eigene Ausbildungs- oder Berufswege zu verfolgen.

2.5.1 Gudrun Dornbacher (19 Jahre alt) und ihre Eltern: Eine Familie, die den Aufstieg schafft und in einem guten Familienklima lebt

2005 bis 2017

In Familie Dornbacher hatte es wegen der Karenz des Vaters, von Beruf KFZ-Mechaniker, in der ersten Erhebungswelle, seine Arbeitslosigkeit in der zweiten und einer abgebrochenen Umschulung zum Bürokaufmann in der dritten sowie einer nicht beendeten Ausbildung zum Krankenpfleger einige finanzielle Engpässe gegeben, die zu Konflikten in der Familie geführt hatten. Danach gelang es Familie Dornbacher, sich aus angespannten finanziellen Bedingungen mehr und mehr zu befreien. Ihre Lebenssituation erwies sich ab der vierten Erhebungswelle - der Vater arbeitete nunmehr in einer Vollzeitanstellung als Technischer Angestellter in einem Betrieb - als stabil und ausgeglichen. Gudrun lebte zusammen mit ihrer drei Jahre jüngeren Schwester in einer Kernfamilie; in der dritten Erhebungswelle hatten ihre Eltern geheiratet. Ihre Mutter, die die Handelsschule abgeschlossen hatte, war über die gesamte Zeit der Erhebung 15 Stunden als Sekretärin in Teilzeit berufstätig. In allen Erhebungswellen wohnte die Familie in einer $80 \mathrm{~m}^{2}$ großen Eigentumswohnung in Stadtnähe, die aufgrund der Vollanstellung des Vaters in der fünften Erhebungswelle abbezahlt war. Mit der Wohnsituation zeigte sich Familie Dornbacher durchgängig zufrieden. Vor allem die gute Anbindung und die Nähe zur Stadt gefielen allen Familienangehörigen. Zum Ende der sechsten Erhebungszeit besuchte Gudrun ein Montessori Oberstufen-Realgymnasium, eine kostenpflichtige Privatschule, mit musischem und kreativem Schwerpunkt; sie hatte sich das Ziel gesetzt, ein Blockflöten-Studium zu beginnen. Um dies zu erreichen, besuchte sie bereits 2016 mit 16 Jahren einen Vorkurs an der Musikhochschule, der sie auf das Aufnahmeverfahren vorbereiten sollte.

Auch noch in der sechsten Erhebungswelle bezeichnete Gudrun das Verhältnis zu ihrer Mutter als sehr eng; ihr Verhältnis zum Vater war zwischenzeitlich weniger innig als zur Mutter. In der dritten Erhebungswelle war Gudrun der Meinung, ihr Vater bevorzuge die jüngere Schwester. Der Vater war in Karenz, als die kleine Schwester auf die Welt kam, diese hat 
dadurch auch eine stärkere Bindung an ihn. In der vierten Erhebungswelle erzählte Gudrun dann, ein gutes Verhältnis zu ihrem Vater zu haben, er arbeite jedoch sehr viel und habe daher kaum Zeit. Eine sehr enge Beziehung hatte Gudruns zu ihrer Großmutter mütterlicherseits. Oma und Enkelin, so ihre Mutter, seien „ganz dick miteinander“, und das Mädchen übernachtete oft bei ihr.

Trotz zeitweiliger Konflikte in der Familie wegen der zwischenzeitlichen beruflichen Unsicherheit des Vaters richteten Gudruns Eltern ihre Handlungsentwürfe so gut wie möglich darauf aus, ein gutes Familienklima herzustellen. Seit der fünften Erhebungswelle standen auch den Kindern eigene Zimmer zur Verfügung, in denen sie sich, wie sie betonten, sehr wohlfühlten. Insgesamt wirkte die Familie entspannter als anfangs und stärker aufeinander bezogen. Seit der fünften Erhebungswelle gelang es auch Gudrun nach einigen Schwierigkeiten zuvor, befriedigende PeerBeziehungen aufzubauen. Schon vor der sechsten Erhebungswelle hatte sie einen festen Freund.

Gudruns Beziehung zu ihren Eltern erschien fast freundschaftlich, in ihrem Vater hatte sie zudem später auch einen Gesprächspartner zu aktuellen politischen Themen, so etwa über die Situation von Geflüchteten in Österreich 2015 und 2016, die Gudrun sehr beschättigte. Zum insgesamt harmonisch wirkenden Familienklima trugen auch gute Kontakte zu den Großeltern sowie Onkeln und Tanten bei. Auch Familie Dornbachers (Medien-)Erziehungsideal beruhte auf der Überzeugung des Miteinander, Regeln setzten die Eltern kaum, vielmehr stand eine freundschaftliche, auf Gespräche setzende Medienerziehung im Vordergrund.

2020: Gudrun Dornbacher und ibre Eltern: Eine vielseitig interessierte junge Frau mit guten Handlungsoptionen und eigenständigen Handlungsentwürfen in einer Aufsteiger-Familie

Zur Zeit der siebten Erhebung ist sich Gudrun nicht sicher, ob sie ihre letzten Matura-Prüfungen wie geplant absolvieren kann, da aufgrund der Covid-19-Pandemie die Schulen geschlossen wurden. In der Regelschule hatte sie es nicht geschaff, das Fach Mathematik zu bestehen und war daher aufs Abendgymnasium gewechselt. Diese Schule ist zwar auch auf Fernunterricht ausgerichtet, doch nicht alle Lehrenden kämen, wie Gudrun bedauert, mit den technischen Anforderungen zurecht. Zudem möchte sie ein Studium zur Flötistinnen-Ausbildung beginnen, in naher Zukunft stehe, wie sie erzählt, dafür die Aufnahmeprüfung an der Universität an. Gudrun ist sich allerdings sehr unsicher darin, ob sie diesen Weg beruflich verfolgen möchte. Daher überlegt sie, ob sie nicht auch 
ein Studium der Literatur- oder Kunstgeschichte beginnen solle. Blockflöte spielt Gudrun weiterhin in einem Ensemble. Um ihren Eltern, bei denen sie noch wohnt, nicht zu sehr „auf der Tasche zu liegen“, hat Gudrun einen Nebenjob in einer Trafik ${ }^{21}$ angenommen. Ihre Mutter erwähnt im Interview, dass Gudrun viel ,gesetzter und ruhiger geworden und nicht mehr so impulsiv" sei. Gudrun selbst erzählt, dass sie sich mit Hilfe einer Magnettafel stets einen Tagesplan mache. Sie hatte, wie ihre Mutter meint, negative Erfahrungen gemacht, der Besuch der Montessori-Schule und die Gestaltung des Tages ohne Planer habe dazu geführt, dass Gudrun ihre Arbeit nicht gut bewältigen konnte. Nun könne sie mit Hilfe einer Magnettafel und eines Planers ihre Gesamtplanung gut organisieren, die Schularbeiten aber, meinte sie, würden schwerer. Doch auch in Mathematik habe sich Gudrun verbessert und sei insgesamt reifer geworden, sie sei „a Stück weit eini g'wachsen mehr“, wie ihre Mutter es beschreibt. Gudrun sei sich bewusst, dass sie auch Dinge bewältigen muss, die nicht spannend sind, um das Ziel des Studierens zu erreichen. Stolz erzählt Frau Dornbacher, dass Gudrun beim Landeswettbewerb als zweitbeste HobbyFlötistin teilgenommen habe. Sie erwähnt auch, dass ihre Tochter einen Mopedführerschein gemacht hat und mit der kleinen Vespa der Mutter zur Schule und zur Musikprobe fährt.

Gudrun liegt sehr am regelmäßigen Austausch mit ihren Freunden und Freundinnen. Daher mache ihr die aktuelle Pandemie-Situation sehr zu schaffen, diese „ziehe sie geradezu herunter“, sie wisse oft nicht, was sie mit ihrer Zeit anfangen solle. Einen großen Stellenwert für Gudrun hat seit Jahren schon ihre „Manga-Anime-Gruppe“, die sich sonst einmal im Monat triff; aus dieser Gruppe hat sie sich einen Freundeskreis geformt. Eine feste romantische Beziehung hat Gudrun aber nicht, sie sei auch nicht „der Beziehungstyp“, sondern ist auch „ganz gern für sich und lieber die beste Freundin von jemanden“. So erwähnt ihre Mutter, dass Gudrun über ihr monatliches Manga-Anime-Treffen auch ihren mittlerweile besten Freund kennengelernt habe, mit dem sie die Wochenenden verbringe. Auch Frau Dornbacher ist davon überzeugt, dass ihre Tochter derzeit „keine Beziehung und auch keine Ambitionen in diese Richtung" hat.

Ihre Mutter ist für Gudrun weiterhin sehr wichtig; wenn es sich um Lebensentscheidungen drehe, hole sie Rat bei ihr, erzählt die 19-Jährige. Mit ihr gemeinsam spielt Gudrun seit zwei Jahren „Impro-Theater“ in einer Gruppe, was beiden sehr viel Spaß macht. Über sich selbst und

21 In Deutschland wird eine Trafik als Kiosk bezeichnet. 
ihren Mann sagt Frau Dornbacher im Interview 2020, dass sie „glücklich verheiratet" seien.

\section{Medien im Leben von Gudrun und ihren Eltern}

Gudrun verfügte schon in der ersten Erhebungswelle über ein umfangreich mit Medien ausgestattetes Zimmer, in dem auch ein Fernseher stand, der jedoch keinen Empfang hatte, sondern nur zum Anschauen von DVDs genutzt werden konnte. Der Computer gewann vor allem zwischen der ersten und zweiten Erhebungswelle mit dem Schuleintritt für Gudrun an Bedeutung, ebenso das Radio. Eine große Rolle spielten für Gudrun seit der dritten Erhebungswelle Musik und Bücher. Sie las gerne Harry Potter, in der vierten Erhebungswelle auch andere Jugendbücher (Isola, TwilightSaga). Die Leidenschaft für das Lesen teilte sie stets mit ihrer Mutter; beide lasen auch in der vierten Erhebungswelle noch gemeinsam. Musik hörte sie im Radio oder über ihren MP3-Player, auf den der Vater ihre Lieblingslieder kopierte. Seit der vierten Erhebungswelle besaß Gudrun ein Handy, mit dem sie mit ihren Freunden und Freundinnen telefonierte und SMS versandte. Das Internet nutzte sie zunächst hauptsächlich für die Schule (Recherche für Referate, Online-Hausübungen) und für kleine Spiele auf Minispiel-Seiten wie Spieleaffe. Gudrun war schon früh fasziniert von Literatur; sie entwickelte sich mit der Zeit geradezu zur „Leseratte“ und schrieb selbst Geschichten auf ihrem Laptop. Sie verfasste auch selbst Buchrezensionen und übte zum Ende der sechsten Erhebungswelle die Aufnahme von Videos. Ihr Wunsch war es, in naher Zukunft selbst eigene Buchbesprechungen auf YouTube hochladen zu können. Daneben rezipierte sie weiterhin zahlreiche Fernsehsendungen, allen voran Krimis und Soaps, oft auch mit den Eltern, und spielte Computerspiele, etwa das Open-World-Spiel Minecraft, das Horror-Spiel One Late Night und das Adventurespiel The Vanishing of Ethan Carter - ihre Eltern schritten dabei nicht ein, obwohl einige Spiele noch nicht für ihre Altersgruppe freigegeben waren. In der fünften Erhebungswelle hatte das Smartphone einen wichtigen Stellenwert eingenommen, ab der sechsten allen voran für WhatsApp.

Familie Dornbacher setzte auch im Umgang mit Medien auf eine offene und partnerschaftliche Erziehung mit viel Freiraum, die Eltern bemühten sich aber um Erklärungen und Mediation. So nutzte Frau Dornbacher Medien oft mit den Kindern gemeinsam und erklärt ihnen später, als das Internet für sie relevant wurde, auch am eigenen Beispiel, wo sie im Internet vorsichtig sein müssen; so thematisierte sie etwa Betrügereien wie versteckte Verträge mit Zahlungsaufforderung. Vor ihrem Einstieg in 
Sozialen Medien-Plattformen habe ihre Mutter sie auch darauf hingewiesen, sich genau zu überlegen, ob sie die Soziale Medien-Kanäle wirklich brauche.

Auch 2020 nutzt Gudrun ein breites Medienrepertoire, Medien sind ihr insgesamt sehr wichtig, wie sie betont. Sie nutzt ihr Smartphone z.B. für die Online-Recherche, um sich Kenntnisse anzueignen, und dies vor allem im Bereich Mediendesign. Bei Facebook schaue sie aber, wie sie erzählt, nur noch alle zwei bis drei Wochen mal hinein; sie verwendet es nur noch, um über Veranstaltungen auf dem Laufenden zu sein. Ursprünglich hatte sie die Idee, über Facebook mit Leuten vom Flötenstudium in Kontakt zu bleiben, die nur Facebook-Messenger verwendeten. Instagram sei ihr wichtiger als Facebook, dort poste sie auch selbst mal ein Bild, und Twitter nutze sie zur Unterhaltung, für „Trump-Postings“. WhatsApp verwendet Gudrun vor allem zum Schreiben von Nachrichten. Sehr wichtig ist ihr aber das Streamen von Let's Plays, wie etwa von Mario-Spielen, auf der Plattform Twitch.tv. Den Stream via Twitch.tv siedelt sie zwischen Arbeit und Hobby an. Gudrun ist auch in der Produktion von Medienangeboten aktiv. Mit drei Freunden betreibt sie einen eigenen Kanal, jeder kümmere sich dabei, je nach Wissen und Können, um unterschiedliche Bereiche. Wie sie erzählt, ist sie für Aufmachung, Display und (Soundund Digital-)Design zuständig. Die nötigen Kenntnisse dafür habe sie sich selbst angeeignet. Der Kanal hat mittlerweile etwa 300 Follower, davon etwa 75 bis 80 Abonnenten, die Gruppe verdient monatlich je 4,90 Euro an ihrer Arbeit dort. Das eingenommene Geld verwendeten sie aber, so Gudrun im Interview, wieder für den Stream, z.B. für den Kauf eines Schnittprogramms oder GreenScreens. Zur Pflege ihrer Freundschaften sind Facetime und Skype für Gudrun relevant. Ihre Handy- und generell ihre Mediennutzung habe sich durch die Krise erhöht, weil man sonst nichts zu tun habe und auf das Handy als Kontaktmöglichkeit beschränkt werde. Aber dies, so bedauert sie im Interview, sei kein Ersatz für realen Kontakt. Gudrun legt Wert darauf, dass ihre Handynutzung nicht ausufert, daher versucht sie, es nicht ständig in die Hand zu nehmen und habe damit auch Erfolg. In der Krise liest sie jetzt auch viel mehr Bücher als sonst, aber das erachtet sie nicht als bedenklich. Außerdem schaut sie vermehrt Filme, teilweise auch mit ihrer Mutter und Schwester, was sie wiederum als schöne Familienaktivität empfindet. Den Abend verbringt Gudrun vor allem mit Lesen, Netflix oder Musikhören. Auf Netflix schaut sie gern Animeserien, vor allem von Freunden empfohlene Serien und Filme unterschiedlicher Genres. 
Gudrun bezeichnet sich als „Fan von Medien“, vor allem von Büchern und Filmen; sie ist überzeugt davon, dass man viel von Medien lernen könne, aber dass es auch Gefahren gebe (in diesem Zusammenhang nennt sie Werbung). Sie erwähnt auch Fake News auf sozialen Medien, aktuell zur Zeit des Interviews im Zusammenhang mit der Pandemie, und sie kritisiert das Verhalten von Leuten, „die aufgrund dieser Fake News in Panikkäufe verfallen“. Im Umgang mit Medien müsse man vorsichtig sein; so konzentriere sie sich auf "die Sachen, die sie wirklich interessieren“. Musikhören ist Gudrun zudem sehr wichtig, vor allem über ihren iPod („Musikhören ist bei jeder Tätigkeit dabei“), aber auch Bücher der deutschen Klassiker- und Weltliteratur (als Reklam-Bücher) sowie Fachbücher zu Themen wie Musik, Philosophie, Anime und Manga, Zeichnen, Kunst. Auch Gudruns Mutter hält Medien als Informationsquelle für sehr wertvoll, aber auch sie betont, dass man unterscheiden müsse, welchen Quellen man glauben kann. Auch sie wies im Interview auf Gefahren durch Fake News hin, z.B. über Engpässe während des „Corona-Lockdowns“. Frau Dornbacher selbst nutzt nach wie vor insbesondere Bücher, die seien für sie „essentiell“, vor allem vor dem Einschlafen im Bett. Die Nutzung aller weiteren Medien schätzt sie eher als gering ein. Fernsehen, Netflix (etwa nachmittags zum Ansehen einer Serie gemeinsam mit ihrer Tochter, von Fantasy- oder Vampir-Serien), Autoradio, Computer, Handy (u.a. für WhatsApp, zum Telefonieren, für ein Spiel, das sie seit Jahren spielt, aber auch um schnell etwas zu googlen) und Bücher, die sie zumeist in der Gemeinde-Bibliothek bestellt, gehören fest zu Frau Dornbachers Repertoire. Herr Dornbacher rezipiert über Netflix vor allem Filme und Crime-Serien. Im Fernsehen, zumeist am Abend, schauen sie Bundeslandnachrichten, die Sendungen Kulturzeit, Millionenshow und Angebote zu Darts. Frau Dornbacher betont in der siebten Erhebungswelle, auch auf die Handynutzung ihrer jüngeren Tochter zu achten; diese dürfe ihr Handy nur drei- bis viermal am Tag für 20 Minuten nutzen. Nachts dürfe sie ihr Handy auch nicht im Zimmer haben.

\section{Fazit}

Familie Dornbacher hat nach einigen sozio-ökonomisch schwierigen Phasen in den Anfangsjahren der Studie einen Aufstieg erlebt. Durch die Festanstellung von Herrn Dornbacher nach Karenz- und Umschulungszeiten und zwischendurch auch einer Zeit der Arbeitslosigkeit - die durchgängige Teilzeitanstellung von Frau Dornbacher reichte nicht aus, um die Familie vor Armutsgefährdung zu schützen - verfügt Familie Dornbacher nunmehr über gute Handlungsoptionen. Trotz konfliktbelasteter Zeiten 
waren Vater und Mutter Dornbacher stets darum bemüht, Gudrun und ihrer zwei Jahre jüngeren Schwester ein möglichst gutes, ausgeglichenes Familienklima zu bieten. Ihre Eltern richteten ihre Handlungsentwürfe über alle Erhebungswellen hinweg auf das Wohlergehen ihrer Töchter aus; vor allem Frau Dornbacher war und ist noch heute eine fördernde und auf die Belange ihrer Kinder eingehende Medienerziehung wichtig.

Gudrun, die sich zum Ende der Erhebung als „Fan von Medien“ bezeichnet, plant ein Studium, wahrscheinlich der Literatur- und Kunstgeschichte, zu beginnen, um ihre Handlungskompetenzen weiter auszubauen, ganz sicher ist sie sich in der Wahl des Studiums noch nicht, denn sie spielt auch sehr gern und erfolgreich Blockflöte und besucht ein Vorstudium an einer Universität. Als eine Ausnahme unter den Panelangehörigen produziert Gudrun selbst mediale Angebote; sie betreibt mit Freunden einen eigenen Internetkanal. Insgesamt weist Gudrun Medien einen groBen Stellenwert in ihrem Alltag zu und nutzt ein breites Repertoire, sie achtet dennoch darauf, dass mediale Aktivitäten keine zu große Bedeutung in ihrem Alltag gewinnen. Wie für ihre Mutter spielen Bücher nach wie vor auch für Gudrun eine besondere Rolle. Zum Abschluss der Studie zeigt sich Gudrun als eine vielseitig interessierte junge Frau mit guten Handlungsoptionen und eigenständigen Handlungsentwürfen.

\subsubsection{Erich Grubert (21 Jahre alt) und seine Eltern: Eine Patchwork- Familie festigt ihre sozio-emotionalen Beziehungsstrukturen und schafft sozio-ökonomische Sicherheit}

2005 bis 2017

Als die Studie begann, lebte Erich (2005 sechs Jahre alt) gemeinsam mit seiner alleinerziehenden Mutter in einem sozial schwachen Viertel einer Großstadt in einer sehr kleinen Mietwohnung $\left(47 \mathrm{~m}^{2}\right)$. Beide waren unzufrieden mit den Wohnverhältnissen und der schlechten Ausstattung. Auch die Wohngegend wurde kritisch betrachtet, insbesondere wegen der fehlenden Freizeitmöglichkeiten und des subjektiv als zu hoch empfundenen Anteils an Migranten und Migrantinnen. In Konsequenz ließ Frau Grubert ihren Sohn nur ungern draußen spielen und vermied den Kontakt zu anderen Bewohnern. So verbrachte Erich seine Freizeit meist in der Wohnung und nutzte Medienangebote, um seine Langeweile zu bekämpfen. Bereits als Sechsjähriger war er leicht übergewichtig, hyperaktiv und konnte sich nicht längere Zeit konzentrieren. Eine Möglichkeit zur Verbesserung der Situation sah Frau Grubert zu diesem Zeitpunkt nicht. 
Sie ist zwar ausgebildete Bürokauffrau, arbeitete jedoch nur 15 Stunden in der Woche als Telefonistin und verfügte entsprechend nur über geringe finanzielle Ressourcen, um Ziele und Pläne ihrer Handlungsentwürfe zu verfolgen.

$\mathrm{Zu}$ Beginn der Studie war das Verhältnis zwischen Erich und seinem leiblichen Vater gut und beide unternahmen regelmäßig etwas zusammen; mit der Zeit wurden die Treffen aber seltener. Einerseits wohnte Erichs Vater in einem anderen Bundesland, anderseits versuchte auch Frau Grubert, Treffen zwischen Sohn und Vater zu verhindern, da dieser angeblich an Tabletten- und Alkoholsucht litt. Ab der fünften Erhebungswelle (2014) bis zum Ende der Studie bestand kein Kontakt mehr zum leiblichen Vater, da dieser sich nicht mehr gemeldet hatte. Laut Frau Grubert litt Erich darunter, während dieser angab, dass sein Vater ihm inzwischen „scheißegal“ sei.

Die Lebenssituation der Familie veränderte sich schließlich grundlegend, als Frau Grubert 2007 eine neue Partnerschaft mit einem Computertechniker und später System-Engineer einging. Dadurch verbesserte sich die finanzielle Situation der neu begründeten Patchwork-Familie sehr, und man bezog eine größere Wohnung in besserer Umgebung. Ab diesem Zeitpunkt äußerten sich sowohl Frau Grubert und ihr Partner als auch Erich regelmäßig positiv und zufrieden über die Wohnung und die Wohngegend. Erich verbrachte nunmehr auch viel Zeit draußen und traf sich dort mit Freunden und Nachbarskindern. Frau Gruberts neuer Lebensgefährte nahm seine Rolle als Stiefvater von Beginn an aktiv an, hatte es jedoch nicht immer leicht, da Erich mit der neuen Situation zunächst noch fremdelte. Obwohl beide anfangs nicht viele Interessen teilten und die Vorstellungen des Stiefvaters von Männlichkeit und Freizeitgestaltung nicht immer mit denen von Erich übereinstimmten, akzeptierte der Junge schließlich dessen Vaterrolle. Ab der vierten Erhebungswelle (2012) verbesserte sich ihr Verhältnis merklich und wurde intensiver. Sie widmeten sich verstärkt gemeinsamen Unternehmungen (Fahrradtouren, Ausflüge, Gesellschafts- und Computerspiele), zudem entwickelte sich der Lebensgefährte zu Erichs bevorzugtem Ansprechpartner für Fragen rund um Technik und Medien. Später realisierten beide gemeinsam auch Projekte, wie etwa das Arbeiten an einem Moped, und das Verhältnis wurde von allen Beteiligten als weitestgehend gut und konfliktfrei beschrieben. Obwohl Erich in seinem Stiefvater eine neue und vertrauenswürdige Vaterfigur gefunden hat, blieb sehr deutlich, wie wichtig seine Mutter als Bezugsperson für ihn war und bis in die Gegenwart ist. Beider Aussagen stimmten in der Regel überein, und es gab weder Widersprüche noch Hinweise 
auf verdeckte Konflikte. Lediglich in der Pubertät gab es alterstypische Geheimnisse zwischen Mutter und Sohn. Erich verheimlichte etwa seine erste Beziehung, da er offenbar noch nicht dazu bereit war, diese Information zu teilen. Jedoch ließ er nie einen Zweifel daran, wie wichtig ihm seine Familie war.

$\mathrm{Ab}$ der fünften Erhebungswelle beginnt eine stetige Verbesserung der sozio-ökonomischen Situation der Familie. Zunächst wechselte der Lebensgefährte von Frau Grubert 2014 den Arbeitgeber und stieg zum System-Engineer auf. Zur gleichen Zeit beendete Erich auf eigenen Wunsch seine Schullaufbahn mit dem Pflichtschulabschluss und begann eine Ausbildung als Koch, da er schnell beginnen wollte, sein eigenes Geld zu verdienen. Der Großteil seiner Freunde, die er zum Teil als „schlaue Leit“ bezeichnete, besuchten dagegen weiterführende Schulen. Dadurch konnte man sich seltener sehen, da Erich oft spät abends arbeitete, und gemeinsame Freizeitaktivitäten wurden schwieriger. Mit der Zeit unternahm Erich auch immer häufiger etwas mit Arbeitskollegen, mit denen er gemeinsame Interessen und eine vergleichbare Lebenswelt teilte. Die Freundschaften seiner Kindheit und Jugend blieben bis zum Ende der Studie wichtig für ihn, jedoch verringerte sich der Kontakt deutlich, und 2017 bezeichnete Erich zeitweise nur mehr einen Jungen aus dieser Zeit als engen Freund. Auch Frau Grubert stockte ihre Arbeitszeit zunächst auf und arbeitete zusätzlich im Service bei Sportveranstaltungen. $2016 \mathrm{nahm}$ sie an einem Umschulungsprogramm teil und verantwortete zum Ende der Studie 2020 im Büro einer Cateringfirma den Bereich der Kommissionierung.

2020: Erich Grubert - ein junger Mann kann sich beruflich etablieren und auch in Krisenzeiten auf seine Patchwork-Familie verlassen

2020 hat sich die Lebenssituation der Gruberts nicht grundlegend geändert. Die Familie bewohnt noch immer die 2007 bezogene Mietwohnung und fühlt sich dort sehr wohl. Erich hat zwar vage Überlegungen, in Zukunft in eine Wohngemeinschaft zu ziehen und später auch einmal im Ausland zu arbeiten, konkret sind diese Pläne jedoch bislang nicht. Seit 2016 teilt die Familie die Wohnung nicht nur mit einer Schlange, einem Hamster und zwei Katzen, sondern auch mit einem Hund. Dessen Versorgung obliegt hauptsächlich Frau Grubert, doch auch Erich nimmt seine Verantwortung wahr und kümmert sich um das Tier.

Dennoch ist Erich 2020, im Vergleich zur telefonischen Nachbefragung 2017, wie ausgewechselt, und er erwähnt diese Veränderung im Interview sofort und voller Enthusiasmus: Nachdem er in den vergangenen Jahren immer mehr zugenommen und sich kaum mehr aktiv betätigt hatte, hat er 
nunmehr 40kg abgenommen, indem er seine Ernährung grundlegend umgestellt hat. Diesen Zustand, so sein Plan und Wunsch, will er unbedingt beibehalten und schlank bleiben. Er sei zwar weiterhin „kein Sportler“, der Gewichtsverlust habe aber dazu geführt, dass er nun „ein ganz anderes Selbstbewusstsein“ habe und sich „einfach viel, viel freier und leichter dadurch“ fühle. In Konsequenz bringt der Gewichtsverlust auch deutliche Veränderungen für Erichs Leben und seine Freizeitgestaltung mit sich. Er traue sich nun auch ins Schwimmbad zu gehen und hat seinen Kleidungsstil verändert. Früher versteckte er sich etwa unter Pullis, jetzt gehe er auch „kurzärmlig“ raus: „des hab“ ich damals ned wollen." Darin zeigt sich deutlich das neu gewonnene Selbstvertrauen, dass sich auch positiv auf Erichs gesamtes Auftreten auswirkt.

Die inzwischen über viele Jahre gefestigten sozio-emotionalen Strukturen innerhalb der neugebildeten Kernfamilie bewähren sich auch 2020 und erlauben es, verschiedenartige Krisensituation zu meistern und gestärkt aus ihnen hervorzugehen. Dazu zählt nicht nur die Covid-19-Pandemie, in der Frau Grubert in ihrem Hauptberuf in Kurzarbeit gehen musste und ebenso wie ihr Partner die Nebenbeschäftigung als Streckenposten im Motorsport verlor und Erich, als gelernter Koch, ebenfalls längere Zeit in Kurzarbeit war und zeitweise gar nicht arbeiten konnte. Auch den Einschnitt einer von Erich begonnenen aber schließlich doch abgebrochenen Ausbildung als Metzger konnte die Familie abfedern. Erich wurde in seinem Betrieb ungerecht behandelt und sollte auf Ausgleichszahlungen verzichten. Schließlich gelang es ihm, die Ausbildung abzubrechen und mit Unterstützung der Arbeiterkammer eine Auszahlung seiner geleisteten Überstunden zu erreichen. In dieser Zeit wurde er von seiner Familie unterstützt, aber auch von seinem ehemaligen Arbeitgeber, einem Gastronomiebetrieb. Dort übernahm man ihn nicht nur erneut in Vollzeit, vielmehr wurde ihm auch eine Perspektive für die innerbetriebliche Fortbildung und den Aufstieg in der Firma gegeben. Derzeit sammelt Erich praktische Erfahrung in den verschiedenen Abteilungen des Unternehmens und soll als zukünftiger Souschef eingesetzt werden. Im Rahmen der ihm zur Verfügung stehenden Handlungsoptionen hat er seine Handlungsentwürfe neu justiert und seine Pläne und Ziele auf den beruflichen Karriereweg als Koch ausgerichtet. Das Überstehen dieser Krise und der erfolgreiche Neustart gelangen Dank seiner Familie im Rücken und durch die Unterstützung seines Arbeitgebers. Diese doppelte Sicherheit als soziale Ressource war für Erich wichtig, um seine Lebensentwürfe umzusetzen und um die nötigen Handlungskompetenzen zu erwerben. 


\section{Medien im Leben von Erich und seiner Familie}

$\mathrm{Ab}$ der zweiten Erhebungswelle war die Familie immer gut mit Medien ausgestattet, was nicht zuletzt auf den Beruf des Stiefvaters zurückgeführt werden konnte. Die Geräte spiegelten dabei in der Regel den neuesten Stand der Technik wider, ein Umstand, der die Familie von den meisten anderen im Panel deutlich unterschied. Erich liebte es, seine Medienausstattung auf dem neuesten Stand zu halten, vor allem als er anfing, sein eigenes Geld zu verdienen und zusätzliche Geräte kaufen konnte. Dabei war er besonders stolz auf seinen Flachbildfernseher, verschiedene Konsolen und eine große Auswahl an Videospielen. Seine Eltern bestätigten, dass er diesbezüglich sehr wählerisch sei, und sie attestierten ihm früh eine klare Vorstellung davon zu haben, was er wolle sowie wo er es bekommen und dabei den besten Preis erzielen könne.

Erichs medienbezogene Interessen wandelten sich im Lauf der Studie kaum, aber die Intensität seiner Mediennutzung änderte sich über die Jahre immer wieder. In der ersten Erhebungswelle war seine Mediennutzung aufgrund fehlender anderer Freizeitaktivitäten und mangelnder Freundschaften sehr intensiv. Erich favorisierte die ihm eigentlich von seiner Mutter wegen ihres gewalthaltigen Inhalts, aber auch wegen seiner ADHS-Erkrankung, verbotenen Anime-Serien, wie etwa $Y u$-Gi-Oh!, und Action-Angebote, da diese, wie seine Mutter seinerzeit betonte, negative Einflüsse auf Erichs Verhalten zeitigten: „Oiso, der Erich, der kann des ned verarbeit'n, wenn der des a Zeiterl anschaut, dann hupft er herum auf da Coach und führt sich voll auf. Der verarbeit' des ned. Des geht eam zu schnell.“ Animes etwa gehörten während der gesamten Studie zu seinen Lieblingsangeboten. Mit dem Umzug in die neue Wohnung erweiterten sich Erichs Freizeitmöglichkeiten deutlich, und in der zweiten und dritten Erhebungswelle ging sein Medienkonsum deutlich zurück. Dies änderte sich jedoch erneut, als er in der dritten und vierten Erhebungswelle in die Phase der Jugend eintrat und zunächst in der Schule und später in der Ausbildung immer größerem Druck ausgesetzt war. Videospiele wurden zu einem wichtigen Ventil für ihn und dienten als zentraler Faktor seiner Freizeitbeschäftigung. Darüber hinaus stellte das Spielen von komplexen Videospielen für Erich eine Möglichkeit dar, sich selbst zu erfahren und Leistung zu zeigen. Seine Mutter vermutete, dass er zeitweise unter einem sehr geringen Selbstwertgefühl litt, da er wohl in der Schule wegen seines hohen Gewichts gemobbt worden war. In diesem Kontext bot der Erfolg in Videospielen auch einen Schlüssel, um sein verletztes Selbstwertgefühl wiederherzustellen. 
Neben Animes und Videospielen gewannen in den letzten beiden Wellen das Internet, Soziale Medien und das Smartphone als Gerät erheblich an Bedeutung für Erich. Jedoch nutzte er Plattformen wie Facebook eher im Modus des Konsumenten und veröffentlichte nur selten eigene Inhalte. Verglichen mit den anderen Jugendlichen im Panel blieb die Zahl seiner Freunde in Sozialen Netzwerken mit etwa 80 auch immer vergleichsweise gering, und Erich nahm immer für sich in Anspruch, dass er alle Sozialen Netzwerk-Freunde auch persönlich kennen würde. Mit dem Eintritt in die Berufsausbildung nahm Erichs Smartphone-Nutzung wieder stark ab, nicht zuletzt, da private Geräte in der Küche seines Arbeitgebers verboten waren. Gleichzeitig wurde das Radio, und dies ist sehr untypisch für junge Menschen in seinem Alter, zu Erichs zentralem Informationsmedium, da es während der Arbeitszeit eigentlich ununterbrochen lief.

Insgesamt waren die Medien für Familie Grubert sehr wichtig. Durch den Beruf des Stiefvaters hatten Mediengeräte auch einen hohen praktischen Wert, da das Einkommen der Familie hauptsächlich auf der Arbeit mit ihnen beruhte. Neben neuen Computern und Smartphones verfügte die Familie aber auch über die gesamte Studiendauer hinweg über ein kostspieliges Abonnement des Pay-TV-Anbieters Sky, auf dessen Angebote Erich jedoch nur sehr vereinzelt zugriff. Medien waren ständige Begleiter und wurden in vielfältiger Weise zur Unterhaltung und zur bequemen Informationsbeschaffung genutzt. Wie Erichs Mutter 2017 betonte, konnte sie sich ein Leben ohne sie nicht vorstellen. Dennoch sahen die Familienmitglieder nur vereinzelt gemeinsam fern oder spielten Computerspiele. Erich führte dies immer wieder auf unterschiedliche Interessen zurück. Während Frau Grubert und ihr Lebensgefährte etwa große Begeisterung für Sportübertragungen im Fernsehen und ganz besonders die Formel 1 zeigten, spielten solche Angebote für Erich nie eine bedeutende Rolle. Daher spielte Erich meist alleine in seinem Zimmer und sah fern, während seine Eltern dafür das Wohnzimmer nutzten.

2020 hat das Interesse von Erich an Medienangeboten erneut stark abgenommen und er bevorzugt es nun verstärkt, sich physisch mit seinen Freunden zu treffen. Diese Veränderung ist nicht zuletzt auf seinen erheblichen Gewichtsverlust zurückzuführen. Dennoch ist er besser als je zuvor mit Medienprodukten ausgestattet: Neben dem Zugriff auf Sky verfügt er nun auch über Abonnements bei Netflix und Amazon Prime und er spielt weiterhin regelmäßig auf seiner PlayStation 4 (Call of Duty, Minecraft, Black Desert). Lineares Fernsehen, Radio, die Zeitung oder Online-Nachrichtenseiten spielen für Erich und sein Informationsbedürfnis keine Rolle mehr, stattdessen bevorzugt er es, Informationen über das Weltgeschehen 
von seinen Arbeitskollegen oder seinem Chef, einem hochrangigen Funktionär in der Wirtschaftskammer, zu erhalten. Auch Soziale Medien spielen kaum mehr eine Rolle in seinem Leben. Erich ist zwar weiterhin auf Facebook angemeldet, rezipiert dort aber lediglich Videos. Für Angebote wie Snapchat, Instagram oder gar TikTok sei er „zu old-school“. Im Vergleich zu ihrem Sohn ist die Mediennutzung von Frau Grubert inzwischen weitaus umfangreicher. Sie nutzt intensiv ihr Smartphone: Es dient ihr zur Informationsbeschaffung (Google News, verschiedene Nachrichten-App und die App der Bundeslandzeitung), für Dienstleistungen wie das OnlineBanking und um über Soziale Medien (Facebook, Facebook Messenger, WhatsApp) zu kommunizieren und sich zu informieren. Auch das Fernsehen nutzt sie sowohl zur Information (n24) als auch zur Unterhaltung. Gemeinsam mit ihrem Partner schaut sie die Formel 1 auf Sky und Dokumentationen. Am wichtigsten ist ihr jedoch die Unterhaltung und dabei eher „keine so hochkomplizierten Filme“, sondern Inhalte, die „nebenher" laufen können. Als Beispiele nennt sie „bayerische Krimiserien“ wie „Rosenheim Cops“ oder „Hubert und Staller“.

\section{Fazit}

Erich und seine Mutter haben zu Beginn der Studie mit stark begrenzten Handlungsoptionen leben müssen. Das Beispiel ihrer erfolgreichen Patchwork-Familie zeigt, wie nachhaltig sich sowohl die sozio-ökonomischen als auch die sozio-emotionalen Perspektiven aufgrund einer harmonischen Partnerschaft der Mutter mit einem finanziell besser gestellten Mann verbessern können. Die stabilen Familienverhältnisse haben sich als Grundlage dafür erwiesen, dass Erich eigene Handlungskompetenzen entwickeln und berufliche Pläne und Ziele verfolgen konnte. Mit der Rückkehr in seinen Ausbildungsbetrieb eröffnen sich ihm nun neue Handlungsoptionen; Erich plant eine innerbetriebliche Fortbildung zum Souschef, um seine mittel- und langfristigen Pläne und Ziele umsetzen zu können: eines Tages im Ausland oder auf einem Kreuzfahrtschiff als leitender Koch arbeiten zu können.

Mit Blick auf Erichs physische Verfassung hat auch sein radikaler Gewichtsverlust zu einer nachhaltigen Veränderung seiner Lebensführung beigetragen. Erst dadurch fühlt er sich selbst in seinem Körper wohl, kann sich frei bewegen und die entsprechenden Freizeitmöglichkeiten wahrnehmen, die ihm sein Lebensumfeld bietet. So hat sich nun im jungen Erwachsenenalter Erichs Medienkonsum reduziert; mittlerweile reserviert er auch Zeit für andere Freizeitaktivitäten. Zuvor, gerade in Phasen des Rückzugs, nahmen Medien, vor allem Bewegtbildangebote und 
Videospiele, für Erich einen sehr hohen Stellenwert in seinem Alltag ein. Auffällig bleibt jedoch auch jetzt noch, dass Erich nahezu keinen Wert auf eine eigene Auseinandersetzung mit medialen Informationsangeboten legt, ihm ist dagegen die Meinung seines Chefs sowie seiner Kolleginnen und Kollegen zum Alltagsgeschehen wichtiger.

\subsubsection{Helmut Pfortner (19 Jahre alt) und seine Eltern: Weitestgehend stabile sozio-emotionale Beziehungen kennzeichnen die Familie, die zunächst herausfordernden sozio-ökonomischen Bedingungen bessern sich}

2005 bis 2017

Helmut und seine ältere Schwester Anneliese (21) waren zu Beginn der Studie vier und sechs Jahre alt. Gemeinsam mit ihren Eltern bildeten sie über den gesamten Erhebungszeitraum hinweg eine Kernfamilie. Seit Beginn der Erhebung wohnte die Familie in einem alten $120 \mathrm{~m}^{2}$ großen Einfamilienhaus am Stadtrand, das allerdings seinerzeit einen schmutzigen, unordentlichen und stark renovierungsbedürttigen Eindruck machte. Zur Dekoration dienten lediglich Gewehre und zahlreiche Trophäen, da Herr und Frau Pfortner regelmäßig mit ihren Kindern auf die Jagd gingen und dazu auch eine eigene Jagd unterhielten. Das Kompressoren-Bauunternehmen von Herrn Pfortner ist ebenfalls im Haus angesiedelt. Neben den Familienmitgliedern waren auch immer Hunde ein Teil der Familie. Die Handlungsoptionen der Familienangehörigen erweiterten sich durch einen deutlichen Anstieg ihrer finanziellen Ressourcen zwischen der zweiten und dritten Erhebungswelle, da Helmuts Mutter eine zusätzliche Teilzeitanstellung als Verkäuferin in einem Drogeriemarkt annahm. 2014 hatte sie eine Zusatzausbildung als Drogistin absolviert. Zuvor hatte sie im Familienbetrieb mitgeholfen, der den Lebensunterhalt der Familie allein nur unzureichend sichern konnte. Auch Helmuts persönliche Situation verbesserte sich seither; in der zweiten Erhebungswelle hatte er wegen einer Teilleistungsschwäche den Schultest nicht bestanden und konnte nicht eingeschult werden, sodass er noch den Kindergarten besuchen musste.

Es wurde zwar nie so bezeichnet, dennoch schien Familie Pfortner schon früh den Plan zu haben, aus ihrem Haus in gewisser Weise ein Mehr-Generationen-Haus zu machen. Dazu zählte es, die Großmutter väterlicherseits aufzunehmen, die ab der dritten Erhebungswelle (2010), bis zu ihrem Tod kurz vor der siebten Erhebungswelle (2020) eine eigene Eta- 
ge in dem Gebäude bewohnte und dort von ihrer Schwiegertochter und ihrem Sohn gepflegt wurde. Zudem wurden auch Baumaßnahmen getroffen, damit Helmut und seine Schwester im jungen Erwachsenenalter und darüber hinaus zu Hause wohnen bleiben können. Zu Beginn der Studie teilten sich die Kinder noch ein Zimmer, ab der zweiten Erhebungswelle (2007) verfügten beide jedoch über jeweils eigene Räume, die sich schnell zu „Lieblingsorten“ entwickelten. Bereits 2008 erhielt die Wohnung eine neue Küche, und ab der vierten Erhebungswelle (2012) wurden die schon früher geplanten Umbau- und Modernisierungsmaßnahmen umgesetzt. Neben einem neuen Bad wurde zu diesem Zeitpunkt auch ein Pool im Garten angelegt. Die Einrichtung blieb zwar auch später noch einfach, wurde aber mit der Zeit in einen besseren Zustand versetzt, zudem machte die Wohnung ab 2010 immer einen sauberen und ordentlichen Eindruck.

Zur fünften Erhebungswelle wurde Helmuts Zimmer mit neuen Möbeln ausgestattet und erhielt eine bunte LED-Beleuchtung am Bett. Darüber hinaus bestanden bereits konkrete Pläne zu einer aufwändigen Neugestaltung des Wohnzimmers und zum Ausbau des Dachgeschosses. 2016 war dieser umfassende Ausbau bereits abgeschlossen, und sowohl Helmut als auch seine Schwester verfügten über eigene, 55 und $50 \mathrm{~m}^{2}$ große Wohnungen, die sie auch zum Ende der Studie noch bewohnten. Die Wohngegend wurde von allen Familienmitgliedern stets positiv bewertet, dazu trugen unter anderem Einkaufsmöglichkeiten, eine gute Verkehrsanbindung und gleichzeitig die Nähe zur Natur bei. Neben vielen Freunden in der Umgebung hoben Frau Pfortner und Helmut auch mehrfach als positiv hervor, dass man in der Umgebung gut spielen und mit dem Hund laufen gehen könne.

Familie Pfortners sozio-emotionale Bedingungen waren über den gesamten Erhebungszeitraum der Studie weitgehend stabil. So war die Familie in der Lage, auch Krisen zu bewältigen, die mit einem erhöhten Alkoholkonsum des Vaters zusammenhingen und die Helmut stark belasteten. In den ersten drei Erhebungswellen hatte Helmut nie von Konflikten mit seinen Eltern berichtet, er hatte jedoch bemängelt, dass sein Vater zu selten Zeit hätte. Dennoch bezeichnete er den Vater stets als sein Vorbild und wendete sich bei Fragen, gerade in technischen Belangen, immer an ihn. Auch sah er seinen Vater stets als Respektsperson und „letzte Instanz“ an, der man „immer und ohne Widerworte gehorchen“ müsse. Das Verhältnis zwischen Mutter und Sohn schien von Beginn an liebevoll und von viel Zuneigung geprägt zu sein. Ab der vierten Erhebungswelle entwickelte sich die Mutter zu einer engen Vertrauensperson für Helmut; er bezeichnete sie auch während der Pubertät als seine erste Ansprechperson, wenn 
es um Fragen rund um Sexualität und körperliche Entwicklung ging. Bis zum Ende der Studie blieb Frau Pfortner, gerade auch im Vergleich zu anderen Müttern im Sample, sehr gut über die Entwicklung und die Belange ihres Sohnes informiert, ohne dass sie ihn kontrollierte oder seine Privatsphäre missachtete.

In der fünften Erhebungswelle hatte sich das Verhältnis zwischen Vater und Sohn stabilisiert, und Herr Pfortner fand die Vorstellung „,cool“, dass sein Sohn eines Tages seinen Betrieb übernehmen würde. Nach einer Lehre in einem anderen Unternehmen wollte er ihm alles zeigen und ihn für später einarbeiten. In der sechsten Erhebungswelle berichtete Frau Pfortner dagegen erneut, dass ihr Mann oft schlechte Laune habe und zu viel getrunken habe. Zudem litt er zu diesem Zeitpunkt unter Burn-out. Herr Pfortner kümmerte sich zwar weiterhin um seinen Sohn, dieser hatte sich jedoch teilweise zurückgezogen und sich bei Fragen, die keine technischen und beruflichen Belange betrafen, eher an seine Mutter gewendet.

Helmut hatte eher einen kleinen und engen Freundeskreis; auch Partys und Alkohol spielten für ihn nach eigener Aussage nur eine sehr geringe Rolle, da sich beides nur schwer mit seinem sportlichen Lebensstil vereinbaren lasse. Von Beginn der Studie an verbrachte er sehr viel Zeit drauBen und trieb Sport, unter anderem Fahrrad fahren, Trampolin springen, Fußball spielen, Snowboard und Longboard fahren und ab 2007 Turnen und später Ringen im Verein. Dies entwickelte sich zu seinem zentralen Hobby; so verbrachte Helmut bis zur sechsten Erhebungswelle 2016 sehr viel Zeit beim Training und wurde von seinen Eltern auch regelmäßig zu Wettkämpfen gefahren. Einige zum Teil international sehr erfolgreiche Ringer in seinem Verein bezeichnete Helmut auch als Vorbilder.

Die Schulzeit war für Helmut nicht immer leicht. Nach seinem wegen einer "Teilleistungsschwäche“ verzögerten Wechsel vom Kindergarten auf die Volksschule litt er dort unter einer Lehrerin, die oft mit ihm geschimptt und ihn sogar geschlagen haben soll. 2012 verbesserte sich die Situation: Nun konnte Helmut wunschgemäß auf eine Sport-Hauptschule wechseln. Dort entwickelte er sich zu einem „Durchschnittsschüler“, wie er sich selbst und auch seine Mutter ihn bezeichneten, und verstand sich nun auch sowohl mit den Lehrerinnen und Lehrern als auch mit seinen Mitschülerinnen und Mitschülern gut. Dennoch fieberte er, wie er betonte, in der sechsten Erhebungswelle bereits dem Ende der Schulzeit entgegen, um endlich seine Ausbildung als Maschinenbautechniker beginnen zu können. 
2020: Helmut Pfortner - ein selbstständiger junger Mann tritt beruflich in die Fußstapfen seines Vaters

Zur siebten und abschließenden Erhebungswelle ist Familie Pfortner mit einigen tiefgreifenden Veränderungen konfrontiert. Nach dem Tod von Herrn Pfortners Mutter zu Beginn des Jahres 2020 und der zuvor anstrengenden häuslichen Pflege - „Es ist eher positiv, weil der Druck weg ist“ - und nach dem kürzlichen Tod einer Urgroßmutter, die aber woanders gelebt hatte, planen Helmuts Eltern, in absehbarer Zeit vom ersten Stock des Hauses in das bislang von Herrn Pfortners Mutter bewohnte Erdgeschoss zu ziehen, damit entweder Helmut oder seine Schwester vom Dachgeschoss in die Wohnung der Familie ziehen könnten. Beide Todesfälle thematisiert Helmut nicht, obwohl die Oma laut Frau Pfortner eine wichtige Bezugsperson war, deren Tod Helmut getroffen habe. Helmut selbst ist derzeit sehr zufrieden mit seiner kleinen Wohnung. Eine gemeinsame Wohnung mit seiner aktuellen Freundin plant er derzeit auch noch nicht, obwohl sie sich, wie er sagt, gut verstehen und schon dreieinhalb Jahren zusammen sind: „Na! Des is noch a bisserl zu friah!“ Einer der Beweggründe ist auch, dass seine Freundin zunächst noch die Schule absolvieren sollte. Helmut wohnt zur Zeit der Erhebung während der Woche als Grundwehrdiener in der Kaserne, danach wird er jedoch seine Tätigkeit als Maschinenbautechniker wieder aufnehmen. Nach dem erfolgreichen Abschluss der Ausbildung wurde er in seinem Lehrbetrieb fest übernommen und möchte sich etablieren. Aktuell plant er, dort noch fünf bis sechs Jahre zu arbeiten und dann die Meisterprüfung abzulegen. Im Anschluss möchte er den Betrieb seines Vaters übernehmen. Helmut kann sich nach wie vor auf seine Familie verlassen, bei der er noch immer ein sicheres Zuhause hat. Obwohl er im Vergleich zu den anderen Jungen im Sample noch recht jung ist, scheint er bereits im Erwachsenenleben angekommen und mit dem Start ins Berufsleben gut zurechtgekommen zu sein: $\mathrm{Zu}$ Beginn sei es zwar „etwas stressig“ gewesen, weil man, im Gegensatz zur Schule, jetzt „wirklich was leisten“ müsse und der Umgangston „etwas rauher" sei, aber daran habe er sich schnell gewöhnt, und er wolle nicht zurück in die Schule. Zu Beginn sei er nach Feierabend sehr erschöptt, „total hinig“, gewesen, aber schließlich sei er doch schnell mit dem neuen Alltag zurechtgekommen. Zu den Vorteilen des Berufslebens zählt Helmut auch die Möglichkeit, dass er sich nun von seinem eigenen Geld Wünsche erfüllen könne. Dazu zählten bereits der Führerschein und ein eigenes Auto. In Zukunft möchte er als "große Pläne“ auch den Motorradführerschein und den Anhängerschein absolvieren und die Jagdprüfung ablegen. 
Das Verhältnis zwischen Mutter und Sohn scheint konfliktfrei zu sein; Frau Pfortner weiß erstaunlich gut über die Belange ihres Sohnes Bescheid, obwohl sie selbst lachend behauptet, dass er jetzt schon groß sei und man als Mutter da nicht mehr so viel wisse. Konflikte zwischen Helmut und seinem Vater scheinen zur Zeit der siebten Erhebungswelle nicht $\mathrm{zu}$ bestehen, allerdings thematisiert Helmut ihn nicht, auch nicht im Kontext der Berufsausbildung und seiner Zukunftspläne. Seine besten Freunde aus Jugendtagen hat Helmut behalten, sein gesamter Freundeskreis sei nahezu „intakt“. Durch die Arbeit habe er aber auch zusätzliche Freunde gewonnen. Noch immer ist Helmut sehr an Sport interessiert, das professionelle Ringen hat er aus zeitlichen Gründen jedoch aufgegeben. Stattdessen hat er mit dem Kickboxen begonnen und sieht darin eine Möglichkeit, um sich „fit zu halten“. An Wettkämpfen nimmt er aber (noch) nicht teil, da er sich noch nicht kompetent genug fühlt. Über seine Freundin spricht Helmut zwar offen, jedoch berichtet er nur von sehr wenigen gemeinsamen Aktivitäten. Unter anderem koche er gerne für beide.

Auch Frau Pfortner hat sich beruflich weiterentwickelt und zwischenzeitlich ihre Arbeitszeit im Drogeriemarkt auf 30h in der Woche aufgestockt; dies sorgt für zusätzliche ökonomische Stabilität in der Familie. So war es der Familie auch möglich, die staatlich verordneten Schließungen während der ersten Corona-Welle und die damit einhergehenden Auftragseinbrüche im Familienunternehmen gut zu überstehen. Ihre Arbeitsstelle in der Drogerie möchte Frau Pfortner auf jeden Fall behalten, obwohl sie, wie sie sagt, „langsam auf die 50" zugehe, sich in einer „Umbruchsphase“ befinde und die Kinder jetzt „komplett flügge san“. Es wird sehr deutlich, dass Frau Pfortner ihre berufliche Tätigkeit und die damit verbundene finanzielle Unabhängigkeit und Sicherheit sehr zu schätzen weiß und stolz darauf ist, sich die neuen Kompetenzen erarbeitet zu haben. Zu viele Pläne wolle sie aber nicht mehr machen, da am Ende „immer alles anders“ käme.

\section{Medien im Leben von Helmut und seinen Eltern}

Familie Pfortner war zu Beginn der Studie eher knapp mit Medien ausgestattet. Zwar gab es bereits seit der ersten Erhebungswelle einen Computer mit Internetanschluss, einen funktionierenden Fernseher jedoch erst $a b$ der zweiten Erhebungswelle. Mit der Zeit wuchs der Bestand an Medien jedoch kontinuierlich an. Ab der fünften Erhebungswelle war die Familie sehr gut ausgestattet, unter anderem mit vier Fernsehgeräten, zwei Laptops, drei Smartphones und einem Tastenhandy für Herrn Pfortner. Die persönliche Medienausstattung von Helmut erschien altersgemäß, im Ver- 
gleich mit den anderen Kindern der Untersuchungspopulation aber eher unterdurchschnittlich, und er wünschte sich in der zweiten Erhebungswelle „alle Medien für sein Zimmer“. Über die Jahre wurde Helmuts Mediennutzungsverhalten komplexer und ausdifferenzierter. Sein erklärtes Lieblingsmedium war zunächst der Fernseher, ab der fünften Erhebungswelle sein Smartphone und ab der sechsten Erhebungswelle zusätzlich auch seine Playstation 3. Im Vergleich zu anderen Jungen im Sample verwendete er jedoch weniger Zeit für die Nutzung des Smartphones und die Nutzung von Computer- und Videospielen, er suchte immer stark den Ausgleich durch sportliche Betätigungen und den Zeitvertreib mit Freunden. Gerade in der fünften Erhebungswelle waren tendenziell gewalthaltige Videospiele wie GTA, Call of Duty oder Assassin's Creed allerdings von großer Bedeutung für Helmut; und er spielte diese oft gemeinsam mit Freunden. Das Smartphone war für Helmut immer ein Kommunikationsgerät, das er ab 2014 für Apps wie WhatsApp, Snapchat, Facebook und Instagram nutzte, um so Kontakt zu seinen Freunden zu halten. Neben der Kommunikation war das Smartphone zudem ein wichtiges Gerät, um Musik zu hören, zu spielen, YouTube zu schauen und ausgewählten YouTubern zu folgen und um sich Informationen zu beschaffen (Google, Wetterbericht, Info-Apps). Als einer der wenigen Jungen im Panel berichtet Helmut zudem tendenziell offen über seine Erfahrungen mit pornografischen Inhalten, die er zum Teil auch als gewalthaltig und verstörend wahrgenommen hatte. Bereits in der vierten Erhebungswelle, mit damals elf Jahren, stieß er in Facebook auf ein Video, das angeblich eine Frau beim Geschlechtsverkehr mit einer Giraffe zeigte. Er wandte sich damals, wie er erzählte, vertrauensvoll an seine Mutter, und beide meldeten gemeinsam das Video. In der fünften Erhebungswelle räumte Helmut ein, pornografische Angebote zwar bewusst, allerdings „nicht oft“ zu rezipieren, allerdings gäbe es „schon gute Sachen“. Über Freunde kam Helmut zudem mehrfach mit extrem gewalthaltigen oder „perversen“ Sex-Videos in Kontakt. In der sechsten Erhebungswelle geschah dies in einem Fall auch als Mutprobe, wie er erzählte, es ging darum, wer sich das Video am längsten ansehen konnte.

In der siebten Erhebungswelle hat sich das Verhältnis von Helmut zu Medien etwas gewandelt. Er räumt ein, dass der Stellenwert der Medien zugenommen habe, sie seien „Alltag gword'n“. Helmut thematisiert dabei weniger eine Zunahme der unterschiedlichen Angebote als vielmehr eine zeitlich intensivere Nutzung. Jedoch betont er, dass er sich weiterhin lieber richtig mit seinen Freunden träfe, als nur über das Smartphone mit ihnen zu kommunizieren. Die bei vielen Jungen beliebten Online-Games sind Helmut dagegen nicht wichtig, da er sich selbst, wie er erzählt, für einen 
sehr schlechten Online-Spieler halte. Insgesamt wäre es "nicht schlimm“ für ihn, wenn er das Handy „ned hätt“. Helmut ist aktuell bei WhatsApp, Facebook, Snapchat und Instagram angemeldet. WhatsApp nutzt er dabei in erster Linie, um sich mit Freunden zu verabreden, aber nicht um dort ausgiebig zu kommunizieren. Bei Sozialen Medien sei er insgesamt „eher der Leser“ und poste nur selten etwas auf Instagram, aber nie auf Facebook. Seine Lieblingsspiele auf der PlayStation sind God of Wars und Tomb Raider, allerdings nur im Single-Player-Modus. Zu Helmuts beliebtesten Medienangeboten zählt der Streaming-Anbieter Netflix, und dabei besonders Serien wie Breaking Bad und Prison Break. Lachend räumt er ein, zum Einschlafen auch gerne „Kindersachen“ wie Family Guy anzusehen, die er schon früher gern mochte. Lineares Fernsehen nutzt Helmut nur eingeschränkt für Dokumentationen und Sendungen auf DMAX. Neben Unterhaltungsangeboten greift er auch auf verschiedene Angebote zur Information zurück. Dazu zählen insbesondere die Lokalzeitung und eine Nachrichten-App. Facebook dient ebenfalls der Information. Helmut betont jedoch, dass man hier gerade in der Covid-19-Pamdemie aufpassen müsse, weshalb er ihm als problematisch erscheinende Informationen auch gerne überprüfe.

Frau Pfortner und ihr Mann nutzten über den gesamten Erhebungszeitraum hinweg verschiedene Medien, sowohl zur Unterhaltung als auch zur Information, wobei Frau Pfortner immer die intensivere und auch innovativere Nutzerin war. Seit dem Beginn der Studie legte sie großen Wert auf Radio, Fernsehen und das Internet, um sich zu informieren. Bereits in der zweiten Erhebungswelle bezeichnete Helmut das Internet als das Lieblingsmedium seiner Mutter. Dort nutzt sie unter anderem Facebook, Wikipedia, Google, YouTube, Amazon, ebay und Mediatheken. Neben der privaten Internetnutzung betreut Frau Pfortner aber auch die Homepage des Familienunternehmens, kommuniziert via E-Mail und nutzt das Intranet der Drogerie, in der sie arbeitet. Herr Pfortner bevorzugt dagegen die Kommunikation per Telefon. $\mathrm{Zu}$ den wichtigen Angeboten im Fernsehen zählten für Frau Pfortner über viele Jahre die Nachrichten, Serien, Talkshows, Spielfilme, Liebesfilme und, wie sie sagt, so genannte „Asselsendungen“ wie Familien im Brennpunkt beim Bügeln, während Herr Pfortner in erster Linie Dokumentationen, Wissenssendungen und Krimis bevorzugte. Beide Elternteile lasen auch immer viele Bücher, Frau Pfortner (historische) Liebesromane, historische Romane und Biografien, Herr Pfortner dagegen Sachbücher über wirtschaftliche Themen, Unternehmer-Biografien, Selbsthilfebücher, Ratgeber und Jagdzeitschriften. In den letzten beiden Erhebungswellen interessiert sich Frau Pfortner auch 
stärker für Dokumentationen; sie beklagt aber, dass diese ihr meist zu spät am Abend kämen. Früher von ihr rezipierte „Vorabendserien“ könne man sich inzwischen „sparen“, und auch die Vielzahl an politischen Talkshows lehnt sie ab. Dagegen haben Informationsangebote für sie stark an Bedeutung gewonnen. Dazu zählen unter anderem Angebote auf Facebook, die Tageszeitung, Radio- und Fernsehnachrichten und Nachrichten-Apps. Gemeinsame Fernsehabende der Familie gebe es, im Gegensatz zu früher, nun jedoch nicht mehr, da jeder seinen eigenen Fernseher habe. Wenn Frau Pfortner jedoch gemeinsam mit ihrem Mann fernsieht, dann gestaltet sich dies, wie sie erzählt, zumeist folgendermaßen: „Also, ich und mein Mann (schauen) natürlich (zusammen fern). Wobei zusammen schauen heißt eher, dass i schau und er schlaft." Zur Kommunikation unterhält die Familie eine recht aktive Familiengruppe auf WhatsApp, die Frau Pfortner sehr wichtig ist.

Fazit

Familie Pfortner bildete von Beginn der Studie an eine sozio-emotional recht stabile Kernfamilie, der es auch gelang, Konflikte innerhalb der Familie und wirtschaftlich schwierige Zeiten zu überstehen. Helmut und seine Schwester konnten sich dabei immer vertrauensvoll an ihre Eltern wenden, denen daran lag, im Rahmen ihrer über die Jahre verbesserten Handlungsoptionen, ihre Handlungsentwürfe auf die Kinder auszurichten, sie zu fördern und ihnen ein möglichst angenehmes Leben zu bieten. Dazu wurden auch kostenintensive Ausbauten des Hauses vorgenommen. Wie lange geplant und auch von Herrn Pfortner gewünscht, ist Helmut schließlich beruflich in die Fußstapfen seines Vaters getreten. Er entwickelte jedoch insofern eigene Handlungsentwürfe als er dazu jedoch den Weg über eine Ausbildung in einer anderen Firma wählte, in der er seine Handlungskompetenzen weiter ausbauen und sich zunächst auch beruflich weiterentwickeln möchte. Dieser Karriereplan wird von Herrn Pfortner akzeptiert und unterstützt, so plant er, seinem Sohn den Betrieb in einigen Jahren zu übergeben. Im Umgang mit Medien erwies sich Frau Pfortner stets als interessierter und elaborierter als ihr Mann; er beschränkte sich auf ein Tastenhandy, auf Bücher und einige wenige Fernsehsendungen. Anders als Helmuts Vater war seine Mutter vergleichsweise gut über den Mediengebrauch ihres Sohnes informiert. Im Vergleich mit vielen anderen Heranwachsenden im Panel spielten Medien bei Helmut erst recht spät eine bedeutendere Rolle - andere Freizeitbeschäftigungen, wie Sport und Freunde zu treffen, waren ihm wichtiger. Mittlerweile zählen sie allerdings zu einem festen Alltagsbestandteil. Sie dienen ihm gleichermaßen zur Un- 
terhaltung, zur Information und zur Kommunikation, wobei es Helmut, wenn immer möglich, vorzieht, sich mit seinen Freunden zu verabreden und persönlich zu treffen, statt sich nur über das Smartphone und Soziale Medien auszutauschen. Hervorzuheben ist Helmuts bewusster und reflektierter Umgang mit verschiedenen Informationsangeboten.

\subsubsection{Susanne Scheib (21 Jahre alt) und ihre ehemals alleinerziehende Mutter: Eine junge Frau mit großem Zugewinn an Handlungsentwürfen und Handlungskompetenzen}

\section{5 bis 2017}

Familie Scheib bestand zu Beginn der Erhebung aus Frau Scheib, Susanne und ihrer ein Jahr jüngeren Schwester. Die Familie lebte in einer sehr schwierigen sozialen Situation und wohnte in einer kleinen, beengt wirkenden Mietwohnung in einem Reihenhaus. Mit einer Teilzeitstelle von 15 Stunden musste Frau Scheib, gelernte Bürokauffrau, ihre Familie allein versorgen. Ihre Handlungsoptionen verbesserten sich erheblich, als Frau Scheib vor der zweiten Erhebungswelle ihren späteren Ehemann, einen in Vollzeit arbeitenden Facharbeiter, kennenlernte und mit ihm und ihren Töchtern in seine kleine Mietwohnung in eine Großstadt nach Deutschland zog. Mit diesen bedeutsamen lebensweltlichen Veränderungen verbesserte sich die sozio-ökonomische Lage für Frau Scheib und ihre Töchter gravierend. Nunmehr wohnte die Familie in einem gepflegten Wohnumfeld. Die deutlich bessere finanzielle Ausstattung bedeuteten für Frau Scheib und ihre Töchter auch einen Zugewinn an Freizeitmöglichkeiten, nun konnte die Familie es sich leisten, häufiger gemeinsam in Urlaub zu fahren. Während der vierten Erhebung wurde die Wohnung umgebaut, um ein zusätzliches Zimmer zu schaffen. Die sozio-ökonomische Lage der Familie verbesserte sich seit der neuen Ehe von Frau Scheib stetig, auch durch ihre eigene Berufstätigkeit. Zuletzt war sie in Teilzeit als Vertriebsassistentin und zudem noch geringfügig in einem Sicherheitsdienst beschäftigt.

Nach dem Umzug nach Deutschland hatte Susanne anfangs darunter gelitten, alle ihre Freundinnen aus dem Kindergarten zurücklassen zu müssen; ihre Traurigkeit darüber versuchte sie, auch mit Hilfe von Medienangeboten, zu bearbeiten, doch bald gelang es ihr, neue Kontakte zu knüpfen. Der Kontakt zum leiblichen Vater der Kinder blieb allerdings über alle Erhebungswellen hinweg bestehen, wenn sie auch damit haderte, nur selten Kontakt zu ihm zu haben. Auch das Verhältnis zu engen 
Verwandten in der Heimatstadt konnte weiter gepflegt werden. Durch die Entfernung sah sie ihren Vater jedoch nicht mehr oft. Allerdings verstand sie sich mit ihm und seiner neuen Frau recht gut. Die Lebensführung von Familie Scheib war nunmehr von Stabilität gekennzeichnet; und mit der Zeit akzeptierten Susanne und ihre Schwester den neuen Partner ihrer Mutter als Vaterfigur. Über alle folgenden Erhebungswellen hinweg erwies er sich als eine wichtige Bezugsperson für Susanne. Die neue Freundin des Vaters, die sie in der sechsten Erhebungswelle kennenlernte, lehnte Susanne ab.

Die Beziehung zwischen Susanne und ihrer Mutter erwies sich über alle Erhebungswellen hinweg als recht ausgeglichen und vertrauensvoll, Susanne schätzte es sehr, dass ihre Mutter und ihr Stiefvater für sie da waren, wenn sie sie brauchte. „Selbst, wenn er in einem abstürzenden Flugzeug sitzen würde, würde der den Fallschirm packen und zu mir runterspringen“, war Susanne in der sechsten Erhebungswelle überzeugt. An ihrem Stiefvater störte sie jedoch, „dass er immer ziemlich beschäftigt ist und eigentlich nie Zeit hat".

Vor der sechsten Erhebungswelle jedoch schien Susanne eine Krise durchlebt zu haben. Sie hatte überraschend wegen Problemen mit dem Lehrpersonal und ihren Peers die Realschule abgebrochen, obwohl Frau Scheib und auch Susanne zuvor stets von guten Noten und Freude an der Schule berichtet hatten. Susanne hatte offenbar psychische Probleme gehabt und eine Psychotherapie besucht und zählte ihre Psychotherapeutin zu ihren wichtigsten Bezugspersonen. Auch das Verhältnis zur Mutter war, möglicherweise infolge der neuen biografischen Herausforderungen, sich von den Eltern abzunabeln und Eigenständigkeit einzuüben - Susanne war mittlerweile 17 Jahre alt -, etwas getrübt; Susanne beklagte, sie würden anders als früher nunmehr sehr unterschiedlich denken.

Bei der telefonischen Nachbefragung machte Susanne, mittlerweile 18 Jahre alt, den Eindruck, ihre Krise überwunden zu haben. Susanne hatte ihren Realschulabschluss an der Volkshochschule in den Fächern Deutsch, Englisch und Mathematik erfolgreich nachgeholt und plante nun, sich dort noch weiter zu bilden, um später eine Fachoberschule zu besuchen - ein Plan, den ihre Mutter als „Trip“ einschätzte, den sie durch ihre Freunde vermittelt bekommen habe. Von der Familie habe sie dies nicht, zumal ihre Tochter „a Gschiss“ mit der Schule hatte, weil ihr „alles zu viel wurde“. 
2020: Susanne Scheib - eine aktive „autobegeisterte“ Frau in stabilen finanziellen und sozio-emotionalen Verhältnissen

In der siebten Erhebungswelle hat sich Susannes Lebensführung erneut sehr verändert. Sie hat nach Abschluss der Mittleren Reife nicht, wie ursprünglich geplant, die Fachoberschule besucht, sondern eine Ausbildung zur Einzelhandelskauffrau in einem Möbelhaus abgeschlossen. Susanne arbeitet nun am Empfang in einem Autohaus - eine Tätigkeit, die ihr gut gefällt. Mittlerweile wohnt Susanne nicht mehr zu Hause, sondern hat zunächst in einer $19 \mathrm{~m}^{2}$ großen Wohnung gewohnt, von der sie später in eine größere, $35 \mathrm{~m}^{2}$ große Wohnung umgezogen ist; mit ihren neuen Wohnverhältnissen ist Susanne nun sehr zufrieden. Seit einem Jahr führt sie, nach einigen gescheiterten kürzeren Beziehungen, eine feste Beziehung mit ihrem langjährig besten Freund, mit dem sie zuvor schon fünf Jahre nur befreundet gewesen war. Susanne hat einen Führerschein gemacht und besitzt ein eigenes gebrauchtes Auto, das ihre Eltern ihr geschenkt haben. Ihre Reparaturrechnungen bezahle sie aber selbst, wie die „autobegeisterte “ junge Frau erzählt.

In ihrer Familie fühlt sich Susanne wohl, ihren Stiefvater nennt sie nun „Papa“. Kontakt zu ihrem leiblichen Vater hat sie „eigentlich nur zu Geburtstagen“; sie zeigt sich enttäuscht darüber, dass er sich nicht öfter meldet, aber, wie sie betont, verstehen sie sich „ganz gut, wenn sie sich dann sehen“. Nicht glücklich war Susanne aber darüber, dass ihre Mutter in die Stadt in Österreich zurückgezogen ist, in der die Familie ursprünglich gewohnt hat. Susanne erzählt, ihre Mutter sei dennoch weiterhin mit ihrem Ehemann zusammen, sie komme noch regelmäßig in die gemeinsame Wohnung. Susanne glaubt, dass ihr Stiefvater auch nach Österreich ziehen werde, wenn er in Rente geht. Mittlerweile, so Susanne, ist es aber für sie „okay“, da sie ihre Mutter trotzdem noch regelmäßig sehe.

Ihre Tätigkeit als Jugendleiterin in einer kirchlichen Einrichtung hat Susanne aufgegeben, da sie nicht mehr hinter der Kirche stehe. Infolge ihres Umzugs in die eigene Wohnung habe sie, wie sie erzählt, „den Kontakt zu den dortigen Freunden verloren"; sie sähe sie noch einmal im Jahr zum Sommerfest. Abgesehen davon ist Susannes Freundeskreis stabil geblieben; hauptsächlich habe sie männliche Freunde, mit denen sie gemeinsam an ihren Autos „rumschraube“.

Susannes Mutter ist zur Zeit der siebten Erhebung als Vertriebsassistenz in Teilzeit beschäftigt; zudem arbeitet sie normalerweise nebenberuflich im Sicherheitsdienst bei Konzerten. Im Interview erzählt Frau Scheib, in ihrem Leben habe es seit der sechsten Erhebung „keinerlei Veränderungen“ gegeben, allerdings seien ihre beiden Töchter mittlerweile ausgezo- 
gen. Ihren Umzug nach Österreich in die Stadt, in der sie zu Beginn der Erhebung 2005 mit ihren beiden Töchtern gelebt hatte, und die damit zumindest räumlich bedingte Trennung von ihrem Mann erwähnt Frau Scheib erstaunlicherweise selbst nicht. Sie äußert sich im Interview vor allem sehr stolz zu ihrer Tochter, „weil sie innerhalb so kurzer Zeit so viel geschafft hat“, und Schule und Ausbildung abgeschlossen habe.

\section{Medien im Leben von Susanne Scheib und ibrer Mutter}

Zur Zeit der sechsten Erhebungswelle hatte Susanne begonnen, sich von ihrer Mutter und ihrem Stiefvater abzunabeln, im Zuge dessen veränderte sich auch Susannes Medienumgang; sie saß nunmehr, wie ihre Mutter berichtete, sieben Stunden am Tag vor dem Fernseher, „wenn sie nicht weiß, was sie mit ihrer Freizeit anfangen soll“. Generell war die Bedeutung von Medien für Susanne schon ab der fünften Erhebungswelle wieder deutlich angestiegen. Neben dem Fernseher wurde das Smartphone zu einem wichtigen Begleiter; Susanne nutzte es vor allem für die Kontaktpflege in Sozialen Netzwerken. Das Fernsehen ist nach der Zeit des sehr intensiven Gebrauchs längst in den Hintergrund gerückt; zur Zeit der siebten Erhebung nutzt Susanne ihr Fernsehgerät nur noch für Netflix, zumeist zum Anschauen von amerikanischen Fernsehserien wie Lucifer, Lost und Riverdale, aber auch Dokumentationen, gemeinsam mit ihrem Freund. Ihr Smartphone ist Susanne dagegen für WhatsApps, E-Mails und fürs Onlinebanking, vor allem aber zum Telefonieren, noch wichtiger geworden. Susanne liest auch Bücher, allen voran Thriller und Kriminalromane. Zeitung liest sie nun aber nicht mehr. Ihren Mediengebrauch schätzt Susanne selbst als „mittel“ ein; sie finde Medien vor allem für die Kommunikation sehr wichtig, aber auf "des ganze Umeinandergeposte“ in Sozialen Netzwerken, wie Facebook (dies nutzt Susanne meistens morgens und abends, eher „schon eine automatisierte Handlung“) und Instagram, das ihr früher sehr wichtig war, und auch darauf, dass sie seinerzeit hohe Likes und Follower-Zahlen erreichte, könne sie im Prinzip verzichten. Ihre Freunde wüssten „eh Bescheid über ihr Leben“, dann brauche sie das dort nicht zu posten. Susanne zieht dieser Kommunikationsform denn auch, wie sie sagt, das Telefonieren vor, denn beim Schreiben könnten eher Missverständnisse entstehen. Fernsehen und Spielen auf der Playstation sieht sie heute als „Zeitverschwendung“.

Susannes Mutter nutzt ein breites Medienrepertoire, allen voran ihr Handy, das aus ihrem Alltag nur noch schwer wegzudenken sei. Gerade für Kommunikation sei es für sie „unabdinglich“, weil das gesamte Umfeld auf Medien zur Kommunikation zurückgreife. Frau Scheib schätzt 
ihren Mediengebrauch eher als gering ein, gibt jedoch zu bedenken, dass dies für ihr Handy nicht zutreffe; dies sei für WhatsApp, Telegram, Onlineshopping und verschiedene Internetrecherchen sehr wichtig. Facebook spielt schon seit zwei Jahren gar keine Rolle mehr für sie, angemeldet sei sie jedoch noch. Zum Musikhören nutzt Frau Scheib gern auch das Radio, auch um nebenbei Nachrichten mitzubekommen. Der Computer sei ihr nur auf der Arbeit wichtig. Bücher liest Susannes Mutter zur Unterhaltung und zur Information, zur Zeit der Erhebung gerade das Buch Das kann doch weg. Das befreiende Gefühl mit weniger zu leben. 55 Tipps für einen minimalistischen Lebensstil.

\section{Fazit}

Susanne Scheibs Leben war in der ersten Erhebungswelle noch stark durch begrenzte Handlungsoptionen der Familie geprägt; die Familie verfügte nur über geringe finanzielle Möglichkeiten, da ihre Mutter sie und ihre jüngere Schwester allein erziehen und ernähren musste. Nach dem Umzug ihrer Mutter zu ihrem neuen, finanziell besser gestellten Lebensgefährten in eine deutsche Großstadt schon vor der zweiten Erhebungswelle hat es sich grundlegend verändert. Durch das Zusammenleben mit dem neuen Mann ihrer Mutter, den sie mittlerweile, wie ihren leiblichen Vater, zu dem sie aber nur noch unregelmäßig Kontakt hat, „Papa“ nennt, haben sich die Handlungsoptionen und Handlungsentwürfe der gesamten Familie deutlich erweitert. Susanne gelingt es - trotz einiger Schwierigkeiten in der Schule und in dem Zusammenhang auch psychischen Problemen zwischen der fünften und sechsten Erhebungswelle -, ihren Weg zu gehen. Sie hat mittlerweile beruflich - sie arbeitet als „autobegeisterte“ junge Frau in einem Autohaus - und privat - sie hat seit einem Jahr einen festen Partner - Fuß gefasst und bewältigt, auch im Umgang mit Medien, ihren Alltag alles in allem recht handlungskompetent. Mit ihrer Familie versteht sich Susanne weiterhin gut, auch mit ihrer Mutter, die vor der siebten Erhebungswelle wieder zurück nach Österreich gezogen war, aber dennoch engen Kontakt zu ihrem noch in einer deutschen Großstadt lebenden Mann und ihren Kindern pflegt. 
2.5.5 Norbert Zarbl (20 Jahre alt) und seine Familie: In einer funktionalen Patchwork-Familie verbesserten sich die sozio-ökonomischen Bedingungen

2005 bis 2017

Norbert und sein jüngerer Bruder Lukas waren zu Beginn der Studie fünf beziehungsweise dreieinhalb Jahre alt. Im Vergleich zu anderen Kindern im Sample sind sie unter stabilen sozio-emotionalen Bedingungen aufgewachsen. Auch die sozio-ökonomischen Bedingungen der Familie erwiesen sich in allen Erhebungswellen als weitestgehend gesichert. Herr Zarbl war zunächst als Versicherungsangestellter tätig, später arbeitete er in den Sommermonaten als Skipper. Ab der zweiten Erhebungswelle 2007 steigerte sich das Familieneinkommen, da Frau Zarbl ihre geringfügige Beschäftigung als Bürokraft ausweitete und später in Teilzeit (30h) als kaufmännische Angestellte arbeitete. Herrn und Frau Zarbl gelang es durch die Abendmatura, ihre Handlungskompetenzen weiter auszubauen; danach verfügten sie über die höchsten Schulabschlüsse unter allen befragten Eltern. ${ }^{22}$ Konkrete Ziele oder Pläne im Zusammenhang mit der erworbenen Weiterqualifikation nannte Frau Zarbl in den Gesprächen jedoch nie.

Die Familie wohnte über den gesamten Erhebungszeitraum hinweg in einem eigenen Haus in der Stadt, das stets in sehr gutem Zustand und mit neuen Möbeln eingerichtet war. Norbert hatte immer ein eigenes Zimmer, das in der sechsten Erhebungswelle von einem Kinderzimmer in ein Jugendzimmer umgestaltet wurde. Ebenso wie das eigene Haus und dessen Ausstattung, etwa ein Fitnessraum im Keller, wurde auch die Wohnregion von allen Familienmitgliedern immer geschätzt, wobei besonders die Parks und Freizeitmöglichkeiten, aber auch die Vielzahl an Freundinnen und Freunden in der Umgebung positiv hervorgehoben wurden.

Zwischen der zweiten und der dritten Erhebungswelle trennte sich Frau Zarbl von ihrem Ehemann. Obwohl beide Elternteile darum bemüht waren, die Trennung einvernehmlich zu gestalten, war das Familienklima zu dieser Zeit belastet. Norbert litt unter den Spannungen und äußerte sich in den Interviews nur sehr ungern und zurückhaltend dazu. Der Kontakt zum leiblichen Vater beschränkte sich nach der Trennung auf monatliche

22 Ein Einschlusskriterium für die Studie war eine niedrige formale Bildung der Eltern. Dies war auch bei Familie Zarbl gegeben, da zum Zeitpunkt der Auswahl beide noch nicht über die Matura verfügten und den angestrebten Abschluss auch nicht erwähnten. Aufgrund der Lebensbedingungen wurde die Familie ins Panel aufgenommen. 
Besuche im Winter, da der Vater im Sommer aufgrund seiner Tätigkeit nicht in Österreich war. Neben unregelmäßigen Treffen bei Familienfeiern gab es aber auch zusätzliche Unternehmungen des Vaters mit seinen Söhnen (Essen, Urlaubstörns, Bogenschießen). In der vierten Erhebungswelle besuchte Norbert seinen Vater regelmäßig alle 14 Tage, beide verstanden sich damals gut und unternahmen gemeinsam verschiedene Dinge (Bogenschießen, Fernsehen, Gesellschaftsspiele). Später gab es zwar keinen Streit, jedoch dünnte der Kontakt immer weiter aus, was der Vater gegenüber seiner Ex-Frau auch bedauerte. Ein Grund lag in dem Umstand, dass Norbert sich mit seinem Stiefvater von Beginn an gut verstand und dieser sich zu einer immer wichtigeren Bezugsperson und zu einem Ansprechpartner bei Fragen und Problemen entwickelte. Bereits in der dritten Erhebungswelle hatte Frau Zarbl einen neuen Lebensgefährten, der eine erwachsene Tochter und einen erwachsenen Sohn mit in die Beziehung brachte. Norbert verstand sich mit beiden gut, besonders mit seiner Stiefschwester. Zwischen der vierten und fünften Erhebungswelle heiratete Frau Zarbl ihren Lebensgefährten, dieser nahm dabei ihren Namen an und zog in das Haus der Familie. Er nahm seine Vaterrolle immer aktiv an und unternahm viel mit Norbert und Lukas, etwa sportliche Freizeitbeschäftigungen, Ausflüge, Fernsehabende und das gemeinsame Schrauben an einem Moped. Norbert schätzte an seinem Stiefvater insbesondere dessen Sportlichkeit und Ehrgeiz sowie dessen technische Fähigkeiten und Humor. Seine Sportbegeisterung machte sich Herr Zarbl später auch beruflich zu Nutze. Von der dritten bis zur sechsten Erhebungswelle arbeitete er in Vollzeit als OP-Pfleger im Krankenhaus, danach reduzierte er dort seine Stundenzahl und machte sich als Gesundheits- und Fitnesstrainer zeitweise selbstständig.

Eine wichtige und zudem beständige Bezugsperson für Norbert war über den gesamten Zeitraum der Studie hinweg sein jüngerer Bruder, mit dem er auch immer viel unternahm, sportliche Aktivitäten, Raufen, PlayStation spielen, Freunde treffen etc. Dennoch war das Verhältnis stets von Spannungen und Rivalitäten gekennzeichnet, gerade da der Altersunterschied nur sehr gering war. Norbert übernahm klar die Rolle des großen Bruders und dominierte den Jüngeren. Frau Zarbl beschrieb das Verhältnis der beiden wie folgt: "Sie lieben sich, so wie sie sich auch hassen." Jedoch berichtete Norbert in allen Interviews von gemeinsamen Unternehmungen und darüber, dass er sich gut mit seinem Bruder verstünde.

In der Schule tat sich Norbert leicht, und er hatte keine Probleme mit seinen Leistungen, wenn er sich auch teilweise nur schwer motivieren konnte zu lernen. Von Beginn an begeisterte er sich für technische Fä- 
cher (Elektronik und Technisches Werken, Informatik, Mathematik) und gehörte dort zu den Besten. Ab der dritten Erhebungswelle besuchte er eine Neue Mittelschule, ab der fünften Erhebungswelle ging er auf eine Höhere Technische Lehranstalt (HTL) mit den Schwerpunkten Elektronik und Technische Informatik. Zu diesem Schulwechsel hatten ihm seine Lehrer geraten, und auch seine Eltern unterstützten Norbert auf diesem herausfordernden Bildungsweg, obwohl sich der größere Lernaufwand und zum Teil seine fehlende Motivation als Schwierigkeiten erwiesen. In der sechsten Erhebungswelle strebte Norbert an, die Schule drei Jahre später mit der Matura erfolgreich abzuschließen. Mit seinen Klassenkameraden verstand er sich dabei immer gut, engere Freundschaften entwickelten sich dort aber kaum. Als Peer-Group-Beziehungen waren ihm immer die Freundschaften in seinem Wohnviertel und auch der enge und freundschaftliche Kontakt mit seinen Cousins wichtiger.

\section{0: Norbert Zarbl - ein behüteter Junge bricht in neue Selbstständigkeit auf}

Auch in der siebten Erhebungswelle erscheint das Leben der Familie sozioemotional sehr stabil. Noch immer leben Norbert und Lukas mit ihrer Mutter und ihrem Stiefvater in einem gemeinsamen Haus. Dennoch kam es zu einigen tiefgreifenden Veränderungen und Neuerungen, welche die Familie herausgefordert haben, die sie aber als Gemeinschaft stemmen konnte. So hatte sich Herr Zarbl nach der sechsten Erhebungswelle erfolgreich als Gesundheits- und Fitnesstrainer selbstständig gemacht und in diese Unternehmung größere Summen investiert. 2020 sah er sich jedoch aufgrund der globalen Covid-19-Pandemie gezwungen, die Selbstständigkeit aufzugeben, um Verluste zu vermeiden. Finanzielle Verwerfungen konnten abgewendet werden, da Herr Zarbl seinen Nebenjob im Krankenhaus aufstocken konnte und dort wieder in Vollzeit arbeitet. Auch Norbert ist von den Folgen der Pandemie betroffen, da er einerseits von zu Hause aus arbeiten muss und sich andererseits in Kurzarbeit befindet. Nach der Matura hatte er zunächst den Wehrdienst abgeleistet und war danach „vergleichsweise einfach“, wie er erzählte, in das Berufsleben gestartet. Als Absolvent einer HTL konnte er zwischen den Angeboten verschiedener Arbeitgeber wählen. Er begann schließlich in der Abteilung für Entwicklung eines Automobilzulieferers und ist mit dieser Entscheidung sehr zufrieden. Durch seine Ausbildung hat er die entsprechenden Handlungskompetenzen erworben, um sich im Unternehmen zu etablieren und dort nun auch seine weiteren Karriereschritte zu planen. Gerade in der Fokussierung auf Forschung und Entwicklung sieht Norbert eine sichere Zukunftsperspektive, und er möchte auch „Verantwortung übernehmen“, 
„vielleicht amal für a paar Projekte“. Sowohl mit seinen Arbeitskollegen als auch mit seinem direkten Vorgesetzten versteht er sich sehr gut und pflegt auch in der Freizeit Kontakt. Insgesamt gefällt ihm das anstrengende Arbeitsleben deutlich besser als die Schule, und wie auch zuvor könne er sich nach Feierabend weiterhin mit seinen Freunden treffen. Obwohl er aufgrund der Kurzarbeit aktuell finanzielle Einbußen hinnehmen muss, betrachtet Norbert seine Situation insgesamt sehr positiv, da er nun mit seinem eigenen Gehalt deutlich mehr Geld zur Verfügung hat als noch als Schüler. Dabei spielt es jedoch auch eine große Rolle, dass Norbert bislang noch mietfrei zu Hause wohnt und somit keinen eigenen Hausstand unterhalten muss. Dies ermöglicht es ihm, sich auch größere Wünsche problemlos erfüllen zu können, dazu zählen unter anderem der Erwerb eines eigenen Autos und eines Motorrades. Doch auch diese Situation soll sich bald ändern: Seit einigen Jahren ist Norbert mit seiner Freundin zusammen, nun wollen sie zusammenziehen und suchen aktuell eine gemeinsame Wohnung in der Stadt. Dies ist für Norbert "ein großer Schritt“, da zusammenziehen „nochmals etwas ganz anderes als die Beziehung an sich" sei. Auch bei diesem Schritt, gewünscht ist eine Zwei-Zimmer-Wohnung mit etwa $65 \mathrm{~m}^{2}$, kann Norbert sich auf die Unterstützung durch seine Familie verlassen. Frau Zarbl gibt an, dass sie und ihr Mann es sich leisten können und wollen, Norbert und seine Freundin sowohl bei der Miete als auch bei der Einrichtung der Wohnung zu unterstützen. Frau Zarbl verknüptt mit dem Auszug aber auch Wünsche für ihre eigene Zukunft gemeinsam mit ihrem Mann. Nach den zuletzt beruflich und durch die Kindererziehung turbulenten und anstrengenden Zeiten wolle sie nun mehr Zeit mit ihm verbringen. Frau Zarbl umschreibt dies als die „Freiheiten“ um die „Elternbeziehung“ mit ihrem Mann zu genießen. Dabei denkt sie besonders an gemeinsame Aktivitäten beim Sport und in der Natur sowie den Besuch kultureller Veranstaltungen am Abend und an Wochenenden. Somit macht Frau Zarbl zum ersten Mal eigene Ziele und Pläne deutlich, die sie in Zukunft verfolgen möchte.

\section{Medien im Leben von Norbert und seiner Familie}

Die gesamte Familie war in allen Erhebungswellen immer sehr gut und umfangreich mit Mediengeräten ausgestattet und machte von diesen Angeboten auch Gebrauch. Dazu zählten gleichermaßen Unterhaltungsangebote wie Fernsehserien, Filme, Computer- und Videospiele, Bücher und Musikangebote (Musikboxen, Radio, MP3-Player, Schallplattenspieler) wie auch Informationsangebote, unter anderem ein Zeitungsabonnement, später ein Online-Abonnement, Nachrichtensendungen, Nachrichten-Apps 
und verschiedene andere Informationsangebote im Internet. Wichtige Geräte, zum Teil auch gemeinschaftlich genutzt, waren CD-Player und Stereoanlage, DVD-Player und Blu-Ray-Player, sowie Computer und iPad. Norbert durfte Medien vergleichsweise umfangreich nutzen, jedoch wurde er dabei, deutlich stärker als andere Heranwachsende im Panel, auch aktiv von seinen Eltern begleitet und angeleitet, die zur Verfügung stehenden Angebote auch als Informationsquellen zu nutzen. Über alle Erhebungswellen hinweg räumte Frau Zarbl Medien einen hohen Stellenwert ein. Bereits in der zweiten Erhebungswelle hob sie die Relevanz des Internets als Informationsquelle hervor und betonte, wie wichtig die Auseinandersetzung mit technischen Neuerungen sei, nicht zuletzt als Qualifikation für das Berufsleben. Darüber hinaus lobte sie auch etwa die entspannende Wirkung des Fernsehens auf die Kinder und noch in der fünften Erhebungswelle betonte sie die gemeinschaftsstiftende Funktion von PlayStation und DVDs innerhalb der Familie. Jedoch stellte Frau Zarbl in der Medienerziehung auch viele und zum Teil strenge Regeln auf, die Norbert oft verwirrten. Bis zur fünften Erhebungswelle gab es auch Verbote und Kontrollen der Inhalte, und Frau Zarbl und ihr Mann kontrollierten einerseits das verbrauchte Datenvolumen und setzten andererseits Filtersoftware ein, um pornografische und gewalthaltige Inhalte zu blockieren. So überrascht es, dass gerade in Familie Zarbl das Smartphone vergleichsweise früh eine wichtige Rolle für die gesamte Familie spielte: WhatsApp fand Verwendung, um zu kommunizieren, Informationen und unterhaltende Inhalte auszutauschen und um Termine zu koordinieren. Darüber hinaus entschied man sich, die abonnierte Tageszeitung durch eine kostenpflichtige App auf den Smartphones der Familie zu ersetzen. Neben dem Elternhaus hatten auch die Schule und die dort zu absolvierenden Aufgaben großen Einfluss auf die Mediennutzung von Norbert und auf seinen Erwerb von Medienkompetenz. Er hatte Informatikunterricht und musste zu Hause sowohl programmieren als auch generell seine Hausübungen zum Teil am Computer erledigen. Zudem nutzte Norbert über viele Jahre intensiv das Internet, um Informationen für die Schule zu recherchieren (Google, Wikipedia) und verwendete dabei auch Angebote wie Übersetzungsprogramme.

Erst ab der sechsten Erhebungswelle, und damit deutlich später als bei anderen Jungen im Panel, lief bei Norbert das Smartphone dem Laptop den Rang als wichtigstes Mediengerät ab. Er nutze es, wie er erzählte, um Musik auf YouTube zu hören, mit Freunden zu kommunizieren und für gratis Online-Spiele, aber auch um zu recherchieren, die Nachrichten-App des ORF zu nutzen, online ein Moped zu kaufen und um pornografische 
Inhalte zu rezipieren. Auch Soziale Medien verwendete Norbert, wobei der Chat mit Freundinnen und Freunden sowie der Austausch von Videos, Bildern und Audiodateien die zentralen Motive waren. Nach Facebook und Twitter legte er später in erster Linie Wert auf Instagram und Snapchat. Im Fernsehen bevorzugte Norbert in erster Linie Angebote aus dem Bereich Action (James Bond, Top Gear, Fluch der Karibik) sowie Sport und Comedy (Two and a half Men, Simpsons), die er auf den Sendern von Sky, DMAX und ProSieben fand. Auch bei Videospielen bevorzugte er Action und spielte neben FIFA in erster Linie Call of Duty: Modern Warfare 3 und Black OPS2 sowie GTA.

In der siebten Erhebungswelle hat sich Norberts Mediennutzung erneut weiterentwickelt. Sowohl das Smartphone als auch seinen Computer nutzt er nun nicht mehr nur privat, sondern intensiv auch für die Arbeit. Er besitzt zwar einen eigenen Fernseher und eine PlayStation 4, die Intensität der Nutzung habe aber etwas nachgelassen, gleiches gilt auch für Facebook und Snapchat. Instagram, Netflix und Amazon Prime spielen dagegen inzwischen eine größere Rolle, wobei Norbert auch gerne Streaming-Angebote gemeinsam mit seiner Freundin nutzt. Auch mit seinem Bruder spielt er noch immer gemeinsam an der Konsole, besonders FIFA und Shooter wie Call of Duty. Hinsichtlich seiner Nutzung von Informationsangeboten unterscheidet sich Norbert deutlich von den anderen jungen Erwachsenen im Panel, und es liegt der Schluss nahe, dass dies auch auf seine Schulbildung zurückzuführen ist. Er greift auf ein breites Repertoire an Informationsangeboten zurück (Tageszeitung, verschiedene Nachrichten-Apps, Radio- und Fernsehnachrichten, zusätzliche Recherchen) und legt dabei ausdrücklich Wert auf zuverlässige Angebote, die er insbesondere bei den Angeboten des öffentlich-rechtlichen ORF verortet. Darin unterscheidet er sich sehr von seiner Mutter, die Informationen inzwischen verstärkt auch in Sozialen Medien-Angeboten sucht. Besonders Facebook hat sich für Frau Zarbl zu einem wichtigen Informations- und Kommunikationskanal entwickelt. Zudem schaut auch sie die Fernsehnachrichten, könnte „aber auf das Fernsehen verzichten". Wichtiger sind ihr inzwischen StreamingAngebote, besonders Netflix und Serien zur Entspannung.

\section{Fazit}

Familie Zarbl gehörte von Beginn an zu den Familien mit vergleichsweise größeren Handlungsoptionen im Panel; ihr gelang es, über die Jahre hinweg, diese sozio-ökonomisch stabilere Stellung zu behaupten und auszubauen. Dies ermöglichte es der Familie, gemeinsame Handlungsentwürfe $\mathrm{zu}$ entwickeln und gemeinsam Ziele zu verfolgen, Krisensituationen zu 
überstehen und die Kinder nicht nur sozio-emotional begleitend, sondern auch finanziell zu unterstützen. Auch die sozio-emotionalen Bedingungen waren und blieben weitgehend stabil, wobei allerdings die Trennung und Scheidung der Eltern einen tiefen Einschnitt markierte. Jedoch gelang es der Patchwork-Familie gut zusammenzuwachsen. Dieser Erfolg beruht auch darauf, dass der Stiefvater seine Rolle aktiv angenommen und sich das Vertrauen von Norbert und seinem Bruder erarbeitet hat. Auch vor dem Hintergrund der eigenen Matura förderte Frau Zarbl Norberts schulische Ausbildung, und er konnte sowohl die Matura als auch seine technische Ausbildung erfolgreich absolvieren. Dieses Vorgehen erwies sich als zentrale Weichenstellung für den Ausbau seiner Handlungsentwürfe und Handlungskompetenzen und damit den erfolgreichen Einstieg in das Berufsleben in einer verantwortungsvollen Tätigkeit, die ihm zudem auch Perspektiven für die Zukunft bietet. Bereits als Kind und Jugendlicher hatte Norbert Zugang zu verschiedenen Medienangeboten. Seine Mediennutzung wurde aber über eine lange Zeit hinweg von seinen Eltern nicht nur kontrolliert, sondern teilweise auch durch Verbote und technische Hilfsmittel eingeschränkt. Infolge dessen wendete er sich beispielsweise erst später als andere Heranwachsende im Panel Videospielen zu. Seine Eltern begleiteten Norbert im Umgang mit Medien, und auch in der Schule wurde Wert auf Vermittlung von Medienkompetenz gelegt. All dies spiegelt sich nun in seinem Mediengebrauch als junger Erwachsener wider: Auch Norbert nutzt verschiedene Medienangebote, wie Streaming und Videospiele, zur Unterhaltung und setzt mit seinen Freunden auf mediale Kommunikation. Ihm bereitet es auch keine Schwierigkeiten, Computer und Internet vielfältig in seinem beruflichen Kontext $\mathrm{zu}$ verwenden und auch für anspruchsvollere Anwendungen zu nutzen. In seinem reflektierten Umgang mit Informationsangeboten unterscheidet sich Norbert aber sehr deutlich von den meisten anderen jungen Erwachsenen im Panel, aber auch von seiner eigenen Mutter. 


\section{Fallübergreifende Entwicklungen im Panel - Ergebnisse der fokussierten Analyse}

Die siebte Erhebungswelle wurde im Frühjahr und Frühsommer 2020 durchgeführt und somit drei Jahre nach der telefonischen Nachbefragung der sechsten Erhebungswelle im Jahr 2017. Durch diesen zeitlichen Abstand war es möglich, nicht nur die Ereignisse und Erlebnisse der Lebensphase Jugend vollständig zu erfassen, sondern auch den Übertritt der Heranwachsenden ins junge Erwachsenenalter und ihre damit verbundenen Herausforderungen und Entwicklungsaufgaben. Dazu zählen in erster Linie die erforderlichen Weichenstellungen für die berufliche Zukunft: Abschluss der Schule und Ausbildung, Einstieg ins Berufsleben oder Übertritt in den tertiären Bildungssektor. Aber auch persönliche Belange wie die Loslösung vom Elternhaus, der Bezug einer eigenen Wohnung und die Begründung von Partnerschaften. Eng mit diesen Entwicklungen verbunden sind mögliche Veränderungen ${ }^{23}$ der Peer-Group-Beziehungen, der präferierten Freizeitaktivitäten, des Mediengebrauchs und der bevorzugten Angebote sowie von Interessen und Einstellungen. In der fokussierten Analyse in Kapitel 3 gilt der Blick den fallübergreifenden Entwicklungen; ausführliche Fallbeschreibungen zu den einzelnen Heranwachsenden und ihren Familien finden sich dagegen in Kapitel 2 in diesem Band.

In einem ersten Schritt werden dazu die Ergebnisse hinsichtlich der Unterschiede zwischen den Geschlechtern betrachtet und dabei auch etwaige Zusammenhänge mit den jeweiligen Zugehörigkeiten der jungen Erwachsenen zu den Familientypen untersucht. Mit Blick auf den Mediengebrauch ist eine solche Zuordnung jedoch nicht möglich, da sich dieser bereits bei der Erstellung und Überarbeitung der Familientypen als zu

23 Stephan Niemand spricht in diesem Zusammenhang von „Alltagsumbrüchen (Transitionen)“; dabei handelt es sich um „ein vielschichtiges Ereignis, das unmittelbar in mehreren Dimensionen der alltäglichen Lebensführung tiefgreifende Umstrukturierungen erforderlich macht" (Niemand 2020: 68). In seiner Studie zu Alltagsumbrüchen und Medienhandeln bei Paaren kommt Niemand zu dem Schluss, dass Alltagsumbrüche das häusliche Medienhandeln dynamisieren. Er stellt fest, dass „technikdeterministische Annahmen, in denen die Technologie als alleinige Ursache für Wandel angesehen wird, (...) zu kurz greifen, denn die Veränderungen des Medienhandelns der Paare ist vorwiegend auf Veränderungen in ihrer Lebensführung zurückzuführen“ (Niemand 2020: 263). 
individuell geprägt präsentiert hat. In einem weiteren Schritt wird - ebenfalls mit Blick auf die Zugehörigkeit zu den Familientypen - der Blick auch auf die Eltern der jungen Erwachsenen und ihre Bedingungen gerichtet: Wie haben sich jeweils ihre Handlungsoptionen, Handlungsentwürfe und Handlungskompetenzen entwickelt, und wie sieht die Lebensführung der Eltern in der erfassten Umbruchphase ihrer Kinder zwischen Jungend und Erwachsenenleben aus? Welche sozio-ökonomischen und sozio-emotionalen Strukturen kennzeichnen nunmehr ihren Alltag und wie sehen ihre jeweiligen Alltags-Bewältigungsstrategien und Zukunftsperspektiven aus? In diesem Schritt gilt die Aufmerksamkeit auch den Strategien des Mediengebrauchs der Eltern. Welche Veränderungen lassen sich identifizieren und mit welchen lebensführungsbezogenen Veränderungen hängen sie jeweils zusammen?

\subsection{Schule, Ausbildung, Studium und erste Arbeitsstelle - der Eintritt in die berufliche Zukunft}

2020 sind alle Teilnehmerinnen und Teilnehmer im Panel volljährig und zwischen 19 und 21 Jahren alt. Somit haben sie die Pflichtschulzeit absolviert und können nun über ihre weitere Ausbildung oder den Einstieg ins Berufsleben entscheiden. Mit nur einer Ausnahme haben 16 der 17 Befragten zumindest die Pflichtschule erfolgreich abgeschlossen; lediglich Timo Landinger hat zwar die Pflichtschulzeit absolviert, die Schule dann jedoch ohne Abschluss verlassen. Nach mehreren erfolglosen Versuchen der dauerhaften Etablierung in einer entsprechenden Einrichtung, wartete er 2020 auf den bereits zugesagten Neustart in einer geschützten Werkstätte im näheren Umkreis seines Elternhauses. Ihm war es nicht gelungen, sich aus seiner Perspektivlosigkeit zu befreien. Wenn sich auch das Verhältnis seiner Eltern untereinander zuletzt verbessert zu haben schien, so sind die Familienstrukturen, gerade mit Blick auf Timo, noch immer schwer belastet (Typ 1). Seine Eltern haben den Wunsch nach einer, wie sie es ausdrücken, „gewöhnlichen“ Lehre für ihren Sohn noch immer nicht gänzlich aufgegeben. Jedoch fehlen Timo dazu nicht nur die kognitiven Voraussetzungen, sondern auch die formalen (Schulabschluss). Seine Eltern engagieren sich zudem nicht bei der Suche nach einer entsprechenden Lehrstelle und bringen sich auch nicht aktiv bei der Suche nach einer für Timo und seine Bedürfnisse passenden Stelle in einer geschützten Werkstätte ein. Sie überlassen es vielmehr den entsprechenden staatlichen Stellen, sich um eine berufliche Verankerung ihres Sohnes zu kümmern. 
Diese Verhaltensweise erstaunt, da Herr Landinger in der Vergangenheit stets vehement gegen staatliche Aktivitäten opponiert hatte.

Mit Blick auf das Gesamtpanel fällt auf, dass die jungen Menschen mehrheitlich mittlere Schulabschlüsse angestrebt und auch erreicht haben: Von den jungen Frauen absolvierten Isabelle Rohringer und Elisabeth Ebner die Hauptschule, Simone Stab eine berufsbildende Bildhauerschule. Susanne Scheib besuchte eine Realschule (in Deutschland) und Viktoria Öllinger eine Handelsschule; sie erreichten somit wirtschaftlich orientierte Abschlüsse.

Fünf der jungen Männer (Alfons Weiss, Helmut Pfortner, Manfred Oblinger, Benedikt Holzner und Erich Grubert) verfügen über einen Abschluss der Hauptschule bzw. der Neuen Mittelschule, und Erich Grubert absolvierte eine Polytechnische Schule, ein berufsvorbereitender Schultyp in Österreich, der der Absolvierung der Unterrichtspflicht bis zum Ende des 15. Lebensjahres dient und der ebenfalls zum Pflichtschulabschluss führt. Auch zwei der jungen Männer erwarben einen wirtschaftlich orientierten Schulabschluss, Torsten Kaiser an einer Handelsschule in Österreich, Gregor Boll an einer Realschule in Deutschland. Manfred Oblinger besuchte zwar kurzzeitig ebenfalls eine Handelsakademie, verließ diese jedoch nach wenigen Monaten. Gregor Boll und Torsten Kaiser entschieden sich gegen einen weiteren Schulbesuch und stattdessen für den schnelleren Eintritt ins Berufsleben; ihr Ziel war es, "Geld zu verdienen“ (Gregor Boll). Damit stehen auch diese beiden jungen Männer exemplarisch für eine Entwicklung im gesamten Panel, denn in früheren Erhebungswellen wurde von vielen Heranwachsenden noch der Wunsch geäußert, weiterführende Schulen zu besuchen, Matura oder Abitur anzustreben und später an eine Universität zu wechseln. Auch gerade bei diesen beiden Jungen wurde in der Vergangenheit immer wieder über eine höhere Schulbildung gesprochen, und beide Mütter unterstützten diese Bestrebungen nachdrücklich. Familie Boll und Familie Kaiser zählen zu den sozio-emotional weniger belasteten, relativ kompetenten Familien, die jedoch sozio-ökonomisch eingeschränkt waren (Тур 3). In beiden Fällen hat sich zwar die sozio-ökonomische Lage in den letzten Erhebungswellen verbessert, allerdings waren die Mütter auch vorher schon, und unabhängig davon, bereit, in die Schulbildung ihrer Söhne zu investieren; es waren letztendlich die beiden Söhne selbst, die sich gegen eine weitere Schulausbildung entschieden. Mit Norbert Zarbl hat lediglich einer der jungen Erwachsenen aus dem Panel zum Zeitpunkt der siebten Erhebungswelle die Matura erfolgreich absolviert. Dieses Ziel hatte die Familie (Typ 4) bereits seit vielen Jahren verfolgt; ihre damit verbundenen Hoffnungen auf 
eine entsprechende Arbeitsstelle haben sich erfüllt. Norbert selbst betonte in seinem letzten Interview, dass er sich die "Arbeitssuche schwieriger vorg'stellt hätt, muss i sag'n, also is eigentlich relativ guad ganga“ und er mit seinem Abschluss an einer Höheren Technischen Lehranstalt (HTL) „einige Vorstellungsgespräche gehabt hat, bei viel Firmen und hab' ma da eigentlich gut aussuchen kenna, wo i jetzt mach'n mecht". Norbert hat sich schließlich für den Einstieg bei einem Automobilzulieferer entschieden und arbeitet dort in der Abteilung für Forschung und Entwicklung; in Zukunft will er sich im Unternehmen weiterentwickeln und auch Projektverantwortung übernehmen.

Von den jungen Frauen absolvierte zudem Gudrun Dornbacher während der siebten Erhebungswelle die Oberstufe einer Allgemeinbildenden Höheren Schule (AHS); auch sie wurde dabei von ihren Eltern (Typ 4) unterstützt. Nachdem sie bereits ein Vorstudium als Konzertflötistin an der Musikhochschule besucht hatte und sich dies lange Zeit auch als Beruf vorstellen konnte, tendierte sie zuletzt jedoch zu einem Studium der deutschen Literatur oder der Kunstwissenschaft. Amelie Aufbauer hatte bereits als Kind den Wunsch geäußert „gern mindestens zwoa Doktortitel“ zu haben, mit dem damit verbundenen Prestige assoziierte sie nicht zuletzt sozialen Aufstieg. Und auch als junge Erwachsene bestätigte sie: „Oiso an hätt' i nach wie vor gern." Um dies zu erreichen, besuchte sie zuletzt das Abendgymnasium, um nach einem zwischenzeitlichen Abbruch der Schule die Matura zu erwerben. Ihre Mutter bestätigte diese Pläne und berichtete, dass ein Medizinstudium das Ziel ihrer Tochter sei. Auffällig in diesem Zusammenhang ist, dass sowohl Norbert Zarbl als auch Gudrun Dornbacher aus Familien des Typs 4 stammen, den relativ kompetenten >Aufsteigern $<$ Diese Familien zeichnen sich dadurch aus, dass sie keine angespannten sozio-ökonomischen Bedingungen mehr haben und ihre sozio-emotionalen Beziehungsstrukturen unbelastet sind. Ebenso wie bei Norbert Zarbl stand es auch bei Familie Dornbacher immer außer Frage, Gudrun auf ihrem Weg zu höherer formaler Bildung zu unterstützen und darin eine entscheidende Perspektive für ihren zukünftigen Lebensweg zu sehen. Familie Aufbauer ist zwar aufgrund ihrer bis zuletzt sozio-ökonomisch eingeschränkten Bedingungen Typ 3 zugeordnet, ihr war es jedoch auch in der Vergangenheit stets daran gelegen, ihre Kinder hinsichtlich ihrer Ausbildung zu unterstützen und ihre Interessen zu fördern. Weiterführende Pläne verfolgt aber auch Simone Stab: Sie will mit der benötigten finanziellen Unterstützung durch das Erasmus+ Programm der Europäischen Union eine Ausbildung zur Steinbildhauerin in Italien absolvieren und im Anschluss nach Österreich zurückkehren, da sie eine Stelle an 
einem Marionettentheater anstrebt. Simones Wunsch nach einer weiterführenden Ausbildung steht möglicherweise auch im Zusammenhang mit den Erfahrungen ihrer Mutter und dem Streben danach, Aufstieg durch formale Bildung zu erreichen. Frau Stab hatte in ihrem Heimatland in Osteuropa zwar die Matura absolviert, jedoch wurde ihr dieser Abschluss in Österreich nicht anerkannt. In Folge dessen arbeitete sie lange Zeit als Putzfrau oder in anderen ihr ohne Ausbildung zugänglichen Beschäftigungen, sie konnte sich aber nicht dazu durchringen, den Abschluss nachzuholen. Bedingt durch das vergleichsweise durchlässig gestaltete Bildungssystem in Österreich, mit zahlreichen Möglichkeiten auf dem Zweiten Bildungsweg, wäre dies ein einfach zu gehender Weg gewesen (siehe dazu die Fallbeschreibung in Kapitel 2).

Den anderen vier jungen Frauen ist über erfolgreiche Ausbildungen der Einstieg in den Beruf bereits geglückt: Susanne Scheib ist Einzelhandelskauffrau und arbeitet festangestellt in Vollzeit am Empfang eines Autohauses. Isabelle Rohringer hat eine Ausbildung als Kindergärtnerin abgeschlossen und leitet inzwischen die Kinderbetreuung eines Hotels. Beiden ist es gelungen, ihren eigenen sozio-ökonomischen Status zu verbessern. Auch Viktoria Öllinger gelang es, sich von ihren belasteten Familienstrukturen (Typ 1) tendenziell zu befreien und eine Ausbildung zur Einzelhandels- und Bürokauffrau zu absolvieren. Sie ist inzwischen, auf eigenen Wunsch in Teilzeit, in der Verwaltung eines großen Krankenhauses tätig. Ihr ist es durch den Berufseinstieg gelungen, sich nicht nur finanziell, sondern auch emotional gegenüber ihrer sie stark einengenden Mutter zu behaupten, wenngleich sich das Parentifizierungsverhältnis zwischen Tochter und Mutter eher noch verstärkt hat; dies allerdings in der Art, dass Viktoria stärker als zuvor von ihrer Mutter Eigenengagement bei der Alltagsbewältigung einfordert. Unerlässlich dafür war und ist bei Viktoria nach wie vor die Unterstützung ihrer Vorgesetzten, die ihre familiären Bedingungen kennen und ihr die benötigten Freiräume hinsichtlich der Arbeitszeiten einräumen. Elisabeth Ebner hat ihre Wunschausbildung zur Floristin absolviert und arbeitete zuletzt festangestellt in einem Blumenladen, nachdem sie zuvor eine Bürotätigkeit in der Floristikabteilung eines großen Discounters übernommen hatte. Sie möchte zwar in ihrem erlernten Beruf bleiben, sich aber gern fortbilden. Dazu hat sie sich nach längeren Überlegungen für eine private Meisterschule angemeldet, obwohl damit große finanzielle Anstrengungen verbunden sind. Da ihre Eltern sie aufgrund mangelnder finanzieller Ressourcen (Typ 3) nicht unterstützen können, hat sie sich schließlich dazu entschlossen, die erforderlichen 15.000 Euro aus ihrem eigenen Ersparten zu nehmen, um damit in ihre 
Zukunft zu investieren: „Weil i ma denk', an der Bildung derf i einfoch net spoar'n und des mecht i einfoch moch'n." Die Verfügbarkeit einer so großen Summe mag zunächst überraschen, lässt sich jedoch erklären. Elisabeth war in den früheren Erhebungswellen eine sparsame Person, zudem hat sie nach dem Abschluss der Lehre auch bereits in Vollzeit gearbeitet. Gleichzeitig hatte sie bislang kaum größere Ausgaben, da sie sowohl bei ihren Eltern als auch bei ihrem Freund immer kostenfrei wohnen konnte.

Heterogener zeigt sich die Situation bei den jungen Männern. Vier von ihnen haben die gewählten Berufsausbildungen erfolgreich abgeschlossen und hatten die Möglichkeit, von ihren Ausbildungsbetrieben auch fest übernommen zu werden; der Weg dorthin verlief jedoch unterschiedlich: Helmut Pfortner und Benedikt Holzner hatten sich beide selbstständig eine Lehrstelle zum Maschinenbautechniker gesucht. Während der siebten Erhebungswelle absolvierten sie den Wehr- bzw. Zivildienst. Bei Helmut Pfortner war dieser Berufsweg gewissermaßen vorgezeichnet, da sein Vater ebenfalls Maschinenbautechniker ist und sein Sohn das Familienunternehmen eines Tages übernehmen soll und will. Gemeinsam hatte die Familie (Typ 4) jedoch vereinbart, dass die Ausbildung in einem externen Unternehmen absolviert werden sollte. Dort wurde Helmut fest übernommen und möchte sich nun etablieren und später auch die Meisterprüfung absolvieren. Benedikt Holzner stammt dagegen aus einer wegen problematischer sozio-emotionaler Beziehungsstrukturen überforderten Familie (Typ 2); er war bei der Berufswahl und der Suche nach einer Ausbildungsstelle gänzlich auf sich allein gestellt. Dennoch war auch er erfolgreich und erhielt das Angebot seines Arbeitgebers, nach der Ausbildung weiterhin dort beschäftigt zu bleiben. Da er aber mit dem Auftreten der Geschäftsführung seines Ausbildungsbetriebs nicht zufrieden war, hat er sich gegen eine Übernahme in dieser Firma entschieden. Er erzählte im Interview, dass er stattdessen entweder bei einer anderen Firma einsteigen oder für einige Jahre bei Verwandten in Kanada wohnen und dort auch arbeiten möchte. Erich Grubert wurde von seinen Eltern (Typ 4) immer sowohl finanziell als auch emotional unterstützt, selbst als er nach dem Pflichtschulabschluss seine Schullaufbahn frühzeitig abbrach und eine Lehre zum Koch aufnahm. Seine Ausbildung hat er inzwischen erfolgreich absolviert und arbeitet nun fest angestellt in diesem Betrieb - und dies mit der Aussicht, intern zum Souschef weitergebildet zu werden. Eine zwischenzeitlich aufgenommene zweite Ausbildung zum Metzger hatte er wegen Differenzen mit seinem Lehrherrn abgebrochen. Auch in dieser schwierigen Phase seines Lebens erhielt er Unterstützung, nicht nur von 
seinen Eltern, sondern auch von seinem jetzigen Chef, der eine wichtige Vorbildfunktion für Erich einnimmt.

Mario Hirtner begann zunächst eine Ausbildung zum Elektrotechniker, diese musste er jedoch aus gesundheitlichen Gründen, seine Höhenangst machte es ihm unmöglich, auf einer Leiter zu arbeiten, wieder abbrechen. Mit Unterstützung des AMS fand er schließlich eine Ausbildungsstelle zum IT-Techniker; seine Lehre hat er inzwischen mit „ausgezeichnetem Erfolg" abgeschlossen und wurde danach vom Betrieb unbefristet in Vollzeit übernommen. So gelang es Mario, sich wirtschaftlich selbständig zu machen und von den belasteten Bedingungen seiner Mutter zu lösen, so dass er selbst nun als >Aufsteiger eingeschätzt werden kann. Sein Beispiel zeigt deutlich, dass die jungen Erwachsenen inzwischen vielfach, aber nicht generell, getrennt von ihren Familien betrachtet werden müssen. Im Gegensatz zu ihrem Sohn blickte Frau Hirtner zuletzt perspektivlos in ihre berufliche Zukunft, da ihr nach längerer Krankheit gekündigt wurde und sie sich erneut arbeitssuchend melden musste (siehe auch Kapitel 2). Ebenso wie Mario Hirtner benötigte auch Manfred Oblinger die externe Unterstützung des AMS, um eine für ihn passende Lehrstelle zu finden und sich aus den vielfältig belasteten Bedingungen seines Elternhauses (Typ 1) zu lösen; dazu war auch ein Umzug in die Nähe einer weiter entfernt gelegenen Großstadt nötig. Dort hat er eine Ausbildungsstelle zum EDV-Kaufmann gefunden; während der siebten Erhebungswelle stand er kurz vor dem erfolgreichen Abschluss seiner Ausbildung. Auch Manfred hat die Aussicht, bei der Firma fest angestellt zu werden. Sein Wunsch ist es, wie er betont, sich im Unternehmen weiterzubilden und zu etablieren. Seine derzeitige Arbeit bezeichnete Manfred selbst als „Traumberuf“; dazu tragen auch das als „hervorragend“ beschriebene Betriebsklima sowie das gute Verhältnis zu seinem Chef und dessen nachhaltige Unterstützung bei.

Auch bei einem dritten jungen Mann, Gregor Boll, spielte die Begleitung durch einen außerfamilialen Unterstützer eine wichtige Rolle: Nach dem Realschulabschluss hatte Gregor zunächst erfolgreich eine Lehre zum Einzelhandelskaufmann in einem Baumarkt abgeschlossen. Das Unternehmen wurde jedoch abgewickelt und die Mitarbeiter wurden abgefunden, da der Inhaber keinen Nachfolger fand. Dennoch gelang Gregor der erfolgreiche Einstieg in das Berufsleben, da ein Bekannter aus dem örtlichen Schützenverein ihm eine Stelle in einem international agierenden Chemiekonzern vermitteln konnte. Dort arbeitete Gregor zuletzt festangestellt als Ungelernter, er stand in der siebten Erhebungswelle jedoch kurz vor dem Beginn einer zweiten Ausbildung im Unternehmen, nach deren Abschluss 
seine Vorgesetzten ihm, wie er erzählte, einen Aufstieg in der Hierarchie Firma zugesagt hätten.

Zwei Ausnahmefälle unter den jungen Männern sind Torsten Kaiser und Alfons Weiss, wobei beide Fälle unterschiedlich gelagert sind. Im Fall von Torsten Kaiser setzt sich auch nach dem Abschluss der Handelsschule, verbunden mit einem Lehrabschluss als Bürokaufmann, seine berufliche Orientierungslosigkeit fort, die sich bereits in früheren Erhebungswellen gezeigt hatte. Der Weg zum Schulabschluss war für Torsten kein leichter, wobei er sich immer auf die sozio-emotionale Unterstützung in seiner Familie (Typ 3) und die damit verbundene Sicherheit verlassen konnte. Seine Mutter sprach auch von einem „schweren Zittern und Dahintun“ und betonte: „aber wir haben's geschaff!“ Zuletzt überlegte Torsten, ob er eine einjährige Zusatzausbildung zum Pflegeassistenten absolvieren sollte, um diese Tätigkeit dann mit den kaufmännischen Kompetenzen aus seiner schulischen Berufsbildung zu verbinden. Tatsächlich, so schien es, war er gänzlich orientierungslos, dennoch betonte er seinen Wunsch nach einer Arbeitsstelle und dem damit verbundenen finanziellen Aufstieg: „Also auf jeden Fall erstamal dass ich an Job find, der was, der auf der einen Seite ein gutes Geld bringt, auf der anderen Seite mir auch an Spaß macht." Im Gegensatz zu Torsten Kaiser hat Alfons Weiss bereits eine Arbeitsstelle, in der Kommissionierung in einem Lagerbetrieb, bekommen. Zwischenzeitlich hatte sich seine Lebenssituation im Vergleich zu den vorherigen Erhebungswellen massiv verschlechtert, was auf einen eskalierenden Streit mit seiner Mutter zurückzuführen war. Familie Weiss zählte zwar nicht mehr zu den sozio-ökonomisch belasteten Familien, die sozio-emotionale Beziehung zwischen Mutter und Sohn blieb hingegen seit vielen Jahren stark beeinträchtigt (Typ 2) und verbesserte sich auch in der siebten Erhebungselle nicht wesentlich. Wegen des ungeklärten Verdachts auf Drogenmissbrauch und Drogenhandel, der junge Mann selbst bestritt dies im Interview vehement, musste Alfons nicht nur seine Ausbildung als Techniker bei der Bahn aufgeben, er lebte auch mehrere Monate auf der Straße, bevor er eine eigene Wohnung beziehen konnte. Dies war ihm durch die Unterstützung einer öffentlichen Stelle gelungen; Alfons hatte sich in seiner Not an den Bürgermeister seiner Heimatgemeinde gewandt. Zuletzt hatte sich das Verhältnis zwischen Mutter und Sohn auf sehr niedrigem Niveau stabilisiert und Alfons äußerte sich positiv über seine Lebenssituation. Er plante, noch etwa zwei Jahre als Lagerarbeiter tätig zu sein und sich dann, dies jedoch sehr unspezifisch, seinen „Traumberuf“ zu suchen, der etwas mit „Traktorfahren“ zu tun haben sollte. 
Abgesehen von den beiden letztgenannten jungen Männern, Torsten Kaiser und Alfons Weiss, sowie von Timo Landinger, der aufgrund seiner sehr belastenden familiären, aber auch ganz persönlichen schweren Bedingungen eine in den letzten Jahren noch weiter gewachsene Perspektivlosigkeit erkennen ließ, ist es inzwischen allen befragten jungen Erwachsenen gelungen, entweder erfolgreich in das Berufsleben einzusteigen, oder aber die Weichen für weiterführende Ausbildungen zu stellen. Diese positive Entwicklung war aufgrund der schlechten sozio-ökonomischen Startbedingungen in den Familien des Panels so nicht zu erwarten gewesen. Auffällig dabei ist, dass es einigen jungen Erwachsenen nur durch außerfamiliäre Unterstützung gelungen ist, wichtige Schritte in ihre berufliche Zukunft $\mathrm{zu}$ unternehmen. Aufgrund der nunmehr besseren Handlungsoptionen und, damit verbunden, auch gewachsenen Handlungskompetenzen konnte es auch den Heranwachsenden aus vielfältig belasteten Familien gelingen, neue Handlungsentwürfe zu entwickeln und diese auch umzusetzen. Diese Entwicklung unterstreicht die Bedeutung sowohl von staatlichen Institutionen als auch von außerfamilialen Bezugspersonen, die Verantwortung für junge Menschen in der wichtigen Lebensphase von der Schule hin zu einer Ausbildung und in eine berufliche Anstellung übernehmen und sie bei ihrem Einstieg ins Berufsleben tatkräftig unterstützen. Andere Heranwachsende, allen voran die jungen Menschen aus den Familien von Typ 4, den relativ kompetenten >Aufsteigern artige Hilfen angewiesen. Ihnen kommt im Panel eine Sonderstellung zu, denn sie erfuhren schon über mehrere Jahre ihrer Sozialisation hinweg die Unterstützung ihrer Familien und mussten nicht länger mit belasteten sozio-ökonomischen Bedingungen zurechtkommen. Ihnen war es möglich, ihre Handlungsentwürfe aufgrund der gewachsenen familiären sozio-ökonomischen und, damit verbunden, auch der größeren sozio-emotionalen Sicherheit, zu verfolgen und erfolgreich umzusetzen.

\subsection{Beziehungen und Pläne für (den Ausbau von) Partnerschaften}

Neben dem Berufseinstieg und entsprechenden Weichenstellungen für weiterführende Ausbildungen zählen eigene Partnerschaften zu den wichtigsten Lebensinhalten junger Erwachsener. Mit deren Etablierung sind zentrale Entwicklungsaufgaben in diesem Lebensabschnitt verbunden, nicht zuletzt da dies häufig mit einer teilweisen Loslösung von der eigenen Kernfamilie und der Begründung eigener Hausstände einhergeht. In diesem Zusammenhang fallen sehr deutliche Unterschiede zwischen den be- 
fragten jungen Frauen und den befragten jungen Männern auf: Während sechs von sieben Frauen sich in der siebten Erhebungswelle in zum Teil bereits langjährigen Beziehungen befanden, so traf dies nur auf vier von zehn Männern zu.

Von den jungen Frauen lebt lediglich Gudrun Dornbacher noch nicht in einer festen Partnerschaft, wobei sie selbst dies nicht problematisch sieht. In ihren eigenen Worten ausgedrückt sei sie „net der Typ, der so in die, diese Schiene Beziehung tendiert“, stattdessen sei sie „liaber beste Freunde mit jemanden“. Frau Dornbacher bestätigte, dass ihre Tochter bislang keinerlei Ambitionen für eine Beziehung zeigte. Die feste Partnerschaft von Amelie Aufbauer war zum Zeitpunkt der Befragung noch relativ frisch, und sie räumte ein, dass sie sich selbst lange dagegen gewehrt hatte, ihre Partnerschaft als feste Beziehung zu bezeichnen. Stattdessen hatte sie mit dem jungen Mann über längere Zeit eine F+, also eine Freundschaft plus unterhalten, in der beide Seiten ihre Freiheiten hatten, denn ihr sei wichtig, dass "jeder hat hoit trotzdem nu' so sei eigenes Ding“. Nach dem offiziellen Beziehungsbeginn verbrachten Amelie und ihr Freund zwar quasi ihre gesamte Freizeit zusammen, jedoch behielten beide ihre eigenen Wohnungen und damit auch ein hohes Maß an Sicherheit und die Option, sich jederzeit zurückziehen zu können. Mit ihrem zögerlichen Beziehungsverhalten und der Entscheidung zu einer sehr unverbindlichen Verbindung, einem unter jungen Menschen heute weit verbreiteten Partnerschaftstypus, gleicht Amelie stark dem Vorbild ihrer Mutter. Auch Frau Aufbauer hatte zwar immer wieder Kontakt zu verschiedenen Männern, jedoch wollte sie sich nicht binden und eine feste Partnerschaft eingehen. Vielmehr trennte sie sich dreimal kurz nach der Geburt ihrer Kinder von den jeweiligen Vätern und suchte auch keinen Kontakt zu diesen. Ähnlich gelagert ist auch die Entwicklung der nunmehr einjährigen Beziehung von Susanne Scheib, mit der diese inzwischen jedoch sehr glücklich ist. Ihr jetziger Partner war zuvor fünf Jahre lang ihr bester Freund gewesen, sie konnten sich jedoch lange nicht zu einer romantischen Beziehung durchringen. Auch die Beziehung von Simone Stab gestaltet sich schwierig, obwohl sie bereits seit über drei Jahren andauert: „Wir san hoit extrem unterschiedlich und es hat extrem vü Turbulenzen g'habt, aber wir merken irgendwie, dass wir ned ganz ohne einander kennan." Dennoch hinterfragt Simone sich selbst und arbeitet an der Frage, „was mecht i für a Beziehung führ' $n$ "? Andere junge Frauen verbinden dagegen ihre Partnerschaften mit dem Gefühl von Sicherheit. So ist etwa Elisabeth Ebner bereits seit über dreieinhalb Jahre mit ihrem Freund zusammen, den auch ihre Eltern sehr schätzen. Sie träumt von einer gemeinsamen Zukunft mit ihm und will 
bald auch fest mit ihm zusammenziehen. Viktoria Öllinger ist dagegen ein Beispiel dafür, dass die Heranwachsenden ihre eigene Beziehung auch als Schlüssel dazu sehen, sich von ihren Elternhäusern zu emanzipieren und sich den belasteten sozio-emotionalen Bedingungen zu entziehen. Das Verhältnis zu ihrer Mutter hat sich zwar verbessert, auch da Viktoria inzwischen deutlich selbstbewusster aufzutreten weiß, dennoch engt sie ihre Mutter mit dem Bedarf nach Unterstützung und Nähe auch weiterhin stark ein. Viktoria spricht im Interview selbst die Notwendigkeit an, dass ihre Mutter sich emanzipieren und wieder eigenständiger werden müsse. Für sich selbst sieht sie die Zukunft aber in ihrer Partnerschaft. Innerhalb der kommenden zwei Jahre, so ihr ausdrücklicher Wunsch, wolle sie von Zuhause ausziehen, später heiraten und langfristig auch eigene Kinder bekommen. Darin zeigt sich deutlich der Wunsch nach stabilen sozio-emotionalen Verhältnissen und einem klassischen Familienbild, wie sie es mit ihrer Mutter nie kannte.

Anders gestaltet sich die Situation bei den jungen Männern, von denen zum Zeitpunkt der siebten Erhebungswelle nur vier eine Beziehung führten. Bei zwei jungen Männern, Alfons Weiss und Erich Grubert, spielte die Frage nach einer Freundin oder einem Freund in den Interviews keine Rolle. Und Torsten Kaiser blockte dieses Thema recht deutlich mit dem Hinweis ab, dass er wegen Führerschein und Arbeitssuche vorläufig „nicht die Zeit hätte, meiner Freundin genug Aufmerksamkeit zu schenken“. Von den befragten Jungen kann Benedikt Holzner als einziger auf eine ehemalige Beziehung zurückblicken. In der sechsten Erhebungswelle 2016 hatte er eine feste Freundin und wurde von deren Familie auch mit offenen Armen zu Hause aufgenommen. Die Ersatzfamilie schenkte ihm, zeitweise, Zuwendung und die sozio-emotionale Geborgenheit, die ihm seine eigene Familie in der Vergangenheit nie zu geben in der Lage war. Diese Beziehung bestand 2020 nicht mehr. Benedikt hat deren Ende jedoch gut verarbeitet, ist glücklich mit seinem Singleleben und will dies auf absehbare Zeit auch nicht ändern. Manfred Oblinger dagegen kann sich in der siebten Erhebungswelle, erstmals seit Beginn der Studie, vorstellen, innerhalb der nächsten fünf Jahre eine Freundin zu haben. Dieser Wunsch geht einher mit einer starken Typveränderung, bedingt durch eine operative Magenverkleinerung und seinen Umzug in die Nähe einer Großstadt:

Des entwickelt sich jetzt langsam zu durchs Fortgeha und so, und ich zwingat mich jetzt manchmal zum Weggehen, ähm entdeck ich des halt grad so für mich, durch des Rausgehen, durch des Freind treffn, des Leit treff'n, und langsam bin i da jetzt zua von eins, da da bietet sich des dann, da steigt des Interesse so ja. (...) Joa eh, i lass 
den Dingen da jetzt seinen Lauf, ich geh jetzt ned irgendwie jedes Wochenende saufen und bagger da jetzt irgendwelche Mädels a, äh ich geh' eigentlich nie saufen. Ich lass den Dingen ihre Zeit.

Timo Landinger äußerte erstmals in der sechsten Erhebungswelle seinen Wunsch nach einer Beziehung, Die Frage, ob er gerne eine Freundin hätte, beantwortete er sehr deutlich: "Sicher, aber i bin a hoffnungsloser Fall.“ $\mathrm{Da}$ er selbst so viele Computerspiele spiele, müsste er ein Mädchen finden, das dies auch täte und dies sei „a bissi schwar“. Er glaubte nicht daran, dass es ein solches Mädchen geben könnte, das ihn auch so akzeptieren würde, wie er ist. Auch in diesem Kontext wurde offenkundig, wie belastet die sozio-emotionalen Beziehungen der Familie Landinger waren, denn seine Eltern wussten um Timos Wunsch, nahmen diesen aber nicht ernst. Vielmehr verhinderten sie, dass ihr Sohn in seiner Freizeit Gleichaltrige traf. Ein gutes Verhältnis zu einer Mitschülerin unterstützten sie nicht, und sein Vater tat es gar mit den Worten ab, dass „des war der Kontakt, weil sie si guad verstanden hom. Zwei Außenseiter bei anand de versteh'n si immer guad. (...) Liebelei dass er do ghob hod, glaub i eher ned, aber vo dera, de hod er hoid wuin und de hod eam anscheinend a megn." In der siebten Erhebungswelle thematisierte Timo Landinger seinen Wunsch nach einer Partnerschaft nicht mehr.

Ebenso wie bei den jungen Frauen gibt es aber auch unter den jungen Männern vier, die bereits langjährige Partnerschaften pflegen. Am längsten, dreieinhalb Jahre, währt jene von Helmut Pfortner. Er äußerte sich auch sehr zufrieden darüber, wollte aber dennoch noch nicht mit seiner Freundin zusammenziehen, da es „noch a bisserl zu früah“ sei. Zur Begründung führte er an, dass sie erst noch die Schule beenden müsse, und ergänzte lachend: „Und naja, i muss ihr noch des Kochen beibringen!“ Dies erklärt sich dadurch, dass Helmut in seiner Freizeit regelmäßig für die beiden kocht. Bei Norbert Zarbl stand das Zusammenziehen mit seiner langjährigen Freundin in der siebten Erhebungswelle dagegen kurz bevor, aber auch er betonte: „Zum Probieren natürlich!“ Wie Helmut Pfortner sieht auch er in einer gemeinsamen Wohnung den deutlich größeren Schritt, als in der Etablierung einer festen Partnerschaft. Zwei der jungen Männer, Gregor Boll und Mario Hirtner, haben diesen Schritt jedoch bereits getan und zeigten sich in den Interviews sehr glücklich darüber, nicht zuletzt, da sie auf diese Weise ihre eigenen Familien gründen und ihre eigenen Handlungsentwürfe für eine selbstständige Zukunft verfolgen konnten. Gregor Boll wohnt bereits seit zweieinhalb Jahren mit seiner Freundin in einer gemeinsamen Wohnung, und beide verfolgen weitere Pläne für ihr gemeinsames Leben. Nach dem Abschluss des Studiums 
seiner Freundin ist geplant, vor Ort ein Grundstück zu erwerben, zu bauen und langfristig zu bleiben, „da will ich eigentlich nie wieder weg!“ Diese Pläne verknüpft Gregor sehr konkret mit einer Erweiterung seiner beruflichen Pläne; davon verspricht er sich nicht nur sozialen Aufstieg, sondern, vor dem Hintergrund seiner eigenen Erfahrungen in der Jugend, auch ökonomische Sicherheit: Er will im Beruf einen Meisterabschluss erreichen, „damit man noch mehr Geld kriegt, auch 'ne Meisterstelle, mehr Verantwortung, damit man es halt nicht immer nur auf der gleichen Stelle rumtritt, dass man sich halt irgendwie wieder was aufbaut.“

\subsection{Wobnbedingungen und Zukunfspläne}

Wie im vorherigen Abschnitt bereits angeklungen ist, hat sich die Wohnsituation von zahlreichen der jungen Erwachsenen seit der sechsten Erhebungswelle verändert. Drei der jungen Frauen und sechs der jungen Männer wohnen bereits nicht mehr zu Hause, bei zwei Frauen und einem Mann ist zudem ein kurz bevorstehender Auszug bereits konkret geplant. Diese Entwicklung im Panel ist insofern bemerkenswert, da zahlreiche der Befragten in oder in der Nähe von Städten wohnen, die für ihre außerordentlich hohen Wohnkosten bekannt sind. Grundsätzlich stellt sich bei allen die Frage nach der Finanzierung der Wohnungen, insbesondere aber bei jenen jungen Erwachsenen, die aus Familien der Typen 1 und 3 stammen, die im Vergleich zu den Familien von Typ 2 und vor allem Typ 4 mit besonders belasteten sozio-ökonomischen Bedingungen leben. Während es nachvollziehbar erscheint, dass sozio-ökonomisch besser gestellte Eltern (Typ 4) ihre Kinder beim Auszug (auch finanziell) unterstützen können, so scheint dies bei den sozio-ökonomisch stärker belasteten Familien zumindest fraglich. Hier bedarf es einerseits individueller Lösungsansätze, andererseits aber auch der Unterstützung durch außerfamiliale Personen oder Organisationen. So zeigten sich einige Fälle, in denen die jungen Erwachsenen sich hinsichtlich ihrer sozio-ökonomischen Bedingungen bereits von ihren zum Teil stark belasteten Elternhäusern lösen konnten und daher nicht länger dem Typ zugeordnet werden können, dem ihre Eltern angehören.

Von den jungen Frauen wohnen Simone Stab, Viktoria Öllinger und Gudrun Dornbacher noch zu Hause. Simone (Typ 3) plante jedoch im Herbst 2020 nach Italien umzuziehen und dort eine Bildhauerschule zu besuchen; alle entsprechenden Verträge waren zum Zeitpunkt der siebten Erhebungswelle bereits unterschrieben. Weder sie selbst noch ihre Mutter 
wären dazu in der Lage, diese Ausbildung, den Umzug und die entstehenden Wohnkosten zu stemmen. Daher hat sie ein Stipendium des Erasmus+ Programms der Europäischen Union zur Förderung der Lernmobilität von Einzelpersonen beantragt und bewilligt bekommen, mit dem sie große Teile der Kosten decken kann. Auch Viktoria (Typ 1) plant den Auszug in eine eigene Wohnung, sie will sich dazu jedoch noch länger, maximal aber zwei Jahre, Zeit lassen. In diesem Schritt sieht sie auch eine nötige Emanzipation von ihrer Mutter. Durch den Berufseinstieg hat sich ihre persönliche sozio-ökonomische Situation etwas verbessert, und sie wäre in der Lage dazu, eine eigene Wohnung zu finanzieren. Dennoch schreckt sie bislang noch vor den Kosten zurück und befürchtet zudem, dass durch ihren Auszug Fördergelder für ihre Mutter, insbesondere für den dringend benötigten Sozialdienstleister, gekürzt werden könnten. Eine Begründung für diese Befürchtung konnte sie jedoch nicht nennen. Lediglich bei Gudrun (Typ 4) ist auf absehbare Zeit kein Auszug geplant. Nach der bevorstehenden Matura will sie in ihrer Heimatstadt ein Studium aufnehmen und währenddessen auch zu Hause wohnen bleiben. Obwohl die Familie sozio-ökonomisch nicht mehr belastet ist, führt ihre Mutter dennoch auch wirtschaftliche Überlegungen für diese Entscheidung an. Da die abbezahlte Eigentumswohnung der Familie zur Verfügung steht, solle diese auch genutzt werden. Auch die sozio-emotionalen Bedingungen von Familie Dornbacher sind gut, insbesondere das Verhältnis von Gudrun zu ihren Eltern; vor diesem Hintergrund werden bislang noch keine Überlegungen dahingehend angestellt, ob ein Auszug zum Studium auch einen wichtigen Entwicklungsschritt darstellen könnte.

Isabelle Rohringer und Elisabeth Ebner sind zwar bereits ausgezogen, können aber dennoch auch weiterhin bei ihren Eltern übernachten. Besonders Isabelle (Typ 2), die mit ihrem Freund zusammenwohnt, macht von dieser Möglichkeit oft Gebrauch, da sich ihre Arbeitsstelle nahe dem Wohnort ihrer Familie befindet und sie sich auf diese Weise, gerade bei schlechtem Wetter, die Anfahrt ersparen kann. Eine wichtige Rolle spielt dabei auch der Umstand, dass sich das schwierige Verhältnis zwischen Isabelle und ihrer sie stark kontrollierenden Mutter seit ihrer Volljährigkeit und einer gesundheitlichen Krise von Frau Rohringer zunehmend entspannt hat. Elisabeth (Тyp 3) verbringt dagegen fast ihre gesamte Zeit bei ihrem Freund, der eine eigene Garconniere bei seinen Eltern hat. Auch sie soll aber in absehbarer Zeit eine eigene Wohnung erhalten, dafür plant die Familie ein bereits bestehendes Garagengebäude für sie in Eigenleistung auszubauen. In etwas fernerer Zukunft, so Elisabeths ausdrücklicher 
Wunsch, wolle sie aber gemeinsam mit ihrem Freund ein Haus bauen, Kinder bekommen und „so richtig Mama sein“.

Unter den befragten jungen Frauen verfügen lediglich Susanne Scheib und Amelie Aufbauer über eine eigene Wohnung, ihr jeweiliger Weg dorthin unterscheidet sich jedoch deutlich. Susanne (Typ 4) hatte bereits während ihrer Ausbildung eine kleine $19 \mathrm{~m}^{2}$ große Wohnung. Zu deren Finanzierung gibt es keine klare Aussage. Da die entsprechenden Mietkosten in ihrer Heimatstadt jedoch das Einkommen einer Auszubildenden weit übersteigen, ist davon auszugehen, dass sie Unterstützung durch ihre Familie erhalten hat. Inzwischen ist sie in eine größere, $35 \mathrm{~m}^{2}$ große Wohnung umgezogen, die sie nach dem Berufseinstieg selbst unterhalten kann. Anders gelagert ist die Situation bei Amelie (Typ 3): Sie ist ebenfalls bereits von zu Hause ausgezogen, was sich positiv auf das Familienklima ausgewirkt hat, und holt derzeit am Abendgymnasium die Matura nach. Über ein eigenes Einkommen verfügt sie nicht. Stattdessen bezieht sie eine staatliche Ausbildungsunterstützung und Wohngeld. Zudem bewohnt sie keine Wohnung, die sie auf dem freien Mietmarkt gefunden hat, sondern eine geförderte Gemeindewohnung. Damit folgt sie dem Beispiel ihrer Mutter. Auch Frau Aufbauer wohnte während der gesamten Erhebungszeit stets in geförderten Mietwohnungen und zog sehr häufig um, wenn deren Förderungen ausliefen, auf diese Weise erneut vergünstigten Wohnraum zu erhalten. Aus diesem Grund wechselte die Familie auch mehrfach die Gemeinde und lebte teils in der Stadt und teils auf dem Land.

Drei der jungen Männer, Timo Landinger, Torsten Kaiser und Erich Grubert, wohnen aktuell noch zu Hause und planen, zumindest kurzfristig, keinen Auszug. Bei Timo (Typ 1) ist dies auch auf seine mangelnde Selbstständigkeit und seine berufliche Perspektivlosigkeit zurückzuführen. Während seiner Schulzeit lebte er bereits zeitweise in einem betreuten Internat, seinen Eltern missfiel dies jedoch, und sie verlängerten seinen Aufenthalt dort nicht, sondern sahen die „bessere“ Lösung für ihren Sohn darin, wenn dieser wieder zu Hause wohnen würde. In der siebten Erhebungswelle gab es keine Überlegungen dahingehend, dass Timo mitteloder langfristig ausziehen und womöglich in einer betreuten Wohngemeinschaft untergebracht werden sollte. Perspektivisch drängt sich somit die Frage auf, was mit Timo geschehen soll, wenn seine Eltern ihn eines Tages nicht mehr versorgen können. Zuletzt hatte sich seine Wohnsituation minimal verbessert, da er zwischen der sechsten und siebten Erhebungswelle von seinem Zimmer im Erdgeschosse des Hauses in das ausgebaute und größere erste Stockwerk ziehen konnte. Damit verbunden ist zumindest ein höheres Maß an Privatsphäre, da er zuvor immer das 
Schlafzimmer seiner Eltern passieren musste, um in sein eigenes Zimmer zu gelangen. Erich (Typ 4) ist bereits seit einiger Zeit fest im Berufsleben integriert und bezieht ein entsprechendes Gehalt, dennoch wohnt er noch immer bei seinen Eltern, in einem vergleichsweise kleinen Zimmer der nur $80 \mathrm{~m}^{2}$ großen Mietwohnung der Familie. Dies scheint ihn jedoch nicht zu stören, und seine Pläne für einen möglichen Umzug klangen zuletzt sehr vage. Demnach würde er gerne am Stadtrand wohnen und wäre dafür auch bereit, einen deutlich längeren Arbeitsweg als bisher in Kauf zu nehmen. Dazu kann er sich vorstellen, mit einem Freund, einem ehemaligen Arbeitskollegen, eine Wohngemeinschaft zu begründen. Auch Torsten (Typ 3) wohnt noch in der Eigentumswohnung seiner Mutter und fühlt sich dort sehr wohl. Das Familienklima in der Kernfamilie Kaiser war in den letzten Jahren immer gut, zudem hat Torsten bislang noch keine konkreten Pläne für den Berufseinstieg. Durch die abbezahlte Wohnung und einen zusätzlichen Nebenverdienst von Frau Kaiser hatten sich die sozio-ökonomischen Bedingungen der Familie im Laufe der Studie verbessert. Dennoch führt Torsten auch ökonomische Gründe für seinen Verbleib zu Hause an: „Wenn ich dann gut schon bisserl was selber verdien, dass ich dann für eine Zeit lang noch bei meiner Mutter wohn'“, zunächst wolle er "gutes Geld“ verdienen und etwas ansparen. Später, so seine Pläne, könne er sich aber eine eigene Wohnung oder den Umzug in eine Wohngemeinschaft vorstellen. Auch Norbert Zarbl (Typ 4) wohnt zwar noch zu Hause, ein Auszug ist aber bereits konkret geplant. Gemeinsam mit seiner langjährigen Freundin sucht er derzeit eine ca. $65 \mathrm{~m}^{2}$ große Wohnung zur Miete, um das Zusammenwohnen auszuprobieren: „Also auf jeden Fall für den Anfang, zum Probieren natürlich, ich mein, wir sind schon länger zam, aber trotzdem amal, zusammen wohnen ist doch nochmals was anderes." Finanziell stellt der Auszug für ihn keine unüberwindbare Hürde dar, er wohnt zwar in einer sehr teuren Stadt, als Hardware-Entwickler bei einem Automobilzulieferer verdient er aber auch gut. Dennoch planen seine Eltern, so die deutliche Aussage seiner Mutter, Norbert und seine Freundin sowohl bei der Miete als auch bei den Kosten für die Einrichtung der Wohnung zu unterstützen.

Die sechs anderen befragten jungen Männer wohnen dagegen bereits in eigenen Wohnungen. Benedikt Holzner, Gregor Boll und Mario Hirtner können diese auch relativ problemlos durch ihr Einkommen unterhalten. Benedikt (Typ 2) ist dabei, wie schon zuvor bei der Suche nach einer passenden Ausbildung, auf sich alleine gestellt, er erhält von seiner Familie weder finanzielle noch emotionale Unterstützung. Während der Schulzeit lebte er in einer betreuten Wohngemeinschaft, verließ diese jedoch nach 
dem Schulabschluss. Bereits als Auszubildender bezog er dann seine eigene Wohnung. Dies war ihm finanziell möglich, da er auf dem Land lebt und die Mietkosten dort vergleichsweise niedrig sind. Während der siebten Erhebungswelle leistete er seinen Zivildienst, in dieser Zeit wurde die Miete, wie gesetzlich vorgesehen, durch den Staat übernommen. Gregor (Typ 3) arbeitet bereits und bezieht ein festes Gehalt. Dennoch unterstützt ihn seine Mutter, die ihre sozio-ökonomischen Bedingungen durch den Erfolg ihrer Hundezucht verbessern konnte, bei größeren Ausgaben, wie etwa dem Erwerb eines Autos. Er bewohnt gemeinsam mit seiner langjährigen Freundin, die noch studiert, eine gut ausgestattete Mietwohnung. In Zukunft planen beide, vor Ort gemeinsam ein Haus zu bauen. Auch Mario (Typ 2) wohnt gemeinsam mit seiner Freundin in einer Mietwohnung. Er arbeitet als IT-Techniker und bezieht ein gutes Gehalt. Sozio-ökonomisch betrachtet ist es ihm gelungen, sich deutlich zu verbessern und von der sozio-ökonomisch angespannten und zuletzt stark verschlechterten Lage seiner Mutter zu entfernen. Die Wohnung wird ihm durch seinen Arbeitgeber vergünstigt zur Verfügung gestellt, dieser Umstand erleichtert die Finanzierung angesichts der sehr hohen Lebenshaltungskosten in seiner Heimatstadt sehr. Ebenso wie Mario wohnt auch Manfred Oblinger (Typ 1) in einer Wohnung seines Arbeitgebers, die dieser ihm sowie zwei weiteren Arbeitskollegen sehr günstig als Wohngemeinschaft zur Verfügung stellt. Dies sei, so Manfred, eigentlich nicht vorgesehen gewesen, sondern es sei ein ausdrückliches Entgegenkommen für besondere Leistungen in der Firma. Im Gegensatz zu Mario könnte Manfred sich sonst auch keine eigene Wohnung oder ein WG-Zimmer vor Ort leisten, da die Mietkosten sowohl in der nahen Stadt als auch im Speckgürtel, in dem die Firma gelegen ist, sehr hoch sind. Auch Manfreds Eltern fehlen die finanziellen Mittel, um ihren Sohn zu unterstützen. Daher musste Manfred zunächst jeden Tag „fünf Stunden“ am Tag zu seiner Ausbildungsstelle pendeln. Die Verbindung war, so Manfred, „recht scheiße“, aber er nahm dies für die Chance auf eine Ausbildung gerne in Kauf. $\mathrm{Zu}$ dieser Zeit lebte er für eineinhalb Monate gemeinsam mit seinem Vater in einem Wohnwagen, den dessen Arbeitgeber ihm als Dienstwohnung zur Verfügung gestellt hatte. Der Umzug wirkte sich schließlich überaus positiv auf Manfred und seine Lebensbedingungen aus, da er nur 120 Euro Miete im Monat zahlen muss. Auch Alfons Weiss (Typ 2) benötigte Hilfe von mehreren Seiten, um seine Wohnung zu bekommen und finanzieren zu können. Nach einem Streit mit seiner Mutter lebte er mehrere Monate auf der Straße und wendete sich schließlich hilfesuchend an den Bürgermeister seiner Heimatstadt. Dieser organisierte für ihn eine geförderte Gemeinde- 
wohnung. Alfons bezieht darüber hinaus monatlich eine staatliche Wohnbeihilfe in Höhe von 180 Euro. Da Alfons beim Einzug mittellos war und zudem wegen Problemen im Umgang mit Finanzen einen gerichtlich bestellten Sachwalter hat, musste dennoch seine Mutter die Kaution für die Wohnung entrichten, da Alfons sonst nicht hätte einziehen dürfen. Dieser Hilfsmaßnahme hat sich Frau Weiss nicht entzogen, obwohl das bereits in früheren Erhebungswellen angespannte Verhältnis zwischen Mutter und Sohn sich nochmals drastisch verschlechtert hatte. Zuletzt konnte Alfons seine Verhältnisse zumindest wieder etwas stabilisieren. Einerseits fühlt er sich in seinen eigenen Wänden sehr wohl, da er dort nicht belästigt wird: „Will meine Ruhe haben! Meine eigene Wohnung.“ Andererseits war er in der Lage, die reduzierten Mietkosten selbst zu tragen. Dazu kann er auf sein Gehalt als Lagerist zurückgreifen und zusätzlich auf eigene Mieteinnahmen, da er gemeinsam mit seinem Halbbruder eine Wohnung von seinem Großvater geerbt hat. Einen Sonderfall hinsichtlich der Wohnsituation stellt Helmut Pfortner (Typ 4) dar. Er wohnte bereits in der sechsten Erhebungswelle mietfrei in einer eigenen, ca. $55 \mathrm{~m}^{2}$ großen Wohnung im ausgebauten Dachgeschoss seines Elternhauses. In gewisser Weise verfolgt Familie Pfortner schon seit geraumer Zeit das Konzept eines Mehr-Generationen-Hauses. Dazu zählt auch, dass die Eltern in absehbarer Zeit ins Erdgeschoss ziehen wollen, damit entweder Helmut oder seine Schwester die Wohnung im ausgebauten Dachgeschoss verlassen und in den ersten Stock des Hauses wechseln können. Dies wurde möglich, da die bislang mit im Haus wohnende Mutter von Herrn Pfortner kurz vor der siebten Erhebungswelle gestorben war. Auf diese Weise soll mehr Wohnraum für die Kinder geschaffen werden, damit diese die Möglichkeit haben, dauerhaft im Elternhaus leben zu können. Während der siebten Erhebungswelle leistete Helmut seinen Grundwehrdienst und wohnte unter der Woche in einer Kaserne; auf eine mögliche Kostenübernahme für die Wohnung durch den Staat hat die Familie verzichtet. Auch Helmut solle, so seine Mutter, erst nach dem Wehrdienst und dem Wiedereinstieg in den Beruf Miete zahlen; darin sieht sie einen wichtigen Schritt zu mehr Selbstständigkeit.

Der Blick auf die Wohnsituation der jungen Erwachsenen zeigt, dass es vielen von ihnen bereits gelungen ist, sich selbstständig zu machen und eine eigene Wohnung, teilweise gemeinsam mit Partnerinnen oder Partnern, zu beziehen. Eine genauere Betrachtung der individuellen Bedingungen verdeutlich aber, dass bei Weitem nicht alle in der Lage sind, dies auch gänzlich eigenständig zu finanzieren. Während sich die Typ 4 Zugeordneten dabei sehr deutlich auf die Unterstützung ihrer Eltern 
verlassen können, stellt dies viele andere vor gewisse Herausforderungen. Gerade Manfred Oblinger (Typ 1), Alfons Weiss (Typ 2) und Amelie Aufbauer (Typ 3) sind maßgeblich auf externe Unterstützungsmaßnahmen angewiesen. Familie Ebner (Typ 3), die auf dem Land lebt, ist dagegen ein Beispiel dafür, wie auch mit eingeschränkten sozio-ökonomischen Bedingungen durch Eigenleistung Wohnraum geschaffen werden kann. Zahlreiche andere junge Erwachsene bestätigen dagegen, wie wichtig die Ausbildung und der erfolgreiche Einstieg in den Beruf für eine Verbesserung der eigenen sozio-ökonomischen aber auch der sozio-emotionalen Bedingungen sind. Ihnen ist es bereits gelungen, aus eigener Kraft heraus ihre erste eigene Wohnung zu beziehen. Die Beispiele von Gudrun Dornbacher und Erich Grubert (beide Typ 4) sowie von Torsten Kaiser (Typ 3) belegen aber, dass es auch junge Erwachsene gibt, die gerne noch zu Hause wohnen wollen und deren Wunsch es nicht ist, möglichst rasch auszuziehen. Dies setzt jedoch in allen drei Fällen stabile sozio-emotionale Bedingungen und ein gutes Verhältnis zwischen den Eltern und ihren nun volljährigen Kindern voraus.

\subsection{Mediengebrauch und Freizeitverhalten}

Wie in den Jahren zuvor verbleibt auch in der siebten Erhebungswelle der Mediengebrauch der befragten nunmehr jungen Erwachsenen auf relativ hohem Niveau und stellt einen wichtigen Teil ihrer Freizeitaktivitäten dar. Auf individueller Ebene lassen sich jedoch zum Teil starke Veränderungen hin zu einer deutlich höheren oder aber niedrigeren Mediennutzung feststellen. Solche erheblichen Veränderungen stehen in vielen Fällen in einem engen Zusammenhang mit dem Eintritt ins Berufsleben. Einerseits nutzen viele der Befragten Medienangebote und Geräte, allen voran Laptop und Smartphone, nun auch beruflich, andererseits verbleibt im Arbeitsalltag weniger Zeit für Freizeitaktivitäten, und die jungen Erwachsenen sehen sich nun verstärkt mit der Herausforderung konfrontiert, ihre begrenzten Zeitbudgets, neben dem Mediengebrauch, auch auf nicht mediale Aktivitäten und Unternehmungen mit Freundinnen und Freunden, Partnerinnen und Partnern sowie der Familie zu verteilen.

Dennoch wollen viele der jungen Erwachsenen nicht von über die Jahre liebgewonnenen Inhalten lassen, auch wenn sie aus diesen inzwischen eigentlich herausgewachsen sind; dazu zählen insbesondere Computerspiele, Fernsehsendungen und Serien, die zumeist über Netflix oder YouTube rezipiert werden. In diesem Zusammenhang ist auch auffällig, dass einige 
der Befragten sich inzwischen verstärkt Informationsangeboten zuwenden, während andere diese Möglichkeit nahezu vollständig ignorieren. Dabei gilt es aber zu beachten, dass der Mediengebrauch, unabhängig von seiner Intensität, sowie die Relevanz verschiedener Medienangebote für Information, Kommunikation und Unterhaltung von den Befragten in dieser letzten Erhebungswelle weitaus reflektierter betrachtet und eingeordnet wurden, als dies noch in der sechsten Erhebungswelle der Fall war.

Neben dem Einfluss auf den Mediengebrauch zeigt sich noch eine andere Folge des erfolgreichen Berufseinstiegs: Ist der Übertritt von Schule und Ausbildung ins Erwerbsleben geglückt, so stehen den jungen Erwachsenen in vielen Fällen weitaus größere finanzielle Mittel zur Verfügung als zuvor. Dieser Effekt ist dabei meist so stark, dass die Befragten finanzielle Einbußen während des Beginns der Covid-19-Pandemie (etwa durch Kurzarbeit) nicht als Einschränkungen wahrgenommen haben. Dabei fällt auf, dass viele zudem auch dazu bereit sind, größere Summen ihres Gehalts in Mediengeräte und -angebote (wie kostenpflichtige Streaming-Angebote) sowie in kostspielige Freizeitbeschäftigungen wie Auto oder Motorrad zu investieren.

Hinsichtlich der Unterschiede zwischen den Geschlechtern fällt auf, dass viele der jungen Männer sehr stark ihre Freunde und ihren Freundeskreis in den Mittelpunkt rücken, auch dann, wenn sie sich in festen Partnerschaften befinden. Die jungen Frauen wenden sich dagegen weitaus deutlicher ihren Partnern zu und thematisieren Aktivitäten mit ihnen auch verstärkt im Kontext von Mediengebrauch und gemeinsamen Unternehmungen. Insgesamt zeigen die Beispiele der 17 befragten jungen Erwachsenen aber zum Teil sehr stark individuell geprägte Entwicklungen in der Zusammenstellung ihres jeweiligen Medienrepertoires.

Während Streaming-Angebote für Norbert Zarbl, ähnlich wie bei vielen anderen, deutlich an Bedeutung gewonnen haben, so hat die Intensität seines Mediengebrauchs insgesamt nachgelassen, besonders mit Blick auf das „Zocken“, TV, Snapchat und Facebook. Andere Angebote, besonders Instagram und Netflix, haben dagegen an Bedeutung gewonnen. Gemeinsam mit seinem Bruder, aber auch mit seiner Freundin, spielt er gerne PlayStation 4. Durch die Beziehung hat er auch begonnen, mit seiner Freundin zusammen Filme zu streamen, um so gemeinsam mehr Zeit verbringen zu können. Gerade sein Smartphone und den Laptop nutzt er inzwischen „sehr viel“, da beide Geräte auch für die Arbeit benötigt werden. In seiner Freizeit dominieren, seit dem Einstieg ins Berufsleben, auch kostspielige Hobbys, wie ein eigenes Auto und Motorrad. Zentral bleiben für ihn jedoch Freundschaften und die gemeinsame Zeit mit 
seinem Bruder. Kostspielige Freizeitaktivitäten kann sich Alfons Weiss aktuell nicht erlauben. Er spielt selten Fußball und geht schwimmen; sein Freizeitverhalten ist stark geprägt von Netflix und WhatsApp, aber er genießt nach der Arbeit auch oft einfach die Ruhe in seiner Wohnung. Auffällig im Vergleich zu anderen Befragten ist seine starke Zuwendung zu Zeitungen (Bildzeitung, Krone am Sonntag) zur Informationsbeschaffung, die er sich regelmäßig kauft. Wie auch in früheren Erhebungswellen interessiert sich Alfons sehr für Sportübertragungen im Fernsehen, jedoch fehlen ihm aktuell die finanziellen Ressourcen, um die dafür notwendigen Abonnements (Sky, DAZN) zu bezahlen, und sein gerichtlich bestellter Sachwalter verhindert entsprechende Verträge. Daher besucht er regelmäßig Sportcafés und Wettbüros, um dort die Übertragungen verfolgen zu können. Computerspiele spielen dagegen, im Gegensatz zu früher, für ihn „keine Rolle mehr“. Ähnlich wie Alfons treibt auch Helmut Pfortner viel Sport (jetzt Kickboxen). Er trifft sich zudem sehr gerne real mit seinen Freunden und nutzt WhatsApp und Telefon in erster Linie, um Verabredungen zu treffen. Dennoch hat er sich während des Lockdowns auch regelmäßig online mit seinen Freunden getroffen und dafür sowohl die Chatfunktion von Computerspielen als auch Apps wie Houseparty verwendet. Er spielt zudem gerne PlayStation, im Gegensatz zu vielen anderen jungen Männern im Panel dagegen nur alleine, da er „voll der schlechte Spieler" sei. Obwohl mediale Unterhaltung für ihn nur eine untergeordnete Rolle spielt, sieht er dennoch eine zunehmende Bedeutung von Medien, da diese „Alltag g'word' $n$ “ seien. Gerade zur Information sind Medien demnach „unerlässlich“, dennoch wäre es „nicht schlimm“ für ihn, wenn er sein Smartphone „ned hätt“. Eine gemeinsame Mediennutzung mit seiner Freundin scheint in seiner Beziehung keine Rolle zu spielen, jedoch genießt es Helmut, sich in seiner Freizeit wieder Serien aus seiner Jugend, insbesondere Family Guy, zuzuwenden. Wie Norbert Zarbl investiert auch Helmut viel Geld in seine Hobbys, seitdem er finanziell dazu in der Lage ist: Dazu zählen neben seinem Auto auch Ausgaben für den Motorradführerschein und die Ausbildung zum Jäger, da er wie seine Eltern die Jagdprüfung ablegen möchte.

Im Gegensatz zu den allmählichen Veränderungen bei Norbert, Alfons und Helmut, haben sich das Freizeitverhalten und der Mediengebrauch von Manfred Oblinger, bedingt durch seinen Umzug in die Nähe einer großen Stadt und die positiven Folgen seiner operativen Magenverkleinerung, grundlegend verändert. Zwar spielt Manfred noch immer gerne Computer- und Videospiele und nutzt diese auch, um mit Freunden zu kommunizieren, jedoch möchte er deren Stellenwert in Zukunft noch 
weiter reduzieren. Inzwischen verbringt er seine Freizeit gerne draußen und fährt auch zum Schwimmen an den See oder in ein Freibad - Aktivitäten, die ihm früher undenkbar waren. Zudem kocht er inzwischen regelmäßig, aufwendig und gesund, sowohl für sich als auch für seine Freunde in der Wohngemeinschaft. Die entsprechenden Rezepte sucht er sich im Internet, vornehmlich auf YouTube. Insgesamt sind ihm Freundschaften inzwischen sehr wichtig geworden, und er genießt es, gemeinsam mit seinen Freunden etwas zu unternehmen, Ausflüge zu machen und Besuche abzustatten. Dazu zählen inzwischen sogar Besuche in Kneipen, dies hatte er früher strikt abgelehnt, und Manfred berichtet in der siebten Erhebungswelle erstmals davon, dass er sein Leben jetzt richtig genießen könne. Ähnlich wie Manfred hat auch Erich Grubert, durch eine Ernährungsumstellung, sehr stark an Gewicht verloren und in Folge sein Freizeitverhalten drastisch verändert. Auch er geht nun schwimmen und verbringt gerne und viel Zeit mit seinen Freunden „unterwegs draußen“. Bedingt durch seine Arbeitszeiten als Koch gehören sehr viele Kolleginnen und Kollegen zu seinen besten Freunden, mit ihnen verbringt er auch seine Freizeit und unternimmt Vieles (Kartfahren, Bowling). Zu seinen wichtigsten Bezugspersonen zählt zudem der Cousin seines Stiefvaters, den er jedoch online über ein gemeinsam genutztes Computerspiel kennengelernt hat. Beide sehen sich oft und besuchen sich gegenseitig. Auch weiterhin haben Computer- und Videospiele sowie Streaming-Inhalte bei Netflix einen hohen Stellenwert bei Erich, jedoch haben sie im Vergleich zu früher an Bedeutung verloren. Dies steht auch in Zusammenhang mit seinem Berufseinstieg und seiner dadurch stark reduzierten Freizeit. Neben der Kommunikation über Telefon und WhatsApp nutzt er daher sein Smartphone auch gerne für kleine Spiele, um sich die Zeit zu vertreiben, wenn einmal Lücken im Arbeitsalltag entstehen. Auch Benedikt Holzner hat seinen Mediengebrauch deutlich verändert. Bedingt durch seine Lehrabschlussprüfungen und die Einbindung in den Zivildienst musste er seine Freizeitaktivitäten stark reduzieren. Noch immer betreibt er gerne Wintersport (Skifahren), jedoch deutlich seltener als früher; generell unternimmt er nun gerne und oft etwas mit Freunden. Soziale Medien, die früher einen sehr großen Stellenwert für ihn hatten, insbesondere zum Identitätsmanagement, haben sehr stark an Bedeutung verloren. Zur Begründung führt er an, dies sei „allweil nimmer das Gleiche“ und deswegen sei er „inaktiv g'word'n“. Von großer Relevanz sind inzwischen dagegen Online-Computerspiele. Diese nutzt er gemeinsam mit Freunden, zudem ist er aber auch in einer wettkampforientierten Online-Liga sehr aktiv. Im Gegensatz zu vielen anderen jungen Erwachsenen im Panel nutzt Benedikt 
kaum Informationsangebote, sondern lässt sich lieber von Freunden und Bekannten über das Weltgeschehen informieren; nur in Ausnahmefällen greift er auf das Online-Portal eines lokalen Anbieters zurück. Auch Torsten Kaiser erlebte in der siebten Erhebungswelle deutliche Veränderungen seines Tagesablaufs. Fragen nach seiner Freizeit beantwortete er nicht mit Aktivitäten, sondern mit dem Hinweis darauf, dass er kaum Zeit habe: Zunächst wegen der Schule, dann wegen der Arbeitssuche und später wegen dem Führerschein. Dies macht in seinen Augen auch eine Beziehung unmöglich. Er nutzt in seiner Freizeit verstärkt Medien, besonders Smartphone und iPad. Während er mit dem Smartphone mit Freunden kommuniziert, dient das Tablet in erster Linie Spielen und der Nutzung von Streaming-Angeboten. Zudem spielt er auch am PC MMORPGs. Auffällig ist, dass Torsten auf YouTube vielen Informations-Kanälen folgt, insbesondere solchen, die sich auch mit politischen Themen befassen wie Mister Wissen2Go und jungdonaiv. Trotz seines, zumindest in der eigenen Wahrnehmung, eingeschränkten Zeitbudgets hat sich Torsten bewusst dafür entschieden, gemeinsam mit seiner Mutter und seinen Brüdern erneut die Patenschaft für ein Tier auf einem Gnadenhof zu übernehmen und dieses auch regelmäßig zu besuchen; zudem übernimmt er auch für den neu angeschafften Familienhund Verantwortung.

Bei zwei jungen Männern, Mario Hirtner und Gregor Boll, zeigt sich, neben den dem Berufseinstieg geschuldeten Veränderungen, auch ein deutlicher Einfluss ihrer Beziehungen auf den Mediengebrauch und das Freizeitverhalten. Mario unternimmt generell viel mit Freunden, unter anderem geht er mit ihnen gerne zum Schwimmen. Zu seinen ausdrücklich erwähnten Freizeitaktivitäten gehört auch das „Shoppen“, wobei er dies als „eigentlich sehr komisch für an Jungen“ bezeichnet. Daher tut er dies auch nicht alleine, sondern lässt sich von seinem Bruder oder seiner Freundin begleiten. Zu Marios großen Leidenschaften gehören auch weiterhin Computerspiele. Dabei spielt er „größere Spiele“ quasi nie alleine, sondern es ist ihm sehr wichtig, dies gemeinsam mit seinen Freunden zu tun und dabei auch intensiv zu kommunizieren. Dazu dient in der Regel das Programm Team-Speak. Soziale Medien seien für ihn dagegen inzwischen „eigentlich gor nicht wichtig“, jedoch legt er großen Wert darauf, über Telefon, SMS und WhatsApp in "ständigem Kontakt" mit seinen Freunden zu stehen. Sowohl für seine Arbeit als IT-Techniker als auch zur Informationsbeschaffung (News-App, DerStandard.at, Krone.at, YouTube, Angebote der Bundesregierung) sind Smartphone und Laptop wichtig für ihn. Eine deutliche Veränderung seines Streaming-Verhaltens auf Netflix hat sich durch seine Beziehung ergeben: Gemeinsam mit seiner 
Freundin entdeckt er dort gerne Neues. Feste bevorzugte Genres haben sie dagegen kaum, beide mögen generell Serien. Seine Freundin liebt zudem Horrorfilme, die beide dann gemeinsam ansehen. Ähnliches berichtet auch Gregor Boll, der ebenfalls gerne und regelmäßig gemeinsam mit seiner Freundin auf Streaming-Dienste zurückgreift. Ihm gefallen nahezu alle Inhalte, insbesondere Actionfilme. Zusammen mit seiner Freundin schaut er aber auch gerne Liebesfilme, was er selbst als „schwul“ bezeichnet: „Ich kuck Action, ich kuck genauso mit meiner Freundin gerne Liebesfilme, so schwul das klingt, aber, alles (lacht), alles kuck ich."Wichtig sind ihm zudem der Kontakt und Treffen mit Freunden und Familie. Während der Covid-19-Pandemie und den zeitweise strengen Kontaktverboten griff er dazu insbesondere auf Telefonie, WhatsApp und die App Houseparty zurück. Außerhalb der Pandemie sind ihm Treffen mit Freunden, gemeinsames „Vorglühen“ sowie regelmäßige Partys und Clubbesuche wichtig. Mit einem Freund bastelt Gregor zudem gerne an Motorrollern, um sich durch deren Verkauf etwas dazuzuverdienen. Eine große Leidenschaft von ihm sind Technik und Medien, unter anderem Smart Home-Angebote von Amazon Alexa, Tablets und ein kostspieliger Gaming PC; gemeinsam mit seiner Freundin hat er kostenpflichtige Abonnements bei Amazon Prime, Netflix und Disney+. Gregor gibt, seitdem ihm dies möglich ist, gerne und viel Geld für Mediengeräte aus und verbindet mit damit auch einen gewissen Status, den er als Jugendlicher vermisst zu haben scheint: „Es ist jetzt nicht so, dass ich immer kucke: Was kommt jetzt für ein neuer heißer Scheiß auf den Markt? Ich hab' ja früher immer gesagt, es ist mir scheiß egal was ich mir hole, Hauptsache es funktioniert halbwegs, mittlerweile ist es aber schon geil, wenn man diesen Luxus an den Mediengeräten hat, ne.“

Ein Ausnahmefall ist, auch in diesem Kontext, erneut Timo Landinger. Er hat weiterhin keinerlei Freunde oder nur Bekanntschaften. Für ihn gibt es nur Medienangebote und Online-Computerspiele, aber keine anderen Freizeitbeschäftigungen. Wie er selbst es ausdrückt: „Immer suchteln, ganz arg!" Jedoch sieht er auch weiterhin keine Veranlassung, oder aber keine Chance, dazu, sein Verhalten zu ändern und sich auch anderen Aktivitäten zuzuwenden. Dennoch fördert die siebte Erhebungswelle auch bei Timo grundlegende Veränderungen seines Mediengebrauchs zu Tage: Früher kontrollierten seine Eltern die von ihm verwendeten Angebote strikt und verwehrten ihm den lang gehegten Wunsch nach einem Smartphone. Diese Kontrollen und Verbote sind nun gefallen. Inzwischen hat auch Timo ein eigenes Smartphone mit Vertrag und kann dort, ebenso wie am Laptop seiner Mutter, das Internet uneingeschränkt nutzen. Von dieser Möglich- 
keit scheint er auch zu Informationszwecken Gebrauch zu machen, wie seine verschwörungstheoretischen Ansichten zur Covid-19-Pandemie unterstreichen. Seine Eltern zeigen dagegen Verständnis für die getroffenen Maßnahmen zur Eindämmung der Pandemie und nehmen diese nicht, wie früher in anderen Kontexten praktiziert, als Anlass zur Kritik am Staat und seinen Maßnahmen „gegen“ die Bevölkerung.

Bei Simone Stab hat ihre berufliche Orientierung, sie möchte Steinbildhauerin werden und an einem Marionettentheater arbeiten, Einfluss auf ihre Freizeitgestaltung und auch auf ihr Beziehungsleben. Ihr Leben ist zwar stark von Freundinnen und Freunden geprägt, mit diesen verbringt sie viel Zeit in der Natur beim Spazierengehen, Schwimmen oder Musikhören, sie zieht sich aber auch am Abend oft zurück und fertigt alleine Zeichnungen und Gemälde an. Zudem verwendet sie auch die Programme InDesign, Photoshop und Illustrator, um Bilder zu bearbeiten, Magazine zu erstellen und Logos zu kreieren. Gemeinsame Aktivitäten mit ihrem Freund thematisiert sie dagegen nicht, jedoch macht sie sehr deutlich, wie wichtig ihr die Beziehung ist, obwohl es immer wieder „Turbulenzen“ gibt. Ihr bewegtes Beziehungsleben hat sie auch thematisch für ihre Abschlussarbeit in der Schule aufgegriffen und zu einem Marionetten-Theaterstück für Kinder verarbeitet. Den Umfang ihrer eigenen Mediennutzung schätzt Simone, wie viele andere der Befragten, als „mittel“ ein, wobei sie gerne YouTube, WhatsApp und Netflix nutzt und besonders auf Instagram auch gerne Selbstdarstellung praktiziert: „I lass' mi gern fotografier'n und lass, wenn i scheene Fotos oder so hab', stell i die gern auf mei Instagram-Seiten. Für Memes oder irgendwelche lustigen Videos.“ Obwohl sie einräumt, dass Medien „sehr in mei Leben integriert“ sind, will sie diesen keinen zu hohen Stellenwert beimessen. Stattdessen solle man, so Simone, seinen Mediengebrauch immer hinterfragen und sich nicht berieseln, ablenken oder manipulieren lassen.

Bei Elisabeth Ebner steht das Beziehungsleben mit ihrem Freund stärker im Vordergrund. Sie fährt fast jeden Tag nach der Arbeit zu ihm und verbringt mit ihm nahezu ihre gesamte Freizeit. Gemeinsam treffen sie auch Freunde und gehen mit dem Hund spazieren. Ähnlich wie auch Simone Stab hat Elisabeth ihre Leidenschaft für das Fotografieren entdeckt, betätigt sich dabei aber in erster Linie als Fotografin und nicht als Model: Sie hat sich bereits eine Spiegelreflexkamera und ein Huawei P30 Pro Smartphone mit besonders guter Kamera gekauft, um noch bessere Fotos machen zu können. Dies tut sie in erster Linie für sich selbst, sie veröffentlicht die Bilder teilweise aber auch bei Instagram, sowohl auf ihrem privaten Account als auch für den Firmen-Account ihres Arbeitgebers. 
Die professionell erstellten Fotos von Blüten und Blumengebinden dienen somit nicht nur ihrem privaten Identitätsmanagement, sondern auch ihrer Positionierung als aufstrebende Floristin. Da dies viel Zeit in Anspruch nimmt, schätzt sie ihre Mediennutzung als „mittel bis hoch“ ein; gerade zur Kommunikation findet sie Medien auch sehr wichtig. Dennoch, so Elisabeth, würde sie gerne einmal einen Monat lang vollständig auf alle Medien- und Kommunikationsangebote verzichten, da sie das Gefühl habe, dass die Menschen, und auch sie selbst, immer abhängiger davon würden, und dies wolle sie nicht.

Gudrun Dornbacher hat zwar im Gegensatz zu Simone Stab keinen festen Freund, sondern einen „best'n Freind“ aber auch er beeinflusst sie stark. Beide haben sich in einem Manga-Anime-Forum kennengelernt, das sich einmal im Monat trifft und einen Fixpunkt für ihre Freizeitgestaltung darstellt. Gemeinsam mit weiteren Freunden aus dieser Gruppe betreibt Gudrun auch aktive Medienproduktion und streamt auf der Plattform Twitch.tv Content zu Nintendo-Spielen. Damit setzt sie eine Entwicklung fort, da sie bereits als Kind und als Jugendliche Rezensionen zu Büchern verfasst und veröffentlicht hatte. Noch immer liest sie sehr gerne Bücher und unterscheidet sich damit deutlich von den anderen Befragten im Panel, wobei auch für sie Netflix und Musikhören sehr wichtig geworden sind. Darüber hinaus nimmt auch das Blockfötenspiel weiterhin einen wichtigen Stellenwert bei Gudrun ein, da sie sich in ihrer Freizeit in einem Ensemble engagiert.

Der Freundeskreis von Isabelle Rohringer hat sich stark vergrößert, seitdem sie seit eineinhalb Jahren mit ihrem Freund zusammenwohnt. Neben der Arbeit und dem Haushalt bleibt ihr nur wenig Freizeit für aufwendige Aktivitäten, aber sie schaut gelegentlich gemeinsam mit ihrem Freund Inhalte auf Amazon Prime an. Ihre Mediennutzung schätzt sie insgesamt als „mittel“ ein. Gerade für die Arbeit wären aber Computer und Smartphone sehr wichtig, damit sie immer erreichbar ist und auch im Privaten betrachte sie Medien für die Kommunikation als „essenziell“. In diesem Kontext verwendet sie auch Snapchat, um mit Freundinnen Bilder zu tauschen und dort kleine Spiele zu spielen.

Auch Viktoria Öllingers Freundeskreis hat sich durch ihre Beziehung stark verändert. Zwar hat sie noch immer ihre beste Freundin von früher, Kontakte zu Schulkameradschaften werden dagegen immer weniger. Vielmehr verlagert sich ihr Freundeskreis immer mehr hin zu männlichen Bekannten und Personen, die sie über ihren Freund kennenlernt. Mit ihnen ist sie gerne draußen unterwegs, geht zum Wandern oder zum Billard Spielen. Für teure Freizeitaktivitäten bleibt ihr, im Gegensatz zu 
anderen der jungen Erwachsenen, trotz ihrer Berufstätigkeit kaum Geld übrig. Dies liegt einerseits daran, dass sie nur in Teilzeit arbeitet, um sich um ihre Mutter kümmern zu können, andererseits würden durch ihren Berufseinstieg verschiedene Fördergelder entfallen. Auch sie weist Medien einen „mittleren“ Stellenwert in ihrem Leben zu und betont in erster Linie deren Bedeutung für die Kommunikation. Jedoch setzt auch sie zur Unterhaltung gerne auf Netflix und YouTube. Gerade die Covid-19-Pandemie habe, so Viktoria, aber auch gezeigt, welche Bedeutung Medien für die Information der Bevölkerung haben.

Auch der Freundeskreis von Susanne Scheib ist, neben ihrem jetzigen Partner und langjährigen besten Freund, stark von männlichen Bekanntschaften geprägt. Dies lässt sich auch darauf zurückführen, dass Susanne, wie einige der befragten männlichen jungen Erwachsenen auch, von Autos begeistert ist und gemeinsam mit ihrem Freund selbst Reparaturen vornimmt und an den Fahrzeugen schraubt. Gemeinsam werden dann auch viele Ausflüge, besonders an verschiedene Seen, unternommen. Nicht nur durch ihre Interessen, sondern auch durch einen anderen Einschnitt in ihrem Leben hat sich Susannes Freundes- und Bekanntenkreis stark verändert: Auf Grund ihrer Unzufriedenheit mit der Institution Kirche hat sie sich aus der Jugendarbeit zurückgezogen, die früher einen großen Teil ihrer Freizeit einnahm. Ihren Medienkonsum schätzt auch sie als „mittel“ ein. Bisweilen schaut sie Inhalte auf Netflix an, und für die Kommunikation sind ihr Smartphone und Medien wichtig. Jedoch, und dies hebt sie hervor, könne sie auf „das ganze Umeinandergeposte“ auf Soziale Medien gut verzichten.

Der Freundeskreis von Amelie Aufbauer unterscheidet sich von jenem der anderen Befragten: Sie konzentriert sich in ihrer Freizeit auf lediglich zwei bis drei sehr enge Freunde und benötigte auch lange Zeit, um sich auf eine feste Partnerschaft mit ihrem langjährigen Freund einzulassen. Inzwischen verbringt sie jedoch sehr viel Zeit mit ihrem Freund und spielt gemeinsam mit ihm oder alleine sehr intensiv das Computerspiel Ark zum Teil die ganze Nacht lang. Dies ist ihre wichtigste Freizeitbeschäftigung. Ansonsten achtet sie auf einen mäßigen Mediengebrauch und legt das Smartphone auch einmal bewusst zur Seite. Jedoch wolle sie nicht auf die zur Verfügung stehenden Möglichkeiten zur Kommunikation verzichten. Zudem hält sie „korrekte Medien“ für sehr wichtig und misst diesen einen hohen Stellenwert für die Gesellschaft bei; gemeint sind damit öffentlich-rechtliche Angebote im Fernsehen, im Radio und in Sozialen Medien sowie private Printmedien, die den Qualitätszeitungen zugeordnet werden können. 


\subsection{Zwischenfazit: Die Lebensführung der jungen Erwachsenen}

Die Ergebnisse der fokussierten Analyse zeigen mit Blick auf die jungen Erwachsenen sehr deutlich, wie individuell sie inzwischen ihr eigenes Leben führen und welche Schwerpunkte sie hinsichtlich ihrer Interessen, ihres Mediengebrauchs, ihres Beziehungslebens sowie ihrer Wünsche, Pläne und Zukunftsperspektiven setzen. Ihre familiären Ausgangspositionen waren dabei unterschiedlich gut ausgestaltet, dennoch ist es einigen von ihnen bereits gelungen, sich aus den zum Teil belasteten Kontexten ihrer Familien zu lösen und ihre Zukunft in die eigene Hand zu nehmen und zu gestalten. Allen individuellen Entwicklungen zum Trotz, lassen sich jedoch auch weiterhin vielfältige Verbindungen der jungen Erwachsenen zu ihren Familien nachweisen, und viele Entscheidungen lassen sich auf ihre Herkunft und die sie prägenden sozio-emotionalen wie sozio-ökonomischen Bedingungen zurückführen. Nicht zuletzt Mediengeräte und -angebote, aber auch kostspielige Hobbys und Freizeitaktivitäten dienen ihnen dabei heute als Statussymbole. Mit Blick auf Partnerschaften fällt auf, dass die jungen Frauen sich auf diese bereits stärker eingelassen haben als die jungen Männer. Dennoch zeugen zahlreiche Beispiele unter beiden Geschlechtern nicht nur von langjährigen, sondern auch von sehr engen Bindungen. Diese Suche nach stabilen Beziehungen kann auch vor dem Hintergrund der jeweiligen Kindheit und Jugend betrachtet werden, die vielfach geprägt waren von wechselnden Partnerschaften und Trennungen der Eltern sowie den damit verbundenen Verlusterfahrungen.

\subsection{Veränderungen im Leben der Eltern}

Während der Übergang von der Jugend ins Erwachsenenalter für Heranwachsende eine Zäsur darstellt, ist diese Phase auch für die involvierten Eltern mit Veränderungen und Herausforderungen, mitunter aber auch mit Chancen, verbunden. Sie müssen damit zurechtkommen, dass die Kinder das Haus verlassen und immer weniger auf ihre Unterstützung angewiesen sind. Dies schafft zusätzlichen Raum für gemeinsame Aktivitäten in der eigenen Partnerschaft; fehlt eine solche jedoch, kann das Fehlen von eingeübten Praktiken und bisher gültigen Lebensinhalten zu einer schwierigen Situation werden. Grundsätzlich ist das Erwachsenwerden der eigenen Kinder eine Gelegenheit, das eigene Leben zu reflektieren und womöglich persönliche Zielsetzungen neu zu justieren und damit stärker eigene Handlungsentwürfe zu entwickeln und ihnen Raum zu geben. Wie 
die Eltern des Panels - und dies mit Blick auf ihre jeweiligen sozio-ökonomischen und sozio-emotionalen Bedingungen - der neuen Lebenssituation ihrer Kinder begegnen und welche Konsequenzen sie damit für ihr eigenes Leben verbinden, steht im Mittelpunkt der folgenden Ausführungen.

Wie die fokussierte Analyse der Mütteraussagen gezeigt hat, haben sich die sozio-ökonomischen und sozio-emotionalen Bedingungen in den $\mathrm{Fa}$ milien weniger stark verändert als bei ihren Kindern durch ihren Eintritt in die Lebensphase junges Erwachsenenalter und die damit verbundenen neuen Entwicklungs- und Lebensaufgaben. Es erscheint daher sinnvoll, die Entwicklungen in den Familien typbezogen vorzustellen und zu diskutieren, um damit sowohl die Veränderungen innerhalb eines Familientyps zu erfassen als auch besser erkennen zu können, inwieweit aktuelle Bewegungen in den sozio-ökonomischen und sozio-emotionalen Bedingungen der Eltern Anlass geben, sie einem anderen Typ zuzuordnen (siehe dazu auch die Fallbeschreibungen in Kapitel 2).

\subsubsection{Typ 1: Massive sozio-ökonomische Probleme als multiple}

Deprivation: Die rundherum überforderten Familien (Landinger, Oblinger, Öllinger) ${ }^{24}$

Bei Familie Oblinger haben sich die sozio-ökonomischen Bedingungen der gesamten Familie Oblinger, von einem sehr niedrigen Ausgangsniveau ausgehend, deutlich verbessert. So haben Manfred und sein jüngerer Bruder erfolgreich eine Berufsausbildung aufgenommen, aber auch Herrn und Frau Oblinger ist es gelungen, wieder in das Arbeitsleben einzusteigen. Damit sind sie, das erste Mal seit vielen Jahren, nicht mehr auf Arbeitslosenunterstützung oder Sozialhilfe angewiesen. Frau Oblinger ist über diese Entwicklung „sehr glücklich“, dennoch trägt sie sich mit dem Gedanken, die als unsicher empfundene Gastronomiebranche, in der sie mittlerweile arbeitet, zu verlassen und sich für die als zukunftssicherer erachtete Pflege umschulen zu lassen. Die verbesserten sozio-ökonomischen Bedingungen der Familie wirken sich auch positiv auf das Familienklima aus, das somit sowohl von Manfred als auch von seiner Mutter zuletzt als sehr harmonisch beschrieben wurde. Diesen positiven Entwicklungen zum Trotz, will Frau Oblinger dennoch keine großen Pläne für die Zukunft schmieden, da sie und ihr Mann auch weiterhin mit vielfältigen gesund-

24 Familie Fein nahm an der siebten Erhebungswelle nicht teil; siehe dazu die Fallbeschreibung in Kapitel 2. 
heitlichen Problemen zu kämpfen haben. Die notwendige Behandlung der Eltern besorgt auch die Kinder sehr. Sie haben daher die Organisation für die anstehenden Behandlungen übernommen, um ihre Eltern zu unterstützen, da diese damit überfordert schienen und gerade während der Covid-19-Pandemie wenig Antrieb dazu zeigten, Entscheidungen zu treffen, um die indizierten Maßnahmen in die Wege zu leiten.

Auch in der noch immer vielfältig belasteten Familie Landinger zeigen sich vorsichtige Entspannungstendenzen, diese sind jedoch vor allem im sozio-emotionalen Zusammenleben der Eltern zu verorten, denn ihre sozio-ökonomische Lage hat sich nicht grundlegend verändert. Herr und Frau Landinger scheinen allerdings ihren Frieden mit sich und ihrer Lebenssituation gefunden zu haben. Gerade das Verhältnis zwischen den Eheleuten wirkt harmonischer und ausgeglichener als in den vorherigen Erhebungswellen. Ihr Sohn Timo profitiert davon jedoch nicht. Während Herr Landinger noch immer arbeitslos ist und geschäftig vorgibt, sich um die Abwicklung des Erbes seiner Mutter kümmern zu müssen, freut sich Frau Landinger auf ihre nun unmittelbar bevorstehende Pensionierung. Sie sei, wie sie im Interview sagt, „im Großen und Ganzen“ zufrieden mit der Gesamtsituation der Familie und möchte eigentlich nichts ändern. Sie will ihren Ruhestand genießen, Zeit in der Natur verbringen und mit dem Hund spazieren gehen; zudem genießt sie es, wenn die Enkelkinder zu Besuch sind und im Garten spielen. Darüberhinausgehende Pläne wolle sie aber nicht machen. Für die Familie habe inzwischen „alles gepasst“ und man warte lediglich auf die endgültige Zusage für eine neue Stelle in einer geschützten Werkstätte für Timo - dessen unsichere Zukunftsaussichten scheinen seine Eltern aber auch weiterhin nicht zu beunruhigen.

Magda Öllingers sozio-ökonomische Lage ist im Wesentlichen gleichgeblieben, und auch ihre gesundheitliche Situation hat sich in der siebten Erhebungswelle nicht verändert. Sie ist weiterhin auf einen Rollstuhl und die Unterstützung durch einen Sozialdienstleister angewiesen. Veränderungen sind jedoch im Familienklima festzustellen und dies - vor allem - bei der schwerbehinderten Frau selbst. So hat sich Frau Öllingers Lebensqualität deutlich verbessert, seitdem ihre Wohnung behindertengerecht umgebaut wurde und sie nun alle Wohnbereiche selbstständig erreichen kann. Auch mit dem beginnenden Abnabelungsprozess ihrer Tochter Viktoria scheint sie inzwischen besser zurecht zu kommen, seit diese deutlich selbstbewusster auftritt, ihre eigene Selbstständigkeit betont und diese auch von ihrer Mutter einfordert. Für die Zukunft wünscht sich Frau Öllinger, endlich wieder arbeiten gehen und ihren Lebensunterhalt selbst verdienen zu können. Dieser Wunsch erscheint jedoch aufgrund 
ihrer gesundheitlichen Situation kaum erfüllbar. So wünscht sich Frau Öllinger letztendlich lediglich eine allgemeine Verbesserung, „dass amoi was bergauf gehen kunt".

3.6.2 Typ2: Die sozio-ökonomisch nicht mehr bzw. wenig belasteten, aber wegen problematischer sozio-emotionaler Beziehungsstrukturen überforderten Familien (Hirtner, Holzner, Rohringer Weiss)

Bei den Familien von Typ 2 lassen sich - bis auf eine Ausnahme, Familie Rohringer - weiterhin zum Teil stark belastete sozio-emotionale Bedingungen erkennen, und die Beziehungen zwischen den (ehemals) alleinerziehenden Müttern und ihren Kindern erweisen sich als nach wie vor stark angespannt. Therese Weiss erklärt im Interview zwar, dass sie mit ihrem Leben zufrieden sei, da „alles passt“ und sie - mit Blick auf ihre derzeitige Arbeitsstelle - bei Bedarf „jederzeit ihre Stunden aufstocken“ könne. Und tatsächlich hat sich ihre sozio-ökonomische Situation nochmals deutlich verbessert, seitdem sie eine Festanstellung als Justizwachbeamtin angetreten hat. Zudem bewohnt sie nun nur noch mit ihrem jüngeren Sohn eine Doppelhaushälfte; dies sei zunächst ungewohnt gewesen, jedoch habe sie sich schnell an die neue Situation, die Ruhe und den vielen Platz gewöhnt, und auch der Haushalt sei nun weniger aufwändig. Aber diese Aussagen können nicht darüber hinwegtäuschen, dass sich die sozio-emotionalen Bedingungen der Familie durch den Streit zwischen ihr und ihrem Sohn Alfons, dessen zwischenzeitlich sehr schwierige finanzielle und auch psychische Lebenssituation eine deutliche Tendenz zu Typ 1 aufweist (siehe dazu die Fallbeschreibung in Kapitel 2), drastisch verschlechtert haben. Frau Weiss bedauert zwar im Interview das Zerwürfnis und versucht auch, ihren Sohn materiell (Kaution) und alltagspraktisch (Waschen) zu unterstützen, gleichzeitig ist sie jedoch nicht in der Lage, sich in Alfons' Lage zu versetzen und zumindest in Ansätzen Verständnis für ihn zu zeigen. Eine Aussprache über die verschiedenen Ansichten und Positionen fand zwischen Mutter und Sohn bis zum Abschluss der siebten Erhebungswelle nicht statt. Vor diesem Hintergrund lässt sich die zunächst etwas irritierende Aussage von Frau Weiss, dass sie mit ihrem Leben zufrieden sei - obwohl die Beziehung zwischen ihr und ihrem Sohn Alfons weiterhin von großen Konflikten geprägt ist - erklären. Schon in den vorherigen Erhebungswellen wurde der Alltag von Frau Weiss vom Thema Geld bestimmt und der steten Sorge, davon nicht genug zu haben (siehe dazu die Ausführungen bei Paus-Hasebrink/ Kulterer/ Oberlinner 2017: 186). An 
Alfons, seiner Entwicklung und seinen Belangen zeigte sie dagegen bereits seit vielen Jahren nur ein sehr eingeschränktes Interesse. Im Gegensatz dazu rückte sie dessen Halbbruder Christian in den Interviews immer wieder, auch ungefragt, in den Mittelpunkt und hob dessen Qualitäten hervor.

Auch das Verhältnis von Heidi Holzner zu ihrem Sohn Benedikt, der selbst nicht länger unter der gestörten Beziehung zu seiner Mutter zu leiden scheint, ist nach wie vor - und dies schon seit vielen Jahren angespannt, wenn nicht zerrüttet. Frau Holzner thematisiert dies im Interview gar nicht; sie scheint sich schlichtweg kaum für ihren Sohn zu interessieren und weiß über dessen positive, auch berufliche, Entwicklung nicht Bescheid. Daher muss ihr Leben - stärker noch als in den Erhebungswellen zuvor - weitgehend losgelöst von Benedikt betrachtet werden. Mit ihrer zweiten Ehe und den beiden nachgeborenen Söhnen scheint Frau Holzner zufrieden zu sein, und auch beruflich laufe es für sie und ihren Ehemann, wie sie im Interview betont, gut. Sie hebt auf die Frage nach Veränderungen seit der vorherigen Erhebungswelle positiv hervor, dass ihr und ihrem Mann in der Wohnung nun deutlich mehr Platz zur Verfügung stehe: „Das Einzige was sich verändert hat ist, dass die Großen jetzt mittlerweile olle weg sand." Den nahezu vollständigen Kontaktabbruch zu ihrem Sohn erwähnt sie dagegen auch in der siebten Erhebungswelle nicht.

Wie bei Frau Holzner und Frau Weiss ist auch das Verhältnis von Frau Hirtner zu ihrem Sohn Mario weiterhin belastet. Sie bedauert immerhin den Auszug ihres „Sohnemannes“ und sei deswegen eine „sehr traurige Mutter“. Diese Äußerung im Interview kann jedoch nicht darüber hinwegtäuschen, dass das Verhältnis zwischen beiden noch immer belastet und nicht endgültig geklärt zu sein scheint. Zudem ist Frau Hirtner die einzige befragte Mutter im Panel, deren sozio-ökonomische Bedingungen sich in der siebten Erhebungswelle im Vergleich zu den letzten Erhebungswellen dramatisch verschlechtert haben; sie lässt damit eine Tendenz zu Typ 1 erkennen. Nach langer Krankheit wurde ihr gekündigt, und sie ist erneut arbeitssuchend. Aufgrund ihrer gesundheitlichen Bedingungen fühlt sie sich perspektivlos, und es sähe, wie sie es im Interview unter Tränen ausdrückt, „scheiße aus“. Auch ihre langjährige Partnerbeziehung sei, wie sie auf die Frage nach den Veränderungen in ihrem Leben erzählt, auseinandergegangen. Aber sie, so versuchte sie sich selbst Mut zuzusprechen, gehe dennoch davon aus, dass es in Zukunft wieder einen Mann und ,auf jeden Fall beruflich wieder etwas für sie geben" werde und sie daher ihren Optimismus nicht verlieren wolle. 
Frau Rohringers, seit ihrer Beziehung zu einem finanziell besser gestellten Mann in der dritten Erhebungswelle, verbesserte sozio-ökonomische Lage hat sich auch in der siebten Erhebungswelle nicht verändert, finanziell kommt sie weiterhin gut zurecht. Ähnlich wie Frau Oblinger, Frau Öllinger und Frau Hirtner hat nun aber auch Frau Rohringer mit gravierenden gesundheitlichen Problemen zu kämpfen. Ihre Krebserkrankung zwischen der sechsten und siebten Erhebungswelle hat sie als ein deutliches Warnzeichen wahrgenommen und dann beschlossen, es nun „ruhiger angehen" zu lassen. Bei ihr wurde ein Gehirntumor diagnostiziert und erfolgreich behandelt: „Da ziaghts da die Schuach aus.“ Ihre Erkrankung habe auch Auswirkungen auf die Familie gehabt und sei in ihrer „Familieng'schicht zusammenschweißend“ gewesen; die „Kinder san schlagartig erwachsen word' $n$ ". Damit verbunden hat sich auch das Familienklima entspannt und die sozio-emotionale Beziehung zu ihrer Tochter Isabelle erscheint nicht mehr in der Weise belastet wie in den vorherigen Erhebungswellen, zumal sich auch Isabelle mittlerweile stärker von ihrer Mutter emanzipiert hat.

\subsubsection{Typ 3: Die zwar sozio-ökonomisch, aber sozio-emotional weniger belasteten, relativ kompetenten Familien (Aufbauer, Boll, Ebner, Kaiser, Stab)}

Seitdem zunächst die älteste Tochter und später auch Amelie ausgezogen waren, hat sich das Familienklima in Familie Aufbauer weiter deutlich verbessert. Danach gelang es der sozio-ökonomisch belasteten alleinerziehenden Mutter besser, als dies bereits in den vorherigen beiden Erhebungswellen der Fall war, ihren Alltag zu organisieren. Mit beiden Kindern hält Frau Aufbauer weiterhin engen Kontakt und unterstützt sie. Die positive Einschätzung teilt sie mit Amelie, die ihren Auszug aus der Wohnung der Mutter als die „beste Entscheidung“ bezeichnet. Frau Aufbauer wohnt nun gemeinsam mit ihren beiden jüngeren Söhnen zusammen und ist mit der Wohnsituation sehr zufrieden. Bereits in „zwei Jahren“ stehe jedoch voraussichtlich erneut ein Umzug an, da der Mietvertrag womöglich nicht verlängert werde. Damit bleibt der ständige Wohnungswechsel eine Konstante im Leben der Familie. In diesem Zusammenhang hofft Frau Aufbauer, dass sie erneut eine geförderte Wohnung erhalten kann, denn anders könne sie sich die Miete kaum leisten. Ihre Arbeit in einem SecondHand-Geschäft sowie ihre Autorinnentätigkeit für eine Kolumne in einem 
Magazin machten ihr zwar Spaß, erlaubten finanziell aber keine großen Sprünge.

Bei Frau Boll und ihrem Sohn haben sich die bisher sozio-ökonomisch angespannten Bedingungen weiter verbessert, Gregor gelang der erfolgreiche Einstieg in eine Anstellung, und die Hundezucht von Frau Boll hat sich nachhaltig positiv entwickelt. Die sozio-emotionalen Bedingungen sind weiterhin stabil geblieben. Gesundheitlich hat Frau Boll jedoch, ähnlich wie auch Frau Oblinger und Frau Öllinger (Typ1) sowie Frau Hirtner und Frau Rohringer (Typ 2) sehr zu kämpfen. Nach ihrer überstandenen Krebserkrankung 2010 bekam sie 2020 eine Embolie, die eine zehntätige intensivmedizinische Behandlung erforderte. Für die noch zu Hause lebenden Kinder bedeutete dies, wie sie erzählte, dass es nun „kein Hotel Mama mehr" gab und sie selbst auch im Haushalt umfassend Verantwortung übernehmen mussten. Auch der Tod ihrer eigenen Mutter sowie der völlig überraschende Tod ihrer besten Freundin seien deutliche Hinweise für sie gewesen, sich nun selbst stärker zurückzunehmen. Nach ihren Erlebnissen auf der Intensivstation, ,an 1.000 Geräten angeschlossen, ja, ach, du liebe Zeit, jetzt geht's dahin", wolle sie daher nun auch keine großen Zukunftspläne mehr schmieden. Bereits seit 2014, zu diesem Zeitpunkt endete eine vorübergehende Partnerschaft und wandelte sich zu einer rein freundschaftlichen Beziehung, ist Frau Boll keine neue Beziehung mehr eingegangen. Es wird zudem sehr deutlich, dass das Verhältnis zu ihrem geschiedenen Mann noch immer auf das Schwerste belastet ist und sie ihre frühere Ehe nicht in Ruhe lässt. So sieht sie dessen nun aufgetretene, akute und wohl lebensbedrohende Krebserkrankung mit unverhohlener Genugtuung: „Aber wie gesagt, irgendwann schließt sich der Kreis, und jetzt ist er selber dran! Und das hat er sich auch verdient!“

Bei Familie Ebner haben sich weder die sozio-ökonomisch eingeschränkte Lage noch ihr sozio-emotional ausgeglichenes Familienleben verändert. Auf die Frage im Interview nach den Veränderungen seit der sechsten Erhebungswelle verneint Kerstin Ebner jegliche Veränderungen, sowohl in ihrem Privat- als auch in ihrem Berufsleben: „Naa, goar nix. Mia san' so im Trott drinnen. (lacht) Es lauft eigentlich jeder Tog so, es gaunze Joahr und... Oiso, es is jetzt nix, wos i wissat, wos jetzt irgendwos Aufregendes is. Wissat jetzt goar nix." Selbst dem Umstand, dass sie zum ersten Mal Großmutter wird, misst sie im Interview keine große Bedeutung bei und verweist auch in diesem Kontext direkt auf ihre Tochter Elisabeth: „Jo naa, verändert hot si net allzu vü. Die, außer i werd“ jetzt dann Oma, im Juli. Von der Elisabeth ihr'm Bruder. Und do g'freit sie si scho' recht, weil sie Tante wird. Und, oba so jetzt vom Ganz'n her." 
Über die Beziehung ihrer Tochter freut sie sich dagegen sehr, da sowohl sie als auch ihr Mann den Partner sehr schätzen. Zudem freut sie sich darüber, dass die Familienbande nicht unter Elisabeths Auszug gelitten haben, Elisabeth komme vielmehr noch immer gern und oft zu Besuch nach Hause. Eigene Zukunftsperspektiven äußert sie nicht.

Bei Frau Kaiser zeigen sich sozio-ökonomische Verbesserungen; sie weist in ihren Lebensverhältnissen mittlerweile eine Tendenz zu Typ 4 auf. Mit ihrem Leben, gemeinsam mit ihren drei Söhnen, sei sie zufrieden; eine neue Partnerschaft gehört für die alleinerziehende Mutter, ähnlich wie auch für Frau Aufbauer, ausdrücklich nicht zu ihren Plänen. Positiv hebt sie hervor, dass sich auch ihre psychische Situation deutlich entspannt habe. Wenn ihre Söhne aus dem Gröbsten heraus seien und dann den Berufseinstieg geschafft hätten, so ihr Wunsch für die Zukunft, wolle sie "gründlich durchschnaufen“ und die Ruhe genießen: „Dass ich wieder mehr Zeit für mich hab'“" Fester Begleiter dabei soll dabei ein bereits angeschaffter Familienhund sein.

Stabilisiert hat sich auch das Leben von Sandra Stab und ihrer Familie. Nach Jahren mit verschiedenen wechselnden Tätigkeiten, arbeitet Frau Stab nun als Gärtnerin in einer von der Europäischen Union geförderten Stelle. Dort verdiene sie zwar nicht viel Geld, aber sie sei zufrieden, da sie Spaß an der Arbeit habe. Auch die in den vorherigen Erhebungswellen belastete Wohnsituation der Familie hat sich durch einen erneuten Umzug entspannt. Die früheren, mit der alten Wohnkonstellation verbundenen Konflikte hatten sie so stark belastet, dass sie stationär behandelt werden musste. Die Beziehung der alleinerziehenden Mutter zu ihren Kindern, insbesondere ihrer Tochter Simone, ist weiterhin ausgeglichen und stabil. Für ihre Zukunft hat Frau Stab allerdings einen ganz expliziten Wunsch, „dass i ein Platzl finde, ein Haus mit Garten. Dass i mi wohl fühle, dass i auch ein paar Tiere hab'n kann, so wie Hühner oder ein Schwein oder so. Des wäre so mein Wunsch. Und eine funktionierende, ein lieber Mann, eine funktionierende Partnerschaft für mein letztes halbes Leben, sozusagen. Des sind so meine Wünsche“. 


\subsubsection{Typ 4: Keine belasteten sozio-ökonomischen Bedingungen mehr und unbelastete sozio-emotionale Beziehungsstrukturen - Die relativ kompetenten `Aufsteiger`(Dornbacher, Grubert, Pfortner, Scheib, Zarbl)}

Den Familien von Typ 4 ist es gelungen, ihre sozio-ökonomisch bereits recht gute Lage weiterhin aufrecht zu halten. Die Frage nach Veränderungen in ihrem Alltag beantwortet Franziska Dornbacher denn auch gelassen. Sie selbst sei mittlerweile ruhiger und gesetzter geworden und nicht mehr so impulsiv wie früher. In näherer Zukunft würde sich für die Familie auch nicht allzu viel ändern, da Gudrun auch während des Studiums zu Hause wohnen bleiben solle; erst danach stünden Veränderungen an. Aber in Zukunft wolle sie alles generell ,in Ruhe und Gemütlichkeit“ angehen, und Dinge nicht mehr auf „Biegen und Brech'n“ erzwingen.

Etwas unklarer erscheint dagegen die Situation bei Gabriele Scheib. Den Auszug ihrer Kinder bezeichnet sie als „amoi gravierende Veränderung“. Entsprechende Konsequenzen thematisiert sie jedoch nicht und zudem verneint sie Veränderungen mit Blick auf ihr eigenes Leben. Aus dem Gespräch mit ihrer Tochter Susanne wird aber deutlich, dass Frau Scheib aus Deutschland zurück in ihre Heimat nach Österreich gezogen ist. Genauere Informationen zu den Beweggründen liegen nicht vor, Susanne betont jedoch direkt, dass es zu keiner Trennung von ihrem Stiefvater gekommen sei; das gute Familienklima scheint dadurch auch nicht belastet zu sein.

Anna Grubert äußert sich sehr positiv über die aktuellen sozio-ökonomischen und sozio-emotionalen Lebensbedingungen ihrer Familien und ihr eigenes Leben. Sie sei sehr zufrieden, sowohl mit ihrer beruflichen als auch ihrer privaten Situation und habe daher keinerlei Änderungswünsche oder Pläne für die Zukunft. Ihre Zufriedenheit unterstreicht Frau Grubert im Interview noch durch die Erwähnung, dass die Familie einen neuen Hund angeschafft habe, der das Familienleben bereichern solle und der den Zusammenhalt innerhalb der Familie noch weiter steigern werde, denn für den Hund müssten schließlich alle in der Familie Verantwortung übernehmen.

Auch Familie Pfortner geht es, ebenso wie den anderen Familien in Typ 4, sozio-ökonomisch und sozio-emotional alles in allem weiterhin gut. Die Familie blickt aber, wie Waltraud Pfortner erzählt, beruflich wie privat, auf anstrengende Zeiten zurück: Wegen der Covid-19-Pandemie musste das Familienunternehmen zwar „etwas kämpfen“, jedoch gelang es, die Krise des ersten Lockdowns gut zu meistern. Zusätzlich ist Frau Pfortner auch in ihrer Nebentätigkeit als Drogerieverkäuferin stark einge- 
bunden; dennoch möchte sie diese nicht missen, da sie ihre Anstellung auch mit Unabhängigkeit und Sicherheit verbindet und beides sehr zu schätzen weiß. Nach dem Tod der Mutter von Herrn Pfortner, die von den Eheleuten zu Hause gepflegt wurde, bräche, wie sie sagt, nun eine „Umbruchphase“ an: Einerseits, da sie auf die 50 zugehe und merke, „dass nicht mehr immer alles so und so perfekt lauft", andererseits, da die Kinder nun „komplett flügge san“. Als einzige konkrete Ziele nennt sie ihren Umzug und den ihres Ehemannes in das Erdgeschoss des Hauses, um im ersten Stock zusätzlichen Platz für eines der Kinder zu schaffen, sowie den Erwerb einer neuen Katze für die Familie.

Die Lebensverhältnisse stellen sich auch in Familie Zarbl als ähnlich gut dar. Wie die anderen Familien aus Typ 4 erleben sie die Phase des Erwachsenwerdens ihrer Kinder durchweg als sehr positiv. Theresa Zarbl betont, dass sich die gelebte „Patchwork-Konstellation“ der Familie zwar bewährt habe und auch weiterhin funktioniere, dennoch freue sie sich auf den bevorstehenden Auszug von Norbert, da sie sich davon mehr „Freiheit“ für sich und die „Elternbeziehung“ mit ihrem Ehemann erhoffe. Neben den Kindern habe auch dessen berufliche Selbstständigkeit in den letzten Jahren das Familienleben eingeschränkt. Nun möchte sie wieder aktiver werden, mit ihrem Mann draußen unterwegs sein, spazierengehen oder mit dem Rad unterwegs sein. Darüber hinaus steht der regelmäßigere Besuch von kulturellen Veranstaltungen, und dies auch ganz explizit am Abend, auf ihrer Agenda.

Familie Zarbl geht es damit ähnlich wie den anderen Familien aus Typ 4 so, dass sie mit den Gedanken an ihre Zukunft keine sozio-ökonomischen Ängste verbinden. Auch die sozio-emotionalen Beziehungen in diesen Familien sind so stabil, dass es ihnen als Eltern nunmehr gelingt, ihre während der vergangenen Jahre stark auf die Unterstützung und Förderung ihrer Kinder gerichteten Handlungsentwürfe stärker auf sich selbst zu beziehen.

\subsection{Veränderungen des Mediengebrauchs der Eltern}

Während Medienangebote im Alltag der Kinder auch noch im jungen Erwachsenenalter fest verankert sind und vielfältigen Funktionen dienen, äußerten sich einige der befragten Mütter in früheren Erhebungswellen immer wieder sehr kritisch über Medien im Allgemeinen und den ausufernden Medienkonsum ihrer Kinder im Speziellen. Dennoch hatten Medien auch bei den Eltern in den meisten Fällen einen wichtigen Stel- 
lenwert, sowohl zur Unterhaltung als auch zur Information und in den letzten Jahren verstärkt auch zur Kommunikation. Daher gilt in diesem abschließenden Abschnitt der Blick nicht nur dem Mediengebrauch der Eltern, sondern auch dessen Veränderungen. Dabei entwickelten sich die Mediengebrauchsweisen der befragten Eltern in den vergangenen Jahren eher kontinuierlich weiter und orientierten sich an ihren jeweils individuellen Bedürfnissen und auch ihren zur Verfügung stehenden Zeitbudgets. $\mathrm{Zu}$ grundlegenden Veränderungen oder gar abrupten Brüchen kam es dagegen nicht. Eine deutliche Ausnahme stellt in diesem Kontext nur Frau Zarbl dar. Sie erzählt, dass sich ihre Einstellung gegenüber Medien seit der letzten Befragung (2016/2017) so fundamental verändert habe, wie sie es selbst nicht für möglich gehalten hätte. Sie verstehe inzwischen die „Abhängigkeit“ junger Menschen von Medien und beobachte, mit Blick auf Facebook, eine solche auch bei sich selbst. So sei es ihr wichtig, sich „mindestens einmal am Tag“ auf den neuesten Stand zu bringen, sonst fehle ihr etwas. Auf Facebook finde sie, gerade in der Covid-19-Pandemie, auch eine „alternative“ Informationsquelle. Darüber hinaus kommuniziere sie inzwischen wie selbstverständlich mit ihren Söhnen zumeist über WhatsApp. Ein Auslöser seien dabei ihre Fahrten zur Arbeit im Bus gewesen. Um sie herum nutzten alle nur noch die weitaus vielfältiger einsetzbaren Handys und Tablets, aber keine Zeitungen mehr. Auch auf den Fernseher könnte sie eigentlich verzichten, da sie dort nur mehr die Nachrichten („Mainstream“) sowie einige Serien zur Entspannung schaue; deutlich wichtiger sei für sie aber inzwischen das Streaming auf Netflix. Letzteres bestätigt nun auch Frau Holzner, die betont, dass man mit diesen Angeboten seine Kinder weitaus besser vor negativen Nachrichten schützen könne als im Fernsehen, da auf Netflix zahlreiche kindgerechte Inhalte zur Verfügung stünden, die man selbst auswählen könne. Solche Überlegungen hatten bei Frau Holzner in den ersten Erhebungswellen in Bezug auf ihre Kinder aus erster Ehe keine Rolle gespielt, diese parkte sie regelmäßig und unbeaufsichtigt vor dem Fernseher und überließ sie so ungeschützt den gesendeten Inhalten.

Deutlich positiv äußert sich auch Frau Ebner, doch sie war bereits in früheren Erhebungswellen neuen Medienangeboten gegenüber offen eingestellt. Inzwischen könne sie sich gar nicht mehr vorstellen, privat und beruflich (Regenradar vor Feldarbeit) auf das Smartphone zu verzichten. Sie äußert sich geradezu begeistert von den vielfältigen Nutzungsmöglichkeiten des Internets. Gefahren sieht sie dagegen im Online-Shopping (Geschäftesterben im Dorf) und im Googlen nach Krankheitssymptomen: 
Man liest do die schlimmst'n Soch'n. (...) Weil, woaßt do steht dann so vü drin und dann mochst da nu mehr Sorg'n und nu mehr Sorg'n und des is a net guat. Des is` wieder. Des is' mit dem, wann irgendwos is', und du schaust, des, und des tuat ma weh, aufs erste kummt a schlimme Krankheit. Krebs oder sunst wos.

Auch Frau Oblingers Einstellung zu Medienangeboten hat sich inzwischen geändert; noch 2016 hatte sie das Fernsehen als „Scheiße“ bezeichnet. Auch heute noch legt sie besonderen Wert auf die "gedruckte“ Tageszeitung und zeigt wenig Verständnis für den Erfolg der Online-Zeitungen. Dennoch haben mittlerweile das Smartphone und besonders WhatsApp einen hohen Stellenwert für sie, um Kontakt mit Freunden und Familie zu halten. Kleinen Social Games und Handy-Spielen spricht Frau Oblinger nach ihrem Schlaganfall, sie sagt zur Konzentrationsübung, zudem eine therapeutische Funktion zu. Ähnlich sieht dies auch Frau Kaiser, die sich daher selbst als „starke“ Mediennutzerin bezeichnet. Sie verfolge im Fernsehen gerne die Nachrichten und schaue Serien. Ihr bevorzugter Sender sei dabei Sat.1 Gold, dessen Angebote sie auch in der entsprechenden Mediathek beziehe. Großen Wert legt sie zudem auf Lektüre der Krone am Sonntag. Ein Angebot, das gerade während der Covid-19-Pandemie für sie stark an Bedeutung gewonnen hat, ist Facetime, da sie so engen Kontakt mit ihrem Sohn in Deutschland halten konnte.

Frau Hirtner dagegen nutzte auch bereits in der Vergangenheit zahlreiche Medienangebote und stand medial vermittelter Information, Kommunikation und Unterhaltung traditionell sehr offen gegenüber. Sie verwendet verschiedene Online-Angebote und ist unter anderem auch seit einigen Jahren bereits sehr intensiv auf Facebook vertreten, nicht zuletzt, um dort auch neue Menschen kennenzulernen. Im Mittelpunkt ihres Interesses stand und steht jedoch das lineare Fernsehen, da sie, auch aus finanziellen Gründen, keinen Netflix-Account habe. Die von ihr rezipierten und von ihr selbst als „lauter Bledsinn“ bezeichneten Unterhaltungsangebote stellen, bedingt durch Arbeitslosigkeit und die bestehenden Kontaktbeschränkungen, ihre einzige Freizeitbeschäftigung dar. Auch Frau Grubert schätzt das Fernsehen als Mittel zur Unterhaltung, sie berichtet jedoch nicht von nennenswerten Veränderungen in ihrem Mediengebrauch. Serien, „bayerische Krimiserien", wie die Rosenheim Cops oder Hubert und Staller, und generell „keine so hochkomplizierten Filme“, könnten aber gut „nebenher" laufen. Auch Frau Öllinger setzt in ihrem Mediengebrauch stark auf Information und Unterhaltung durch Fernsehangebote sowie auf das Smartphone: „Fernseher immer. Und des Handy hob i sowieso bei mir.“ Auf Grund ihrer körperlichen Einschränkungen blieben ihr lange Zeit nur 
wenige Beschäftigungen, jedoch nimmt seit 2019 der Rollstuhltanz einen wichtigen Stellenwert in ihrem Freizeitrepertoire ein; der Sport sowie der soziale Austausch vermitteln ihr, wie sie deutlich macht, die dringend benötigte Lebensfreude. Frau Boll ist ebenfalls traditionell eine intensive Mediennutzerin, jedoch sind ihre Interessen ganz anders gelagert. Sie nutzt das Fernsehen, um sich sowohl über Österreich als auch über Deutschland zu informieren. Zudem schätzt sie viele Angebote des öffentlich-rechtlichen Rundfunks, insbesondere Polit-Talkshows, und greift regelmäßig auch auf die Mediatheken zu. Abgesehen vom Tatort meide sie aber Serien und Unterhaltungsangebote, da sie sich nicht „abhängig machen“ lassen wolle. Nachdem Smartphone und WhatsApp bereits wichtig für sie waren, um sich mit der Familie auszutauschen und das Leben der verstreut lebenden Kinder verfolgen zu können, hat sich Frau Boll zuletzt auch dazu entschieden, in ihrer Hundezucht stärker auf Online- und Mobil-Kommunikation zu setzen, um von den entsprechenden Möglichkeiten profitieren zu können.

Ganz anders präsentiert sich die Situation bei Frau Weiss. Sie äußert sich weder positiv noch negativ über Medien im Allgemeinen, sondern spricht sich lediglich gegen deren exzessive Nutzung aus. Sie selbst gebrauche Angebote nur zur Information und Kommunikation, denn sie habe „des eigentlich gar ned g'braucht, die Unterhaltung mit Medien“. Arbeit, Nebenbeschäftigung, Freizeit und Freunden stünden im Mittelpunkt. Ähnlich äußert sich auch Frau Landinger, die Medien nur mehr einen „mittleren“ Stellenwert zubilligt, da diese „nicht das Wichtigste im Leben“ seien. Jedoch verfolge sie gerne die Nachrichten im Fernsehen und im Internet, suche sich Basteltipps auf YouTube und halte über WhatsApp Kontakt mit Freunden und Familie. Frau Stab schätzt ihre eigene Mediennutzung als gering ein, erkennt jedoch die Vorteile der Medien zu Vernetzung der Welt und zum Austausch von Informationen. Ihr persönlich seien dies aber bereits zu viele Informationen, weshalb sie lieber weniger Medien nutze. Den Fernseher habe sie bereits „seit 15 Jahren nicht mehr“ und inzwischen überlege sie sich auch, auf das Smartphone zu verzichten: „Also ich denke manchmal, dass i auch das Handy ausschalte und einfach kein's mehr habe." Reduziert präsentiert sich auch der Mediengebrauch von Frau Dornbacher. Ebenso wie für ihre Tochter sind aber auch für sie Bücher essenziell. Insgesamt schätzt sie ihre Nutzung aber als ,gering“ ein. Dennoch hält sie Medien für wertvolle Informationsquellen, man müsse aber genau prüfen und unterscheiden, welchen Quellen man Glauben schenken dürfe und welchen nicht. 
Auch Frau Pfortner steht Medien offen gegenüber, sie räumt jedoch kritisch ein, nur noch selten fernzusehen, da es zwar sehr viele Sender aber „nix G'scheits“ mehr gäbe. Zudem schlafe ihr Mann regelmäßig beim gemeinsamen Fernsehen ein. Medien misst sie zwar keinen großen Stellenwert bei, dennoch verwendet sie Homepage, E-Mail und Smartphone regelmäßig in ihren beruflichen Kontexten. Privat wendet sie sich ebenfalls verschiedenen Online-Angeboten (u.a. Facebook und YouTube) sowie ausgewählten Fernsehsendungen, der Zeitung und vielen Büchern zu. Als einzige der befragten Mütter im Panel weiß sie überraschend gut über den Mediengebrauch ihres Sohnes Bescheid. Dennoch räumt sie lachend ein, dass eine Mutter auch nicht alles wissen müsse, zumal Helmut ja nun auch erwachsen sei.

Sehr kritisch gegenüber Medien und dem Mediengebrauch im Allgemeinen äußert sich dagegen Frau Scheib, die damit recht deutlich von ihren Positionen in der sechsten Erhebungswelle abweicht. Sie wirft in diesem Zusammenhang die Frage auf, „ob des digitale Zeitalter, ob des wirklich jetzt so a Bereicherung is“". Gerade bei Unternehmen und Angeboten wie Facebook, Google und WhatsApp sieht sie die Gefahr der Datenweitergabe sowie eine Einschränkung der Meinungsfreiheit durch Zensur und kritisiert die „Zusammenhänge“; gemeint sind damit wohl deren kommerzielle Strukturen und die Vernetzungen zwischen den einzelnen Angeboten. Ihrer eigenen Skepsis zum Trotz nutze sie gerade das Smartphone jedoch sehr viel. Daher wolle sie das eigene Medienverhalten zukünftig wieder stärker kontrollieren, testweise auf das Internet verzichten und „ma moi s WLAN ausschalt'n. Mal 24 Stunden". Ganz ähnlich positioniert sich auch Frau Rohringer: Sie bekennt sich sowohl beruflich (Organisatorisches schnell und digital erledigen) als auch privat (Smartphone, Amazon Alexa, Fernsehen, Radio) zu einem sehr intensiven Mediengebrauch. Jedoch hält sie sowohl ihren eigenen als auch den allgemeinen Mediengebrauch in der Gesellschaft für zu intensiv. Daher sei sie inzwischen dazu übergegangen, das Smartphone zuweilen auf lautlos zu setzen oder ganz abzuschalten und es zu ignorieren, wenn es zu oft klingele. Auch aus ihrer Erkrankung an einem Hirntumor hat sie Konsequenzen gezogen; so achte sie nun verstärkt auf Strahlenbelastungen. Dazu zählt es auch, das WLAN zu Hause am Abend konsequent abzuschalten. Ein ähnliches Paradoxon beschreibt auch Frau Aufbauer, die sowohl ihren eigenen Mediengebrauch („Jo, a zvü.“) als auch den ihrer Kinder („A zvü. A zvü. Sicher a zvü.“) als viel zu hoch bezeichnet. Dennoch räumt sie ein, Medien gerne zu nutzen und sie billigt diesen einen hohen Stellenwert in allen Lebenslagen zu. Für sie stünden dabei insbesondere WhatsApp, Instagram und YouTube 
(Informationssuche, Tutorials), Online-Recherchen (Rezepte) und OnlineZeitungen (DerStandard.at, Presse.com) im Mittelpunkt. Gerade mit Blick auf die Zeit des Lockdowns in der Covid-19-Pandemie seien aber alle Grenzen überschritten worden: „Naa, wenn'st des jetzt in Corona fragst, dann kann i eich sag'n a überhohe (Mediennutzung). Oiso, des is total eskaliert. In alle Richtungen."

Die Aussagen der Eltern in der siebten Erhebungswelle zeigen, dass viele von ihnen sich inzwischen ebenfalls sehr stark mit Medien befassen und diese sowohl zur Information und Unterhaltung als auch zur Kommunikation gebrauchen. Nur wenige berichten von einem reduzierten Mediengebrauch oder davon, sich selbst konsequent zu reglementieren und den selbst gesetzten Zielen zu folgen. Vielmehr fällt auf, dass viele von ihnen die Begeisterung ihrer Kinder für Medien inzwischen nachvollziehen können und deren Beispielen teilweise auch folgen. Jedoch kann auch festgehalten werden, dass viele der Eltern ihren Mediengebrauch, die Chancen und Risiken von Medienangeboten und auch strukturelle $\mathrm{Zu}$ sammenhänge inzwischen deutlich stärker hinterfragen und einzuordnen versuchen, als dies in früheren Erhebungswellen noch der Fall war.

\subsection{Fazit}

Der fallübergreifende Blick der fokussierten Analyse auf die jungen Erwachsenen (zur weiteren Einordnung der Befunde siehe Kapitel 6) legt offen, dass die Entwicklung der jungen Menschen in einem engen Zusammenhang mit ihren Entwicklungsaufgaben steht, allen voran der Loslösung vom Elternhaus. So orientieren sie sich weniger als noch zum Ende der sechsten Erhebungswelle (2016) an den Handlungsentwürfen ihrer Eltern und setzen sich mit ihnen darüber auseinander, vielmehr folgen sie, entsprechend den für sie aktuell zur Auseinandersetzung und Bewältigung anstehenden Entwicklungsaufgaben, sei es beim Aufbau oder der Pflege einer romantischen Beziehung, sei es im Umgang mit der Peer-Group privat oder am Arbeitsplatz oder der Bewältigung alltäglicher Aufgaben, eigenen Handlungsentwürfen, dies jedoch vor dem Hintergrund ihrer jeweiligen Handlungsoptionen und damit auch ihrer Handlungskompetenzen. Aus den Jugendlichen sind junge Erwachsene geworden, denen vielfach bereits der erfolgreiche Einstieg in das Berufsleben gelungen ist und die zum Teil schon feste und langjährige Partnerschaften begründet haben. Anderen stehen diese Weichenstellungen noch bevor. Hinsichtlich der sozio-emotionalen und sozio-ökonomischen Bedingungen zeigt die Auswer- 
tung, dass es vielfach zu Stabilisierungen und Verbesserungen gekommen ist. Es muss aber festgehalten werden, dass zum Teil auch dramatische Verschlechterungen zu verzeichnen sind und einige der Befragten auch heute noch in eine mehr als ungewisse Zukunft blicken - nicht zuletzt wiederum eine Folge ihrer familiären Verankerung und damit der sozioökonomischen und, mehr noch, ihrer sozio-emotionalen Bedingungen, unter denen sie aufgewachsen sind.

Neben den jungen Erwachsenen sehen sich auch deren Eltern in der Notwendigkeit, neue Lebensaufgaben zu bewältigen, auch sie erleben eine Phase des Umbruchs mit neuen Herausforderungen. So sehen sie sich damit konfrontiert, ihre Kinder langsam gehen lassen zu müssen und in ihrem eigenen Leben den Fokus nun verstärkt wieder auf andere Belange zu richten. Dazu zählen insbesondere berufliche Weiterentwicklung, Partnerschaften und das Verfolgen von eigenen Interessen. Viele der Eltern freuen sich bereits auf oder über diese neue Freiheit und haben entsprechende Zukunftspläne entworfen, ihnen ist der notwendige Schritt bereits gelungen. Manche hadern aber noch mit dieser Entwicklung und wollen ihre Kinder nur ungern in die Unabhängigkeit entlassen. Es finden sich aber auch Beispiele für solche Familien, in denen ein Abnabelungsprozess noch nicht begonnen hat und die jungen Erwachsenen noch in einem hohen Maße abhängig von ihren Eltern beziehungsweise der Familie sind.

Über die letzten Jahre hinweg war in vielen Fällen, mit zum Teil auch sehr deutlichen Ausnahmen, eine Verbesserung der sozio-ökonomischen und sozio-emotionalen Bedingungen in den Familien zu beobachten. Dennoch zeigen sich in vielen Familien und gerade auch unter den jungen Erwachsenen, selbst dann, wenn sie bereits ihr eigenes Leben führen, zahlreiche Verhaltensweisen und Orientierungen, die auf in Kindheit und Jugend erfahrene Einschränkungen, Ängste, Sorgen, Verlust- und Trennungserfahrungen zurückzuführen sind.

Die grundlegenden Unterschiede zwischen den Familientypen schlugen sich unter anderem darin nieder, dass für einige junge Menschen aus Familien von Typ 1 und Typ 2, zum Teil auch von Typ 3, Vermittlungsleistungen und Unterstützung von Institutionen oder Personen außerhalb der Familie nötig waren. Bei ihnen konnte der Einstieg in eine Lehre oder eine Arbeitsstelle nur deshalb gelingen, weil sie die dafür erforderliche nötige Hilfe erhielten. Hingegen brauchten alle jungen Männer und Frauen aus den >Aufsteiger-Familien (Typ 4) keine Vermittlungshilfe von Einrichtungen oder Personen außerhalb der Familie. Ihnen gelang es nicht nur, ihre schon früh geäußerten Ziele in der Schule und damit verbundene weitere (Aus-)Bildungswege stets mit Unterstützung ihrer Eltern anzustreben, son- 
dern auch den Weg tatsächlich in eine Lehre, eine weiterführende Ausbildung bzw. ein Studium oder eine gewünschte Arbeitsstelle zu erreichen.

Richtet man den Blick auf den Mediengebrauch der jungen Erwachsenen, so zeigt sich, dass dieser ebenfalls im Kontext der Entwicklungsaufgaben der jungen Menschen zu verorten ist. So sind sie, wie Hurrelmann und Quenzel (2016, siehe dazu ausführlicher Kapitel 1) deutlich machen, herausgefordert sich zu qualifizieren, zu binden, zu konsumieren und nicht zuletzt $\mathrm{zu}$ partizipieren, sei dies in privaten Kontexten, in der Ausbildung bzw. bei der Arbeitsstelle oder auf gesellschaftlicher Ebene. Der Mediengebrauch der jungen Menschen ist eng mit diesen Herausforderungen verbunden, er spielt für sie sowohl für das Informations- als auch für das Beziehungs- und Identitätsmanagement (Paus-Hasebrink/ Schmidt/Hasebrink 20112), sprich zur Information, Kommunikation und nicht zuletzt zur Selbstreflexion und Selbstvergewisserung, eine bedeutende Rolle. Im Mediengebrauch findet das Bemühen der jungen Erwachsenen seinen je individuell geprägten und im Zusammenhang mit ihren jeweiligen Bedürfnissen stehenden Ausdruck. Damit setzt sich eine Entwicklung fort, die schon in Ansätzen in der Jugendphase der Panelangehörigen zu beobachten war. Zwei Aspekte lassen sich jedoch deutlich stärker als in der Jugend phase, und dies fallübergreifend bei fast allen 17 jungen Frauen und Männern, feststellen: 1) Im Vergleich zu früher blicken sie reflektierter auf ihren eigenen Mediengebrauch, hinterfragen und bewerten ihr Tun und versuchen zum Teil auch, Kommunikation und Medien sowie deren gesamtgesellschaftliche Funktion einzuordnen. 2) Bedingt durch ihre zunehmenden zeitlichen Belastungen durch Ausbildung und Beruf, in einigen Fällen auch durch die Pflichten der eigenen Haushaltsführung, bleibt deutlich weniger Raum für Freizeitaktivitäten und in Folge dessen auch für den privaten Mediengebrauch. Stärker als früher müssen Abwägungsentscheidungen zu Gunsten der einen und gegen die andere Freizeitaktivität getroffen werden.

Wie ihre Kinder, blicken auch viele der befragten Mütter inzwischen reflektierter und kritischer auf ihren eigenen Mediengebrauch; dies schlägt sich jedoch nur in sehr wenigen Fällen in einer bewusst reduzierten Nutzung nieder. Vielmehr lässt sich beobachten, dass der Mediengebrauch der Mütter sich insgesamt jenem der jungen Erwachsenen angeglichen hat. Diese Entwicklung bezieht sich dabei weniger auf den Gebrauch von gleichen Angeboten und die Präferenz für gleiche Inhalte. Vielmehr berichten die Mütter in der siebten Erhebungswelle deutlich häufiger als in früheren Erhebungswellen, verstärkt von den Möglichkeiten digitaler Kommunikationswege und Medienangebote Gebrauch zu machen und diese in ihr Medienhandeln einzubinden. 


\section{Perspektiven sozial benachteiligter junger Menschen und ihrer Eltern auf gesellschaftliche Krisen und die Rolle von Medien}

Wie gehen sozial benachteiligte Familien, Jugendliche bzw. junge Erwachsene und ihre Eltern, mit gesellschaftlichen Krisen um? Wie verhalten sich die diejenigen, die es geschafft haben, sich sozio-ökonomisch zu stabilisieren? Lassen sich Unterschiede zwischen den jungen Leuten und ihren Eltern erkennen? Und welche Rolle spielen in diesen Zusammenhang mediale Angebote? Diese Fragen stehen im Mittelpunkt des folgenden Kapitels. Zwei große gesellschaftliche Krisensituationen, die auch in Österreich über längere Zeit die mediale Aufmerksamkeit in besonderer Weise beherrscht haben und die zu stark kontroversen Perspektiven in der Bevölkerung geführt haben - zum einen die Diskussion um Migration und Flucht in den Jahren 2015 und 2016 sowie die seit März 2020 andauernde Covid-19-Pandemie - boten den Hintergrund für zwei spezielle Zusatzbefragungen im Rahmen der (Medien-)Sozialisationsstudie. In der sechsten Erhebungswelle wurden Fragen zur Wahrnehmung und zum Umgang mit Geflüchteten und die Rolle von Medien in die Leitfäden der Interviews mit den Jugendlichen und zumindest einem Elternteil integriert. In ähnlicher Weise wurden in der siebten Erhebungswelle die jungen Erwachsenen sowie zumindest ein Elternteil zur Wahrnehmung und zum Umgang mit der Covid-19-Pandemie und der Nutzung von Medien in diesem Kontext befragt.

\subsection{Perspektiven sozial benachteiligter Familien auf Geflüchtete ${ }^{25}$}

Die Diskussion um Migration und Flucht nimmt in der aktuellen gesellschaftspolitischen Debatte einen großen Stellenwert ein. Sie ist nach der im Jahr 2015 vielfach proklamierten so genannten Willkommenskultur, die

25 Die Analyse zu Migration und Flucht beruht auf einem Beitrag von Paus-Hasebrink, der in kommunikation.medien veröffentlicht wurde (siehe Paus-Hasebrink 2020). Die Befragungsergebnisse zu diesem Thema wurden im Rahmen seiner Projektmitarbeit an der (Medien-)Sozialisationsstudie im Wesentlichen von Andreas Oberlinner ausgewertet. Ihm gebührt dafür der Dank der Autorin. 
sich nach den Vorfällen am Silvesterabend desselben Jahres in Köln und dessen medialer Aufbereitung rapide ins Gegenteil zu verkehren schien (Herrmann 2015, 2016), nicht nur rund um die Wahlen etwa in Österreich und Deutschland oder auch zum Europaparlament hoch virulent, sie erweist sich vielmehr in allen Lebensbereichen als ein relevantes Thema. Zumeist dominieren Emotionen die Diskussion, die sich, oft populistisch orientiert, auf tatsächliche oder auch nur vermeintliche Ängste und Sorgen in der Bevölkerung bezieht und in einem Kessel von Argumenten und Behauptungen zusammenfließt, der als wohlfeile Fundgrube nicht zuletzt für Soziale Medien-Plattformen dient, auf denen sich die emotionsdominierte Debatte - geradezu selbstreferentiell - zu verstärken scheint. Evidenzbasierte Forschung erscheint vor diesem Hintergrund besonders wichtig.

Zwar genießt in der sozialwissenschaftlichen Forschung das Themenspektrum zu Flucht, Geflüchteten und ihre Integration in die Gesellschaft, nicht zuletzt mit Blick auf ihre mediale Aufbereitung, wieder eine gestiegene Bedeutung. Seit 2016 wird auch eine verstärkte Forschungsförderung in Europa proklamiert, etwa vom Bundesministerium für Bildung und Forschung (BMBF) in Deutschland ${ }^{26}$, und das Thema facettenreich, z.B. von der Bundeszentrale für Politische Bildung ${ }^{27}$ diskutiert. ${ }^{28}$ Zudem liegen mittlerweile zahlreiche medienpädagogisch bzw. sozialpädagogisch orientierte Berichte und Konzepte vor, wie z.B. Integration unterstützt werden kann (siehe dazu etwa die Forschungen von Kutscher 2017 sowie Kutscher/ Kreß 2015, 2017), wie Kinder und Jugendliche die Berichterstattung in Medien wahrnehmen (Goetz/ Holler 2016; Holler 2016), oder auch Studien zur Mediennutzung Geflüchteter, etwa unbegleiteter Minderjähriger (Kutscher/ Kreß 2015). Auch die wissenschaftliche Auseinandersetzung mit der Frage, wie die Bevölkerung eines Landes selbst Geflüchtete in ihrer Lebenswelt wahr- und aufnimmt und wie sie ihnen im Alltag begegnet, hat zunehmend Beachtung gefunden. So wurde etwa in dem vom deutschen Bundesministerium für Bildung und Forschung (BMBF) geförderten Forschungsprojekt „Flucht als Krise?“ untersucht, wie über Flucht betreffende Themen berichtet wurde und wie Kinder und Jugendliche sie wahrnehmen und sich aneignen (Brüggen et al. 2021). Im Fokus standen

26 Siehe http://bmbf.bmbfcluster.de/de/mehr-forschung-zu-flucht-migration-und-int egration-3594.html.

27 Siehe https://www.bpb.de/gesellschaft/medien-und-sport/medienpolitik/172752/ migration-integration-und-medien? $\mathrm{p}=$ =all.

28 Stellvertretend für die auch in Österreich breitgefächerte sozialwissenschaftliche Forschung zum Themenkomplex siehe etwa Reinprecht/ Latcheva (2016). 
somit Heranwachsende - eine mit Blick auf ihre politische Sozialisation besonders relevante und doch selten untersuchte Altersgruppe. Die Frage aber, wie sozial benachteiligte Menschen, die in der Gefahr stehen, an den Rand der Gesellschaft gedrängt zu werden, mit den Themen Migration und Flucht sowie mit Geflüchteten umgehen und welche Rolle in diesem Zusammenhang unterschiedlichen Medien zukommt, ist bislang noch wenig erforscht. Diese Frage wird auf Basis von Daten der Panel-Langzeitstudie zur Rolle von Medien in der Sozialisation sozial benachteiligter Heranwachsender diskutiert. Zunächst wird ein Blick auf die gesamte Erhebungszeit von 2005 bis 2016 geworfen und anschließend die Frage näher beleuchtet, wie die Angehörigen der Panel-Studie die Geflüchteten während der politisch zugespitzten Situation ab 2015 wahrnahmen, welche Einstellung sie ihnen gegenüber erkennen ließen und welche Rolle in diesem Zusammenhang Medien spielten.

Das Herzstück des Kapitels bezieht sich auf die aus unterschiedlichen Untersuchungsschritten - einer fokussierten und einer kontextuellen Analyse - gewonnenen Ergebnisse zur Wahrnehmung der Themen Flucht und Migration. Bereits in den ersten fünf Erhebungswellen zeigte sich, dass das Thema Migration für einige Familien des Panels eine wichtige Rolle spielte, da sie oft in direkter Nachbarschaft mit Menschen mit Migrationshintergrund wohnten. Vor und während der sechsten Erhebungswelle 2015/2016 kamen zahlreiche Geflüchtete nach Europa und speziell auch nach Österreich; viele waren auf dem Weg nach Deutschland. Daher wurden Fragen zu diesem wichtigen Thema in die sechste Erhebungswelle der Studie integriert. Die Jugendlichen des Panels und ihre Eltern wurden gefragt, wie sie diese Situation wahrnehmen und wie ihre Perspektiven auf Geflüchtete aussehen. Zudem wurden sie gebeten, Auskunft über eventuelle Begegnungen und Erlebnisse mit Geflüchteten zu geben und darzulegen, welche Rolle unterschiedliche Medien für sie dabei spielen. Oftmals wurde das Thema von den Jugendlichen und ihren Eltern wegen der hohen Brisanz und Aktualität zum Zeitpunkt der Interviews auch selbst angesprochen.

4.1.1 Wahrnehmung und Perspektiven zum Thema Migration und Flucht - Erfahrungen in den ersten fünf Erhebungswellen (2005 bis 2014)

In den meisten Panelfamilien zeigte sich in den ersten fünf Erhebungswellen kaum Interesse an politischen Themen, außer wenn es sich um Themen mit unmittelbarer Relevanz für die eigene Lebensführung handelte. 
So wurde von einigen Panelangehörigen insbesondere darüber geklagt, dass sie häufig in Siedlungen mit einem hohen Anteil an Familien mit Migrationshintergrund wohnten. Die Wohnsituation gewann auch in der Sozialisation der Kinder eine zentrale Bedeutung; um mit anderen Kindern draußen spielen zu können, waren sie auf Kinder aus der Nachbarschaft angewiesen. Da viele Eltern jedoch einen negativen Einfluss von Nachbarskindern mit Migrationshintergrund auf ihre Söhne und Töchter befürchteten bzw. ihn zu erkennen glaubten, missfiel ihnen eine Freundschaft oder auch nur der Kontakt zu ihnen, und sie versuchten, ihn zu unterbinden. Einige Familien nahmen die in ihrer Wohngegend lebenden Familien mit Migrationshintergrund, darunter vor allem türkischstämmige muslimische Familien, gar als eine Bedrohung für die Erziehung der eigenen Kinder wahr, sie waren besorgt, dass sich ihre Kinder von diesen Kindern in der Nachbarschaft ungute Verhaltensweisen abschauen könnten. Einige schätzten sie dezidiert als „schlechten Umgang“ für ihre eigenen Kinder ein. Manfred Oblingers Eltern (siehe dazu ausführlicher die Fallbeschreibung in Kapitel 2) etwa verboten ihrem Sohn aus diesem Grund anfangs jeglichen Kontakt zu den türkischstämmigen Kindern in der Nachbarschaft (siehe dazu insbesondere Paus-Hasebrink/ Bichler 2008: 224f.). Als Ausgleich für mangelnde Spielkontakte in der Nachbarschaft besorgte seine Mutter ihrem Sohn, seiner ausgeprägten Leidenschaft für Computerspiele entsprechend, gewalthaltige Computerspiele für 16bis 18-Jährige.

Einige Eltern berichteten über gewaltbereite Migranten in ihrer Wohngegend. Vor allem Mütter äußerten Skepsis und Besorgnis gegenüber Gruppenbildungen in der Schule und beklagten Sprachprobleme durch zu viele Kinder aus Familien mit Migrationshintergrund.

Für die Kinder des Panels selbst waren die Kontakte zu Peers und Gleichaltringen, auch die zu Kindern aus Migrationsfamilien, hingegen hochwillkommen; sie genossen eine hohe Bedeutung für sie, wie etwa Benedikt Holzner in der zweiten Erhebungswelle eindrucksvoll deutlich machte: „Weil ich sie gern mag, und weil die können mir am meisten erklären." Mit zunehmendem Alter kamen die Kinder allerdings stärker in Kontakt mit Kindern aus Familien mit Migrationshintergrund und Geflüchteten und berichteten nun vereinzelt selbst von Problemen mit „Ausländerkindern“; einige Kinder schlossen jedoch auch Freundschaften mit Kindern anderer Nationalitäten. Manfred selbst, der sich als einziger Junge des Panels bereits sehr früh für politische Themen interessierte, wetterte eine Zeitlang gegen Menschen mit Migrationshintergrund, die Familien wie der seinen Ressourcen wegnähmen. Manfred schwärmte in dieser 
Zeit für den rechtspopulistischen österreichischen Politiker Jörg Haider. Er hing der Phantasie nach, dass Haider die Grenzen für Menschen mit Migrationshintergrund schließen würde. Unter Jörg Haiders plötzlichem Unfalltod im Herbst 2008 litt der Junge sehr. Selbst hegte er lange den Plan, ein „Bundesheerlermann“ zu werden, um in dieser Funktion „für Ordnung sorgen zu können“. Später freute sich Manfred über den Wahlsieg von Barack Obama, den er als schwarzen Politiker in einer Außenseiterposition wahrnahm und der es dennoch mit seinem Sieg „allen gezeigt“ hatte. In dieser Zeit phantasierte sich Manfred für die Zukunft in eine ähnlich mächtige Rolle für seine Heimat hinein, wie diese Obama in den Vereinigten Staaten innehatte. In der fünften Erhebungswelle interessierte sich Manfred - in dieser Zeit hatte er eine Zeit lang einen muslimischen Freund - stark für den Islam und sympathisierte vorübergehend mit dem sogenannten „Islamischen Staat“.

$\mathrm{Ab}$ der vierten Erhebungswelle, also mit zwölf Jahren, zeigte sich auch bei einigen anderen Kindern der Panelfamilien Interesse am Weltgeschehen. So wurden vor allem Entwicklungen im Zusammenhang mit dem Syrien-Krieg und dem sogenannten „Islamischen Staat“ medial verfolgt.

\subsubsection{Der Alltag mit Geflüchteten in der sechsten Erhebungswelle - Themen in den Panelfamilien}

Mit den vielen vor allem aus Syrien und Afghanistan stammenden Menschen, die 2015/2016 durch Österreich zogen, änderten sich die Wahrnehmung und der Umgang mit Geflüchteten in den Panelfamilien nachhaltig. Migration, Flucht und Geflüchtete waren nun in beinahe allen Familien ein Thema, dies war insbesondere bei den Familien der Fall, die direkt mit Geflüchteten auf ihrem Weg durch Österreich konfrontiert wurden. Insbesondere bei den Eltern dominierten dabei die Themen Konkurrenz und Ungerechtigkeit. Viele Panelangehörige nahmen Geflüchtete als eine bedrohliche Konkurrenz für sich selbst wahr, genannt wurden insbesondere als ungerecht empfundene finanzielle Leistungen oder auch Sachleistungen, die diese Menschen vom Staat erhielten, wie dies zahlreiche Familien beklagten. Viele von ihnen hatten das Gefühl, selbst um ihre Existenz bangen zu müssen, und empfanden die Geldzahlungen an Geflüchtete, ohne dass diese, wie sie betonten, vorher Leistungen erbracht hätten, als ungerecht. Negativ wurde auch bewertet, dass ihnen „so viel Essen geschenkt“ wurde, dass diese „viel davon einfach wegwarfen“ und sie selbst „das Geld abzählen“ müssten, um ihren eigenen Einkauf bewältigen zu 
können. Kritisch erwähnten einige Eltern in diesem Zusammenhang, dass viele Geflüchtete „einfach achtlos“ durch ihre Wohngebiete zogen und dabei sehr viel Müll zurückließen. Sie fürchteten auch allgemein, dass die Geflüchteten den größten Teil der finanziellen Zuwendungen erhielten und sie selbst dahinter dann zurückstehen müssten; manche klagten, dass sie die große Solidarität, die Geflüchteten entgegengebracht werde, selbst bräuchten, aber im Gegensatz zu diesen Menschen nicht erhielten. Frau Weiss etwa fühlte sich Geflüchteten gegenüber benachteiligt; sie kritisierte, dass es zu viele „Ausländer“ gebe und diese zu viel kosteten und dass sie dafür bezahlen müsse. Einige Eltern sorgten sich auch darum, dass die jungen Geflüchteten ihren Kindern später Arbeitsplätze wegnehmen könnten, dies war auch bei einigen Jugendlichen der Fall. So betonte zwar der 16-jährige Alfons Weiss, eigentlich „nichts gegen Flüchtlinge zu haben“, er zeigte sich aber in Bezug auf Arbeitsplatzverluste für Österreicherinnen und Österreicher hoch besorgt. „Flüchtlinge sind schon arm“, doch er sei dagegen, „dass ihnen alles bezahlt wird“. Viele teilten das Klischee, „dass die Geld wollen, ohne dafür arbeiten“.

Ein weiteres Thema, durch die Ereignisse in Köln in der Silvesternacht 2015/2016 befeuert, wurde in den Familien zumeist von den Eltern, allen voran den Müttern, geäußert, die Sorge und Angst um ibre Töchter. Unter den Geflüchteten seien „zu viele Männer, die Frauen nicht achten“. Insgesamt ließen sich in den Familien Sorgen um die kulturelle Zukunft und die Freiheit von Frauen erkennen. So erzählten einige Eltern mit Töchtern und manche Mädchen selbst, große Angst vor den vielen jungen, männlichen muslimischen Geflüchteten zu haben. Diese Sorgen äußerten sie vor allem nach der Berichterstattung über die Ereignisse zu Silvester in Köln. Einige Mütter und Töchter berichteten jedoch auch von unangenehmen und negativen Begegnungen mit ihnen in ihrer Wohngegend, insbesondere in der Nähe von Bahnhöfen, etwa von den jungen Männern angestarrt zu werden und sich belästigt zu fühlen. So erzählte etwa Frau Hirtner, die in der Nähe eines Bahnhofs wohnt, dass sie vor ihrer Wohnungstür von einem Geflüchteten überfallen worden sei. Die räumliche Nähe zu Geflüchteten stellte für viele Familien ein Problem dar. So kritisierte etwa Frau Holzner, dass in ihrer Wohngegend viele Asylbewerber bzw. Asylbewerberinnen seien und bezeichnete sie als „Schurken“; einen Moment später bemerkte sie, nicht pauschalisieren zu wollen, weil es auch „schlimme Schicksale“ gebe, „aber die Flüchtlinge“ führten sich „teilweise sehr auf“. Zudem missfiel ihr, „dass sie in Gruppen auftreten und ganz nah wohnen“. Hinzu kämen noch „bettelnde Rumänen und Bulgaren“. 
Ängste wurden auch in Bezug auf Terroristen und Terroristinnen sowie auf Anhängerinnen und Anhänger des sogenannten „Islamischen Staates" geäußert, die einige Panelangehörige unter den Geflüchteten vermuteten. Als problematisch empfanden die Panelfamilien daher vor allem die unmittelbare räumliche Nähe ihrer Wohnungen zu Unterkünften für Geflüchtete. Einige wenige Familien zeigten sich jedoch auch engagiert in der Unterstützung von Geflüchteten und halfen bei der Organisation von Hilfsangeboten für sie.

\subsubsection{Gemeinsamkeiten und Unterschiede in den Wahrnehmungen und Perspektiven zwischen Eltern und Kindern}

In nahezu allen Familien war das Thema Flucht und Geflüchtete ein wichtiges Gesprächsthema, das nicht selten zwischen den Eltern und ihren Kindern zu heftigen Diskussionen und Streit führte. Zumeist bemühten sich die Eltern, ihre Kinder von der eigenen Einstellung zu überzeugen. Insgesamt herrschte die Überzeugung, dass man nur noch in der eigenen Familie seine Meinung offen äußern könne, öffentlich sei das Thema zu schwierig und kaum mehr ansprechbar. Viele Jugendliche berichteten von Begegnungen mit sehr negativ eingestellten Menschen und dass es oft nötig sei, die eigene Meinung zur Streitvermeidung zurückzuhalten.

Zwischen den Eltern der Panelstudie und ihren Kindern ließen sich Unterschiede im Umgang mit den Themen Flucht und Migration sowie mit Geflüchteten feststellen. ${ }^{29}$ So zeigten sich die Eltern durchweg stärker an diesen Themen interessiert als ihre Kinder, diese fühlten sich infolge der häufigen Konfrontation mit dem Themenkomplex „genervt“, wie dies Gregor auf den Punkt brachte: „Flüchtlinge, muss ich sagen, hat mich anfangs interessiert, aber momentan hört man nur darüber, und äh es geht mir langsam aufn, aufn Zeiger“. Viele Jugendliche antworteten auf die von ihnen als Überflutung wahrgenommenen Informationen und Gespräche mit Desinteresse.

Eltern und Kinder verfügten zu den Themen Migration, Flucht und Geflüchtete über unterschiedliche Informationsquellen. So bezogen die Eltern ihre Informationen oft von Verwandten und Bekannten, aber auch von

29 Wie Gniewosz und Noack (2015) feststellten, zeigen sich zwischen Eltern und ihren jüngeren Kindern in Bezug auf die Einstellung der Kinder Migrantinnen und Migranten gegenüber deutliche Einflüsse; diese halten etwa bis zum 16 . Lebensjahr an. 
Informationsabenden, die Kinder hingegen zumeist aus der Schule, in die, wie einige Kinder erzählten, auch Geflüchtete eingeladen worden waren, um von ihren Erlebnissen und Erfahrungen in ihrem Heimatland und während der Flucht zu berichten:

Ähm, letztens bei uns in der Schule, am vorletzten Schultag“, erzählte z.B. Gregor, „war einer, äh, Flüchtling bei uns, der sehr, sehr gut Englisch sprach, auch irgendwie war der an 'ner Uni und war da Übersetzer, als Translator ähm, und hat uns auch alles erzählt. Hat uns alles erzählt, wie die Flucht war und was alles passiert ist und wie der halt hier lebt und was er halt zukünftig machen will, und ja dadurch hat man halt noch 'nen viel besseren Einblick bekommen, was die halt durchgemacht haben und alles.

Insgesamt war das Thema Geflüchtete in den Schulen an der Tagesordnung. Zuweilen gerieten Heranwachsende, wie sie berichteten, durch die Gespräche dort, in denen zumeist versucht wurde, die Lage der Geflüchteten einzuordnen und um Verständnis für sie zu werben, in eine emotional belastende Situation ihren Eltern gegenüber, da in den meisten Familien eher kritisch, wenn nicht ablehnend, von den Geflüchteten gesprochen wurde.

Einige Eltern und Kinder erwiesen sich als abwägend und in ihrer Haltung differenziert; sie unterschieden etwa zwischen geflüchteten Frauen und Kindern, die ihnen willkommen seien, und den vielen geflüchteten jungen Männern, denen sie eine eher ablehnende Haltung entgegenbrachten. Unterschieden wurde zudem zwischen „Kriegsflüchtlingen“, die ebenfalls willkommen seien, und „Wirtschaftsflüchtlingen“, die ihnen nicht recht waren und deren Gründe sie nicht akzeptierten.

Einige wenige Familien ließen eine insgesamt positive Haltung den Geflüchteten gegenüber erkennen; sie bekundeten Interesse an den Menschen und ihren Geschichten und betonten die Notwendigkeit der Hilfe aus humanitären Gründen. Einige Familienangehörige waren auch bereit, Geflüchtete engagiert zu unterstützen.

Vor allem die sozio-ökonomisch und sozio-emotional besonders stark belasteten Eltern, die in der Bewältigung ihres Alltags überfordert waren, zeigten sich von der aufwühlenden Thematik überwältigt und gaben an, das Thema (zeitweise) nicht mehr verfolgen zu können und aus diesem Grund auch ihre Kinder in der Auseinandersetzung mit den Themen nicht begleiten zu können. 


\subsubsection{Umgang mit Medien und Kritik an ihrer Berichterstattung}

Eine große Rolle wurde sowohl von den Eltern als auch ihren Kindern der Information über Medien zugewiesen. Viele Eltern schrieben Medien einen sehr großen Einfluss auf die Sichtweise und Haltung ihrer Kinder Geflüchteten gegenüber zu (siehe dazu auch Goetz/ Holler 2016; Flimmo 2016). Besondere Bedeutung wurde in diesem Zusammenhang der umfangreichen medialen Berichterstattung über die Ereignisse in der Silvesternacht 2015 auf 2016 in Köln zugewiesen. Im Vordergrund standen dabei Meinungsäußerungen in Sozialen Medien-Netzwerken mit zuweilen ausgeprägten „Horrormeldungen“ zu Geflüchteten, wie sie von einigen Panelangehörigen bezeichnet wurden.

Wirft man einen Blick auf die Nutzung von Medienangeboten, so zeigte sich, dass Informationen zu Geflüchteten zum einen aus dem Radio bezogen wurden; als Grund dafür wurde zumeist Gewohnheit genannt; so sei während der Autofahrten das Autoradio eingeschaltet, und auch bei der Arbeit liefe das Radio. Im Vordergrund standen regionale Radiosender, die mit Bezug zur eigenen Wohngegend über Geflüchtete berichteten. Einige wenige hörten sich zudem gezielt Talksendungen zum Thema an. Zur Rezeption von Fernsehnachrichten zogen die Panelangehörigen vornehmlich RTL, aber zuweilen auch den ORF sowie allgemein politische Talkshows heran. Viele Eltern und ihre Kinder verfolgten zum Thema auch die regionalen Nachrichten-Portale sowie die Online-Auftritte von Bild.de und Krone.at; Zeitungsabonnements fanden sich in den Familien der Sozialisationsstudie nur sehr selten. In diesen Fällen rezipierten die Heranwachsenden jedoch lediglich die Überschriften der Artikel. Auffällig war, dass viele Eltern zur Information auch Facebook heranzogen; besonders mit Bekannten wurden die Berichte über negatives Verhalten von Geflüchteten geteilt, da von ihnen keine negativen Sanktionen zu befürchten waren. Die Heranwachsenden selbst nutzten zur Information und zum Austausch über das Thema häufig Online-Medien, allen voran WhatsApp, zuweilen auch für gelegentliche Kontakte mit Geflüchteten. Zudem spielte YouTube für viele Heranwachsende eine Rolle; sie zogen etwa das Soziale Medien-Angebot von LeFloid zur Orientierung heran. Einige wenige mieden jedoch auch wegen schlechter Erfahrungen gezielt Diskussionen auf Plattformen in den Sozialen Medien.

Die meisten Panelangehörigen, Eltern wie Kinder, äußerten Kritik an der medialen Berichterstattung zu Geflüchteten. Frau Pfortner brachte die Kritik vieler Panelangehörigen auf den Punkt: So bezeichnete sie einerseits die Berichterstattung als „zu schön“, was für sie bedeutete, dass man 
nichts gegen Geflüchtete sagen dürfe, um nicht „gleich als ein Rassist“ abgestempelt zu werden. ${ }^{30}$ Andererseits war sie davon überzeugt, dass es letztendlich gut sei, wenn nicht alles wahrheitsgemäß berichtet werde, denn sonst „griagat ma eh a Massenpanik“. Diese Einschätzung korrespondiert mit aktuellen Untersuchungen zum sogenannten Hostile Media Effect, der sich in besonderem Maße bei den Gegnerinnen und Gegnern der Willkommenskultur nachweisen lässt (siehe dazu Merten/ Dohle 2019). Die negativen Wahrnehmungen korrelieren, so die Autorin und der Autor, „mit einem geringeren Vertrauen in die Medien, einer schlechteren Bewertung der Qualität der themenspezifischen Berichterstattung und erhöhter Wut" (Merten/ Dohle 2019: 274). Danach tendieren die Anhängerinnen und Anhänger einer bestimmten Position dazu, die mediale Berichterstattung zu einem betreffenden Themenbereich bzw. Gegenstand als einseitig, unfair und zu Ungunsten ihrer eigenen Einstellung wahrzunehmen (ebd.: 275f.).

Ein eindrucksvolles Beispiel bietet die 15-jährige Olivia Fein. Sie nutzte Medien, um sich zu informieren, äußerte jedoch auch deutlich Zweifel an den Aussagen. So erzählte sie im Interview, „letztens hot ma bei ZIB oder so glesen, dass die Flüchtlinge uns voi attackieren woin, und donn auf oamoi, a poa Stunden spada, ahm bei ZIB2, hot's ghaßn, dass' voi liab san und dass sie uns voi megn und so. Und do stimmt irgendwos ned!" Auf Nachfrage, was sie eher glaubte, was ihrer Meinung nach stimmte, antwortete sie: „Das erste (lacht)“.

Eine unausgewogene Berichterstattung wurde jedoch in der Sozialisationsstudie nicht nur von den Gegnern und Gegnerinnen der Geflüchteten kritisiert, sondern auch von denen, die diesen positiver gegenüberstanden. Auch diese Wahrnehmung lässt sich als ein Hostile Media Effect identifizieren (siehe dazu auch Merten/ Dohle 2019). Merten und Dohle kommen jedoch zu dem Schluss, dass sich dieser häufiger bei den Gegnern und Gegnerinnen der Geflüchteten nachweisen lässt (ebd.: 274). Auch die wenigen Befürworter und Befürworterinnen von Geflüchteten unter den Panelangehörigen der Sozialisationsstudie beklagten sich über die Berichterstattung; sie hielten sie für unzureichend, so fände man kaum Diskussionen zur Wohnungs- und Arbeitsmarktproblematik für Einheimische, die durch die Zunahme von Geflüchteten zunähmen; zudem würden wichtige Information zum Fehlverhalten der Geflüchteten verschwiegen, etwa zu den gro-

30 So betonte die 15-jährige Olivia Fein vor dem Interview besorgt, sie wolle nichts Falsches sagen, und fragte die Interviewerin, wie diese gegenüber „Ausländern“ eingestellt sei: „Jo, i wü jetzt nix Folsches song. I waß ned, ob du Ausländer mogst oder ned, aber mogst du de? Ausländer?“ 
ßen Mengen an zurückgelassenem Müll. Lediglich in einigen sehr wenigen Fällen fühlten sich die Panelangehörigen von Medienberichten bestätigt; in diesen Fällen wurde ihnen hohe Glaubwürdigkeit zugeschrieben.

\subsubsection{Wahrnehmung von Geflüchteten mit Blick auf die Familientypologie}

Nach der Auswertung der Interviewdaten der Panelangehörigen zu ihren Wahrnehmungen von Geflüchteten schien es lohnenswert, sich diese Aussagen mit Blick auf die Familientypologie der Studie anzusehen. Dabei zeigte sich, dass die Haltungen der Panelangehörigen zu Geflüchteten in den vier Typen nicht klar unterscheidbar waren, sich aber dennoch Tendenzen identifizieren ließen, die insbesondere Zusammenhänge mit den Handlungsoptionen und Handlungsentwürfen und damit ihren sozioökonomischen und sozio-emotionalen Bedingungen ihrer Lebensführung erkennen ließen. Das Analysekonzept der Handlungskompetenzen war mit Blick auf die Wahrnehmungen von Geflüchteten nur in Ansätzen fruchtbar. Es hätte eine längerfristige Untersuchung der erst in der letzten Erhebungsphase eingebrachten Fragen bedurft, um stichhaltige Zusammenhänge etwa zwischen der politischen Sozialisation der Heranwachsenden und ihrem elterlichen Vorbild und den Einschätzungen der Kinder zu identifizieren bzw. die Handlungskompetenzen der Eltern valide zu beurteilen.

So wurde deutlich, dass die Angehörigen von Typ 1 Geflüchteten gegenüber eher negativ eingestellt waren; es schien, als verhinderten ihre belasteten sozio-ökonomischen und sozio-emotionalen Bedingungen und damit ihre stark eingeschränkten Handlungsoptionen und Handlungsentwürfe Offenheit und Verständnis für Geflüchtete. Eine Ausnahme bildete dabei lediglich Frau Oblinger. Sie arbeitete während der Zeit ehrenamtlich für eine gemeinnützige Organisation, in der sie auch mit Geflüchteten in Kontakt kam und eine differenziertere Sichtweise auf Geflüchtete entwickelte. Ihr Sohn Manfred hingegen nahm Geflüchtete als Konkurrenz in Bezug auf Arbeitsstellen wahr. Mit seiner Mutter war er in der Überzeugung einig, dass man zwar „Frauen und Kinder holen“, vor allem die alleinstehenden Männer aber zurückschicken sollte. Manfred bezeichnete Geflüchtete „als Gesindel“; er war der Meinung, dass Österreich von Politikern „niedergetreten werde“.

Auch die Angehörigen von Typ 2, insbesondere die Eltern, zeigten sich Geflüchteten gegenüber skeptisch. Bei ihnen machte es den Eindruck, dass 
die Erinnerung an das Erleben eigener sozio-ökonomischer Einschränkungen, das sich sowohl in ihren Handlungsoptionen als auch Handlungsentwürfen niederschlug, die Abwehrhaltung Geflüchteten gegenüber mitbegründete.

Die Familien von Typ 3 erschienen zwar größtenteils recht zufrieden mit ihrer Lebenssituation. Darin unterscheiden sie sich klar von den Familien aus Typ 1, aber auch aus Typ 2. Geflüchteten gegenüber ließen diese Panelfamilien - die Eltern wie zumeist auch die Kinder - aber deutliche Ressentiments erkennen; auch sie betonten wie die Angehörigen von Typ 1 und 2, es gebe zu viele Geflüchtete, von denen die Mehrzahl „Wirtschaftsflüchtlinge" seien und dass diese die eigene Bevölkerung vor allem finanziell belasten würden. Ihre eingeschränkten Handlungsoptionen und Handlungsentwürfen führten zu einer kritischen Perspektive auf Geflüchtete.

Zusammenfassend lässt sich sagen, dass die meisten Familien vor allem von Typ 1, aber auch von Typ 2 und Typ 3, den Zustrom der Geflüchteten als eine bedrohliche Konkurrenz für sich selbst wahrnahmen. Sie ließen zumeist große Ressentiments und auch Abwehr Geflüchteten gegenüber erkennen, sie nahmen sich selbst als benachteiligt wahr und kritisierten die staatliche Hilfe den Geflüchteten gegenüber. Auffällig war, dass es sich in vielen Fällen der Familien um alleinziehende Mütter handelte und diese generell eine ablehnende Haltung gegenüber Migranten und Migrantinnen sowie Geflüchteten zeigten. Diese seien zu viele und kosteten zu viel, und sie selbst müssten noch für sie bezahlen. Konkurrenz und das Gefühl, ungerecht behandelt zu werden sind vorherrschend. Die negative Haltung der meisten Familien von Typ 1, 2 und 3 Geflüchteten gegenüber kulminierte in der Einschätzung, das „Boot“ sei „voll“, wie dies etwa Frau Boll (Typ 3) formulierte. Viele dieser Panelangehörigen übten deutliche Kritik an den Politikern und Politikerinnen des Landes und empfanden die politische Lage als „außer Kontrolle“. In diesen Familien standen auch Argumente wie etwa „Frauenverachtung" durch Muslime und die insgesamt nicht passende Religion bzw. Kultur im Vordergrund.

Lediglich die Familien in Typ 4 nahmen Geflüchtete wesentlich offener wahr und zeigten Verständnis für ihre Lage und die damit verbundenen Nöte; einige engagierten sich auch selbst in der Hilfe von Geflüchteten. Kritik an der großen Zahl der Geflüchteten und die Sorge, diese Lage politisch nicht bewältigen zu können, wurde jedoch auch in einigen der Familien von Typ 4 geäußert. „Rassistische“ Äußerungen, wie etwa Frau Pfortner von Typ 4 betonte, seien aber in jedem Fall völlig unangebracht. 


\subsubsection{Ausgewählte Fallbeispiele}

Im Folgenden wird anhand von Beispielen aus den beiden am stärksten kontrastierenden Familientypen, Typ 1 und Typ 4, jeweils ein charakteristisches Fallbeispiel mit Bezug zur Wahrnehmung von Geflüchteten vorgestellt.

\subsubsection{Familien von Typ 1: Das Fallbeispiel Familie Öllinger}

Ein eindringliches Beispiel für die Familien von Typ 1 ist Familie Öllinger (siehe dazu ausführlicher Kapitel 2.2.4). Frau Öllinger hatte kurz vor der sechsten Erhebungswelle eine Privatinsolvenz hinter sich gebracht und ist aufgrund schwerer Krankheiten erwerbsunfähig. Das Thema Flucht und Geflüchtete spielte bei ihr und ihrer Tochter eine große Rolle. In der Wahrnehmung von Geflüchteten unterschieden sich Mutter und Tochter jedoch. Frau Öllinger zeigte große Ressentiments ihnen gegenüber; sie vermutete, dass sie deutlich mehr staatliche Unterstützung erhalten als sie und ihre Tochter gemeinsam, eine Situation, die sie für sehr ungerecht hielt. So war Frau Öllinger davon überzeugt, dass Geflüchtete pro Person zumindest 900 Euro erhielten. Ihre angespannte sozio-ökonomische Situation ließ sie um die Frage kreisen, warum für sie nicht mehr Geld vorhanden sei als für Geflüchtete. Mit ihrer Tochter Viktoria diskutierte sie viel über dieses Thema; beide suchten Informationen dazu insbesondere über Fernseh- und Radiosendungen. Frau Öllinger betonte, dass die Berichte in den Medien sie stets sehr aufwühlten und sie immer wieder Pausen von der Rezeption brauchte. Große Angst äußerte sie im Interview vor den vielen männlichen Geflüchteten; viele von ihnen seien ganz in der Nähe ihrer Wohnung untergebracht. Frau Öllinger befürchtetet eine Gefährdung für Viktoria, die sie durch die Berichterstattung zu den Silvester-Vorfällen in Köln bestätigt sah. Viktoria hingegen nahm Geflüchtete nicht als Bedrohung wahr. So erzählte sie, selbst einmal Geflüchtete besucht zu haben, um diese näher kennenzulernen, sie hätte keine Angst vor ihnen und finde es auch „nicht schlimm“, dass sie da seien. Viktorias Verhalten erweckte den Anschein, dass ihr der eigene Blick auf Geflüchtete wichtig war, um sich von ihrer Mutter abzusetzen und sich zumindest in diesem Punkt einen kleinen Freiraum zu schaffen. ${ }^{31}$

31 Eine ähnliche Verhaltensweise zeigte sich auch bei Frau Fein aus Typ 1. Sie erzählte, dass sie sich eigentlich „nicht mit solchen Themen“ befassen könne; diese 
Wie bei den meisten Angehörigen von Typ 1 drängte sich auch bei Frau Öllinger der Eindruck auf, dass ihre eingeschränkten Handlungsoptionen und Handlungsentwürfe und damit zusammenhängend auch ihre mangelnden Handlungskompetenzen im Zusammenhang mit ihren negativen Perspektiven auf das Thema Flucht und Geflüchtete stand und sie aufgrund von sozio-ökonomischer und sozio-emotionaler Überforderung nicht in der Lage waren, die Lage von Geflüchteten differenzierter zu beurteilen.

\subsubsection{Familien von Typ 4: Das Fallbeispiel Familie Dornbacher}

Familie Dornbacher (siehe dazu ausführlicher Kapitel 2.5.1) beschäftigte sich intensiv mit dem Thema Flucht und Geflüchtete; sie empfand diese auch nicht als Konkurrenz für sich selbst. Gudrun engagierte sich selbst in der Hilfe für Geflüchtete, als Grund gab sie an, mit ihnen Mitleid zu haben. Sie erzählte, dass an ihrer Schule zwischenzeitlich eine Initiative zum Thema eingerichtet worden sei, bei der nicht nur über Geflüchtete gesprochen worden sei, sondern auch Ideen entwickelt wurden, wie man Geflüchtete aktiv unterstützen könnte. Gudrun half einigen etwa am Bahnhof, den Weg zur S-Bahn zu finden. Einige Mitschüler und Mitschülerinnen, aber auch andere Menschen aus ihrer Nähe, hätten sie daher schon beschimptt. Gudrun betonte im Interview, sie sei davon überzeugt, dass man „zamhelfen“ müsse, da die Politik versagt habe. So habe sie auch eigene Kontakte zu Geflüchteten aufgebaut, um sich auf diese Weise besser über ihre Lage informieren zu können. ${ }^{32}$ Die Angehörigen von Typ 4 verfügen über größere Handlungsoptionen und Handlungsentwürfen; ihnen war es möglich, ihre Handlungskompetenzen zur differenzierteren Wahrnehmung und in einigen Fällen auch zur Unterstützung von Geflüchteten einzusetzen.

wären ihr psychisch „zu viel“. Frau Fein war auch der Überzeugung, dass ein Dritter Weltkrieg bevorstehe und wehrte insgesamt politische Themen möglichst ab. Geflüchteten gegenüber ließ sie starke Aggressionen erkennen.

32 Wie Familie Dornbacher betonten auch Erich Grubert, seine Mutter und sein Stiefvater ihr Mitgefühl Geflüchteten gegenüber. Erich war im Rahmen seiner Jugendgruppe aktiv in der Hilfe von Geflüchteten tätig; er habe dort gelernt, dass sie arm seien. 


\subsubsection{Zusammenfassung}

Das Thema Flucht und Geflüchtete wird aus unterschiedlichen Perspektiven und auf unterschiedlichen Ebenen wissenschaftlich beleuchtet; ${ }^{33}$ selten allerdings kommen dabei Menschen aus der Bevölkerung eines Landes, in dem sich viele Geflüchtete aufhalten, direkt selbst zur Wort, noch seltener rücken dabei speziell Angehörige sozio-ökonomisch schlechter ausgestatteter Milieus mit ihren Wahrnehmungen und Perspektiven auf Geflüchtete in den Fokus der Aufmerksamkeit. ${ }^{34}$ Als im Jahr 2015 und 2016 zahlreiche Geflüchtete aus Syrien, Afghanistan und anderen Ländern, in denen Krieg herrschte, und Menschen, die aufgrund ihrer Rasse, ihrer Religion oder politischen Einstellung verfolgt und bedroht wurden, in großer Zahl ihr Land verlassen mussten und in diesem Zusammenhang in Österreich Asyl suchten oder auf dem Weg in andere europäische Länder durch Österreich flohen, wurden im Rahmen der sechsten Erhebungswelle der Studie die Heranwachsenden und ihre Eltern aus den Panelfamilien darum gebeten, Auskunft über ihre Wahrnehmungen von Geflüchteten, ihre Perspektiven auf sie und ihre Begegnungen und Erlebnisse mit ihnen zu geben und zu berichten, welche Bedeutung sie in diesem Kontext Medien zuschreiben.

Das Thema Flucht und Geflüchtete fand bei den Panelfamilien große Aufmerksamkeit. Geflüchtete waren in den Schulen, in der Ausbildung der Heranwachsenden, den Arbeitsstellen der Eltern, bei nahezu allen

33 Siehe hierzu etwa die Zusammenstellung von Beiträgen von Rohmann/ Stürmer 2018 sowie den Beitrag von De Coninck/ Ogan/ d'Haenens (2021).

34 Friedrichs/ Leßke/ Schwarzenberg (2019: 350) weisen darauf hin, dass es schwierig sei, den Einfluss des sozialen Status der Bewohnerschaft etwa auf die Toleranz Geflüchteten gegenüber nachzuweisen, und beklagen in diesem Zusammenhang den Mangel an entsprechenden empirischen Studien. In der Zusammenfassung zu ihrer Untersuchung in Bezug auf den Zusammenhang von Wohngebiet und Einstellung zu Geflüchteten heben sie hervor, dass mit steigender Schulbildung, in etwas geringerem Maße mit steigendem Einkommen, Vorurteile gegenüber Geflüchteten abnähmen (ebd.: 363). In einer Studie von De Coninck/ Ogan/ d'Haenens (2021) in Belgien, Frankreich, den Niederlanden, Schweden und der Türkei wurde deutlich, dass der sozio-ökonomischen Lage der Befragten hohe Relevanz zukommt: „Educational attainment seems to only play a role in Europe: those with a secondary or tertiary degree are found to experience significantly less threat than the lower educated. However, economic class is relevant in both Western Europe and Turkey: those individuals in higher socio-economic classes experience less threat, both economic and quality-of-life, than those from the lowest socio-economic class.“ (ebd.: 231) 
Begegnungen mit Bekannten und Freunden, aber auch in den Gemeinden nahezu tägliches Gesprächsthema. Einige wenige Familien des Panels beteiligten sich auch selbst an der Hilfe für Geflüchtete. Allerdings wurde von den meisten Panelangehörigen der Alltag mit Geflüchteten mit negativen Begegnungen in Verbindung gebracht; insbesondere Frauen, allen voran Mütter, aber auch Töchter, berichteten von negativen Erlebnissen mit männlichen Geflüchteten oder generell von der Angst vor ihnen. Viele Ängste sowohl der Eltern, vor allem der Mütter, aber auch der Töchter kreisten nach den Ereignissen in der Silvesternacht in Köln um die Sorge, von den vielen Männern belästigt zu werden. Angst galt auch Terroristen und Terroristinnen sowie Anhängerinnen und Anhängern des sogenannten „Islamischen Staates“, die sie unter den Geflüchteten vermuteten. Die oft als zu groß empfundene Nähe der eigenen Wohnung zu den Unterkünften der Geflüchteten wurde von vielen als besonders unangenehm und sogar besorgniserregend wahrgenommen.

Bei den meisten Eltern ließ sich eine negative Sichtweise auf Geflüchtete finden; bei ihnen dominierte die Haltung, das „Boot ist voll“, es gebe zu viele Geflüchtete. Sie empfanden die Situation als „außer Kontrolle“ und äußerten deutliche Kritik an der Politik. Eine zentrale Sorge galt der Verachtung von Frauen und der „nicht passenden Religion bzw. Kultur“. Bei einigen Eltern ließ sich eine stärker abwägende Perspektive erkennen; bei ihnen waren Frauen und Kinder tendenziell willkommen, allerdings die jungen Männer nicht. Menschen, die wegen eines Krieges in ihrem Land geflüchtet waren, wurden mehr Sympathie und mehr Verständnis entgegengebracht als den von vielen als „Wirtschaftsflüchtlinge“ bezeichneten Geflüchteten. Einige wenige Familien zeigten eine positive Einschätzung der Lage und äußerten Interesse an den Menschen und ihren Geschichten; sie hielten Hilfe aus humanitären Gründen für nötig und zeigten dabei zuweilen auch eigenes Engagement.

Viele stark sozio-ökonomisch und sozio-emotional belastete Eltern, die ohnehin in ihren Bewältigungsstrategien überfordert waren, zeigten sich von der aufwühlenden Thematik überwältigt; sie fühlten sich zeitweise nicht mehr in der Lage, das Thema zu verfolgen, sie sahen sich zudem außer Stande, ihre Kinder bei der Thematik zu begleiten.

Viele Familien ließen deutliche Konkurrenzgefühle erkennen; Eltern und Kinder äußerten die Besorgnis, dass Arbeitsplätze und Wohnungen knapper würden; sie machten ihrem Unmut darüber Luft, dass zu viele soziale Leistungen vom Staat für Geflüchtete aufgewendet würden und sie selbst nicht ausreichende staatliche Unterstützung bekämen. Sie empfanden sich selbst als ungerecht behandelt. 
Die Ergebnisse zeigen, dass auch in der Frage nach der Wahrnehmung von Geflüchteten die Panelangehörigen differenzierter betrachtet werden müssen; auch zwischen den Eltern und ihren 2016 nahezu erwachsenen Kindern wurden Unterschiede deutlich. Das Thema Flucht und Geflüchtete führte in vielen Panelfamilien zu Diskussionen und Streit; zumeist bemühten sich die Eltern, ihre Kinder von der eigenen Einstellung zu überzeugen. Einige Kinder übernahmen die Einstellungen der Eltern, andere zeigten aber auch eine distanziertere oder gelassenere Haltung. Doch auch einige Heranwachsende brachten ihren Eindruck zum Ausdruck, nur noch in der Familie die eigene Meinung äußern zu können, da sie das Thema öffentlich entweder zu schwierig oder gar nicht ansprechbar empfanden.

Als wichtigste Informationsquellen zum Thema dienten den Panelangehörigen Verwandte und Bekannte, bei den Kindern aber auch Lehrpersonen und Mitschüler und Mitschülerinnen; in einigen Gemeinden und Schulen fanden Informationsabende statt. Zudem war das Thema Flucht und Geflüchtete in den Medien allgegenwärtig. Besonders Online-Medien, etwa Bild.de und Krone.at, wurden von den meisten Familien zur Information zum Thema genutzt, dazu Nachrichten und Talkshows im Fernsehen, etwa auf RTL, aber auch im ORF, und insbesondere regionale Radiosender. Die Eltern zogen als alternative Informationsquellen häufig Facebook heran, die meisten Kinder informierten sich hingegen über YouTube, etwa bei LeFloid, der vielen als Orientierungsangebot diente. Waren die meisten Eltern stark an Informationen über Geflüchtete interessiert, führte die häufige Konfrontation mit dem Thema bei manchen Heranwachsenden zu Desinteresse.

Eltern und Kinder übten zuweilen harsche Kritik an der Berichterstattung der Medien; es würden wichtige Informationen verschwiegen und heikle Debatten, etwa über die Wohnungs- und Arbeitsmarktproblematik für Einheimische, gar nicht thematisiert. Die Wahrheit werde entweder vertuscht oder beschönigt. Analog zu Untersuchungen zum Hostile Media Effect schrieben im Rahmen der Sozialisationsstudie sowohl Befürwortende als auch Kritikerinnen und Kritiker von Geflüchteten Medien nur eine geringe Glaubwürdigkeit zu.

Unterschiede ließen sich mit Blick auf die (Bildung von) Familientypen im Hinblick auf sozio-ökonomische und sozio-emotionale Bedingungen der Panelfamilien feststellen. Vor allem die Eltern, die nach wie vor auf staatliche Hilfe angewiesen waren (allen voran von Typ 1, aber auch Typ 3), jedoch auch diejenigen, die sich zwar im Laufe der Studie sozio-ökonomisch etwas stabilisierten, aber nach wie vor sozio-emotional in ihrer 
Lebensführung belastet fühlten (Typ 2), sahen sich in einer unmittelbaren Konkurrenzsituation mit den Geflüchteten. Sie waren aufgrund mangelnder Handlungsoptionen und damit zusammenhängend eingeschränkten Handlungsentwürfen und -kompetenzen überfordert, anteilnehmendes Interesse an den Geflüchteten zu empfinden, sie fürchteten, mit ihren Nöten und Sorgen selbst nicht ausreichend öffentlich berücksichtigt zu werden. Lediglich die Familien von Typ 4, die sich während der Laufzeit der Panelstudie sozio-ökonomisch und sozio-emotional deutlich verbessert hatten und als >Aufsteiger bezeichnet werden konnten, nahmen Geflüchtete nicht als Belastung wahr; sie zeigten nicht nur Verständnis und Mitgefühl für sie; einige von ihnen engagierten sich auch selbst in der Hilfe für Geflüchtete.

\subsubsection{Reflexion und Conclusio zu den Wahrnehmungen und Perspektiven auf Geflüchtete}

Mit Hilfe des in der sechsten Erhebungswelle aufgenommenen Erhebungsschritts bot sich die Möglichkeit, den Wahrnehmungen und Perspektiven von sozial benachteiligten Heranwachsenden und ihren Eltern auf Geflüchtete und die Rolle von Medien dabei nachzugehen. So konnten die Themen von Eltern und Kindern in Bezug auf Geflüchtete identifiziert werden, Gemeinsamkeiten und Unterschiede zwischen den Eltern des Panels, aber auch zwischen den Eltern und ihren Kindern, eruiert und erste Erkenntnisse über Zusammenhänge von sozio-ökonomischen und sozioemotionalen Bedingungen der Panelfamilien und ihren Wahrnehmungen von Geflüchteten und Perspektiven auf sie gesammelt werden. Die beiden ausgewählten Fallbeispiele der am stärksten unterschiedlichen Familientypen 1 und 4 ließen Zusammenhänge zwischen den Handlungsoptionen, Handlungsentwürfen und in Ansätzen auch den Handlungskompetenzen der Familienmitglieder und ihren Wahrnehmungen von und Sichtweisen auf Geflüchtete(n) erkennen. Diese ersten Erkenntnisse verlangen nach weiterer Forschung und einer auch quantitativ verankerten Studie. Deutlich wurde jedoch bereits auf Basis der in dieser Studie gewonnenen Ergebnisse, dass sozial schlechter gestellte Familien im Zuge der Debatte um Migration und Flucht nicht in Vergessenheit geraten dürfen. Sie zeigen sich aufgrund ihrer schlechteren sozio-ökonomischen Lage und infolgedessen auch oft aufgrund einer belasteten sozio-emotional angespannten Lebensführung mit eingeschränkten Handlungsoptionen und Handlungsentwürfen besonders sensibel; sie empfinden Hilfe für Andere tendenziell 
als ungerecht und nehmen alle, die staatliche Hilfe beanspruchen dürfen, als potenzielle Konkurrentinnen und Konkurrenten wahr, die die Ressourcen schmälern, die sie selbst dringend bräuchten. Es bedarf des allgemeinen gesellschaftlichen, das heißt auch politischen, Willens und der Anstrengung, die Lebenslagen und damit verbundenen Probleme sozial benachteiligter Familien wahrzunehmen und damit auch Wege zur besseren sozialen Partizipation zu ermöglichen. Auch Medienanbieter, nicht zuletzt so genannter Sozialer Medien, sind vor diesem Hintergrund herausgefordert, ihre Berichterstattung mit Blick auf die Klientel sozial benachteiligter Familien verantwortlich zu gestalten und in diesem Sinne journalistisch aufklärend zu arbeiten.

\subsection{Perspektiven auf die Covid-19-Pandemie im Kontext sozialer Benachteiligung}

Seit Anfang 2020 hält die Covid-19-Pandemie die ganze Welt in Atem. Die Furcht vor einer Ansteckung beziehungsweise die Folgen einer solchen Erkrankung (vom Orde 2020: 17-18; Schnetzer et al. 2020: 5; Blom et al. 2020: 14) sowie die staatlichen Gegenmaßnahmen zur Eindämmung der Pandemie beeinträchtigen nahezu alle Bereiche des gesellschaftlichen Lebens (Blom 2020). Während Abstands- und Kontaktregeln sowie die Pflicht zum Tragen eines Mund-Nasen-Schutzes den eher milderen Maßnahmen zuzurechnen sind, so haben die Absage nahezu aller Veranstaltungen und die verordnete Schließung nicht systemrelevanter Geschäfte massive Auswirkungen auf das ökonomische Gesamtgefüge im Staat (Papier 2020; Ther 2020), Beschäftigungsverhältnisse und das verfügbare Einkommen der Menschen. Und nicht zuletzt die Ausdünnung von frühkindlicher, (berufs)schulischer und universitärer Ausbildung, beziehungsweise deren Verlagerung oder Kompensation durch Online-Angebote und Fernlehre, beeinträchtigt die Entwicklung nachfolgender Generationen (Wrase/ Allmendinger 2020; Schubarth 2020).

In besonderem Maße von diesen Einschränkungen und Maßnahmen betroffen erscheinen sozial benachteiligte Haushalte und formal niedriger gebildete Eltern (siehe dazu ausführlich Steiber/ Liedl/ Molitor 2020: 2; Blom 2020). Gerade weniger qualifizierte Anstellungen im Handel und in der Gastronomie sind besonders von Schließungen betroffen; dies betriff auch Schülerinnen und Schüler sowie Studierende, die in diesen Bereichen in Nebenjobs tätig waren. Zudem besteht im Handels- und Dienstleistungssektor, im Gegensatz zu anderen Berufszweigen, nur sehr eingeschränkt 
die Möglichkeit, berufliche Aufgaben strukturiert von zu Hause aus zu erledigen (Blom et al. 2020: 10f.). Die Folge sind einerseits eine stärkere Risikoexposition und andererseits eine stärkere Betroffenheit von Kurzarbeit und Arbeitslosigkeit (Bonacini/ Gallo/ Scicchitano 2021: 306f.). Durch die Notwendigkeit weiter der Arbeit nachzugehen, können viele sozial benachteiligte Eltern sich oft auch nur vermindert der Betreuung ihrer Kinder zuwenden, deren Bildungseinrichtungen geschlossen sind (siehe dazu Tengler/ Schrammel/ Brandhofer 2020: 10-13; Huebener/ Schmitz 2020: 2f.; Huber et al. 2020: 7f.; Teach for Austria 2020; Wrase/ Allmendinger 2020; Rohmann/ Stürmer 2018). In diesem Kontext manifestiert sich auch eine Benachteiligung gegenüber formal höher gebildeten Eltern, die in vielen Fällen die ausfallende professionelle Schulbildung besser durch persönliches Engagement kompensieren können. Ein weiteres Element ungleicher Betroffenheit basiert auf der Möglichkeit finanziell besser gestellter Familien, privat bezahlte Zusatzleistungen im Bildungsbereich zu finanzieren. „,(Bildungs-)Verliererinnen und -verlierer' in der aktuellen Situation sind wahrscheinlich Schülerinnen und Schüler aus sozio-ökonomisch (hoch) benachteiligten Elternhäusern“" (Huber et al. 2020: 108).

\subsubsection{Rahmung der Erhebung}

Vor diesem Hintergrund wurde die im Frühjahr und Frühsommer 2020 durchgeführte siebte Erhebungswelle der Langzeitstudie zur Mediensozialisation sozial benachteiligter Heranwachsender um einen zusätzlichen Themenblock in den Leitfadeninterviews ergänzt, der sich mit der Covid-19-Pandemie und ihren Folgen befasste. Dieser beinhaltete folgende vier Hauptpunkte: 1) Persönliche Betroffenheit, 2) Informations- und Kommunikationsverhalten (Veränderung der Mediennutzung sowie medialer und non-medialer Informations- und Kommunikationsgebrauch zur Auseinandersetzung mit der Covid-19-Pandemie), 3) Perspektiven auf die Covid-19-Pandemie und deren Folgen sowie 4) Sichtweise auf und Bewertung von staatlichen Maßnahmen zur Eindämmung der Covid-19-Pandemie. Die Interviews fanden in den Monaten März, April und Mai 2020 statt und somit während des Höhepunkts und der Endphase des ersten Lockdowns in Österreich und Deutschland, wobei während der später durchgeführten Interviews bereits einige Lockerungen erfolgt waren, etwa die schrittweise Öffnung von Geschäften. Die Gespräche wurden entsprechend den geltenden Hygienevorschriften per Telefon, WhatsApp-Call oder Skype fernmündlich geführt, transkribiert, mit Hilfe von MAXQDA 
codiert und unter Verwendung von fallspezifischen Matrizen ausgewertet. Bedingt durch den frühen Erhebungszeitpunkt bietet die (Medien-)Sozialisationsstudie einen Überblick über die Wahrnehmung und Einstellung der Panelangehörigen zur ersten Pandemie-Welle. So berichteten die Befragten bereits über die von ihnen erlebten Einschränkungen sowohl im Berufs- als auch im Privatleben, weitreichende Konsequenzen sowie nachhaltige Beeinträchtigungen ihrer eigenen Lebensbedingungen wurden allerdings erst selten thematisiert. Während der ersten Welle der Pandemie wurde noch bezweifelt, dass die Regierung ein zweites Mal vergleichbar harte Maßnahmen wie im Frühjahr beschließen könnte, selbst wenn der Herbst eine zweite Welle bringen würde; begründet wurde dies mit den möglichen Folgen für die Wirtschaft.

Anders als dies bei den Perspektiven der Befragten auf Geflüchtete der Fall war, zeigte die Auswertung des Themenkomplexes zur Covid-19-Pandemie keine klaren typbezogenen Zusammenhänge zwischen sozio-ökonomischen und sozio-emotionalen Bedingungen und den Bewältigungsstrategien der mit Covid-19 verbundenen Herausforderungen im Alltag; dies war möglicherweise eine Folge des frühen Erhebungszeitraums, zu dem vielen Befragten die volle Schwere der Pandemie noch nicht bewusst bzw. vorstellbar war. Die Ergebnisse der Auswertungen werden im Folgenden in zwei Schritten dargestellt. Zunächst werden verschiedene lebensweltliche Herausforderungen behandelt, mit denen die Familen des Panels in der Pandemie konfrontiert waren. Danach geht es speziell um das pandemiebezogene Informations- und Kommunikationsverhalten.

\subsubsection{Lebensweltliche Herausforderungen in der Pandemie}

Alle befragten Personen sind mehr oder weniger stark von den Auswirkungen der Pandemie betroffen, darin spiegelt sich zum Teil auch wider, wie zufrieden sie etwa mit der Gesamtsituation, mit dem eigenen Verhalten, mit den Maßnahmen der Regierung und den sich ihnen bietenden Handlungsmöglichkeiten sind (siehe dazu für die österreichische Bevölkerung während der ersten Corona-Welle auch Willems et al. 2020). Als erste Gruppe, die aus rein beruflicher beziehungsweise wirtschaftlicher Perspektive unter besonderem Druck steht, zeigen sich jene, die sich nicht in einem Angestelltenverhältnis befinden, sondern entweder als selbstständige Unternehmer tätig sind oder im Rahmen von Events tätig werden. Als zweite wirtschaftlich stark betroffene Gruppe erweisen sich jene, die aus gesundheitlichen Gründen zur Risikogruppe gehören und aus diesem 
Grund aktuell ihrer Arbeit nicht nachgehen können oder gar ihre Arbeitsstelle verloren haben (siehe dazu auch Blom et al. 2020: 11). Anhand der folgenden Beispiele wird dabei deutlich, welchen Einfluss die familialen Strukturen haben können, um auch unvorhersehbare Belastungen wie die Covid-19-Pandemie erfolgreich überstehen zu können.

Besonders betroffen von der Pandemie waren die wenigen selbstständig tätigen Personen. Familie Pfortner führt seit Beginn der Untersuchung ein Unternehmen für Kompressorentechnik. Die Pandemie führte hier zeitweise zu einem völligen Einbruch beim Auftragseingang, da die Kunden stark verunsichert waren. Nur auf sehr geringem Niveau konnten die bestehenden Projekte fortgesetzt werden. Diese Phase, Frau Pfortner bezeichnet sie retrospektiv scherzhaft als „tote Hose“, konnte aber nach einiger Zeit überwunden werden. Mit ihren Einkünften aus einer Nebentätigkeit in einer Drogeriekette gelang es Frau Pfortner, die Familieneinkünfte abzusichern, wobei sie bei voller Lohnfortzahlung in großem Umfang Überstunden und Resturlaub abgebaut hat. Die gewonnene Freizeit zur Erholung sieht Frau Pfortner als eine ganz klar positive Folge der Pandemie: „Eigentlich hab i a sehr gute Zeit g'habt muss ich sagen (lacht)! “

In ähnlicher Weise, wenn auch in verschärfter Form, war auch Familie Zarbl betroffen. Während Frau Zarbl selbst beruflich nicht durch die Pandemie betroffen war, hatte sie jedoch für ihren Ehemann und somit auch die gesamte Familie weitreichende Konsequenzen. Herr Zarbl musste aufgrund von Corona seine Selbstständigkeit als Gesundheits- und Fitnesstrainer aufgeben, um weitere Verluste zu vermeiden. Zusätzliche Kredite sollten nicht aufgenommen werden. Bislang aufgelaufene Schulden durch den Aufbau der Selbstständigkeit mussten noch bedient werden. Er hatte bisher im Nebenerwerb auch im Krankenhaus gearbeitet und konnte sein Arbeitspensum dort direkt auf $100 \%$ aufstocken. So war die Entwicklung (auch finanziell) zwar keine Katastrophe, denn man sei „im Prinzip relativ unbeschadet bislang“ - „emotional“ sei die Entwicklung jedoch sehr belastend (siehe zu psychischen Belastungen durch Arbeitsplatzverlust und Arbeitslosigkeit aufgrund der Covid-19- Pandemie in Österreich auch Vlasak/ Barth 2020).

Frau Scheib hat zwar bislang ihren Hauptberuf als Vertriebsassistentin in Teilzeit nicht verloren, dennoch ist auch sie von Covid-19 und den Auswirkungen betroffen und muss finanzielle Einbußen hinnehmen. Denn normalerweise arbeitet sie im Nebenerwerb auch für einen Sicherheitsdienst als Ordnungskraft bei Konzerten. Diese Zusatzeinnahmen fallen aufgrund von Corona aktuell komplett weg, da alle Konzerte abgesagt wurden und es für den Ausfall keine Ersatzleistungen gibt. 
In ganz ähnlicher Weise ist auch Familie Grubert von Corona betroffen: Sowohl Frau Grubert als auch ihr langjähriger Lebenspartner arbeiten neben ihren Hauptberufen als Streckenposten bei Motorsportveranstaltungen. Diese entfallen derzeit ersatzlos. Zudem ist Frau Grubert aufgrund der Corona-Pandemie in Kurzarbeit und hat dadurch Gehaltseinbußen von mehreren Hundert Euro im Monat. Dies sieht sie aber nicht problematisch, zumal ihr Partner weiterhin als IT-Techniker arbeiten und den Verlust ausgleichen kann. Ähnlich wie auch Frau Pfortner genießt sie die zusätzliche Freizeit und findet es „super“, aktuell nur jede zweite Woche arbeiten zu müssen (siehe zu Aspekten des Verhältnisses zwischen Arbeit und Freizeit in der Covid-19-Pandemie auch Schnetzer et al. 2020: 8; Schnetzer/ Hurrelmann 2020: 9). Ihr Sohn Erich ist ausgebildeter Koch; auch er hatte erhebliche finanzielle Einbußen durch die Kurzarbeit und konnte teilweise gar nicht arbeiten. Zeitweise stand auch die Kündigung aller Mitarbeiterinnen und Mitarbeiter bei seinem Arbeitgeber im Raum, dieser Schritt konnte aber noch abgewendet werden (siehe zu besonders belasteten Branchen in Österreich auch Steiber/ Liedl/ Molitor 2020: 2). Schließlich hat das Restaurant einen erfolgreichen „Lieferservice und Take-away für Mittagessen" gestartet, wodurch sich die Situation erheblich gebessert habe. Diese an sich einschneidende Erfahrung thematisieren aber weder Erich noch seine Mutter als eine problematische Situation. Erich steht damit einerseits exemplarisch für eine Gruppe von jungen Erwachsenen im Panel, die sich zwar beruflich bereits etabliert, in ihrer Lebensführung aber noch nicht gänzlich von ihren Eltern gelöst haben. Sie wohnen noch zu Hause und zahlen dort keine oder nur eine sehr geringe Miete. Durch dieses familiale Sicherheitsnetz gelingt es ihnen, die wirtschaftlichen Folgen der Schutzmaßnahmen gegen die Pandemie abzumildern. Erich, der aus einer sowohl sozio-ökonomisch als auch sozioemotional gefestigten Familie stammt, bietet andererseits aber auch ein Beispiel dafür, wie eine große Mehrheit der Jugendlichen und jungen Erwachsenen in Österreich die erste Phase der Covid-19-Pandemie erlebt hat. Schnetzer et al. (2020: 6) schreiben dazu:

Die allermeisten Jugendlichen und jungen Erwachsenen in Österreich sind also privat gut verankert und lassen sich auch durch eine große Gesundheitskrise mit sehr starken Einschnitten in das alltägliche Leben nicht irritieren. Obwohl sie sich in einer Umbruchsituation ihres Lebens befinden und viele schwierige Übergänge von der Schule in die Ausbildung oder das Studium und anschließend in den Beruf bewältigen müssen, können sie sich demnach flexibel auf die veränderte Lebenssituation einstellen. Die gute Verankerung im familiären Kreis, 
in der Freundesgruppe und in der Region gibt ihnen offenbar den nötigen Halt.

Auch Helmut Pfortner wohnt noch in seinem Elternhaus, wenn auch in einer ausgebauten Wohnung, dort soll er erst nach dem Wehrdienst, den er während des Höhepunktes der ersten Coronawelle angetreten hat, eine erhöhte Miete zahlen. Helmut ist, wie sein Vater, ausgebildeter Maschinenbautechniker und wurde nach der Lehre von seinem Ausbildungsbetrieb sofort unbefristet in Vollzeit übernommen. Wie auch seine Mutter fühlt er sich selbst durch die Covid-19-Pandemie nicht wirklich eingeschränkt in seiner Lebensgestaltung. Er hat so stattdessen viel Zeit, um Sport zu machen (siehe auch Jugendkultur.at 2021: 1). In der Arbeit hatte er zunächst drei Wochen Urlaub, anschließend hat er viel von zu Hause ausgearbeitet. Finanzielle Einbußen hatte er bislang nicht, da er durch eine Vereinbarung mit seinem Chef Überstunden und Resturlaub abbauen konnte. Ähnlich erging es Norbert Zarbl, der nach der Matura an einer HTL zunächst den Wehrdienst abgeleistet hatte und dann in Vollzeit in der Entwicklungsabteilung eines Automobilzulieferers begonnen hat. Im Gegensatz zu Helmut Pfortner ist er zwar von Kurzarbeit betroffen, dies sieht er aber als nicht so problematisch an, da er einerseits noch mietfrei zu Hause wohnen kann und andererseits durch sein nun erstes eigenes Einkommen „eh viel mehr Geld hat als zuvor als Schüler“ zur Verfügung habe (siehe auch Schnetzer et al. 2020: 6; Großegger 2019: 3). Zudem geht er davon aus, dass sich die Situation bald wieder bessern wird und er, im Gegensatz zu Kollegen in der Fertigung, zur Not auch von zu Hause aus arbeiten könne (Vlasak/ Barth 2020). Erneute scharfe Einschnitte, wie sie mit dem zweiten und dritten Lockdown in Österreich im Winter eingetreten sind, konnte er sich zum Zeitpunkt des Interviews nicht vorstellen.

Auch Susanne, die Tochter von Frau Scheib, äußert sich positiv über die hinzugewonnene Freizeit. Zu Beginn empfand sie die starken Einschränkungen als schlimm und belastend, mit deren Andauern hat sie sich mit der Gesamtsituation aber gut arrangiert. Nach ihrer erfolgreich abgeschlossenen Ausbildung zur Einzelhandelskauffrau arbeitet Susanne inzwischen am Empfang eines großen Autohauses. Aufgrund von Corona ist sie derzeit in Kurzarbeit und muss, im Gegensatz zu Erich, Helmut und Norbert, ihre eigene kleine Wohnung unterhalten. Dies stellte aber bislang kein Problem dar, und zudem kann sie sich auch auf Unterstützung durch ihre Eltern verlassen.

Neben der Studienteilnehmerin Amelie Aufbauer konnte sich in der Covid-19-Pandemie auch ihre ältere Schwester Agnes auf die Unterstüt- 
zung durch ihre Mutter verlassen, obwohl auch in dieser Familie die wirtschaftliche Situation angespannt ist. Frau Aufbauer ist in Kurzarbeit, noch gelingt es der Familie jedoch, dies abzufedern und füreinander einzustehen. Agnes lebte zuvor in London, hat das Vereinigte Königreich aufgrund der Pandemie aber fluchtartig verlassen. Ihre Mutter unterstützt sie nun dabei, wieder in Österreich Fuß zu fassen, dennoch hat die Covid-19-Pandemie zu einem massiven Bruch in ihrem Leben geführt (siehe zu solchen Umbrüchen auch Schnetzer et al. 2020: 7). Amelie selbst und ihr jüngerer Bruder Florian besuchen beide noch die Schule und sind von Distanz-Lehre betroffen. Wie auch einige andere der befragten jungen Erwachsenen (etwa Torsten Kaiser, Frau Weiss für ihren jüngeren Sohn, Gudrun Dornbacher, Simone Stab) äußern sie sich kritisch über die praktische Umsetzung dieser neuen Unterrichtsform und die Kompetenz der Lehrkräfte (siehe auch Huber et al. 2020: 86-97, 105; Tengler/ Schrammel/ Brandhofer 2020: 24f.). In erster Linie sieht sich Amelie jedoch davon betroffen, ihre Freunde nicht mehr treffen zu können (siehe auch Schnetzer et al. 2020: 4; Großegger 2020b: 5), obwohl sie gleichzeitig angibt, die Ruhe in der Pandemie zu genießen (zur Bedeutung des „chillens“ in der Pandemie siehe Jugendkultur.at 2021: 1). Um dies zu kompensieren, hat sie, besonders zu Beginn der Maßnahmen zur Eindämmung der Pandemie, intensiv mit Freundinnen und Freunden telefoniert: „Drei, vier Stund'n einfach schau, weil ma hoit nix anders zum toa hat." Im Verlauf der Pandemie habe sie jedoch versucht, dieses Zeitpensum wieder zu reduzieren. Jedoch spricht Amelie mit der Trennung von Freunden ein Thema an, das insgesamt die befragten Mädchen (sehr deutlich auch bei Gudrun Dornbacher) stärker zu belasten scheint als die Jungen. Jedenfalls wird dieser Umstand von ihnen häufiger als problematisch angesprochen, während die Jungen (etwa Gregor Boll, Helmut Pfortner, Mario Hirtner) eher positiv von pragmatischen Lösungen über medial vermittelte Kontakte berichten, etwa über Messenger-Dienste, Videotelefonie oder Gruppentreffen via Houseparty (siehe auch Jugendkultur.at 2021: 3; vom Orde 2020: 19). Zwar nutzen auch die Mädchen solche Angebote, ihre Sichtweise scheint jedoch kritischer. So betont etwa Gudrun Dornbacher, dass Chats und Videotelefonie einfach „kein wirklicher Ersatz für reale Treffen“ seien. Diese Perspektiven und der Unterschied zwischen den Geschlechtern findet sich auch in anderen Studien:

WhatsApp, TikTok und Co. schienen vielen zunächst ein Rettungsanker in der sozialen Isolation. Doch schon bald verdichtete sich das Gefühl, dass Social Media den direkten persönlichen Kontakt mit FreundInnen nicht ersetzen können. Selbst eingefleischte Social-Media-Kids 
fühlten sich in den eigenen vier Wänden eingesperrt und von der Gesellschaft der Altersgleichen entkoppelt. (Großegger 2020b: 5)

Anders als bei den fünf zuvor beschriebenen jungen Erwachsenen (Erich Grubert, Helmut Pfortner, Norbert Zarbl, Susanne Scheib und Agnes Aufbauer) zeigt sich die Lage bei Mario Hirtner und seiner Mutter. Mario selbst ist aus wirtschaftlicher Perspektive, anders als seine Mutter, von der Covid-19-Pandemie kaum betroffen. Er hat zwischen der sechsten und siebten Erhebungswelle seine Ausbildung erfolgreich abgeschlossen, wurde von seinem Ausbildungsbetrieb unbefristet übernommen, und ihm wurde darüber hinaus von seinem Arbeitgeber eine günstige Werkswohnung zur Verfügung gestellt, die er gemeinsam mit seiner Freundin bewohnt. Dies sorgt, vor dem Hintergrund der sehr hohen Mitpreise in seiner Heimatstadt, für zusätzliche ökonomische Sicherheit. Auf Grund der Pandemie hat er keinerlei finanzielle Einbußen, und er geht seiner Tätigkeit weiterhin in Vollzeit nach. Während der Phase der pandemiebedingten Schließungen hat er seinen Arbeitsplatz nach zu Hause verlegt und hielt über Online-Kommunikationstools und Telefon engen Kontakt mit den Kunden und seinem Team. Um einerseits seine berufliche Tätigkeit und andererseits seine Freizeitaktivitäten am Computer weiterhin gut trennen zu können, hat er sich extra zwei separate Arbeitsplätze eingerichtet. Das beschriebene Ausmaß an sowohl ökonomischer als auch emotionaler Sicherheit ist neu für Mario. Über den bisherigen Erhebungszeitraum der Langzeitstudie hinweg lebte er gemeinsam mit seiner Mutter und seinem jüngeren Bruder in einer geförderten Sozialwohnung. Zudem hatte Mario nie Kontakt zu seinem leiblichen Vater, und wechselnde Lebensgefährten seiner Mutter, die wichtige Bezugspersonen waren, verschwanden nach der Trennung wieder aus seinem Leben. Seine Mutter hatte bereits in der Vergangenheit regelmäßig wechselnde Arbeitsstellen und war immer wieder arbeitslos, so auch während der Befragung zur siebten Erhebungswelle. Zuletzt war sie an der Frischetheke in einem Bio-Supermarkt tätig gewesen, aufgrund einer Asthmaerkrankung war sie jedoch längere Zeit krankgeschrieben und verlor schließlich ihre Arbeitsstelle. Die im Lebensmitteleinzelhandel geltenden Hygienevorschriften schränken sie bei der Jobsuche sehr stark ein, und sie ist erneut abhängig von Hilfszahlungen des AMS (Steiber/ Liedl/ Molitor 2020: 2). Dadurch fühle sie sich aktuell sehr überfordert und perspektivlos und könne sich kaum Vorstellungen für eine bessere Zukunft machen (siehe zu entsprechenden Belastungen auch Schedlich 2020; Vlasak/ Barth 2020; Blom et al. 2020: 12f.). Gerade Menschen mit sehr niedrigem verfügbaren Einkommen geben in Österreich an, nun öfters Angst zu verspüren als vor der Covid-19-Pandemie, 
zudem berichten Arbeitslose in der aktuellen Situation deutlich häufiger von depressiven Symptomen als alle anderen Berufsgruppen (Braun et al. 2020: 6, 9). Mario thematisiert im Interview die Notlage seiner Mutter nicht. Auch eine mögliche Unterstützung der Mutter wird weder von Frau Hirtner noch von Mario angesprochen.

Darin unterscheidet sich die Lage deutlich von der Situation der Familie Oblinger. Zwar geht es auch Manfreds Eltern nicht gut, doch anders als Mario Hirtner sorgt er sich in der Pandemie um sie. Über viele Jahre hinweg gehörte Familie Oblinger zu den wirtschaftlich am stärksten belasteten Familien im Panel. Doch bereits in der sechsten Erhebungswelle hatte sich die ökonomische Lage der Familie gebessert, und erstmals seit 2005 war sie nun nicht mehr auf staatliche Transferleistungen angewiesen. Manfred hat nach langer Suche mittlerweile eine Ausbildungsstelle als EDV-Kaufmann und damit seinen „Traumberuf“ gefunden (siehe zur Berufswahl beziehungsweise zur Wahl der passenden Ausbildung Großegger 2019: 3f.). Es gelang ihm so, seine weit abgelegene Heimatregion zu verlassen und sich im Zentralraum zu etablieren. Während der Pandemie hält Manfred engen Kontakt zu seinen Eltern; er telefoniere viele Male am Tag mit ihnen, und man besuche sich auch gegenseitig, wie es in den Interviews heißt. Von der Covid-19-Pandemie war er selbst bislang nur durch größere Abstandsregeln im Betrieb und durch Distanz-Lehre in der Schule betroffen; diese Form des Lernens gefällt ihm aber gut. Obwohl sein Arbeitgeber Spezialsoftware für die Hotellerie anbietet, sieht Manfred in naher Zukunft keine beruflichen Risiken auf sich zukommen. Anders seine Eltern, die beide in der Gastronomie tätig sind und daher in der Covid-19-Pandemie nicht mehr arbeiten konnten, sondern von Kurzarbeitergeld abhängig sind. Zudem leiden sowohl Herr als auch Frau Oblinger unter verschiedenen Vorerkrankungen und haben auch aktuell Bedarf an medizinischen Eingriffen, die jedoch verschoben oder verlegt werden mussten (siehe zum Recht auf gesundheitliche Versorgung Krennerich 2020). In dieser Situation wendet sich Manfred zwar äußerst scharf gegen die Maßnahmen der Regierung, dennoch ist er, gemeinsam mit seinen Geschwistern, in der Pandemie für seine Eltern da und hat sie bei der Suche und Organisation einer alternativen Behandlungsmöglichkeit unterstützt.

In ganz ähnlicher Weise ist auch Frau Öllinger betroffen. Sie würde zwar gerne wieder eine Arbeit aufnehmen, dies erscheint aber auf absehbare Zeit unmöglich, und sie lebt von 909 Euro Berufsunfähigkeitspension im Monat. Seit einer misslungenen Lumbalpunktion im Jahr 2018 ist sie querschnittgelähmt und sitzt im Rollstuhl. Sie ist abhängig von einem häuslichen Sozialdienst (Pflegestufe 4), der ihr einerseits bei der allgemei- 
nen Körperhygiene behilflich ist und zudem die professionelle Pflege ihres Dauerkatheters übernimmt. Darüber hinaus erfährt sie umfangreiche Unterstützung durch ihre Tochter Viktoria, die noch zu Hause wohnt, um ihrer Mutter zur Seite zu stehen, obwohl sie sich für beide Seiten mehr Selbstständigkeit und Unabhängigkeit wünscht und gerne ausziehen und eine eigene Familie gründen würde. Viktoria arbeitet in Teilzeit (30h in der Woche) im Personalmanagement eines Krankenhauses und ist gerade in der Covid-19-Pandemie froh um ihren sicheren Arbeitsplatz. Zudem haben ihre Vorgesetzten Verständnis für die besondere Situation der Familie und drängen sie nicht, ihre Arbeitszeit aufzustocken. Die derzeitige Notwendigkeit, von zu Hause aus zu arbeiten, sorgt dagegen für besondere Herausforderungen, da Viktoria in der Pandemie zusätzliche Pflegeaufgaben übernommen hat, da der Sozialdienstleister nicht mehr täglich, sondern nur noch dreimal in der Woche zu ihrer Mutter kommt. Wie Manfred Oblinger tut sie dies zwar gerne für ihre Mutter, dennoch versucht sie, sie zu mehr Selbstständigkeit zu erziehen, um für beide eine höhere Lebensqualität zu erreichen. Neben den beruflichen und gesundheitlichen Einschränkungen leidet Frau Öllinger in der Pandemie zusätzlich unter der Stigmatisierung, als Pflegefall angesehen zu werden, da das Personal des Sozialdienstes und des Krankentransportdienstes Schutzkleidung und Masken trägt und sie angestarrt werde.

Auch die beiden jüngeren Geschwister von Benedikt Holzner sorgen sich um ihre Eltern, besonders um die Mutter. Während Benedikt sich ein eigenes Leben weit weg von der Familie aufgebaut hat und weder in der Berufsschule noch im Ausbildungsbetrieb von der Covid-19-Pandemie betroffen war, verbrachten seine beiden deutlich jüngeren Brüder die gesamte Zeit der ersten Corona-Welle zu Hause, da ihr Kindergarten beziehungsweise die Grundschule im Zuge der Maßnahmen zur Pandemie-Einschränkung geschlossen waren. Beide litten sehr darunter, weder ihre Freunde auf dem Spielplatz noch ihre Klassenkameraden sehen zu können (siehe auch Sindelar 2020; Schnetzer et al. 2020: 7; siehe zu den Auswirkungen der Distanz-Lehre in Grundschulen und zu Hause auch Trültzsch-Wijnen/ Trültzsch-Wijnen 2021). Frau Holzner war nicht von beruflichen Einschränkungen betroffen, während ihr Mann in Kurzarbeit war und sich daher mehr als sonst um die Kinder kümmern konnte (Tengler/ Schrammel/ Brandhofer 2020: 24). Dennoch ist diese Familie ein Beispiel dafür, wie sehr die Pandemie gerade auch die Jüngsten belastet. Besonders am jüngeren der beiden Brüder, so Frau Holzner, habe die Situation sehr "genagt" und er habe immer wieder darum gebeten, dass seine Mutter nicht arbeiten gehen solle, um sich nicht in Gefahr zu 
begeben. Beiden Elternteilen war es in dieser Situation ein Anliegen, die Söhne vor zu vielen Nachrichten über die Pandemie zu schützen (siehe Ortner/ Kovacs/ Jadin 2020 zum Medienumgang von Kindern in der Covid-19-Pandemie). Daher nutzen sie verstärkt Netflix, um die Kinder vor Fernsehnachrichten „abzuschirmen“ und dort Sendungen zu suchen, die „eine kindliche Version des Virus“ zeigen.

Für einige der befragten jungen Erwachsenen erwies sich, wie bereits im Fall von Amelie Aufbauer und ihrem Bruder thematisiert, die Umstellung des Unterrichts als schwierig oder zumindest als „Herausforderung“ (Tengler/ Schrammel/ Brandhofer 2020: 6ff.; siehe auch Maaz/ Diedrich 2020), während für andere, wie etwa für Manfred Oblinger die Vorteile des Online-Unterrichts (mehr Freizeit, keine Anfahrt) überwogen (siehe zur Einschätzung der schulischen und beruflichen Situation während der ersten Corona-Welle auch Schnetzer et al. 2020: 6; Huber et al. 2020: 107). Auch in breit angelegten Studien zeigt sich eine Zweiteilung zwischen „Schülerinnen und Schülern, die sich sehr positiv über das Homeschooling und die damit verbundenen Chancen äußern“ sowie jenen, „die sich über die Situation des Homeschooling kritisch äußern und sie als hohe Belastung erleben" (Huber et al. 2020: 106). Familien, in denen die Probleme der Distanz-Lehre stark thematisiert wurden, zeichneten sich auch dadurch aus, dass sie wirtschaftlich von der Pandemie kaum oder gar nicht betroffen waren. So konnten sie sich stärker mit diesem Aspekt der Auswirkungen befassen. Sehr kritisch, wenn auch reflektiert, äußerte sich Torsten Kaiser. Als Schüler im Abschlussjahrgang störte es ihn besonders, dass zunächst einige Wochen völlige Unklarheit darüber herrschte, welche eLearning-Plattformen verwendet werden sollen (Microsoft Teams, MNS und andere) und die Lehrer sich nicht einigen konnten. Diese kritische Bewertung ist besonders vor dem Hintergrund bedeutsam, dass die technische Ausstattung für webbasierte Lehre von Schulleitungen und Lehrenden an Schulen in Österreich signifikant besser eingeschätzt werden als in Deutschland (ebd.: 97f.). Die Umstellung auf Online-Unterricht selbst sei für ihn sehr anspruchsvoll gewesen, da es einerseits sehr viel Stoff zu bewältigen gab und die Unterrichtsformen sehr neu und ungewohnt waren und sich andererseits die Lehrenden (siehe zu deren Perspektiven auf die Distanz-Lehre auch Trültzsch-Wijnen/ Sturm 2021) nicht gut mit den Plattformen auskannten. Das schließlich gewählte Programm Microsoft Teams bezeichnet er zwar als „sehr einfach“, dennoch seien insbesondere die ersten zwei Wochen „ein rechtes Chaos“ gewesen. Auch Amelie Aufbauer befürchtete zunächst, dass die Matura-Prüfungen aufgrund der Pandemie und der Distanz-Lehre auf das nächste Jahr ver- 
schoben werden könnten, diese Sorge erwies sich retrospektiv jedoch als unbegründet (siehe auch Schnetzer et al. 2020: 7 zu drohenden Brüchen in der Bildungsbiografie). Während Frau Kaiser, durchaus nicht ohne Stolz, betont, wie gut Torsten und auch sein jüngerer Bruder Jakob mit dem Online-Unterricht zurechtgekommen seien und sich in die Programme „eingefuchst“ hätten (siehe auch Huber et al. 2020: 93; vom Orde 2020: 19), stellte diese Umstellung für andere eine große Herausforderung dar. So berichtet etwa Frau Weiss von großen Belastungen in diesem Kontext. Zwar hatte sie als Bedienstete im Justizwesen (sie arbeitet als Justizwachbeamtin) keine finanziellen Einbußen in der Pandemie und verfügte zudem über viel Freizeit, dennoch forderte sie der Online-Unterricht ihres jüngeren Sohnes sehr. Computer, Drucker und E-Mail mussten eingerichtet werden, obwohl weder Mutter noch Sohn sich mit der Materie gut auskannten (siehe ebd.: 92; Tengler/ Schrammel/ Brandhofer 2020: 24; Teach for Austria 2020).

Ebenso wie Amelie Aufbauer befürchtete auch Gudrun Dornbacher zunächst, dass die Covid-19-Pandemie und die Einschränkungen Auswirkungen auf die letzten Matura-Prüfungen haben könnten, obwohl sie zum Zeitpunkt des Interviews bereits einige der Abschlussprüfungen absolviert hatte. Sie besuchte ein Abendgymnasium, das auch in normalen Zeiten eigentlich auf Fernunterricht ausgerichtet sei, dennoch hatten die Lehrer zum Teil große Schwierigkeiten damit, den "technische Anforderungen“ gerecht zu werden. Die von Amelie geäußerten Sorgen über eine zeitliche Verzögerung oder gar einen Bruch in ihrer Bildungsbiografie sind dabei nicht unbegründet. Jeweils über $30 \%$ der 14- bis 39-Jährigen in Österreich geben an, dass sich durch die Covid-19-Pandemie ihre Zukunftsperspektiven beziehungsweise ihre schulische oder berufliche Situation verschlechtert hat (Schnetzer et al. 2020: 6). Die Autoren und Autorinnen (ebd.: 7) weisen in diesem Zusammenhang besonders darauf hin, dass es gerade die jungen Erwachsenen sind, die „in einer empfindlichen Phase des Lebenslaufs getroffen" werden und deren Übergang zum Erwachsenenleben erheblich beeinträchtigt werden kann.

Drei Familien waren zum Zeitpunkt der Befragung kaum wirtschaftlich durch die Pandemie und die Gegenmaßnahmen betroffen: Die Eltern von Elisabeth Ebner betreiben eine eigene Landwirtschaft und hier müsse auch in der Pandemie ,alles seinen Gang“ gehen. Auch das Logistikunternehmen von Frau Rohringer, der Mutter von Isabelle Rohringer, und ihrem Lebensgefährten war zunächst nicht eingeschränkt. Und Frau Stab, die Mutter von Simone Stab, hatte zwischen der sechsten und siebten Erhebungswelle ihren Beruf als Hausmeisterin aufgegeben und eine neue 
Tätigkeit als Gärtnerin aufgenommen. In diesem Beruf verdiene sie zwar generell nicht viel Geld, und sie war auch zwei Monate in Kurzarbeit, damit sei sie aber gut zurechtgekommen und habe die viele Freizeit genossen. Alle drei Töchter dieser Familien haben große Pläne und waren auch zum Zeitpunkt der Befragung optimistisch, diese trotz Allem umsetzen zu können (siehe zu möglichen Zukunftsperspektiven Schnetzer et al. 2020: 6f.; Großegger 2020a: 1f.; Karmasin et al. 2020: 10-13). Isabelle Rohringer hatte ihre Ausbildung als Kinderpflegerin bereits erfolgreich abgeschlossen und sich im Berufsleben etabliert. Sie leitet die Kinderbetreuung in einem großen Hotel und hoffe, diese Position auch behalten zu können, obwohl die Hotellerie scharfen Einschränkungen unterworfen ist. Elisabeth Ebner und Simone Stab planten beide, nachhaltig in ihre berufliche Zukunft $\mathrm{zu}$ investieren. Elisabeth ist ausgebildete Floristin und möchte ab 2021 eine private Meisterklasse in diesem Bereich besuchen. Dafür ist sie bereit, 15.000 Euro aus ihrem Ersparten in die eigene Ausbildung zu investieren. Simone Stab hatte gerade die Schule mit ausgezeichnetem Erfolg abgeschlossen und plante im September 2020 nach Südtirol umzuziehen, um dort, gefördert durch das Erasmus+ Programm der Europäischen Union, eine Beschäftigung in einem Marmorbetrieb aufzunehmen und sich als Steinmetzin ausbilden zu lassen. Beide waren zum Zeitpunkt der Befragung vorsichtig optimistisch, ihre Pläne der Covid-19-Pandemie zum Trotz umsetzen zu können, da alle entsprechenden Verträge bereits unterschrieben waren. Dieser, wenn auch gedämpfte, Optimismus findet sich auch in österreichweit angelegten Studien wie etwa "Generation Corona" des Instituts für Jugendkulturforschung: „In den ausbildungs- und berufsbezogenen Lebenszielen geben sich junge Menschen heute ambitioniert und setzen auf Eigenverantwortung sowie Selbstbehauptung“ (Großegger 2020a: 1). Dennoch weist die Autorin (ebd.: 2f.) darauf hin, dass Corona auch bei den Ansichten der jungen Menschen Spuren hinterlassen hat, „zumindest kurzfristig“. Deutlich kritischer äußern sich Schnetzer et al. (2020: 8), die gerade die sich verschlechternden Zukunftsperspektiven der jüngeren Generationen als „ein ernstzunehmendes Bedrohungsszenario für die Entwicklungszukunft der Gesamtgesellschaft" bezeichnen.

Vergleichsweise glimpflich stellten sich die Folgen der Pandemie auch bei Familie Boll dar, da weder Gregor Boll noch seine Mutter von der Covid-19-Pandemie wirtschaftlich betroffen sind. Gregor hat nach seiner Ausbildung zum Einzelhandelskaufmann eine Tätigkeit in einem Chemieunternehmen in seiner Heimatregion aufgenommen, ist mit seinem Arbeitgeber sehr zufrieden und möchte sich im Betrieb weiterentwickeln (siehe zu solchen Aufstiegsperspektiven Großegger 2019: 3). Gemeinsam 
mit seiner Freundin hat er eine Wohnung ganz in der Nähe bezogen und beide möchten dort in Zukunft auch bauen und sich etablieren. Bei seinem Arbeitgeber gab es bislang weder Kurzarbeit noch die Veranlassung, von zu Hause aus zu arbeiten. Die einzige Einschränkung, so Gregor, bestand darin, dass sich im Raucherraum nur noch eine begrenze Anzahl an Personen aufhalten durfte und Mindestabstände eingehalten werden mussten. „Belastet“ sah sich Gregor aufgrund der Pandemie nur, da er sich acht Wochen lang an alle Regeln gehalten und seine Freunde nicht getroffen habe (siehe dazu Schnetzer/ Hurrelmann 2020: 3, 5f.; Blom et al. 2020: 4f.). Dadurch vermisste er „Normalität“, jedoch habe der Freundeskreis darauf reagiert und regelmäßig Treffen per Telefon, WhatsApp und Houseparty organisiert (Großegger 2020b: 5; vom Orde 2020: 19). Auch Frau Boll sah sich durch die Pandemie nicht negativ betroffen, obwohl sie als ehemalige Krebspatientin und im Zustand nach einer schweren Embolie klar zur Risikogruppe zählt (siehe zur Risikowahrnehmung Blom et al. 2020: 14f.). Lediglich auf eine Reise zur Hochzeit eines Sohnes in Österreich habe sie aus Sicherheitsgründen verzichtet. Finanziell betrachtet sich Frau Boll dagegen sogar als eine „Gewinnlerin“ der Krise. Sie bezieht seit vielen Jahren eine Erwerbsunfähigkeitsrente aus Österreich, und diese werde auch weiterhin regelmäßig ausgezahlt. Zudem verdient sich Frau Boll seit vielen Jahren mit einer Hundezucht etwas hinzu. Der Verkauf habe seit Beginn der Pandemie deutlich angezogen, und alle Würfe seien immer ausverkauft. Frau Boll führt dies, wohl zu Recht, auf die Kontaktbeschränkungen und mangelnde Freizeitmöglichkeiten im Lockdown sowie auf die Arbeit von zu Hause aus zurück, weshalb viele Menschen sich ein Haustier anschaffen würden. Dadurch stünde ihr in der Covid-19-Pandemie nun deutlich mehr Geld zur Verfügung als zuvor.

Familie Landinger kommt im Panel eine Sonderrolle zu. Seit Beginn der (Medien-)Sozialisationsstudie im Jahr 2005 war die Familie immer von staatlichen Hilfsmaßnahmen abhängig, und Herr Landinger war, mit wenigen kurzen Unterbrechungen, über den gesamten Zeitraum hinweg arbeitslos. Frau Landinger war zum Zeitpunkt der letzten Befragung als Bürokauffrau tätig und stand kurz vor der lang ersehnten Pensionierung. Eine Verschlechterung der wirtschaftlichen Situation brachte die Covid-19Pandemie für die Familie nicht. Insbesondere auch Timo bedurfte aufgrund seiner kognitiven Einschränkungen stets intensiver Betreuung und der Unterbringung in verschiedenen Einrichtungen zur Förderung von Behinderten. In vergangenen Erhebungswellen berichtete Herr Landinger in diesem Kontext regelmäßig über, so seine Sichtweise, Probleme mit verschiedenen staatlichen Stellen und deren Inkompetenz. Vergleichbare 
Aussagen sind in der siebten Erhebungswelle erstmals nicht gefallen. Dies ist insbesondere bemerkenswert, da Timo zu diesem Zeitpunkt seine gesamte Zeit ohne Tätigkeit zu Hause verbrachte. Aufgrund von nicht näher benannten Mobbingvorfällen, Timo möchte sich dazu ausdrücklich nicht äußern, hatte er seine bisherige Betriebsstätte verlassen. Zum Zeitpunkt der Befragung lag zwar bereits die Zusage für eine neue Stelle in einer anderen Behinderteneinrichtung vor, bedingt durch die Covid-19-Pandemie konnte er sie bislang jedoch nicht antreten. Timo selbst sieht oder thematisiert keinen Zusammenhang zwischen seiner eigenen Situation und der herrschenden pandemischen Lage, somit kritisiert er auch seine aktuelle Arbeitslosigkeit nicht. Dennoch äußert er sich überaus kritisch über die Maßnahmen der Österreichischen Bundesregierung (siehe zu Einstellungen der Bevölkerung in Österreich Willems et al. 2020: 3f.), die er "scheiße“ findet und an die er sich nur halte, weil er "kei Wahl ned“ hat. Auf welchen Informationen seine Meinung beruht, bleibt unklar, da Timo bisher in allen Erhebungswellen keine Informationsmedien rezipiert hatte und auch aktuell verneint, solche Angebote zu konsumieren, obwohl ihm inzwischen uneingeschränkter Zugang zu den Angeboten im Internet zur Verfügung stünde. Dennoch wendet er sich insbesondere gegen die Maskenpflicht und die Einschränkungen der Wirtschaft und prangert die Geldvernichtung an: „Ich find des is einfach nur Schwachsinn. Und generell, wenn ma scho alles schließt, des komplette Land, da gehen doch nur, mehrere Milliarden Euro kaputt, weil des macht...macht ja keiner die Arbeit. Find's scheiße!“ Mit seiner Haltung zählt Timo zu einer nicht unerheblichen Gruppe gerade jüngerer Menschen in Österreich, die die Maßnahmen zur Einschränkung der Pandemie und individuellen Verzicht kritisieren oder verweigern (Schnetzer et al. 2020: 4f.). Mit dieser Haltung bestätigt er auch Ergebnisse aus Deutschland, wonach eher formal niedriger gebildete Männer aus abseits von Großstädten gelegenen Wohnorten die Maßnahmen und Rücksichtnahme ablehnen (Schnetzer/ Hurrelmann 2020: 7).

Seine Eltern scheinen nicht die Auslöser für diese Aussagen zu sein. Denn Frau Landinger bedauert zwar, aktuell ihre Enkel kaum sehen zu können, sieht sich darüber hinaus aber kaum eingeschränkt, da Lebensmittelläden weiterhin offen seien. Auch in normalen Zeiten lebt die Familie weitestgehend zurückgezogen und pflegt keine sozialen Kontakte mit Freunden oder Nachbarn. Dieses Verhalten wurde in der Covid-19-Pandemie beibehalten und Kontakte vermieden (siehe dazu auch Moser 2020): "Ja dass man nicht so wirklich unterwegs sein kann, außer mit dem Hund halt, weil der muss aussi." Insgesamt scheinen sich Herr und Frau Landin- 
ger mit ihrer Lebenssituation abgefunden zu haben und das Beste daraus zu machen.

Die Erfahrungen, die Familie Zarbl, die insgesamt zu den wirtschaftlich besser gestellten Familien im Panel gehört und in ihrer Patchwork-Konstellation über starke und belastbare sozio-emotionale Strukturen verfügt, mit der Pandemie gemacht hat, sind ambivalent. Frau Zarbl selbst hat bislang keine persönlichen Einschränkungen durch die Covid-19-Pandemie erlitten, und auch ihr Sohn Norbert hat sich nach der erfolgreich abgeschlossenen Matura mit technischer Ausbildung erfolgreich im Beruf etabliert (siehe zu solchen Bildungsbiografien Großegger 2019: 2f.). Aktuell habe er keine Sorgen um seinen Arbeitsplatz, obwohl er sich zum Zeitpunkt des Interviews in Kurzarbeit befand und von zu Hause aus arbeiten musste (siehe dazu Blom 2020; Steiber/ Liedl/ Molitor 2020: 2). Stattdessen kann er weiterhin mietfrei zu Hause wohnen, und seine Eltern planen, ihn beim Auszug mit seiner Freundin umfangreich finanziell zu unterstützen. Hart getroffen wurde dagegen Herr Zarbl, der aufgrund der Covid-19-Pandemie seine Selbstständigkeit als Gesundheits- und Fitnesstrainer aufgeben musste. Glücklicherweise, so Frau Zarbl, hatte er auch bisher im Nebenerwerb zusätzlich im Krankenhaus gearbeitet und konnte dort direkt wieder auf $100 \%$ der wöchentlichen Arbeitszeit aufstocken. So erwies sich die Entwicklung finanziell zwar nicht als „Katastrophe“, denn man sei „im Prinzip relativ unbeschadet bislang" hindurchgekommen, so Frau Zarbl, „emotional“ war das Erlebnis jedoch sehr belastend (Schedlich 2020; Vlasak/ Barth 2020).

\subsubsection{Information und Kommunikation über die Covid-19-Pandemie}

Die Covid-19-Pandemie, Berichte über Infektionszahlen und Todesfälle, Informationen zu neuen, verschärften oder gelockerten staatlichen Gegenmaßnahmen, Berichte über deren Auswirkungen, Pressekonferenzen und Interviews mit Politikerinnen und Politikern sowie Prognosen und Einschätzungen von Expertinnen und Experten haben sich seit dem Frühjahr $2020 \mathrm{zu}$ ständigen Begleitern entwickelt. Es ist inzwischen kaum mehr möglich, den Fernseher oder das Radio einzuschalten, die Zeitung aufzuschlagen, auf Online-Seiten zu surfen oder Soziale Medien zu nutzen, ohne, gewollt oder ungewollt, mit der Thematik konfrontiert zu werden (siehe etwa Aigelsreiter 2020; Mitschka/ Unterberger 2020; Großegger 2020b; vom Orde 2020: 18). Selbst die beliebte Videoplattform YouTube 
präsentiert prominent auf der Startseite neben den Angeboten verschiedener Nachrichtenkanäle auch offizielle Videos der jeweiligen Regierungen.

Diesem umfangreichen Informationsangebot steht auf Seiten der Bevölkerung ein großes Informationsbedürfnis gegenüber (Großegger 2020b; Aigelsreiter 2020; Mitschka/ Unterberger 2020; vom Orde 2020; für internationale Perspektiven auf die Rolle von Medien für junge Menschen in der Pandemie siehe Katz/ Bond 2021; Ortner/ Kovacs/ Jadin 2020); dies zeigt sich auch bei den Befragten des Panels. Eine Aussage von Alfons Weiss mag beispielhaft dafür stehen, wie die Befragten sich über die Covid-19-Pandemie informiert haben: Über die Maßnahmen habe er sich „wie eh jeder" in den Medien informiert (siehe auch Mitschka/ Unterberger 2020; Großegger 2020a, 2020b; Aigelsreiter 2020; Jugendkultur.at 2021; Marx 2020; Heinzlmair 2020; vom Orde 2020). Dazu zählen für ihn die Fernsehnachrichten des österreichischen öffentlich-rechtlichen Rundfunks, mit einem besonderen Blick auf die Regionalnachrichten, und die Bildzeitung, um auch über die Entwicklung in Deutschland informiert zu sein, da er sehr grenznah wohnt. So wie Alfons verfügen alle Befragten über mehr oder weniger umfangreiche Medienrepertoires, in denen sich die eigene Lebenssituation und die individuellen Informationsbedürfnisse widerspiegeln und die durch zahlreiche unterschiedliche Informationsquellen geprägt werden.

\subsubsection{Fernsehen und öffentlich-rechtlicher Rundfunk}

Während einige der jungen Erwachsenen Nachrichten im Fernsehen ausdrücklich ignorierten (Gregor Boll, Erich Grubert, Torsten Kaiser, Helmut Pfortner) spielten diese für die anderen Befragten eine wichtige Rolle. Besonders häufig wurden dabei Sendungen des öffentlich-rechtlichen Fernsehens (siehe auch Heinzlmair 2020: 119f.; Mitschka/ Unterberger 2020; vom Orde 2020: 18) und regionale Fernsehnachrichten der Landesstudios genannt (siehe zur Bedeutung der Arbeit der Landesstudios während der Pandemie Stadtherr/ Schmidinger 2020). Als Grund hierfür nennt beispielsweise Norbert Zarbl ausdrücklich dessen Glaubwürdigkeit, eine Sichtweise, die etwa seine eigene Mutter zumindest teilweise in Zweifel zieht. Auch Frau Holzner gibt an, hauptsächlich einen anderen Sender zu rezipieren: Ihre wichtigste Informationsquelle war der von Dietrich Mateschitz betriebene Privatsender Servus-TV, weil „dort halt noch so die unzensiertesten Sachen (lacht) kommentiert werden“. Diese Aussage zeugt zwar von einem gewissen Misstrauen gegenüber dem öffentlich-rechtli- 
chen Rundfunk, jedoch machte Frau Holzner in diese Richtung sonst keine anderen Andeutungen und nutzte stattdessen auch intensiv die Online-Angebote des ORF, um sich über die Covid-19-Pandemie auf dem Laufenden zu halten. Insgesamt wurde das vielfältige und über zahlreiche Distributionskanäle verbreitete Angebot des ORF stark angenommen. Dazu zählen, neben den Fernsehnachrichten und Diskussionsrunden, auch die Homepage und die Nachrichten-App des ORF (Frau Pfortner, Norbert Zarbl) und der Instagram-Account der ZIB (Frau Aufbauer, Amelie Aufbauer). Neben den Sendern und Nachrichten des ORF wurden aber auch zahlreiche private, auch deutsche, Fernsehsender als Informationsquellen genannt: nTV, n24, Servus-TV, Sat.1 GOLD, Puls4. Im Gegensatz zu vorherigen Erhebungswellen wurden keine Informationsangebote des öffentlich-rechtlichen Rundfunks aus Deutschland angeführt, obwohl einige der Befragten ausdrücklich auch über die Lage im Nachbarland informiert sein wollten.

\subsubsection{2 (Online-)Zeitungen mit besonderem Blick auf regionale Angebote}

Neben dem Fernsehen waren gedruckte Zeitungen (Frau Oblinger, Helmut Pfortner, Frau Zarbl, Norbert Zarbl) und die Online-Angebote von Zeitungen und Magazinen für fast alle Befragten von großer Bedeutung. Auffällig dabei ist, dass mit Mario Hirtner nur eine einzige Person das Online-Angebot einer überregionalen Qualitätszeitung (DerStandard.at) und einer Boulevardzeitung (Krone.at) genannt hat. Österreichweit war Der Standard bei jungen Menschen während der ersten Corona-Welle dagegen deutlich beliebter als die Kronenzeitung (Heinzlmair 2020: 120; Großegger 2020b: 9). Zusätzlich verweist Alfons Weiss auf die ebenfalls überregionale Bildzeitung (Print). Eine besondere Bedeutung für die Information über die Covid-19-Pandemie kommt unter den Befragten den Online-Angeboten der österreichischen Bundesländerzeitungen (Frau Boll, Frau Holzner, Frau Zarbl, Norbert Zarbl, Frau Aufbauer) sowie noch stärker regional orientierten Informationsplattformen (Helmut Pfortner, Frau Pfortner) zu.

\subsubsection{YouTube als wichtige Informationsplattform}

Unter den Soziale Medien-Angeboten hat sich gerade YouTube als wichtige Anlaufstelle erwiesen (siehe dazu auch Marx 2020: 27; vom Orde 2020: 
18). Dabei ist zu betonen, dass nur Torsten Kaiser angibt, hier bewusst themenspezifischen Kanälen zu folgen. Er nennt zwei Kanäle, die sich unter anderem mit politischen und gesellschaftlichen Themen befassen: Mister Wissen2go und jungdonaiv. Auch Frau Weiss informierte sich, zumindest zu Beginn der Pandemie, über YouTube, und Simone Stab sieht hier Videos an, die ihr eine Freundin empfohlen hat. Die tatsächliche Bedeutung von YouTube in der Pandemie streicht aber Mario Hirtner heraus: Die Plattform präsentiert nämlich auf der Startseite Videos der Österreichischen Bundesregierung, unabhängig von den auf Grund eigener Suchanfragen vorgeschlagenen Videos. In Deutschland wird dieser Platz mit Angeboten der Bundeszentrale für gesundheitliche Aufklärung (BZgA) bespielt. Auf diese Weise erreichen die Informationsangebote breitere Zielgruppen. Auch Frau Aufbauer und ihre Tochter Amelie verweisen ausdrücklich auf diese Inhalte. Die YouTube-Kanäle von Fernsehsendern, Zeitungen und Medienhäusern werden von den Befragten darüber hinaus jedoch nicht thematisiert.

\subsubsection{Soziale Medien und ihre Risiken}

Neben YouTube wird insbesondere Facebook auch mehrfach von den Befragten als Informationsquelle genannt. Hierbei muss zwischen Newsartikeln von Medienhäusern, die dort vorgeschlagen werden, und der Diskussion in Gruppen unterschieden werden. Einige der Befragten nutzen durchaus Facebook und seine Vorschläge, um auf journalistische Beiträge aufmerksam zu werden (Frau Grubert, Frau Hirtner, Frau Holzner, Benedikt Holzner, Frau Pfortner, Helmut Pfortner, Frau Weiss). Insbesondere Frau Pfortner und ihr Sohn Helmut verweisen dabei auf das mangelnde Vertrauen in Informationen, die auf Facebook gefunden wurden, und geben an, dass man solche bei Bedarf durch eigene Recherchen überprüfen müsse. Ohne Facebook als Plattform konkret zu nennen, verweist auch Frau Dornbacher auf die Verbreitung von „fake news“ über Covid-19 in den sozialen Medien. Vergleichbar mit Artikeln auf Facebook sind auch Informationskanäle auf Instagram, Frau Aufbauer und ihre Tochter Amelie folgen hier dem Angebot ZIB des ORF. Andere Befragte, wie Frau Landinger und Norbert Zarbl, lehnen Soziale Medien zur Information über Covid-19 grundsätzlich und ausdrücklich ab.

Soziale Medien ermöglichen aber auch die Kommentierung von Beiträgen und den diskursiven Austausch in Gruppen. Davon machen die Befragten im Panel aber nur sehr eingeschränkt Gebrauch: Frau Grubert 
etwa kommentiert vereinzelt Beiträge und tauscht sich mit anderen über den Facebook-Messenger aus. Frau Weiss beteiligte sich an einer WhatsApp-Gruppe unter Bekannten zum Austausch über Covid-19, reduzierte ihr Engagement hier aber aus zeitlichen Gründen und mangels Interesses wieder. Lediglich für Frau Zarbl stellen die Diskussionen und Informationen aus Facebook-Gruppen einen wichtigen Teil ihres Informationsrepertoires dar. Sie sieht hierin einen wichtigen Pool für alternative Meinungen.

\subsubsection{Das Radio - ein Informationsmedium}

In der Covid-19-Pandemie hat sich auch das Radio wieder verstärkt als Informationsmedium unter den Befragten des Panels etabliert (siehe dazu auch Mitschka/ Unterberger 2020; Aigelsreiter 2020; Großegger 2020b: 9; vom Orde 2020: 20). Während einige der befragten Mütter auch in der Vergangenheit regelmäßig Radio gehört hatten, spielte dieses Medium bei den jungen Erwachsenen in den vorherigen Erhebungswellen nahezu keine Rolle mehr. Dies hat sich nun, auch auf Grund der neuen Lebensund Arbeitsbedingungen, verändert. Ein Grund dafür ist die Möglichkeit, im Auto Radio zu hören. Frau Stab sowie Norbert Zarbl und Gregor Boll hören auf dem Weg zur Arbeit Radionachrichten im Auto. Für Gregor war dies, neben Nachrichtenzusammenfassungen auf Amazon Alexa, sogar die einzige mediale Informationsquelle über die Covid-19-Pandemie. Auch Frau Holzner, Frau Scheib und Manfred Oblinger setzten auf verschiedene Radiosender zur Information. Gerade Manfred zeigte sich dabei sehr unzufrieden mit den auch im Radio verbreiteten Inhalten. Beispielhaft dafür griff er ein Interview heraus, das ihn und seine Eltern persönlich stark betroffen und wütend gemacht hätte: Der Leiter eines Unikrankenhauses verkündete dort demnach öffentlich, wie gut, trotz der Covid-19-Pandemie, vielen Menschen ambulant geholfen werden könne, wenn sie Schmerzen haben (siehe dazu auch Wrase/ Allmendinger 2020). Für ihn sorgte dies für ein „sehr ungutes Gefühl“, da zeitgleich seine Eltern den ganzen Tag bei jeder Bewegung starke Schmerzen hatten und sie dennoch keine Hilfe bekamen, sondern stattdessen von eben diesem Krankenhaus abgewiesen worden wären, obwohl sie einen vereinbarten Behandlungstermin gehabt hätten. So führte die mediale Berichterstattung in diesem Fall zu einer Verstärkung der persönlich empfundenen Benachteiligung. 


\subsubsection{Persönliche Informationsquellen}

Neben der mediengestützten Auseinandersetzung mit Covid-19 haben sich auch persönliche Kontakte als wichtige Informationsquellen für die Befragten im Panel erwiesen. Dabei lassen sich zwei Tendenzen feststellen: 1) Die Mütter nannten in diesem Kontext ausgewählte Personen, die durch ihre Funktion über besondere Kenntnisse verfügen. Dazu zählen etwa eine Mitarbeiterin im Magistrat der Stadt (Frau Holzner), ein Polizist, der „immer über aktuelle Krankheitsfälle und Todesfälle informiert“ war (Frau Kaiser) sowie Vorgesetzte am Arbeitsplatz, die mit der Umsetzung von Hygieneregeln betraut sind (Frau Kaiser, Frau Pfortner, Frau Holzner). 2) Bei den jungen Erwachsenen nennt mit Erich Grubert nur ein Junge einen solchen Funktionsträger als wichtige Informationsquelle. Dabei handelt es sich um einen führenden Funktionär der Wirtschaftskammer, der an Verhandlungen mit der Regierung über Beschränkungen und Hilfsmaßnahmen beteiligt war und zugleich Erichs Chef ist. Alle anderen befragten jungen Erwachsenen beziehen sich bezüglich Informationen über Covid-19, wie auch Erich selbst, dagegen auf den Austausch mit ihren Arbeitskollegen. So bezeichnet etwa Gregor Boll den Informationsaustausch bei der Arbeit als „Live-Ticker“, und auch Benedikt Holzner, Manfred Oblinger und Norbert Zarbl verweisen auf die Arbeitsumgebung als Informationsquelle. Bei den jungen Frauen dagegen werden keine Kontakte bei der Arbeit genannt, sondern Freundinnen, die sie mit Informationen versorgen. Simone Stab etwa rezipiert YouTube-Videos und liest OnlineNachrichten, die ihr eine Freundin vorgeschlagen hat. Darüber hinaus sucht sie dann bei Google gezielt nach Informationen. Auch Susanne Scheib nutzt, nur wenn es nötig wird, Google, denn sie sieht eigentlich keinen Anlass dazu, sich aktiv über Covid-19 zu informieren. Als Grund dafür gibt sie an, über Freunde alle Änderungen der Maßnahmen auch so mitzubekommen.

Die besondere Thematik der Pandemie scheint aber zum Teil auch zu einer Vergiftung persönlicher Gespräche zu führen, weshalb Covid-19 in Unterhaltungen im Freundeskreis teilweise bewusst ausgeklammert wird. Anschaulich schildert dies Erich Grubert: Im Freundeskreis gab es viele Unterhaltungen über Covid-19, und es sei alles „schon zu Tode geredet worden" (siehe auch Großegger 2020b: 5 zur Bedeutung der Covid-19Pandemie und zum Wunsch nach realen Kontakten mit den Peers). Die Thematik führte schließlich immer zu einer schlechten Stimmung. Daher habe er begonnen, das Thema zu meiden. Eine ähnliche Konsequenz zog auch Frau Weiss, wenn auch aus anderen Gründen: Zu Beginn der Pande- 
mie informierte sie sich über Facebook und YouTube und rezipierte dort „interessante Beiträge“. Ergänzend dazu tauschte sie sich in einer WhatsApp-Gruppe mit zwölf Freunden über die Pandemie aus. Beides stellte sie jedoch im weiteren Verlauf ein, einerseits aus zeitlichen Gründen und andererseits, weil die Thematik sie nicht mehr so sehr interessierte. Tatsächlich wurden Frau Weiss fehlende Informationen zum Verhängnis, da sie nicht über die bestehenden Ein- und Ausreisebestimmungen Bescheid wusste und auf Grund einer Fahrt nach Deutschland in mehrtägige Quarantäne musste. In diesem Kontext beklagt sie, darüber nicht zumindest von der Grenzpolizei informiert worden zu sein.

\subsubsection{Informationsvermeidung und Kritik}

Bei einigen der Befragten, mehrheitlich handelt es sich dabei um junge Erwachsene, führt die aus ihrer Sicht überbordende Berichterstattung über die Pandemie zu einer bewussten Abkehr von nahezu allen Medienangeboten, manche meiden auch speziell solche Angebote, die sie mit Information in Verbindung bringen (ähnliche Perspektiven finden sich auch bei Großegger 2020a: 5). Viktoria Öllinger etwa bezeichnet die Berichterstattung über Covid-19 als zu „omnipräsent“ und würde „am liebsten alles ausschalten“. Eine gänzliche Abkehr von Informationen ist ihr auf Grund ihrer Arbeit im Krankenhaus und der Fürsorge für ihre kranke Mutter so jedoch nicht möglich. Anders reagiert ihre Mutter selbst, die zwar wegen Covid-19 „Angst vor der Mediennutzung" hat und sich damit auf Schreckensszenarien bezieht. Sie will dennoch informiert sein (siehe zu den Informationsbedürfnissen junger Frauen Großegger 2020b: 8, 12) und greift dabei besonders auf Fernsehnachrichten (vom Orde 2020: 20) und einen lokalen Online-Informationsdienst zurück. Auch Gudrun Dornbacher hat in der Pandemie ihren Mediengebrauch bewusst verändert und greift verstärkt auf ihr Smartphone zurück. Jedoch geht es ihr dabei kaum um Information, sondern darum, mit Freunden und Bekannten in Kontakt zu bleiben. Darüber hinaus nutzt sie die ihr zur Verfügung stehende Zeit, um mehr Bücher zu lesen und, wie auch früher schon, gemeinsam mit ihrer Mutter und ihrer Schwester Filme zu schauen. Am deutlichsten äußert sich Benedikt Holzner, der von sich aus behauptet, auch generell „wenig Information“ $\mathrm{zu}$ „machen“. Als einzige verwendete Informationsquelle nennt er nur Facebook (siehe zur Relevanz von Sozialen Medien Großegger 2020c: 70f.; vom Orde 2020: 19). Allerdings zeigt er sich auch dort genervt von der überbordenden Kommunikation über 
die Covid-19-Pandemie: „Wennst bei Facebook eini gehst, ist eh das erste was'd zehn Minuten sigst Corona, Corona, Corona und des wird einfach nur anstrengend."

Die Abkehr von Informationsangeboten geht einher mit zunehmender Skepsis gegenüber den politischen Maßnahmen zur Eindämmung der Pandemie. Die Interviews der siebten Erhebungswelle fanden im Frühjahr und Frühsommer 2020 statt, während des Höhepunkts und der Endphase des ersten Lockdowns. An weitere, langandauernde Lockdowns in Deutschland beziehungsweise in Österreich sowie die mehrfache Verlängerung der staatlichen Maßnahmen zur Eindämmung der Pandemie war zu diesem Zeitpunkt für viele der Befragten noch nicht zu denken. Auf die mögliche Entwicklung der pandemischen Lage im Herbst und Winter wurde zwar von Wissenschaftlerinnen und Wissenschaftlern frühzeitig hingewiesen, und eine drohende zweite Welle wurde auch in den Medien und von der Politik thematisiert, frühzeitige Maßnahmen oder längerfristige Strategien wurden jedoch lange Zeit nicht in Angriff genommen, wohl auch aus Sorge vor einem Kippen der Stimmung (siehe zu Erosionen in der Gesellschaft auch Hummel/ Graf Strachwitz 2021). Norbert Zarbl fasst dieses Spannungsverhältnis aus seiner Perspektive wie folgt zusammen: Die Maßnahmen bezeichnete er als "für unsere Wirtschaft a g'scheider Dämpfer", und er konnte sich nicht vorstellen, dass im Herbst erneut vergleichbare oder gar schärfere Maßnahmen getroffen werden könnten, sollte das Corona-Virus dann zurückkommen. Er thematisiert dabei auch die Frage der erneuten Akzeptanz von Einschränkungen durch die Bevölkerung (siehe auch Willems 2020). Dem frühen Erhebungszeitraum zum Trotz waren bereits damals bei vielen der Befragten deutliche Ermüdungserscheinungen ob der langanhaltenden Pandemie und der damit verbundenen Einschränkungen zu bemerken. Dies zeigt sich auch deutlich daran, dass viele der Befragten anschaulich davon berichten, wie sie zu Beginn der Pandemie sehr regelmäßig nach neuen Nachrichten gesucht haben und tagesaktuell über Infektionszahlen in der eigenen Umgebung, in angrenzenden Gemeinden und Landkreisen sowie bundes- und weltweit informiert sein wollten. Neben Elisabeth Ebner, die mit Spannung und Sorge die Entwicklung der Fallzahlen in Stadt und Land verfolgte, waren es in erster Linie sechs Mütter (Frau Boll, Frau Grubert, Frau Zarbl, Frau Zarbl, Frau Pfortner, Frau Scheib), die sich zunächst sehr intensiv mit den aktuellen Entwicklungen auseinandergesetzt haben. Im Verlauf der Pandemie, so berichten viele der Befragten, fand aber aus verschiedenen Gründen eine Reduktion der Informationsbeschaffung oder gar eine Abkehr statt (siehe dazu auch Großegger 2020b: 12). Frau Hirtner etwa ist 
gesundheitlich stark angeschlagen, war lange krankgeschrieben und hat im Zuge der Covid-19-Pandemie ihren Arbeitsplatz verloren und wurde arbeitslos, dies belastet sie sehr (siehe dazu auch Vlasak/ Barth 2020). Sie informierte sich sowohl über Fernsehnachrichten auf ORF 2 und nTV als auch über Online-Artikel, die ihr auf Facebook vorgeschlagen wurden. Inzwischen aber habe sie kaum noch Interesse daran, etwas über die Covid-19-Pandemie zu hören. Das Thema bezeichnet sie als „Horror“ (siehe zu derartigen Wahrnehmungen und psychosozialen Herausforderungen Schedlich 2020) und die ausufernde Berichterstattung mache sie „schon wahnsinnig“. Frau Holzner und Frau Pfortner haben dagegen mit der Zeit einfach das Interesse verloren. Wobei auch Frau Pfortner darauf verweist, dass die andauernde Auseinandersetzung mit Covid-19 einen auch „hysterisch" machen könne und es inzwischen nicht mehr zu viel verlangt sei, wenn man die Hygieneregeln kenne und sich an diese auch halte.

Weitaus kritischer zu betrachten sind die Beweggründe von Frau Stab, ihre Informationsbeschaffung lediglich auf Radionachrichten im Autoradio und Artikel auf der Seite ihres E-Mail-Anbieters GMX zu beschränken: Sie wolle sich nicht mehr weitergehend mit der Thematik auseinandersetzen, weil sie die Covid-19-Pandemie weitgehend für „Angst- und Panikmacherei" halte. Ganz ähnlich positioniert sich auch Frau Oblinger, die an sich über alle Erhebungswellen hinweg sehr besonnene Positionen in Bezug auf Politik und Gesellschaft eingenommen hatte, während ihr Sohn Manfred, gerade in jüngeren Jahren, zum Teil radikale politische Positionen angedeutet hatte. In Bezug auf Information konzentriert sie sich damals wie heute stark auf die Fernsehnachrichten im ORF und die Lektüre der regionalen Tageszeitung, jedoch wolle auch sie sich inzwischen mit der Covid-19-Pandemie eigentlich nicht mehr befassen. Frau Oblinger ist sowohl beruflich als auch gesundheitlich stark von der Pandemie betroffen. Sie arbeitet in der Gastronomie und befindet sich daher in Kurzarbeit, zudem wurde eine geplante und dringend benötigte Operation im Krankenhaus ohne Begründung abgesagt, während diese in einem anderen Krankenhaus dann problemlos möglich war (siehe dazu auch Wrase/ Allmendinger 2020). Frau Oblingers Unmut richtet sich daher auch weniger gegen die Eindämmungsmaßnahmen per se, sondern gegen die aus ihrer Sicht schlechte Informationspolitik, wozu sie auch die Irritationen über den so bezeichneten Feiertagserlass zu Ostern und zum 1. Mai zählt, die sie eine „reine Verarsche“ nennt (zur Bewertung der Maßnahmen und der Kommunikationsstrategien durch die Bevölkerung siehe Willems et al. 2020). Zudem kritisiert sie, dass sich Ausländer frei im Land bewegen könnten, während die einheimische Bevölkerung stark eingeschränkt sei 
und die „Leit deppert gemacht wern“ durch die „Panikmache“ der Regierung. Dabei stellt sie sich auch die Frage, ob Leute an oder mit Covid-19 sterben und zieht die Höhe der Todeszahlen in Zweifel. Sie begründet dies auch damit, dass die jährlichen Todeszahlen durch die „wirkliche Grippe“ viel höher seien als durch Covid-19. In ihrer Argumentation zeigt Frau Oblinger auch Nähe zu Verschwörungserzählungen: „Also i sag: Die Wirtschaft ist von der Regierung in den Ruin getrieben worden. Das war der Plan." Obwohl Frau Oblinger in der siebten Erhebungswelle ebenso wie auch in den Erhebungswellen zuvor offenherzig erzählt, lässt sich nicht klären, woher sie diese Ansichten bezieht. Eine Ursache könnte in der Unzufriedenheit mit der eigenen wirtschaftlichen Lage und Verlustängsten liegen (siehe auch Blom 2020), jedoch hat sich die finanzielle Situation der gesamten Familie und insbesondere auch der Kinder in den letzten Jahren deutlich verbessert. Auch ihr Sohn Manfred, der inzwischen vom Land in den Zentralraum gezogen ist, erfolgreich eine Ausbildung absolviert und sich etabliert hat, zieht, ähnlich wie seine Mutter, die Risiken durch Covid-19 in Zweifel. Er nennt für seine Sichtweise keine Quellen in Medien oder im Internet, sondern verweist auf seine eigenen Erfahrungen, wonach auch ältere Menschen Covid-19 zum Teil problemlos überstehen würden, während er auch junge Leute im Alter von 18 oder 19 Jahren kenne, denen es „richtig scheiße ganga is“. Auch er kritisiert die „Panikma-

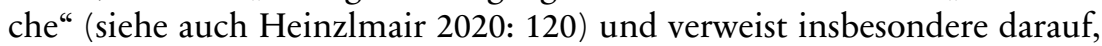
dass jeden Tag im Fernsehen ,irgendein Experte“ über die Gefährlichkeit und die Lebensbedrohlichkeit von Covid-19 spräche, als „wär das jetzt die Pest 2.0". All dies führt auch bei ihm zu einer gewissen Abkehr von Informationsangeboten im Fernsehen und zu einer stärkeren Hinwendung zu Unterhaltungsangeboten.

Auch Frau Zarbl berichtet, die gesamte Situation rund um die Pandemie habe ihr stark zu denken gegeben, und sie bezeichnet sich inzwischen selbst als „zwiegespalten“ mit Blick auf die Maßnahmen gegen Covid-19 und die daraus resultierenden Folgen. Zunächst sei sie klar dafür gewesen und habe überzeugt hinter den Maßnahmen gestanden. Nun aber sähe sie verstärkt auch die wirtschaftlichen Folgen und sei sich unsicher darüber, wie lange es noch so weitergehen könne, was dies mit dem Land mache und ob die Maßnahmen inzwischen nicht „zu krass“ seien. Sie bemerke auch in ihrem Umfeld eine zunehmend kritische Sichtweise, wobei sie sich selbst noch eher als Befürworterin empfinde, aber dennoch in eine „Findungsphase“ eingetreten sei.

Diese Unsicherheit spiegelt sich auch in ihrer Mediennutzung wider: Frau Zarbl nutzt die Zeitung, das Fernsehen (ORF) und Gruppen auf 
Facebook zur Information. Facebook sieht sie dabei ausdrücklich als Alternative, als „Kontrast“ zu Fernsehen und zu den Printmedien, wo nur mehr der Mainstream oder die Meinung der Regierung gezeigt würden: „also Printmedien und und und ORF im Prinzip, da is man eh jetzt so eher auf einer Linie, also da ist man jetzt eh von der Meinung her." Die Informationen in Gruppen böten dagegen eine breitere Sicht auf die Dinge und auch alternative Meinungen, um sich ein eigenes Bild der Lage machen zu können: „da kriegt man dann eigentlich so a bissel, a bissel was anders, a bissel was anders präsentiert.“ In ihrer Bewertung der Medienqualitäten positioniert sich Frau Zarbl somit entgegen der klaren Mehrheit der Österreicherinnen und Österreicher (Heinzlmair 2020: 120; Mitschka/ Unterberger 2020; Großegger 2020b).

Die Ansichten von Frau Zarbl sind bislang jedoch noch nicht so extrem wie jene von Frau Oblinger, die bereits deutlich Verschwörungserzählungen zuneigt. Jedoch muss es als Warnsignal gewertet werden, wie schnell eine Person aus einer mittlerweile sozio-ökonomisch eher besser gestellten Familie das Vertrauen in den Staat und die Unabhängigkeit der Medien in einer Krisensituation wie der gegenwärtigen verliert. Die entsprechenden Aussagen von Norbert Zarbl unterstreichen in diesem Kontext, wie stark sich einige der jungen Erwachsenen inzwischen von ihren Eltern gelöst haben und eigenständige Meinungen vertreten; auch dann, wenn sie noch weiter zu Hause wohnen. Er teilt die Ansichten seiner Mutter nicht, und auch sein Informationsverhalten unterscheidet sich deutlich von ihrem. Insgesamt hat er viele Informationen aus traditionellen Medien rezipiert: Dazu zählen insbesondere die Bundeslandzeitung in gedruckter Form, die Smartphone-Apps der Bundeslandzeitung und des ORF und „hauptsächlich" auch das Fernsehprogramm des ORF. Als Grund dafür nannte er ausdrücklich die Glaubwürdigkeit des öffentlich-rechtlichen Rundfunks. Ergänzend hört er Radionachrichten auf dem Weg zur Arbeit. Angebote Sozialer Medien spielten für seine Information dagegen ganz bewusst keine Rolle. Zudem fühlte Norbert sich auch über seinen Stiefvater gut informiert, der als Unternehmer persönlich betroffen war und sich schnell mit den sich verändernden Begebenheiten arrangieren musste.

\subsection{Fazit zum Blick der Befragten auf gesellschaftliche Krisen}

Die Flucht- und Migrationsbewegungen der Jahre 2015 und 2016 sowie die seit Anfang 2020 andauernde globale Covid-19-Pandemie sind einschneidende Ausnahmesituationen, die im gesamtgesellschaftlichen Dis- 
kurs verhandelt wurden und werden. Mit Blick auf beide Krisen zeigt sich die überragende Bedeutung sowohl der medialen Berichterstattung als auch des persönlichen kommunikativen Austauschs über medial vermittelte Angebote. Gerade vor dem Hintergrund sozialer Benachteiligung gewinnen solche Krisen an Bedeutung, erlauben sie doch, wie durch ein Brennglas betrachtet, den Blick auf mögliche Verschiebungen im sozialen Gefüge der Gesellschaft und den Kitt, der sie zusammenhält. Wie nehmen gerade besonders vulnerable Gruppen tatsächliche Bedrohungsszenarien oder als solche empfundene Situationen wahr? Bedingt durch die unterschiedlichen Erhebungszeitpunkte und die jeweiligen Besonderheiten der beiden hier untersuchten Krisen führen die Auswertungen zu unterschiedlichen Erkenntnissen.

Anhand der Diskussion um Migration und Flucht wird deutlich, wie unsicher sich die Befragten ob ihrer eigenen Stellung in der Gesellschaft sind und wie sehr sie den Zuzug von Hilfesuchenden als Bedrohung und Gefahr für ihren eigenen Besitzstand wahrnehmen. Gerade auch die Rolle der Medien und deren Berichterstattung werden kritisiert. Lediglich die >Aufsteiger der Typ 4 zugeordneten Familien betrachten die Situation differenzierter und zeigen Verständnis und zum Teil auch Engagement für die Geflüchteten. Dieser Befund wiegt umso schwerer, da „Ausländer“, also Menschen mit Migrationshintergrund, auch bereits in früheren Erhebungswellen oft sehr negativ betrachtet wurden und deren Anwesenheit als Malus der eigenen Wohnumgebung wahrgenommen wurde. Im Gegensatz dazu wurden die direkten Auswirkungen der Covid-19-Pandemie durch die Befragten zwar bereits im Frühjahr und Frühsommer 2020 wahrgenommen und diskutiert, jedoch noch nicht in ausgeprägter Weise auf die eigene Lebenssituation bezogen und als Bedrohung eingeschätzt. Dieser Auswertungsteil rückt einen anderen Aspekt in den Fokus: die Bedeutung der vielfältigen Medienkanäle und -angebote in der Gesellschaft für die Information der Bevölkerung und den kommunikativen Austausch zur Verhandlung von Themen. Dies wird von nahezu allen befragten jungen Erwachsenen und ihren Eltern betont. Deutlich stärker als in früheren Erhebungswellen und in anderen Kontexten wird nun davon berichtet, wie stark und welche Informationsangebote in Anspruch genommen werden. In diesem Zusammenhang wird auch deutlich, wie heterogen die Ansichten im Panel sind und wie gering zum Teil das Vertrauen in die Medien und auch die Politik ausgeprägt ist. Während vielfach die Rolle und Qualität öffentlich-rechtlicher Angebote hervorgehoben wird, finden sich auch zahlreiche Stimmen, die dort, aber auch bei den privaten Qualitätsmedien, gleichgeschalteten „Mainstream“ vermuten und sich abwenden. 
Als vermeintlich unabhängige Alternative werden verschiedentlich Soziale Medien und namentlich Facebook erwähnt. Die Auswertung wirft dabei auch ein Schlaglicht darauf, wie weit Verschwörungserzählungen bereits in die Gesellschaft vorgedrungen sind und dass sich auch sozial etwas Bessergestellte diesen zuwenden - möglicherweise Ausdruck der Sorge, die mühsam errungene sozio-ökonomische Stabilität wieder zu verlieren. Die Erkenntnisse beider Auswertungen unterstreichen somit das Anliegen der Gesamtstudie als engagierte Sozialforschung: Es zeigt sich, wie wichtig es ist, dass sich Politik und Zivilgesellschaft stärker als bisher den Sorgen und Bedürfnissen aller Bevölkerungsgruppen, insbesondere sozial Benachteiligter zuwenden, diese ernst nehmen und konstruktive Lösungsvorschläge zur Verbesserung von Lebensbedingungen entwickeln. 


\section{Qualitative Längsschnittforschung: Vielfältige Herausforderungen auf unterschiedlichen Ebenen}

Kommunikationswissenschaftlich ausgerichtete Forschung ist allzu oft $\mathrm{Ta}$ gesgeschäft. Um den Forschungsaufwand aus arbeitstechnischen, sprich auch aus Kostengründen, möglichst gering zu halten, aber auch aus publikationsstrategischen Gründen - Aktualität wird hoch bewertet werden insbesondere Querschnittuntersuchungen durchgeführt. Erste Einschätzungen und Trends lassen sich damit in jedem Fall formulieren. Medienhandeln jedoch ist tief in den Alltag von Menschen und damit in die ich prägenden lebensweltlichen und gesellschaftlichen Veränderungsprozesse integriert, eine Querschnitterhebung greift allein aus diesen Gründen bereits zu kurz. Prozesse der Habitualisierung, lebensweltlich geprägte Umgangsweisen mit medialen Angeboten lassen sich auf diese Weise kaum identifizieren; denn langfristige Kommunikationsprozesse verlaufen individuell sehr unterschiedlich und verändern sich mit der Zeit teilweise grundlegend in ihrer Struktur und Funktion. Subjektive Wahrnehmungsund Verarbeitungsprozesse und somit Bedeutungszuschreibungen nachzeichnen zu wollen, setzt daher eine methodisch anspruchsvoll angelegte Langzeitforschung voraus. Diese ist zudem notwendig, um den fortschreitenden Mediatisierungsprozessen nicht hinterher zu hinken; denn niemals zuvor waren Medien unterschiedlicher Art so tief in den Alltag von Menschen integriert und unterliegen in ihren Angebotsweisen einem derartig schnellen Wandel, wie dies mittlerweile der Fall ist. Dennoch spielen Längsschnittstudien, obwohl sie besonders aussagekrättig sind, in der Mediengebrauchsforschung bisher keine ihnen gebührende Rolle. Komplexe Prozesse der Sinnorientierung, Individualität, Situativität und ökologische Kontextualität der Medien-Interaktion im Zeitverlauf erfassen und analysieren zu wollen, ${ }^{35}$ erfordert zudem oft einen qualitativen Zugang und damit den unmittelbaren Kontakt zu der Klientel, die im Zentrum des Forschungsanliegens steht. Wenn dies Kinder sind, ergeben sich noch weitere Herausforderungen, wenn nicht Erschwernisse. Heranwachsende befinden

35 Siehe dazu auch die fünf von Stephan Niemand (2020) generierten handlungsleitenden Prinzipien: Ganzheitlichkeit, Kontextorientierung, Prozessorientierung, Offenheit der Entwicklungsrichtung sowie Nutzerzentrierung zur Untersuchung von lebenswelt-, speziell lebensführungsbezogenen Handlungen von Individuen. 
sich noch in der Entwicklung; ihr Medienhandeln zu verstehen, fordert erwachsene Forscher und Forscherinnen in besonderer Weise heraus. Qualitative Forschung muss, um lebensnahe Ergebnisse zu erhalten, in die Lebenswelt der Untersuchten hineingehen, um nachvollziehen zu können, wie Menschen in ihrem Alltag leben und wie sie ihren Alltag gestalten, welcher Praktiken sie sich bedienen, um ihr Leben sinnvoll zu leben. Lebensnähe in der Forschung heißt auch, und dies insbesondere in der Forschungsarbeit mit Kindern, dass sich erwachsene Sozialwissenschaftler und Sozialwissenschaftlerinnen stets ihre Doppelnatur vergegenwärtigen müssen. Sie sind herausgefordert, sich in der Erhebungsphase dem Standpunkt ihrer Klientel anzunähern, aber dennoch die nötige Distanz zu wahren, die geboten ist - denn Eingreifen in das Leben der Beforschten ist zum einen aus ethischen Gründen nicht erlaubt, zum anderen würde dies einen möglichst unvoreingenommenen Blick auf den Forschungsprozess und das daraus hervorgehende Forschungsmaterial vermutlich stark behindern. Dies bedeutet auch, sich stets kritisch mit den Aspekten der sozialen Erwünschtheit auseinanderzusetzsen und dieser durch eine geeignete Methodenkombination entgegenzuwirken. ${ }^{36}$ Ebenso notwendig ist es, sich möglicher so genannter Paneleffekte bewusst zu sein. Im folgenden Kapitel werden die Konsequenzen einer qualitativen Panel-Langzeitforschung aus zwei unterschiedlichen Perspektiven reflektiert, zum einen aus dem Blickwinkel der Forschenden, zum anderen aus der Erfahrung der Beforschten selbst, der nunmehr jungen Erwachsenen und ihrer Eltern aus der über 15 Jahre durchgeführten (Medien-)Sozialisationsstudie.

\subsection{Jahre Panel-Langzeitstudie: Herausforderungen für die Forschung}

Erhebung und Auswertung qualitativer Langzeitdaten stellen Forscher und Forscherinnen vor eine Reihe großer Herausforderungen (siehe zu den folgenden Ausführungen Paus-Hasebrink 2017b sowie Paus-Hasebrink/ Kulterer/ Sinner 2019). Um qualitative Daten eines, wie im vorliegenden Fall über 15 Jahre beforschten, Panels möglichst transparent und intersubjektiv nachvollziehbar auszuwerten, war ein triangulativ ineinandergreifendes, sich gegenseitig validierendes und über die Jahre sensibel

36 So war es vor allem, als die Kinder noch sehr jung waren, wichtig, sowohl Eltern- als auch Kinder-Interviews zu führen, da Eltern hin und wieder dazu neigten, Strategien ihrer (Medien-)Erziehung im Sinne sozialer Erwünschtheit zu beschönigen. 
weiter auszubauendes Methodendesign von Nöten. Damit allein ist es jedoch nicht getan. Über die Jahre stellte sich für das Forschungsteam eine Fülle von Herausforderungen: So war das Team infolge der als Langzeitforschung angelegten Studie mit einer Vielzahl interner und externer Probleme konfrontiert. Allein die Rekrutierung und die Panelpflege war mit einem nicht zu unterschätzenden Aufwand verbunden: Es war äußerst mühsam, die Kinder, Jugendlichen und zum Schluss die jungen Erwachsenen und ihre Eltern in der Regel nach etwa zwei Jahren (zwischen der telefonischen Nachbefragung der sechsten und der siebten Erhebungswelle waren etwas mehr als drei Jahre vergangen) erneut zu erreichen und zu einem Interview zu bewegen - Telefonnummern und E-Mail-Adressen änderten sich, Briefe wurden (gerade in der untersuchten Klientel) häufig nicht beachtet. Oft waren daher auch persönliche Besuche zur Anbahnung einer erneuten Terminvereinbarung nötig - ein erfolgreiches Vorgehen, das jedoch mit nicht unerheblichen Kosten und zeitlichem Aufwand verbunden war. Zahlreiche Umzüge und tiefgreifende Veränderungen in den Familienkonstellationen erschwerten die Situation zusätzlich.

Darüber hinaus ergaben sich Herausforderungen auf Seiten des Forschungsteams selbst: Die Länge des Projektverlaufs ging einher mit zahlreichen personellen Wechseln - sei es aufgrund der Befristung von Qualifikationsstellen, persönlichen Veränderungen oder durch den Abschluss des Studiums bei studentischen Hilfskräften. Infolge dessen änderten sich die subjektiven Perspektiven und die konkreten Arbeitsweisen der Beteiligten. All dies hatte vor allem Konsequenzen für die intersubjektive Nachvollziehbarkeit der Auswertung der über die Jahre erheblichen Datenmengen aus unterschiedlichen Datenquellen und für ihre Vergleichbarkeit, das für eine Langzeitforschung zentrale Gütekriterium! Insofern bedurfte es großer Anstrengungen, dem Kriterium der intersubjektiven Nachvollziehbarkeit in der empirischen Sozialforschung gerecht zu werden.

In der (Medien-)Sozialisationsstudie dienten dazu vielfältige Maßnahmen: Durch eine gewisse Standardisierung auch in der qualitativen Forschung wurde versucht, ein möglichst hohes Maß an Vergleichbarkeit zu ermöglichen. So wurden etwa Codewortbäume nur sehr behutsam verändert und thematisch strukturierte Matrizen mit fest vorgegebenen Kategorien verwendet. Gerade die sorgfältige und mehrfach gesicherte Dokumentation des Vorgehens erwies sich als unausweichlich. Sämtliche Vorgaben wie Transkriptions- und Anonymisierungsregeln und Beschreibungen der Codes wurden exakt festgehalten und jeweils aktualisiert, so dass auch nach einigen Jahren das Vorgehen noch nachvollziehbar war. Ein zentrales Instrument der Vermittlung dieser Grundlagen waren Interview- und 
Codier-Schulungen durch gut in die Materie eingearbeitete Personen, die neben Fakten auch Einblicke in die Atmosphäre der jeweiligen Familien vermitteln konnten. Diese Schulungen mussten bei jeder Welle wiederholt werden und umfassten alle Ebenen des Forschungsprozesses (Paus-Hasebrink et al. 2018). Hilfreich waren außerdem regelmäßige und detaillierte Zwischenberichte und Zusammenfassungen, wie sie u.a. an den Förderer der Studie, den Jubiläumsfonds der Oesterreichischen Nationalbank geschickt wurden; sie sorgten für Kontinuität und bildeten die Basis für die jeweils folgende Untersuchungswelle.

Wer einen so tiefgehenden Zugang zur Lebenswelt von Menschen erhält, wie dies im Falle dieser Studie über etwa 15 Jahre der Fall war, sieht sich in besonderer Weise mit ethischen Fragen und Problemen konfrontiert. Wie sind Menschen anzusprechen, zu Beginn der Studie und während der unterschiedlichen Erhebungsphasen? Wie geht man mit Fragen von Kindern, Jugendlichen und Eltern um, die die Forschenden nicht nur als befragende Personen wahrnehmen, sondern diese gleichermaßen auch als Experten bzw. Expertinnen und potenzielle Ratgeber betrachten? Wie ist mit offenkundig werdenden Problemen oder Verwerfungen in einer Familie umzugehen, wie etwa schweren psychischen oder psychosomatischen Erkrankungen oder gar Missbrauchserfahrungen oder dem Verdacht, dass Missbrauch vorliegt? Wann müssen ein Forscher oder eine Forscherin ihre Rolle als zwar teilnehmende, aber doch zur Distanz verpflichtete Person, die nicht unmittelbar in lebensweltliche Kontexte eingreifen sollte, verlassen und aus Gründen der Fürsorge invasiv aktiv werden?

Entsprechende Konfliktsituationen traten im Verlauf der Studie mehrfach auf; in diesen Situationen galt es jeweils, im Team sorgfältig, sensibel und verantwortungsvoll abzuwägen, welche gegebenenfalls mit Eingriffen in das familiäre System verbundenen Maßnahmen ergriffen werden sollten. Dabei ist es unter Umständen erforderlich, vertrauenswürdige Personen aus dem Umfeld der Betroffenen oder auch versierte Fachleute hinzuzuziehen. Besonders gravierende Herausforderungen ergaben sich in der sechsten Erhebungswelle 2016 bei den Familien Fein und Öllinger (siehe dazu die Fallbeschreibungen in Kapitel 2). In beiden Fällen kontaktierte die Leiterin der Studie die Universitätspsychologin und erarbeitete mit ihr Strategien, wie den Familien am ehesten geholfen werden kann, ohne 
einen direkten Eingriff durch das Forschungsteam in das familiäre System vorzunehmen. ${ }^{37}$

\subsection{Jahre Panel-Langzeitstudie: Was sagen die Panel-Angehörigen selbst dazu?}

Eine Längsschnittstudie über 15 Jahre fortzuführen, bringt nicht nur zahlreiche Herausforderungen für das Forschungsteam mit sich. ${ }^{38}$ Auch - und dies nicht zuletzt - sind die Menschen gefordert, die im Mittelpunkt des Forschungsprozesses stehen, die Kinder, später die Jugendlichen und jungen Erwachsenen und ihre Eltern, denn um sie ging es in den 15 Jahren in der im Sinne von Norbert Elias (1987) als engagierte Sozialforschung betriebenen (Medien-)Sozialisationsstudie (siehe dazu ausführlicher Paus-Hasebrink/ Bichler 2008 sowie Paus-Hasebrink/ Kulterer 2014). Kernziel war es, die Aufmerksamkeit auf die Belange von sozial benachteiligten Heranwachsenden zu lenken, aber darüber hinaus auch zu erforschen, wie sich Sozialisation als Prozess vollzieht und welche Aspekte dabei in welcher Weise im Kontext der Sozialisation der Heranwachsenden und

37 Siehe dazu auch die beiden Best Practice Guides des Forschungsnetzwerks EU Kids Online (Lobe et al. 2008, darin speziell die Schilderungen von Paus-Hasebrink (S. 57) zum Umgang mit ethischen Konfliktsituationen, sowie Ólafsson/ Livingstone/ Haddon 2013).

38 Siehe dazu die Langzeitstudie von Annette Lareau (2011), insbesondere die Kapitel 14 (Reflections on longitudinal ethnography and the families reaction on „Unequal Childhoods“). Darin diskutiert die Autorin, wie die Angehörigen ihrer Langzeitstudie zehn Jahre später im Rahmen einer qualitativen Befragung auf die damalige Erhebung reagiert haben und wie sie nunmehr die Studie einschätzen. Annette Lareau konfrontierte die Familien, die sie 2001 und 2002 besucht hatte, auch mit den Ergebnissen ihrer Studie und bat sie um Stellungnahmen dazu. Lareau hatte eine quantitative Befragung bei „Middle-Class“-Familien und „Working-Class"-Familien zu ihren Lebensverhältnissen durchgeführt. Mit ihrer Studie ging es der Autorin darum zu erforschen, welche Auswirkungen ihre Zugehörigkeit zur „Middle-Class“ oder zur „Working-Class“ für das Aufwachsen der Kinder hat. Lareau ging davon aus, dass unabhängig von der Rasse (sie untersuchte weiße Amerikaner und afrikanisch-stämmige Amerikaner) der sozioökonomische Status bestimmt, wie Kinder in der Lage waren, ihre Fähigkeiten zu entwickeln und ihre Zukunft zu gestalten. Lareau kam zehn Jahre später nach ihrer erneuten Befragung der Familien zu dem Schluss, dass der "Social Class" in Bezug auf die Eheschließung, die berufliche Stellung und den Wohnort eine große Bedeutung zugekommen sei. „Social Class" habe dabei besonders Einfluss auf das Leben der Kinder genommen und weniger auf das Leben als Familie. 
später der jungen Erwachsenen interagieren. Um dies leisten zu können, mussten die Angehörigen des Panels nicht nur bereit sein, über sich selbst und ihre Lebensführung Auskunft zu geben, sondern auch bereit sein, dies immer wieder in bestimmten zeitlichen Abständen zu tun und dazu auch zu erlauben, dass die Mitglieder des Forschungsteams sie in ihren unmittelbaren privaten Räumen, ihren Wohnungen besuchen und, in Bezug auf die Kinder und Jugendlichen, ihre jeweiligen eigenen Zimmer betreten.

Was hat dies im Rückblick für die jungen Erwachsenen und ihre Eltern bedeutet? Was haben sie selbst zum Ablauf der Studie zu sagen, welche Kritik und welches Lob äußern sie, aber auch welche Hinweise geben sie dem Forschungsteam zur Verbesserung ihres Vorgehens, und interessieren sie sich und, wenn ja, in welcher Weise für die Ergebnisse der Langzeitstudie?

Diese Fragen bildeten den Abschluss des Leitfadenkatalogs in der siebten Erhebungswelle. Zum Ende eines jeden Interviews baten die Interviewer und Interviewerinnen die jungen Männer und jungen Frauen sowie ihre Eltern - in der letzten Befragung waren dies ausschließlich Mütter - um Antworten.

Zur Auswertung der Antworten, die wie alle anderen auch mit Hilfe von MAXQDA codiert wurden, diente ein Kriterienkatalog, der sich zum einen deduktiv aus den Leitfragen und zum anderen - dies war der bei weitem bedeutendere Anteil - induktiv aus der ersten Durchsicht der Antworten und den daraus gefilterten, über die Leitfragen hinausgehenden Aspekten der Befragten speiste. ${ }^{39}$

39 Der Kriterienkatalog zur Analyse der Meta-Fragen zur Langzeitstudie enthält folgende Aspekte: Bewertung des Projekts; Angebot zur Selbstreflexion (in Bezug auf Medienangebote, eigenen Mediengebrauch sowie den der Kinder; Möglichkeit zur Reflexion der jeweils eigenen Entwicklung (Kinder/Eltern); vice versa zur Entwicklung des Kindes bzw. der Eltern; Wahrnehmung des Forschungsteams; Wahrnehmung der Mitglieder des Forschungsteams als Gesprächspartner/innen; wahrgenommenes Gefühl, ernst genommen worden zu sein; Wahrnehmung, dass eigene Belange relevant waren; Verbesserungsvorschläge zur Studie; Interesse an den Ergebnissen der Studie allgemein; Interesse persönlich an den Ergebnissen (z.B. was man als Teilnehmer bzw. Teilnehmerin selbst vor Jahren gesagt hat); Bereitschaft zur erneuten Teilnahme im Rückblick. 
5.2.1 „Nie jemand für eigene Wünsche interessiert“: Elternaussagen zur Langzeitstudie

Auffällig ist, dass sich alle in der siebten Erhebungswelle zum Schluss des Interviews nach ihren Wahrnehmungen und Einschätzungen der Langzeitstudie befragten Mütter (nur ein Vater äußerte sich im Hintergrund des Telefongesprächs mit der Mutter) ( $n=17)$ positiv zur Studie äußerten. Sie beurteilten diese entweder als „,interessant“, „Neuland“, „spannend“ (etwa Frau Kaiser: „Jedes Mal spannend“; Frau Rohringer: „Jo, wor a coole Erfahrung"), ,angenehm“, „sehr positiv“ und geeignet, selbst etwas zu lernen. So betonte etwa Frau Öllinger, sie habe „de Studie sehr guad g'funden“; sie sei „sehr interessant" gewesen, „weil, es waren oft Fragen dabei, de wos / ja, es is ${ }^{6}$ ned nur um Medien ganga, es is ${ }^{6}$ a um private Sachen g'angen". Sie meinte, sie empfehle jedem und jeder, an einem solchen Projekt teilzunehmen, „da man ja nie auslernt“.

Die weitaus meisten Mütter $(n=16)$ bekundeten auf die Frage, ob sie sich auch jetzt nach 15 Jahren Langzeitstudie noch vorstellen könnten, erneut teilzunehmen, dass sie sich sehr gern nochmals für die Teilnahme entscheiden würden. Nur eine Mutter (Frau Aufbauer) war sich nicht ganz sicher, ob sie dafür nochmals Zeit aufbringen würde. „Oiso mi haut's scho ziemlich umadum mit meiner Orbeit und mit oillm. Oiso, woaß i net, ob i da jetzt sofort wieder sagat, jawohl da bin i dabei. Aber vielleicht scho". Frau Hirtner würde ebenfalls wieder teilnehmen, wenn sie auch, wie sie sich erinnert, den ersten Besuch als etwas „komisch“ empfunden habe, da sie nicht so recht gewusst habe, was sie sagen sollte oder was sie vielleicht auch eher nicht sagen sollte. „Danach war es aber okay und hat immer gepasst“.

Alle befragten Mütter waren auch mit dem Verlauf der Teambesuche bzw. dem Verlauf der Interviews einverstanden; Kritik geäußert wurde kaum. Lediglich eine Mutter (Frau Aufbauer) machte, dies allerdings mit einem halb-ironischen Unterton, die Anregung, „mehr zahl'n hätt's kinna. (lacht) Mehr Geld, Geld ist alles“40. Dagegen betonte Frau Scheib, dass die im Rahmen der Studie jeweils für die Teilnahme an einer Untersuchungswelle gezahlte Aufwandsentschädigung in keinem Fall der ausschlaggebende Punkt gewesen sei: „Des allein war's mit Sicherheit net. Klar, war's

40 Mit dieser Äußerung nahm Frau Aufbauer Bezug auf die Aufwandsentschädigung in Höhe von 50 Euro bei Besuchen und in Höhe von 20 Euro bei der telefonischen Befragung, die den Eltern und in der siebten Erhebungswelle auch den jungen Erwachsenen für ihre Teilnahme an der Studie ausgezahlt wurde. 
nett a bissl a Taschengeld zusätzlich, aber des war sicherlich net der Beweggrund.“

Zur Frage, wie sie das Forschungsteam wahrgenommen haben, äußerten die Mütter, sie hätten das Team stets als „total nett“, „höflich“, „respektvoll“ und „total vertrauenswürdig“ empfunden. Frau Weiss erzählte, sie habe aber zuweilen dennoch ein unangenehmes Gefühl gehabt, da man „fremden Menschen sehr private Dinge“ erzähle. Sie bemerkte jedoch, es auch als ,ihre Pflicht“ empfunden zu haben, dennoch die „Wohrheit zu sag'n“ und „ned auch irgendwas zu erzählen“. „Aber“, so erinnerte sie sich, „momentan denkt ma sich halt, man erzählt dann schon sehr viel“.

Für viele Mütter bot die Langzeitstudie die Möglichkeit zur Reflexion über vergangene Lebensphasen, entweder über eigene Veränderungen in der eigenen Lebensführung, zur Auseinandersetzung mit eigenen Wünschen und Zielen allgemein, aber auch ganz explizit im Umgang mit Medien: „Dass ma des dann numoi reflektiert und, ahjo, genau des hot si scho massiv verändert oder so irgendwie, sog i jetzt amoi. Weil ma genauer des reflektiert und nach, bei sich selber nachhakt" (Frau Dornbacher). Aber die Studie habe auch die Chance geboten, die Entwicklung ihrer Kinder Revue passieren zu lassen. So meinte etwa Frau Öllinger, dass sich ihre Tochter verändert habe, ,also es is von kloa auf jugendlich gangen, auf Erwachsene nachhan, ja, ma sieht sicher vui bei eich, wos sich g'ändert hod alloa scho". Einige Mütter bekundeten, die Studie habe ihnen die Möglichkeit geboten, eigene Erinnerungen aufzufrischen; so fand Frau Zarbl die Interviews „zum Teil auch lustig, weil man sich so daran erinnern konnte, wie alles angefangen hat, als die Kinder noch klein waren“. Frau Kaiser meinte, die Fragen, die Reflexion und der Blick von außen seien spannend gewesen; sie fügte hinzu, dass außer den Mitgliedern des Forschungsteams sie „einfach noch nie jemand gefragt (habe), was ich denn persönlich für Wünsche hab!“ Noch leiser als zuvor schob sie im Interview nach „für mich!“

Die letzten Fragen des Interviews beinhalteten die Bitte, Aspekte zu nennen, was das Forschungsteam in Bezug auf den Ablauf der Studie, bei den jeweiligen Besuchen in den Familien und Interviews hätte besser machen sollen und was nicht gut verlaufen sei. Die weitaus meisten Befragten $(\mathrm{n}=13)$ antworteten, dass sie keine Verbesserungsvorschläge hätten, da alles sehr gut gelaufen sei und das Team gewusst habe, was es tue. Lediglich drei Mütter äußerten Anregungen bzw. Kritik; diese bezog sich zum einen auf den Umgang mit Daten und zum anderen auf die Kenntnisnahme der Studienergebnisse. So hätte es etwa Frau Scheib gut gefunden, etwas mehr über die Art der Auswertung zu erfahren: „Wos natürlich fehlt, aber des 
wird wahrscheinlich auch a Zeitfrage sein, weiß net äh, also von der Transparenz her. ${ }^{41}$ Man wünscht sich halt schon irgendwo, wie jetzt des genau ausgewertet wurde oder dass ma selber amal für sich auch irgendwelche Daten hat, wo ma dann sagn kann: Schau, so war's vor acht Jahr'n. Ja (lacht). Aber des is wahrscheinlich irgendwie schwer, des da wieder rauszuzieh'n. Weil diese Studie, die, was ihr da habt, da sind ja wahrscheinlich ganz viele Leute involviert." Frau Weiss meinte, sie hätte gerne schon früher genauer erfahren, wie strikt mit den Daten umgegangen werde. Dass es „anonym“ sei, sei ihr zwar gesagt worden, aber „das hab“ ich gar ned so gewusst, dass Sie da ganz andere Namen hernehmen." Dies sei ihr erst später durch einen Interviewer so verständlich erklärt worden, aber vielleicht habe sie „da aber auch mal etwas überhört“. ${ }^{42}$ Frau Grubert und Frau Zarbl hätten es gern gesehen, wenn das Team die Ergebnisse ihnen gegenüber kommuniziert hätte, allerdings betonte sie auch, selbst nie nach Informationen gesucht zu haben. Frau Zarbl bedauerte es jedoch im Nachhinein, selbst nicht intensiveres Interesse an den Ergebnissen geäußert zu haben.

Unklar war zwei Müttern, weshalb das Forschungsteam überhaupt eine Studie dieser Art durchgeführt hat; so meinte Frau Stab, die Besuche seien immer "ganz nett" gewesen, sie habe sie als „freundlich" und „pünktlich" empfunden, sie wisse „aber nicht, was das (die Studie, Anm. die Verf.) für euch (das Forschungsteam, Anm. die Verf.) bringt." Frau Weiss erzählte, sie habe sich darüber gewundert, dass sie für die Studie befragt worden sei, weil Medien „in ihrem Leben ja keine so große Rolle spielen, wie dies bei anderen Menschen der Fall ist“.

\subsection{2 „Es war vor allem auch mal was anderes“: Wie blicken die jungen} Erwachsenen auf ihre Teilnahme an der Studie zurück?

Mit Ausnahme von Olivia Fein konnten alle 17 jungen Erwachsenen erreicht und für eine Teilnahme an der Studie gewonnen werden. Drei der jungen Männer (Gregor Boll, Manfred Oblinger, Helmut Pfortner)

41 Auf Nachfrage hat das Team den Beteiligten Daten zur eigenen Familie zur Verfügung gestellt. In der siebten Erhebungswelle kam das Erhebungsteam selbst auf die Ergebnisse der Studie zu sprechen und gab den Interessierten Hinweise auf Publikationen (Link zur Projektseite auf der Homepage der Universität sowie Angaben und Link zur aktuellsten Open Access-Publikation der Studie).

42 Die Interviewten wurden zu Beginn jedes Interviews bzw. zu Beginn jedes Besuches darüber informiert, dass sämtliche Angaben absolut vertrauenswürdig und anonym behandelt werden. 
waren über die erneute, und für sie unerwartete, Kontaktaufnahme durch das Forschungsteam so erfreut, dass sie umgehend ihre Mütter über die erhaltene Interviewanfrage informierten. Auch Alfons Weiss, der zwischen der sechsten und siebten Erhebungswelle auf Grund eines gravierenden Konflikts mit seiner Mutter mehrere Monate auf der Straße gelebt hatte, ließ sich von einer Teilnahme überzeugen. Um ihn zu erreichen und befragen zu können, war es jedoch erforderlich, zunächst seine neuen Kontaktdaten über Frau Weiss zu beziehen und von seinem gerichtlich bestellten Sachwalter die Zustimmung für das Interview einzuholen. Obwohl Alfons eigentlich nur für „maximal fünf Minuten“ zur Verfügung stehen wollte, dauerte das Gespräch schließlich doch knapp 20 Minuten und bot substanzielle Erkenntnisse. Dabei machte er sehr deutlich, dass ihm die Interviews immer gut gefallen hätten und er sich sogar zwischen den Erhebungswellen einen noch engeren Kontakt mit dem Interviewer ${ }^{43}$ gewünscht hätte: „Ja, so ab und zu was Aktuelles reden. So ja.“ Ein zu enger Kontakt oder gar die Übernahme der Rolle eines Freundes oder einer Freundin, wie von manchen Heranwachsenden gewünscht, verbot sich, wie eingangs thematisiert; gerade in einer qualitativen Panel-Langzeitstudie mit mehreren Erhebungswellen war es nötig, stets professionelle Distanz zu wahren. Mit seinem Wunsch nach einem engeren Kontakt war Alfons Weiss denn auch nicht allein. Einige weitere Befragte, in erster Linie junge Männer - ihnen schien es an Ansprechpartnern zu fehlen -, betrachteten die Möglichkeit, etwas von sich zu erzählen, als sehr positiv und begründeten dies auf vielfältige Weise. ${ }^{44}$ Torsten Kaiser freute sich, dass sich für seine Ansichten „auch Leit interessieren“ und schätzte es immer sehr, „wenn ich mal mit wem drüber reden ko“. Manfred Oblinger betonte, dass ihm die Gespräche gerade in der Pubertät wichtig gewesen

43 Wie bereits in allen vorherigen Erhebungswellen wurde auch dieses Mal darauf geachtet, dass ein männliches Teammitglied mit den Jungen und später jungen Männern sowie ein weibliches Teammitglied mit den Mädchen und jungen Frauen sprach.

44 Bei den folgenden Aussagen ist zu beachten, dass vor allem die jungen Erwachsenen, bei denen es sich im Sinne der Forschungsfragen um besonders aussagekräftige Fallbeispiele handelte, über viele Jahre hinweg stets von den gleichen Interviewern besucht und befragt wurden. Dies war jedoch nicht bei allen Familien und nicht über den gesamten Erhebungszeitraum möglich. Manche Familien mussten, z.B. aus organisatorischen Gründen (etwa Personalwechsel und geschlechtsspezifische Zuordnung), von zwar intensiv geschulten, aber wechselnden Interviewenden befragt werden. 
seien. So habe er damals den Interviewer ${ }^{45}$ „von der erst' $n$ Sekunden an als voll als den lässigen, coolen Typen erkannt“. Dieser habe ihn mit seinen Fragen „herausgefordert“, zum Nachdenken angeregt und sich in einer Weise für ihn als Person interessiert, wie er es zuvor außerhalb der Familie nicht kannte. Auch Helmut Pfortner schätzte die Gespräche mit dem Interviewer und hob hervor, dass es für ihn Bedeutung hatte, von einem Außenstehenden befragt zu werden, den er mit der Zeit kannte, alles andere wäre „komisch“ gewesen. Helmut schien es ebenfalls angenehm empfunden zu haben, dass er seit der vierten Erhebungswelle stets vom selben Interviewer befragt worden ist: „Also, wenn das irgendeiner gewesen wäre, dann wär's vielleicht nicht so cool g'wen." Aber auch ein Mädchen, Elisabeth Ebner, führt aus, wieso die langjährigen Erfahrungen mit dem Team so besonders für sie waren:

Des woar hoit voi cool für mi. Dann hob i des amoi erst verstand, warum ihr überhaupt kemmt's und wos ihr mocht's und so. Und jo, ihr woart's immer total nett und jo. I find des cool, dass des einfoch g'mocht wird a. Dass des so aufgschrieb'n wird, und i bin froh, dass i mitmoch'n hob kenna. Es woar immer nett. I hob mi nie unwohl g'fühlt, es woar, woaß i net, wie i s jetzt $\operatorname{sog}^{\text {'‘ }}$ soi. I bin total z'fried'n g'wesn, weil's einfoch a scheene Erfahrung woar.

Neben den beiden genannten jungen Männern, Manfred Oblinger und Helmut Pfortner, beschrieben auch vier weitere (Torsten Kaiser, Erich Grubert, Norbert Zarbl, Erich Hirtner) die Interviews als „cool“, „nett“, „immer nett“, „eigentlich immer ganz nett“ (lachend) und „sehr nett“. Mario Hirtner räumte jedoch ein, dass er es auch als „sehr, sehr komisch irgendwie“ empfunden habe, „so viel Privates eben preiszugeben“ - dennoch konnte er sich eine erneute Teilnahme vorstellen. Timo Landinger

45 Es ist anzumerken, dass Manfred Oblinger zwar von der vierten bis zur siebten Erhebungswelle (2012 bis 2020) immer von demselben und hier adressierten Interviewer befragt wurde, in den ersten drei Erhebungswellen (2005 bis 2010) hatte ihn aber ein anderer Mitarbeiter aus dem Forschungsteam besucht. Diesen Wechsel schien er rückblickend vergessen zu haben, wobei zu bedenken ist, dass er zu Beginn der Studie erst knapp sechs Jahre alt war. Auch andere der befragten Heranwachsenden räumten ein, sich an die ersten Jahre der Studie nur eingeschränkt erinnern zu können: „Ma, i muaß ehrlich sagn, i hab die Interviews immer wieder und wieder vergessen (lacht). Dass moi wer da war und gfragt hat.“ (Simone Stab) „Oiso, i muss ganz ehrlich sogn, i konn mi a an ganz viel nimmer erinnern, nur von de Erzählungen her.“ (Isabelle Rohringer) „Jo, oiso domois im Kindergart'n hob i des jo net wirklich mitkriagt, wieso. Und es woar hoit einfoch, ihr woarts hoit do.“ (Elisabeth Ebner) 
bezeichnete die Interviews lediglich als „normales Gespräch halt. Besuche halt. Wie man sich das des immer so vorstellt halt." Obwohl ihn längere Gespräche anstrengten, er sich nach einer gewissen Zeit nicht mehr konzentrieren konnte und sich lieber wieder mit seinen Computerspielen beschäftigt hätte, schloss er dennoch eine erneute Teilnahme in der $\mathrm{Zu}$ kunft nicht kategorisch aus. Auf die Frage des Interviewers, ob er auch ein weiteres Mal wieder mitmachen würde, antworte er: „Ja. Also, i glaub, ja.“

Auch sechs der sieben befragten jungen Frauen bezeichneten ihre Beteiligung als „cool“. Lediglich Amelie Aufbauer verwendete diese Umschreibung nicht, aber auch für sie waren die Besuche und Interviews grundsätzlich eine positive Erfahrung: „Für mi war des jetzt net irgendwie a besonderer Aufwand und hab da nie negative Erfahrungen g'macht."

Insgesamt zeigten sich auch alle anderen befragten jungen Erwachsenen offen dafür, auch in Zukunft wieder an einer Befragung teilzunehmen. Während manche Antworten nüchtern („Klar“ ... „I würd's wieder duan“ (Helmut Pfortner)) oder auch gönnerhaft ausfielen - Torsten Kaiser etwa konnte sich die Teilnahme gut vorstellen „wenn ich Zeit habe“, da er „immer recht offen“ und „auch sehr kooperativ“ sei - so fielen andere Antworten geradezu euphorisch aus: „Oiso, i würd immer wieder mitmachen!“ (Viktoria Öllinger), „Ja, auf jeden Foil!““ (Elisabeth Ebner), „Ja klar!“ (Gudrun Dornbacher), „Falls i des noch amoi derf, bin i gern dabei ja!“ (Manfred Oblinger). Gregor Boll bot seine Bereitschaft zu weiteren Erhebungswellen gar ungefragt an. Neben Timo Landinger zeigte sich nur Alfons Weiss, der auch längere Gespräche sichtbar scheute, zunächst skeptisch. Doch auch er bekundete Bereitschaft an einer erneuten Teilnahme, falls die Interviews nicht zu lange dauern würden. Im Gegensatz zu den befragten Müttern spielte die gezahlte Aufwandsentschädigung oder deren Höhe bei den jungen Erwachsenen keine Rolle. Niemand von ihnen sprach diese an oder knüptte gar eine erneute Teilnahme daran. Überraschend positiv fiel dagegen die Rückmeldung auf die kleinen Geschenke (Büromaterial, Sticker oder Gummibären im Wert weniger Euros) aus, die die Kinder und später Jugendlichen in früheren Erhebungswellen als kleines Dankeschön erhalten haben. Mehrere der jungen Erwachsenen hoben hervor, dass sie sich darüber immer besonders gefreut hätten und die Auswahl immer als sehr passend und altersgerecht empfunden hätten; sehr deutlich ausgeprägt war dies bei Elisabeth Ebner und Manfred Oblinger. Zusammenfassend lässt sich sagen, dass sich insgesamt alle 17 befragten jungen Erwachsenen, ebenso wie ihre Mütter, positiv über die Teilnahme äußerten. 
Ein weiterer Aspekt, den die Befragten im Interview hervorhoben, war, dass es ihnen über die unterschiedlichen Erhebungswellen hinweg möglich war, über ihre eigene Entwicklung nachzudenken. Im Kindergartenalter sei es allerdings noch schwierig oder fast unmöglich gewesen, die Interviewsituation zu verstehen und einzuordnen. Dennoch wurde auch die damalige Situation mehrfach als "cool“ und "besonders" empfunden, ja geradezu „aufregend“, da „jemand Fremdes“ zu einem gekommen sei und Fragen gestellt habe. Diese Einschätzung blieb bei den meisten konstant. Aus welchen Gründen ihr die Teilnahme an der Studie wichtig war, zeigt die Äußerung von Gudrun Dornbacher:

Ah, denk i, es is a guate Abwechslung g'wes'n. Es war was Neiches, i bin sowieso immer für was Neiches offen. Mir is` ma immer mit sehr vü Respekt und Höflichkeit entgegenkummen. Es war immer nett, ma hat a moi lachen kennan und so. Oiso, des hab' i überhaupt net, hab $\mathrm{i}$ hab $\mathrm{i}$ ois positiv in Erinnerung. I hab des, glaub i, ois Kind sogar recht cool g'fund'n. Es kumman Leit zu mir, die was i net kenn, die intressier'n si für mi ähm die woll'n mi jetzt immer seg'n, die red'n mit mir über Medien und so. Des hab' i scho ganz cool g'fund'n, glaub i. So wie i mit der Mama letztens amoi g'redt hab, die hat g'sagt: Naa, da warst du immer ganz stolz, wenn da Leit da wor'n, die was du net kennt hast.

Auch andere Befragte hoben hervor, dass sie die Besuche und dabei das Auftreten der Interviewenden als angenehm empfunden haben. Eine wichtige Rolle spielte demnach, dass die Teammitglieder den Interviewten stets mit Respekt gegenüber getreten seien, und dies nicht nur als jungen Erwachsenen; bereits im frühen Kindesalter, wie Mario Hirtner erzählt, habe er sich ernstgenommen gefühlt:

Ich finde, es war immer sehr, sehr nett irgendwie. Ich finde, es war an sich sehr gut, wie ihr, also ich war ja früher mal ein Kind (lacht), wie ihr eben auf Kinder zugegangen seid, ihr wart's immer sehr nett und sehr offen und ähm, ihr habt's ned unbedingt ähm, ja ähm so, ihr habt's ned a Kind anders behandelt, als wenn ihr einen Erwachsenen fragen würdet's und das hab ich sehr gut g'funden und ihr wart's eigentlich immer sehr nett, ich hab da also eigentlich gar nichts zum Aussetzen gehabt.

Dennoch machte Mario Hirtner im Interview deutlich, dass es in der Vergangenheit zum Teil schwierig für ihn gewesen sei, über private Belange mit einer Person von außerhalb der Familie offen zu sprechen. 
Viele der Befragten thematisierten, es sei für sie ein besonderes Erlebnis gewesen, dass sich jemand außerhalb der Familie für sie interessiert habe und sie nach ihren persönlichen Belangen und Sorgen befragt hätte: „Also, eigentlich ganz cool allweil. Es war eigentlich immer ganz cool, wenn a'mal wer kummt, der wo einen ausfragt.“ (Helmut Pfortner) „Es war vor Allem auch mal was Anderes. Ich bin bei sowas eh gern dabei, weil ich mein, es hilft sicher auch für irgendwas.“ (Erich Grubert) „Wenn man Fragen gestellt kriegt, dann ist es gleich a'mal selbst interessanter, wenn man sich mal Gedanken drüber macht, würd' i sag'n." (Norbert Zarbl) „Vor allem, ich hab“ mich da so interessant gefühlt. (lacht) Hat mir gefallen.“ (Susanne Scheib) „I find's a richtig cool, mei Leben is jo net so unspannend, und dass es irgendwie aufzeichnet word' $\mathrm{n}$ is, des find i scho cool.“ (Viktoria Öllinger) „Und i find des generell so cool, wenn ma so vü Sach'n über sei Leben g'fragt wird, dann hoit vü mehr von aner, anderen Perspektive die Sachen betrachtet." (Simone Stab)

Neben der Selbstreflexion wurde aber auch der Lerneffekt der Teilnahme an solch einer Studie betont, etwa von Manfred Oblinger: „Es ist, man hat, i hab viel gelernt. (...) War auch irgendwie lehrreich für mi.“ Im jungen Erwachsenenalter zeigten die Befragten dann auch ein gesteigertes Interesse daran, einerseits mehr über die Gesamtstudie und die Ergebnisse zu erfahren und andererseits auch einen genaueren Einblick in ihre eigene Entwicklung im Studienverlauf zu bekommen. Noch in der sechsten Erhebungswelle (2016) hatten die Jugendlichen daran kein Interesse gezeigt. So gab es seinerzeit kaum Resonanz auf das Angebot, Ergebnisse zu erhalten. In der siebten Erhebungswelle sprachen einige der jungen Erwachsenen ihr Interesse an Unterlagen sogar von sich aus an (Susanne Scheib, Viktoria Öllinger, Simone Stab, Elisabeth Ebner, Mario Hirtner, Gregor Boll).

Neben den Fragen nach ihrer Wahrnehmung der Teilnahme wurden die jungen Erwachsenen auch darum gebeten, bei Bedarf Kritik zu üben und, wenn möglich, Verbesserungsvorschläge zu formulieren, damit zukünftige Studien davon profitieren könnten. Zusammenfassend lässt sich sagen, dass die jungen Erwachsenen, ebenso wie ihre Mütter, nur sehr wenig Grund zur Kritik sahen; auch Verbesserungsvorschläge wurden nur sehr selten geäußert - insgesamt habe es für alle, wie Amelie Aufbauer es formuliert, „voi passt“. Dennoch gaben fünf von ihnen ein substanzielles Feedback: Gregor Boll kamen die Interviews als Kind rückblickend sehr lang vor, und gerade in der Pubertät hätte er eigentlich andere Prioritäten gehabt:

Ja, ich sag mal so als Kind, da war es mir glaube ich relativ egal. Also da habe ich nicht besonders viel von gehalten. Als ich dann so in 
die Pubertät kam, ich glaube, da hatte ich einmal wirklich überhaupt keinen Bock auf dieses stundenlange Gespräch. Aber kann ich jetzt, ich kann jetzt wirklich nicht sagen, warum ich da keinen Bock draufhatte, ich weiß nicht. Ich glaube, da hatte ich gerade meine PlayStation gekriegt und hab' die ganze Zeit nur gezockt und hatte keine Lust auf irgendwas anderes.

Später habe er aber Freude an den Gesprächen gewonnen, und auch er betrachtete die damit verbundene Reflexionsmöglichkeit als positiv. Eine ähnliche Kritik äußerte auch Alfons Weiss. Er empfand die Interviews zwar als „absolut nicht unangenehm“, dennoch seien die Gespräche ihm in der Tendenz zu lang vorgekommen. Diese Aussage steht dabei in einem gewissen Widerspruch zu seinem Wunsch in der siebten Erhebungswelle nach noch stärkerer Einbindung und seinem großen Gesprächsbedarf in früheren Erhebungswellen. Timo Landinger verbalisierte zwar keine direkte Kritik an der Länge der Interviews, jedoch ließ auch er durchblicken, dass ihm kürzere Gespräche besser gefallen hätten. In seinem Fall war auch deutlich erkennbar, dass seine Konzentrationsfähigkeit nach einer gewissen Zeit stark nachließ.

In eine andere Richtung weisen die Kritik und der Verbesserungsvorschlag von Manfred Oblinger. Er zeigte zwar Verständnis für die Ausrichtung der Studie, gab aber zu bedenken, dass Themenkomplexe wie „Politik“, „Nachrichten“ sowie breite und vielfältige Medienrepertoires nach seinem Verständnis womöglich zu intensiv behandelt worden seien. Stattdessen hätte der Fokus stärker auf Soziale Medien gelenkt werden sollen: „Denn i glab, jetzt so die Jugendlichen bzw. Teenager, Jugendlichen bis junge Erwachsene, wo ich jetzt einifall, ähm, die ham, die ham des so gar nimmer mit Zeitung lesen oder Nachrichten schau'n. "46 Mehrere Befragte machten deutlich, dass sie gerade als Kinder nur wenig mit der Befragung hätten anfangen können. So sei anfangs der Wunsch ihrer Mütter ausschlaggebend gewesen: „Ja, mei, i find's cool, dass die Mama des überhaupt mit mir durchg'führt hot. Und der Mama wor des a immer sehr wichtig, dass ma sowos moch'n.“ (Isabelle Rohringer) „Die Mama hot hoit

46 Manfred Oblinger erwies sich damit als einer der wenigen Befragten, die im Interview Tendenzen des Wandels im Mediengebrauch Jugendlicher und junger Erwachsener thematisierten. Grundsätzlich wurden alle Teilnehmende offen nach den von ihnen genutzten und präferierten Medienangeboten und -geräten gefragt. Im Verlauf des jeweiligen Gesprächs lag der Schwerpunkt dann darauf; eine allgemeine Diskussion auch über den Mediengebrauch anderer wurde mit den Befragten dagegen nicht geführt. 
g'sogt, i soi mit eich red'n. Und am Anfong woar's so unverständlich für mi. Oba es woar net unangenehm oder irgendwos, es woar einfoch so, es woar immer voi toll.“ (Elisabeth Ebner) Elisabeth räumte aber ein, dass sie als kleines Kind besorgt über das Audio-Aufnahmegerät war, weil sie dessen Zweck und Funktionsweise nicht verstanden hatte. Erst später, als ihr die Bedeutung der Aufnahme und der Transkription bewusst wurden, war ihr die Aufzeichnung "hoit natürlich wurscht.“ Einen anders gelagerten Kritikpunkt äußerte Torsten Kaiser zur Interviewführung in der siebten Erhebungswelle. Ihm wäre ein persönliches Treffen, wie in den ersten sechs Erhebungswellen, lieber gewesen. Allerdings zeigte er Verständnis dafür, dass dies während der Covid-19-Pandemie nicht möglich war. Insofern bezeichnet er das Vorgehen als „angemessen“ und betonte, dass die technisch vermittelte Art der Gesprächsführung sich nicht negativ auf den Verlauf des Interviews und die behandelten Themen ausgewirkt hätte.

\subsection{Fazit}

$\mathrm{Zu}$ den großen, gleichermaßen organisatorischen wie wissenschaftlichen Herausforderungen der (Medien-)Sozialisationsstudie zählte es, dies lässt sich zusammenfassend sagen, das Panel über einen so langen Zeitraum von 15 Jahren zusammenzuhalten und immer wieder Zugang zu den Eltern und ihren Kindern zu bekommen, sei es in herausfordernden Entwicklungsphasen, wie etwa der Pubertät, oder auch zur Zeit der siebten Erhebungswelle, die für die Befragten unerwartet kam, da sich das Forschungsteam bereits nach der sechsten Erhebungswelle bei allen Familien verabschiedet und ihnen abschließend für ihre Teilnahme an der Studie gedankt hatte.

Zwar hatte es während der Laufzeit der Studie zwischendurch Familien gegeben, die aus unterschiedlichen Gründen nicht bereit waren, an einer Erhebungswelle teilzunehmen, der qualitative, lebensweltbezogene Forschungsansatz erlaubte es dann aber, in einem späteren Interview zurückzublicken und darin die zurückliegenden Ereignisse zu erfahren und damit die Erkenntnisse in den Datenkorpus der Studie zu integrieren.

Mit Blick auf die Aussagen der Befragten, Kinder wie Eltern, zeigte sich, dass nahezu alle ihre Beteiligung an der Studie im Rückblick als positiv bewerteten. Einige Mütter hatten in den Interviewern und Interviewerinnen willkommene Gesprächspartner und -partnerinnen gesehen, denen sie einmal aus ihrer Perspektive schildern konnten, was ihren Alltag ausmachte. Insbesondere die jungen Männer des Panels hoben hervor, 
dass sie ihren Interviewer, insbesondere in der Pubertät, als ein wichtiges Gegenüber wahrgenommen hatten; einige hätten sich, wie sie betonten, gern auch einen engeren Kontakt zu ihm gewünscht. Diese Erfahrung legt den Schluss nahe, dass es vor allem Jungen an (externen) Gesprächspartnern fehlt, mit denen sie über sich selbst und ihre Anliegen sprechen können. Zwar äußerten auch die jungen Frauen im Rückblick auf die Langzeitstudie, dass sie diese sehr positiv und mit zunehmendem Alter auch als bereichernd empfunden haben, es war jedoch keine darunter, die der jeweiligen Interviewerin einen ähnlichen Stellenwert zuschrieben, wie dies bei einigen der jungen Männer der Fall war. Insgesamt erwähnten vor allem die jungen Menschen, wie dies einige von ihnen deutlich hervorhoben, dass sie es als etwas Besonderes erlebt hatten, in welcher Weise ihnen die Teammitglieder schon in der frühen Kindheit begegnet waren, mit Interesse an ihren Belangen und ihrer Sichtweise, mit Aufmerksamkeit und, wie dies einige betonten, mit Respekt.

Interessant war, dass die jungen Menschen erstmals in der siebten Erhebungswelle Interesse an der Gesamtkonzeption der Studie erkennen ließen und dass es für sie bedeutsam war, auf diese Weise auf ihre eigene Entwicklung von der Kindheit bis zum jungen Erwachsenenleben zurückzublicken. So betonten einige der jungen Erwachsenen mehrfach, dass schon der Rückblick auf die Zeit zwischen den Erhebungswellen zu Beginn eines jeden Interviews ein besonderes Erlebnis für sie gewesen sei. Sie erlebten sich auf diese Weise selbst als die Experten und Expertinnen ihres Alltags und, darin integriert, ihres Mediengebrauchs; der Ansatz, sie von der ersten Erhebungswelle an als solche wahrzunehmen und ernst zu nehmen, erwies sich auch mit Blick darauf als ein guter Forschungsweg. Dies ermöglicht dem Forschungsteam nun, die Panel-Langzeitstudie auf Stärken und Schwächen hin kritisch zu beleuchten und die Rückmeldungen und Einschätzungen der jungen Erwachsenen und ihrer Eltern als Potenzial für weitere Forschungsvorhaben zu nutzen.

Eine Längsschnittstudie über insgesamt 15 Jahre fortzuführen, dies lässt sich abschließend feststellen, bedeutet die Bereitschaft, sich stets aufs Neue um möglichst adäquate Lösungsstrategien zur Weiterbearbeitung des Forschungsgegenstands im Sinne der zentralen Forschungsfrage( $\mathrm{n}$ ) zu bemühen; jede neue Erhebungs-, mithin auch Auswertungsphase ist eine methodische Gratwanderung zwischen Kontinuität und Wandel. Außerdem gilt es, im Rahmen einer derartig langen Forschungsarbeit stets auf Neue und dies mit Blick auf die Verantwortung den Panelangehörigen gegenüber ethische Fragen zu reflektieren. Sie stellt sich außerdem als eine Gratwanderung dar zwischen dem Erkenntnisinteresse der Forschenden auf der 
einen Seite und der Notwendigkeit, auf der anderen Seite, sich stets bewusst zu machen, dass es keine Selbstverständlichkeit ist, Einblicke in den Alltag von Menschen zu erhalten. ${ }^{47}$ Eine Panel-Langzeitstudie erfordert es stets, sich der Verantwortung als Wissenschaftler und Wissenschaftlerin, sowohl dem Forschungsgegenstand als - vor allem - den Menschen in ihrem Alltag gegenüber, die diesen Forschungsgegenstand ausmachen, bewusst zu sein und sie konkret wahrzunehmen. Dies bedeutet, ihnen mit Sensibilität zu begegnen und alle Schritte sorgsam zu gehen; das heißt auch, dem eigenen Tun gegenüber kritisch zu bleiben und zu beherzigen, was vor Jahren Nico Fried in einem Leitartikel der Süddeutschen Zeitung mit Blick auf Politiker und Politikerinnen betonte, als er an die Worte erinnerte, die seinerzeit der Vater des deutschen Politikers Peter Struck seinem Sohn als Kind mit auf den Weg gegeben hatte: „Egal, was Du machst, und wenn Du nur den Hof fegst - mach' es ordentlich.“

47 Dies wurde auf eindrucksvolle Weise in den Äußerungen von Frau Weiss und Frau Hirtner deutlich. So gab Frau Weiss zu bedenken, dass man, wenn man sich dazu bereiterklärt habe, an einer solchen Studie teilzunehmen, auch die Wahrheit sagen und nichts beschönigen sollte. Frau Hirtner befand sich während der siebten Erhebungswelle in einer schweren gesundheitlichen und auch finanziellen Krise, über die zu sprechen sie während des Interviews mehrfach zum Weinen brachte. Dennoch schilderte sie ihre Situation: „Na, es schaut scheiße aus!“ Und fügte an: „Ja sicher, warum soll i di anlügen? Es ist ja die Wahrheit.“ 


\section{Zusammenfassung, Diskussion und Konsequenzen}

Mehr als 15 Jahre Langzeitforschung - das bietet Gelegenheit zurückzublicken und noch einmal über einige wichtige Aspekte in der langen Zeit der Forschung nachzudenken: Was waren die Ziele der Panel-Studie? Auf welchen theoretischen und methodischen Füßen steht sie? Welche Ergebnisse und welche Erfahrungen sind mit der Studie verbunden und - dies vor allem - welche Konsequenzen lassen sich nach einer so langen Forschungszeit zur Verbesserung der sozialen Lage für junge Menschen aus sozial benachteiligten Lebenslagen benennen?

\subsection{Von der allmählichen Verfertigung eines Forschungsansatzes}

\subsubsection{Ausgangspunkt und Ziele}

Am Anfang der Studie zur Rolle von Medien in der Sozialisation sozial benachteiligter Heranwachsender 2005, im Sinne von Norbert Elias (1987) als engagierte Sozialforschung angelegt, das heißt als eine Studie mit einem wissenschaftlichen und einem sozialen Anliegen, standen Beobachtungen, dass sich „die Schere zwischen den Gewinnern und Verlierern im Prozess des heutigen Aufwachsens weiter öffnet" (Rauschenbach 2011: 5). Insbesondere Vertreter und Vertreterinnen der Politischen Ökonomie mahnten zu Beginn der 2000er Jahre eindringlich, dass die Dynamik von Inklusions- und Exklusions-Prozessen in engem Zusammenhang mit den Rechten auf Kommunikation und gesellschaftliche Partizipation betrachtet werden muss und dass in diesem Kontext auch der Umgang mit neuen Medien Beachtung verdient (Murdock/ Golding 2004). Mit Blick auf die große Bedeutung einer funktionierenden Öffentlichkeit für die Zivilgesellschaft diskutierte Peter Dahlgren schon früh die Rolle des Internets und seiner unterschiedlichen Formen netzbasierter Kommunikation (Dahlgren 2000a \& 2000b). Überlegungen dieser Art dienten als Hintergrund für die Formulierung der zentralen Forschungsfragen der Studie: Was heißt Aufwachsen in sozial benachteiligten Lebenslagen für Kinder und Heranwachsende, für ihre Sozialisation, ihre Partizipationschancen an der Gesellschaft? Und: Welche Rolle spielen Medien in diesem Zusammenhang? Denn Aufwachsen hieß auch zur Zeit der Planung der Studie 2004 längst 
Aufwachsen mit Medien. So war der Alltag der Kinder schon zu Beginn der Panelstudie 2005 von Medien unterschiedlicher Couleur durchdrungen. Das Team der (Medien-)Sozialisationsstudie sah sich daher mit einer doppelten, eng miteinander verflochtenen Dynamik konfrontiert, zum einen der sich im Rahmen medial-technischer Wandlungsprozesse (etwa der Digitalisierung und der Konvergenz) dynamisch verändernden Mediendienste und Medienangebote und zum anderen der sich dynamisch vollziehenden Entwicklung von Kindern im Rahmen ihrer Sozialisation.

\subsubsection{Aufriss der theoretischen Fundierung}

Aus dieser Feststellung erwuchs die Konzeption der Studie, auf die im Folgenden in einem kurzen Aufriss Bezug genommen werden soll (zur Gesamtkonzeption siehe insbesondere Paus-Hasebrink 2017a, 2019; PausHasebrink/ Bichler 2008; Paus-Hasebrink/ Kulterer 2014). Zum einen erschien es sinnvoll, Theorien der Mediatisierung zu berücksichtigen und Medien als Teil alltäglicher Lebenspraktiken zu verstehen, da Menschen ihren Alltag mehr und mehr mit Hilfe von Medien leben und gestalten. Alltagspraktiken, die als kommunikative Muster verstanden werden können (siehe Paus-Hasebrink/ Hasebrink 2014), werden durch neue Kommunikationstools und Technologien (Lundby 2014: 3) transformiert, indem Grenzen zwischen Diensten und Formen der interpersonalen und der Massenkommunikation weiter verfließen und die Ausdifferenzierung hochspezialisierter Dienste fortschreitet. Aber die Ausgangsperspektive der (Medien-)Sozialisationsstudie zielte nicht in erster Linie auf die Medien und ihre transformative Kraft. Vielmehr liegt der Studie die Überzeugung zu Grunde, dass nicht als erstes den Medien die Aufmerksamkeit gebührt, sondern dem Einzelnen, dem Kind, in seiner Sozialisation. Das zentrale Forschungsziel richtete sich daher vielmehr auf die Frage nach dem subjektiven Sinn des Medienhandelns schon von Kindern im Rahmen ihrer Sozialisation und darauf, zu eruieren, worin dieser Sinn jeweils konkret besteht, um so erklären zu können, wie Medien zur Bearbeitung spezifischer Lebensherausforderungen, biografisch mitbedingter Entwicklungs- bzw. Lebensaufgaben, beitragen. Um dies zu ermöglichen, waren weitere Theorieperspektiven nötig, so eine entwicklungspsychologische Perspektive, die Entwicklung nicht als Reifung begreift, sondern als tätige Auseinandersetzung des Individuums mit den sozialen Aufgaben und Bedingungen seiner alltäglichen Lebensführung an einem je spezifischen sozialen Ort. An diesem Punkt setzte in der (Medien-)Sozialisationsstudie 
der zentrale Aspekt der sozialen Ungleichheit an und, damit verbunden, der Blick auf die Bedeutung des sozialen Orts. Damit war es möglich, die Identifizierung „handlungsleitender Themen“48 der Kinder und ihrer Eltern nicht allein über die Perspektive einer verengten Entwicklungspsychologie zu modellieren, sondern mit Hilfe soziologischer Theorien der Sozialisationsforschung eine ganzheitliche und kontextuelle Perspektive auf Sozialisationsprozesse zu entwickeln - und den Zusammenhang zwischen Mediatisierung und Mediensozialisation in den Blick zu nehmen (siehe dazu auch Hoffmann/ Krotz/ Reißmann 2017).

Als weiterer wichtiger theoretischer Baustein diente Bourdieus Theorie der Praxis (1977) und seine Kategorie des Habitus. Bourdieus Habitus-Kategorie bot das Instrument, um den inneren Zusammenhang von Eigenschaften der sozialen Lage, den ihr zugehörigen Ressourcen und den diesen Ressourcen eingeschriebenen Regeln und Optionen des Handelns in Mustern subjektiver Sinngebung, die für den Mediengebrauch prägend werden, beschreibbar zu machen. Mit dem Blick auf das soziale Milieu und auf seine phänomenologische Ausprägung, den Habitus, wird die zentrale Lebensbasis reflektierbar, und die Verflochtenheit der genannten Aspekte kann erfasst werden. Bourdieu versteht den Habitus als „inkorporierte soziale Struktur“ (Weiß 1997: 246). Er erwächst aus der körperlichen Teilhabe an einer gemeinschaftlichen Handlungspraxis, das heißt, in der kulturell gewachsenen Eingebundenheit in Routinisierungen und Habitualisierungen. Dem Habitus als „Erzeugerprinzip“ ist die Abstammung aus einer Position im „sozialen Raum“ anzusehen. Diese ist ihrerseits durch die Struktur sozial ungleich zugemessener Bedingungen der Lebensführung bestimmt“ (ebd.). Mit dem Blick auf die „Methoden der Sinngebung“, das Tableau alltagspraktischer Orientierungsmuster, wie Ralph Weiß dies nennt, d.h. wie Menschen - auch mit Hilfe von Medien - ihren Handlungen im Alltag Sinn geben, tut sich eine Perspektive auf, die das je individuelle, aber dennoch über die subjektive Repräsentation hinausweisende Lebensumfeld und die jeweilige darin eingelagerte Lebensführung in den Blick nimmt. Die Lebensführung eines Individuums vollzieht sich vor dem Hintergrund seines sozialen Milieus, also der sozialen Räume, die dem bzw. der Einzelnen tatsächlich oder symbolisch

48 Diesem Begriff liegt die Erkenntnis zu Grunde, dass die Themen von Kindern von ihrem Entwicklungsstand und ihren individuellen Erfahrungen mitbestimmt werden und dass diese ihre Handlungen im Kontext ihres Alltags leiten. Danach steht, so Charlton und Neumann (1986: 31) „das Thema (...) vor der Handlungsausführung”. 
zur Verfügung stehen, den Räumen, in denen der bzw. die Einzelne seine Kapitalien möglichst sinnvoll einsetzt.

Nach Ralph Weiß sind die Umgangsweisen von Menschen mit Medien dies gilt in besonderer Weise mit und im Kontext von Sozialen Medien - als „Tätigkeiten“ zu verstehen (Weiß 2000, 2001; siehe auch Weiß 2013); Tätigkeiten, die die Nutzer und Nutzerinnen je nach Kommunikationsmodus einnehmen ${ }^{49}$, etwa eher als Film-Sehen, als Fern-Sehen, als Radio-Hören bzw. als Handeln im und mit dem Internet bzw. im und mit dem Sozialen Netz. Diese Nutzungsmodi sind in die alltägliche Lebensführung der Menschen eingebettet und nehmen von dort ihren Ausgangspunkt. Konkret bedeutet dies, von der Lebenswelt eines Kindes in seinem zentralen Sozialisationskontext, seiner Familie bzw. Kernbeziehungsgruppe und dessen spezifischem Milieu, auszugehen. Im Mittelpunkt steht also die alltägliche Lebensführung (Kudera 2001) eines Kindes von seiner frühen Kindheit an und wie sich sein Mediengebrauch ${ }^{50}$ - und auch der seiner Eltern - im Sinne einer aktiven Tätigkeit in seiner Familie ausprägt. Es geht damit also um die Arrangements der alltäglichen Lebensführung, in der sich die Handlungsweisen von Individuen - in diesem Fall des Kindes und seiner zentralen Bezugspersonen - ausbilden und in denen sich auch, als integraler Bestandteil, ihre Mediengebrauchsweisen als Teil ihrer Kommunikationspraktiken entwickeln; denn erst in der alltäglichen Lebensführung erhält der Mediengebrauch eines Individuums Struktur und Sinn.

Um die Verzahnung subjektiver und struktureller Kontexte deutlich zu machen, wurden in der Studie drei Handlungskonzepte als Analysekonzepte entwickelt: Handlungsoptionen, Handlungsentwürfe und Handlungskompetenzen.

- Handlungsoptionen bezeichnen das für das Individuum, ein Kind, seine Geschwister und seine Eltern in der Familie, faktisch existierende Arrangement der objektiven Merkmale der sozialen Lebenslage - eine Anordnung von Ermöglichungen und Beschränkungen.

49 Mit Hilfe des von Hasebrink (2013) vorgeschlagenen Konzepts der Kommunikationsmodi können die medialen Handlungsweisen von Menschen mit den diversen Mediendiensten konkreter erfasst und analysiert werden. Vor diesem Hintergrund lassen sich dann die individuellen Gebrauchsweisen der medial Handelnden in Rückbindung an ihre Lebensführung im Alltag, die in der Bewältigung ihrer konkreten mit ihrer sozialen Situation unmittelbar verbundenen Entwicklungs- und Lebensaufgaben (siehe dazu Paus-Hasebrink 2010) besteht, erfassen, beschreiben und verstehen.

50 Siehe zum Verständnis des Medienhandelns als Gebrauch Paus-Hasebrink (2017a) sowie Kapitel 1, Fußnote 3. 
- Handlungsentwürfe kennzeichnen die Ziele und Pläne, die sich bei einem Kind und seinen Bezugspersonen in seiner Familie eruieren lassen. Dies sind die als subjektive Transformation der objektiven Merkmale seiner sozialen Lage gebildeten Anschauungsweisen von Welt, die Orientierungen, die einem Individuum helfen, die Welt wahrzunehmen und zu deuten und Sinn im eigenen Leben herzustellen. Handlungsentwürfe sind also die subjektiven Wahrnehmungen dieser Struktur im Zusammenspiel der Verzahnung von Praxis und handlungsleitender Anschauung, verbunden mit dem jeweiligen sich aus diesem Zusammenspiel entwickelnden Eigensinn des Einzelnen in seinem unmittelbaren Umfeld.

- Handlungskompetenzen bezeichnen, wie sich - im Sinne Bourdieus die dem Subjekt zugänglichen materiellen, kulturellen und sozialen Ressourcen seines Milieus zur Umsetzung seiner Lebensentwürfe in den kognitiven und motivationalen Voraussetzungen seines Handelns niedergeschlagen haben.

Vor diesem Hintergrund ließ sich ein wesentliches bereits in den ersten beiden Panelphasen identifiziertes Phänomen verstehen, nämlich, dass Medien und ihr Gebrauch nicht als Verursacher von Problemen zu sehen waren, sondern vielmehr als Ausdruck von Lebensbedingungen. (Medien-)Sozialisation, dies wurde schon früh deutlich, stellt sich, je nach untersuchter Familie und ihren ganz spezifischen Handlungsoptionen, Handlungsentwürfen und Handlungskompetenzen im Zusammenspiel aller Familienmitglieder, mit zunehmendem Alter eines Kindes auch von Peers und Freunden, als ein komplexes und veränderbares Zusammenspiel vieler Faktoren dar. Als wichtige Erkenntnis in diesem Zusammenhang schien dabei auf, dass auf allen Ebenen des im Falle der Langzeitstudie qualitativen Forschungsprozesses stets der Problematik Rechnung getragen werden musste, dass es um die Interpretationen von Handlungen in ihren Praxiskontexten gehen muss. Handlungen sind also in ,ihrer Verkettung und Folgewirkung“, wie dies Hörning nennt, zu betrachten, „dies aber ohne deren sinnhafte, auf Deutungsmustern, biografischen Schemata u.ä. basierende Einordnung zu ignorieren " (Hörning 2001: 158 zit. nach Lange 2003: 106). Als sehr wichtig erwies es sich, die große Rolle von individualbiografischen Faktoren stets mit zu bedenken. Sie bestimmen das Zusammenspiel von milieu- bzw. habitus-geprägten sowie (medien-)biografischen psycho-sozialen Prozessen mit. Mediales wie non-mediales Handeln ist so zum einen eng verbunden mit den im Rahmen medial-technischer Wandlungsprozesse verfügbaren Medien und zum anderen mit der sich dynamisch vollziehenden Identitätsgenese von Individuen, den Kindern 
und ihren unmittelbaren Bezugspersonen, in der Sozialisation, die sich ihrerseits im Kontext ihrer jeweiligen milieugeprägten Lebensführung und den davon geprägten Alltagspraktiken von Individuen an ihrem je spezifischen sozialen Ort vollzieht.

Vor diesem Hintergrund konnte und kann die Erforschung der Rolle von Medien in der Sozialisation dieser Herausforderung nur dann gerecht werden, dies sei zusammenfassend betont, wenn sie stets mit Blick auf den gesellschaftlich zunehmend relevanter werdenden transformativen Metaprozesses der Mediatisierung und seine Auswirkungen auf die Sozialisation von Individuen erfolgt. Dabei wurde sehr deutlich, dass Lebenspraxis aber nur mit offenem Blick auf die gelebten Praktiken der Handelnden und durch die Konzentration auf ihren jeweiligen subjektiven Sinn im Rahmen ihrer täglichen Lebensführung erfasst werden kann. Bourdieu versteht Wirklichkeit - ähnlich wie Berger und Luckmann (1967) und Giddens (1984) - als „Spiel“ zwischen „symbolischer Beherrschung und praktischer Handhabung" (Bourdieu 1982: 40f). Auch nach Überzeugung des Symbolischen Interaktionismus, wie ihn Mead geprägt hat, sowie nach Schütz ist Wirklichkeit keine Summe von zusammenhängenden Fakten; vielmehr stellt sie sich als kommunikative Konstruktion dar, die soziale Konstitution in Gang bringt (Flick 2005: 22; Schweizer 2007: 280). Im Kontext der als praxeologisch verstandenen Sozialisationsstudie wird Praxis daher nicht als ein Reservoir verstanden, das den Sinn enthält, der dann von den Handelnden lediglich, wie dies Knoblauch als Kritik zu Schatzki anmerkt, „abgerufen“ wird, so, „als wären sie Animateure ihres eigenen Tuns" (Knoblauch 2008: 220). In der (Medien-)Sozialisationsstudie geht es vielmehr stets im Sinne Bourdieus um die Frage, wie sie pointiert Peter Lunt (2020: 2946) formuliert, „of how subjectivity and the material world are constituted and sustained through practice, how meaning and intelligibility are grounded in use and performance, in the acknowledgment of the dialectic". Gesellschaftliche Praktiken werden als im Prozess der Sozialisation in Subjekten kulturell eingeprägte, von ihnen verinnerlichte, aber auch durch ihren jeweiligen individuellen Vollzug veränderbare Praktiken verstanden.

Im Rahmen der Panel-Langzeitstudie verfertigte sich der theoretische Zugang zur (Medien-)Sozialisation sozial benachteiligter Kinder mit den in ihrem Mittelpunkt stehenden Begriffen Lebenswelt, soziales Milieu und Habitus, Lebensführung sowie Orientierungssuche und Identitätskonstruktion eines Kindes und seiner Eltern (siehe Paus-Hasebrink/ Bichler 2008: 85ff.) im Prozess der Forschung selbst. Es lag der Studie also ganz zu Beginn kein einmal festgefügter Theorie-Ansatz zu Grunde, vielmehr 
handelte es sich dabei um einen sich in seinen Facetten erst in der Forschungspraxis weiter schärfenden Zugang - ganz im Sinne von Elias und Bourdieu, nach denen Theorien nicht auf Basis von Momentaufnahmen entstehen können, sondern sich vielmehr in der Forschungspraxis selbst entwickeln und entfalten müssen.

\subsubsection{Zur methodischen Fundierung}

Fest stand jedoch von Anfang an die Entscheidung, die Studie als eine qualitative Panelstudie anzulegen; sie erschien für die Rekonstruktion der subjektiven Bedeutungen der Medien in den jeweiligen Phasen der Sozialisation unter Berücksichtigung der sozialen Herkunft der Heranwachsenden sinnvoll. Den Kern des Untersuchungsdesigns bildete daher eine qualitative Panelstudie bei Kindern ab dem Alter von fünf Jahren, die in ihren unterschiedlichen Lebensphasen mit Blick auf ihren zentralen Sozialisationskontext, also ihre Familie bzw. Kernbeziehungsgruppe, über insgesamt 15 Jahre jeweils mit Blick auf wichtige Entwicklungsphasen hinweg untersucht wurden. Als zentrales Erhebungsinstrument der triangulativ ausgerichteten Studie ${ }^{51}$ diente das teilstandardisierte Leitfadeninterview, das durch eine standardisierte Fragebogenerhebung der Eltern und eine teilnehmende Beobachtung ergänzt wurde (siehe dazu und zu den weiterführenden Methoden Kapitel 1.4). Da die hier gezielt zu untersuchenden subjektiven Bedeutungs- und Funktionszuschreibungen an Familie, Umwelt oder Medien von Person zu Person und vor allem von Kind zu Erwachsenen stark variieren können - jeder Mensch ist schließlich Experte für seine eigenen Bedeutungsgehalte (Mayring 2002: 66) -, wurden für die Untersuchung konkreter Familien sowohl die Kinder als auch deren Eltern berücksichtigt. In der siebten Erhebungswelle fanden keine persönlichen Besuche statt, die Leitfadeninterviews sowohl mit den jungen Erwachsenen als auch mit ihren Eltern wurden per Telefon bzw. Skype oder WhatsApp-Call durchgeführt (siehe dazu Kapitel 1.4).

51 Es fand eine Triangulation sowohl der Theorien als auch der Perspektiven von Forschern und Forscherinnen im Team statt als auch und insbesondere eine Triangulation der Erhebungs- und Auswertungsmethoden (siehe dazu Paus-Hasebrink et al. 2018). Siehe zur Anlage von Mehr-Ebenen- und Mehr-Methoden-Studien und speziell zur Triangulation auch Trültzsch-Wijnen (2018: 24ff. und 231f.) sowie Trültzsch/ Wijnen/ Dürager 2013: 112f.). 
Im Hinblick auf die methodische Anlage soll ein wichtiger Aspekt einer wie oben beschrieben praxeologisch ausgerichteten qualitativen $\mathrm{Pa}$ nel-Langzeitstudie, die auch, wenngleich nicht nur, auf biografische, lebensgeschichtliche Veränderungen zielt, nicht unerwähnt bleiben. So äuBerte Pierre Bourdieu Bedenken gegen eine rein biografisch ausgerichtete Herangehensweise; er hegte gegenüber lebensgeschichtlichen Erzählungen große Skepsis und bezeichnete sie in einer Schrift als „Biographische Illusion" (Bourdieu 1990; siehe dazu auch Griese/ Schiebe 2018). Schließlich können mit narrativ angelegten Befragungen Schwierigkeiten wie etwa das Problem von "Lebenslügen' und 'Angeberei“" (ebd.; siehe auch Beck/ Büser/ Schubert 2016: 81) verbunden sein. „Bourdieu plädiert dafür“, darauf weisen Beck, Büser und Schubert (2016: 81) in ihrer Studie zu Mediengenerationen hin, „Lebensgeschichte nicht nur in ihrer zeitlichen Dimension, sondern vor allem in ihren positionellen Relationen als, gesellschaftliche Laufbahn' innerhalb eines Prozesses des sozialen Alterns und den damit verbundenen Veränderungen im sozialen Raum zu betrachten“. Die praxeologisch ausgerichtete, subjektive wie objektive Faktoren der Sozialisation gleichermaßen in den Blick nehmende Forschung zielte daher auf eine Verbindung von makro-, meso- und mikrostrukturellen Aspekten. Sie berücksichtigt, wie Bourdieu fordert, „das Ensemble der objektiven Beziehungen, die den betreffenden Akteur - mindestens in einer gewissen Zahl anhaltender Zustände - vereinigt haben mit der Gesamtheit der anderen Akteure, die im selben Feld engagiert sind und die demselben Möglichkeitsraum gegenüberstehen“ (Bourdieu 1990: 80f.). Auf der Makro-Ebene wurden die von politischen, wirtschaftlichen und kulturellen Kontexten eines Landes, wie etwa familienpolitischen Entscheidungen und Gesetzen, institutionellen Bildungseinrichtungen, aber auch der Infrastruktur an Freizeiteinrichtungen mitbestimmten Aspekte berücksichtigt; auf der Meso-Ebene galt der Blick zunächst insbesondere den für die Familie relevanten sozial-strukturellen Faktoren wie Einkommen und Bildung der Eltern. Das Gesamtkonstrukt der Lebensführung in einer Familie und des sozialen Netzwerks, in dem sich Eltern, Geschwister und vor allem die Kinder selbst bewegen, stellt die Basis etwa für den Umgang eines Kindes mit Medien dar. Später spielen dann zunehmend Peers und Freunde eine Rolle sowie institutionelle Einrichtungen wie Kindergarten und Schule, Lehre, weiterführende Schulausbildung, Studium oder gleich Beruf, Lebenspartner bzw. Partnerin etc. In diese Zusammenhänge bringt sich das Individuum aus einer bestimmten Position ein - abhängig von Alter und Geschlecht und seiner damit verbundenen kognitiven, sozialen und emotionalen Entwicklung und von den jeweiligen Anliegen der Selbst-, Sozi- 
al- und Sachauseinandersetzung (Neumann-Braun 1992; Paus-Hasebrink/ Schmidt /Hasebrink 2011: 27ff.), als Mikro-Ebene modelliert. Nicht vergessen werden durfte aber auch der spezifische Eigen-Sinn des Individuums, d.h. wie dieses die sozial-strukturell relevanten Faktoren wie Einkommen und Bildung und damit die von seiner sozialen Lage geprägten Lebensbedingungen wahrnimmt und seine eigenen Interessen einbringt. Die Auswahl der Panel-Familien orientierte sich an der Definition von sozialer Benachteiligung und folgte den Kriterien von Hradil (1999: 11).

Nicht unterschlagen werden darf bei einer derartigen, qualitativ angelegten Panel-Studie das in der Sozialforschung in Bezug auf reaktive Methoden zu Recht die intensiv diskutierten Phänome der sozialen Erwünschtheit ${ }^{52}$ und der so genannten Paneleffekte. In diesem Zusammenhang galt es jedoch stets, Stärken und Schwächen der methodischen Vorgehensweise gegeneinander abzuwägen und nicht nur in Kauf zu nehmen, dass die Befragten „Experten ihres Lebens“ sind - schließlich verbietet sich eine lebenslange Beobachtung von Menschen bereits aus ethischen Gründen -, sondern gerade diesen Umstand als eine Chance zu nutzen, näher in die Bedeutungs- und Sinnkonstruktionen des Mediengebrauchs in den verschiedenen Alltagskontexten vorzudringen. Nötig ist jedoch auch stets die genannten Phänomene Erwünschtheit und eventuelle Paneleffekte zu diskutieren. Durch entsprechende Breite in den Interview-Themen, die den Entwicklungs- und Lebensaufgaben der Befragten angepasst wurden, und eine gut abgestimmte Methoden-Triangulation ließ sich diesen Aspekten jedoch entgegenwirken (siehe dazu auch Kapitel 5).

\subsection{Von der (Medien-)Sozialisationsforschung als integrativer Familienforschung bin zu einer breiteren Perspektive auf Sozialisationskontexte}

\subsubsection{Familie im Zentrum der Sozialisationskontexte von Kindern und Jugendlichen}

Im Rahmen der Leitfadeninterviews mit den Kindern und zumindest einem Elternteil, und insbesondere auch in der teilnehmenden Beobachtung wurde schon in den ersten beiden Panelwellen 2005 und 2007

52 Siehe dazu auch die Ausführungen und Reflexionen von Beck, Büser und Schubert (2016) im Rahmen ihrer Untersuchung zu biografischen und kollektivbiografischen Mustern des Medienhandelns. 
deutlich, dass der Mediengebrauch eines Kindes in einem unmittelbaren Zusammenhang mit seinem lebensweltlichen Kontext in der Familie und den (Medien-)Erziehungsstrategien seiner Eltern und ebenfalls dem eigenen Mediengebrauch der Eltern, aber auch der Geschwister, gesehen und eingeordnet werden muss. Als sehr bedeutsam erwies sich dabei das Familienklima, das heißt wie Eltern und Kinder miteinander umgehen, wie sie zueinanderstehen und aufeinander eingehen oder auch aneinander vorbeileben. So zeigte sich deutlich, dass wesentliche Merkmale des Medienumgangs - etwa der technische Zugang, die Nutzungsintensität oder Vorlieben für bestimmte Inhalte - nicht systematisch von den sozio-ökonomischen Bedingungen der Familie und ihren Praktiken der Lebensführung bestimmt werden, sondern dass diese vielmehr auch mit sozio-emotionalen Bedingungen in der Familie, aber auch ganz individuellen Interessen und Wünschen im Kontext des Eigen-Sinns eines Kindes oder auch seiner Eltern zusammenhingen. Auch wenn somit keinesfalls ein direkter oder gar kausaler Einfluss von lebensweltlichen Bedingungen auf den Mediengebrauch eines Individuums auf seine Sozialisation konstatiert werden durfte, wurde während des Forschungsprozesses der enge Zusammenhang zwischen dem Mediengebrauch und den ganz spezifischen Handlungsoptionen, Handlungsentwürfen und Handlungskompetenzen der Heranwachsenden und ihrer Familien offenkundig. Es zeigte sich deutlich, dass Medien $^{53}$ zumeist dann besondere Relevanz für die Heranwachsenden erlangten, wenn ihre Sozialisation von erschwerten Familienkontexten geprägt war und es ihren Eltern nicht oder nur schlecht gelang, ihren Kindern genügend Aufmerksamkeit zu geben und sie so zu begleiten, dass sich die Kinder bei ihren Eltern aufgehoben fühlten. Die Jungen und Mädchen, die in ihren Familien Geborgenheit und emotionale Nähe erlebten, waren dagegen den Herausforderungen ihres Alltags gegenüber besser gewachsen, dies fand seinen Niederschlag auch im Mediengebrauch. Diese Kinder entwickelten anspruchsvollere Handlungsentwürfe, z.B. in Bezug auf ihre Freizeitbeschäftigungen, aber auch ihre Erwartungen an die Schule, und hatten es leichter, ihre kognitiven Fähigkeiten zu entfalten und Handlungskompetenz aufzubauen, als diejenigen Heranwachsenden, die sich in ihren Familien nicht aufgehoben und angenommen wussten und, damit stärker auf sich gestellt, mit den Herausforderungen ihres Alltags, einschließlich ihres Umgangs mit Medien, weitgehend allein zurechtkom-

53 Printmedien spielten bei den Kindern kaum eine Rolle. Sie bevorzugten crossmedial vermarktete Medienangebote, deren primäres Ausgabemedium das Fernsehen war (siehe ausführlicher Paus-Hasebrink/ Bichler 2008: 135). 
men mussten. Medien waren zumeist dann, dies soll noch einmal explizit betont werden, ein sehr relevanter Alltagsbegleiter für die Kinder und Jugendlichen, wenn Konflikte in ihren Familien auftraten und wenn es ihren zentralen Bezugspersonen aufgrund ihrer schwierigen sozio-ökonomischen und dann auch oft sozio-emotionalen Lebensbedingungen nicht gelang, ihren Kindern genügend Aufmerksamkeit und Unterstützung zu geben. So suchten sich die Kinder und Jugendlichen zur Auseinandersetzung mit ihren Entwicklungsaufgaben und alltäglichen Herausforderungen in Kindergarten und Schule entsprechend ihrem Geschlecht und ihrem Alter Orientierung und Unterstützung in Medienangeboten.

Trotz der technischen Aufrüstung durch die Eltern zu Beginn der Schulzeit zeigte sich, dass viele Eltern im Panel ihren Kindern im Umgang mit Medien kaum Anleitung und Unterstützung boten bzw. bieten konnten. Insbesondere die in ihrer Lebensführung belasteten Familien waren überfordert und zeigten kaum Kapazitäten, sich um die Mediennutzung ihrer Kinder zu kümmern, ihnen Hintergrundkenntnisse zu vermitteln oder mit ihnen über ihre Medienthemen und -anliegen zu sprechen. Des Öfteren blieb es in den Familien, auch bereits als die Kinder noch jünger waren, bei einer gemeinsamen Nutzung von Fernsehprogrammen, allen voran von Programmen, die sich nicht speziell an Kinder richteten, und dies zumeist an Wochenenden. Aufgrund der Überforderung der Eltern überließen diese ihre Kinder bei der Mediennutzung oft sich selbst, andere Eltern reagierten sehr restriktiv und verboten die Nutzung. Mit Zunahme der Internetnutzung ihrer Kinder war zwar in manchen Familien von Datenschutz und der Sicherung der Privatsphäre die Rede, es mangelte den überforderten Eltern aber selbst an entsprechenden Kenntnissen und Medienkompetenz. Stattdessen ließen manche Eltern selbst einen unvorsichtigen Umgang mit sozialen Medien erkennen, indem sie etwa Bilder auf Facebook stellten, die den Kindern sehr unangenehm waren.

Im Rückblick lässt sich sagen, dass sich das Verhalten der Eltern des Panels ihren Kindern gegenüber, ob sie Zuwendung, Gleichgültigkeit oder Ablehnung bzw. gar Aggression zeigten, als hochrelevant für die Sozialisation der Heranwachsenden erwies; ihr emotionales Verhältnis zu ihren Kindern bestimmte auch die Art ihrer Medienerziehung mit. So wurde über die Jahre eindrucksvoll deutlich, dass elterliches Desinteresse an ihren Kindern oder gar die Ablehnung eines Kindes mit erheblichen Konsequenzen für die jeweiligen Kinder verbunden war und gar zu traumatisierenden Erlebnissen für sie führte. Doch auch ein Übermaß an Kontrolle in Bezug auf den Umgang ihrer Kinder mit Medien, die sich als Zeichen unzureichenden Vertrauens der Eltern in ihre Kinder erwies, 
oder der übermäßige Wunsch nach Nähe, von den Kindern als erdrückend empfunden, wirkten sich als Hemmnis in der Sozialisation der Kinder aus und behinderten sie im Aufbau eigener Handlungskompetenzen (siehe dazu ausführlicher Paus-Hasebrink 2018).

So wurde bereits im Laufe der ersten und zweiten Panelwelle unmissverständlich deutlich - und diese Erkenntnis bestätigte sich auch in der dritten und vierten Panelwelle, d.h. in den Wellen zur Zeit der mittleren Kindheit und der Adoleszenz -, dass die Frage nach der Rolle von Medien, das heißt, wie sich mediale, aber auch non-mediale kommunikative Praktiken entwickeln, nach einer integrativen Familienforschung verlangte. Denn die sozio-ökonomischen und, damit oft eng verbunden, sozioemotionalen Lebensbedingungen der Familie oder Kernbeziehungsgruppe und des engsten sozialen Netzwerks, in dem ein Kind aufwächst, stellen die Basis für (Medien-)Sozialisation dar. Ohne den Blick auf die Familie und ihre Lebensbedingungen zu richten, kann deshalb (Medien-)Sozialisationsforschung im Kindes- und Jugendalter nicht erfolgreich sein. Aus diesem Grund wurden nach Beendigung der vierten Erhebungswelle Familientypen gebildet. Denn bereits nach den ersten vier Erhebungswellen war deutlich geworden, dass sich bei den Familien der Panelstudie keine durchgängigen Muster in der Mediensozialisation der Kinder identifizieren ließen. Diese erschien vielmehr als ein von einer Fülle von Einflüssen mitbestimmtes, multifaktorielles Phänomen, das sich in jeder Familie, je nach Zusammenspiel meso-struktureller und mikro-struktureller Faktoren, unterschiedlich ausgestaltete. Zwar waren zu Beginn der Studie alle Panelfamilien sozio-ökonomisch schlechter ausgestattet, wie aber die Familienmitglieder ihr Leben anschließend führten, schlug sich nieder in ihren sozio-emotionalen Familienstrukturen, ihrem doing family fu $^{54}$ das ihr Zusammenleben als Familie prägte. So konnten in den Panelfamilien deutliche Unterschiede, aber auch Gemeinsamkeiten identifiziert werden, die die Basis für eine Typologie aller Familien bildete. Mit Blick auf die jeweiligen sozio-ökonomischen und sozio-emotionalen Bedingungen sowie die damit eng verbundenen Strategien der Familien zur Bewältigung ihres Alltags konnten vier Typen generiert werden (siehe dazu Paus-Hasebrink/ Kulterer/ Oberlinner 2017: 129ff. sowie Kapitel 1 und Kapitel 2).

Auch in der Jugendzeit (fünfte und sechste Panelwelle) zeigte sich noch die große Bedeutung der Familie bzw. der Kernbeziehungsgruppe.

54 Siehe dazu ausführlicher Paus-Hasebrink 2017a sowie Paus-Hasebrink 2019. Zur neueren Forschung zu Familie und doing family siehe Krinninger (2020) sowie explizit zum Zusammenhang von Medien und doing family Lange (2020). 
Die Heranwachsenden des Panels durchlebten in diesen Erhebungswellen wichtige Phasen; sie waren herausgefordert, die insbesondere mit der $\mathrm{Pu}$ bertät verbundene Bewältigung einer Reihe von Entwicklungsaufgaben, etwa der Festigung der eigenen Identität, der Partizipation an Peer-Groups sowie im weiteren Sinne an der Gesellschaft und allen voran der Abgrenzung von ihren Eltern sowie allgemein der Auseinandersetzung und Bearbeitung ihrer alltäglichen Herausforderungen, zu leisten. Zur Befriedigung ihrer vielfältigen Bedürfnisse setzten die Heranwachsenden des Panels nun ein breites Spektrum von Medienangeboten und Mediendiensten ein, in deren Mittelpunkt Anwendungen der Sozialen Medien standen; diese boten den Jugendlichen als symbolische wie reale Spielräume Möglichkeiten zur Bearbeitung ihrer Entwicklungsaufgaben. Sie nutzten seine Foren, sei dies Facebook und Instagram oder YouTube - bei den Mädchen waren vor allem Beauty-Channels beliebt, bei den Jungen Channels und Foren zu Computerspielen. Diese Foren dienten den Heranwachsenden dazu, sich spielerisch auszuprobieren und zu präsentieren und den Austausch mit Peers und Freunden bzw. Freundinnen zu pflegen. Für einige waren ihre Stars jedoch auch Beispiele für eine erfolgreiche und finanziell lukrative Karriere, die sie auch selbst gern einmal hätten.

In diesen beiden Erhebungswellen fiel auf, dass sich einige der Jugendlichen aus den sowohl sozio-ökonomisch als auch sozio-emotional belasteten und in ihren Bewältigungsstrategien im doing family inkompetenten Familien (Typ 1), aber auch aus den Familien, die sich insbesondere aufgrund ihrer schwierigen sozio-emotionalen Bedingungen im doing family ebenfalls als inkompetent erwiesen hatten (Typ 2), trotz ihrer mittlerweile gestiegenen Mobilität sozial weitgehend isolierten. Ihnen stand - anders als den Jugendlichen aus Familien von Typ 3 und vor allem Typ 4 - kein tragfähiges soziales Netz aus Verwandten oder Freunden der Familie zur Verfügung ${ }^{55}$. Diesen Jugendlichen, es handelte sich vor allem um Jungen, fiel es schwer, mit ihren Peers in der Entwicklung Schritt zu halten, sie erlebten sich oft als Außenseiter in ihren Klassen. Sie hatten entweder gar keine Freunde mehr oder nur sehr wenige, mit denen sie ihre eigenen Interessen teilen konnten. Ihre freundschaftlichen Kontakte bewegten sich, angestoßen von ihren Lieblingscomputerspielen, in der Online-Welt.

55 Siehe dazu ausführlicher Paus-Hasebrink und Oberlinner 2017: 241ff. 


\subsubsection{Die jungen Erwachsenen und ihr Beziehungsnetzwerk}

Im Rückblick auf die ersten sechs Erhebungswellen wird deutlich, dass die Handlungsoptionen, Handlungsentwürfe und Handlungskompetenzen der Heranwachsenden in engem Zusammenhang mit der Lebensführung der Familien bzw. der Kernbeziehungsgruppe standen, in denen sie aufwuchsen. Mit der Zeit aber - besonders augenfällig in der siebten Erhebungswelle - verlor die Familie bzw. die Kernbeziehungsgruppe den Stellenwert für die Heranwachsenden des Panels als der ihren Alltag unmittelbar prägende Kontext an Bedeutung. Waren zunächst insbesondere die Eltern, in einigen Fällen auch Stiefväter, und in einigen Familien auch andere Familienmitglieder, wie etwa Großmütter, Tanten oder Onkel, für die Kinder die entscheidenden Bezugspersonen gewesen, gewannen in der Pubertät der Heranwachsenden auch andere Personen, seien dies Peers, Freunde und Freundinnen und bei einigen auch bereits erste Partnerschaften an Relevanz für die eigene Lebensführung. Bei den nunmehr jungen Erwachsenen zeigte sich mit großer Deutlichkeit, dass ihre Lebensführung von wichtigen, mit dem Eintritt in eine Lehrstelle bzw. eine Arbeitsstelle und/oder auch dem Umzug in eine eigene Wohnung, der Aufnahme oder Festigung einer Partnerschaft einhergehenden Abnabelung von den Eltern verbundenen Strukturveränderungen geprägt wurde. Nun gewannen auch Personen außerhalb der ursprünglichen Familie als Rollenvorbilder, Bezugspersonen und Mentoren an Bedeutung, seien dies z.B. bei den jungen Frauen, die schon mit ihrem Partner zusammenlebten, die Partner selbst oder bei den jungen Männern neben den eigenen Partnerinnen auch deren Väter. Dies war insbesondere bei den jungen Männern der Fall, die bei einer allererziehenden Mutter aufgewachsen waren oder bei denen später die Ehe ihrer Eltern geschieden wurde und die Mütter fortan allein lebten. In der siebten Erhebungswelle wurde unmissverständlich deutlich, dass die jungen Erwachsenen, die sich bereits fest gebunden hatten, nun auch ihrem jeweiligen Partner bzw. ihrer Partnerin einen bedeutenden Part in ihrem Leben zuwiesen. Dies zeigte sich deutlicher bei den jungen Frauen, während die jungen Männer sich etwas zurückhaltender äußerten und insbesondere dem Schritt zusammenzuziehen größere Bedeutung beimaßen und diesen aus verschiedenen Gründen noch hinauszögerten.

Da die Bedeutung des Lehr- und Ausbildungssektors in dieser Lebensphase gewachsen war, nahm damit einhergehend auch die persönliche Relevanz eines Arbeitgebers oder einer Arbeitgeberin für einen jungen Mann oder eine junge Frau zu. Diese wurden in einigen Fällen zu wichtigen Orientierungs- und Ratgebern, sowohl bei den jungen Männern 
als auch den jungen Frauen. Vorgesetzte bei der Arbeitsstelle oder auch andere, außerhalb der Familie stehende Personen, wie etwa Kollegen und Kolleginnen, Freunde und Freundinnen wurden wichtig. So stellten sich die jungen Menschen nicht nur mehr und mehr sozio-ökonomisch auf eigene Beine; sie ordneten auch ihre sozio-emotionalen Beziehungen neu, oft verbunden mit einer Emanzipierung von der Familie bzw. der Kernbeziehungsgruppe.

\subsubsection{Relevanz individueller Passungen beim Einstieg ins Erwerbsleben der jungen Erwachsenen}

Als besonders relevant erwies sich in der siebten Erhebungswelle für die jungen Erwachsenen der Einstieg in eine Lehre oder eine Arbeitsstelle. Damit dieser überhaupt gelingen konnte, waren für einige junge Erwachsene Vermittlungsleistungen von Institutionen oder Personen außerhalb der Familie von entscheidender Relevanz. Dies war etwa bei einigen jungen Menschen aus Familien mit massiven sozio-ökonomischen Problemen und einer insgesamt multiplen Deprivation, etwa in Folge schwerer Krankheiten, der Fall, die mit der Bewältigung ihres Alltags überfordert waren (Typ 1). Dies war aber auch bei einigen jungen Menschen aus Familien der Fall, die zwar sozio-ökonomisch nicht mehr bzw. nur noch wenig belastetet waren, aber wegen problematischer sozio-emotionaler Beziehungsstrukturen Überforderung in der Alltagsbewältigung erkennen ließen (Typ 2), sowie aus Familien, die zwar weiterhin sozio-ökonomisch belastet waren, aber sozio-emotional besser miteinander zurechtkamen und daher in der Alltagsbewältigung relativ kompetent erschienen (Typ 3, siehe dazu die Fallbeschreibungen in Kapitel 2 sowie die fokussierte Analyse in Kapitel 3). Diese jungen Erwachsenen benötigten nicht nur in früheren Jahren ihres Aufwachsens, wenn auch auf unterschiedlichen Ebenen und mit unterschiedlicher Intensität und auch unterschiedlichem Erfolg, die Unterstützung außerfamilialer Einrichtungen, wie etwa der Kinder- und Jugendfürsorge und eine damit verbundene zeitweilige oder auch andauernde Unterbringung und Unterstützung in einer betreuten Wohneinrichtung oder auch die Aufnahme in einer geschützten Werkstatt. Im jungen Erwachsenenalter waren diese jungen Erwachsenen auf die Aufmerksamkeit und Übernahme von Verantwortung durch Arbeitgeber, den Einsatz eines Gemeindebürgermeisters, die Hilfe eines Bekannten aus einem Verein oder auch die Möglichkeit, eine Lehre im Rahmen des Erasmus+ Programms der Europäischen Union zu absolvieren, angewiesen. Eine wichtige Rolle 
bei der Suche nach einer passenden Lehrstelle spielte mehrfach auch der Arbeitsmarktservice der Republik Österreich (AMS), dessen Konzeption mit der Agentur für Arbeit in Deutschland vergleichbar ist. Für alle diese jungen Männer und Frauen waren dies allerdings keine vorhersagbaren Wege zu einer persönlichen Verbesserung der Lebensumstände, nötig war vielmehr eine geglückte individuelle Passung, das heißt zur rechten Zeit auch die nötige Unterstützung zu erhalten, um auch ohne elterliche Hilfe eigene Wege beschreiten zu können. Auffällig ist, dass alle jungen Männer und Frauen aus den >Aufsteiger-Familien (Typ 4) eine Sonderrolle im Panel einnehmen. Ihnen gelang es nicht nur, ihre schon früh geäußerten Ziele in der Schule und damit verbundene weitere (Aus-)Bildungswege, stets mit Unterstützung ihrer Eltern, anzustreben, sondern auch den Weg tatsächlich in eine Lehre, eine weiterführende Ausbildung bzw. ein Studium oder eine gewünschte Arbeitsstelle zu erreichen, ohne dafür an irgendeiner Stelle ihres Lebens eine Vermittlungshilfe von Einrichtungen oder Personen außerhalb der Familie zu benötigen. Insgesamt muss jedoch gesagt werden, dass eine, wie von Arnett für die Vereinigten Staaten von Amerika identifizierte Perspektivlosigkeit, wenn nicht gar Hoffnungslosigkeit junger Menschen aus „lower social classes“ (Arnett 2016: 227, siehe dazu Kapitel 1) bei ihren Bemühungen um den Einstieg in den Arbeitsmarkt für die jungen Erwachsenen im Panel nicht in gleichem Maße festgestellt werden konnte. Dennoch sind auch viele junge Erwachsene aus Österreich - und dies insbesondere die aus Typ 1 und 3, aber auch Typ 2, wie die (Medien-)Sozialisationsstudie zeigt, auf Unterstützung und - dies nicht zuletzt - auf eine glückliche Passung angewiesen, um Zugang zu einer Ausbildungs- und späteren Arbeitsstelle zu bekommen, die tatsächlich ihren Neigungen und Kompetenzen entspricht.

\subsection{Die jungen Erwachsenen und ibr Mediengebrauch}

In den ersten sechs Erhebungswellen diente der Mediengebrauch den Kindern und Jugendlichen als unmittelbar wichtiges Orientierungsangebot; die Medienfiguren und -protagonisten und -protagonistinnen gar als Vorbild und als symbolische Stellvertreter und Stellvertreterinnen, als direkte, gut erreichbare Gefährten und Gefährtinnen in der Strukturierung des eigenen Alltags. In einigen Fällen avancierten Medien gar zu einer Art Kompensationshilfe in einem mehrfach belasteten (Familien-)Alltag bzw. als willkommene Mittel zum Füllen von Leer-Stellen, etwa dann, wenn die Kinder versuchten, mit ihrer Hilfe Defizite in unmittelbaren Eltern-, 
Geschwister- oder auch Peer-Beziehungen auszugleichen. Medien nahmen damit in der Sozialisation von Heranwachsenden geradezu Lehr-Stellen ein und dienten allen voran in den ersten beiden Erhebungswellen den seinerzeitigen Kindergarten- und Grundschulkindern als maßgebliche Kontexte ihrer Sozialisation.

Wenn auch die Mediatisierung des Alltags weiter zugenommen hat und insbesondere digitale Medien in einem besonders hohen Maße schon während der ersten Phase der Covid-19-Pandemie im Frühjahr und beginnenden Sommer 2020 eine in vorher nie dagewesener Weise alltagsstrukturierende Rolle spielen, zeigt sich mit Blick auf die jungen Erwachsenen dennoch in der siebten Erhebungswelle kein genereller Anstieg ihrer Mediennutzung. Vielmehr lassen die jungen Erwachsenen einen in jeder Hinsicht stark individuell geprägten Mediengebrauch erkennen, der in unmittelbarem Zusammenhang mit ihrem aktuellen Lebensalltag steht. Dieser Befund lässt darauf schließen, dass der Mediengebrauch der jungen Erwachsenen - wie auch schon in ihrer Kindheit und Jugend - im Kontext ihrer jeweils zur Bewältigung anstehenden Entwicklungs- bzw. Lebensaufgaben zu verorten ist und nunmehr zum einen im Kontext ihrer Aus- und Weiterbildung, ihrer Lehre und ihrem Einstieg in den Arbeitsalltag, und zum anderen ihrer privaten Partnerschafts- und Freundschaftsbeziehungen steht. Das Medienrepertoire der jungen Menschen richtet sich klar nach ihren alltäglich anstehenden beruflichen und privaten Herausforderungen. Und wie auch in den vorherigen Erhebungswellen wird der jeweilige Mediengebrauch auch der jungen Erwachsenen durch ihre spezifischen sozio-ökonomischen und sozio-emotionalen Bedingungen grundiert - etwa indem bestimmte kostspielige Angebote nicht genutzt werden können oder gerade auf eine nahezu exzessive Medienausstattung Wert gelegt wird, um auf diese Weise Sozialprestige im Freundeskreis zu generieren. Insgesamt lassen die Mediengebrauchsweisen der jungen Menschen dennoch auch in der siebten Erhebungswelle keinen linearen Zusammenhang zu den Familientypen erkennen. Dies gilt nun umso mehr, als die Eltern der jungen Erwachsenen auf den Medienumgang ihrer nunmehr volljährigen Kinder keinen unmittelbaren Einfluss mehr nehmen und Medien zwischen den Kindern und ihren Eltern weniger denn je überhaupt Thema sind. Wie auch in den vorherigen Erhebungswellen zeigt sich in der siebten Erhebungswelle in den Medienumgangsweisen der Familien, den (Medien-)Erziehungsweisen der Eltern sowie dem Mediengebrauch der Kinder selbst kein direkter Einfluss. Die Mediengebrauchsweisen von Kindern und Eltern, auch im Zusammenleben als Familie, ihrem doing family, konnten jedoch in den ersten sechs Erhebungswellen stärker als nunmehr 
als ein Ausdruck der Bewältigung spezifischer sozio-ökonomischer und sozio-emotionaler Herausforderungen angesehen werden, als dies bei den jungen Erwachsenen der Fall ist. Dennoch wurden diese Aspekte auch in der siebten Erhebungswelle mitreflektiert und, wo (noch) relevant, benannt (siehe dazu Kapitel 2).

Stärker als in den vorherigen Erhebungswellen lassen die jungen Erwachsenen nunmehr einen individuellen Mediengebrauch erkennen, der sich klar nach ihren spezifischen Bedürfnissen und Interessen, ihrer zur Verfügung stehenden Zeit und ihren sozialen Beziehungen richtet und der deutlich geprägt ist von ihren beruflichen und privaten Herausforderungen und Lebenssituationen; in sie webt sich die Mediennutzung mit ein. Mit Blick auf die Veränderungen zwischen der Jugendphase und der Phase des jungen Erwachsenalters wird deutlich, dass die Befragten aber insgesamt kritischer als in den Jahren zuvor mit Medien umgehen und ihre Mediennutzung stärker reflektieren. So schätzen einige junge Erwachsene, vor allem junge Frauen, aber auch einige wenige junge Männer, ihren Mediengebrauch explizit als „zu hoch“ ein und betonen, ihn reduzieren zu wollen, da er „vom Eigentlichen“ ablenke. Besonders mit Blick auf den Umgang mit Sozialen Medien ${ }^{56}$ fällt auf, dass Angebote im Sozialen Netz bei den jungen Erwachsenen keinen großen Stellenwert mehr einnehmen. Zwar nutzen auch die jungen Erwachsenen diese Angebote weiterhin, allen voran die Messenger-Dienste WhatsApp und Facetime sowie Instagram, mit Abstrichen aber auch Snapchat und Facebook sowie gerade in der Covid-19-Pandemie auch Angebote wie Houseparty - und dies nahezu habituell -, aber einen besonderen Stellenwert messen sie ihnen in ihrem Alltag nicht mehr zu, so wie dies noch einige der Jugendlichen in den vorherigen Erhebungswellen, insbesondere der fünften und sechsten, in besonderer Weise getan haben. Dieser Befund entspricht den Ergebnissen der Studie Heranwachsen mit dem Social Web (Hasebrink/ Paus-Hasebrink/ Schmidt 2010). In beiden Studien stechen vor allem Jugendliche im Alter von 15 bis 17 Jahren (in der fünften und sechsten Erhebungswelle) hervor;

56 In diesem Zusammenhang ist daran zu erinnern, dass Soziale Medien in den ersten Erhebungswellen der Panelfamilien noch keine Rolle spielten, ganz anders als sich dies bei den Kindern im Kindergarten- und insbesondere im Grundschulalter heute darstellt. Da es sich bei der Sozialisationsstudie nicht um eine Kohortenstudie handelt, kann dieses Phänomen fortgeschrittener Mediatisierung im Kinderalltag - dies eine Limitierung der Studie - nicht durch eigene Daten belegt werden; siehe dazu die Daten der KIM-Studie 2020 sowie die miniKIM-Studie 2014 des Medienpädagogischen Forschungsverbundes Südwest (MPFS 2014, 2020). 
für sie spielt das Soziale Netz eine enorm wichtige Rolle, und dies vor allem für die Kommunikation, aber auch - insbesondere bei den Jungen - für Online-Spiele. Für die jungen Erwachsenen nehmen dagegen die direkten Freundschaftskontakte in Form persönlicher Treffen und gemeinsamer Unternehmungen eine größere Bedeutung ein als der nach wie vor bestehende Kontakt über Soziale Medien-Kanäle. Dieser Befund zeigt sich in der siebten Erhebungswelle insbesondere bei den jungen Männern. Bei den jungen Frauen sticht hervor, dass sie sich in ihrer jeweiligen Medienauswahl, der Zusammenstellung ihres Medienrepertoires und der konkreten Mediennutzung stark nach ihren Partnern richten. Allerdings sind auch einige junge Männer, die in festen Partnerschaften leben, bereit, in Bezug auf ihre Medienaktivitäten auf die Interessen ihrer Freundinnen Rücksicht zu nehmen und sich ihnen anzupassen. Wie bereits auch in der Studie Heranwachsen mit dem Social Web festzustellen war, betätigen sich nur sehr wenige junge Menschen der Sozialisationsstudie aktiv als Producer. Auffällig ist dabei insbesondere eine junge Frau, die mit drei Freunden einen eigenen Kanal auf Twitch.tv betreibt. ${ }^{57}$ Computerspiele genießen bei den jungen Erwachsenen, insbesondere den jungen Männern, weiterhin einen hohen Stellenwert in ihrem Mediengebrauch. Allerdings finden sich in der siebten Erhebungswelle bis auf einige wenige Ausnahmen kaum mehr junge Männer, die sich - wie noch in den beiden Erhebungswellen zuvor - in Online-(Spiele-)Welten zurückziehen. Der Beginn einer Lehre bzw. einer Ausbildung geht mit einer tendenziellen Verschiebung von Prioritäten im Alltag einher. Ehemalige Leer-Stellen, seinerzeit von medialen Angeboten besetzt, werden nunmehr durch neue Handlungsoptionen, etwa die Chance auf eine Verstetigung eines Arbeitsverhältnisses bzw. auf eine Weiterbildung in einer Firma oder auch (neue) Freundschaften und Partnerschaften, gefüllt.

Zusammenfassend lässt sich mit Blick auf den Mediengebrauch der jungen Erwachsenen sagen, dass weniger die sozio-ökonomischen Bedingungen als vielmehr die mit der Lebensphase der jungen Menschen im $\mathrm{Zu}$ sammenhang stehende Auseinandersetzung und Bewältigung ihrer jeweiligen Entwicklungs- bzw. Lebensaufgaben, der Weg in den Beruf und damit einhergehend auch die finanzielle Eigenständigkeit, das Sicht-Einfinden in eine Partnerschaft und in diesem Zusammenhang auch oft der Bezug einer gemeinsamen Wohnung, wie sie in Kapitel 1 als zentrale Merkmale der Sozialisation junger Erwachsener beschrieben sind, den Mediengebrauch prägen.

57 Siehe dazu die Fallbeschreibung von Gudrun Dornbacher (Typ 4) in Kapitel 2.5. 


\subsection{Gesellschaftliche Konsequenzen aus der Panel-Langzeitstudie}

Die vorliegende Studie zeigt, dass gesellschaftlicher Handlungsbedarf auf vielfältigen Ebenen besteht und dass es einer großen, nicht nur finanziellen Anstrengung der Gesellschaft bedarf, um Kindern in sozial benachteiligten Familien das ihnen - wie anderen Kindern und Jugendlichen zustehende Recht auf Entwicklung, Integration und Partizipation zu gewährleisten, bzw. dass es überfällig ist, Konstellationen zu schaffen, die die Einlösung dieses Rechtsanspruchs erst ermöglichen. „Denn obwohl sich vor allem Eltern aus niedrigen Einkommensgruppen und Migrantenfamilien Aufstiegschancen für ihre Kinder erhoffen, erleben sie häufiger, dass sie ihr Kind zu Hause nicht ausreichend fördern können“ (Walper/ Müller 2021, S. 47). Nötig sei es daher, wie Walper und Müller mit Blick auf den Neunten Familienbericht des Deutschen Bundesfamilienministeriums hervorheben, „Unterstützungsangebote für Familien im gesamten Bildungsverlauf der Kinder zu etablieren, beispielsweise über Familienzentren an Schulen. Außerdem sollten multiprofessionelle Teams an Schulen mit Sozialarbeiterinnen und -arbeitern sowie Gesundheitsfachkräften die Vermittlung insbesondere gesundheitsbezogener, digitaler, sozialer und persönlicher Kompetenzen der Kinder fördern“ (ebd.). Wie die Ergebnisse der siebten Erhebungswelle der (Medien-)Sozialisationsstudie deutlich zeigen, ist es sowohl auf staatlicher als auch auf zivilgesellschaftlicher Ebene überfällig, umfassende Integrationskonzepte zur Unterstützung und Begleitung für junge Menschen zu entwickeln, in deren Umsetzung alle relevanten Anspruchsgruppen $^{58}$ - von den Kindergärten und Schulen über Familienämter und Einrichtungen des Kindes- und Jugendwohls bis hin zu Jugendhilfe- und zu Elternbildungseinrichtungen - miteinbezogen werden müssen. Von zentraler Bedeutung ist es, jungen Menschen - und dies vor allem, wenn sie in ihren Elternhäusern aufgrund erschwerter sozio-ökonomischer, aber auch sozio-emotionaler Bedingungen in besonderer Weise darauf angewiesen sind - nach der Schule den Weg zu einer Ausbildung oder Lehre oder zu einem Studium zu ermöglichen bzw. sie zu beraten und zu unterstützen, damit es ihnen gelingt, einen ihren Handlungsentwürfen und Handlungskompetenzen entsprechenden beruflichen Weg zu beschreiten. So braucht es beispielsweise Mentoring-Einrichtungen, die als Brückenbauer

58 Als derartige Anspruchsgruppen bzw. Stakeholder gelten lokale und nationale Behörden, Verbände und Interessen-Lobbys, politische Parteien, Bürgerinitiativen sowie die allgemeine Öffentlichkeit, aber auch ausländische und internationale Organisationen (siehe dazu Murdock 2010). 
zwischen Schule, Weiterbildungseinrichtungen und Betrieben fungieren können und die als Anlauf- und Beratungsstellen für junge Menschen agieren. ${ }^{59}$

Es bedarf einer gesteigerten Aufmerksamkeit und eines deutlich stärkeren Verantwortungsbewusstseins der Gesellschaft, um Voraussetzungen für die Multi-Optionalität zu schaffen, die nötig ist, jungen Menschen, und nicht zuletzt sozial benachteiligten jungen Menschen - auch und gerade in Krisenzeiten, wie sie die Fluchtbewegung 2015 und 2016 sowie die Covid-19-Pandemie 2020 und 2021 darstellen - die ihnen zustehenden Partizipationsmöglichkeiten zu gewährleisten. Damit verbunden sind große staatliche, aber auch zivilgesellschaftliche Herausforderungen - Aufgaben, die sich für die Zukunft nicht nur junger Menschen lohnen, sondern die nötig sind, um den gesellschaftlichen Zusammenhalt ${ }^{60}$ auch in hoch entwickelten und verhältnismäßig reichen Ländern wie Deutschland und Österreich zu ermöglichen. Denn, wie Deitelhoff et al. (2020: 23) mit Bezug auf Reckwitz (2017) zu bedenken geben, fordert

die Zunahme von ökonomischen und sozialen Ungleichheiten den Zusammenhalt in besonderer Weise heraus, indem sie sozialen Vergleich, Statuskonkurrenz und Statusstress befördert und damit möglicherweise Solidarität und wechselseitige Verständigung zwischen den auseinanderdriftenden Bevölkerungsgruppen (erschwert). Diese soziostrukturellen Makroentwicklungen rahmen soziale Beziehungen und Praktiken auf der Mikroebene, die Formen sozialen Zusammenhalts generieren.

Vor diesem Hintergrund erscheint es notwendig, das Phänomen soziale Ungleichheit - und dies insbesondere mit Blick auf ihre Folgen für Sozialisation junger Menschen - nicht nur ernst zu nehmen, sondern soziale Ungleichheit auch politisch zu bekämpfen und damit Kindern und Jugendli-

59 Siehe dazu etwa die Social-Start-up-Einrichtung Sindbad; sie hat sich zum Ziel gesetzt, Jugendliche beim Übergang von der Pflichtschule in die weiterführende Ausbildung zu unterstützen und richtet ihr Augenmerk dabei insbesondere auf die Unterstützung und Begleitung sozial benachteiligter junger Menschen (Sindbad 2020).

60 Siehe zur Entstehung und Problematisierung der „neuen gesellschaftlichen Leitvokabel“ bzw. des „neuen politischen Leitbegriffs „Gesellschaftlicher Zusammenhalt" den Forschungsaufriss von Deitelhoff et al. (2020: 9f.) sowie die entsprechende Publikation des Forschungsinstituts Gesellschaftlicher Zusammenhalt (https://www.fgz-risc.de/) und dazu auch die Diskussion um die Rolle von Medien (Hasebrink et al. 2020). 
chen und nicht zuletzt jungen Erwachsenen, die besondere Unterstützung bei ihren ersten Schritten in eine berufliche und, damit eng verbunden, auch in ihre private Zukunft benötigen, die ihnen zustehenden gleichen Partizipationschancen auf allen Ebenen der Gesellschaft zu ermöglichen. Dazu zählt auch - und nicht zuletzt - die Stärkung der Handlungskompetenz junger Menschen und in diesem Kontext die vom Kindergarten an notwendige Förderung eines kritisch-kompetenten Mediengebrauchs junger Menschen. 


\section{Literaturverzeichnis}

Aigelsreiter, Hannes (2020): Berichterstattung im Ausnahmezustand. In: Medienqualität in Zeiten von Corona. Public Value Texte 24. Wien: ORF, S. 37-39.

Alikhani, Behrouz (2020): Sozialisierung aus figurationssoziologischer Perspektive. In: Grundmann/ Matthias/ Höppner, Grit (Hrsg.): Dazwischen - Sozialisationstheorien reloaded. Weinheim und Basel: Juventa, S. 106-124.

Alkemeyer, Thomas (2020): Soziale Ordnungen und ihre Subjekte. Überlegungen zum Verhältnis von Sozialisation und praxeologischer Subjektivierungstheorie 1. In: Grundmann/ Matthias/ Höppner, Grit (Hrsg.): Dazwischen - Sozialisationstheorien reloaded. Weinheim und Basel: Juventa, S. 81-105.

Arnett, Jeffrey, J. (2000): Emerging Adulthood: A Theory of Development from the Late Teens through the Twenties. In: American Psychologist, 55. Jg., H. 5, S. 469-480.

Arnett, Jeffrey, J. (2004): Emerging Adulthood: The Winding Road from Late Teens Through the Twenties. Oxford: Oxford University Press.

Arnett, Jeffrey J. (2016): Does Emerging Adulthood Theory Apply across Social Classes? National Data on Persistent Questions. In: Emerging Adulthood, 4. Jg., H. 4, S. 227-235.

Aufenanger, Stefan (2008): Mediensozialisation. In: Sander, Uwe/von Gross, Friederike/Hugger, Kai-Uwe (Hrsg.): Handbuch Medienpädagogik. Wiesbaden: VS Verlag, S. 87-92.

Baumrind, Diana (1991): Effective Parenting During the Early Adolescent Transition. In: Cowan, Philip, A./ Hetherington, Ellen, M. (Hrsg.): Family Transitions. New York, NY: Routledge, S. 111-163.

Beck, Klaus/ Büser, Till/ Schubert, Christiane (2016): Mediengenerationen. Biografische und kollektivbiografische Muster des Medienhandelns. Konstanz: UVK.

Berger, Peter L./ Luckmann Thomas (1967): The Social Construction of Reality. A Treatise in the Sociology of Knowledge. London: Penguin Group.

Bettinger, Patrick/ Hugger, Kai-Uwe (2020): Praxistheorien in der Medienpädagogik - Einleitung. In Bettinger, Patrick/ Hugger, Kai-Uwe (Hrsg.): Praxistheorien in der Medienpädagogik Wiesbaden: Springer VS, S. 1-18.

Blom, Annelies G. (2020): Zum gesellschaftlichen Umgang mit der Corona-Pandemie. Ergebnisse der Mannheimer Corona-Studie. In: Aus Politik und Zeitgeschichte, H. 35-37/2020. Bonn: bpb. Online unter: https://www.bpb.de/apuz/31 4345/zum-gesellschaftlichen-umgang-mit-der-corona-pandemie (abgerufen am 25.06.2021). 


\section{Literaturverzeichnis}

Blom, Annelies G./ Wenz, Alexander/ Rettig, Tobias/ Reifenscheid, Maximilian/ Naumann, Elias/ Möhring, Katja, Lehrer, Roni/ Krieger, Ulrich/ Juhl, Sebastian/ Friedel, Sabine/ Fikel, Marina/ Cornesse, Carina (2020): Die Mannheimer Corona-Studie: Das Leben in Deutschland im Ausnahmezustand. Bericht zur Lage vom 20. März bis 09. Juli 2020. Mannheim: Universität Mannheim. Online unter: https://www.uni-mannheim.de/media/Einrichtungen/gip/Corona_Studie /10-07-2020_Mannheimer_Corona-Studie_-_Bericht_zur_Lage_in_den_Tagen_2 0_Mrz-09_Jul_2020.pdf (abgerufen am 25.06.2021).

Böhnisch, Lothar/ Lenz, Karl (2015): Erwachsenensozialisation. In: Hurrelmann, Klaus/ Bauer, Ullrich/ Grundmann, Matthias/ Walper, Sabine (Hrsg.): Handbuch Sozialisationsforschung. Weinheim und Basel: Beltz, S. 871-884 (8. Auflage).

Bonacini, Luca/ Gallo, Giovanni/ Scicchitano, Sergio (2021): Working from Home and Income Inequality: Risks of a 'New Normal' with COVID-19. In: Journal of Population Economics, 34. Jg., S. 303-360.

Bourdieu, Pierre (1977): Outline of a Theory of Practice. New York, NY: Cambridge University Press.

Bourdieu, Pierre (1982): Die feinen Unterschiede. Kritik der gesellschaftlichen Urteilskraft. Frankfurt/Main: Suhrkamp.

Bourdieu, Pierre (1989): Social Space and Symbolic Power. In: Sociological Theory, 7. Jg., H. 1, S. 14-25.

Bourdieu, Pierre (1990): Die Biographische Illusion. In: BIOS - Zeitschrift für Biographieforschung, Oral History und Lebensverlaufsanalysen, 3. Jg, H. 1, S. 7581.

Bourdieu, Pierre (1996): Distinction: A Social Critique of the Judgement of Taste. London: Routledge (8. Auflage).

Bovill, Moira/ Livingstone, Sonia (2001): Bedroom Culture and the Privatization of Media Use. LSE Research Online. London: LSE. Online unter: http://eprints.lse. ac.uk/archive/00000672 (abgerufen am 24.06.2021).

Braun, Marlies/ Kirchner, Stefanie/ Niederkrotenthaler, Thomas/ Till, Benedikt (2020): SARS CoV-2: Mental Health in Österreich. Ausgewählte Ergebnisse zur vierten Befragungswelle, 26.06.-08.07.2020. Wien: MedUniWien. Online unter: http://www.suizidforschung.at/wp-content/uploads/2020/10/Bericht-Welle-4.pdf (abgerufen am 25.06.2021).

Brüggen, Niels/ Dohle, Marco/ Kelm, Ole/ Müller, Eric (Hrsg.) (2021): Flucht als Krise? Flucht, Migration und Integration in den Medien sowie die themenbezogene Aneignung durch Heranwachsende. München: KoPäd.

Charlton, Michael/ Neumann, Klaus (1986): Medienkonsum und Lebensbewältigung in der Familie. Methode und Ergebnisse der strukturanalytischen Rezeptionsforschung - mit fünf Fallbeispielen. München und Weinheim: Psychologie Verlags Union.

Dahlgren, Peter (2000a): Media, Citizens and the Civic Culture. In: Gurevitch, Michael/ Curran, James (Hrsg.): Mass Media and Society. London: Edward Arnold, S. 310-328. 
Dahlgren, Peter (2000b): The Internet and the Democratization of Civic Culture. In: Political Communication, 17. Jg., H. 4, S. 335-340.

De Coninck, David/ Ogan, Christine/ d'Haenens, Leen (2021): Can 'the Other' ever become 'One of Us'? Comparing Turkish and European Attitudes toward Refugees: A Five-Country Study. In: The International Communication Gazette, 83. Jg., H. 3, S. 217-237.

Deitelhoff Nicole/ Groh-Samberg, Olaf/ Middell, Matthias/ Schmelzle, Cord (2020): Gesellschaftlicher Zusammenhalt - Umrisse eines Forschungsprogramms. In: Deitelhoff Nicole/ Groh-Samberg, Olaf/ Middell, Matthias (Hrsg.): Gesellschaftlicher Zusammenhalt. Ein interdisziplinärer Dialog. Frankfurt am Main und New York, NY: Campus Verlag, S. 9-40.

Dresing, Thorsten/ Pehl, Thorsten (2015): Praxisbuch Interview, Transkription \& Analyse. Anleitungen und Regelsysteme für qualitativ Forschende. Marburg: Audiotranskription (6. Auflage).

Elias, Norbert (1987): Engagement und Distanzierung. Arbeiten zur Wissenssoziologie I. Frankfurt am Main: Suhrkamp.

Elias, Norbert (2006): Was ist Soziologie? Gesammelte Schriften. Herausgegeben von Blomert, Reinhard/ Hammer, Heike/ Heilbron, Johan/ Treibel, Annette/ Wilterdink, Nico. Band 5. Frankfurt am Main: Suhrkamp.

Flick, Uwe (2005): Wissenschaftstheorie und das Verhältnis qualitativer und quantitativer Forschung. In: Mikos, Lothar/ Wegener, Claudia (Hrsg.): Qualitative Medienforschung. Ein Handbuch. Konstanz: UVK - UTB, S. 20-28.

Flick, Uwe (2013): Qualitative Sozialforschung. Ein Handbuch. Reinbek bei Hamburg: Rowohlt.

Flimmo-Kinderbefragung (2016): Wie informieren sich Kinder über das Thema Flucht? München: Flimmo.

Fortuin, Janna/ van Geel, Mitch/ Vedder, Paul (2016): Peers and Academic Achievement: A Longitudinal Study on Selection and Socialization Effects of in-class Friends. In: The Journal of Educational Research, 109. Jg., H. 1, S. 1-6.

Friedrichs; Jürgen/ Leßke, Felix/ Schwarzenberg, Vera (2019): Die Akzeptanz von Flüchtlingen. Eine vergleichende Studie sechs deutscher Wohngebiete. In: Raumforschung und Raumordnung | Spatial Research and Planning, 22. Jg., H. 4, S. 349-366.

Fromme, Johannes (2007): Sozialisation in einer sich wandelnden Mediengesellschaft. In: Lauffer, Jürgen/ Röllecke, Renate (Hrsg.): Dieter Baacke Preis - Handbuch 2. Mediale Sozialisation und Bildung. Bielefeld: GMK, S. 12-29.

Giddens, Anthony (1984): Die Konstitution der Gesellschaft. Grundzüge einer Theorie der Strukturierung. Frankfurt am Main: Suhrkamp.

Gniewosz, Burkhard/ Noack, Peter (2015): Parental Influences on Adolescents' Negative Attitudes Towards Immigrants. In: Journal of Youth and Adolescence, 44. Jg., H. 9, S. 1787-802.

Goetz, Maja/ Holler, Andrea (2016): Wie Kinder und Jugendliche das Thema Geflüchtete verstehen. Eine Studienreihe zu Wissen, Mediennutzung, Emotionalen Einstellungen und Emotionen. In: TelevIZIon, 29. Jg., H. 2, S. 4-10. 


\section{Literaturverzeichnis}

Griese, Birgit/ Schiebe, Martina (2018): Bourdieu und die Biographieforschung. In: Lutz, Helma/ Schiebel, Martina/ Tuider, Elisabeth (Hrsg.): Handbuch Biographieforschung. Wiesbaden: Springer VS, S. 115-126 (2. Auflage).

Großegger, Beate (2019): Zielgruppe „BerufseinsteigerInnen“. Der erste Job: Herausforderung für junge Menschen und ihre ArbeitgeberInnen. Wien: Institut für Jugendkulturforschung. Online unter: https://jugendkultur.at/wp-content /uploads/Zielgruppe_Berufseinsteiger_Grossegger_2019.pdf (abgerufen am 25.06.2021).

Großegger, Beate (2020a): Generation Corona: Rückzug vom Rückzug? Kommentar von Dr. Beate Großegger zuraktuellen Eigenstudiedes Instituts für Jugendkulturforschung. Wien: Institut für Jugendkulturforschung. Online unter: https://jugendkultur.at/wp-content/uploads/Kommentar_Generation_Corona_ Gro\%C3\%9Fegger2020.pdf (abgerufen am 25.06.2021).

Großegger, Beate (2020b): Alles sehr unklar und neu... Corona-News aus Sicht des jungen weiblichen Publikums. In: Medienqualität in Zeiten von Corona. Public Value Texte 24. Wien: ORF, S. 5-13.

Großegger, Beate (2020c): Junge „News-Avoider/innen“ als Zielgruppe. In: Informationsdeprivation \& News-Avoiding. Eine Herausforderung für Demokratie und öffentlich-rechtliche Medien. Publik Value Studie. Wien: ORF, S. 67-97.

Grundmann, Matthias (2015): Das Modell von Sozialisation als Beziehungspraxis. In: Hurrelmann, Klaus/ Bauer, Ullrich/ Grundmann, Matthias/ Walper, Sabine (Hrsg.): Handbuch Sozialisationsforschung. Weinheim und Basel: Beltz, S. 162179 (8. Auflage).

Grundmann, Matthias/ Höppner, Grit (2020): Einleitung: Aspekte einer transtheoretischen Bestimmung von Sozialisation. In: Grundmann, Matthias/ Höppner, Grit (Hrsg.): Dazwischen - Sozialisationstheorien reloaded. Weinheim/ Basel Juventa, S. 7-16.

Guglhör-Rudan, Angelika (2012): Zwischen Elternhaus und Partnerschaft: Kulturvergleichende Analysen von Skalen zu Autonomie und Verbundenheit junger Erwachsener in Deutschland, Italien und Schweden. Inaugural-Dissertation zur Erlangung des Doktorgrades der Philosophie an der Ludwig-Maximilians-Universität München: LMU. Online unter: https://edoc.ub.uni-muenchen.de/17741/ 2/Guglhoer-Rudan_Angelika.pdf (abgerufen am 24.06.2021).

Habermas, Jürgen (1988): Theorie des kommunikativen Handelns. 2. Band. Frankfurt am Main: Suhrkamp (Nachdruck der 4. durchgesehenen Auflage).

Habermehl, Werner (1992): Angewandte Sozialforschung. München: Oldenbourg Verlag.

Hasebrink, Uwe (2013): Modi audiovisueller Kommunikation. In: Wijnen, Christine/ Trültzsch, Sascha/ Ortner, Christina (Hrsg.): Medienwelten im Wandel. Kommunikationswissenschaftliche Positionen, Perspektiven und Konsequenzen. Wiesbaden: VS Verlag, S. 55-70. 
Hasebrink, Uwe (2014): Die kommunikative Figuration von Familien: Medien, Kommunikation und Informationstechnologie im Familienalltag. In: Rupp, Martina/ Kapella, Olaf/ Schneider, Norbert F. (Hrsg.): Die Zukunft der Familie. Anforderungen an Familienpolitik und Familienwissenschaft. Opladen: Verlag Barbara Budrich, S. 225-239.

Hasebrink, Uwe/ Domeyer, Hanna (2012): Media Repertoires as Patterns of Behaviour and as Meaningful Practices. A Multimethod Approach to Media Use in Converging Media Environments. In: Participations. Journal of Audience \& Reception Studies, 9. Jg., H. 2, S. 757-779.

Hasebrink, Uwe/ Paus-Hasebrink, Ingrid/ Schmidt, Jan-Hinrik (2010): Das Social Web in den Medienrepertoires von Jugendlichen und jungen Erwachsenen. In: Hepp, Andreas/ Höhn, Marco/ Wimmer, Jeffrey (Hrsg.): Medienkultur im Wandel. Konstanz: UVK, S. 331-347.

Hasebrink, Uwe/ Schmidt, Jan-Hinrik/ Loosen, Wiebke/ Schulz, Wolfgang (2020): Medien und gesellschaftlicher Zusammenhalt. In: Deitelhoff, Nicole/ GrohSamberg, Olaf/ Middell, Matthias (Hrsg.): Gesellschaftlicher Zusammenhalt. Ein interdisziplinärer Dialog. Frankfurt am Main und New York, NY: Campus Verlag, S. 333-348.

Havighurst, Robert J. (1948): Developmental Tasks and Education. New York, NY: Longmans, Green \& Company (1. Auflage).

Havighurst, Robert J. (1972): Developmental Tasks and Education. New York, NY: McKay (3. Auflage).

Heckhausen, Jutta/ Schulz, Richard (1999): Biological and Societal Canalizations and Individuals' Developmental Goals. In: Brandtstädter, Jochen/ Lerner, Richard (Hrsg.): Action and Self-Development: Theory and Research Through the Life-Span. Thousand Oaks, CA: Sage, S. 67-103.

Heinz, Walter, R. (2003): From Work Trajectories to Negotiation Careers. The Contingent Life Work Course. In: Mortimer, Jeylan, T/ Shanahan, Michael, J. (Hrsg.): Handbook of the Life Course. New York, NY: Kluwer Academic Plenum, S. 185-204.

Heinzlmair, Bernhard (2020): Eine Generation im Bildersturm. Über das Mediennutzungsverhalten der Jugend. In: Gallner-Holzmann, Katharina/ Hug, Theo/ Pallaver, Günther (Hrsg.): Jugendliche Mediennutzung und die Zukunft des Qualitätsjournalismus. Innsbruck: Innsbruck University Press, S. 117-126.

Heismann, Christiane (2012): Persönliche Ziele im frühen Erwachsenenalter. Eine längsschnittliche Analyse. Inaugural-Dissertation an der Philosophischen Fakultät und Fachbereich Theologie der Friedrich-Alexander-Universität. Erlangen und Nürnberg: FAU. Online unter: https:/opus4.kobv.de/opus4-fau/files/2783/ ChristianeHeismannDissertation (abgerufen am 24.06.2021).

Hepp, Andreas/ Hasebrink, Uwe (2014): Human Interaction and Communication Figurations. In Lundby, Knut (Hrsg.): Mediatization of Communication. Handbook of Communication Science. Berlin: Walter de Gruyter, S. 249-271.

Herrmann, Friederike (2015): Das Märchen vom überkochenden Brei - Narrative in der medialen Berichterstattung zum Flüchtlingsthema im Herbst 2015. In: Communicatio Socialis, 49. Jg., H. 1, S. 6-20. 


\section{Literaturverzeichnis}

Herrmann, Friederike (2016): Von der Willkommenskultur zum Problemdiskurs: Wie Medien zwischen April 2015 und Juli 2016 über Flüchtlinge berichteten. In: merz - medien + erziehung, 60. Jg., H. 5, S. 12-19.

Hofer, Manfred/ Pikowsky, Birgit (2002): Familien mit Jugendlichen. In: Hofer, Manfred/ Wild, Elke/ Noack, Peter (Hrsg.): Lehrbuch Familienbeziehungen. Göttingen: Hogrefe, S. 241-264.

Hoffmann, Dagmar/ Krotz, Friedrich/ Reißmann, Wolfgang (Hrsg.) (2017): Mediatisierung und Mediensozialisation. Prozesse - Räume - Praktiken. Wiesbaden: VS Verlag.

Holler, Andrea (2016): Geflüchtete im TV: Wahrnehmung und Wünsche aus Kinder- und Jugendsicht. In: TelevIZIon, 29. Jg., H.2, S. 17-20.

Hörning, Karl H. (2001): Experten des Alltags. Die Wiederentdeckung des praktischen Wissens. Weilerswist: Velbrück.

Hradil, Stefan (1999): Soziale Ungleichheit in Deutschland. Opladen: Leske+Budrich.

Hradil, Stefan (2008): Soziale Ungleichheit, soziale Schichtung und Mobilität. In: Korte, Hermann/ Schäfers, Bernhard (Hrsg.): Einführung in die Hauptbegriffe der Soziologie. Opladen: Leske+Budrich, S. 211-234.

Huber, Stephan Gerhard/ Günther, Paula Sophie/ Schneider, Nadine/ Helm, Christoph/ Schneider, Julia A./ Pruitt, Jane (2020): COVID-19 - Herausforderungen in Schule und Bildung. Erste Befunde des Schul-Barometers in Deutschland, Österreich und der Schweiz. Münster und New York, NY: Waxman.

Huebener, Mathias/ Schmitz, Lisa (2020): Corona-Schulschließungen: Verlieren leistungsschwächere SchülerInnen den Anschluss? In: DIW aktuell. H. 30. Online unter: https://www.diw.de/documents/publikationen/73/diw_01.c.758242.d e/diw_aktuell_30.pdf (abgerufen am 25.06.2021).

Hummel, Siri/ Graf Strachwitz, Rupert (2021): Zivilgesellschaft und gesellschaftlicher Zusammenhalt. In: Aus Politik und Zeitgeschichte, H. 13-15/2021. Bonn: bpb. Online unter: https://www.bpb.de/apuz/im-dienst-der-gesellschaft-2021/ 329324/zivilgesellschaft-und-gesellschaftlicher-zusammenhalt (abgerufen am 25.06.2021).

Hurrelmann, Klaus (1990): Die Einführung in die Sozialisationstheorie. Über den Zusammenhang von Sozialstruktur und Persönlichkeit. Weinheim und Basel: Beltz (3. Auflage).

Hurrelmann, Klaus/ Bauer, Ullrich (2015): Das Modell des produktiv realitätsverarbeitenden Subjekts. In: Hurrelmann, Klaus/ Bauer, Ullrich/ Grundmann, Matthias/ Walper, Sabine (Hrsg.): Handbuch Sozialisationsforschung. Weinheim und Basel: Beltz, S. 144-161 (8. Auflage).

Hurrelmann, Klaus/ Bauer, Ullrich (2021): Einführung in die Sozialisationstheorie. Das Modell der produktiven Realitätsverarbeitung. Weinheim und Basel: Beltz Verlag (14. Auflage).

Hurrelmann, Klaus/ Bauer, Ullrich/ Grundmann, Matthias/ Walper, Sabine (Hrsg.) (2015): Handbuch Sozialisationsforschung. Weinheim und Basel: Beltz (8. Auflage). 
Hurrelmann, Klaus/ Quenzel, Gudrun (2016): Lebensphase Jugend. Eine Einführung in sozialwissenschaftliche Jugendforschung. Weinheim und Basel: Beltz Juventa (13. Auflage).

Jugendkultur.at (2021): Neue Jugendfreizeitstudie des Instituts für Jugendkulturforschung gibt Einblicke in die Freizeitbedürfnisse Jugendlicher im Lockdown. Wien: Institut für Jugendkulturforschung. Online unter: https://jugendkultur. at/wp-content/uploads/Presseinformation_Studie_Freizeit-im-Lockdown.pdf (abgerufen am 25.06.2021).

Kammerl, Rudolf/ Müller, Jane/ Lampert, Claudia /Rechlitz Marcel/ Potzel, Katrin (2020): Kommunikative Figurationen - ein theoretisches Konzept zur Beschreibung von Sozialisationsprozessen und deren Wandel in mediatisierten Gesellschaften? In: van Ackeren, Isabell/ Bremer, Helmut/ Kessl, Fabian/ Koller, Hans Christoph/ Pfaff, Nicolle/ Rotter Caroline/ Klein, Dominique/ Salaschek, Ulrich (Hrsg.): Bewegungen - Beiträge zum 26. Kongress der Deutschen Gesellschaft für Erziehungswissenschaft. Opladen: Verlag Barbara Budrich, S. 377-388.

Karmasin, Sophie/ Gangl, Katharina/ Walter, Anna/ Spitzer, Florian/ Kocher, Martin (2020): Das neue Retro? Österreich nach der Corona-Krise - wie sich unser Land verändern wird. Wien: Karmasin Research \& Identity, Insight Austria. Online unter: https://irihs.ihs.ac.at/id/eprint/5328/7/karmasin-gangl-walter-s pitzer-kocher-2020-das-neue-retro.pdf (abgerufen am 25.06.2021).

Katz, Vikki S./ Bond, Bradley J. (2021): Introduction. Children, Media, and the Clarity of Crises in 2020. In: Journal of Children and Media, 5. Jg., H. 1, S. 1-5.

Kluge, Susann (2000): Empirisch begründete Typenbildung in der qualitativen Sozialforschung. In: Forum Qualitative Sozialforschung, 1. Jg., H. 1. Online unter: http://nbn-resolving.de/urn:nbn:de:0114-fqs0001145 (abgerufen am 24.06.2021).

Knoblauch, Hubert (2008): Sinn und Subjektivität in der qualitativen Forschung. In: Kalthoff, Herbert/ Hirschauer, Stefan/ Lindemann, Gesa (Hrsg.): Theoretische Empirie. Zur Relevanz qualitativer Forschung. Frankfurt am Main: Suhrkamp, S. 210-234.

Kowal, Sabine/ O'Connell, Daniel C. (2005): Zur Transkription von Gesprächen. In: Flick, Uwe/ Kardorff, Ernst von/ Steinke, Ines (Hrsg.): Qualitative Forschung. Ein Handbuch. Reinbek bei Hamburg: Rowohlt, S. 437-447.

Krampen, Günther/ Reichle, Barbara (2008): Frühes Erwachsenenalter. In Oerter, Rolf/ Montada, Leo (Hrsg.): Entwicklungspsychologie. München und Weinheim: Psychologie Verlags Union, S. 333-365 (6. Auflage).

Krennerich, Michael (2020): Gesundheit als Menschenrecht. In: Aus Politik und Zeitgeschichte, H. 51/2020. Bonn: bpb. Online unter: https://www.bpb.de/apu z/weltgesundheit-2020/318302/gesundheit-als-menschenrecht (abgerufen am 25.06.2021).

Krinninger, Dominik (2020): Medium und Tool. Doing family und digitale Medien. In: Wiesemann, Jutta/ Eisenmann, Clemens/ Fürtig, Inka/ Lange, Jochen/ Mohn, Bina Elisabeth (Hrsg.): Digitale Kindheiten. Medien der Kooperation Media of Cooperation. Wiesbaden: Springer Nature, S. 109-128. 


\section{Literaturverzeichnis}

Krotz, Friedrich (2017): Sozialisation in mediatisierten Welten. Mediensozialisation in der Perspektive des Mediatisierungsansatzes. In: Hoffmann, Dagmar/ Krotz, Friedrich/ Reißmann, Wolfgang (Hrsg.): Mediatisierung und Mediensozialisation. Wiesbaden: Springer VS, S. 21-40.

Krotz, Friedrich/ Hepp, Andreas (2013): A Concretization of Mediatization: How Mediatization Works and Why ,Mediatized Worlds' are a Helpful Concept for Empirical Mediatization Research. In: European Journal for the Philosophy of Communication, 3. Jg., H. 2, S. 37-152.

Kübler, Hans-Dieter (2010): Medienwirkungen versus Mediensozialisation. In: Vollbrecht, Ralf/ Wegener, Claudia (Hrsg.): Handbuch Mediensozialisation. Wiesbaden: VS Verlag., S. 17-31.

Kudera, Werner (2001): Anpassung, Rückzug oder Restrukturierung - zur Dynamik alltäglicher Lebensführung in Ostdeutschland. In: Lutz, Burckart (Hrsg.): Entwicklungsperspektiven von Arbeit. Ergebnisse aus dem Sonderforschungsbereich 333 der Universität München. Berlin: Akademie Verlag, S. 46-82.

Kulterer, Jasmin/ Paus-Hasebrink, Ingrid (2017): Familientypen als Sozialisationskontexte. In: Paus-Hasebrink, Ingrid (Hrsg.): Langzeitstudie zur Rolle von Medien in der Sozialisation sozial benachteiligter Heranwachsender. Lebensphase Jugend. Lebensweltbezogene Medienforschung: Angebote - Rezeption - Sozialisation, Band 5. Baden-Baden: Nomos, S. 69-81.

Kutscher, Nadia (2017): Geflüchtete als Zielgruppe medienpädagogischer Arbeit in der digitalisierten Gesellschaft. In: Eder, Sabine/ Mikat, Claudia/ Tillmann, Angela (Hrsg.): Software takes command. München: kopaed, S. 203-217.

Kutscher, Nadia/ Kreß, Lisa-Marie (2015): Internet ist gleich mit Essen. Empirische Studie zur Nutzung digitaler Medien durch unbegleitete minderjährige Flüchtlinge. Vechta: Deutsches Kinderhilfswerk und Universität Vechta. Online unter: https://images.dkhw.de/fileadmin/Redaktion/1.1_Startseite/3_Nachrichten/Stud ie_Fluechtlingskinder-digitale_Medien/Studie_digitale_Medien_und_Fluechtlin gskinder_Langversion.pdf (abgerufen am 24.06.2021).

Kutscher, Nadia/ Kreß, Lisa-Marie (2017): Zur doppelten Funktion digitaler Medien für junge Geflüchtete. In: von Gross, Friederike/ Röllecke, Renate (Hrsg.): Medienpädagogik der Vielfalt. Integration und Inklusion. Dieter Baacke Preis Handbuch 12. München: KoPaed, S. 53-57.

Lange, Andreas (2003): Theorieentwicklung in der Jugendforschung durch Konzeptimport. Heuristische Perspektiven des Ansatzes „Alltägliche Lebensführung“. In: Mansel, Jürgen/ Griese, Hartmut/ Scherr, Albert (Hrsg.): Theoriedefizite der Jugendforschung. Standortbestimmung und Perspektiven. Weinheim/ München: Juventa, S. 102-118.

Lange, Andreas (2020): Doing Family durch Medien und Kommunikationstechnologien. Systematisierungen und Forschungsüberblick. In: Jurczyk, Karin (Hrsg.): Doing und Undoing Family. Konzeptionelle und empirische Entwicklungen. Weinheim und Basel: Beltz Juventa, S. 355-375.

Lareau, Annette (2011): Unequal Childhoods. Class, Race, and Family Life, with an Update a Decade Later. Berkeley, CA: University of California Press. 
Lincoln, Siân (2013): Media and Bedroom Culture. In: Lemish, Dafna (Hrsg.): The Routledge International Handbook of Children, Adolescents and Media. New York, NY: Routledge, S. 315- 321.

Livingstone, Sonia/ Lunt, Peter (2014): Mediatization: an Emerging Paradigm for Media and Communication Research. In: Lundby, Knut (Hrsg.): Mediatization of Communication: Handbooks of Communication Science, Vol. 21. Berlin: De Gruyter Mouton, S. 703-723.

Lobe, Bojana/ Livingstone, Sonia/ Ólafsson, Kjartan/ Simões, José Alberto (Hrsg.) (2008): Best practice research guide. How to research children and online technologies in comparative perspective. London: LSE. Online unter: http://eprints .lse.ac.uk/21658/. Darin: Paus-Hasebrink, Ingrid: FAQ 28: What shall I do if a child respondent seems to be at risk? (abgerufen am 24.06.2021).

Lundby, Knut (2014): Mediatization of Communication. In: Lundby, Knut (Hrsg.): Mediatization of Communication: Handbooks of Communication Science, Vol. 21. Berlin: De Gruyter Mouton, S. 3-35.

Lunt, Peter (2020): Beyond Bourdieu: The Interactionist Foundations of Media Practice Theory. In: International Journal of Communication, 14. Jg., S. 29462963.

Maaz, Kai/ Diedrich, Martina (2020): Schule unter Pandemiebedingungen: „Lockdown“ - „Hybridmodell“ - „Normalbetrieb“. In: Aus Politik und Zeitgeschichte, H. 51/2020. Bonn: bpb. Online unter: https:/www.bpb.de/apuz/schule-2020/ 322683/schule-unter-pandemiebedingungen (abgerufen am 25.06.2021).

Marx, Martina (2020): Nachrichten via Instagram, YouTube und Tiktok - Journalistische Angebote für junge Menschen. In: Gallner-Holzmann, Katharina/ Hug, Theo/ Pallaver, Günther (Hrsg.): Jugendliche Mediennutzung und die Zukunft des Qualitätsjournalismus. Innsbruck: Innsbruck University Press, S. 23-36.

Mascheroni, Giovanna/ Ponte, Cristina/ Jorge, Anna (Hrsg.) (2018): Digital Parenting. The Challenges for Families in the Digital Age. Göteborg: Nordicom.

Mayring, Philipp (2002): Einführung in die Qualitative Sozialforschung. Weinheim und Basel: Beltz (5. Auflage).

Mayseless, Ofra/ Keren, Einat (2014): Finding a Meaningful Life as a Developmental Task in Emerging Adulthood: The Domains of Love and Work Across Cultures. In: Emerging Adulthood, 2. Jg., H. 1, S. 63-73.

Mead, Georg H. (1988): Geist, Identität und Gesellschaft. Frankfurt am Main: Suhrkamp (7. Auflage).

Medienpädagogischer Forschungsverbund Südwest (MPFS) (2014): minKim-Studie 2014. Kleinkinder und Medien. Stuttgart: MPFS. Online unter: https://www.mp fs.de/studien/minikim-studie/2014/ (abgerufen am 24.06.2021):

Medienpädagogisches Forschungsverbund Südwest (MPFS) (2020): KIM-Studie 2020. Kindheit, Internet, Medien. Basisuntersuchung zum Medienumgang 6bis 13-Jähriger. Stuttgart: MPFS. Online unter: https://www.mpfs.de/studien/ki m-studie/2020/ (abgerufen am 24.06.2021). 


\section{Literaturverzeichnis}

Merten, Milena/ Dohle, Marco (2019): Wie beurteilen unterschiedliche Meinungslager die Medienberichterstattung zur „Flüchtlingskrise“? Ergebnisse einer Untersuchung zu Hostile-Media-Wahrnehmungen. In: Studies in Communication and Media, 8. Jg., H. 2, S. 272-285.

Mitschka, Konrad/ Unterberger, Klaus (2020): Zahlen im ORF im Zusammenhang mit Corona. In: Medienqualität in Zeiten von Corona. Public Value Texte 24. Wien: ORF, S. 40-41.

Moser, Evelyn (2020): Rückzug des Politischen? Beobachtungen zur politischen Soziologie der Corona-Pandemie. In: Aus Politik und Zeitgeschichte, H. 3537/2020. Bonn: bpb. Online unter: https:/www.bpb.de/apuz/314347/rueckzug-d es-politischen (abgerufen am 25.06.2021).

Murdock, Alex (2010): Stakeholders. In: Anheier, Helmut, K./ Toepler Stefan (Hrsg.): International Encyclopedia of Civil Society. New York, NY: Springer.

Murdock, Graham/ Golding, Peter (2004): Dismantling The Digital Divide: Rethinking the Dynamics of Participation and Exclusion. In: Calabrese, Andrew/ Sparks, Colin (Hrsg.): Towards a Political Economy of Culture: Capitalism and Culture in the Twenty First Century. Lanham, MD: Rowman and Littlefield, S. 244-260.

Neumann-Braun, Klaus (1992): Medienwelten - Medienkinder. In der Glitzerwelt der Erwachsenenunterhaltung. In: TelevIZIon, 5. Jg., H. 2, S. 4-7.

Niemand, Stephan (2020): Alltagsumbrüche und Medienhandeln. Eine qualitative Panelstudie zum Wandel der Mediennutzung in Übergangsphasen: Wiesbaden: Springer VS.

Ólafsson, Kjartan/ Livingstone, Sonia/ Haddon, Leslie (2013): How to research children and online technologies? Frequently asked questions and best practice. London: LSE. Online unter: http://eprints.lse.ac.uk/50437/ (abgerufen am 24.06.2021).

Ortner, Christina (2014): Wie junge Erwachsene die EU sehen und was die Medien dazu beitragen können. Zur Bedeutung medienvermittelter Erfahrungen für die Entwicklungen von Orientierungen gegenüber der EU. Lebensweltbezogene Medienforschung: Angebote - Rezeption - Sozialisation, Band 3. Baden-Baden: Nomos.

Ortner, Christina/ Kovacs, Carrie/ Jadin, Tanja (2020): Kinder und Medien in der Covid-19-Krise. Österreich im internationalen Vergleich. In: Medienimpulse, 58. Jg., H. 3, S. 1-40.

Papier, Hans-Jürgen (2020): Umgang mit der Corona-Pandemie: Verfassungsrechtliche Perspektiven. In: Aus Politik und Zeitgeschichte, H. 35-37/2020. Bonn: bpb. Online unter: https://www.bpb.de/apuz/314341/verfassungsrechtliche-pers pektiven (abgerufen am 25.06.2021).

Paus-Haase, Ingrid/ Hasebrink, Uwe/ Mattusch, Uwe/ Keuneke, Susanne/ Krotz, Friedrich (1999): Talkshows im Alltag von Jugendlichen. Der tägliche Balanceakt zwischen Orientierung, Amüsement und Ablehnung. Schriftenreihe Medienforschung der Landesanstalt für Rundfunk Nordrhein-Westfalen (LfR), Band 32. Opladen: Leske+Budrich. 
Paus-Hasebrink, Ingrid (2010): Lebens-Herausforderungen: Medienumgang und Lebensaufgaben. Was muss kommunikationswissenschaftliche Forschung leisten? In: Hartmann, Maren/ Hepp, Andreas (Hrsg.): Die Mediatisierung der Alltagswelt. Wiesbaden: VS Verlag, S. 195-210.

Paus-Hasebrink, Ingrid (2017a): Zur Entwicklung der praxeologischen Perspektive auf die Rolle von Medien in der Sozialisation. In: Paus-Hasebrink, Ingrid (Hrsg.): Langzeitstudie zur Rolle von Medien in der Sozialisation sozial benachteiligter Heranwachsender. Lebensphase Jugend. Lebensweltbezogene Medienforschung: Angebote - Rezeption - Sozialisation, Band 5. Baden-Baden: Nomos, S. 22-46.

Paus-Hasebrink, Ingrid (2017b): Die Langzeit-Panelstudie: Diskussion und Fazit. In: Paus-Hasebrink, Ingrid (Hrsg.): Langzeitstudie zur Rolle von Medien in der Sozialisation sozial benachteiligter Heranwachsender. Lebensphase Jugend. Lebensweltbezogene Medienforschung: Angebote - Rezeption - Sozialisation, Band 5. Baden-Baden: Nomos, S. 271-286.

Paus-Hasebrink, Ingrid (2018): Mediation Practices in Socially Disadvantaged Families. In: Mascheroni, Giovanna/ Ponte, Cristina/ Jorge, Ana (Hrsg.): Digital Parenting. The Challenges for Families in the Digital Age. Göteborg: Nordicom, S. 51-60.

Paus-Hasebrink, Ingrid (2019): The Role of Media within Young People's Socialization: A Theoretical Approach. In: Communications, 44. Jg., H. 4, S. 407-426.

Paus-Hasebrink, Ingrid (2020): Perspektiven sozial benachteiligter Familien auf Geflüchtete und die Rolle von Medien. Befunde einer Langzeitstudie in Österreich. In: kommunikation.medien. Jg. 2020, H. 12. Online unter: http://eplus.uni-salzb urg.at/ (abgerufen am 24.06.2021).

Paus-Hasebrink, Ingrid (2021): Medien und Sozialisationsforschung - ein praxeologischer Ansatz. Langzeitstudie zur Rolle von Medien bei sozial benachteiligten Heranwachsenden. In: Bettinger, Patrick/ Hugger, Kai-Uwe (Hrsg.): Praxistheorien in der Medienpädagogik. Wiesbaden: Springer VS, S. 147-161.

Paus-Hasebrink, Ingrid/ Bichler, Michelle (2008): Mediensozialisationsforschung. Theoretische Fundierung und Fallbeispiel sozial benachteiligter Kinder (unter Mitarbeit von Christine Wijnen). Innsbruck/Wien/ Bozen: StudienVerlag.

Paus-Hasebrink, Ingrid/ Hasebrink, Uwe (2014): Kommunikative Praxen im Wandel. Eine Verknüpfung von Sozialisations- und Repertoireperspektive. In: Medien Journal. Zeitschrift für Kommunikationskultur, 38. Jg., H. 1, S. 4-14.

Paus-Hasebrink, Ingrid/ Kulterer, Jasmin (2014): Praxeologische Mediensozialisationsforschung. Langzeitstudie zu sozial benachteiligten Heranwachsenden (unter Mitarbeit von Philip Sinner). Reihe: Lebensweltbezogene Medienforschung: Angebote - Rezeption - Sozialisation. Band 2. Baden-Baden: Nomos.

Paus-Hasebrink, Ingrid/ Kulterer, Jasmin/ Oberlinner, Andreas (2017): Familientypen als Sozialisationskontexte. In: Paus-Hasebrink, Ingrid (Hrsg.): Langzeitstudie zur Rolle von Medien in der Sozialisation sozial benachteiligter Heranwachsender. Lebensphase Jugend. Lebensweltbezogene Medienforschung: Angebote - Rezeption - Sozialisation, Band 5. Baden-Baden: Nomos, S. 129-240. 


\section{Literaturverzeichnis}

Paus-Hasebrink, Ingrid/ Kulterer, Jasmin/ Sinner, Philip (2019): Social Inequality, Childhood and the Media. A Longitudinal Study of the Mediatization of Socialisation. Transforming Communications - Studies in Cross-Media Research, Band 4. London: Palgrave Macmillan.

Paus-Hasebrink, Ingrid/ Lampert, Claudia/ Hammerer, Eva/ Pointecker, Marco (2004): Medien, Marken, Merchandising in der Lebenswelt von Kindern. In: Paus-Hasebrink, Ingrid/ Neumann-Braun, Klaus/ Hasebrink, Uwe/ Aufenanger, Stefan (Hrsg.): Medienkindheit - Markenkindheit. Untersuchung zur multimedialen Verwertung von Markenzeichen für Kinder. Schriftenreihe der LPR Hessen, Band 18. München: KoPaed, S. 133-179.

Paus-Hasebrink, Ingrid/ Oberlinner, Andreas (2017): Sozialisation in unterschiedlichen Sozialisationskontexten. In: Paus-Hasebrink, Ingrid (Hrsg.): Langzeitstudie zur Rolle von Medien in der Sozialisation sozial benachteiligter Heranwachsender. Lebensphase Jugend. Lebensweltbezogene Medienforschung: Angebote Rezeption - Sozialisation, Band 5. Baden-Baden: Nomos. S. 241-269.

Paus-Hasebrink, Ingrid/ Schmidt, Jan-Hinrik/ Hasebrink, Uwe (2011): Zur Erforschung der Rolle des Social Web im Alltag von Heranwachsenden. In: Schmidt/ Jan-Hinrik/ Paus-Hasebrink, Ingrid/ Hasebrink, Uwe (Hrsg.): Heranwachsen mit dem Social Web. Zur Rolle von Web 2.0-Angeboten im Alltag von Jugendlichen und jungen Erwachsenen. Schriftenreihe Medienforschung der LfM, Band 62. Berlin: Vistas, S. 13-40 (2. unveränderte Auflage).

Paus-Hasebrink, Ingrid/ Sinner, Philip/ Kulterer, Jasmin/ Oberlinner, Andreas (2017): Methodologische und methodische Herausforderungen: Zum Design der Langzeitstudie. In: Paus-Hasebrink, Ingrid (Hrsg.): Langzeitstudie zur Rolle von Medien in der Sozialisation sozial benachteiligter Heranwachsender. Lebensphase Jugend. Lebensweltbezogene Medienforschung: Angebote - Rezeption - Sozialisation Band 5. Baden-Baden: Nomos, S. 45-67.

Paus-Hasebrink, Ingrid/ Sinner, Philip/ Prochazka, Fabian/ Kulterer, Jasmin (2018): Auswertungsstrategien für qualitative Langzeitdaten: Das Beispiel einer Langzeitstudie zur Rolle von Medien in der Sozialisation Heranwachsender. In: Scheu, Andreas (Hrsg.): Auswertung qualitativer Daten. Strategien, Verfahren und Methoden der Interpretation nicht-standardisierter Daten in der Kommunikationswissenschaft. Wiesbaden: Springer VS, S. 209-225.

Rauschenbach, Thomas (2011): Aufwachsen unter neuen Vorzeichen. In: DJI Impulse 1/2011, H. 92/93, S. 4-7.

Reckwitz, Andreas (2017): Die Gesellschaft der Singularitäten. Zum Strukturwandel der Moderne. Frankfurt am Main: Suhrkamp.

Reichertz, Jo (1998): Kommunikations- und/ oder Medienwissenschaft? AVISO 22/1998, S. 8-9.

Reinders, Heinz (2003): Jugendtypen. Ansätze zu einer differenziellen Theorie der Adoleszenz. Opladen: Leske+Budrich.

Reinprecht, Christoph/ Latcheva, Rossalina (2016): Migration: Was wir nicht wissen. Perspektiven auf Forschungslücken In: Österreichische Zeitschrift für Soziologie, 41 Jg., H. 2, S. 1-13. 
Rohmann, Anette/ Stürmer, Stefan (Hrsg.) (2018): Die Flüchtlingsdebatte in Deutschland - Sozialpsychologische Perspektiven. Unter Mitarbeit von Helen Landmann. Reihe Beiträge zur Angewandten Psychologie, Band 2. Berlin: Peter Lang.

Röser, Jutta/ Müller, Kathrin Friederike/ Niemand, Stephan/ Roth, Ulrike (2019): Das mediatisierte Zuhause im Wandel. Eine qualitative Panelstudie zur Verhäuslichung des Internets. Wiesbaden: Springer VS.

Schatzki, Theodore, R. (2002): The Site of the Social. A Philosophical Account of the Constitution of Social Life and Change. University Park, PA: The Pennsylvania State University Press.

Schedlich, Claudia (2020): Psychosoziale Herausforderungen in der COVID-19Pandemie. In: Bering, Robert/ Eichenberg, Christiane (Hrsg.): Die Psyche in Zeiten der Corona-Krise. Herausforderungen und Lösungsansätze für Psychotherapeuten und soziale Helfer. Stuttgart: Klett-Cotta, I.1.

Schneewind, Klaus, A. (1999): Familienpsychologie. Stuttgart: Kohlhammer (2. Auflage).

Schnetzer, Simon/ Herczeg, Heinz/ Hurrelmann, Klaus/ Leibovici-Mühlberger, Martina (2020): Jugend und Corona in Österreich: Junge Österreicher im Lockdown und Wege aus der Corona-Krise. In: Die Studie "Junge Österreicher 2021“. Kempten: DATAJOCKEY.

Schnetzer, Simon/ Hurrelmann, Klaus (2020): Jugend und Corona: Wie rücksichtsvoll verhalten sich die jungen Generationen? In: Die Studie "Junge Deutsche 2021“. Kempten: DATAJOCKEY.

Schubarth, Wilfried (2020): „Wir wollen wieder in die Schule“. Schule als sozialen Ort (wieder)entdecken. In: Aus Politik und Zeitgeschichte, H. 51/2020. Bonn: bpb. Online unter: https:/www.bpb.de/apuz/schule-2020/322688/schule-als-sozi alen-ort-wiederentdecken (abgerufen am 25.06.2021).

Schulz, Marlen/ Ruddat, Michael (2012): „Let's Talk about Sex!“ Über die Eignung von Telefoninterviews in der qualitativen Sozialforschung. In: Forum Qualitative Sozialforschung. 13. Jg., H. 3. Online unter: http://nbn-resolving.de/urn:nbn: de:0114-fqs120329 (abgerufen am 24.06.2021).

Schütze, Yvonne (1977): Innerfamiliale Kommunikation und kindliche Psyche. Eine exemplarische Analyse der Kommunikations- und Rollenstrukturen zweier Familien. Berlin: Schriften des Max-Planck-Instituts für Bildungsforschung.

Schweizer, Herbert (2007): Soziologie der Kindheit. Verletzlicher Eigen-Sinn. Wiesbaden: VS Verlag.

Sindbad (2020): Jahresbericht 2020 und Ausblick 2021. Wien: Sindbad. Online unter: https://www.sindbad.co.at/ (abgerufen am 24.06.2021).

Sindelar, Brigitte (2020): Kinder- und Jugendlichenpsychotherapie Online in Zeiten der Corona-Krise. In: Bering, Robert/ Eichenberg, Christiane (Hrsg.): Die Psyche in Zeiten der Corona-Krise. Herausforderungen und Lösungsansätze für Psychotherapeuten und soziale Helfer. Stuttgart: Klett-Cotta, II.2. 


\section{Literaturverzeichnis}

Stadtherr, Lisa/ Schmidinger, Paul (2020): ORF-Landesstudios essenziell in der Krise. In: Medienqualität in Zeiten von Corona. Public Value Texte 24. Wien: ORF, S. 42-44.

Steiber, Nadia/ Liedl, Bernd/ Molitor, Philipp (2020): Infektionsrisiko am Arbeitsplatz: Das Risiko für eine Corona-Infektion ist ungleich verteilt. Wien: A\&W Blog. Online unter: https://irihs.ihs.ac.at/id/eprint/5633/1/steiber-et-al-2020-infek tionsrisiko-am-arbeitsplatz.pdf (abgerufen am 25.06.2021).

Süss, Daniel (2004): Mediensozialisation von Heranwachsenden. Dimensionen, Konstanten, Wandel, Wiesbaden: VS Verlag für Sozialwissenschaften.

Tasheva, Gallina (2020): Von der Intersubjektivität zum Mitsein. Eine Genealogie der Sozialisation. In: Grundmann, Matthias/ Höppner, Grit (Hrsg.): Dazwischen - Sozialisationstheorien reloaded. Weinheim und Basel: Juventa, S. 146-167.

Teach for Austria (2020): Homeschooling benachteiligter Kinder. Wien: TFA. Online unter: https:/www.teachforaustria.at/story/homeschooling-benachteiligter -kinder/ (abgerufen am 25.06.2021).

Tengler, Karin/ Schrammel, Natalie/ Brandhofer, Gerhard (2020): Lernen trotz Corona. Chancen und Herausforderungen des distance learnings an österreichischen Schulen. In: Medienimpulse, 58. Jg, H. 2, S. 1-37.

Ther, Philipp (2020): Wettbewerb der Systeme. Die Corona-Pandemie als Herausforderung für Demokratie und europäische Integration. In: Aus Politik und Zeitgeschichte, H. 35-37/2020. Bonn: bpb. Online unter: https://www.bpb.de/a puz/314353/die-corona-pandemie-als-herausforderung-fuer-demokratie-und-euro paeische-integration (abgerufen am 25.06.2021).

Thomae, Hans (1956): Psychologische Probleme des Erwachsenenalters. In: Haseloff, Otto Walter/ Stachowiak, Herbert (Hrsg.): Moderne Entwicklungspsychologie. Berlin: Lüttke Verlag, S. 104-113.

Thomae, Hans (1988): Das Individuum und seine Welt. Eine Persönlichkeitstheorie. Göttingen: Hogrefe.

Trültzsch-Wijnen, Christine/ Sturm, Wolfgang (2021): Lehren während des COVID-19 Lockdowns: die Perspektive österreichischer Lehrerinnen auf den Fernunterricht: österreichische KiDiCoTi-Teilstudie. Salzburg: Universität Salzburg. Online unter: https://eplus.uni-salzburg.at/obvusboa/content/titleinfo/5743308 (abgerufen am 25.06.2021).

Trültzsch-Wijnen, Sascha (2018): Identität, Orientierung und Lebenswelt: Ein mehrdimensionaler Blick auf situatives Aushandeln von Privatheit vor dem Hintergrund von Kommunikation und Selbstpräsentation im Social Web. Lebensweltbezogene Medienforschung: Angebote - Rezeption - Sozialisation, Band 7. Baden-Baden: Nomos.

Trültzsch-Wijnen, Sascha/ Trültzsch-Wijnen, Christine (2021): Kids Digital Lives in Covid-19 Times. Salzburg: Universität Salzburg. Online unter: https://kowi.uni-s alzburg.at/covid19familien/ (abgerufen am 25.06.2021). 
Trültzsch, Sascha/ Wijnen, Christine W./ Dürager, Andrea (2013): Methodenvielfalt in der Forschung zu Audiovisueller und Online-Kommunikation. In: PausHasebrink, Ingrid/ Trültzsch, Sascha/ Pluschkowitz, Alois/ Wijnen, Christine (Hrsg.): Integrative AV- und Online-Kommunikationsforschung. Lebensweltbezogene Medienforschung: Angebote - Rezeption - Sozialisation, Band 1. BadenBaden: Nomos, S. 100-147.

Veith, Hermann (2015): Zur Geschichte sozialisationstheoretischer Fragesellungen. In: Hurrelmann, Klaus/ Bauer, Ullrich/ Grundmann, Matthias/ Walper, Sabine (Hrsg.): Handbuch Sozialisationsforschung. Weinheim und Basel: Beltz, S. 1649 (8. Auflage).

Vlasak, Thomas/ Barth, Alfred (2020): Arbeitslosigkeit als Risikofaktor für die psychische Gesundheit während der Corona-Krise - ein Überblick. In: Bering, Robert/ Eichenberg, Christiane (Hrsg.): Die Psyche in Zeiten der Corona-Krise. Herausforderungen und Lösungsansätze für Psychotherapeuten und soziale Helfer. Stuttgart: Klett-Cotta, III.3.

Vollbrecht, Ralf/ Wegener, Claudia (Hrsg.) (2010): Handbuch Mediensozialisation. Wiesbaden: VS Verlag.

Vom Order, Heike (2020): Youth, Well-being and the Media in Times of Covid-19. What we Know so far from International Research. In: TelevIZIon, 2020, 33, 17-20. Online unter: https:/www.br-online.de/jugend/izi/english/publication/te levizion/33_2020_E/vom_Orde-Youth_well-being_and_the_media_in_times_of _COVID-19.pdf (abgerufen am 25.6.2021).

Walper, Sabine/ Müller, Dagmar (2021): Familien krisenfest machen. In: DJI Impulse 1/2021, H. 125, S. 46-47.

Weiß, Ralph (1997): Auf der Suche nach kommunikativen Milieus. Plädoyer für eine sozialstrukturell interessierte Fragehaltung der Nutzungsforschung. In: Scherer, Helmut/ Brosius, Hans-Bernd (Hrsg.): Zielgruppen, Publikumssegmente, Nutzergruppen. Beiträge aus der Rezeptionsforschung. München: Reinhard Fischer, S. 239-261.

Weiß, Ralph (2000): „Praktischer Sinn“, soziale Identität und Fern-Sehen. Ein Konzept für die Analyse der Einbettung kulturellen Handelns in die Alltagswelt. In: M\&K. Medien und Kommunikationswissenschaft, 48. Jg., H. 1, S. 42-62.

Weiß, Ralph (2001): Fern-Sehen im Alltag. Zur Sozialpsychologie der Medienrezeption. Wiesbaden: Springer VS.

Weiß, Ralph (2013): Theoretische Grundlagen des praxeologischen Kontextualismus bei Ingrid Paus-Hasebrink. In: Wijnen, Christine/ Trültzsch, Sascha/ Ortner, Christina (Hrsg.): Medienwelten im Wandel. Kommunikationswissenschaftliche Positionen, Perspektiven und Konsequenzen. Wiesbaden: VS Verlag, S. 25-38.

Willems, Jürgen/ Knassmüller, Monika/ Ebinger, Falk/ Dinhof, Katharina/ Schmid, Moritz (2020): COVID-19 Pandemie in Österreich - Zufriedenheit der Bürgerinnen mit Maßnahmen und Kommunikation. Wien: WU. Online unter: https:/e pub.wu.ac.at/7587/1/COVID-19\%20Pandemie\%20in\%20\%C3\%96sterreich.pdf (abgerufen am 25.06.2021). 
Wrase, Michael/ Allmendinger, Jutta (2020): Das Recht auf Bildung verwirklichen. Herausforderungen für Bildung und Bildungspolitik in Deutschland. In: Aus Politik und Zeitgeschichte, H. 51/2020. Bonn: bpb. Online unter: https:/www.b pb.de/apuz/schule-2020/322695/das-recht-auf-bildung-verwirklichen (abgerufen am 25.06.2021). 